\title{
Conceptual Design Report
}

\section{NUCLEAR MATERIALS STORAGE FACILITY RENOVATION}

Part V - Structural / Seismic Investigation Section B Renovation Calculations/ Supporting Data

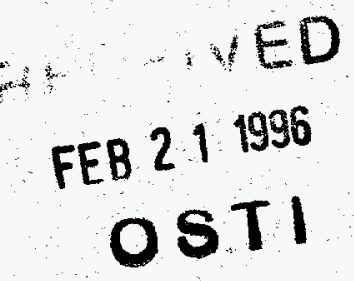

Los Alamos

National Laboratory

July 14, 1995 


\section{DISCLAMMER}

Portions of this document may be illegible in electronic image products. Images are produced from the best available original document. 
FOR

LINE ITEM PROJECT

NUCLEAR MATERIALS STORAGE FACILITY RENOVATION

AT THE

LOS ALAMOS NATIONAL LABORATORY

Project identification Number 11818

Technical Area 55

Building PF-41

\author{
Prepared by \\ ICF KAISER ENGINEERS, INC. \\ 1900 Diamond Drive \\ Los Alamos, NM 87544 \\ and
}

University of California

Los Alamos National Laboratory
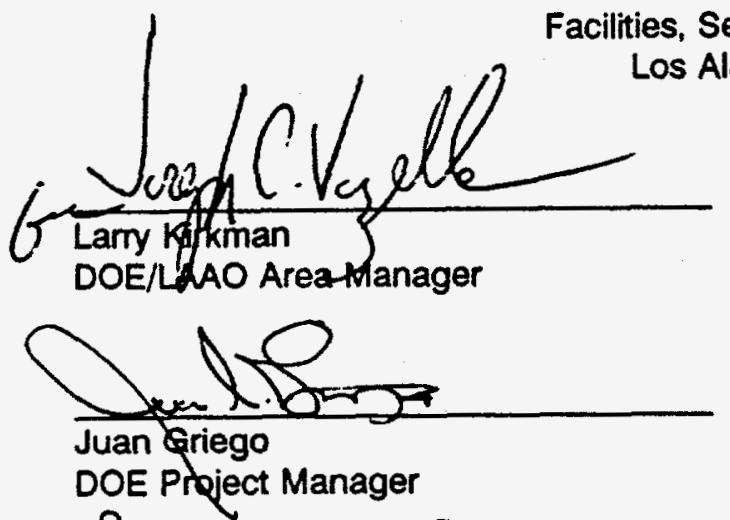

T. J. Trapp

Nuclear Materials Program Manager

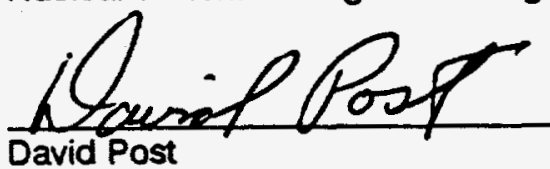

NMT-8 Group Leader/TA-55 Facility Manager

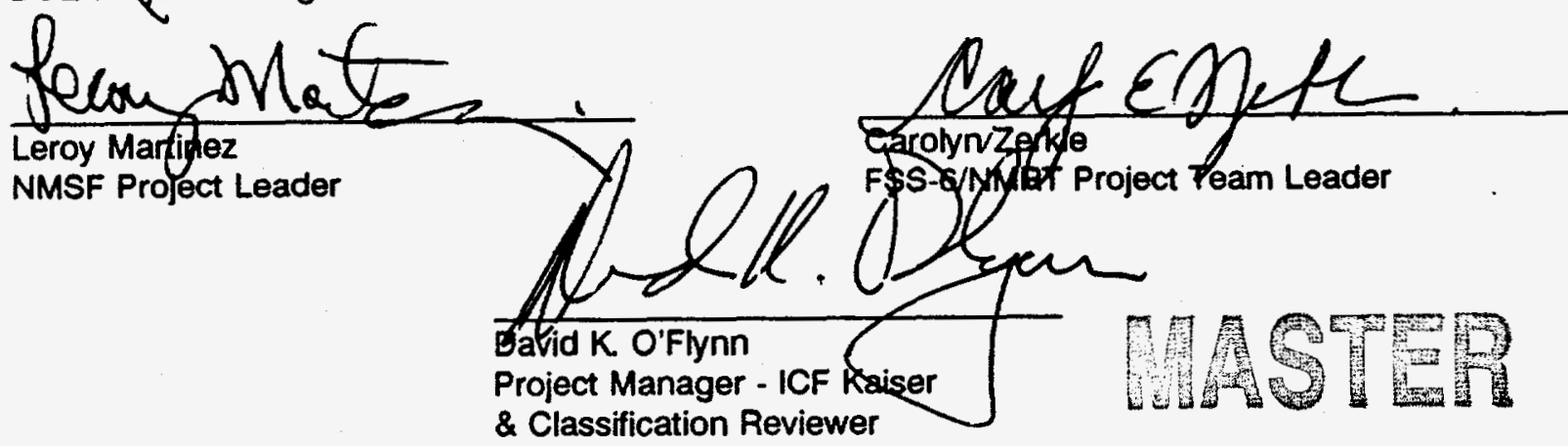

\title{
DISCLAIMER
}

This report was prepared as an account of work sponsored by an agency of the United States Government. Neither the United States Government nor any agency thereof, nor any of their employees, makes any warranty, express or implied, or assumes any legal liability or responsibility for the accuracy, completeness, or usefulness of any information, apparatus, product, or process disclosed, or represents that its use would not infringe privately owned rights. Reference herein to any specific commercial product, process, or service by trade name, trademark, manufacturer, or otherwise does not necessarily constitute or imply its endorsement, recommendation, or favoring by the United States Government or any agency thereof. The views and opinions of authors expressed herein do not necessarily state or reflect those of the United States Government or any agency thereof. 
NMSF Conceptual Design Report

July 14, 1995

Page i

TABLE OF CONTENTS

\section{PART I - DESIGN CONCEPT}

A. GENERAL DESCRIPTION OF PROJECT

B. JUSTIFICATION

C. RELATIONSHIP TO OTHER PROJECTS

D. ALTERNATIVES

E. DESIGN CONCEPT

\section{SECTION}

A

B

C

D

E

1. Project Design Description

2. Energy Conservation Analysis

3. Utility Assessment

4. Environmental Considerations

5. Facility and Equipment Maintenance and Operation Considerations

6. Safety Considerations

7. Security Considerations

8. Site Development Plan Coordination

9. Outline Specifications and Standards

10. Space Program

F. QUALITY ASSURANCE

$\mathbf{F}$

G. SCHEDULES

G

H. KEY MILESTONES

H

I. COST ESTIMATES AND CONTINGENCY

J. ACRONYMS AND ABBREVIATIONS J

\section{PART II - PROJECT MANAGEMENT}
A. PROJECT MANAGEMENT ORGANIZATION
A
B. PROJECT MANAGEMENT ELEMENTS
B 
PART II - PROJECT MANAGEMENT (Cont'd)

C. PROJECT CONTROLS AND REPORTING SYSTEM

D. SPECIAL PLANS

PART III - SUPPLEMENTAL INFORMATION

A. RENEWABLE ENERGY SYSTEMS ANALYSIS

A

B. CALCULATIONS AND SUPPLEMENTAL DATA

B

1. Civil/Miscellaneous Structural

2. Architectural

3. Mechanical

4. Electrical

5. Special Facilities and Storage Array Equipment

6. Thermal Analysis

7. Criticality

8. Radiological / Shielding

9. Material Handling Data

10. Process Flow

C. PRELIMINARY FIRE HAZARDS ANALYSIS

D. EXISTING CONDITION SURVEY

E. DRAFT Q/A PLAN

PART IV - CONCEPTUAL DRAWINGS

PART V - STRUCTURAL / SEISMIC INVESTIGATION

A. EXISTING CONDITION

A

B. RENOVATION

\section{SECTION}

C

D

A

B

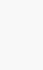


BASIS AND ASSUMPTIONS

TRACKING EXAMPLE

CONVENTIONAL ACQUISITION STRATEGY
A. TEC AND TPC SUMMARIES
B. CONTINGENCY ANALYSIS
B
C. ESCALATION ANALYSIS
C
D. LABORATORY PROCUREMENT BURDEN
D
E. OTHER PROJECT COSTS
F. FIELD OVERHEAD AND EXPENSES
$\mathbf{F}$
G. ED\&1
G
H. A\&E COST OF SERVICES
$H$
I. DETAILED CONSTRUCTION ANALYSIS

A

DESIGN/BUILD ACQUISITION STRATEGY
A. TEC AND TPC SUMMARIES
A
B. CONTINGENCY ANALYSIS
B
c. ESCALATION ANALYSIS
C
D. LABORATORY PROCUREMENT BURDEN
D
E. OTHER PROJECT COSTS
F. FIELD OVERHEAD AND EXPENSES
$\mathbf{F}$
G. ED\&!
G 
NMSF Conceptual Design Report

July 14, 1995

Page iv

H. A\&E COST OF SERVICES

H

I. DETAILED CONSTRUCTION ANALYSIS

I

SUPPORTING INFORMATION 


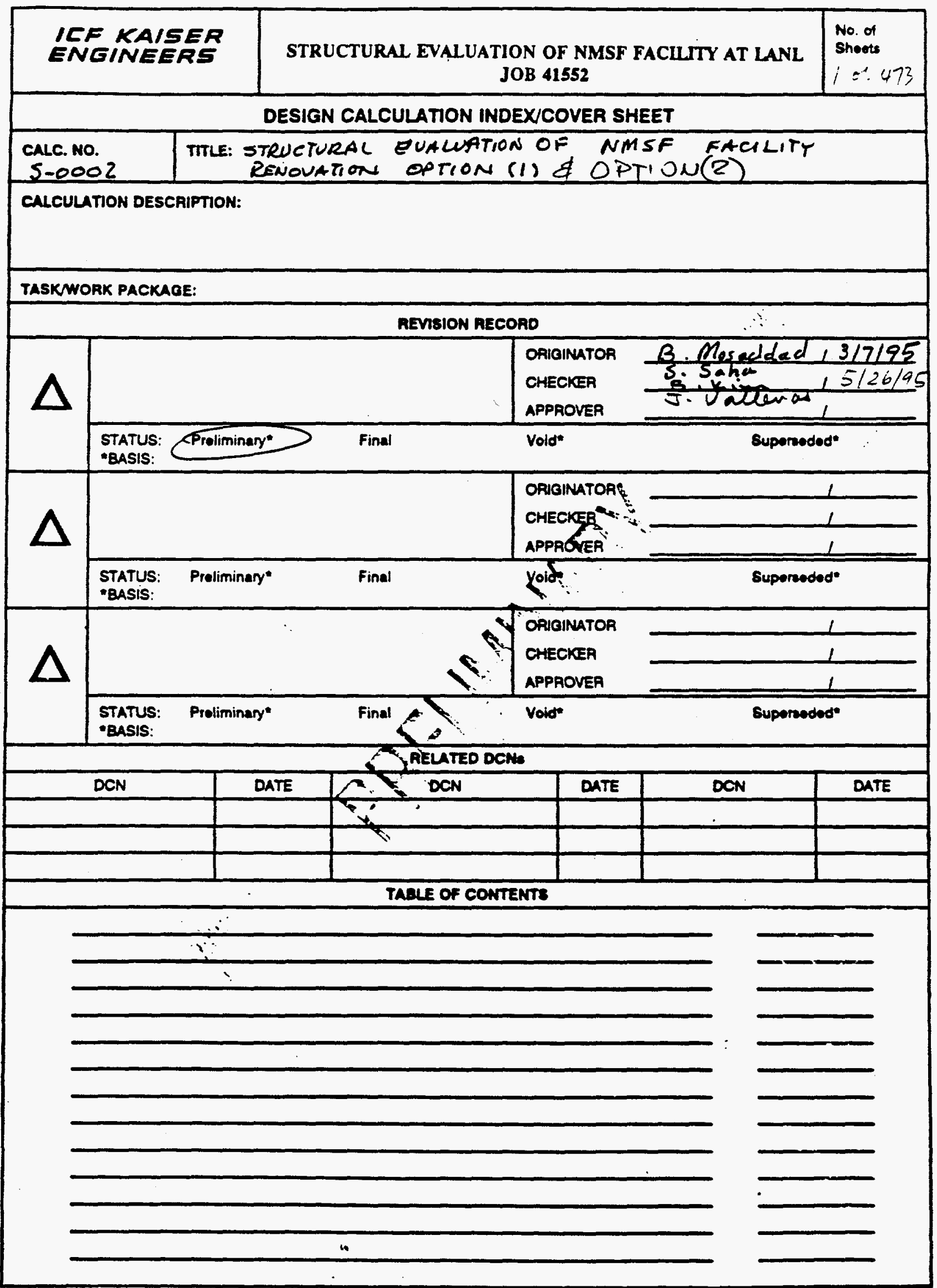

Form 3.3-1 


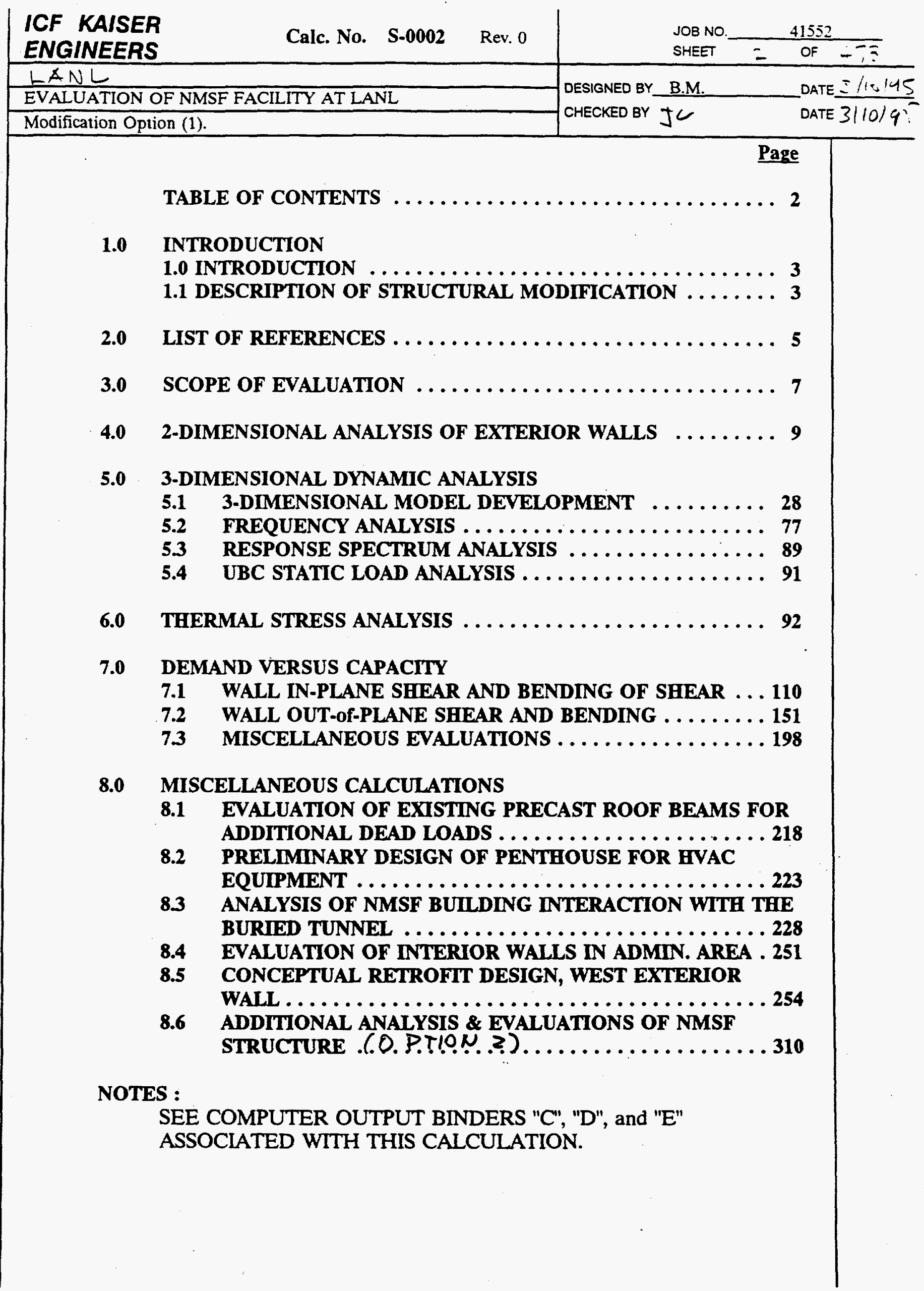




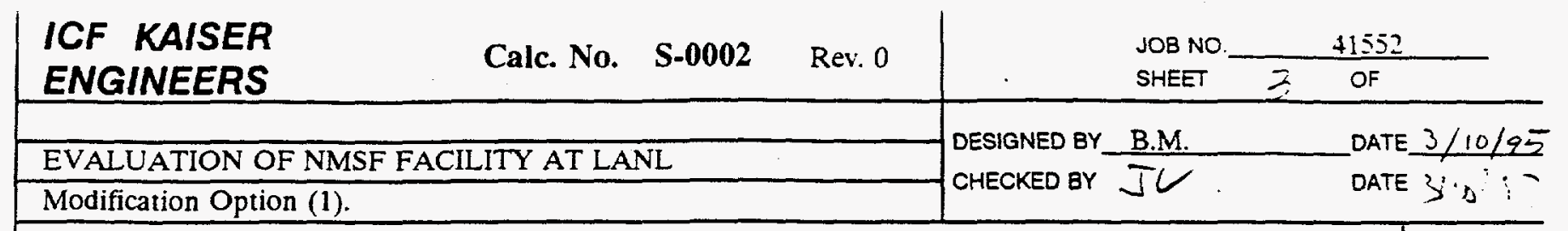

\subsection{INTRODUCTION}

\subsection{INTRODUCTION}

The NMSF building consists of a two story storage area and a single story section including mechanical and administration area. The 1st floor of the storage area continues on grade to form the administration building, See figure 1. The basement of the storage building is buried in the soil. The 1st floor walls of the storage area is also completely embedded on east side, north side and south side of the building.

This calculation documents the details of the analysis procedure and structural evaluation of the renovated (With modification Option 1) NMSF building in accordance with the LANL, DOE and associated industry standard design codes $(1,2,3,4)$. For a complete presentation of the evaluation criteria and presentation of the summary of evaluation results refer to the report titled:

"Structural Evaluation of the NMSF Building at LANL, Volume I Report"

\subsection{DESCRIPTION OF STRUCTURAL MODIFICATIONS}

The proposed modifications to the As-Built structure of the NMSF Facility Includes but is not limited to the following major items:

1) The 1st floor slab between lines 1 and 3 is removed.

2) A New deck, 30 inches thick charge deck in added at about 3 feet bellow the existing 1st floor level between lines 1 and 2 .

3) North/South wall on line 1.5 in the basement is removed.

4) The existing Mezzanine slab between lines A and B is removed.

5) A new Mezzanine level at 10 feet above the basement slab is added between lines 2 and 3 .

6) New 1.5 feet thick concrete Shielding walls are added below charge deck at lines 1 and 2 . These walls are added to existing 1.5 feet thick and 1 foot thick walls at these locations, respectively. 


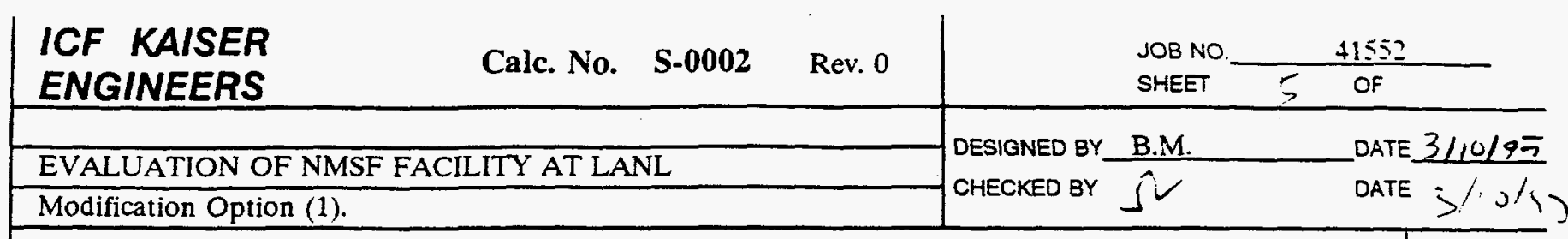

\subsection{LIST OF REFERENCES}

(1) Los Alamos Facilities Engineering Standard- Structural

(2) "Natural Phenomena Hazards Design and Evaluation Criteria for Department of Energy Facilities", DOE Standard DOE-STD-1020-94, April 1994.

(3) "Minimum Design Loads for Buildings and Other Structures", ASCE Standard ASCE-7.

(4) Uniform Building Code (UBC), 1991 edition.

(5). Dames \& Moor Geotechnical report, 1972

(6) EPRI Report NP-6041-SL Rev 1.

(7) Soil Dynamics, By Shamsher Parkash

(8) "Seismic Analysis of Safety-Related Nuclear Structures and Commentary on Standard for Seismic Analysis of Safety Related Nuclear Structures", ASCE Standard ASCE-4-86, September 1986.

(9) Nuclear Regulatory Guide NUREG-098

(10) "A Series of Computer Programs for Static and Dynamic Finite Element Analysis of Structures", SAP90 User's Manual, Computers and Structures, Inc., Berkeley, California.

(11) Soil-Structure-Interaction in Time Domain, by Wolf.

(12) Burns and Roe Drawings

DOE DRG. NO.: LA-ZJ-B.A.1 Through LA-ZJ-B.A.25

and

DOE DRG NO.: LA-ZJ-B.S.1 Through LA-ZJ-B.S.11

(13) ICF KAISER Preliminary (0\%) Architectural drawings of NMSF

Facility, Sheets A1 to A5, Received by Oakland/KAISER Office on Jan 13, 1995.

(14) "Advanced Value Engineering Study for NMSF at LANL", Volume II (PART G): Supporting Data," Prepared by ICF Kaiser Engineering and University of California Los Alamos National Laboratory, October 6, 1994. 


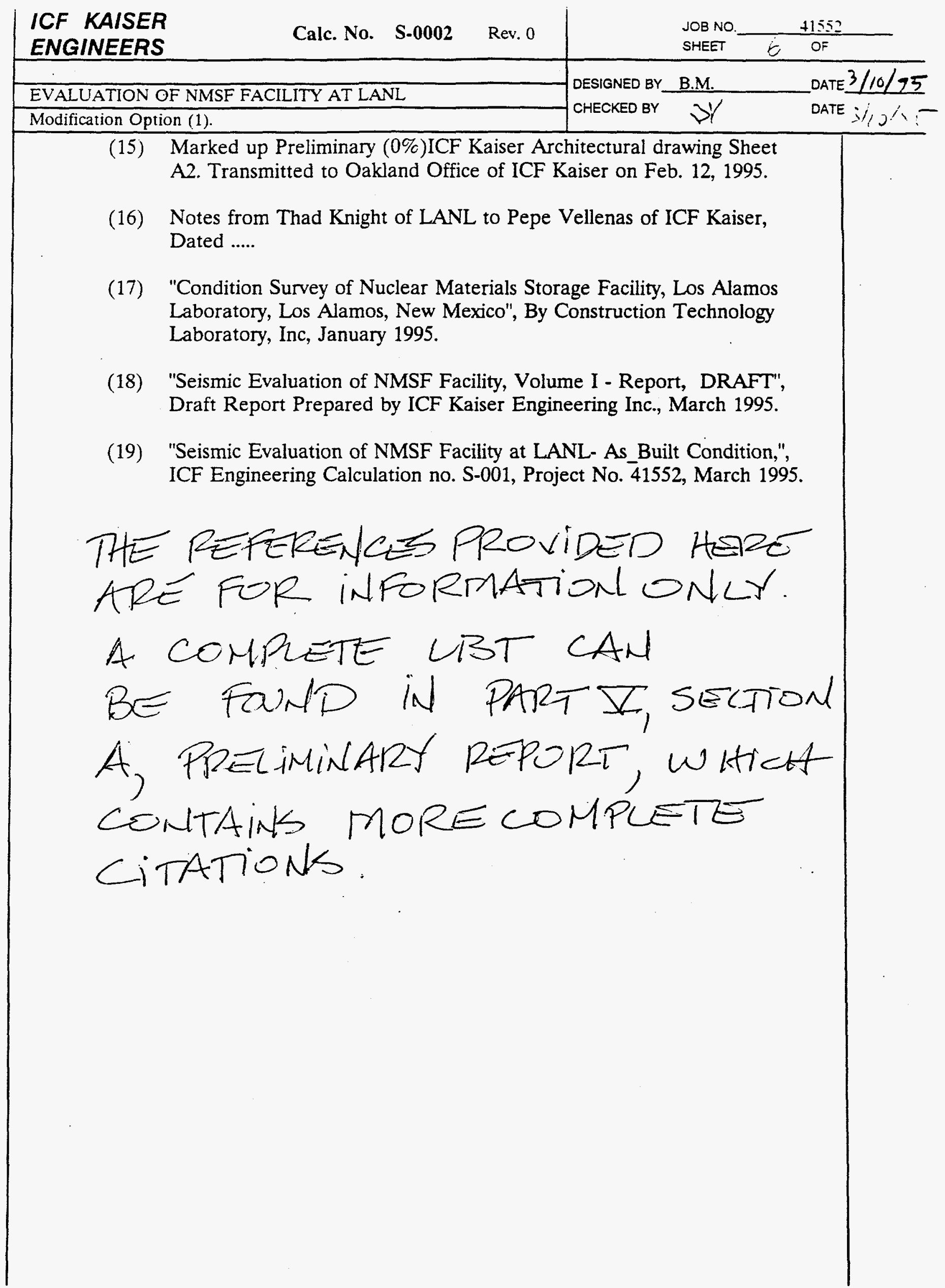




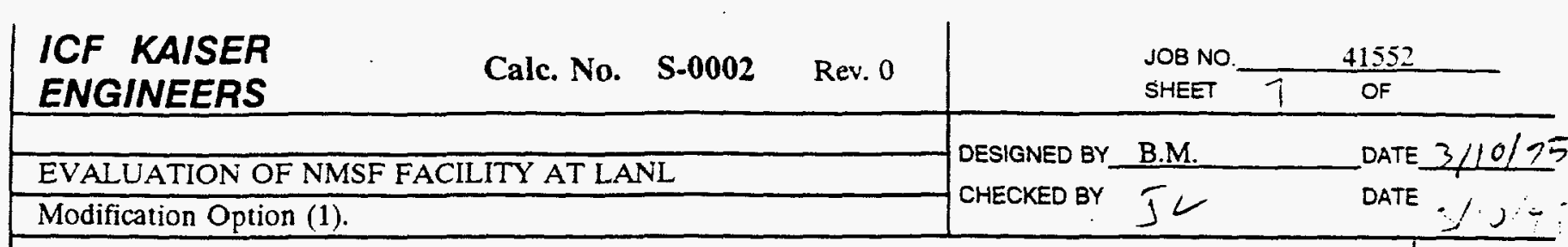

3.0 SCOPE OF EVALUATION

A preliminary evaluation of the exterior walls are first performed using a simple two-dimensional analysis of typical sections of the perimeter walls, subjected to lateral soil pressures due to earthquakes. In this evaluation the out-of-plane bending and shear demand on the walls are estimated and compared with corresponding capacities. The results from this evaluation is presented in section 4.0 of this calculation.

A three dimensional dynamic analysis of the Modified structure of the NMSF building is performed using Computer program SAP90 (10). The finite element model used in evaluation of as-built NMSF building was modified to incorporate the proposed structural changes described in section 1.1 of this calculation. The detail of model modifications and associated calculations are presented in section 5.1 of this calculations.

The finite element model includes the soil-structure-interaction effects using a lumped mass approach per guidelines of ASCE 4-86(8). The static and dynamic lateral soil pressure on the embedded perimeter walls are included as equivalent static loads in the 3-dimensional model. The accidental torsional effects are accounted for by imposing a deliberate additional 5\% eccentricity of floor masses. A frequency analysis of the model was performed to compute natural frequency and mode shapes of the modified NMSF Building. The results of frequency analysis is presented in section 5.2.

Response spectrum analysis is performed using 5\% damped NUREG-98 (9) acceleration response spectrum applied in three orthogonal directions simultaneously. The magnitude of the vertical components of the earthquake is assumed to be $2 / 3$ of the horizontal component. The peak ground acceleration of $0.3 \mathrm{~g}$ is used in these analysis. The structural modal damping used in response spectrum analysis is assumed to be equal to $7 \%$ of the critical damping. In addition to dynamic loads the NMSF building is also evaluated for UBC code specified static load combinations including dead,live and soil pressure loads. The In-Plane shears and out-of-plane moments for the critical wall segments are summarized and compared to their capacities to obtain Demand/Capacity (D?C) ratios in sections 7.0 of this calculations.

Thermal stress analysis of the concrete walls surrounding the proposed nuclear material storage area in the basement of NMSF facility was also performed as part of the current evaluation. In this evaluation thermal gradients of 20 and 40 degrees of are assumed for the west and east walls and the charge deck per recommendations from LANL Staff (16). A future heat transfer analysis is needed to determine a more accurate estimate of the thermal gradient through the concrete walls during operation and accidental failure of the proposed passive cooling system of the storage area. The results from this analysis is presented in section 6.0 of this calculation. 


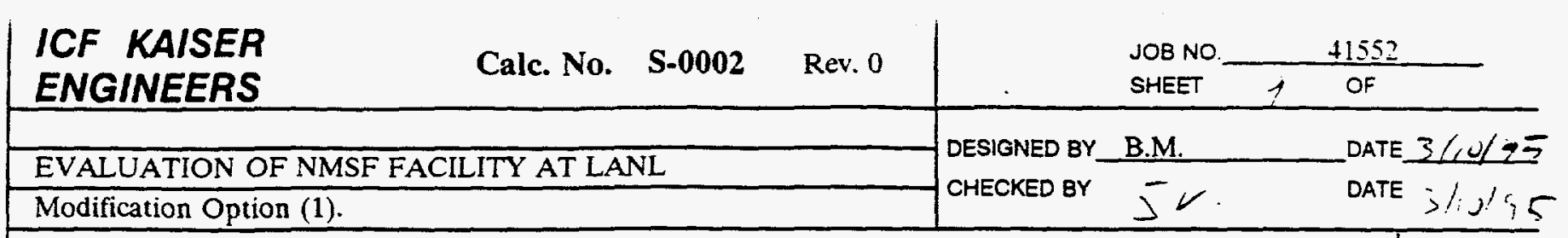

\subsection{2-DIMENSIONAL ANALYSIS OF EXTERIOR WALLS}

The purpose of this analysis was to perform a preliminary evaluation of the exterior walls of the NMSF building that are buried in the ground and/or are backfilled with soil on for sides of the western portion of the building.

In this analysis a 1 foot slice of the typical walls are modeled using beam elements of Computer program SAP90. The loadings consist of static and dynamic soil pressures. The dynamic soil pressure is estimated using Mononobe-Okabe Formulas from reference provided by reference $(6,7,8)$ using a PGA of equal to $0.3 \mathrm{~g}$.

A summary of computed demands and capacities of different walls are tabulated on next page. The results indicate that the all exterior walls have adequate strength to withstand the lateral soil pressure caused by the postulated DBE earthquake except the wall at the west side of the building which shows significant overstress between the charge deck and roof elevations.

The 2-dimensional analysis is conservative because it ignores the 3-dimensional response of the wall to lateral loads and also can not account for the support of newly added buttress along the height of the west wall. A more realistic D/C ratios results from three dimensional analysis of the NMSF building, described next. 


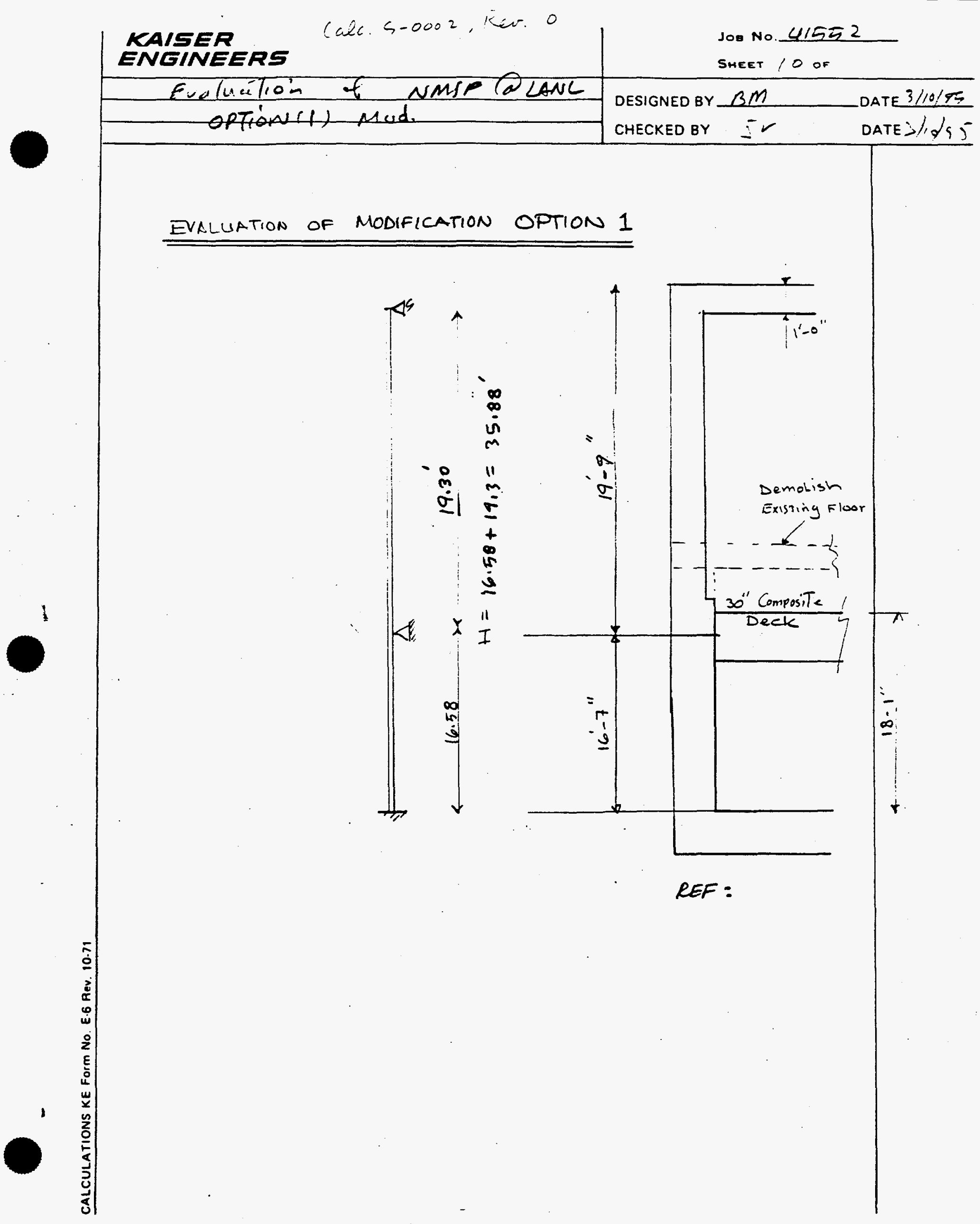




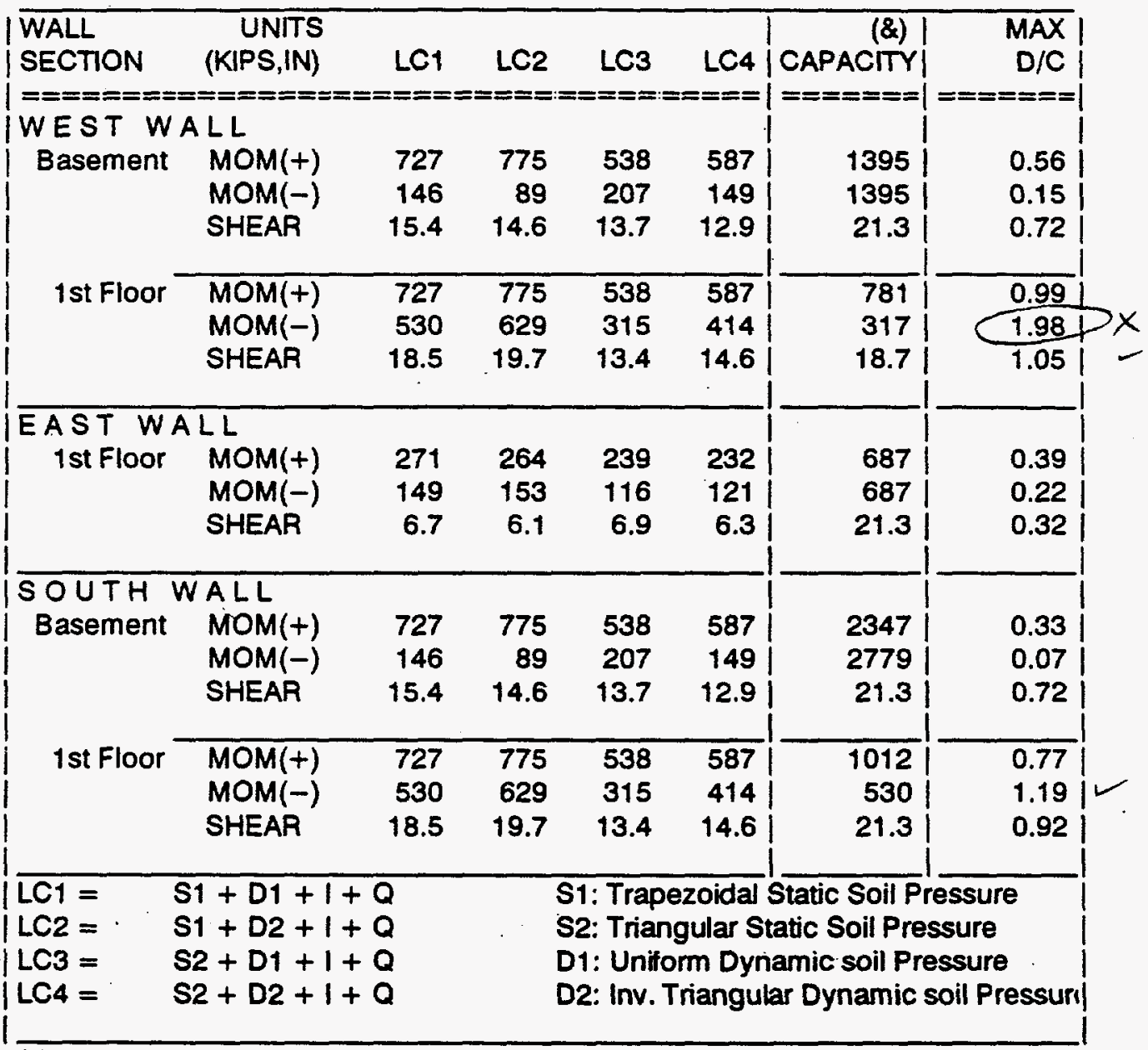

(*) - Reference SAP90 Runs:

WEST4, EAST2

(\&) - As Built Wall Capacities 


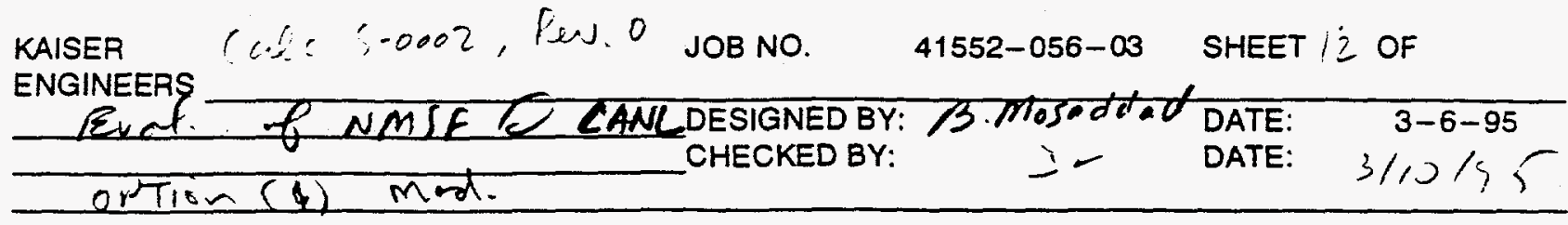

\begin{tabular}{|c|c|c|c|c|c|c|}
\hline$\overline{\text { WALL }}$ & & & UNITS: & S,II & & $\left({ }^{*}\right) 1$ \\
\hline SECTION & & Rebar & As & & a & CAPACITY \\
\hline l = = = = = = = = & $=====$ & $=====$ & $====$ & 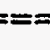 & $====$ & $======-1$ \\
\hline & & & & & & \\
\hline Basement & $\mathrm{Mu}(t)$ & \#10@9 & 1.693 & 33 & 2.49 & 2904 \\
\hline & $\operatorname{Mu}(-)$ & \#10@9 & 1.693 & 33 & 2.49 & 2904 \\
\hline & & & & 33 & & $42.6 \mid$ \\
\hline 1st Floor & $M u(+)$ & \#8@9 & 1.053 & 15 & 1.55 & 809 \\
\hline & $\mathrm{Mu}(-)$ & \#5@9 & 0.413 & 15 & 0.61 & 328 \\
\hline & Vc & & & 15 & & $19.4 \mid$ \\
\hline$\overline{E A S T}$ WA & & & & & & \\
\hline 1st Floor & $\mathrm{Mu}(+)$ & \#7@9 & 0.8 & 33 & 1.18 & 1400 \\
\hline & $M u(-)$ & \#7@9 & 0.8 & 33 & 1.18 & 1400 \\
\hline & Vc & & & 33 & & 42.6 \\
\hline SOUTH V & $L L$ & & & & & \\
\hline Basement & $\operatorname{Mu}(+)$ & \#10@5 & 3.048 & 15 & 4.48 & 2100 \\
\hline & $\mathrm{Mu}(-)$ & \#11@5 & 3.744 & 15 & 5.51 & 2476 \\
\hline & Ve & & & 15 & & 19.4 \\
\hline 1st Floor & $\mathrm{Mu}(+)$ & \#9@10 & 1.2 & 15 & 1.76 & 915 \\
\hline & $\mathrm{Mu}(-)$ & \#5@10 & 0.612 & 15 & 0.90 & 481 \\
\hline & Vc & & & 15 & & 19.4 \\
\hline$f^{\prime} c=$ & 4 & ksi & $f y=$ & & & \\
\hline $\begin{array}{l}M u=0.95 \\
V_{c}=0.85\end{array}$ & $\begin{array}{l}s^{*} \text { is * } \\
\text { * Sqrt( } f^{\prime}\end{array}$ & $\begin{array}{l}d-a / 2) \\
c * b w\end{array}$ & & As & $s /(.85$ & $\left.* f c^{\star} b\right)$ \\
\hline
\end{tabular}




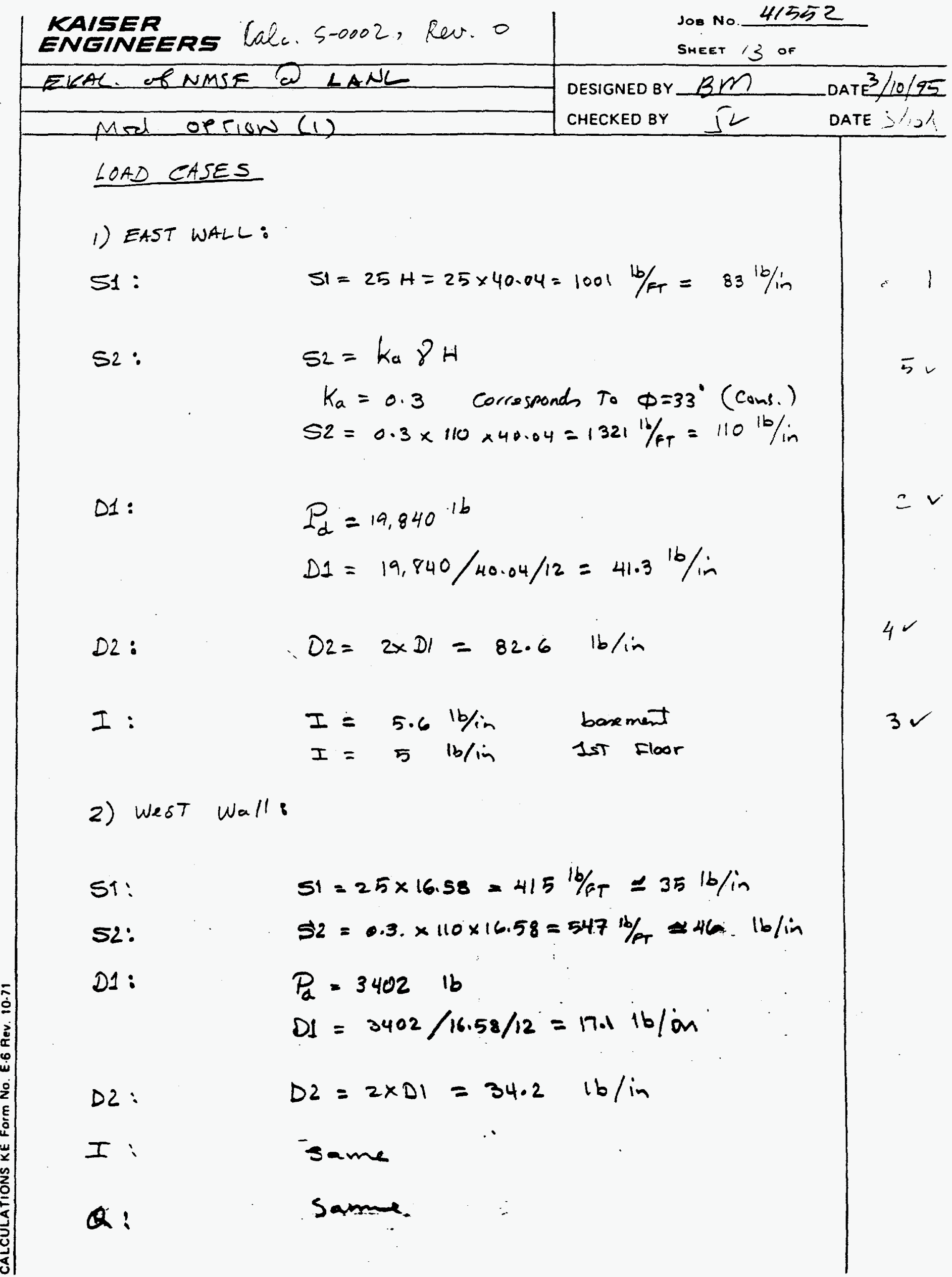




\begin{tabular}{|c|c|c|c|c|}
\hline Parameter & & (1) & (2) & (3) \\
\hline S Slope of Back of Wall to Vertical (rad): & Beta $=$ & 0 & 0 & \\
\hline Slope of Ground Behind Wall (rad): & $i=$ & 0 & 0 & \\
\hline Angle of Friction - Soil to Wall (rad): & Delta $=$ & 0 & 0 & \\
\hline | Internal Angle of Friction - Soil (deg): & Phi $=$ & 40 & 40 & \\
\hline (rad): & $\therefore \mathrm{Phi}=$ & 0.698 & 0.698 & \\
\hline | Unit Weight of Soil & Gamma & 110 & 110 & 110 \\
\hline Wall Height & $H=$ & 16.58 & 16.58 & 16.58 \\
\hline Horizontal Ground Acceleration (g): & $\mathrm{Kh}=$ & 0.3 & 0.3 & 0.3 \\
\hline Vertical Ground Acceleration $\quad$ (g): & $\mathrm{Kv}=$ & 0.2 & -0.2 & \\
\hline chi $=\operatorname{atan}(K h /(1-K v))$ & chi $=$ & 0.359 & 0.245 & \\
\hline Total Soil Pressure & $\mathrm{pae}=$ & 677 & 794 & \\
\hline Static Soil Pressure (psf): & $\mathrm{pa}=$ & 397 & 397 & \\
\hline Dynamic Soil Pressure Increment (psf): & $p d=$ & 281 & 397 & 410 \\
\hline Static Soil Pressure & $\mathrm{ps}=$ & 414.5 & 414.5 & 414.5 \\
\hline $\begin{array}{l}\text { Total Dynamic Force } \\
\text { Total Static Force }\end{array}$ & $\begin{array}{l}\mathrm{Pd}= \\
\mathrm{Ps}=\end{array}$ & $\begin{array}{l}2328 \\
5498\end{array}$ & $\begin{array}{l}3295 \\
5498\end{array}$ & $\begin{array}{l}3402 \\
5498\end{array}$ \\
\hline
\end{tabular}


West

Mononobe Okabe Analysis of Typical East Wall Section (Line 1 between B and G) - OPTION 1

\begin{tabular}{|c|c|c|c|c|}
\hline Parameter & & (1) & (2) & (3) \\
\hline Slope of Back of Wall to Vertical (rad): & Beta $=$ & 0 & 0 & \\
\hline | Slope of Ground Behind Wall (rad): & $i=$ & 0 & 0 & \\
\hline Angle of Friction - Soil to Wall (rad): & Delta $=$ & 0 & 0 & \\
\hline Internal Angle of Friction - Soil (deg): & $\begin{array}{l}\mathrm{Phi}= \\
\mathrm{Phi}=\end{array}$ & $\begin{array}{r}40 \\
0698\end{array}$ & $\begin{array}{r}40 \\
0609\end{array}$ & \\
\hline $\begin{array}{ll}\text { Unit Weight of Soil } & \text { (rad): }\end{array}$ & $\begin{array}{l}\text { Phi }= \\
\text { Gamma }\end{array}$ & $\begin{array}{r}0.698 \\
110\end{array}$ & $\begin{array}{r}0.698 \\
110\end{array}$ & 110 \\
\hline Wall Height & $H=$ & 40.04 & 40.04 & 40.04 \\
\hline Horizontal Ground Acceleration (g): & $\mathrm{Kh}=$ & 0.3 & 0.3 & 0.3 \\
\hline Vertical Ground Acceleration (g): & $\mathrm{Kv}=$ & 0.2 & -0.2 & \\
\hline $\operatorname{chi}=\operatorname{atan}(\mathrm{Kh} /(1-\mathrm{Kv}))$ & chi $=$ & 0.359 & 0.245 & \\
\hline Total Soil Pressure & $\mathrm{pae}=$ & 1636 & 1918 & \\
\hline $\begin{array}{l}\text { Static Soil Pressure (psf): } \\
\text { Dynamic Soil Pressure Increment (psf): }\end{array}$ & $\begin{array}{l}\mathrm{pa}= \\
\mathrm{pd}=\end{array}$ & $\begin{array}{l}958 \\
678\end{array}$ & $\begin{array}{l}958 \\
960\end{array}$ & 991 \\
\hline Static Soil Pressure & $\mathrm{ps}=$ & 1001 & 1001 & 1001 \\
\hline $\begin{array}{l}\text { Total Dynamic Force } \\
\text { Total Static Force }\end{array}$ & $\begin{array}{l}\mathrm{Pd}= \\
\mathrm{Ps}=\end{array}$ & $\begin{array}{l}13576 \\
32064\end{array}$ & $\begin{array}{l}19216 \\
32064\end{array}$ & $\begin{array}{l}19840 \\
32064\end{array}$ \\
\hline
\end{tabular}


cake. S-0002, kes.0, J06*41552, $\therefore t, 15=$

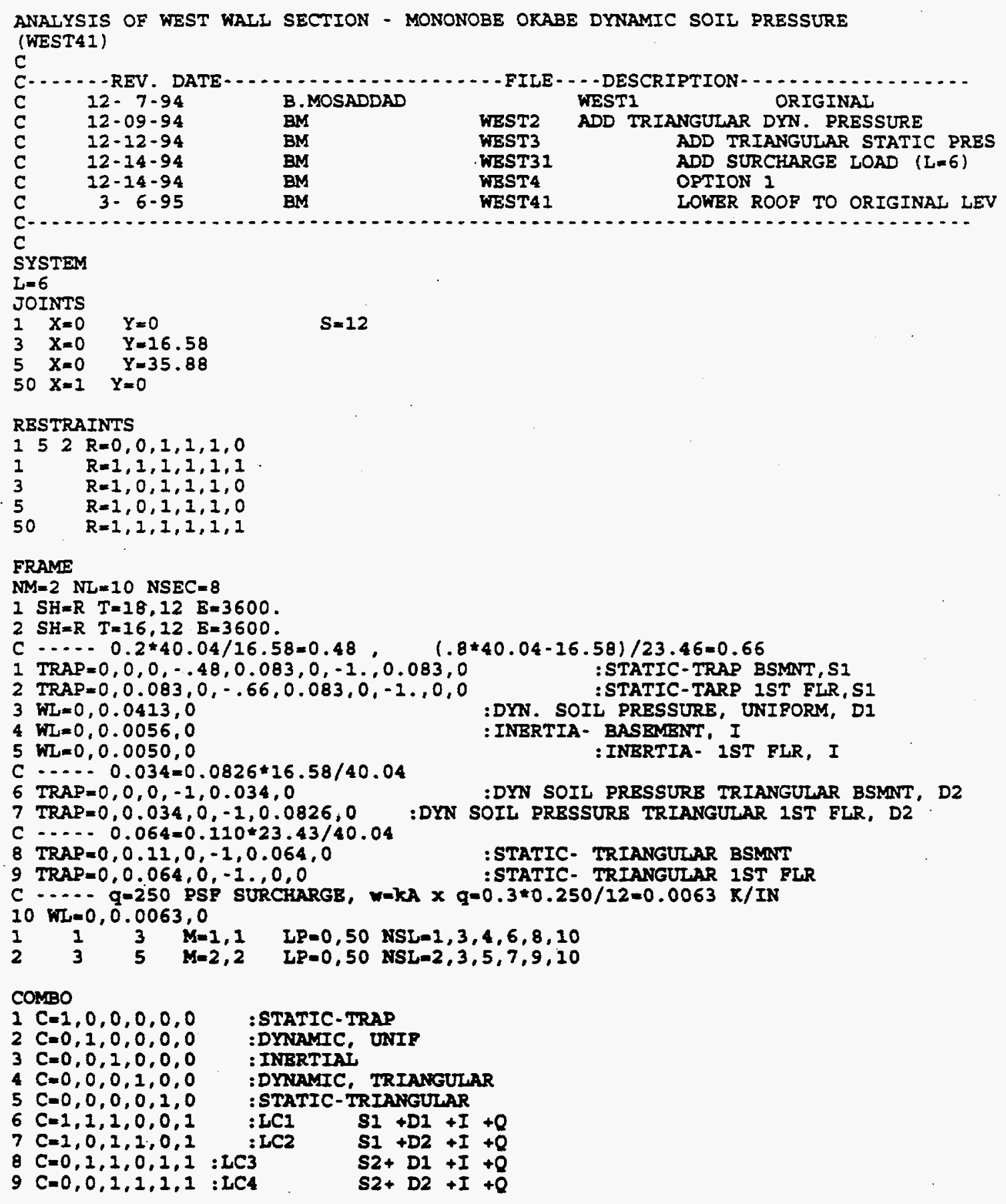


ale. S-0002, Her. $0,506 \times 4 i s 2$ $\therefore \quad \bar{i}=$

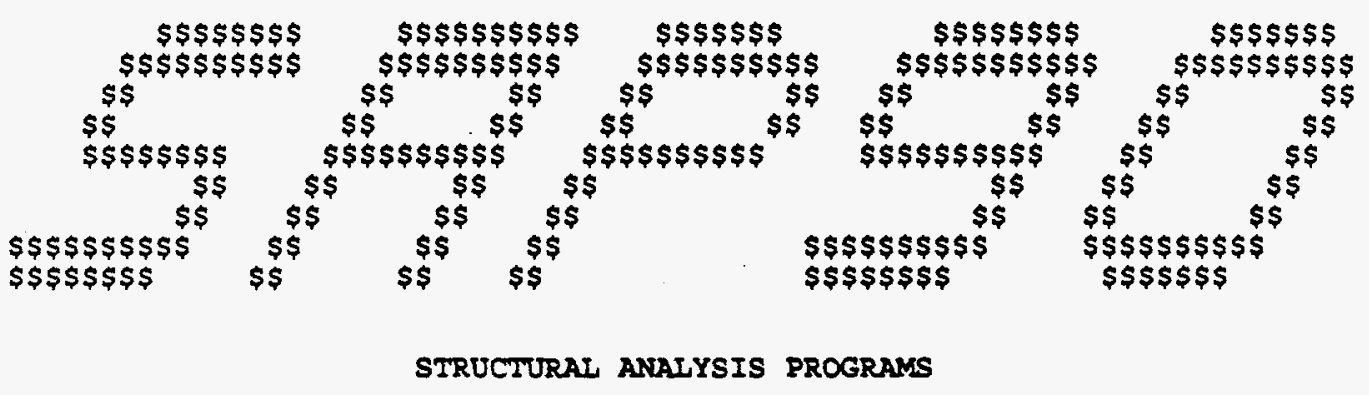

VERSION P5.40

Copyright (C) $1978-1992$

EDWARD I. WILSON

All rights reserved 
CS I / S A P 90 - - FINITE ELEMENT ANALYSIS OF STRUCTURES PAGE 1 PROGRAM : SAPGO/FILE : WEST41.F3F

FRAME ELEMENT FORCES

\begin{tabular}{|c|c|c|c|c|c|c|c|}
\hline ELT & $\begin{array}{ll}\text { LOAD } & \text { DIST } \\
\text { COMB } & \text { ENDI }\end{array}$ & SHEAR & $\begin{array}{l}\text { PLANE } \\
\text { MOMENT }\end{array}$ & $\begin{array}{l}\text { AXIAL } \\
\text { FORCE }\end{array}$ & $\operatorname{SHEAR}^{1-3}$ & $\begin{array}{l}\text { PLANE } \\
\text { MOMENT }\end{array}$ & $\begin{array}{l}\text { AXIAI } \\
\text { TORQ }\end{array}$ \\
\hline 1 & $\begin{array}{r}0.000 \\
0.000 \\
28.423 \\
56.846 \\
85.269 \\
113.691 \\
142.114 \\
170.537 \\
198.960 \\
198.960\end{array}$ & $\begin{array}{r}-3.326 \\
-2.975 \\
-1.922 \\
-0.167 \\
2.147 \\
4.506 \\
6.865 \\
9.224\end{array}$ & $\begin{array}{r}112.205 \\
20.991 \\
-50.267 \\
-81.613 \\
-53.963 \\
40.585 \\
202.185 \\
430.837\end{array}$ & 0.000 & . & & \\
\hline & $2 \begin{array}{r}0.000 \\
0.000 \\
28.423 \\
56.846 \\
85.269 \\
113.691 \\
142.114 \\
170.537 \\
198.960 \\
198.960\end{array}$ & $\begin{array}{r}-3.392 \\
-2.218 \\
-1.044 \\
0.130 \\
1.304 \\
2.478 \\
3.651 \\
4.825\end{array}$ & $\begin{array}{r}89.307 \\
9.587 \\
-36.768 \\
-49.759 \\
-29.385 \\
24.353 \\
111.456 \\
231.923\end{array}$ & 0.000 & & & \\
\hline & 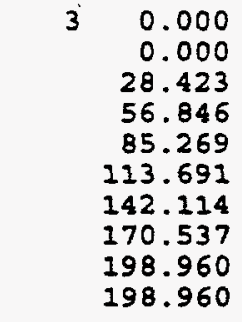 & $\begin{array}{r}-0.480 \\
-0.321 \\
-0.162 \\
-0.003 \\
0.156 \\
0.315 \\
0.475 \\
0.634\end{array}$ & $\begin{array}{r}13.456 \\
2.062 \\
-4.808 \\
-7.154 \\
-4.976 \\
1.725 \\
12.951 \\
28.701\end{array}$ & 0.000 & & & \\
\hline & $4 \begin{array}{r}0.000 \\
0.000 \\
28.423 \\
56.846 \\
85.269 \\
113.691 \\
142.114 \\
170.537 \\
198.960 \\
198.960\end{array}$ & $\begin{array}{l}0.582 \\
0.651 \\
0.858 \\
1.203 \\
1.686 \\
2.308 \\
3.067 \\
3.964\end{array}$ & $\begin{array}{r}-59.583 \\
-42.386 \\
-21.266 \\
7.702 \\
48.441 \\
104.876 \\
180.931 \\
280.529\end{array}$ & 0.000 & & & \\
\hline & $5 \quad \begin{array}{r}0.000 \\
0.000 \\
28.423 \\
56.846 \\
85.269\end{array}$ & $\begin{array}{l}-9.793 \\
-6.760 \\
-3.914 \\
-1.254\end{array}$ & $\begin{array}{r}316.639 \\
81.841 \\
-69.401 \\
-142.395\end{array}$ & 0.000 & & & \\
\hline
\end{tabular}




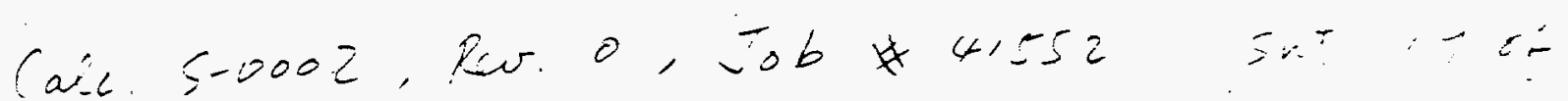

CSI / SAP 90 - - FINITE ELEMENT ANALYSIS OF STRUCTURES PAGE 2 PROGRAM: SAP9O/FILE : WEST 4 1.F3F

FRAMETLEMENT EORCES

\begin{tabular}{|c|c|c|c|c|c|c|}
\hline $\begin{array}{l}\text { ELT LOAD } \\
\text { ID COMB }\end{array}$ & $\begin{array}{r}\text { DIST } \\
\text { ENDI } \\
113.691 \\
142.114 \\
170.537 \\
198.960 \\
198.960\end{array}$ & $\begin{array}{l}{ }^{1-2} \\
\text { SHEAR } \\
1.219 \\
3.505 \\
5.6004 \\
7.517\end{array}$ & $\begin{array}{r}\text { PLANE } \\
\text { MOMENT } \\
-142.452 \\
-74.879 \\
55.014 \\
241.919\end{array}$ & $\begin{array}{l}\text { AXIAL } \\
\text { FORCE }\end{array}$ & 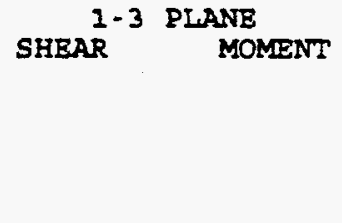 & $\begin{array}{l}\text { AXIAL } \\
\text { TORQ }\end{array}$ \\
\hline 6 & $\begin{array}{r}0.000 \\
0.000 \\
28.423 \\
56.846 \\
85.269 \\
113.691 \\
142.114 \\
170.537 \\
198.960 \\
198.960\end{array}$ & $\begin{array}{r}-7.716 \\
-5.853 \\
-3.287 \\
-0.020 \\
3.806 \\
7.677 \\
11.548 \\
15.419\end{array}$ & $\begin{array}{r}228.591 \\
34.102 \\
-97.452 \\
-146.117 \\
-92.807 \\
70.378 \\
343.593 \\
726.839\end{array}$ & 0.000 & & \\
\hline 7 & $\begin{array}{r}0.000 \\
0.000 \\
28.423 \\
56.846 \\
85.269 \\
113.691 \\
142.114 \\
170.537 \\
198.960 \\
198.960\end{array}$ & $\begin{array}{r}-3.742 \\
-2.984 \\
-1.385 \\
1.053 \\
4.188 \\
7.507 \\
10.964 \\
14.558\end{array}$ & $\begin{array}{r}79.702 \\
-17.871 \\
-81.950 \\
-88.656 \\
-14.980 \\
150.901 \\
413.068 \\
775.444\end{array}$ & 0.000 & & \\
\hline 8 & $\begin{array}{r}0.000 \\
0.000 \\
28.423 \\
56.846 \\
85.269 \\
113.691 \\
142.114 \\
170.537 \\
198.960 \\
198.960\end{array}$ & $\begin{array}{r}-14.183 \\
-9.637 \\
-5.279 \\
-1.107 \\
2.878 \\
6.676 \\
10.287 \\
13.712\end{array}$ & $\begin{array}{r}433.025 \\
94.953 \\
-116.586 \\
-206.899 \\
-181.297 \\
-45.086 \\
196.422 \\
537.921\end{array}$ & 0.000 & & \\
\hline 9 & $\begin{array}{r}0.000 \\
0.000 \\
28.423 \\
56.846 \\
85.269 \\
113.691 \\
142.114 \\
170.537 \\
198.960 \\
198.960\end{array}$ & $\begin{array}{r}-10.209 \\
-6.768 \\
-3.377 \\
-0.034 \\
3.260 \\
6.506 \\
9.703 \\
12.851\end{array}$ & $\begin{array}{r}284.136 \\
42.979 \\
-102.084 \\
-149.438 \\
-103.470 \\
35.437 \\
265.897 \\
586.526\end{array}$ & 0.000 & & \\
\hline
\end{tabular}


Sake. S-0002, kw. $0,-506+1=2$

C SI / S P 90 - - FINITE ELEMENT ANALYSIS OF STRUCTURES PAGE 3 PROGRAM: SAPSO/FILE: WEST4 I.P3F

FRAME ELEMENT FORCES

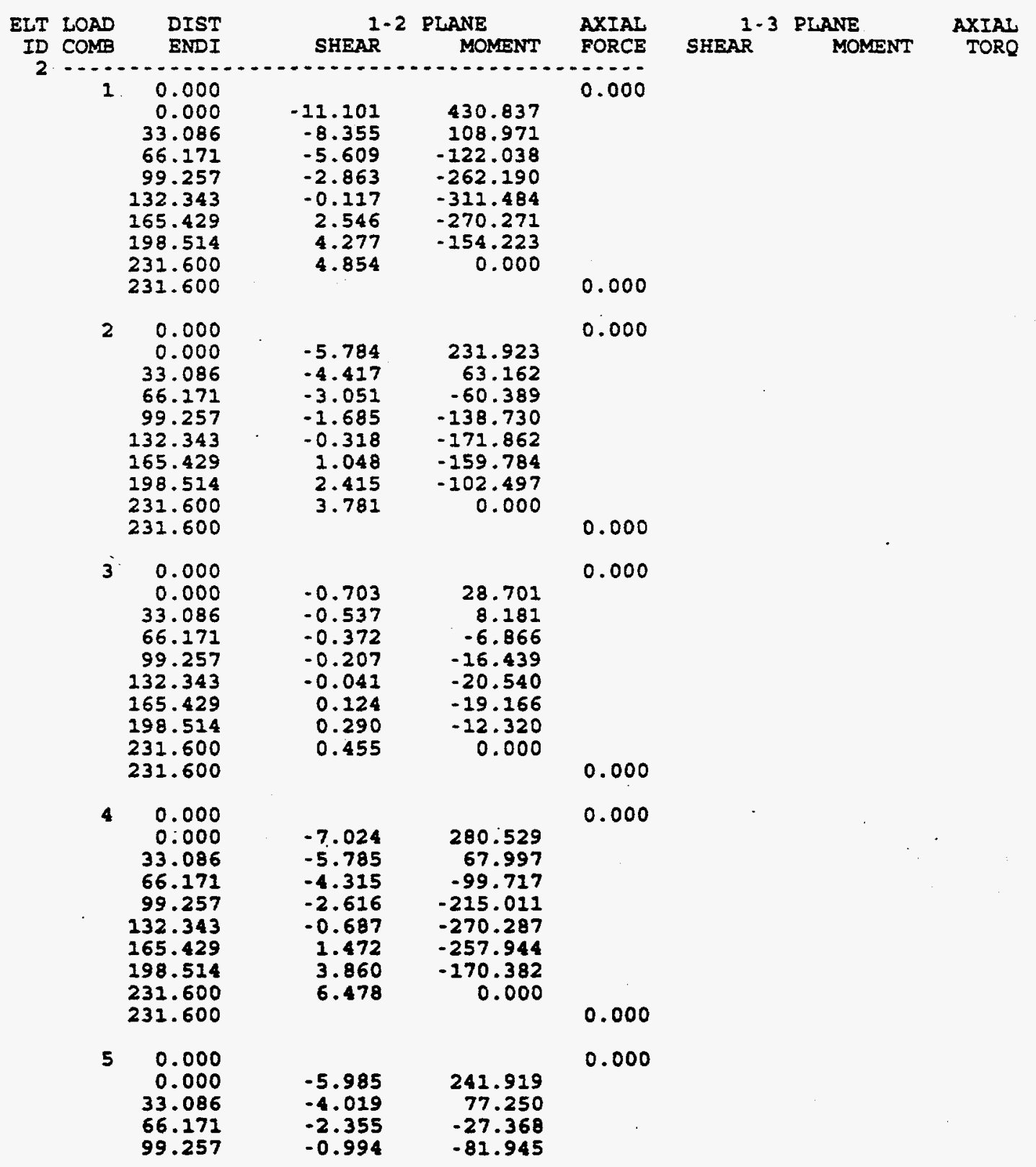


rete S-0002, Fis. 0 , Job 41552 .

CSI / S A P 90 - - FINITE ELEMENT ANALYSIS OF STRUCTURES PAGE 4 PROGRAM:SAP9O/FILE: WEST41.F3F

FRAME ELEMENT FORCES

\begin{tabular}{|c|c|c|c|c|c|c|c|}
\hline $\begin{array}{l}\text { ELT LOAD } \\
\text { ID COMB }\end{array}$ & $\begin{array}{r}\text { DIST } \\
\text { ENDI } \\
132.343 \\
165.429 \\
198.514 \\
231.600 \\
231.600\end{array}$ & $\begin{array}{l}{ }^{1-2} \\
\text { SHEAR } \\
0.065 \\
0.821 \\
1.275 \\
1.426\end{array}$ & $\begin{array}{l}\text { PLANE } \\
\text { MOMENT } \\
-96.488 \\
-81.006 \\
-45.507 \\
0.000\end{array}$ & 0.000 & SHEAR & $\begin{array}{l}\text { PLANE } \\
\text { MOMENT }\end{array}$ & $\begin{array}{l}\text { AXIAL } \\
\text { TORQ }\end{array}$ \\
\hline 6 & $\begin{array}{r}0.000 \\
0.000 \\
33.086 \\
66.171 \\
99.257 \\
132.343 \\
165.429 \\
198.514 \\
231.600 \\
231.600\end{array}$ & $\begin{array}{r}-18.470 \\
-13.984 \\
-9.498 \\
-5.011 \\
-0.525 \\
3.878 \\
7.349 \\
9.667\end{array}$ & $\begin{array}{r}726.839 \\
189.949 \\
-198.505 \\
-438.522 \\
-530.102 \\
-473.595 \\
-284.675 \\
0.000\end{array}$ & 0.000 & & & \\
\hline 7 & $\begin{array}{r}0.000 \\
0.000 \\
33.086 \\
66.171 \\
99.257 \\
132.343 \\
165.429 \\
198.514 \\
231.600 \\
231.600\end{array}$ & $\begin{array}{r}-19.711 \\
-15.351 \\
-10.762 \\
-5.943 \\
-0.894 \\
4.302 \\
8.795 \\
12.363\end{array}$ & $\begin{array}{r}775.444 \\
194.783 \\
-237.833 \\
-514.803 \\
-628.527 \\
-571.755 \\
-352.560 \\
0.000\end{array}$ & 0.000 & & & \\
\hline 8 & $\begin{array}{r}0.000 \\
0.000 \\
33.086 \\
66.171 \\
99.257 \\
132.343 \\
165.429 \\
198.514 \\
231.600 \\
231.600\end{array}$ & $\begin{array}{r}-13.355 \\
-9.648 \\
-6.244 \\
-3.142 \\
-0.343 \\
2.153 \\
4.347 \\
6.239\end{array}$ & $\begin{array}{r}537.921 \\
158.228 \\
-103.835 \\
-258.277 \\
-315.106 \\
-284.331 \\
-175.959 \\
0.000\end{array}$ & 0.000 & . & & \\
\hline & $\begin{array}{r}0.000 \\
0.000 \\
33.086 \\
66.171 \\
99.257 \\
132.343 \\
165.429 \\
198.514 \\
231.600 \\
231.600\end{array}$ & $\begin{array}{r}-14.595 \\
-11.015 \\
-7.508 \\
-4.074 \\
-0.712 \\
2.576 \\
5.792 \\
8.936\end{array}$ & $\begin{array}{r}586.526 \\
163.062 \\
-143.163 \\
-334.558 \\
-413.531 \\
-382.490 \\
-243.844 \\
0.000\end{array}$ & 0.000 & & & \\
\hline
\end{tabular}




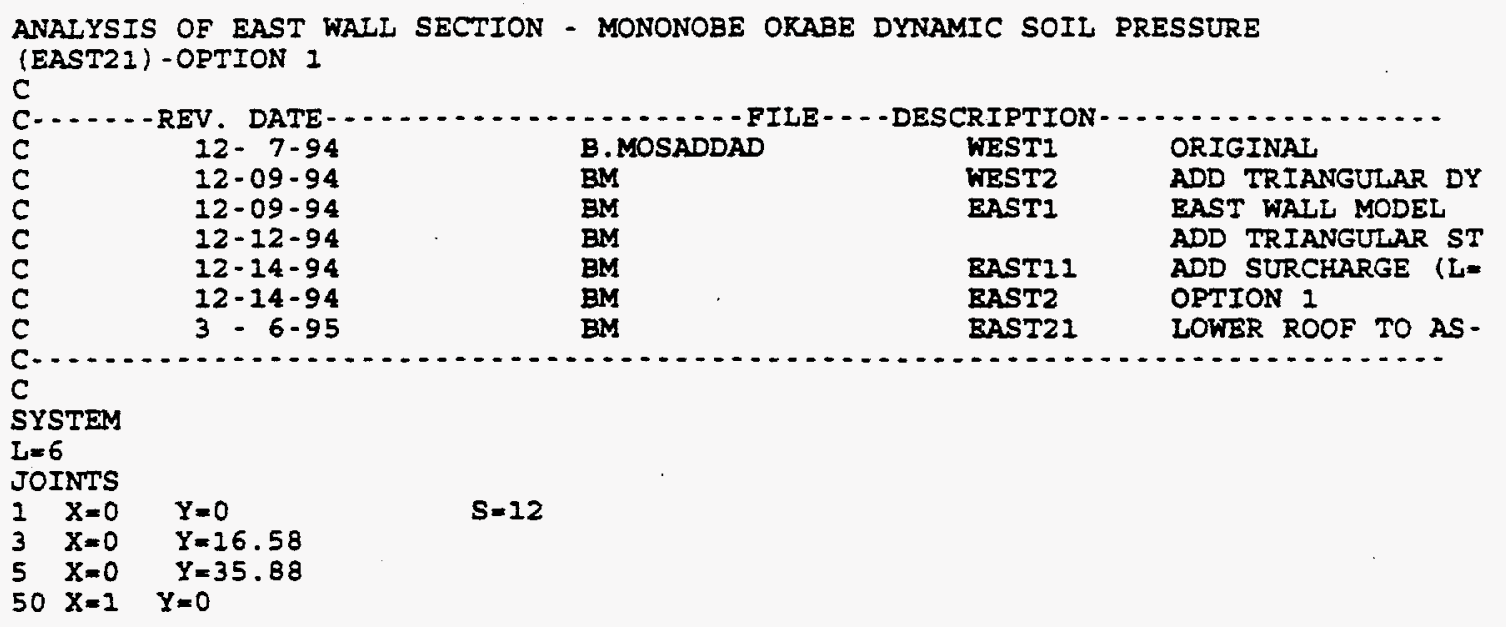

RESTRAINTS

$\begin{array}{lll}152 & R=0,0,1,1,1,0 \\ 1 & & R=1,1,1,1,1,1 \\ 3 & & R=1,0,1,1,1,0 \\ 5 & & R=1,0,1,1,1,0 \\ 50 & R & =1,1,1,1,1,1\end{array}$

FRAME

$N M=2 \quad N L=6 \quad N S E C=8$

$1 \mathrm{SH}=\mathrm{R} \quad \mathrm{T}=18,12 \quad \mathrm{E}=3600$.

$2 \mathrm{SH}=\mathrm{R} \quad \mathrm{T}=16,12 \quad \mathrm{E}=3600$.

1 TRAP $=0,0,0,-.2,0.035,0,-.8,0.035,0,-1 \ldots 0,0 \quad$ :STATIC-TRAP, S1

2 KL $=0,0.0171,0$

$3 \mathrm{WL}=0,0.0056,0$ :DYN. SOIL PRESSURB, UNIPORM, DI

IINERTIA- BASEMENT, I

4 TRAP $=0,0,0,-1,0.0342,0 \quad:$ DY. SOIL PRESSURB TRIANGULAR, D2

5 TRAP $=0, .046,0,-1,0.0$ :STATIC- TRIANGULAR, S2

C SURCHARGE OF 250 PSE, $Q=0.3 * 0.250 / 12=0.0063$ $6 \mathrm{WL}=0,0.0063,0$

$13 \quad 1 \quad 3 \quad M=1,1 \quad$ LP $=0,50$ NSL $=1,2,3,4,5,6$

COMBO

I $C=1,0,0,0,0,0 \quad$ :STATIC-TRAP

$2 C=0,1,0,0,0,0 \quad$ :DYNAMIC, UNIF

$3 C=0,0,1,0,0,0 \quad$ :INBRTIAL

$4 \mathrm{C}=0,0,0,1,0,0 \quad$ :DYNAMIC, TRIANGULAR

$5 \mathrm{C}=0,0,0,0,1,0 \quad$ :STATIC-TRIANGULAR

$6 C=1,1,1,0,0,1 \quad$ :LC1 $\quad S 1+D 1+I+0$

$7 C=1,0,1,1,0,1 \quad: L C 2 \quad S 1+D 2+I+Q$

$8 \mathrm{C}=0,1,1,0,1,1=I C 3 \quad S 2+D 1+I+Q$

$9 \mathrm{C}=0,0,1,1,1,1: L C 4 \quad S 2+D 2+I+Q$ 


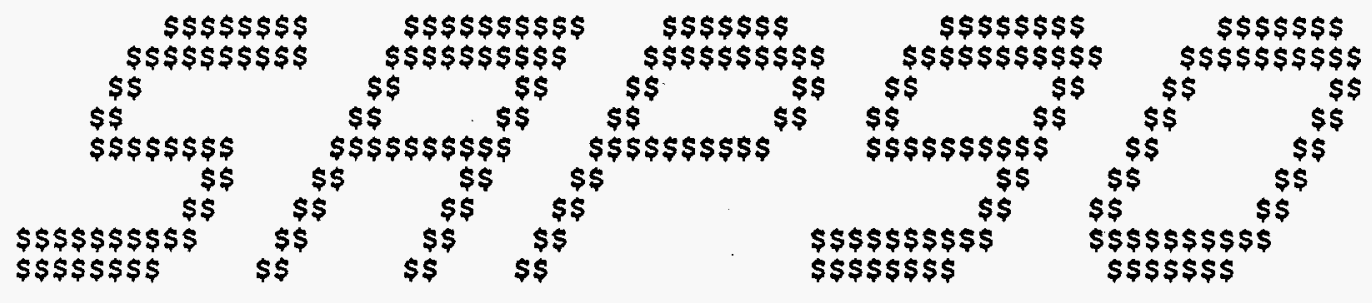

STRUCTURAL ANALYSIS PROGRAMS

VERSION P5.40

Copyright (C) 1978-1992

EDWARD L. WILSON

All rights reserved 
lake $5-0002,4$, 0 , vor $4: 55=$

C $S I / S A P 90$ - - FINITE ELEMENT ANALYSIS OF STRUCTURES PAGE 1 ANALYSIS OF EAST WALL SECTION - MONONOBE ORABE DYNAMIC SOIL PRESSURE ER A E E LEMENT FORCES

\begin{tabular}{|c|c|c|c|c|c|c|c|}
\hline ELT & $\begin{array}{l}\text { LOAD } \\
\text { COMB }\end{array}$ & SHEAR & $\begin{array}{l}\text { PLANE } \\
\text { MOMENT }\end{array}$ & $\begin{array}{l}\text { AXIAI } \\
\text { FORCE }\end{array}$ & SHEAR ${ }^{1-3}$ & $\begin{array}{l}\text { PLANE } \\
\text { MOMENT }\end{array}$ & $\begin{array}{l}\text { AXIAL } \\
\text { TORQ }\end{array}$ \\
\hline 1 & $\begin{array}{r}0.000 \\
0.000 \\
28.423 \\
56.846 \\
85.269 \\
113.691 \\
142.114 \\
170.537 \\
198.960 \\
198.960\end{array}$ & $\begin{array}{r}-3.335 \\
-2.980 \\
-2.042 \\
-1.047 \\
-0.053 \\
0.942 \\
1.880 \\
2.235\end{array}$ & $\begin{array}{r}143.153 \\
51.717 \\
-20.250 \\
-64.157 \\
-79.789 \\
-67.146 \\
-26.444 \\
33.728\end{array}$ & 0.000 & & & \\
\hline & $2 \begin{array}{r}0.000 \\
0.000 \\
28.423 \\
56.846 \\
85.269 \\
113.691 \\
142.114 \\
170.537 \\
198.960 \\
198.960\end{array}$ & $\begin{array}{r}-1.991 \\
-1.505 \\
-1.019 \\
-0.533 \\
-0.047 \\
0.439 \\
0.926 \\
1.412\end{array}$ & $\begin{array}{r}75.367 \\
25.694 \\
-10.165 \\
-32.209 \\
-40.439 \\
-34.855 \\
-15.456 \\
17.757\end{array}$ & 0.000 & & & \\
\hline & $3 \begin{array}{r}0.000 \\
0.000 \\
28.423 \\
56.846 \\
85.269 \\
113.691 \\
142.114 \\
170.537 \\
198.960 \\
198.960\end{array}$ & $\begin{array}{r}-0.652 \\
-0.493 \\
-0.334 \\
-0.174 \\
-0.015 \\
0.144 \\
0.303 \\
0.462\end{array}$ & $\begin{array}{r}24.682 \\
8.414 \\
-3.329 \\
-10.548 \\
-13.243 \\
-11.414 \\
-5.062 \\
5.815\end{array}$ & 0.000 & & & \\
\hline & $\begin{array}{r}0.000 \\
0.000 \\
28.423 \\
56.846 \\
85.269 \\
113.691 \\
142.114 \\
170.537 \\
198.960 \\
198.960\end{array}$ & $\begin{array}{r}-1.370 \\
-1.300 \\
-1.092 \\
-0.745 \\
-0.259 \\
0.366 \\
1.130 \\
2.033\end{array}$ & $\begin{array}{r}68.063 \\
29.796 \\
-4.525 \\
-30.952 \\
-45.538 \\
-44.336 \\
-23.399 \\
21.220\end{array}$ & 0.000 & & & \\
\hline & 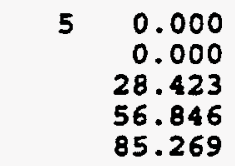 & $\begin{array}{l}-3.513 \\
-2.299 \\
-1.272 \\
-0.431\end{array}$ & $\begin{array}{r}111.194 \\
29.042 \\
-21.258 \\
-45.014\end{array}$ & 0.000 & & & \\
\hline
\end{tabular}


'ale S-0002, hes. $0, j=b \times 41552$

C S I S A P 90 - - FINITE ELEMINT ANALYSIS OF STRUCTURES PAGE 2 ANALYSIS OF EAST WALL SECTION - MONONOBE ORABE DYNAMIC SOII PRESSURE

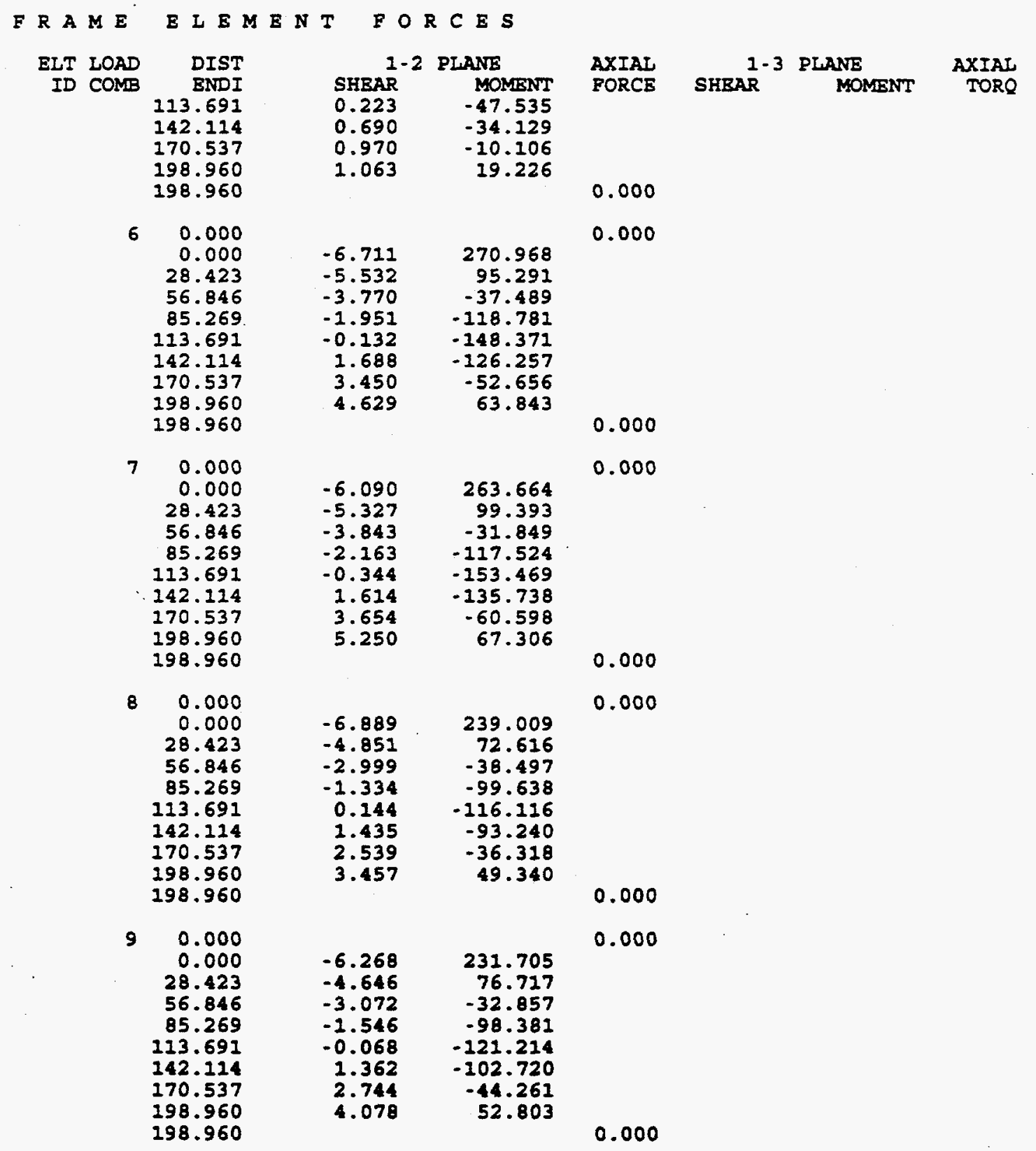


lake. 5-0002, fur. 0, vob 41552

CSI / S A P 90 - - FINITE BLEMENT ANALYSIS OF STRUCTURES PAGE 33 ANALYSIS OF EAST WALI SECTION - MONONOBE ORABE DYNAMIC SOIL PRESSURE

FRAME ELEMENT FORCES

\begin{tabular}{|c|c|c|c|c|c|c|c|}
\hline ELT & $\begin{array}{ll}\text { LOAD } & \text { DIST } \\
\text { COMB } & \text { ENDI }\end{array}$ & SHEAR & $\begin{array}{l}\text { PIANE } \\
\text { MOMIENT }\end{array}$ & $\begin{array}{l}\text { AXIAL } \\
\text { FORCE }\end{array}$ & SHEAR & $\begin{array}{l}\text { PLANE } \\
\text { MOMENT }\end{array}$ & $\begin{array}{r}\text { AXIAL } \\
\text { TORQ }\end{array}$ \\
\hline 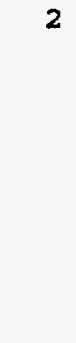 & $\begin{array}{r}0.000 \\
0.000 \\
33.086 \\
66.171 \\
99.257 \\
132.343 \\
165.429 \\
198.514 \\
231.600 \\
231.600\end{array}$ & $\begin{array}{l}-0.146 \\
-0.146 \\
-0.146 \\
-0.146 \\
-0.146 \\
-0.146 \\
-0.146 \\
-0.146\end{array}$ & $\begin{array}{r}33.728 \\
28.910 \\
24.092 \\
19.273 \\
14.455 \\
9.637 \\
4.818 \\
0.000\end{array}$ & 0.000 & & & \\
\hline & $2 \begin{array}{r}0.000 \\
0.000 \\
33.086 \\
66.171 \\
99.257 \\
132.343 \\
165.429 \\
198.514 \\
231.600 \\
231.600\end{array}$ & $\begin{array}{l}-0.077 \\
-0.077 \\
-0.077 \\
-0.077 \\
-0.077 \\
-0.077 \\
-0.077 \\
-0.077\end{array}$ & $\begin{array}{r}17.757 \\
15.220 \\
12.684 \\
10.147 \\
7.610 \\
5.073 \\
2.537 \\
0.000\end{array}$ & 0.000 & & & \\
\hline & $3 \begin{array}{r}0.000 \\
0.000 \\
33.086 \\
66.171 \\
99.257 \\
132.343 \\
165.429 \\
198.514 \\
231.600 \\
231.600\end{array}$ & $\begin{array}{l}-0.025 \\
-0.025 \\
-0.025 \\
-0.025 \\
-0.025 \\
-0.025 \\
-0.025 \\
-0.025\end{array}$ & $\begin{array}{l}5.815 \\
4.984 \\
4.154 \\
3.323 \\
2.492 \\
1.661 \\
0.831 \\
0.000\end{array}$ & 0.000 & & & \\
\hline & $\begin{array}{r}0.000 \\
0.000 \\
33.086 \\
66.171 \\
99.257 \\
132.343 \\
165.429 \\
198.514 \\
231.600 \\
231.600\end{array}$ & $\begin{array}{l}-0.092 \\
-0.092 \\
-0.092 \\
-0.092 \\
-0.092 \\
-0.092 \\
-0.092 \\
-0.092\end{array}$ & $\begin{array}{r}21.220 \\
18.189 \\
15.157 \\
12.126 \\
9.094 \\
6.063 \\
3.031 \\
0.000\end{array}$ & 0.000 & . & & \\
\hline & $5 \quad \begin{array}{r}0.000 \\
0.000 \\
33.086 \\
66.171 \\
99.257\end{array}$ & $\begin{array}{l}-0.083 \\
-0.083 \\
-0.083 \\
-0.083\end{array}$ & $\begin{array}{l}19.226 \\
16.479 \\
13.733 \\
10.986\end{array}$ & 0.000 & & & \\
\hline
\end{tabular}




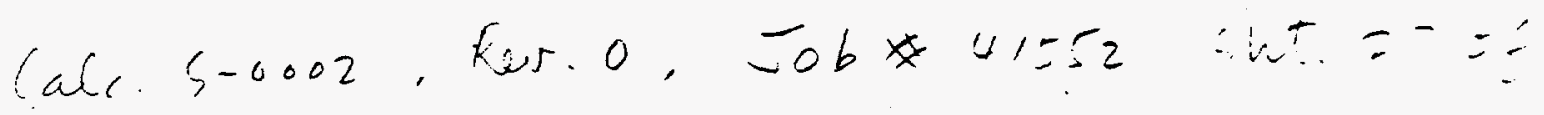

CS I / S A P 90 - - FINITE ELEMENT ANALYSIS OF STRUCTURES PAGE 4 ANALYSIS OF EAST WALL SECTION - MONONOBE OKABE DYNAMIC SOIL PRESSURE

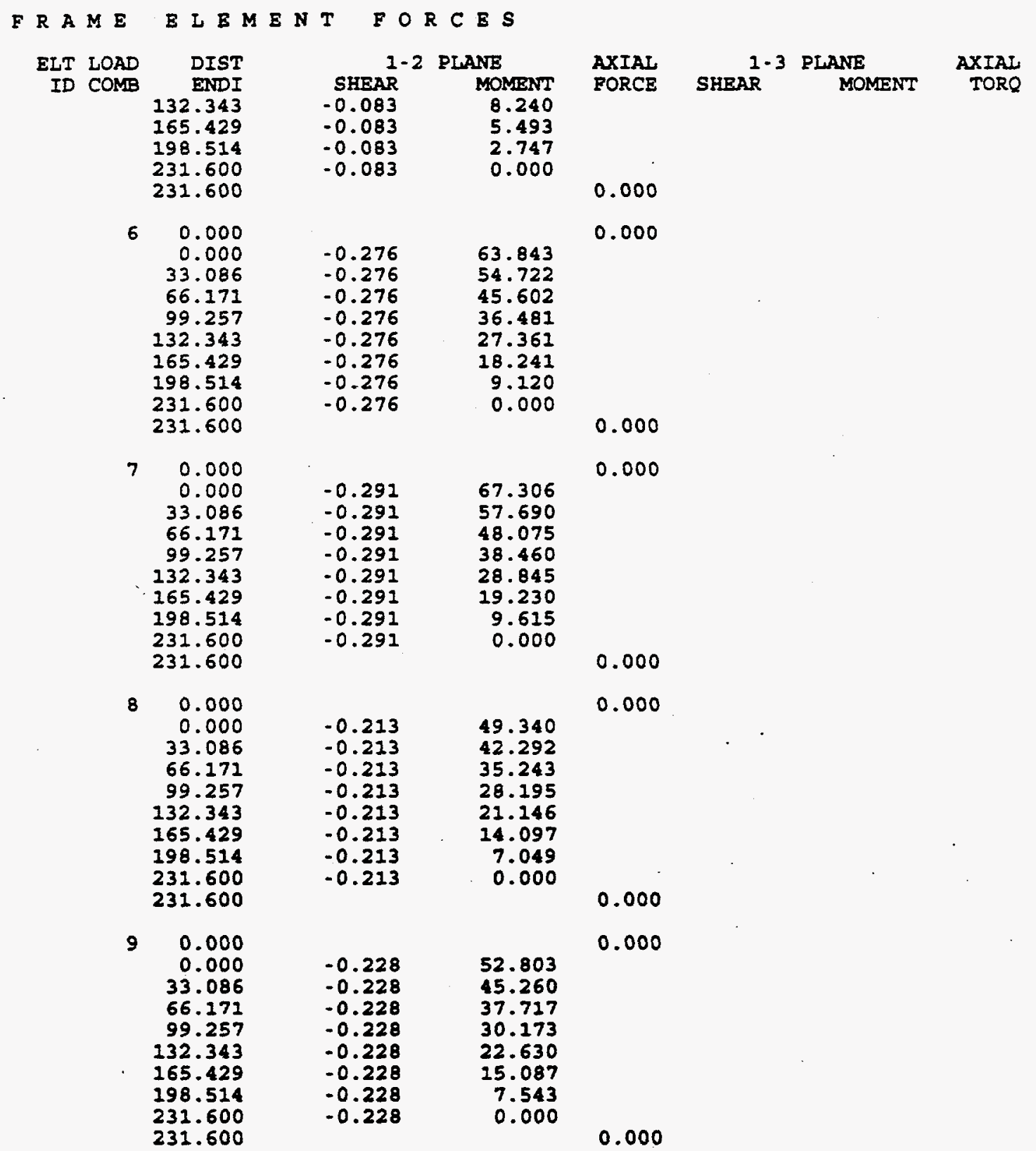




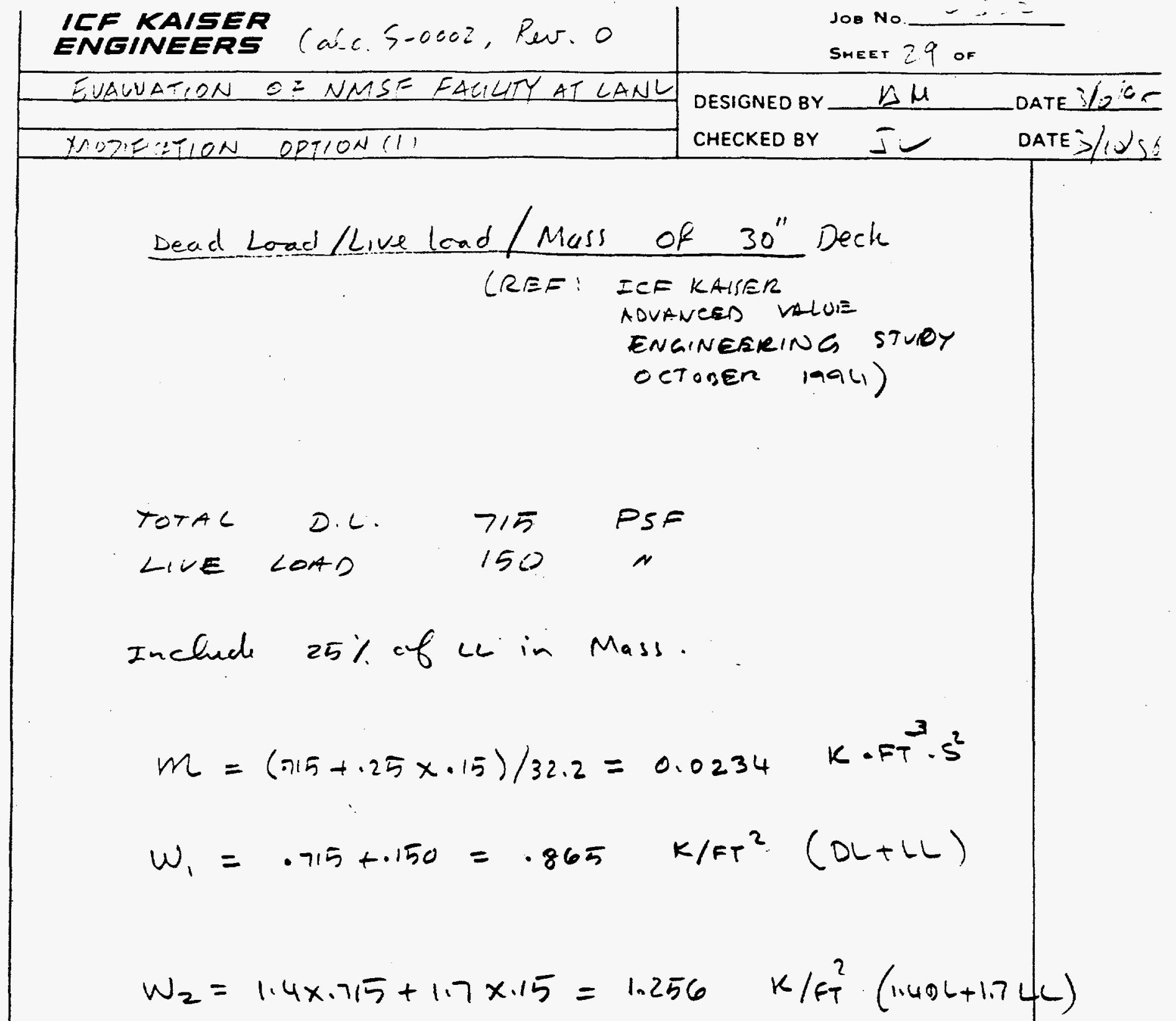




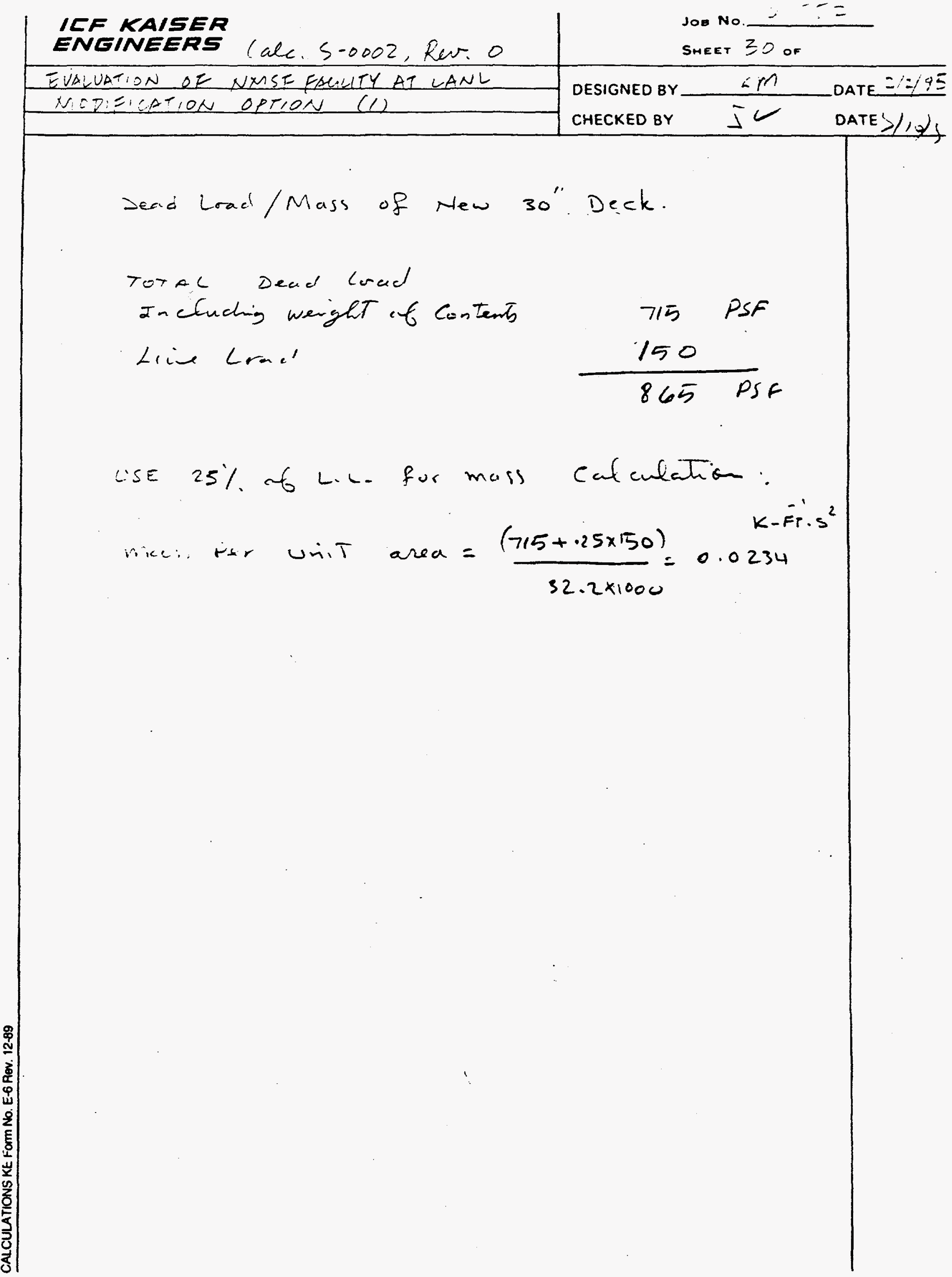


$696^{-14 n \cdot 6}+9,6$
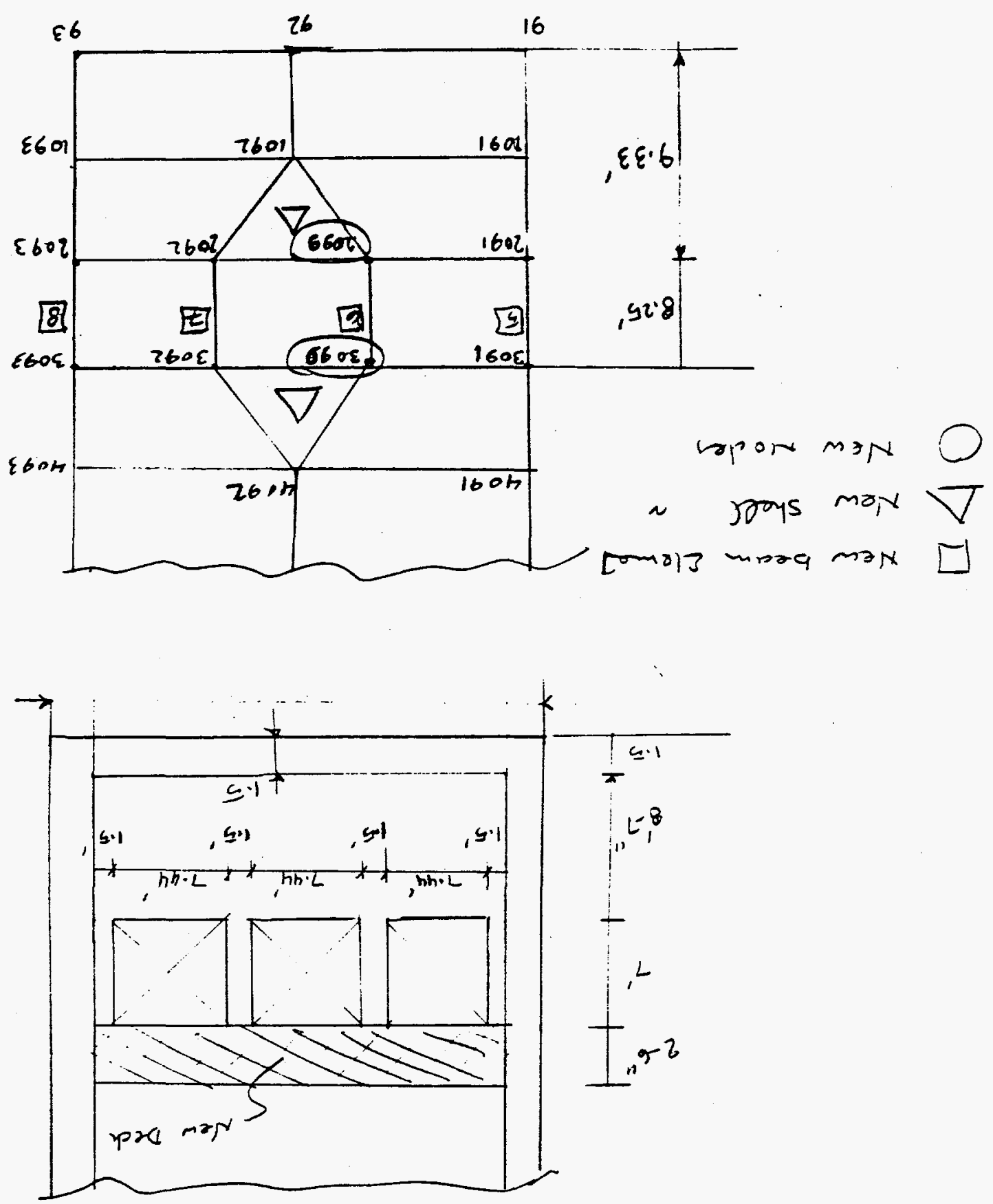

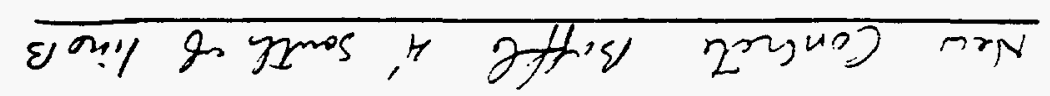

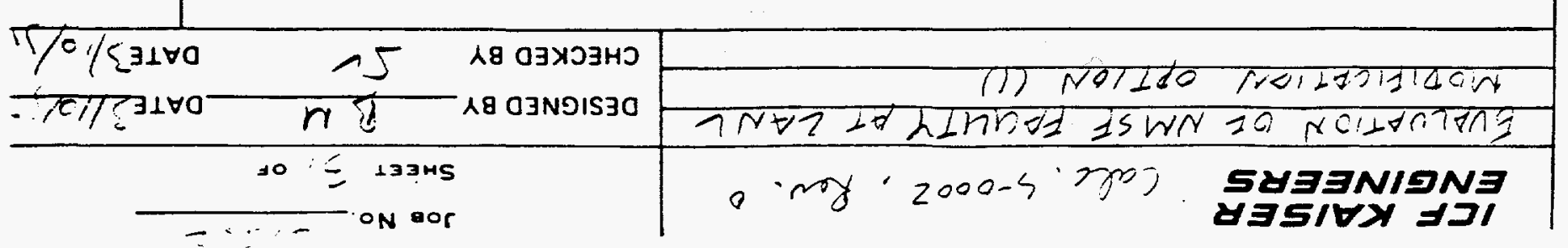




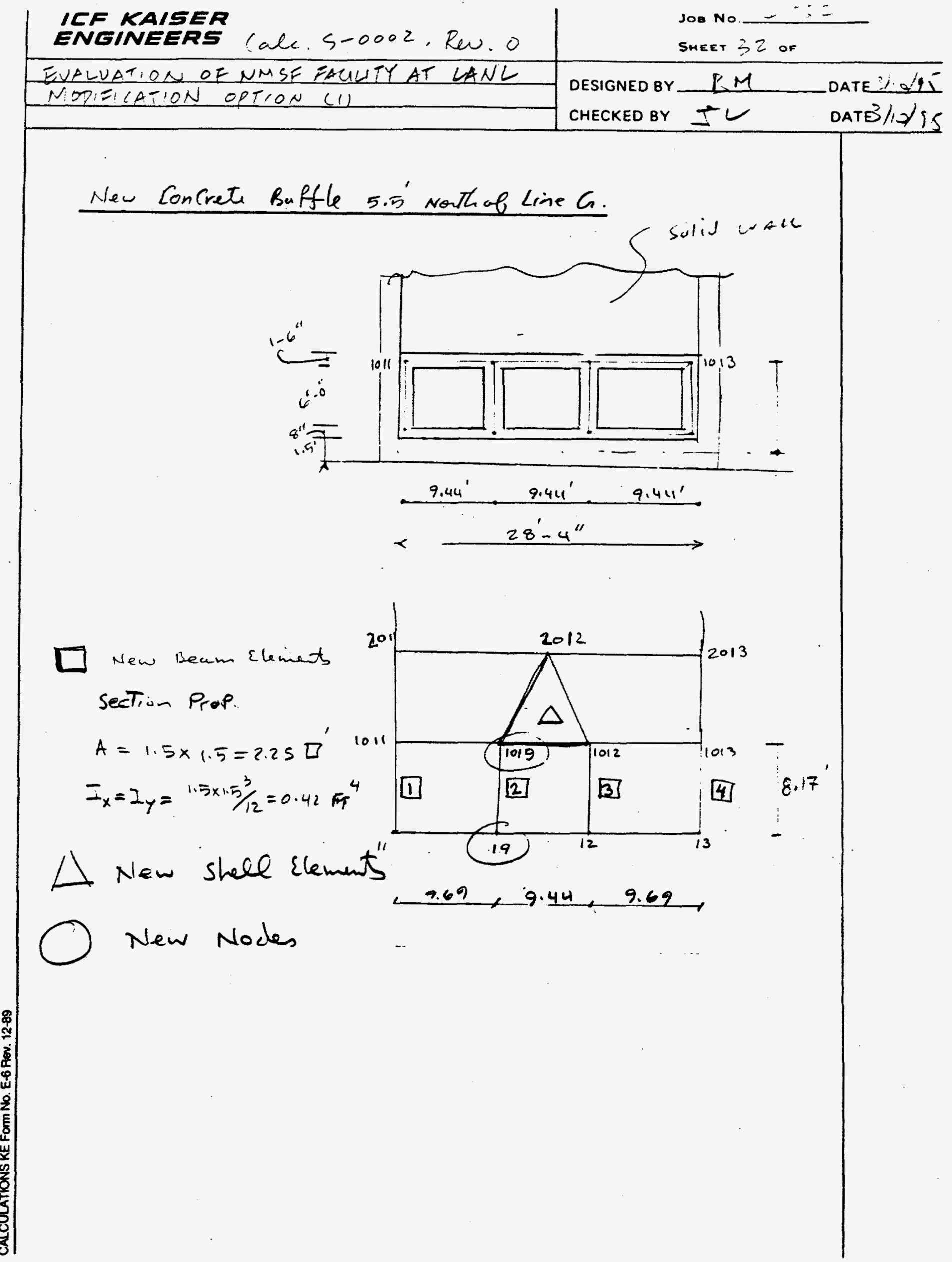




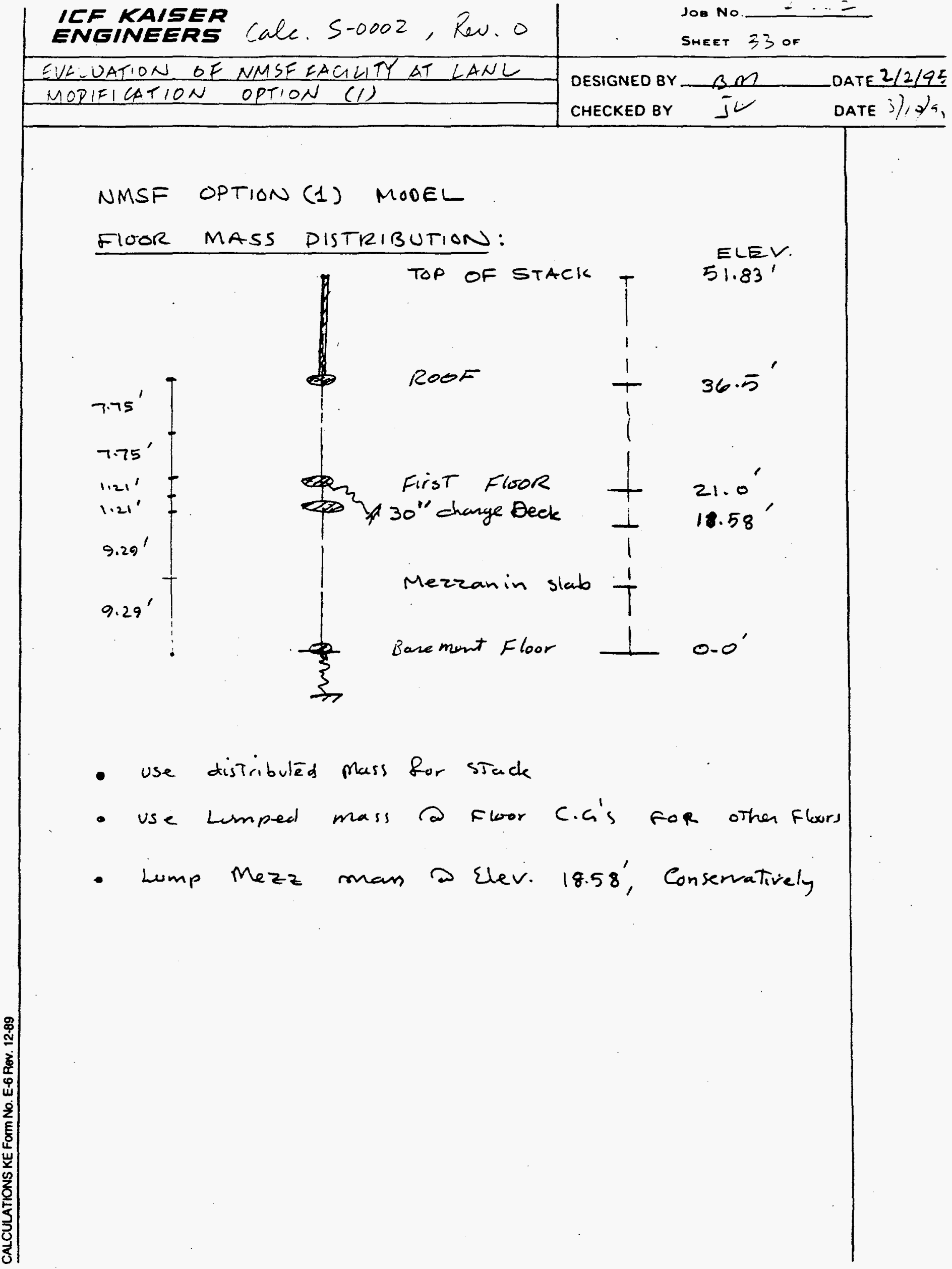




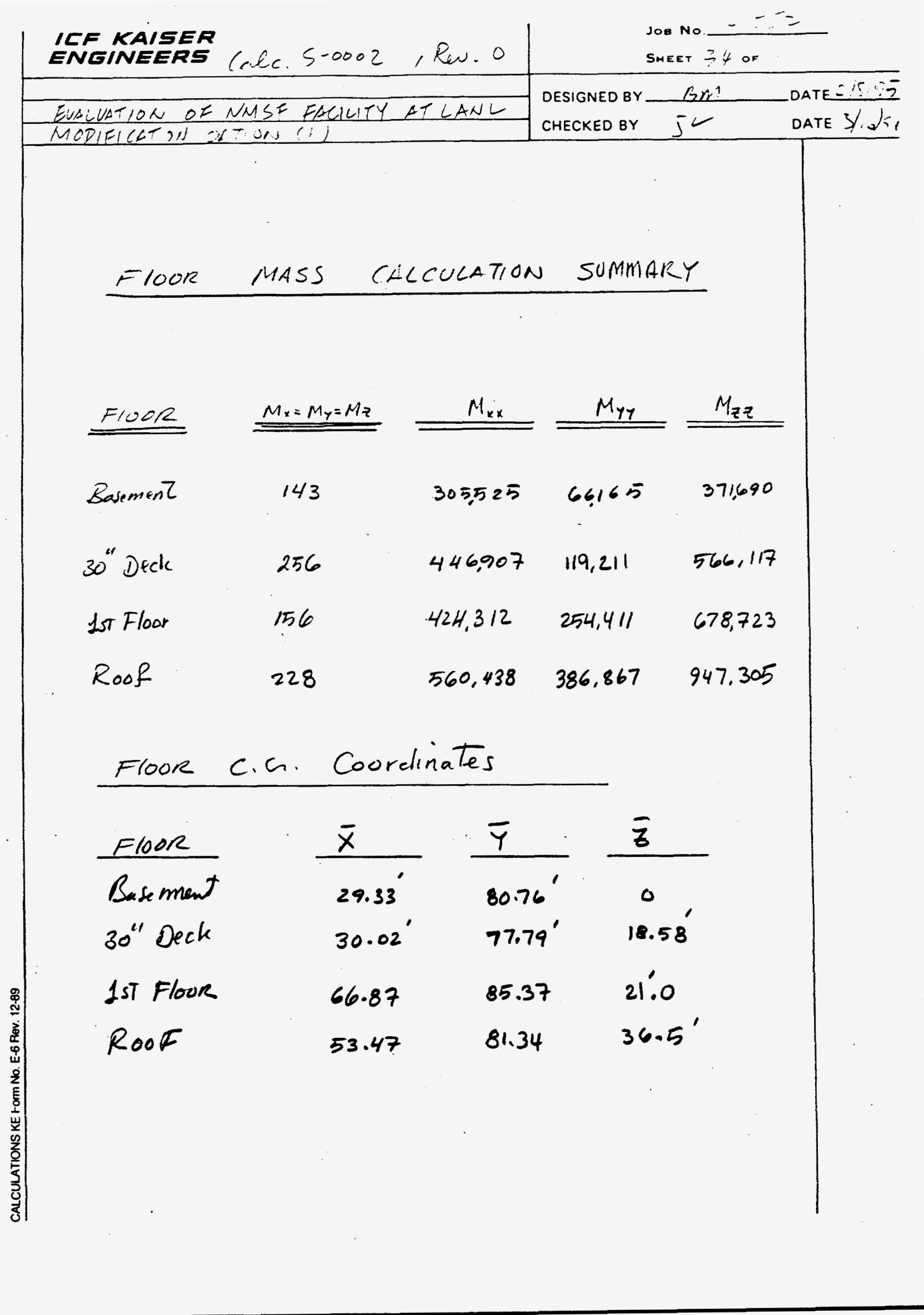


IICF KaISER Cale. S-0002, Reu. IENGINEER

SUBJ-D-Basement Floor Mass DESIGNED BY:

1 - - - - OPTION (1)

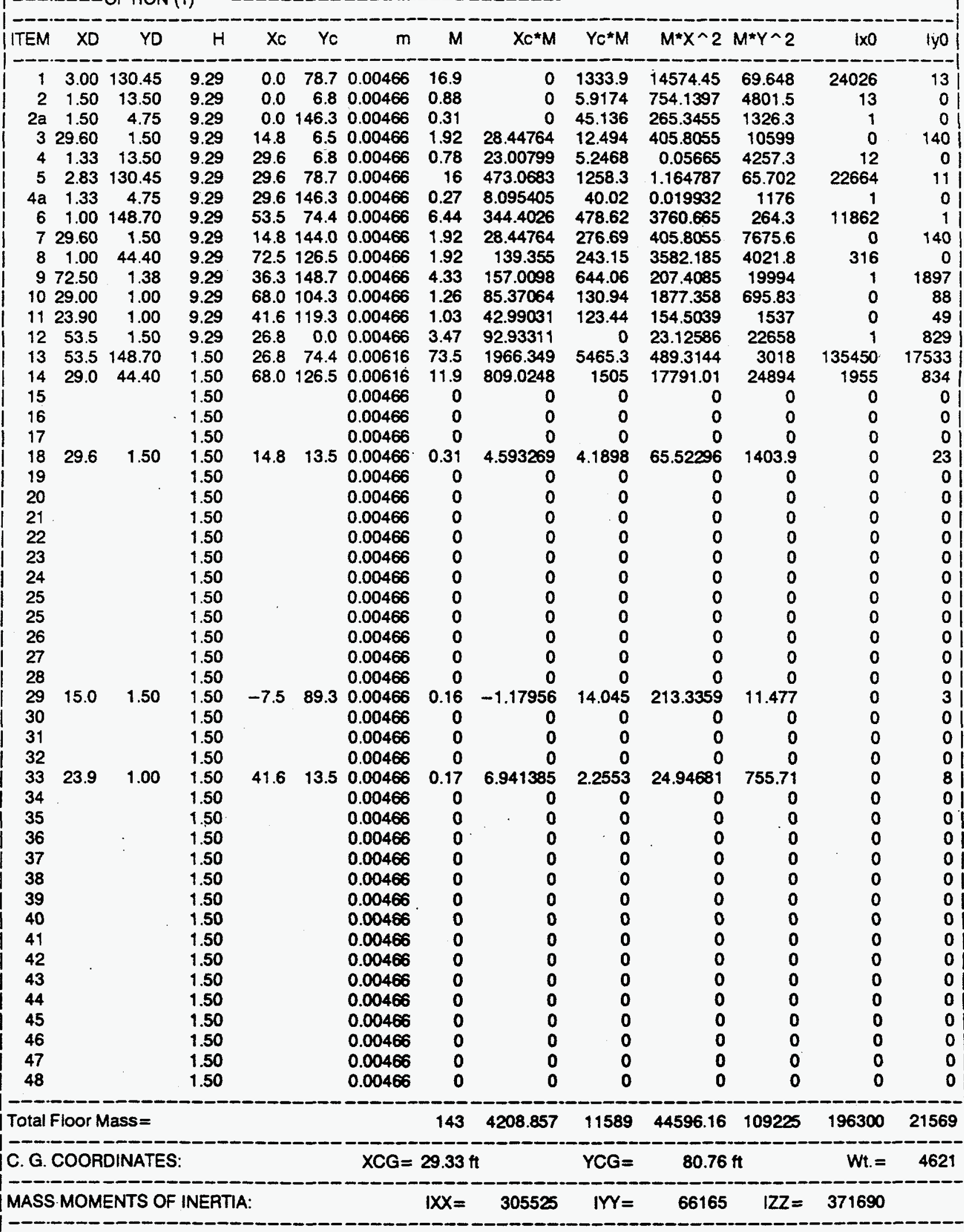

Units: Kips, Feet, Second
JOB NO.: $\quad 4:=$

DATE:

DATE:
SHEET: $3:-$ OF 


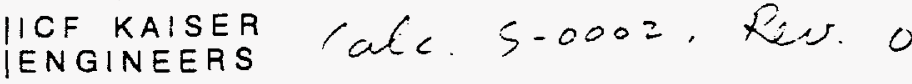

SUBJ---CHARGE DECK ELEV

1------OOPTION (1)

\begin{tabular}{|c|c|c|c|c|c|c|c|c|c|c|c|c|c|}
\hline ITEM & $X D$ & YD & $H$ & $x_{c}$ & Yc & $m$ & $M$ & $X c * M$ & $Y C \star M$ & $M=X \wedge 2$ & $M^{*} Y \wedge 2$ & $1 \times 0$ & lyo \\
\hline 1 & 3.00 & 130.45 & 9.29 & 0.0 & 78.7 & 0.00466 & 16.9 & 0 & 1333.9 & 8.31 & 107 & 4026 & 13 \\
\hline 2 & 1.50 & 13.50 & 9.29 & 0.0 & 6.8 & 0.00466 & 0.88 & 0 & 5.9174 & 790.0427 & 423.6 & 13 & 0 \\
\hline $2 a$ & 1.50 & 4.75 & 9.29 & 0.0 & 146.3 & 0.00466 & 0.31 & 0 & 45.136 & 277.978 & 1449.2 & 1 & 0 \\
\hline 3 & 29.60 & 1.50 & 10.50 & 14.8 & 6.5 & 0.00466 & 2.17 & 32.15288 & 14.121 & 503.26 & 11040 & 0 & 159 \\
\hline 4 & 1.33 & 13.50 & 9.29 & 29.6 & 6.8 & 0.00466 & 0.78 & 23.00799 & 5.2468 & 0.137172 & 3922.3 & 12 & 0 \\
\hline 5 & 2.83 & 130.45 & 9.29 & 29.6 & 78.7 & 0.00466 & 16 & 473.0683 & 1258.3 & 2.820397 & 14.251 & 22664 & 11 \\
\hline $4 a$ & 1.33 & 4.75 & 9.29 & 29.6 & 146.3 & 0.00466 & 0.27 & 8.095405 & 40.02 & 0.048264 & 1285 & 1 & 0 \\
\hline 6 & 1.00 & 148.70 & 9.29 & 53.5 & 74.4 & 0.00466 & 6.44 & 344.4026 & 478.62 & 3548.997 & 75.989 & 11862 & 1 \\
\hline 7 & 29.60 & 1.50 & 10.50 & 14.8 & 144.0 & 0.00466 & 2.17 & 32.15238 & 312.73 & 503.26 & 9510.5 & 0 & 159 \\
\hline 8 & 1.00 & 44.40 & 9.29 & 72.5 & 126.5 & 0.00466 & 1.92 & 139.355 & 243.15 & 3468.581 & 4561.4 & 316 & 0 \\
\hline 9 & 72.50 & 1.38 & 9.29 & 36.3 & 148.7 & 0.00466 & 4.33 & 157.0098 & 644.06 & 168.1058 & 21781 & 1 & 1897 \\
\hline 10 & 29.00 & 1.00 & 9.29 & 68.0 & 104.3 & 0.00466 & 1.26 & 85.37064 & 130.94 & 1810.955 & 2.59 & 0 & 88 \\
\hline 11 & 23.90 & 1.00 & 9.29 & 41.6 & 119.3 & 0.00466 & 1.03 & 42.99031 & 123.44 & 472 & 1783.2 & 0 & 49 \\
\hline 12 & 53.5 & 1.50 & 9.29 & 26.8 & 0.0 & 0.00466 & 3.47 & 92.93311 & 0 & 37.15055 & 21021 & 1 & 829 \\
\hline 13 & & & & & & & 0 & 0 & 0 & 0 & 0 & 0 & 0 \\
\hline 14 & & & & & & & 0 & 0 & 0 & 0 & 0 & 0 & 0 \\
\hline 15 & 23.9 & 148.70 & 1.00 & 65.5 & 74.4 & 0.00616 & 21.9 & 1432.845 & 1627.7 & 27480.82 & 258.42 & 40339 & 1042 \\
\hline 16 & 3.0 & 130.45 & 1.21 & 29.6 & 78.7 & 0.00466 & 2.21 & 65.31732 & 173.72 & 417 & 468 & 3129 & 2 \\
\hline 17 & 1.5 & 4.75 & 1.21 & 29.5 & 146.3 & 0.00466 & 0.04 & 1.185163 & 5.8786 & 0.010867 & 188.73 & 0 & 0 \\
\hline 18 & 29.6 & 1.50 & 9.29 & 14.8 & 13.5 & 0.00466 & 1.92 & 28.44764 & 25.949 & 653 & 3.5 & 0 & 140 \\
\hline 19 & 2.8 & 143.95 & 1.21 & 0.0 & 72.0 & 0.0 & 2.3 & 0 & 165.33 & 109 & 558 & 3967 & 2 \\
\hline 20 & 1.5 & 6.50 & 1.21 & 29.6 & 3.3 & 466 & 0.05 & 1.6273 & 0.1787 & 702 & .43 & 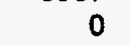 & 0 \\
\hline 21 & 1.3 & 148.70 & 1.21 & 53.5 & 74.4 & 466 & 1.05 & 56.072 & 77.924 & 103 & 372 & 1931 & 0 \\
\hline 22 & 1.0 & 15.00 & 1.21 & 72.5 & 111.8 & 466 & 0.08 & 6.131978 & 59 & 264 & 855 & 2 & 01 \\
\hline 23 & 1.3 & 4.75 & 1.21 & 0.0 & 146.3 & 0.00466 & 0.04 & 0 & 5.2124 & 32.10262 & 167.34 & 0 & 01 \\
\hline 24 & 11.0 & 1.00 & 1.21 & 137.0 & 128.7 & 0.00466 & 0.06 & 8.49737 & 7.9826 & 709.8531 & 160.78 & 0 & 11 \\
\hline 25 & 0.9 & 128.70 & 1.21 & 131.5 & 64.4 & 0.00466 & 0.62 & 82.06804 & 40.16 & 6427.002 & 112.66 & 861 & 01 \\
\hline & & & & & & 0.00000 & 0 & 0 & 0 & 0 & 0 & 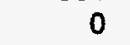 & 01 \\
\hline 26 & 1.0 & 20.00 & 1.21 & 142.5 & 138.7 & 0.00466 & 0.11 & 16.07001 & 15.641 & .761 & .45 & 4 & 0 \\
\hline 27 & 72.5 & 1.12 & 1.21 & 36.3 & 148.7 & 466 & 0.46 & 16.59722 & 68.083 & 016 & 2.5 & 0 & 201 \\
\hline 28 & 70.0 & 1.00 & 1.21 & 107.5 & 148.7 & 0.0 & 0.39 & 42.43047 & 58 & .45 & 34.9 & 0 & 161 \\
\hline 29 & 15.0 & 1.50 & 10.50 & -7.5 & 89.3 & 0.0 & 1.1 & -8.25694 & 13 & .835 & 145.96 & 0 & 21 \\
\hline 30 & 78.0 & 0.84 & 1.21 & 92.5 & 59.3 & 0.00466 & 0.37 & 34.1733 & 21.908 & 1442.202 & 126.25 & 0 & 187 \\
\hline 31 & & & & & & 466 & 0 & 0 & 0 & 0 & 0 & 0 & 0 \\
\hline 32 & & & & & & 0.00466 & 0 & 0 & 0 & 0 & 0 & 0 & 0 \\
\hline 33 & 23.9 & 1.00 & 10.50 & 41.6 & 13.5 & 0.00466 & 1.17 & 48.58969 & 15.787 & 623 & 4832.8 & 0 & 56 \\
\hline 34 & 53.5 & 1.33 & 1.21 & 26.8 & 0.0 & 0.00466 & 0.4 & 10.73249 & 0 & 4.290375 & 2427.6 & 0 & 96 \\
\hline 35 & 78.0 & 1.00 & 1.21 & 92.5 & 0.0 & 0.0 & 0.44 & 40.6825 & 0 & 1716.907 & 2661.1 & 0 & 223 \\
\hline 36 & 19.0 & 0.67 & 1.21 & 63.0 & 104.3 & 0.00466 & 0.07 & 4.522101 & 7.4866 & 78.07261 & 50.461 & 0 & 2 \\
\hline 37 & & & & & & 0.00466 & 0 & 0 & 0 & 0 & 0 & 0 & 0 \\
\hline 38 & & & & & & 0.00466 & 0 & 0 & 0 & 0 & 0 & 0 & 0 \\
\hline 39 & & & & & & 0.00465 & 0 & 0 & 0 & 0 & 0 & 0 & 0 \\
\hline 40 & & & & & & 0.00466 & 0 & 0 & 0 & 0 & 0 & 0 & 0 \\
\hline 41 & & & & & & 0.00466 & 0 & 0 & 0 & 0 & 0 & 0 & 0 \\
\hline 42 & & & & & & 0.0 & 0 & 0 & 0 & 0 & 0 & 0 & 0 \\
\hline 43 & & & & & & 0.00466 & 0 & 0 & 0 & 0 & 0 & 0 & 0 \\
\hline 44 & & & & & & 0.00465 & 0 & 0 & 0 & 0 & 0 & 0 & 0 \\
\hline 45 & 53.5 & 130.45 & 1.00 & 26.8 & 78.7 & 0.02340 & 163 & 4368.552 & 12857 & 1746.354 & 144.08 & 231590 & 38953 \\
\hline 46 & & & & & & 0.00468 & 0 & 0 & 0 & 0 & 0 & 0 & 0 \\
\hline 47 & & & & & & 0.00466 & 0 & 0 & 0 & 0 & 0 & 0 & 0 \\
\hline 48 & & & & & & 0.0 & 0 & 0 & 0 & 0 & 0 & 0 & 0 \\
\hline otal & Floor A & Aass $=$ & & & & & 256 & 7686.824 & 19918 & 74920.3 & 106186 & 340721 & 44290 \\
\hline \multicolumn{5}{|c|}{ C. G. COORDINATES: } & \multicolumn{4}{|c|}{$X C G=30.02 \mathrm{ft}$} & $Y C G=$ & \multicolumn{2}{|c|}{$77.79 \mathrm{ft}$} & $W \mathrm{t} .=$ & 8245 \\
\hline & MON & ENIS & & & & & $1 X X=$ & 446907 & IYY $=$ & 119211 & $\mathbf{I Z Z}=$ & 566117 & \\
\hline
\end{tabular}

| Units: Kips, Feet, Second

SHEET: $2 G \quad$ OF

JOB NO: $4: \div=$

DATE:

DATE: 


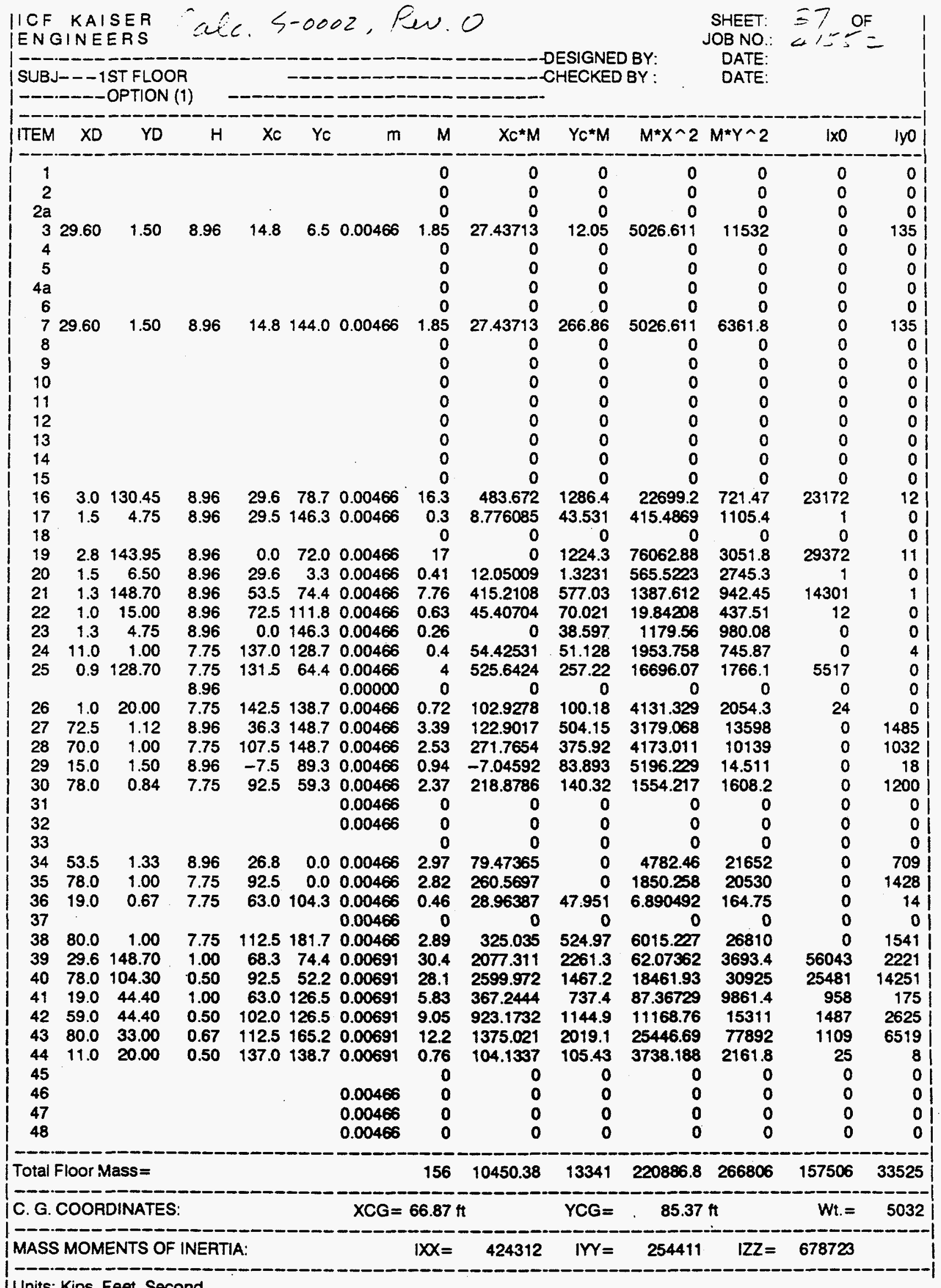


IICF KAISER

IENGINEERS

SUBJ--

| SUBJ- - ROOF

- --_--OPTION (1)

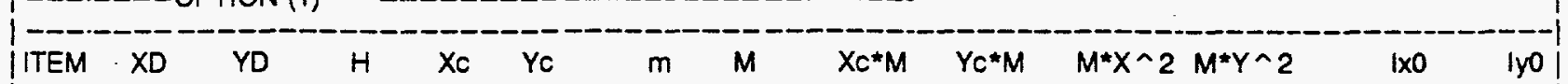$$
\left\{\begin{array}{l}
1 \\
\hdashline
\end{array}\right.
$$$$
2
$$$$
\begin{array}{|lllllll}
2 \mathrm{a} & & & & & & \\
3 & 29.60 & 1.50 & 7.75 & 14.8 & 6.5 & 0.00466
\end{array}
$$$$
5
$$$$
6
$$$$
17
$$$$
8
$$$$
9
$$$$
10
$$$$
11
$$$$
12
$$$$
13
$$$$
14
$$$$
15
$$$$
116
$$$$
\begin{array}{r}
17 \\
18
\end{array}
$$$$
20
$$$$
12
$$$$
123
$$$$
\begin{array}{lll}
23 & 1.3 & 4.75
\end{array}
$$$$
25
$$$$
126
$$$$
\text { I }
$$$$
128
$$$$
130
$$$$
1
$$$$
1
$$$$
\{3
$$$$
335
$$$$
3.0 \quad 130.45
$$$$
\begin{array}{lr}
1.5 & 4.75
\end{array}
$$

$\begin{array}{llll}29.6 & 78.7 & 0.00466\end{array}$ $\begin{array}{llll}29.5 & 146.3 & 0.00466\end{array}$

$\begin{array}{llll}0.0 & 72.0 & 0.00466\end{array}$ $\begin{array}{lll}29.6 & 3.3 & 0.00466\end{array}$ $\begin{array}{llll}53.5 & 74.4 & 0.00466\end{array}$ $\begin{array}{llll}72.5 & 111.8 & 0.00466\end{array}$ 0.0146 .30 .00466 137.0128 .70 .00466 $131.5 \quad 64.4 \quad 0.00466$

$$
0.00000
$$

$\begin{array}{llll}142.5 & 138.7 & 0.00466\end{array}$

36.3148 .70 .00466

$\begin{array}{llll}107.5 & 148.7 & 0.00466\end{array}$

$\begin{array}{llll}-7.5 & 89.3 & 0.00466\end{array}$

$\begin{array}{llll}92.5 & 59.3 & 0.00466\end{array}$

$\begin{array}{llll}26.8 & 74.4 & 0.01183\end{array}$

$\begin{array}{llll}92.5 & 74.4 & 0.00578\end{array}$

$\begin{array}{llll}26.8 & 0.0 & 0.00466\end{array}$

$\begin{array}{llll}92.5 & 0.0 & 0.00466\end{array}$

$\begin{array}{llll}63.0 & 104.3 & 0.00466\end{array}$

$\begin{array}{llll}112.5 & 165.0 & 0.00578\end{array}$

$\begin{array}{llll}112.5 & 181.7 & 0.00466\end{array}$

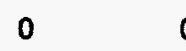

0

6$$
0
$$

0

0

1.6

1.6
0

$$
0
$$$$
0
$$$$
0
$$$$
0
$$$$
0
$$

0

0.26

0

14.7

0.35

6.71

0.54

0.23

0.4

4
0

0.72

2.93

0.81

2.37

94.1

57.7

0

2.57

0.46

13.1

2.89
SHEET: 38 OF JOBNO.: 41552

DATE:

DATE:

0

3.73189

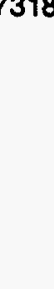

418.3547

7.590922<smiles>CCC</smiles>

10.42279

359.1388

39.27506

54.42531

525.6424

102.9278

106.3045

271.7654

$-6.09441$

218.8786

2517.522

5333.025

68.74116

260.5697

28.96387

1476.328

325.035

10.4

0
0

0

0

0

0

$$
\begin{array}{rr}
023 & 2398.044 \\
0 &
\end{array}
$$$$
\begin{aligned}
& 0 \\
& 0 \\
& 0
\end{aligned}
$$

$\begin{array}{rr}0 & 0 \\ 23082 & 2398.044\end{array}$

2398.044
0

$$
\begin{aligned}
& 0 \\
& 0 \\
& 0 \\
& 0 \\
& 0 \\
& 0 \\
& 0 \\
& 0
\end{aligned}
$$

1112.7

37.652

1058.9

1.1444

499.1

60.565

33.385

51.128

257.22

100.18

436.07

375.92

72.564

140.32

7002

4289.5

0
0
0

47.951

2165.3

524.97

41.73946

$$
1478
$$

147.8666

42066.24

200.6591

0.005376

196.1457

652.3503

2771.709

24337.1

5724.97

869.7526

7379.522

3020.833

3604.299

67201.3

87819.53

1834.937

4290.832

45724.69

10066.96
0
0
0
8982
0
0
0
0

6285.1

0

0

0
0

0

0

0

96.878

1086.6

1291.2

2147.4

328.28

502.52

963.42

890.94

1154.3

2376.2

13305

11470

51.447

1149.8

4536.9

2779.3

17003

18639

242.29

91840

29099

$-$

$$
01
$$

39

40

| 41

| 42

43

| 44

45

46

47
48

0.00466

0.00466

0.00466

Total Floor Mass=

C. G. COORDINATES: 
IICF KAISER
IENGINEERS lale. \$-000Z, Kin. O

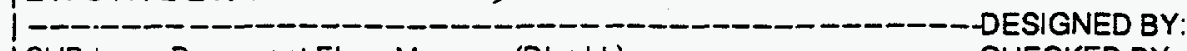

SUBJ-- -Basement Floor Mass (DL+LL)

1--.--OPTION (1)

(1---

ITEM XD YD H XO YC $m$ M M

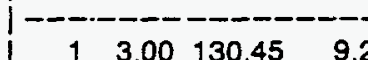

1

1

1

1

$14 a$

1

CHECKED BY

$Y c * M$

0.88

o 5.9174

0.31

28.44764

45.136

0.78

23.00799

12.494

$\begin{array}{rr}16 & 473.0683 \\ 0.27 & 8.095405\end{array}$

$6.44 \quad 344.4026$

40.02

\begin{tabular}{ll}
$1.92 \quad 28.44764$ \\
\hline
\end{tabular}

478.62

139.355

6.69

4.33

157.0098

644.06

\section{1}

$\mid \begin{array}{llll}17 & & & 1.50 \\ 18 & 29.6 & 1.50 & 1.50\end{array}$

$\begin{array}{lll}19 & 29.6 & 1.50 \quad 1.50 \\ 20 & 1.50\end{array}$

20

2

22

23

24

25

| 25

26

| 27

28

| 29

\begin{tabular}{|}
30 \\
$\quad 31$
\end{tabular}

I 32

3

\begin{tabular}{l}
34 \\
\hline
\end{tabular}

35
36

36
37

| 38

| 39

\begin{tabular}{l}
40 \\
\hline
\end{tabular}

I 41

| 42

I 44

45
46

\begin{tabular}{l}
46 \\
\hline
\end{tabular}

\begin{tabular}{l}
47 \\
\hline
\end{tabular}

$\begin{array}{ll}47 & 1.50 \\ 48 & 1.50\end{array}$

Total Floor Mass=

1.50

1.50

1.50

1.50

1.50

1.50

1.50

1.50

1.50

1.50

$\begin{array}{lll}15.0 & 1.50 \quad 1.50\end{array}$

1.50

1.50

1.50

$\begin{array}{lll}23.9 & 1.00 \quad 1.50\end{array}$

1.50

1.50

1.50

1.50

1.50

1.50

1.50

1.50

1.50

1.50

1.50

1.50

$\begin{array}{ll}0.00466 \\ -7.5 & 89.30 .00465\end{array}$

$\begin{array}{lll}-7.5 & 89.3 & 0.00466\end{array}$

0.00466

0.00466

0.00466

$\begin{array}{lll}41.6 & 13.5 & 0.00466\end{array}$

0.00466

0.00466

0.00466

0.00466

0.00466

0.00466

0.00466

0.00466

0.00466

0.00466

0.00466

0.00466

0.00466

0.00466

0.00466

SHEET:

JOB NO:

DATE:

DATE: $\div i$ OF

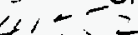

\section{G COORDINATES. \\ C. G. COORDINATES:}

$197 \quad 5957.877$

$M^{\star} X \wedge 2 \quad M^{\star} Y \wedge 2$

IxO

|yo

$\begin{array}{lllll}6.9 & 0 & 1333.9 & 15445.4 & 86.6\end{array}$

799.206

86.638

86.638
4831.9

24026

281.2021

1316.8

455.4802

0.273962

10666

5.632951

4284.3

0.096394

81.728

3496.713

1167.6

455.4802

3440.29

7619

3980.8

85.37064

130.94

158.868

1794.438

19857

1.0342 .99031

123.44

133.4366

682.08

1518.4

$\begin{array}{lllll}92.93311 & 0 & 41.19951 & 22789\end{array}$

$\begin{array}{llll}120 & 3205.527 & 8909.6 & 1421.088\end{array}$

5285.5

$\begin{array}{|lllllll|}9.4 & 1318.866 & 2453.5 & 27721.76 & 40168 & 3186 & 1359\end{array}$

$\begin{array}{|lllllll|}9.4 & 1318.866 & 2453.5 & 27721.76 & 40168 & 3186 & 1359\end{array}$

$\begin{array}{|lllllll|}9.4 & 1318.866 & 2453.5 & 27721.76 & 40168 & 3186 & 1359\end{array}$

220809

$\begin{array}{|lllllll|}9.4 & 1318.866 & 2453.5 & 27721.76 & 40168 & 3186 & 1359\end{array}$

8583

$\mathrm{XCG}=30.19 \mathrm{ft}$

YCG

55969.11126804

$282891 \quad 33144$

I MASS MOMENTS OF INERTIA:

$1 X X=409695$

$\mathrm{YCG}=$

$80.99 \mathrm{tt}$

$W t=6354$

Units: Kips, Feet, Second 
IICF KAISER Calc. S-0002, Res. 0
IENGINEERS SUBJ-- Basement Floor Mass (1.4DL + 1.7LL) 1-----OOPTION (1)

| - - - - - - - - - - - - - - -

IITEM XD YD H $X_{C} Y_{C} \quad m$ M $X_{C}$

\begin{tabular}{|c|c|c|c|c|c|c|}
\hline 1 & 3.00 & 130.45 & 9.29 & 0.0 & 78.7 & 0.00652 \\
\hline 2 & 1.50 & 13.50 & 9.29 & 0.0 & 6.8 & 0.00652 \\
\hline $2 a$ & 1.50 & 4.75 & 9.29 & 0.0 & 146.3 & 0.00652 \\
\hline 3 & 29.60 & 1.50 & 9.29 & 14.8 & 6.5 & 0.00652 \\
\hline 4 & 1.33 & 13.50 & 9.29 & 29.6 & 6.8 & 0.00652 \\
\hline 5 & 2.83 & 130.45 & 9.29 & 29.6 & 78.7 & 0.00652 \\
\hline $4 a$ & 1.33 & 4.75 & 9.29 & 29.6 & 146.3 & 0.00652 \\
\hline 6 & 1.00 & 148.70 & 9.29 & 53.5 & 74.4 & 0.00652 \\
\hline 7 & 29.60 & 1.50 & 9.29 & 14.8 & 144.0 & 0.00652 \\
\hline 8 & 1.00 & 44.40 & 9.29 & 72.5 & 126.5 & 0.00652 \\
\hline 9 & 72.50 & 1.38 & 9.29 & 36.3 & 148.7 & 0.00652 \\
\hline 10 & 29.00 & 1.00 & 9.29 & 68.0 & 104.3 & 0.00652 \\
\hline 11 & 23.90 & 1.00 & 9.29 & 41.6 & 119.3 & 0.00652 \\
\hline 12 & 53.5 & 1.50 & 9.29 & 26.8 & 0.0 & 0.00652 \\
\hline 13 & 53.5 & 148.70 & 1.50 & 26.8 & 74.4 & 0.01562 \\
\hline 14 & 29.0 & 44.40 & 1.50 & 68.0 & 126.5 & 0.01562 \\
\hline 15 & & & & & & \\
\hline 16 & & & & & & \\
\hline 17 & & & & & & \\
\hline 18 & 29.6 & 1.50 & 1.50 & 14.8 & 13.5 & 0.00652 \\
\hline 19 & & & & & & \\
\hline 20 & & & & & & \\
\hline 21 & & & & & & \\
\hline 22 & & & & & & \\
\hline 23 & & & & & & \\
\hline 24 & & & & & & \\
\hline 25 & & & & & & \\
\hline $\begin{array}{l}25 \\
26\end{array}$ & & & & & & \\
\hline 27 & & & & & & \\
\hline
\end{tabular}

30

$\begin{array}{lllllll}15.0 & 1.50 & 1.50 & -7.5 & 89.3 & 0.00652 & 0.22\end{array}$

31

32

34

23.

1.00

$\begin{array}{lllll}1.50 & 41.6 & 13.5 & 0.00652 & 0.23\end{array}$

\section{7}

.23

\section{.43}

32.

32.8267

7.3455

11.33357

482.1636

39.8267

195.097

219.8137

119.5189

60.18643

130.1064

4986.455

2051.603

0

0
0.43

0
0
0

6.43057

0
076

YC*M

0

0
8657

0

0

0

0

0
35
36

I 37

38

39

40
41

42

43

44

I 45

46

47

48

$M^{*} X^{\wedge} 2 M^{*} Y \wedge 2$

$20404.23 \quad 97.508$

055.796

371.4836

6722.1

1856.8

14839

14839
5960.3

$\begin{array}{lll}1761.6 & 1.630702 & 91.982\end{array}$

$\begin{array}{lll}56.028 & 0.027905 & 1646.3\end{array}$

$\begin{array}{lll}670.07 & 5264.931 & 370.02\end{array}$

$\begin{array}{lll}387.37 & 568.1277 & 10746\end{array}$

$\begin{array}{lll}340.41 & 5015.059 & 5630.6\end{array}$

$\begin{array}{lll}901.69 & 290.3719 & 27992\end{array}$

$\begin{array}{lll}183.32 & 2628.302 & 974.16\end{array}$

$\begin{array}{lll}172.81 & 216.3055 & 2151.8\end{array}$

$\begin{array}{ll}0 & 32.37621\end{array}$

$13860 \quad 1240.85$

$3816.6 \quad 45116.14$

31721

7653.4

63128

0
0

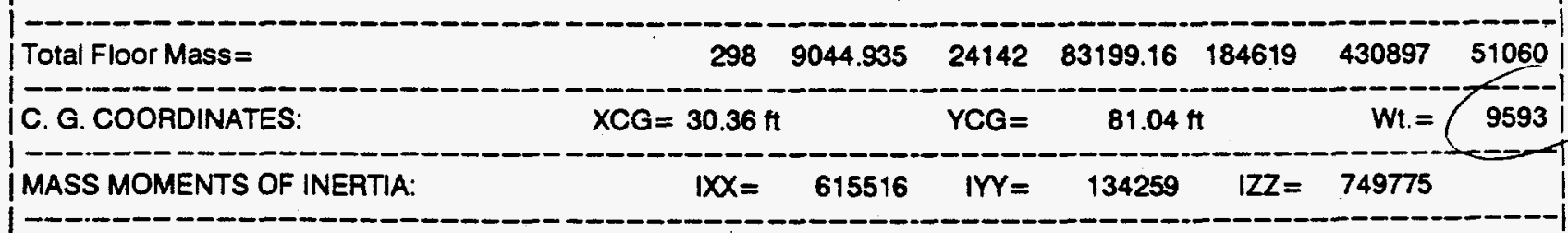

I Units: Kips, Feet, Second 
IICF KAISER

IENGINEERS (akC. S-0002, kin. 0

SUBJ-- CHARGE DECKELEV. (DL+LL)

SHEET:

JOB NO: $\angle \square-\angle O=$

DATE:

- $-----O O P T I O N(1)$

|

IITEM XD YD $H$ XC YC $m \quad M \quad X C * M \quad Y_{C}^{*} M \quad M * X \sim 2 M * Y \wedge 2$

\begin{tabular}{|c|c|c|c|c|c|c|c|c|c|c|c|c|c|}
\hline 1 & 3.00 & 130.45 & 9.29 & 0.0 & 78.7 & 0.00466 & 16.9 & 0 & 1333.9 & 15268.31 & 15.107 & 24026 & 13 \\
\hline 2 & 1.50 & 13.50 & 9.29 & 0.0 & 6.8 & 0.00466 & 0.88 & 0 & 5.9174 & 790.0427 & 4423.6 & 13 & 0 \\
\hline $2 a$ & 1.50 & 4.75 & 9.29 & 0.0 & 146.3 & 0.00466 & 0.31 & 0 & 45.136 & 277.978 & 1449.2 & 1 & 0 \\
\hline 3 & 29.60 & 1.50 & 10.50 & 14.8 & 6.5 & 0.00466 & 2.17 & 32.15288 & 14.121 & 503.26 & 11040 & 0 & 159 \\
\hline 4 & 1.33 & 13.50 & 9.29 & 29.6 & 6.8 & 0.00466 & 0.78 & 23.00799 & 5.2468 & 0.137172 & 3922.3 & 12 & 0 \\
\hline 5 & 2.83 & 130.45 & 9.29 & 29.6 & 78.7 & 0.00466 & 16 & 473.0683 & 1258.3 & 2.820397 & 14.251 & 22664 & 11 \\
\hline $4 a$ & 1.33 & 4.75 & 9.29 & 29.6 & 146.3 & 0.00466 & 0.27 & 8.095405 & 40.02 & 0.048264 & 1285 & 1 & 0 \\
\hline 6 & 1.00 & 148.70 & 9.29 & 53.5 & 74.4 & 0.00466 & 6.44 & 344.4026 & 478.62 & 3548.997 & 75.989 & 11862 & 1 \\
\hline 7 & 29.60 & 1.50 & 10.50 & 14.8 & 144.0 & 0.00466 & 2.17 & 32.15288 & 312.73 & 503.26 & 9510.5 & 0 & 159 \\
\hline 8 & 1.00 & 44.40 & 9.29 & 72.5 & 126.5 & 0.00466 & 1.92 & 139.355 & 243.15 & 3468.581 & 4561.4 & 316 & 0 \\
\hline$\theta$ & 72.50 & 1.38 & 9.29 & 36.3 & 148.7 & 0.00466 & 4.33 & 157.0098 & 644.06 & 168.1058 & 21781 & 1 & 1897 \\
\hline 10 & 29.00 & 1.00 & 9.29 & 68.0 & 104.3 & 0.00466 & 1.26 & 85.37064 & 130.94 & 1810.955 & & 0 & 88 \\
\hline 11 & 23.90 & 1.00 & 9.29 & 41.6 & 119.3 & 0.00466 & 1.03 & 42.99031 & 123.44 & 137.5472 & 1783.2 & 0 & 49 \\
\hline 12 & 53.5 & 1.50 & 9.29 & 26.8 & 0.0 & 0.00466 & 3.47 & 92.93311 & 0 & 37.15055 & 21021 & 1 & 829 \\
\hline 13 & & & & & & & 0 & 0 & 0 & 0 & 0 & 0 & 0 \\
\hline 14 & & & & & & & 0 & 0 & 0 & 0 & 0 & 0 & 0 \\
\hline 15 & 23.9 & 148.70 & 1.00 & 65.5 & 74.4 & 0.01198 & 42.6 & 2787.298 & 3166.3 & 53458.15 & 502.7 & 78472 & 2027 \\
\hline 16 & 3.0 & 130.45 & 1.21 & 29.6 & 78.7 & 0.00466 & 2.21 & 65.31732 & 173.72 & 0.389417 & 1.9468 & 3129 & 2 \\
\hline 17 & 1.5 & 4.75 & 1.21 & 29.5 & 146.3 & 0.00466 & 0.04 & 1.185163 & 5.8786 & 0.010867 & .73 & 0 & 0 \\
\hline 18 & 29.6 & 1.50 & 9.29 & 14.8 & 13.5 & 0.0 & 1.92 & 28.44764 & 49 & 445.2653 & 3.5 & 0 & 140 \\
\hline 19 & 2.8 & 143.95 & 1.21 & 0.0 & 72.0 & 0.00466 & 2.3 & 0 & 33 & 109 & 58 & 3967 & 2 \\
\hline 20 & 1.5 & 6.50 & 1.21 & 29.6 & 3.3 & 466 & 0.05 & 1.6273 & 0.1787 & 0.009702 & 43 & 0 & 0 \\
\hline 21. & 1.3 & 148.70 & 1.21 & 53.5 & 74.4 & 0.00466 & 1.05 & 56.072 & 77.924 & 577.8103 & 72 & 1931 & 0 \\
\hline 22 & 1.0 & 15.00 & 1.21 & 72.5 & 111.8 & 0.00466 & 0.08 & 6.131978 & 9.4 & 152.6264 & 55 & 2 & 0 \\
\hline 23 & 1.3 & 4.75 & 1.21 & 0.0 & 146.3 & 0.00466 & 0.04 & 0 & 5.2124 & 32.10262 & 167.34 & 0 & 0 \\
\hline 24 & 11.0 & 1.00 & 1.21 & 137.0 & 128.7 & 0.00466 & 0.06 & 8.49737 & 7.9826 & 709.8531 & 160.78 & 0 & 1 \\
\hline 25 & 0.9 & 128.70 & 1.21 & 131.5 & 64.4 & 0.00466 & 0.62 & 82.06804 & 40.16 & 6427.002 & 112.66 & 861 & 0 \\
\hline & & & & & & 0.00000 & 0 & 0 & 0 & 0 & 0 & 0 & \\
\hline 26 & 1.0 & 20.00 & 1.21 & 142.5 & 138.7 & 0.00466 & 0.11 & 16.07001 & 841 & 761 & .45 & 4 & 0 \\
\hline 27 & 72.5 & 1.12 & 1.21 & 36.3 & 148.7 & 466 & 0.46 & 9722 & 83 & 016 & 2.5 & 0 & 201 \\
\hline 28 & 70.0 & 1.00 & 1.21 & 107.5 & 148.7 & 0.00466 & 0.39 & 3047 & 92 & .45 & 4.9 & 0 & 161 \\
\hline 29 & 15.0 & 1.50 & 10.50 & -7.5 & 89.3 & 0.00466 & 1.1 & -8.25694 & 13 & 1549.835 & .96 & 0 & 21 \\
\hline 30 & 78.0 & 0.84 & 1.21 & 92.5 & 59.3 & 0.00466 & 0.37 & 34.1733 & 21.908 & 1442.202 & 126.25 & 0 & 187 \\
\hline 31 & & & & & & 0.00466 & 0 & 0 & 0 & 0 & 0 & 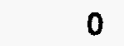 & 0 \\
\hline 32 & & & & & & 0.00466 & 0 & 0 & 0 & 0 & 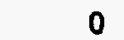 & 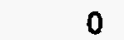 & 0 \\
\hline 33 & 23.9 & 1.00 & 10.50 & 41.6 & 13.5 & 0.00466 & 1.17 & 48.58969 & 15.787 & 155.4623 & 4832.8 & 0 & 56 \\
\hline 34 & 53.5 & 1.33 & 1.21 & 26.8 & 0.0 & 0.00466 & 0.4 & 10.73249 & 0 & 4.290375 & 7.6 & 0 & 96 \\
\hline 35 & 78.0 & 1.00 & 1.21 & 92.5 & 0.0 & 0.00466 & 0.44 & 40.6825 & 0 & 1716.907 & 1.1 & 0 & 223 \\
\hline 36 & 19.0 & 0.67 & 1.21 & 63.0 & 104.3 & 0.00466 & 0.07 & 4.522101 & 7.4866 & 78.07261 & 50.461 & 0 & 2 \\
\hline 37 & & & & & & 0.00466 & 0 & 0 & 0 & 0 & 0 & 0 & 0 \\
\hline 38 & & & & & & 0.00466 & 0 & 0 & 0 & 0 & 0 & 0 & 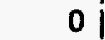 \\
\hline 39 & & & & & & 0.00466 & 0 & 0 & 0 & 0 & 0 & 0 & \\
\hline 40 & & & & & & 0.00465 & 0 & 0 & 0 & 0 & 0 & 0 & 0 \\
\hline 41 & & & & & & 0.00466 & 0 & 0 & 0 & 0 & 0 & 0 & \\
\hline 42 & & & & & & 0.00466 & 0 & 0 & 0 & 0 & 0 & 0 & 0 \\
\hline 43 & & & & & & 0.00466 & 0 & 0 & 0 & 0 & 0 & 0 & 0 \\
\hline 44 & & & & & & 0.00466 & 0 & 0 & 0 & 0 & 0 & 0 & \\
\hline 45 & 53.5 & 130.45 & 1.00 & 26.8 & 78.7 & 0.02689 & 188 & 5020.808 & 14776 & 2007.097 & 165.59 & 266169 & 44769 \\
\hline 46 & & & & & & 0.00466 & 0 & 0 & 0 & 0 & 0 & 0 & \\
\hline 47 & & & & & & 0.00466 & 0 & 0 & 0 & 0 & 0 & 0 & 0 \\
\hline 48 & & & & & & 0.00466 & 0 & 0 & 0 & 0 & 0 & 0 & \\
\hline
\end{tabular}

Total Floor Mass $=$

$\begin{array}{lllllll}301 & 9693.533 & 23376 & 101158.4 & 106452 & 413431 & 51091\end{array}$

C. G. COORDINATES:
MASS MOMENTS OF INERTIA: $\mathrm{XCG}=32.19 \mathrm{ft}$

I Units: Kips, Feet, Second 


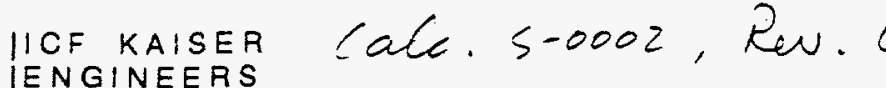
IENGINEERS

-

SUBJ---CHARGE DECK ELEV. (1.4DL + 1.7LL)---_-_--CHECKED BY :

SHEET: - ¿ OF

JOBNO: $\angle: \div=$

DATE:

- - OPTION (1)

DATE:

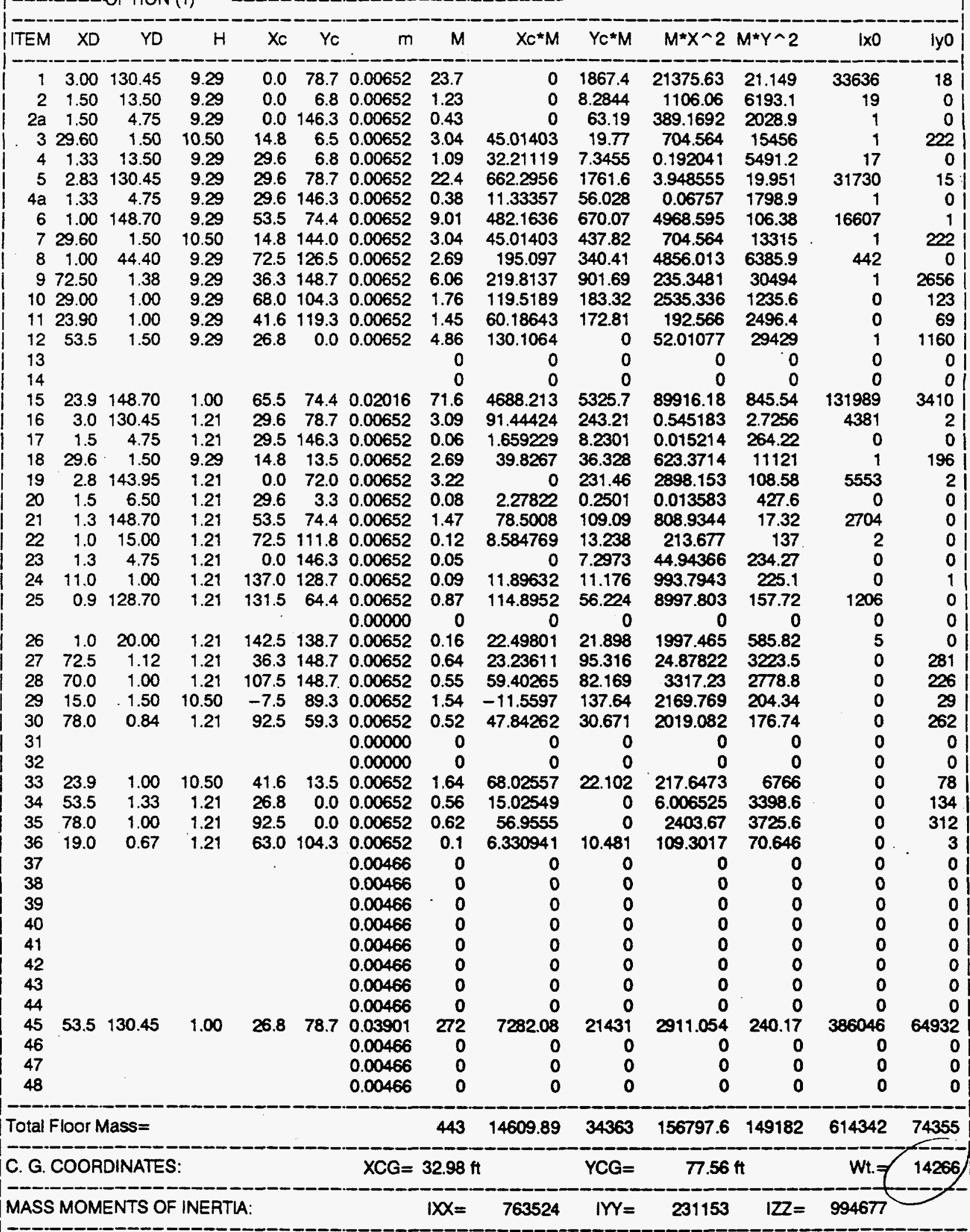

Units: Kips, Feet, Second 
IICF KAISER

IENGINEERS. (ale,,-0002 , Ris. 0

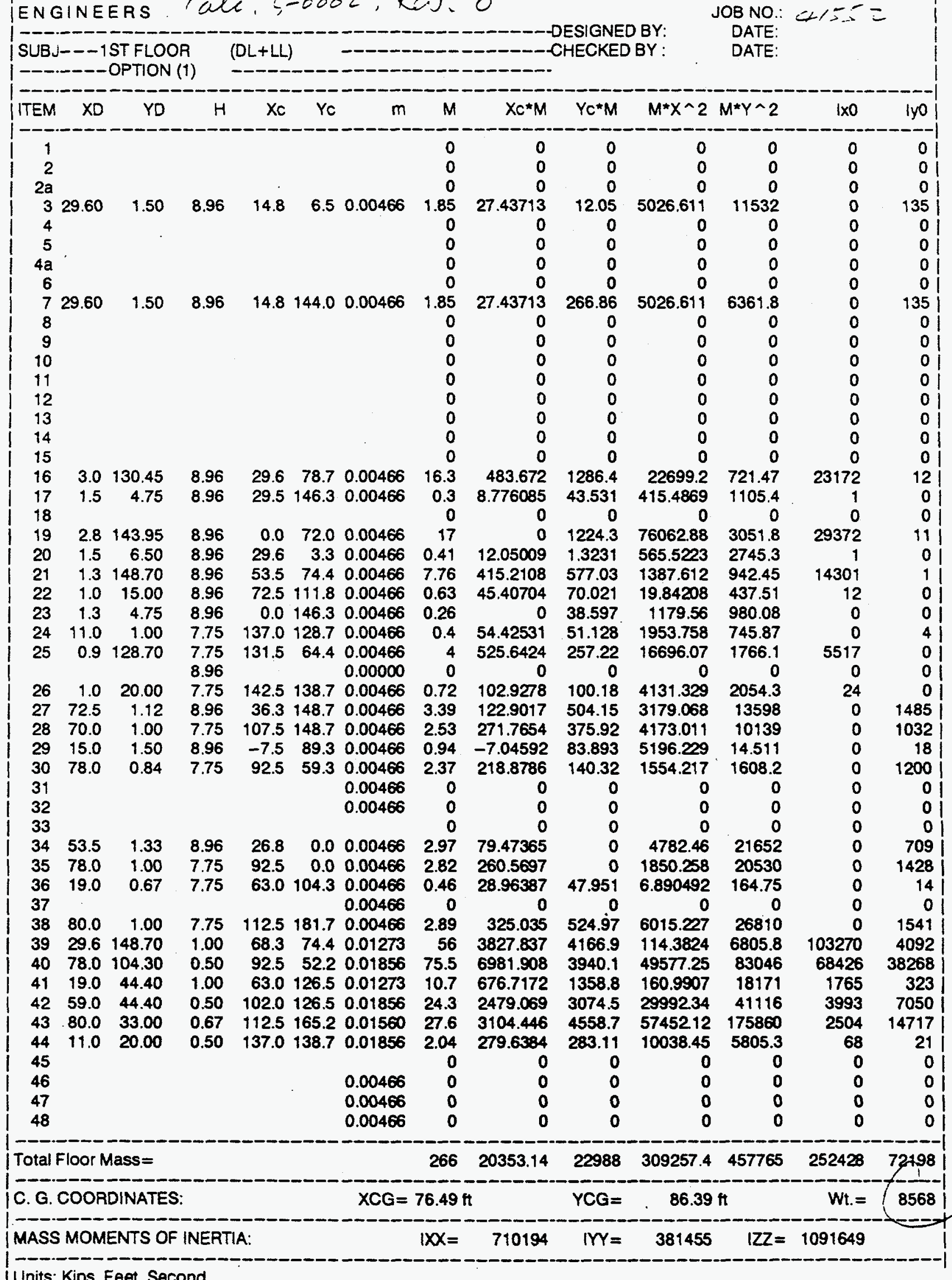

Units: Kips, Feet, Second 


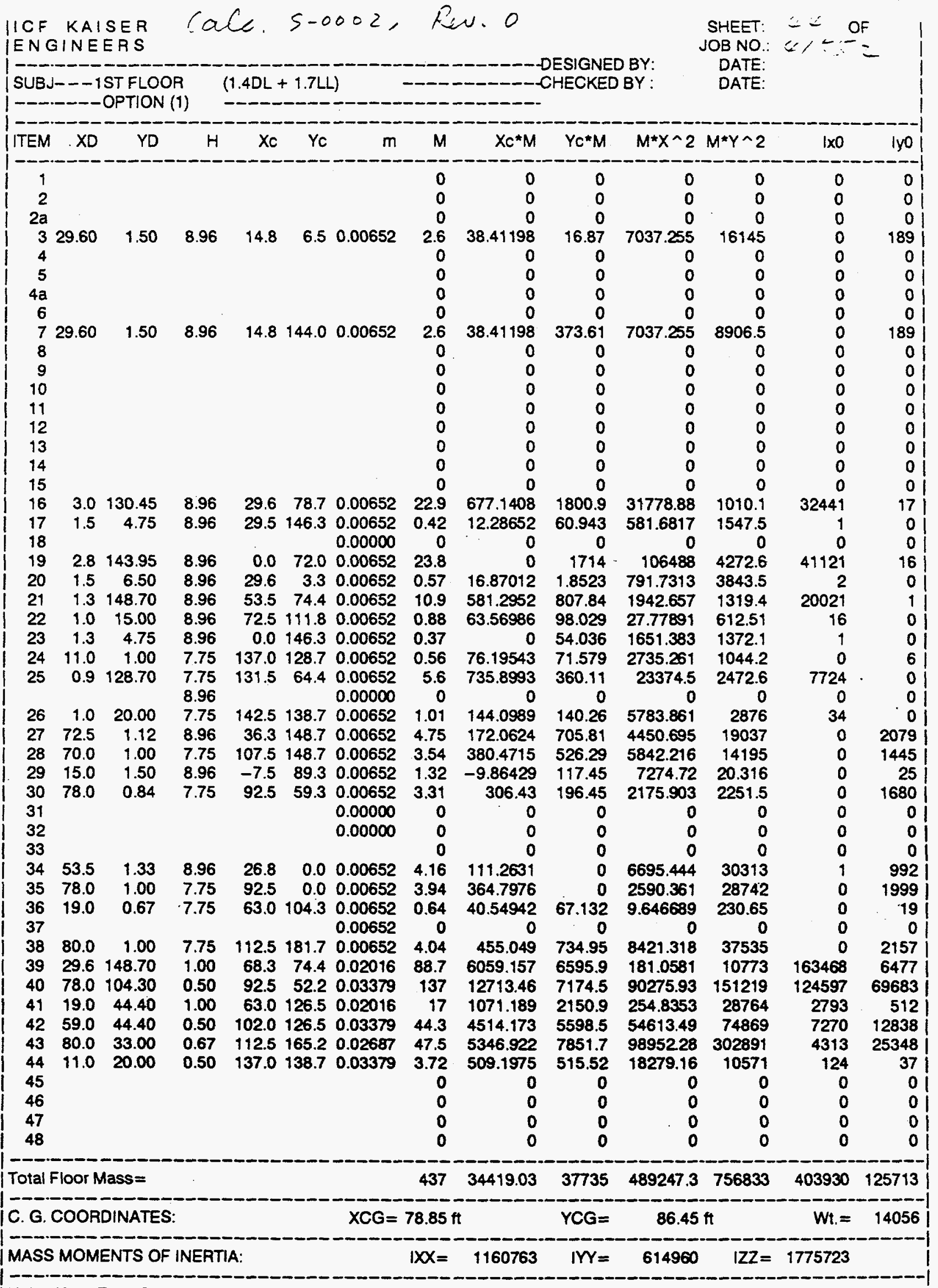

Units: Kips, Feet, Second 


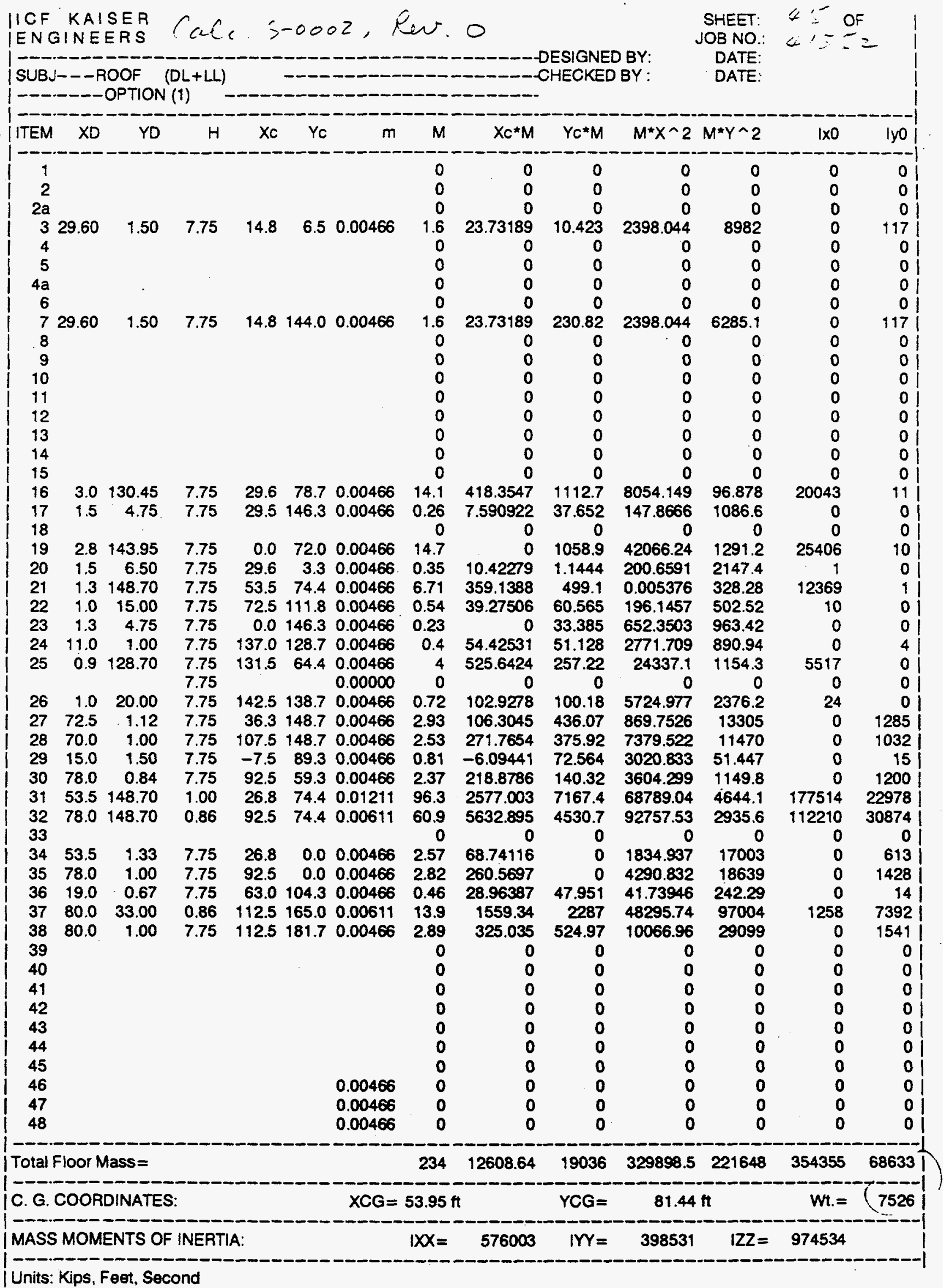


IICF KAISER
IENGINEERS ?ale. S-0002, Kis. o

|

SUBJ--DOOF (1.4DL + 1.7LL)--

1---- - OPTION (1)

\begin{tabular}{|c|c|c|c|c|c|c|c|c|c|c|c|c|c|}
\hline ITEM & XD & YD & $\mathrm{H}$ & $X c$ & Yc & $m$ & $M$ & $X_{C} * M$ & $Y C * M$ & $M * X^{\wedge} 2$ & $M^{\star} Y \sim 2$ & $1 \times 0$ & lyo \\
\hline 1 & & & & & & & 0 & 0 & 0 & 0 & 0 & 0 & 0 \\
\hline 2 & & & & & & & 0 & 0 & 0 & 0 & 0 & 0 & 0 \\
\hline $2 a$ & & & & & & & 0 & 0 & 0 & 0 & 0 & 0 & 0 \\
\hline 3 & 29.60 & 1.50 & 7.75 & 14.8 & 6.5 & 0.00652 & 2.24 & 33.22464 & 14.592 & 3357.262 & 12575 & 0 & 164 \\
\hline 4 & & & & & & & 0 & 0 & 0 & 0 & 0 & 0 & 0 \\
\hline 5 & & & & & & & 0 & 0 & 0 & 0 & 0 & 0 & 0 \\
\hline $4 a$ & & & & & & & 0 & 0 & 0 & 0 & 0 & 0 & 0 \\
\hline 6 & & & & & & & 0 & 0 & 0 & 0 & 0 & 0 & 0 \\
\hline 7 & 29.60 & 1.50 & 7.75 & 14.8 & 144.0 & 0.00652 & 2.24 & 33.22464 & 323.15 & 3357.262 & 8799.2 & 0 & 164 \\
\hline 8 & & & & & & & 0 & 0 & 0 & 0 & 0 & 0 & 0 \\
\hline 9 & & & & & & & 0 & 0 & 0 & 0 & 0 & 0 & 0 \\
\hline 10 & & & & & & & 0 & 0 & 0 & 0 & 0 & 0 & 0 \\
\hline 11 & & & & & & & 0 & 0 & 0 & 0 & 0 & 0 & 0 \\
\hline 12 & & & & & & & 0 & 0 & 0 & 0 & 0 & 0 & 0 \\
\hline 13 & & & & & & & 0 & 0 & 0 & 0 & 0 & 0 & 0 \\
\hline 14 & & & & & & & 0 & 0 & 0 & 0 & 0 & 0 & 0 \\
\hline 15 & & & & & & & 0 & 0 & 0 & 0 & 0 & 0 & 0 \\
\hline 16 & 3.0 & 130.45 & 7.75 & 29.6 & 78.7 & 0.00652 & 19.8 & 585.6966 & 1557.7 & 11275.81 & 135.63 & 28060 & 15 \\
\hline 17 & 1.5 & 4.75 & 7.75 & 29.5 & 146.3 & 0.00652 & 0.36 & 10.62729 & 52.713 & 207.0133 & 1521.2 & 1 & 0 \\
\hline 18 & & & & & & & 0 & 0 & 0 & 0 & 0 & 0 & 0 \\
\hline 19 & 2.8 & 143.95 & 7.75 & 0.0 & 72.0 & 0.00652 & 20.6 & 0 & 1482.5 & 58892.74 & 1807.7 & 35568 & 14 \\
\hline 20 & 1.5 & 6.50 & 7.75 & 29.6 & 3.3 & 0.00652 & 0.49 & 14.5919 & 1.6022 & 280.9228 & 3006.4 & 2 & 01 \\
\hline 21 & 1.3 & 148.70 & 7.75 & 53.5 & 74.4 & 0.00652 & 9.4 & 502.7944 & 698.74 & 0.007526 & 459.6 & 17317 & 11 \\
\hline 22 & 1.0 & 15.00 & 7.75 & 72.5 & 111.8 & 0.00652 & 0.76 & 54.98509 & 84.791 & 274.604 & 703.52 & 14 & 0 \\
\hline 23 & 1.3 & 4.75 & 7.75 & 0.0 & 146.3 & 0.00652 & 0.32 & 0 & 46.739 & 913.2904 & 1348.8 & 1 & 01 \\
\hline 24. & 11.0 & 1.00 & 7.75 & 137.0 & 128.7 & 0.00652 & 0.56 & 76.19543 & 71.579 & 3880.392 & 1247.3 & 0 & $6 i$ \\
\hline 25 & 0.9 & 128.70 & $\begin{array}{l}7.75 \\
7.75\end{array}$ & 131.5 & 64.4 & $\begin{array}{l}0.00652 \\
0.00000\end{array}$ & $\begin{array}{r}5.6 \\
0\end{array}$ & $\begin{array}{r}735.8993 \\
0\end{array}$ & $\begin{array}{r}360.11 \\
0\end{array}$ & $\begin{array}{r}34071.95 \\
0\end{array}$ & $\begin{array}{r}1616 \\
0\end{array}$ & $\begin{array}{r}7724 \\
0\end{array}$ & $\begin{array}{l}0 \\
0\end{array}$ \\
\hline 26 & 1.0 & 20.00 & 7.75 & 142.5 & 138.7 & 0.00652 & 1.01 & 144.0989 & 140.26 & 8014.968 & 3326.7 & 34 & 01 \\
\hline 27 & 72.5 & 1.12 & 7.75 & 36.3 & 148.7 & 0.00652 & 4.11 & 148.8263 & 610.5 & 1217.654 & 18627 & 0 & 1798 \\
\hline 28 & 70.0 & 1.00 & 7.75 & 107.5 & 148.7 & 0.00652 & 3.54 & 380.4715 & 526.29 & 10331.33 & 16057 & 0 & 1445 \\
\hline 29 & 15.0 & 1.50 & 7.75 & -7.5 & 89.3 & 0.00652 & 1.14 & -8.53217 & 101.59 & 4229.167 & 72.025 & 0 & 21 \\
\hline 30 & 78.0 & 0.84 & 7.75 & 92.5 & 59.3 & 0.00652 & 3.31 & 306.43 & 196.45 & 5046.018 & 1609.7 & 0 & 1680 \\
\hline 31 & 53.5 & 148.70 & 1.00 & 26.8 & 74.4 & 0.01724 & 137 & 3667.969 & 10202 & 97910.68 & 6610.1 & 252664 & 32706 \\
\hline 32 & 78.0 & 148.70 & 0.86 & 92.5 & 74.4 & 0.00888 & 88.6 & 8195.131 & 6591.5 & 134950.2 & 4270.9 & 163251 & 44918 \\
\hline 33 & & & & & & & 0 & 0 & 0 & 0 & 0 & 0 & 0 \\
\hline 34 & 53.5 & 1.33 & 7.75 & 26.8 & 0.0 & 0.00652 & 3.6 & 96.23762 & 0 & 2568.912 & 23805 & 1 & 858 \\
\hline 35 & 78.0 & 1.00 & 7.75 & .92 .5 & 0.0 & 0.00652 & 3.94 & 364.7976 & 0 & 6007.164 & 26095 & 0 & 1999 . \\
\hline 36 & 19.0 & 0.67 & 7.75 & 63.0 & 104.3 & 0.00652 & 0.64 & 40.54942 & 67.132 & 58.43524 & 339.21 & 0 & 19 \\
\hline 37 & 80.0 & 33.00 & 0.86 & 112.5 & 165.0 & 0.00888 & 20.2 & 2268.637 & 3327.3 & 70264.03 & 141129 & 1830 & 10755 \\
\hline 38 & 80.0 & 1.00 & 7.75 & 112.5 & 181.7 & 0.00652 & 4.04 & 455.049 & 734.95 & 14093.74 & 40738 & 0 & 2157 \\
\hline 39 & & & & & & & 0 & 0 & 0 & 0 & 0 & 0 & 0 \\
\hline 40 & & & & & & & 0 & 0 & 0 & 0 & 0 & 0 & 0 \\
\hline 41 & & & & & & & 0 & 0 & 0 & 0 & 0 & 0 & 0 \\
\hline 42 & & & & & & & 0 & 0 & 0 & 0 & 0 & 0 & 0 \\
\hline 43 & & & & & & & 0 & 0 & 0 & 0 & 0 & 0 & 0 \\
\hline 44 & & & & & & & 0 & 0 & 0 & 0 & 0 & 0 & 0 \\
\hline 45 & & & & & & & 0 & 0 & 0 & 0 & 0 & 0 & 0 \\
\hline 46 & & & & & & & 0 & 0 & 0 & 0 & 0 & 0 & 0 \\
\hline 47 & & & & & & & 0 & 0 & 0 & 0 & 0 & 0 & 0 \\
\hline 48 & & & & & & & 0 & 0 & 0 & 0 & 0 & 0 & 0 \\
\hline \multicolumn{7}{|c|}{ Total Floor Mass= } & 334 & 18106.9 & 27192 & 471203.5 & 315899 & 506468 & 8722 \\
\hline \multicolumn{6}{|c|}{ C. G. COORDINATES: } & \multicolumn{3}{|c|}{$X C G=54.28 \mathrm{ft}$} & $Y C G=$ & \multicolumn{2}{|c|}{$81.52 \mathrm{ft}$} & $W_{t}=$ & 10741 \\
\hline \multicolumn{7}{|c|}{ MASS MOMENTS OF INERTIA: } & $1 X X=$ & 822367 & $r Y=$ & 569925 & $\mid Z Z=$ & 3922292 & \\
\hline
\end{tabular}

Units: Kips, Feet, Second
SHEET: 26 OF

JOB NO.: $\angle: \div=$

DATE:

DATE: 


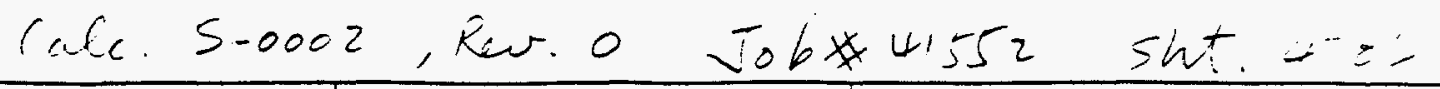

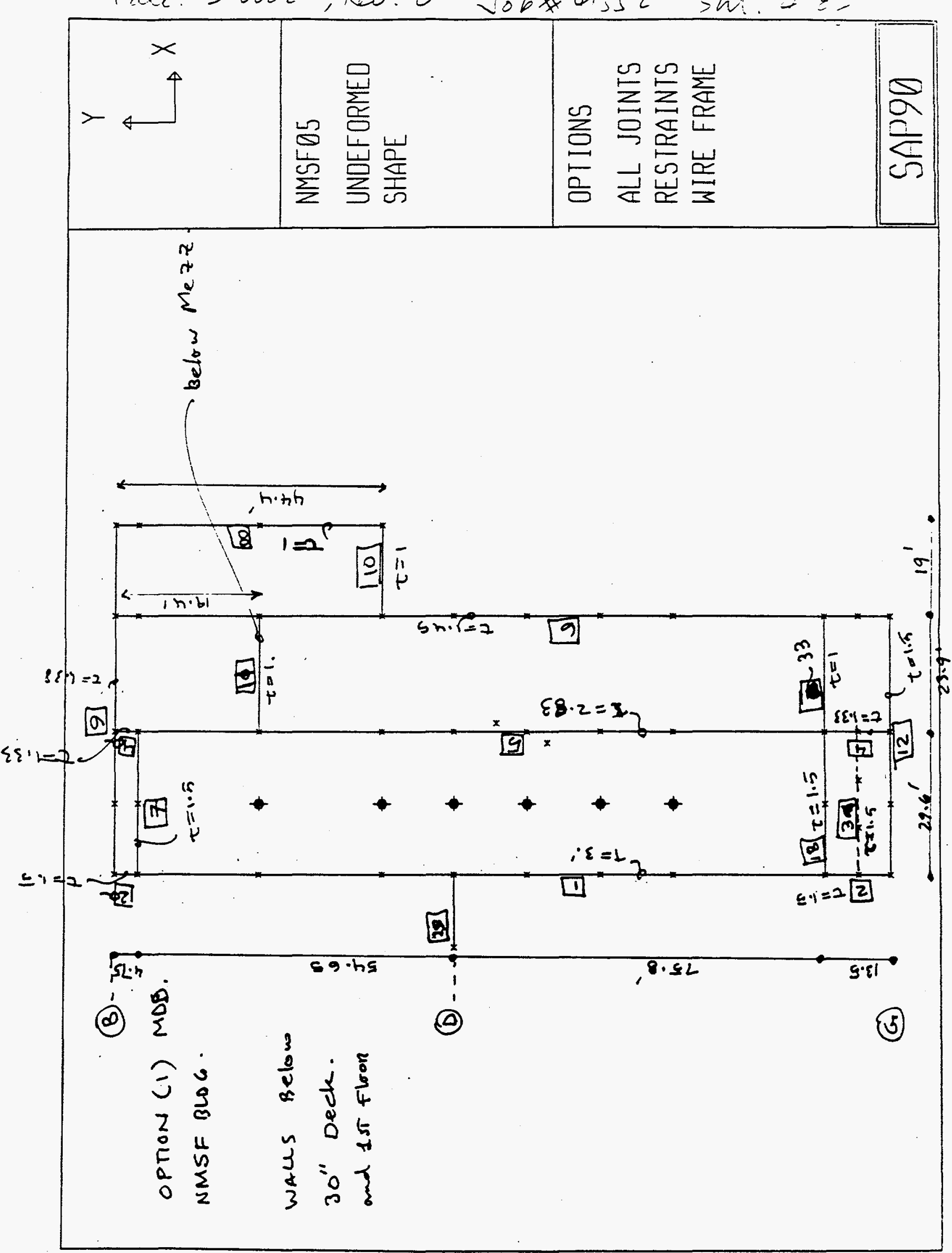


ase S-0002, Res. O Vob 4552

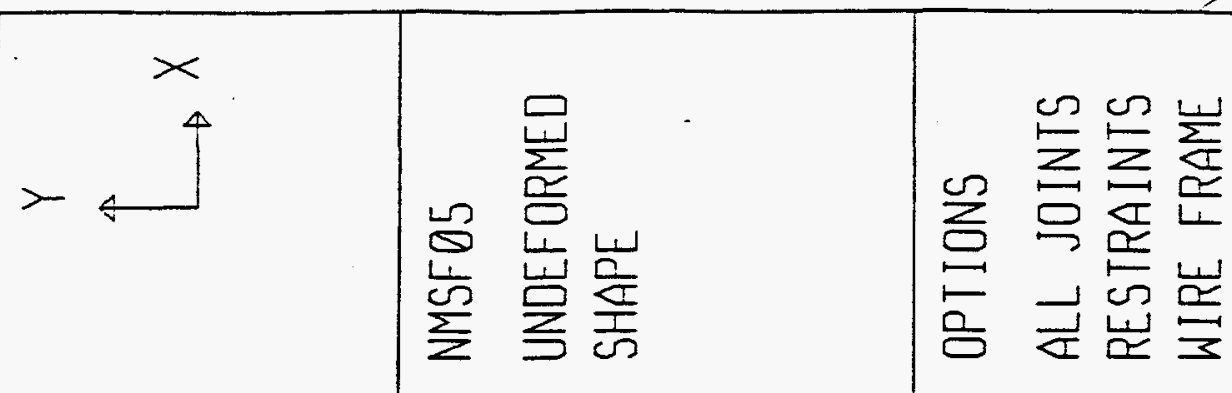

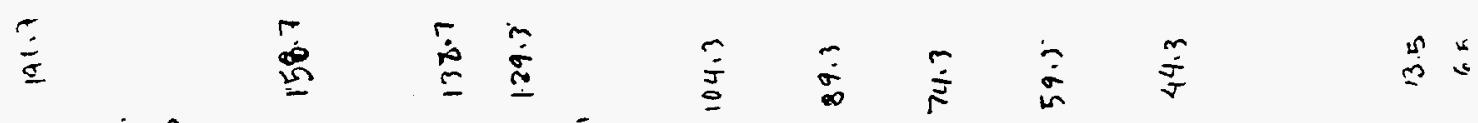

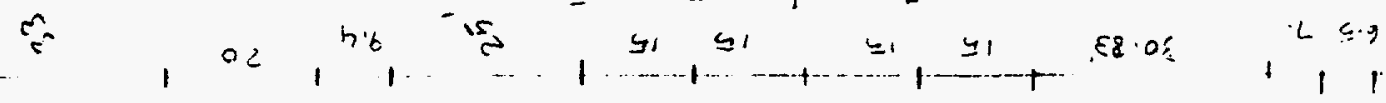

15

-

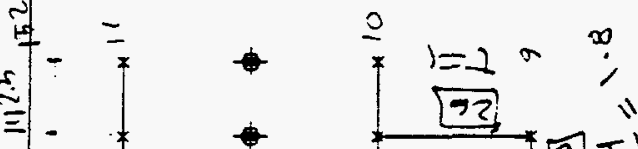

$\stackrel{\sigma}{0}-\left[\begin{array}{l}\infty \\ m\end{array}\right.$

$1=2$ से

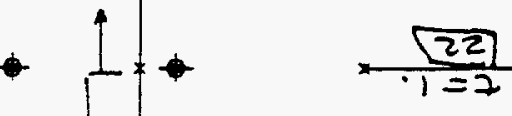

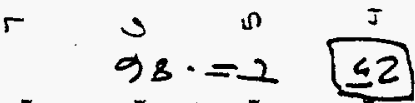

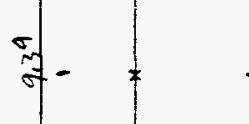

$\stackrel{\sim}{\sim}-$

is.

-

$\sqrt{1=2}$

स]

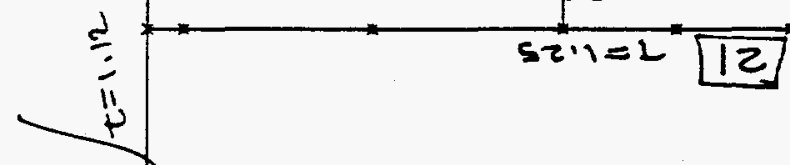

$+5_{\infty}^{5}$

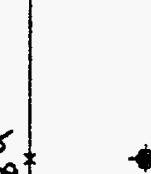

8

$\stackrel{n}{n}$

in $0.25=2$ in

$+4$

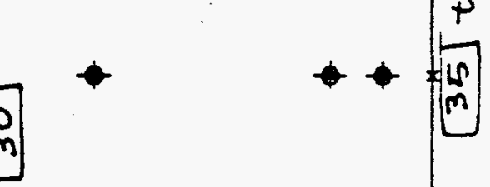

$-\left(\left\{E^{\prime}\right)=2\right)$

$\varepsilon 2$

जิ

b] $88 \cdot T=2$

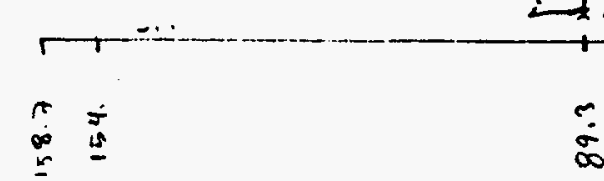




$$
7
$$


cake. S-0002, Rev. 0 Tob $\times 41552$

$1+5=2-2$

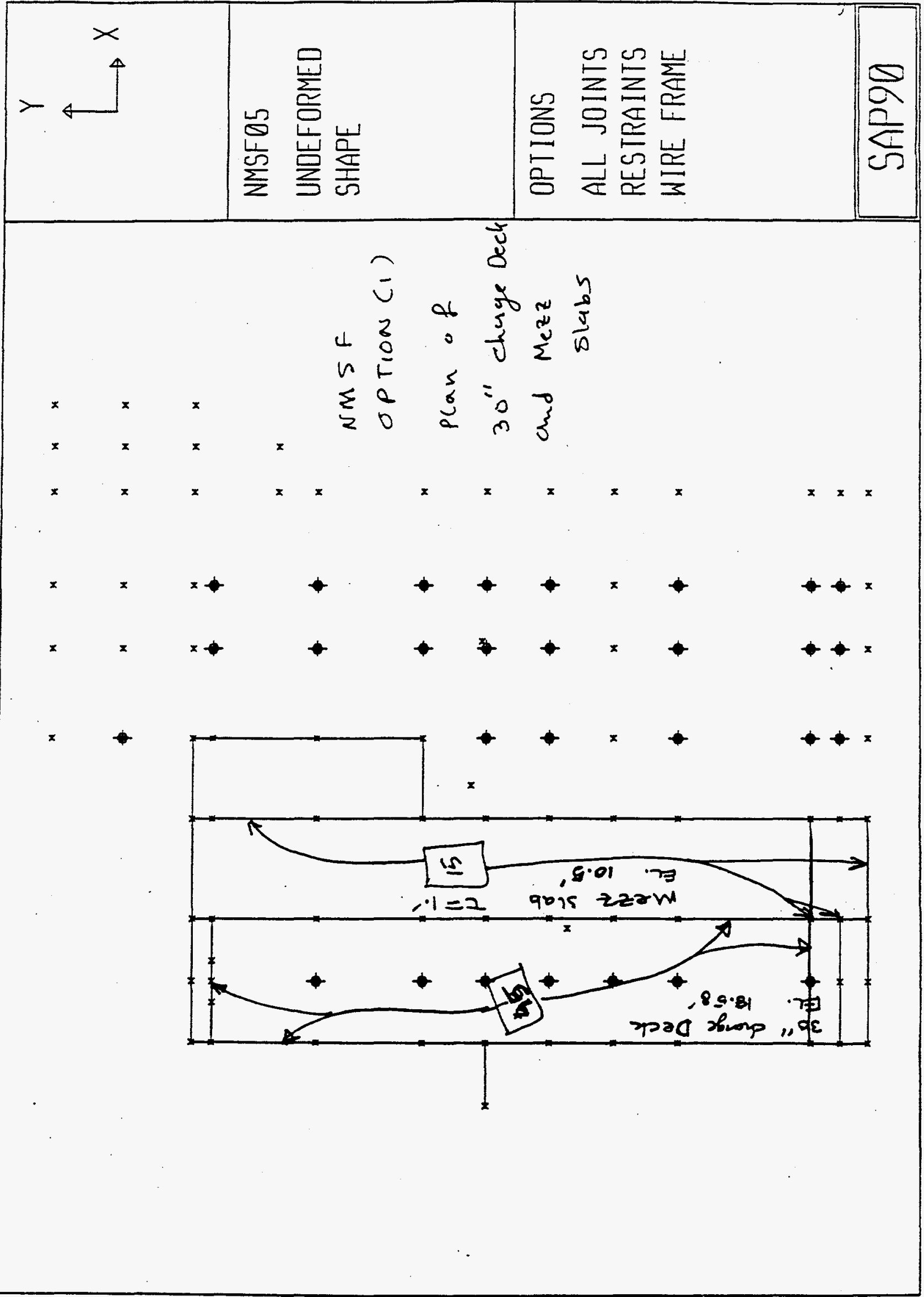



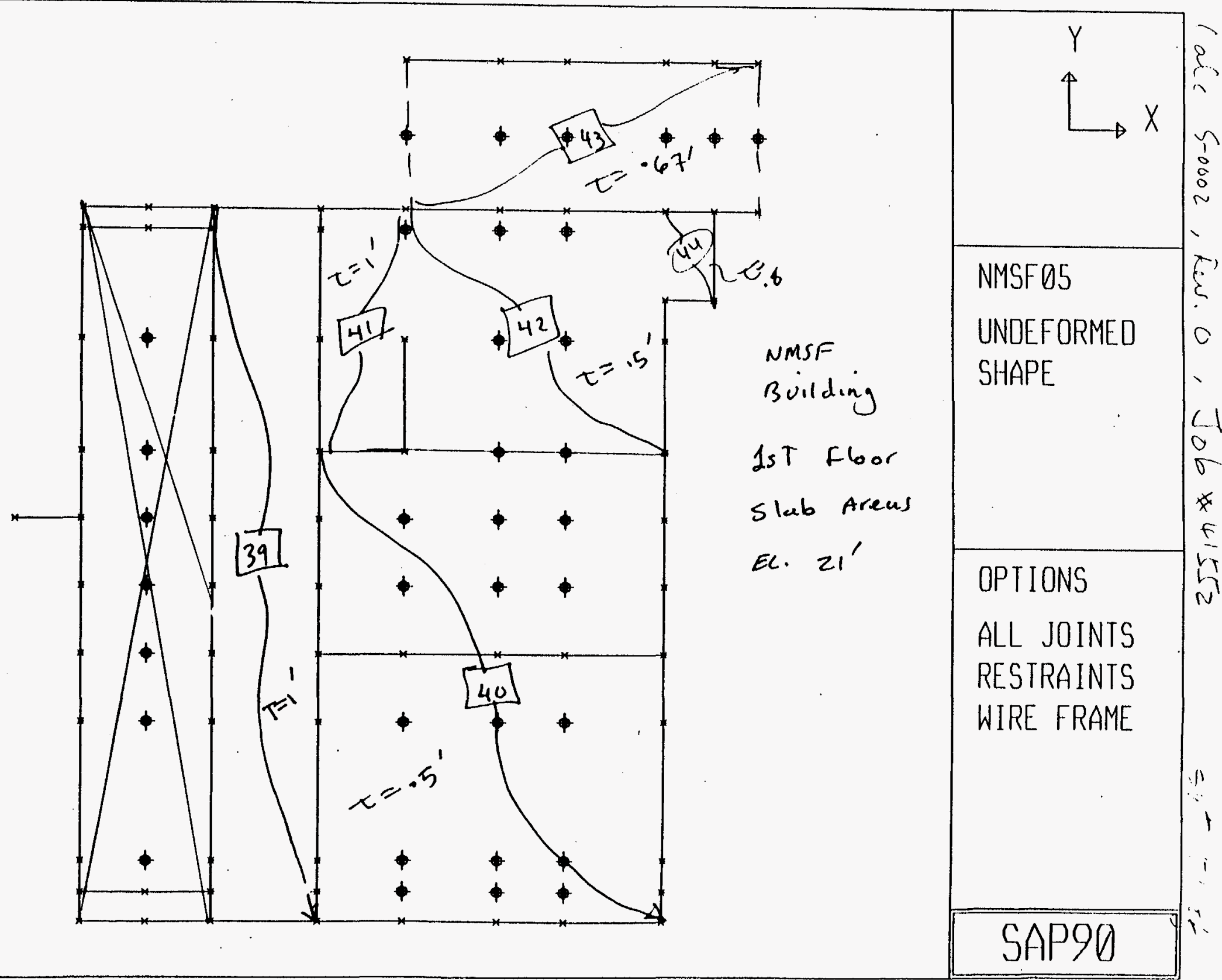


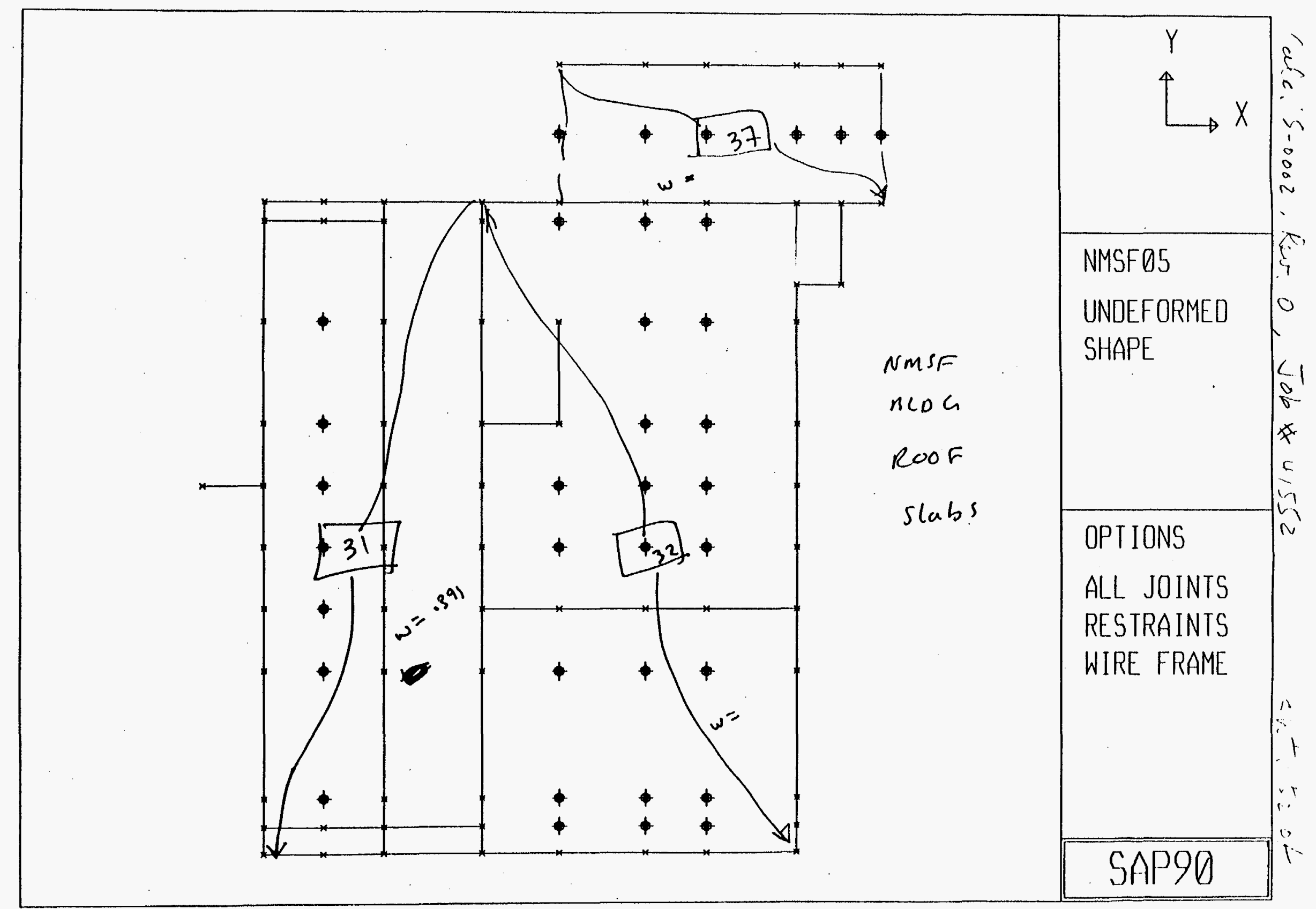


ENGINEERS (ale S.000Z, Riv. O

Jos No.

SMEET $\therefore$ OF

EVAL of NMSF D LANL

MEIFICATIOL $3^{\circ}$.S

DTe... Sol trassur DISTRIBUTION

DESIGNED BY DMATE $3 / 1 / 2=$

App'y $C_{2}$ wast, seoth on Nowth wall.
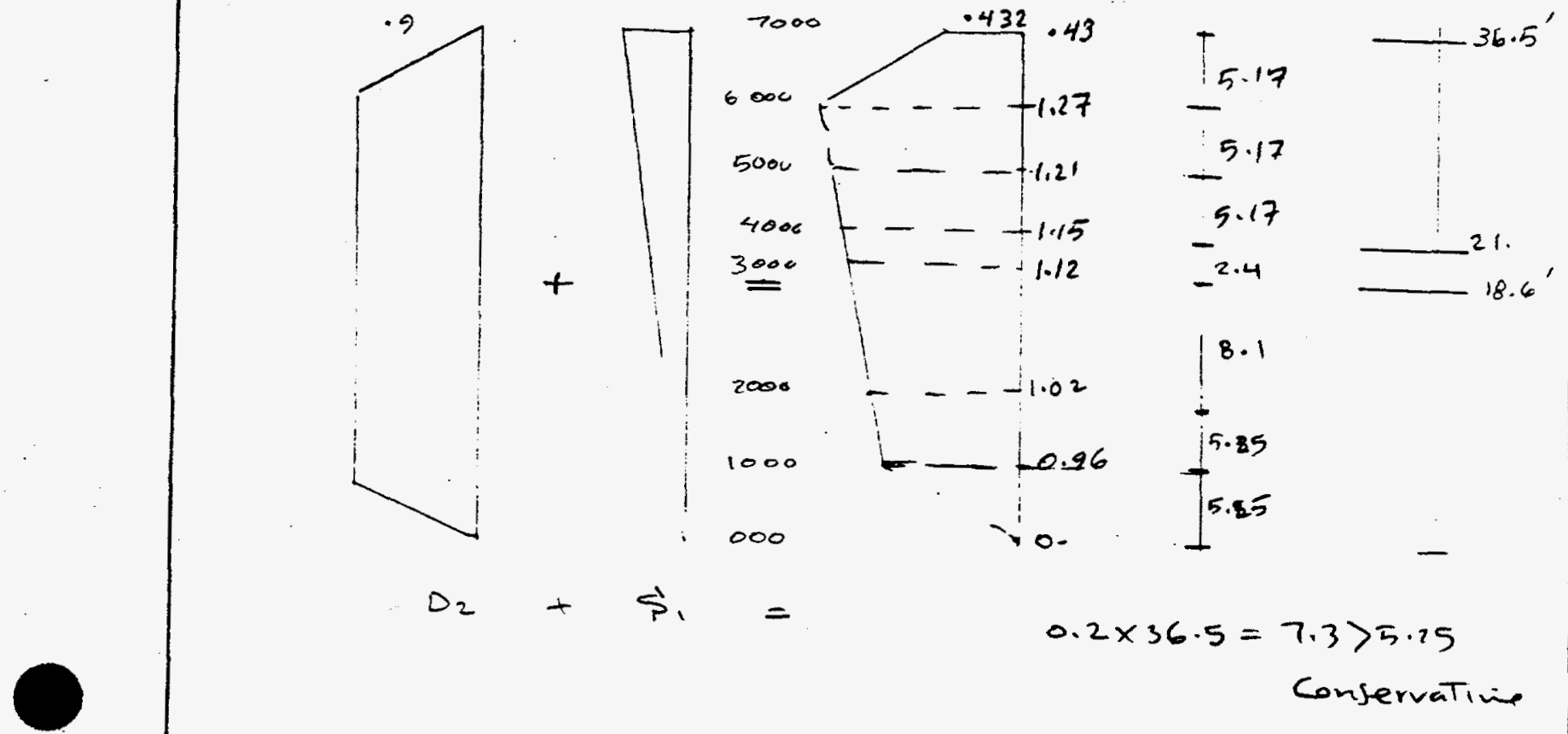

$D_{2}+S_{1}=$

$$
0.2 \times 36.5=7.3>5.15
$$




\begin{tabular}{|c|c|c|c|}
\hline 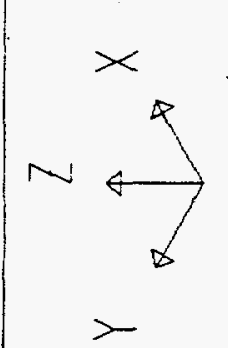 & 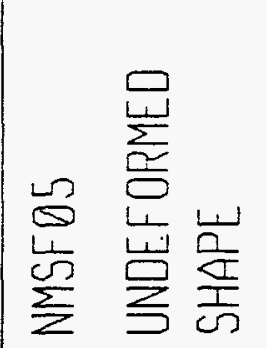 & 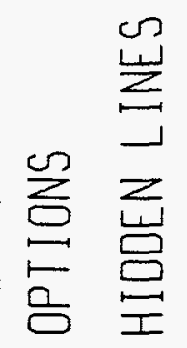 & 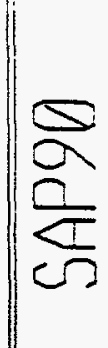 \\
\hline
\end{tabular}

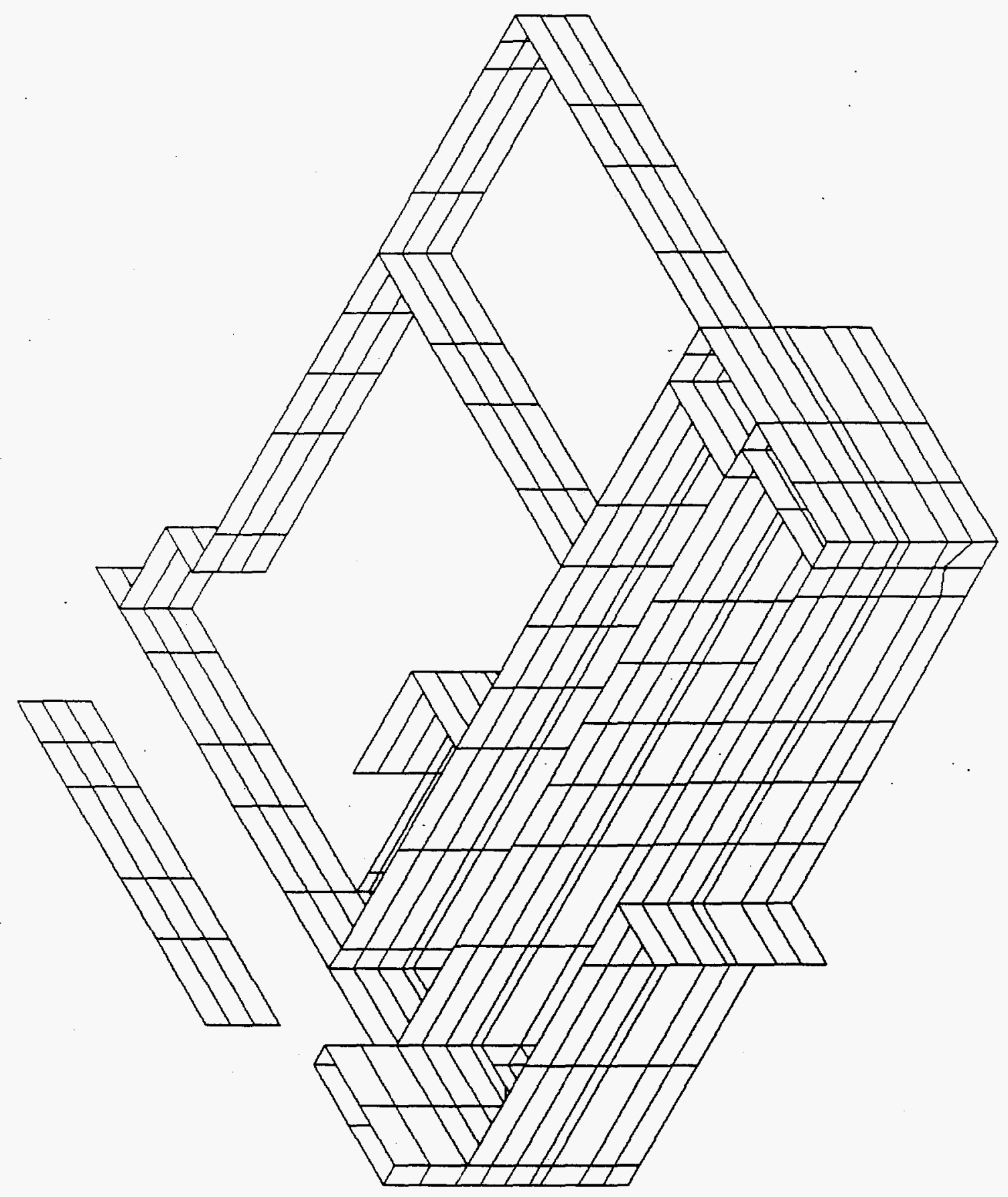




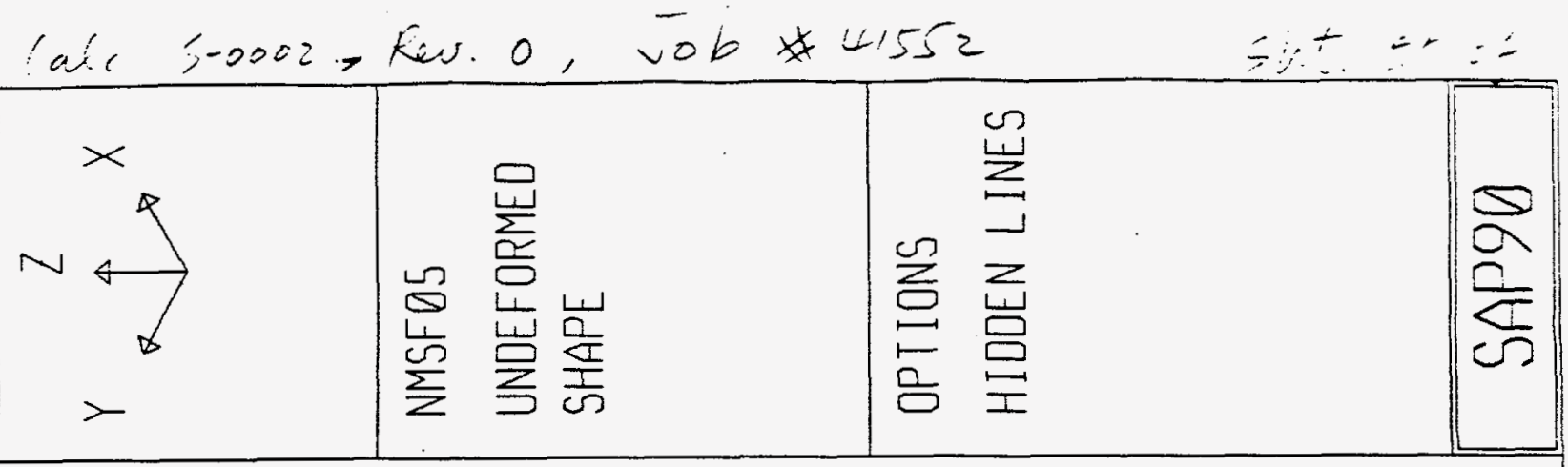

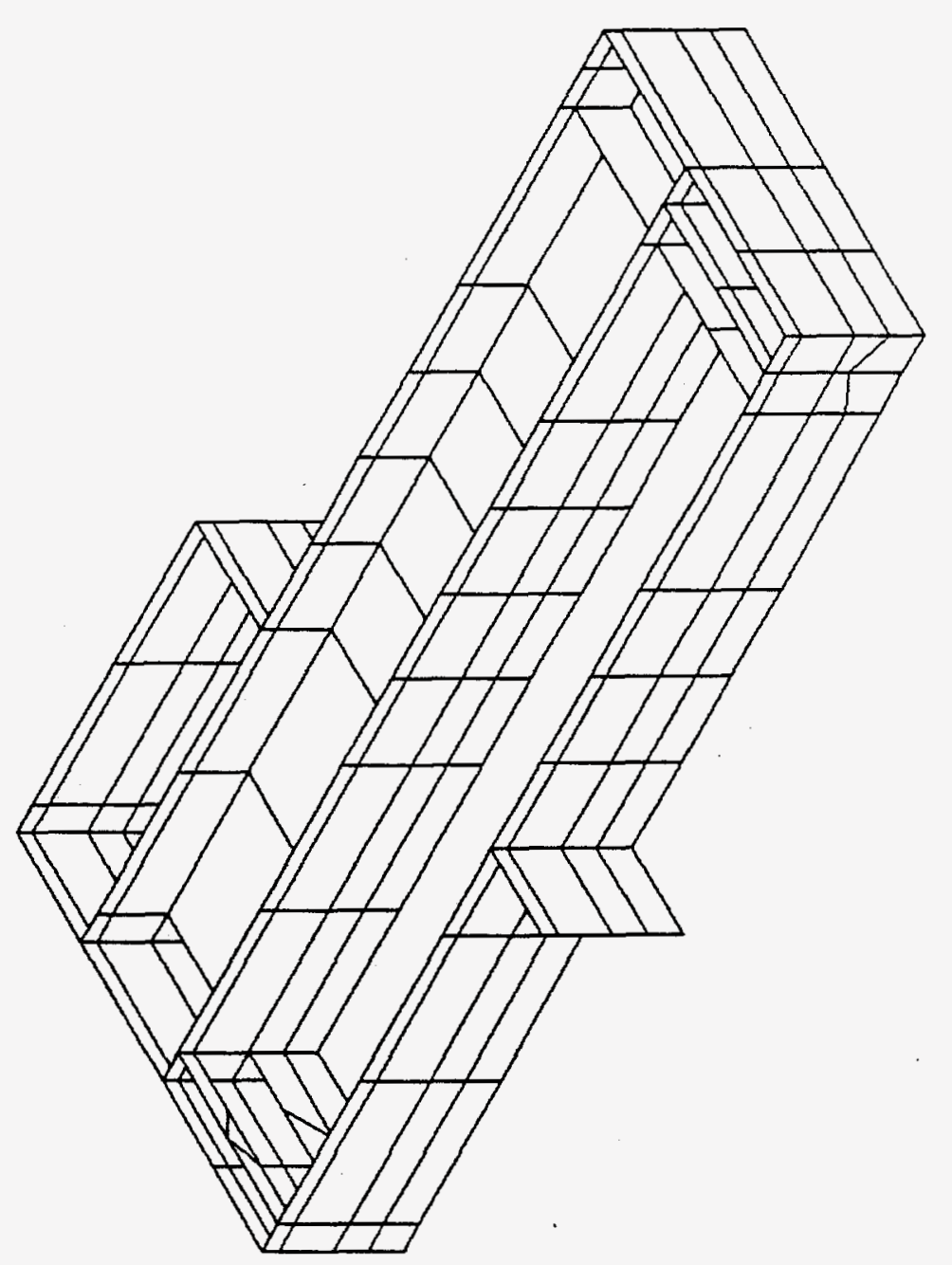


Calc. S-0002, Res. o, Tob $\$ 41552$

$\therefore-5=$

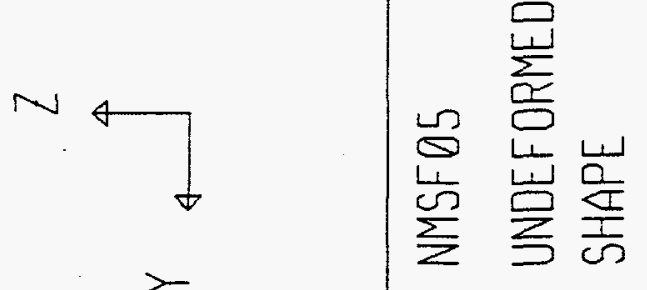

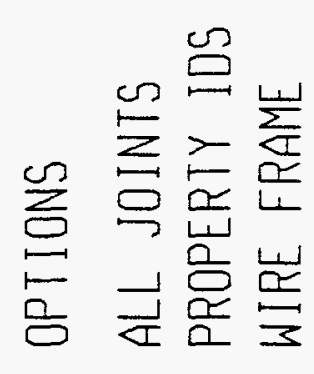

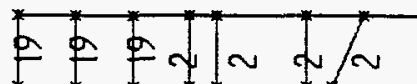

$20+-t-$

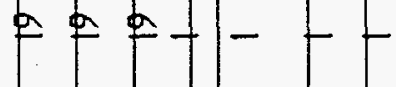

$=20 \sim a v$

$202-1-$

$2 a-1-t$

$a^{2}=-5$

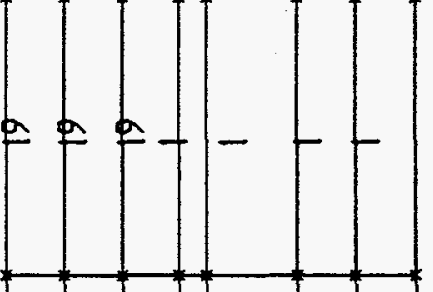

$2=2-5$

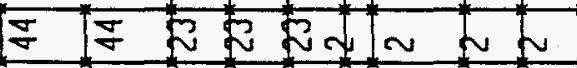




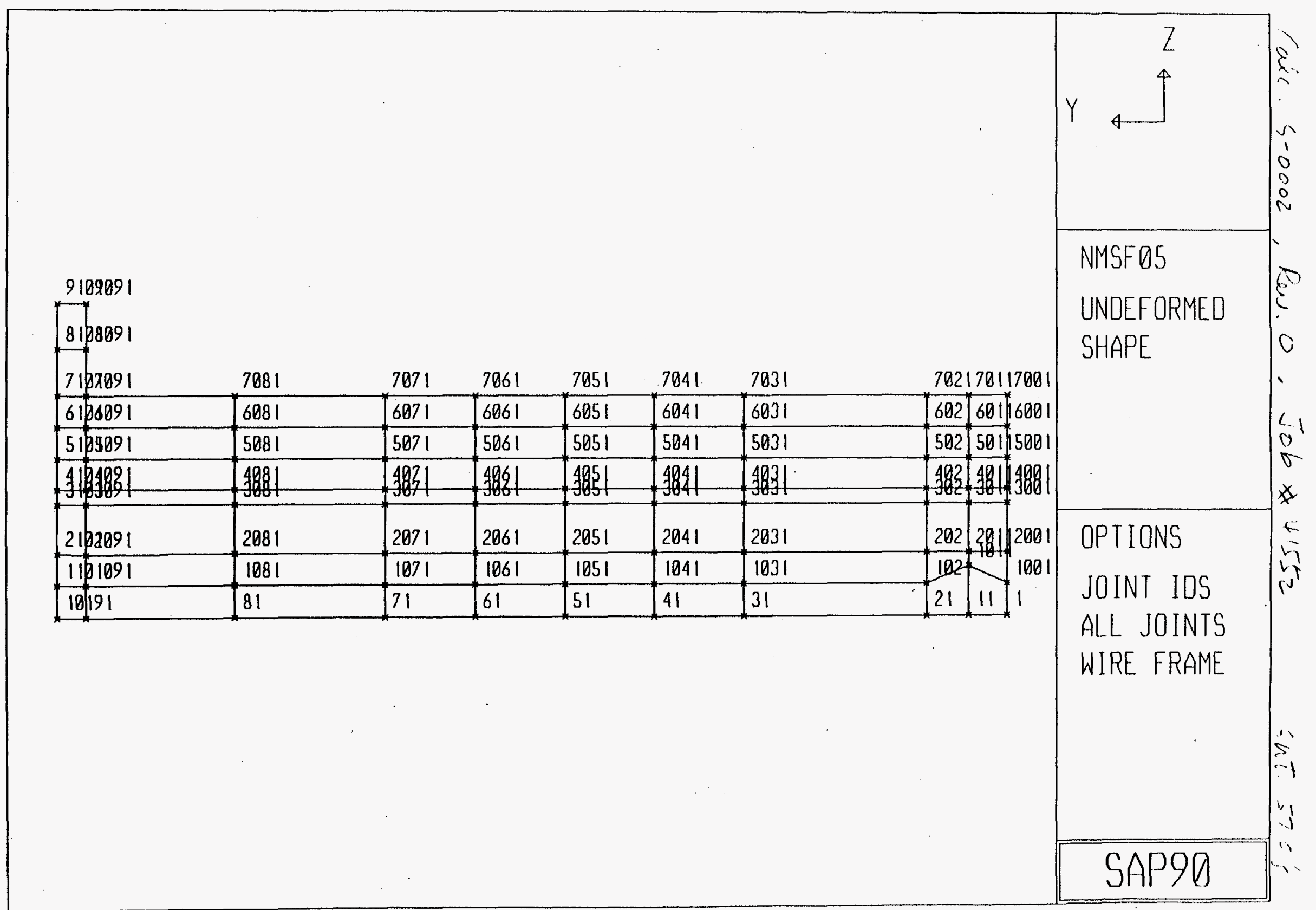




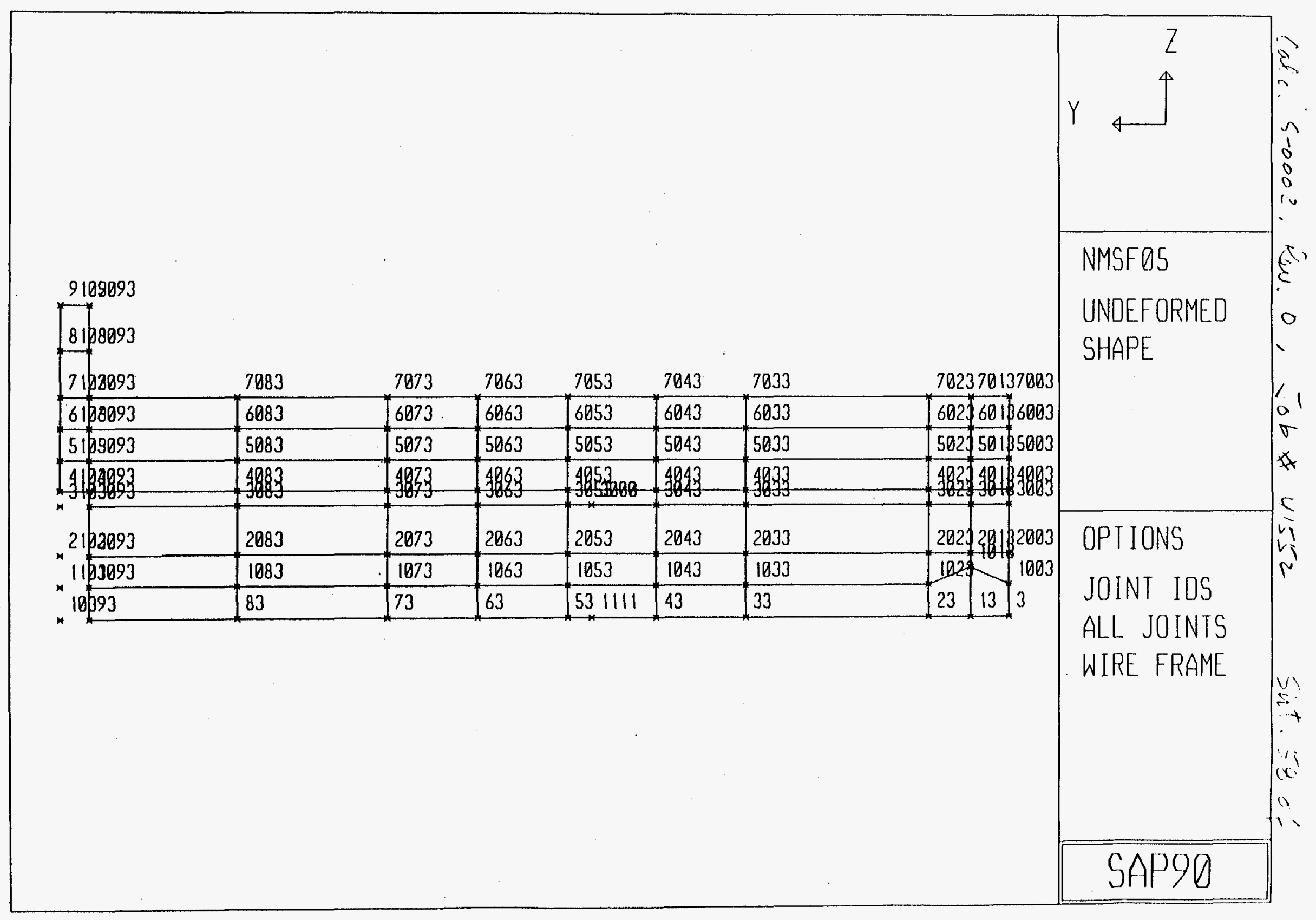




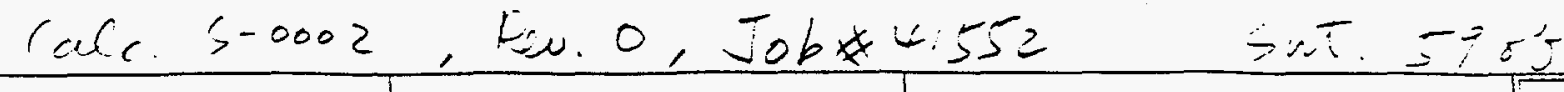

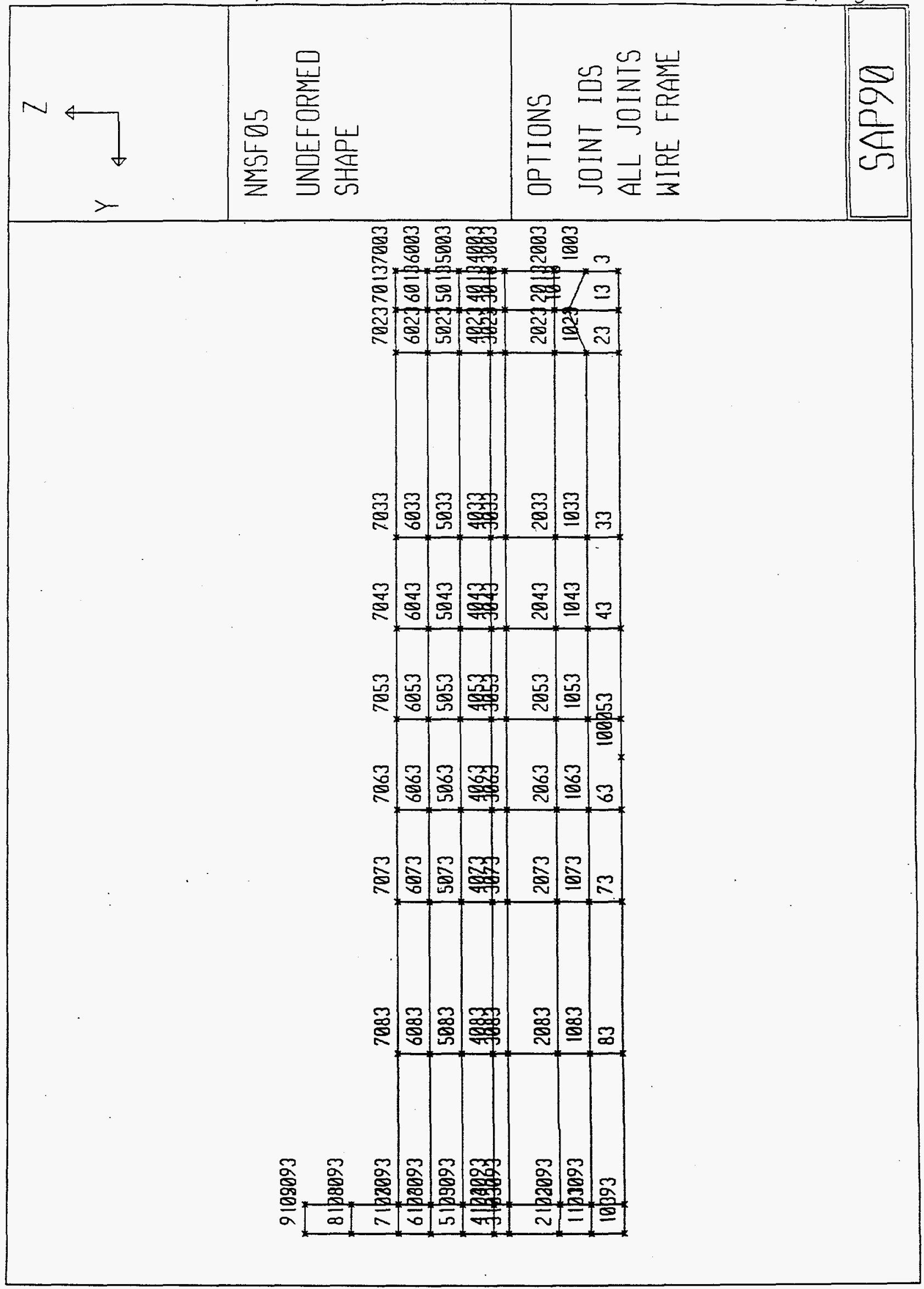




\begin{tabular}{|c|c|c|c|c|c|c|c|c|}
\hline & & & & & & & & $\begin{array}{r}Z \\
Y \\
Y \quad 4\end{array}$ \\
\hline 7107094 & 7084 & 7074 & 7064 & 7054 & 7044 & 7034 & 702470147004 & \multirow[t]{5}{*}{$\begin{array}{l}\text { JMSFD5 } \\
\text { INDEFORMED } \\
\text { HAPE }\end{array}$} \\
\hline 6196094 & 6084 & 6074 & 6064 & 6054 & 6044 & 6834 & 602460146004 & \\
\hline 51$) 8094$ & 5084 & 5074 & 5064 & 5054 & 5044 & 5034 & 502450145004 & \\
\hline 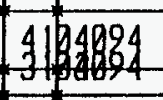 & 4888 & 4834 & 48664 & 3854 & 4864 & 4834 & 482448 & \\
\hline 2192094 & 2084 & 2074 & 2064 & 2054 & 2044 & 2834 & 202420142004 & \\
\hline 1164094 & 1884 & 1074 & 1064 & 1054 & 1044 & 1034 & $102 / 101 / 1004$ & \\
\hline 10194 & 84 & 74 & 64 & 54 & 44 & 34 & \begin{tabular}{|l|l|l}
24 & 14 & 4 \\
\end{tabular} & UPT IUIVS \\
\hline & & & & & & & & $\begin{array}{l}\text { JOINT IDS } \\
\text { ALL JOINTS } \\
\text { WIRE FRAME }\end{array}$ \\
\hline & & & & & & & & SAP9D \\
\hline
\end{tabular}




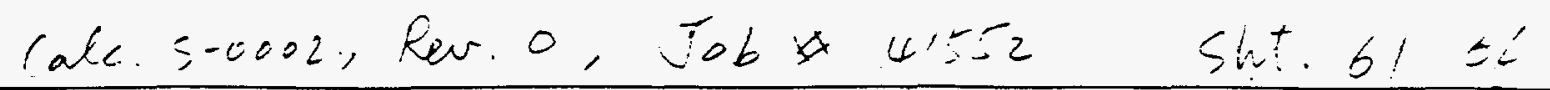

\begin{tabular}{|c|c|c|c|}
\hline 4 & 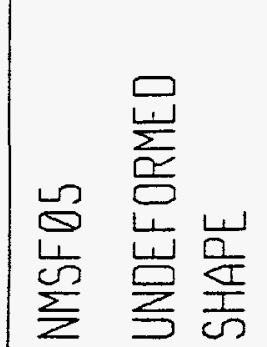 & 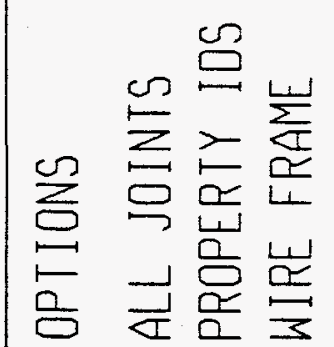 & 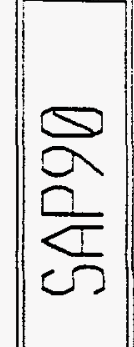 \\
\hline
\end{tabular}

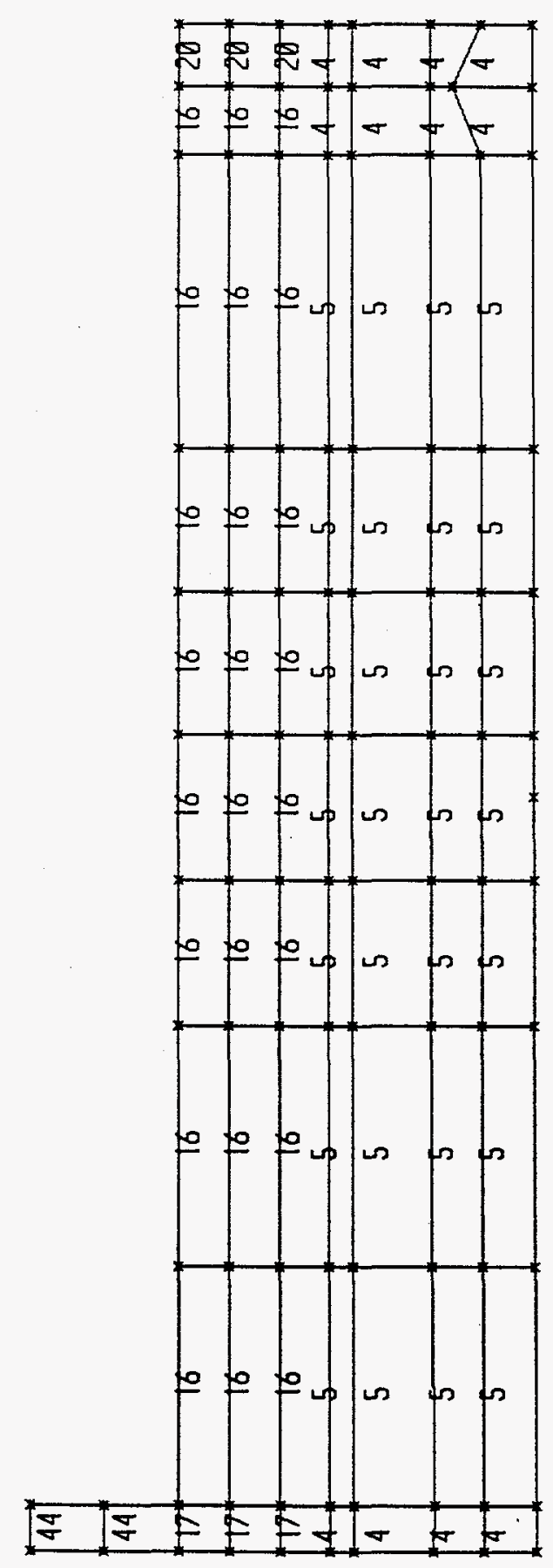




\begin{tabular}{|c|c|c|c|}
\hline Fid. & & & \\
\hline$N \leftarrow$ & 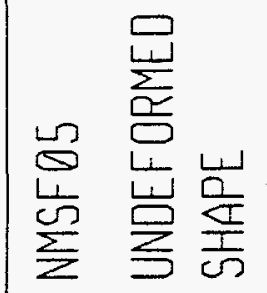 & 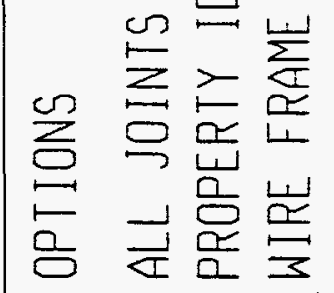 & 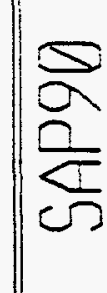 \\
\hline
\end{tabular}

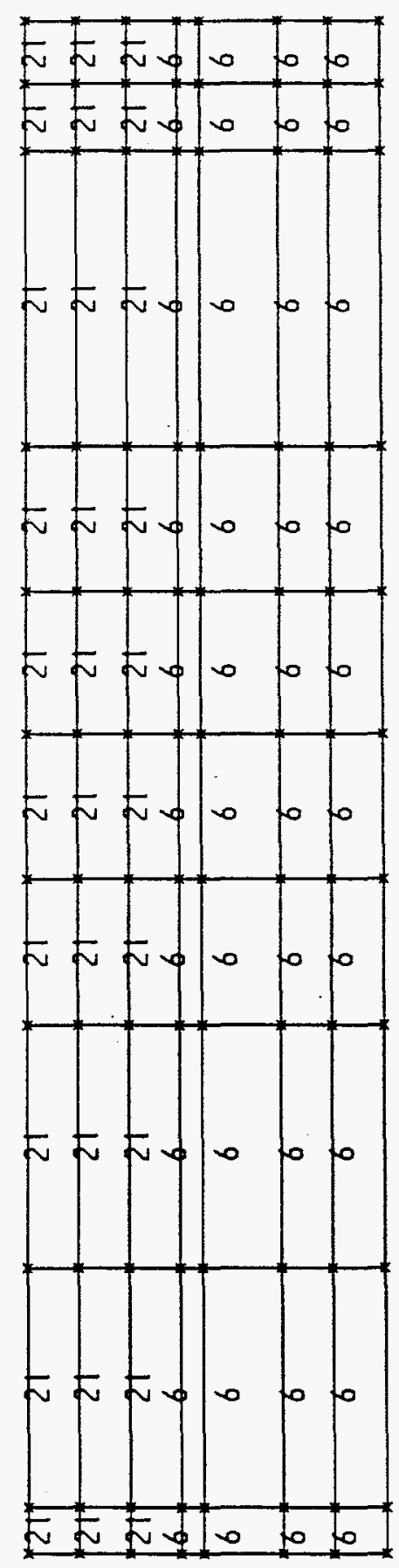




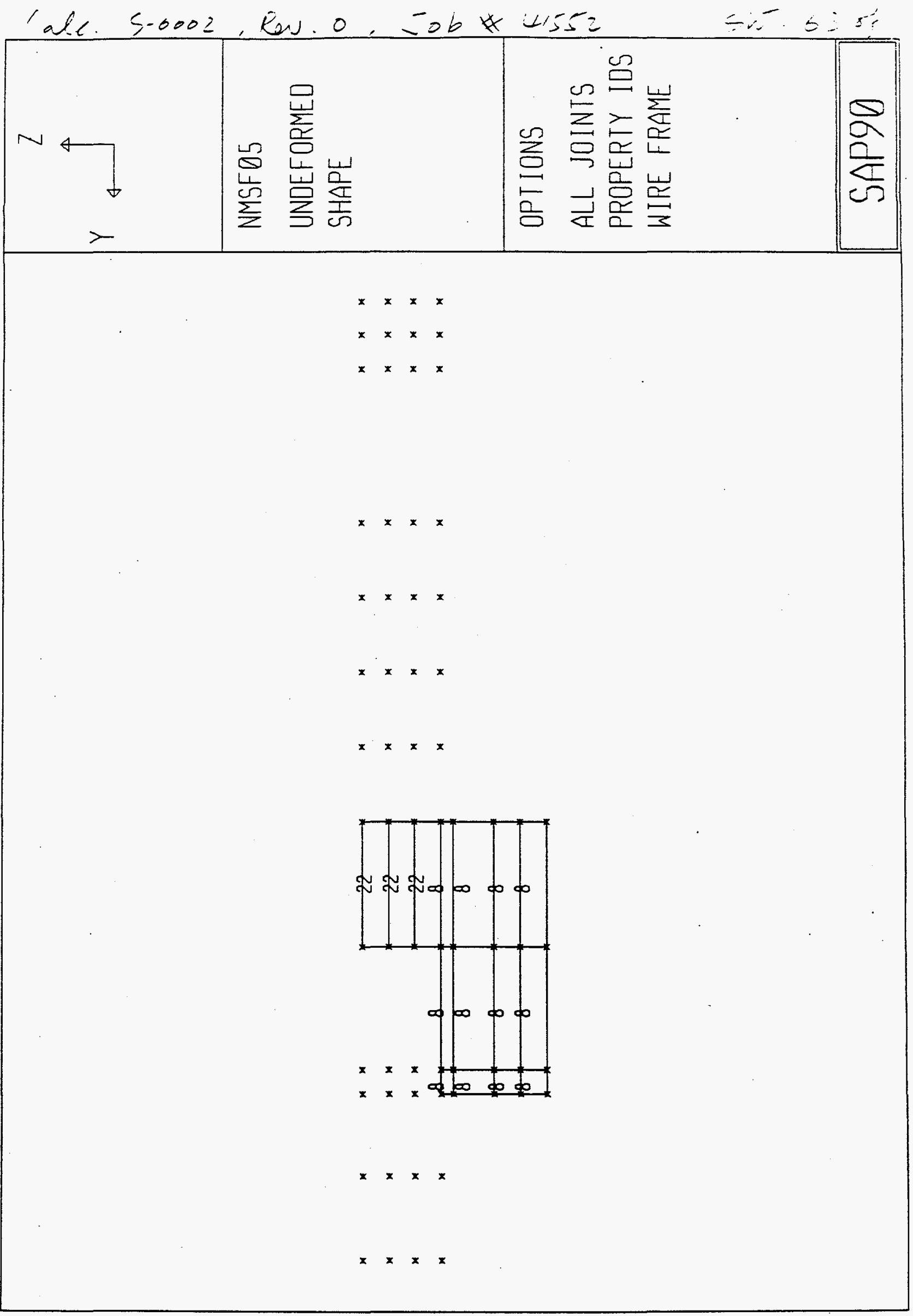


lale $5-0002$ Rex.o, Tob *41552

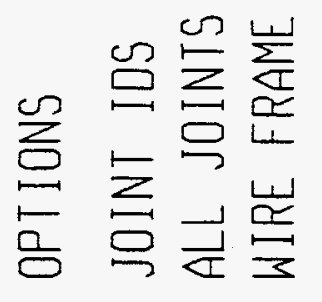

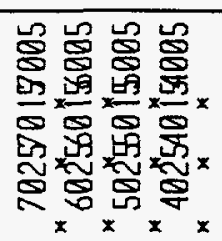

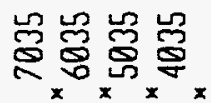

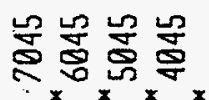

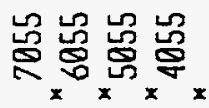

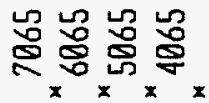

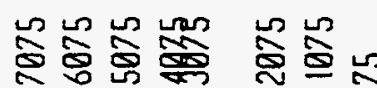

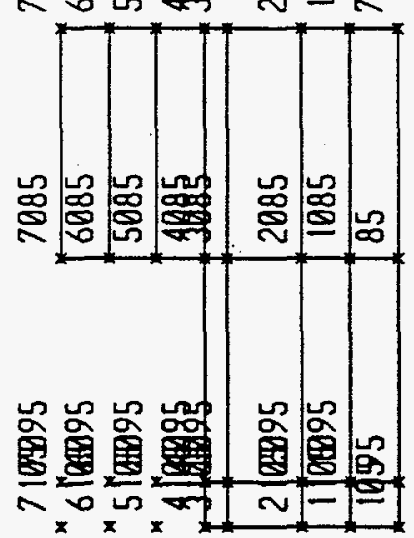

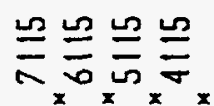

$\cong \simeq \cong$

$\mp \frac{1}{6} \equiv$ 
lale. S-0002, Rev. 0, Tob *41552

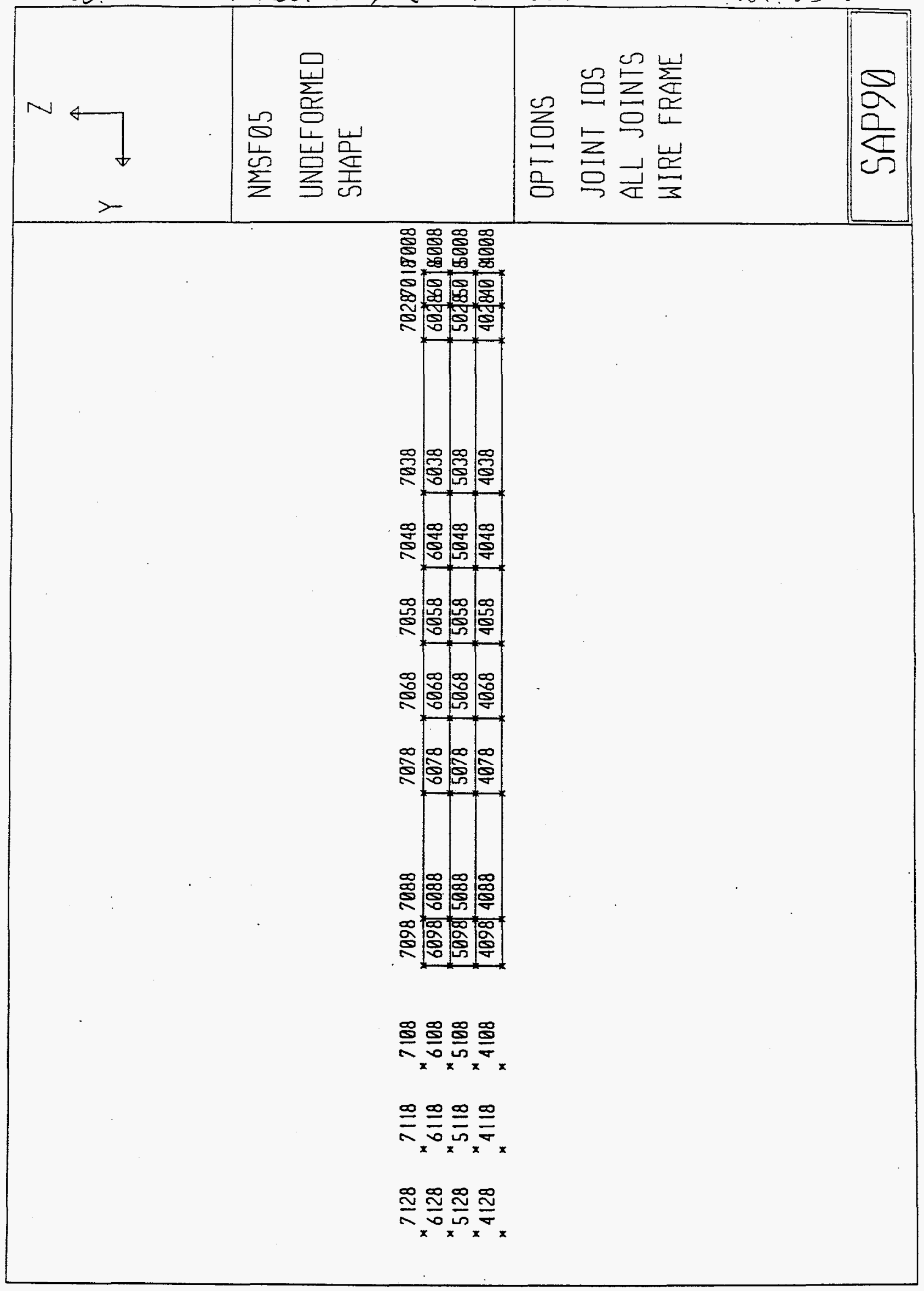


(ase 4-000z, Rew $0, \quad==b * 41552$ $-2 c^{t}=0=1$

\begin{tabular}{|c|c|c|c|}
\hline $\begin{array}{c}N+ \\
\\
\\
\succ\end{array}$ & 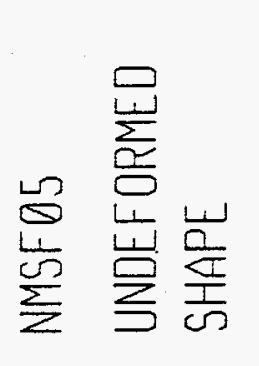 & 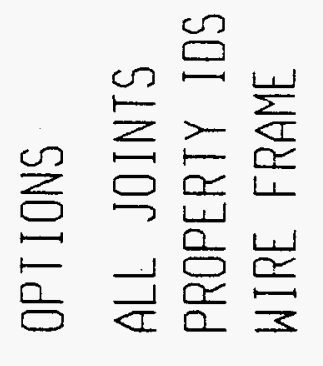 & $\frac{s}{a}$ \\
\hline
\end{tabular}

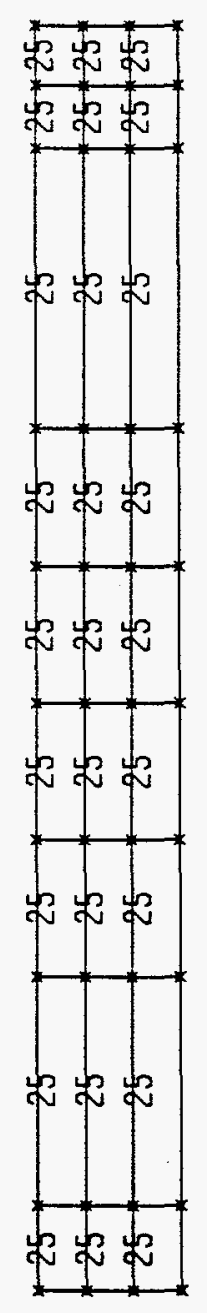

....

...

... 
Iale S-0002, Rew 0, Tob *41552 SAt =TE-

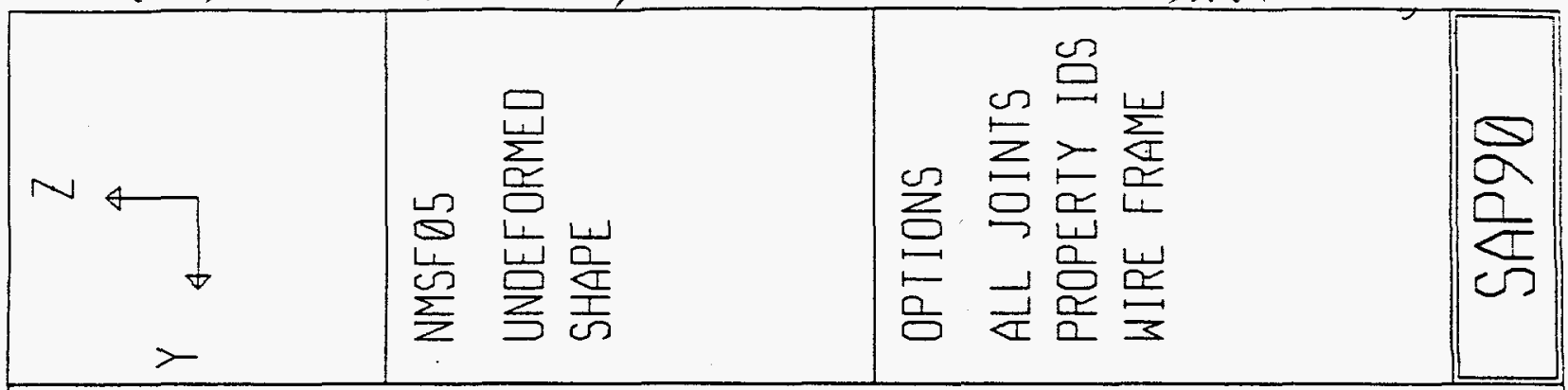

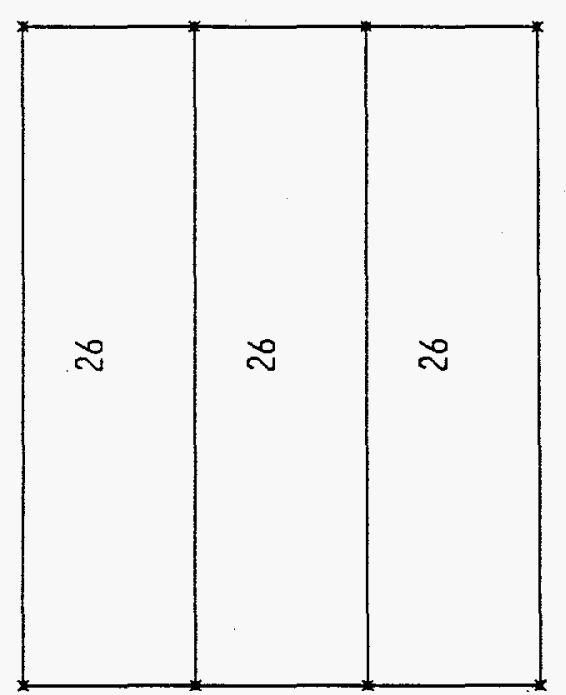

$\mathbf{x}$

$\mathbf{x}$

$\times$

$\mathbf{x}$

$\mathbf{x}$

x

$x$ 


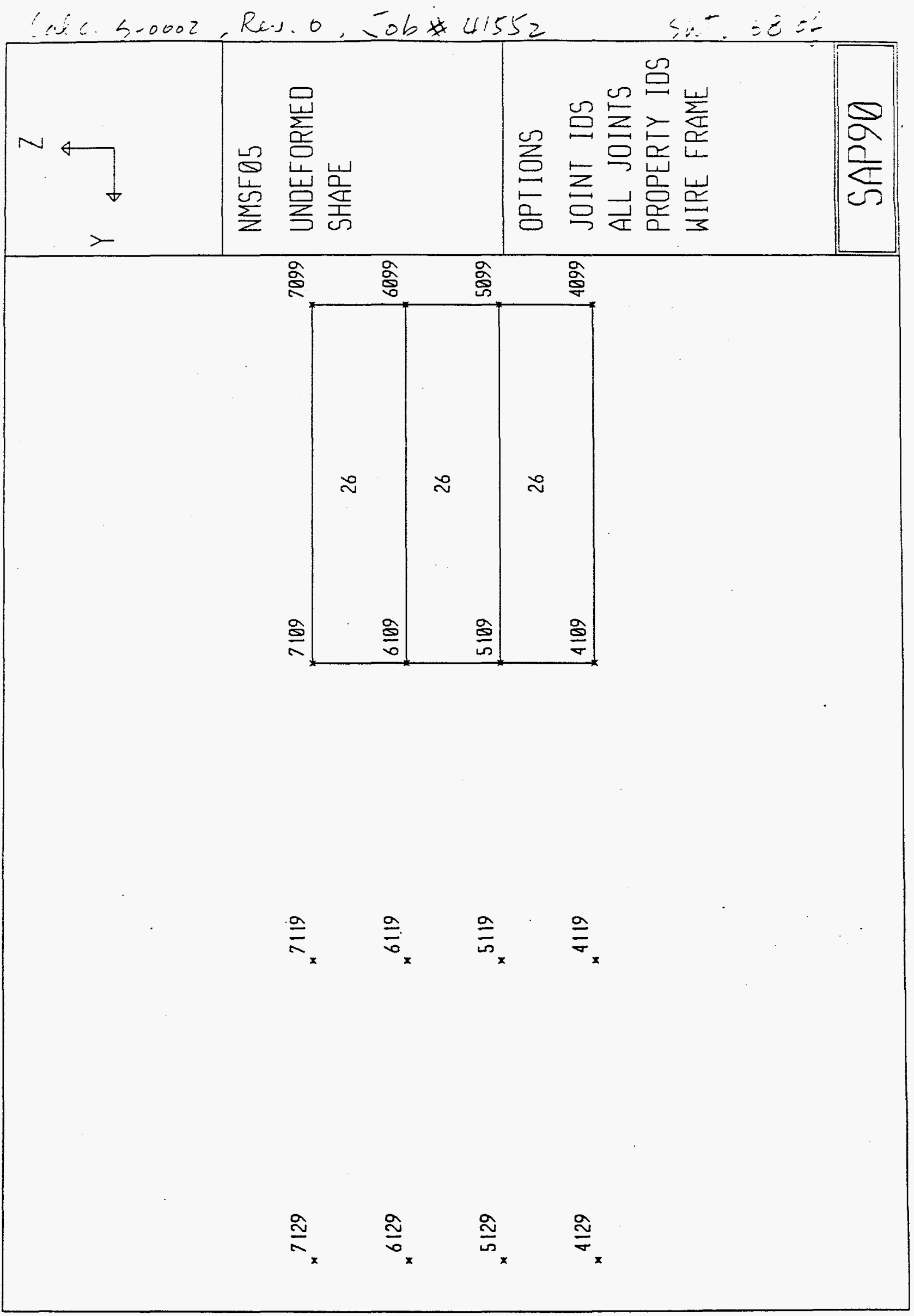


1a1c. S-0002, Kes, 0, Tob $\times 41552$

$-x^{+}=1:-$

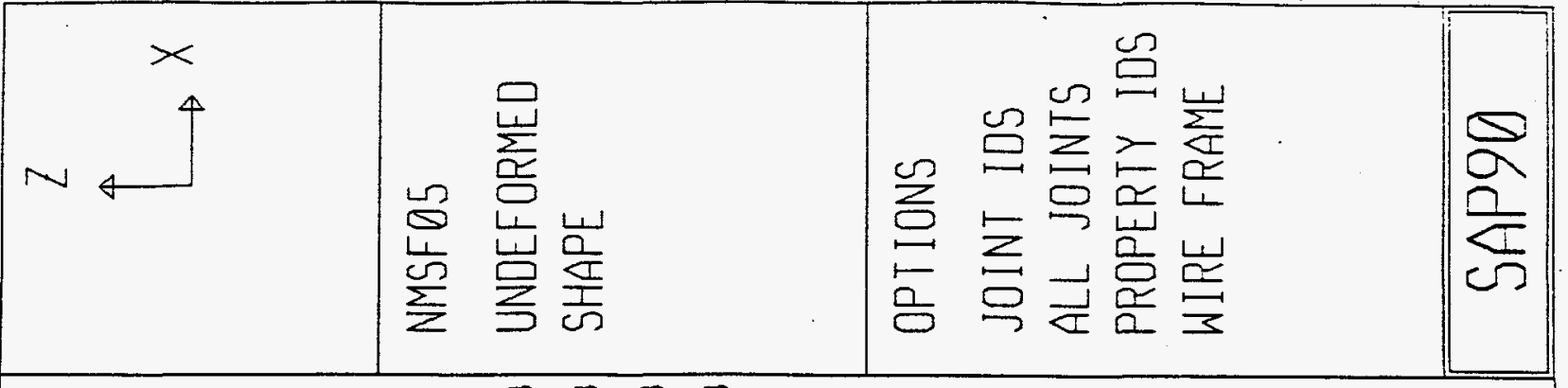

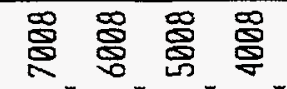

今)

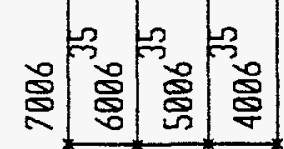

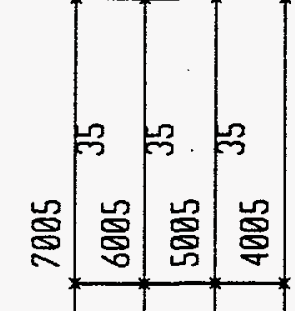

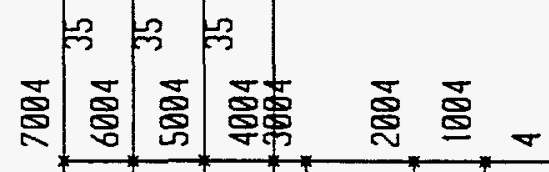

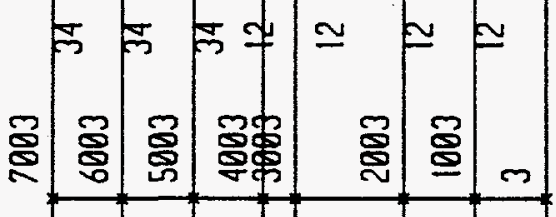

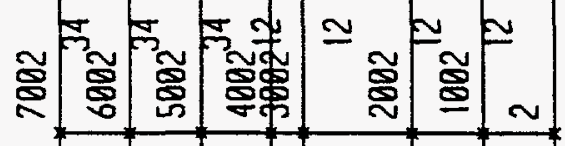

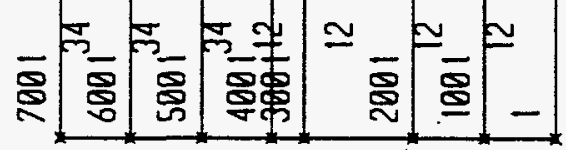


(alc: 5-0002, Tou. 0, Tob 441552

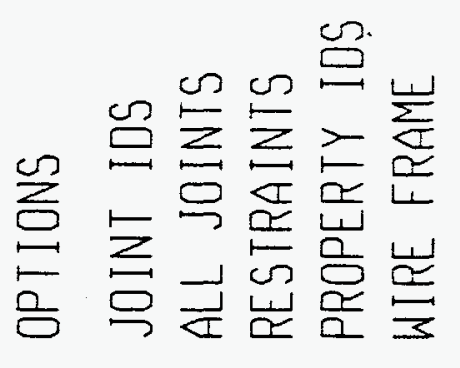

㪉高高高

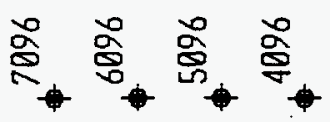

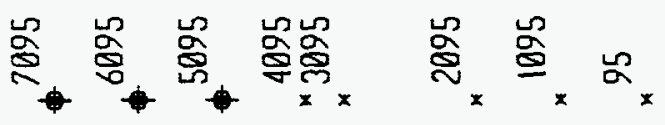

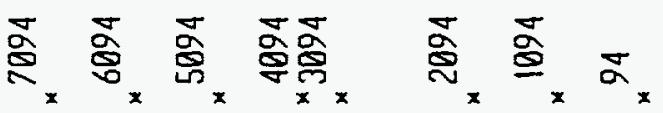

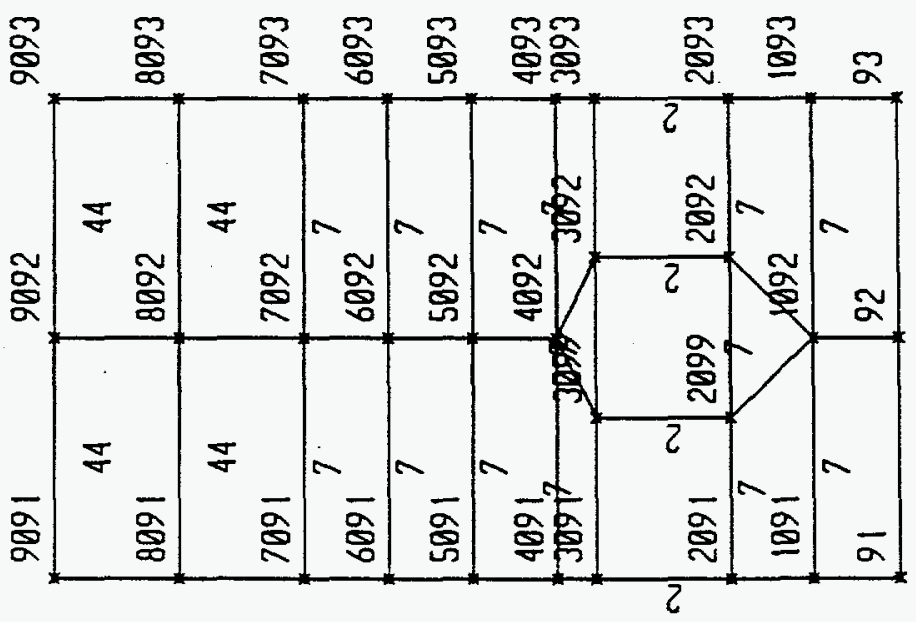




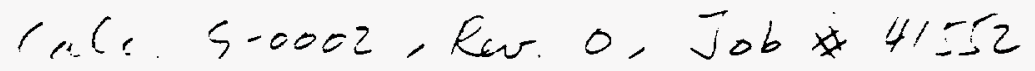

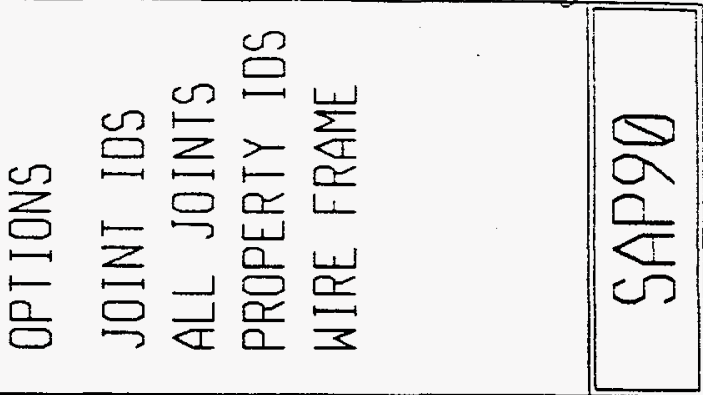

$\sum_{x}^{\infty} \sum_{x}^{\infty} \sum_{x}^{\infty} \sum_{x}^{\infty} \frac{\infty}{b}$

बे के

$\frac{0}{\infty} \frac{\infty}{\infty} \frac{\infty}{\infty} \frac{0}{\infty}$

$\frac{2}{0} \frac{\pi}{0} \sum_{x}^{\frac{n}{0}} \frac{\pi}{0}$

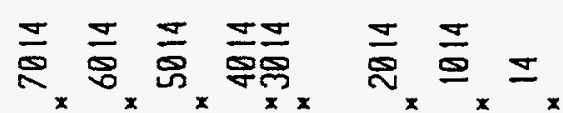

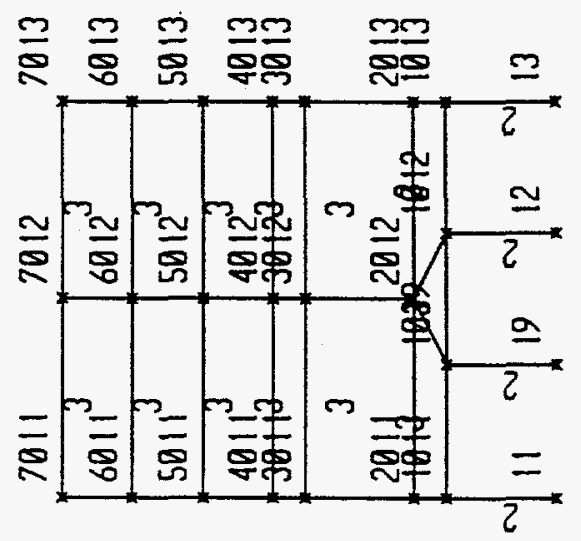


Calc. 5-0002, Ees.0, Job *41552

Lit - : :

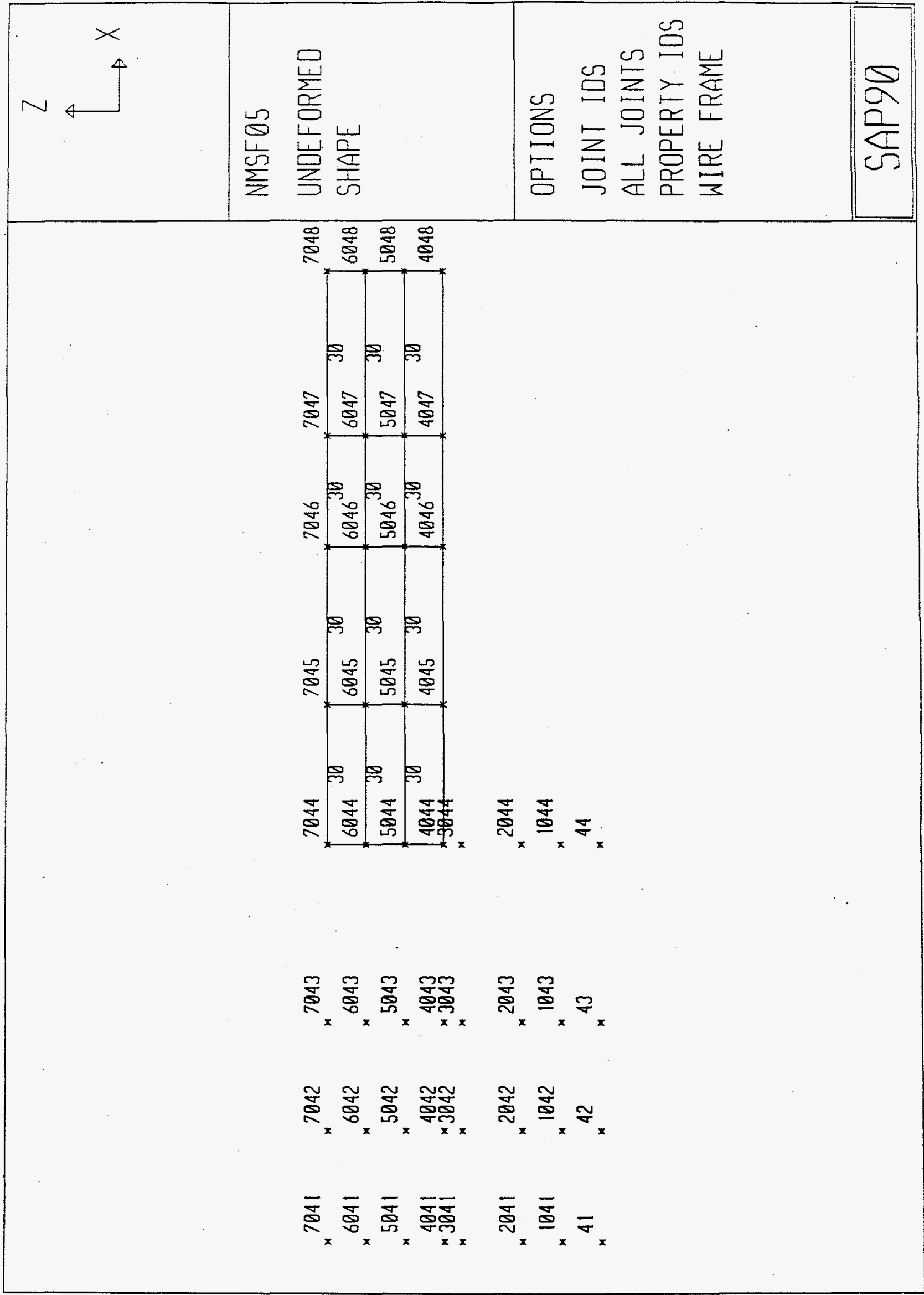


wie s-0002, Ker. 0, Job $\$ 41552$ Sw- -

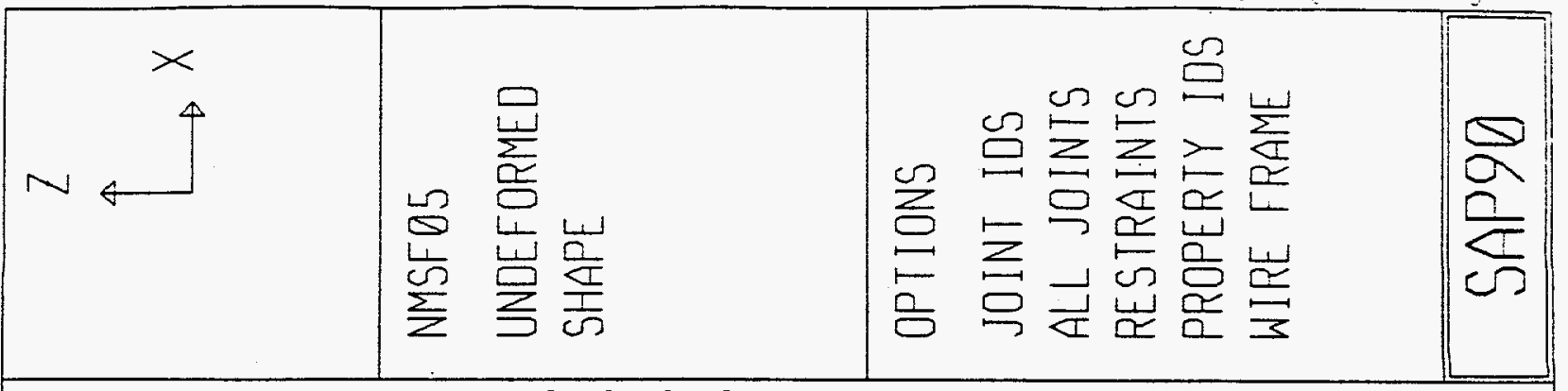

बे बे बे बे

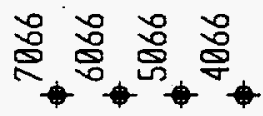

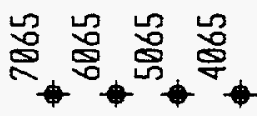

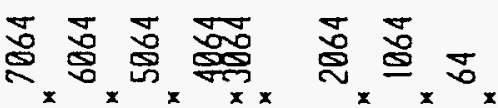

হి

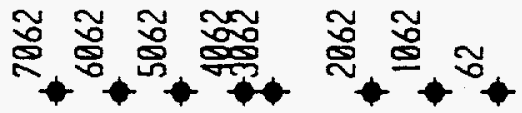

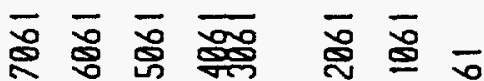

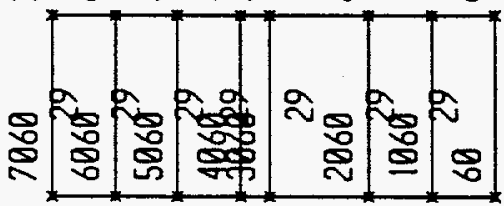


lale 5-0002, Rav. 0, Job $\times 44552$

\begin{tabular}{|c|c|c|c|}
\hline $\begin{array}{l}x_{4} \\
N_{4}\end{array}$ & 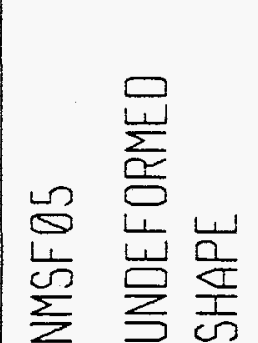 & 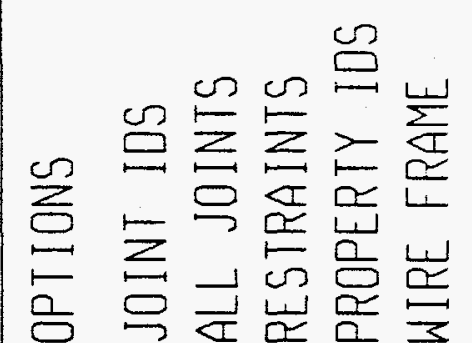 & $\frac{5}{\frac{5}{2}}$ \\
\hline
\end{tabular}

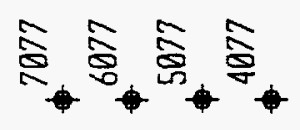

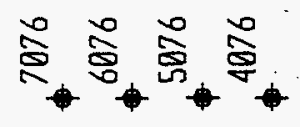

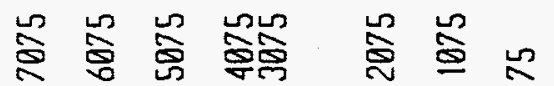

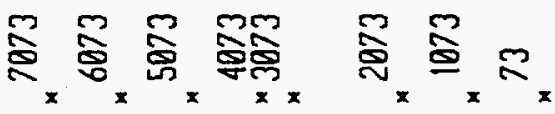

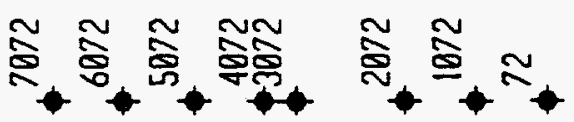

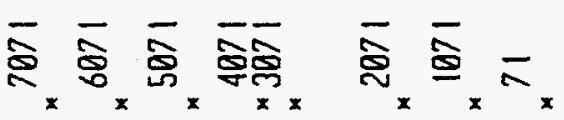




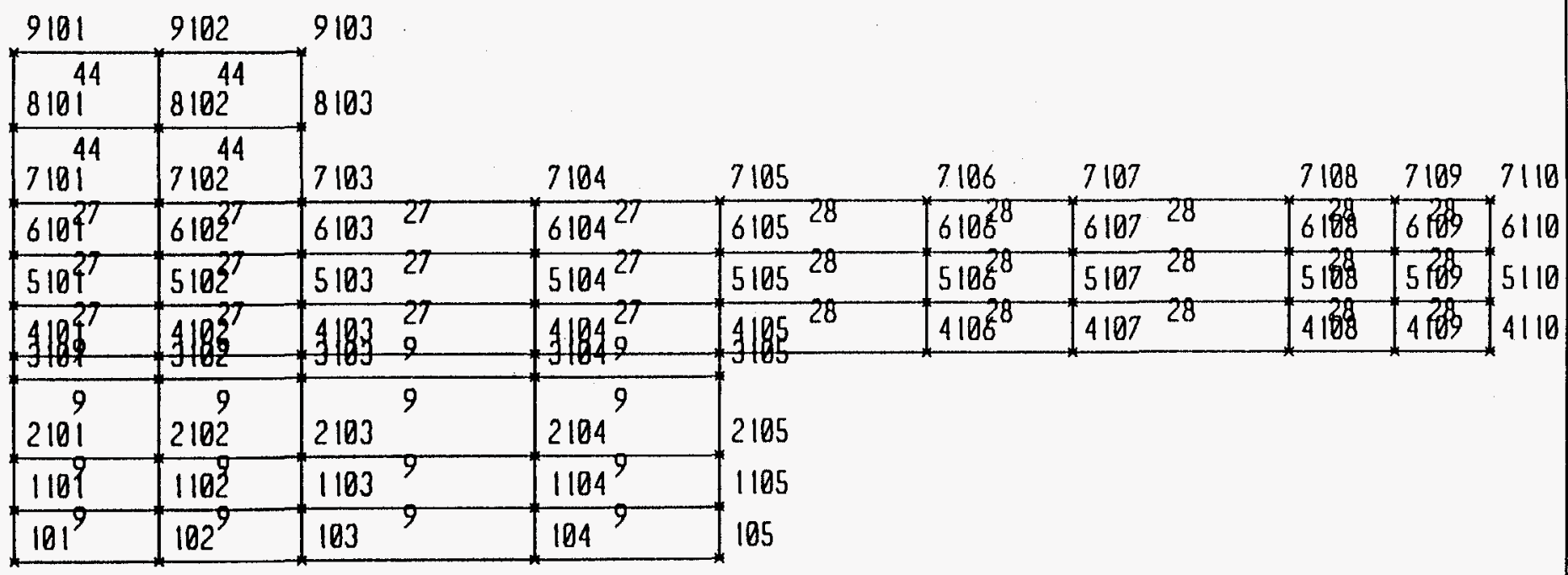

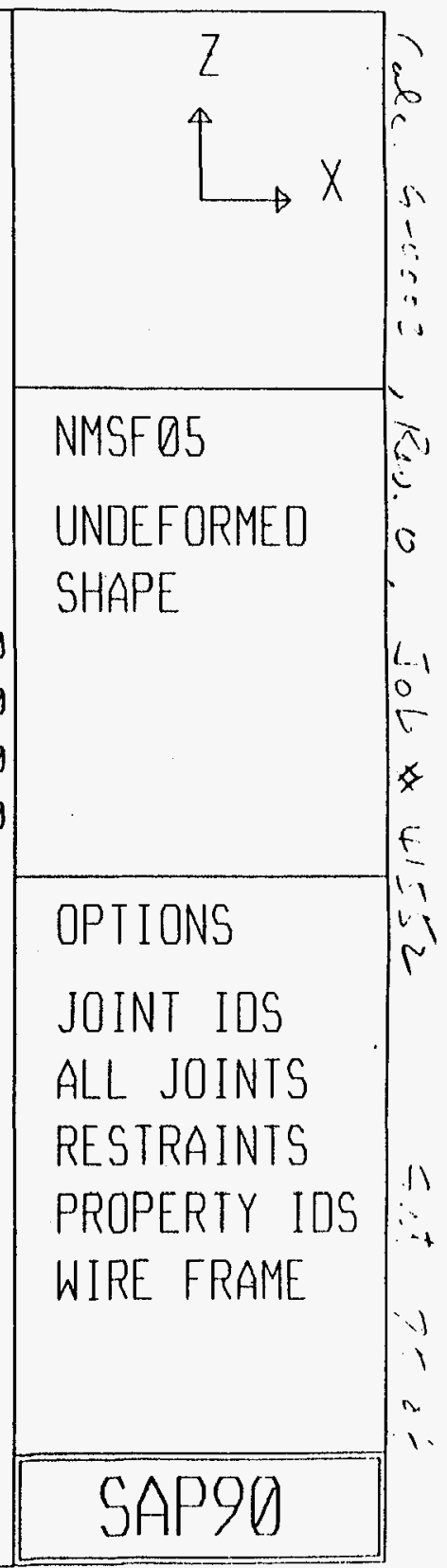


Calc. S-0002, Eev 0, Job *41552

$\sin 7 \cdot 2-2$

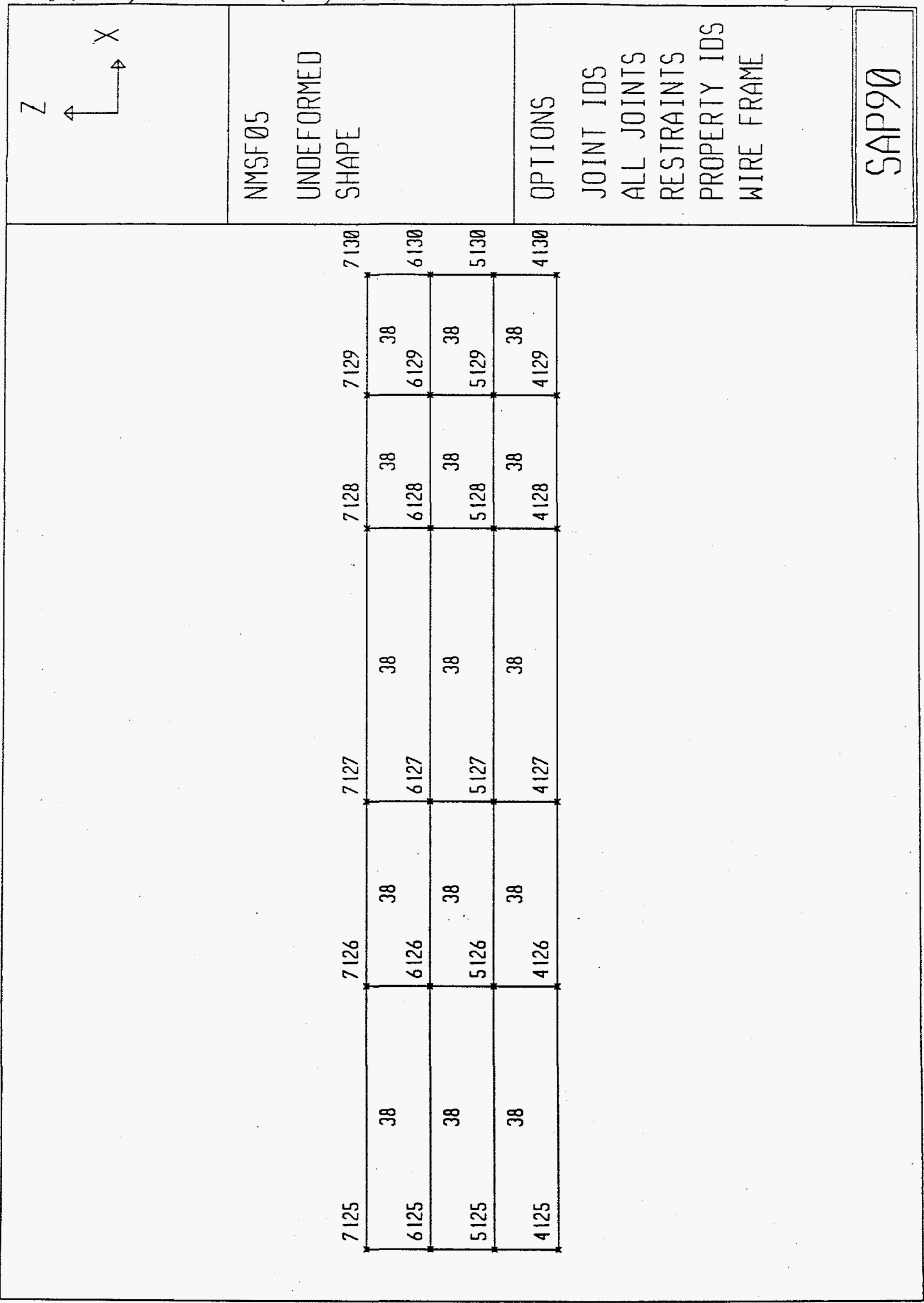




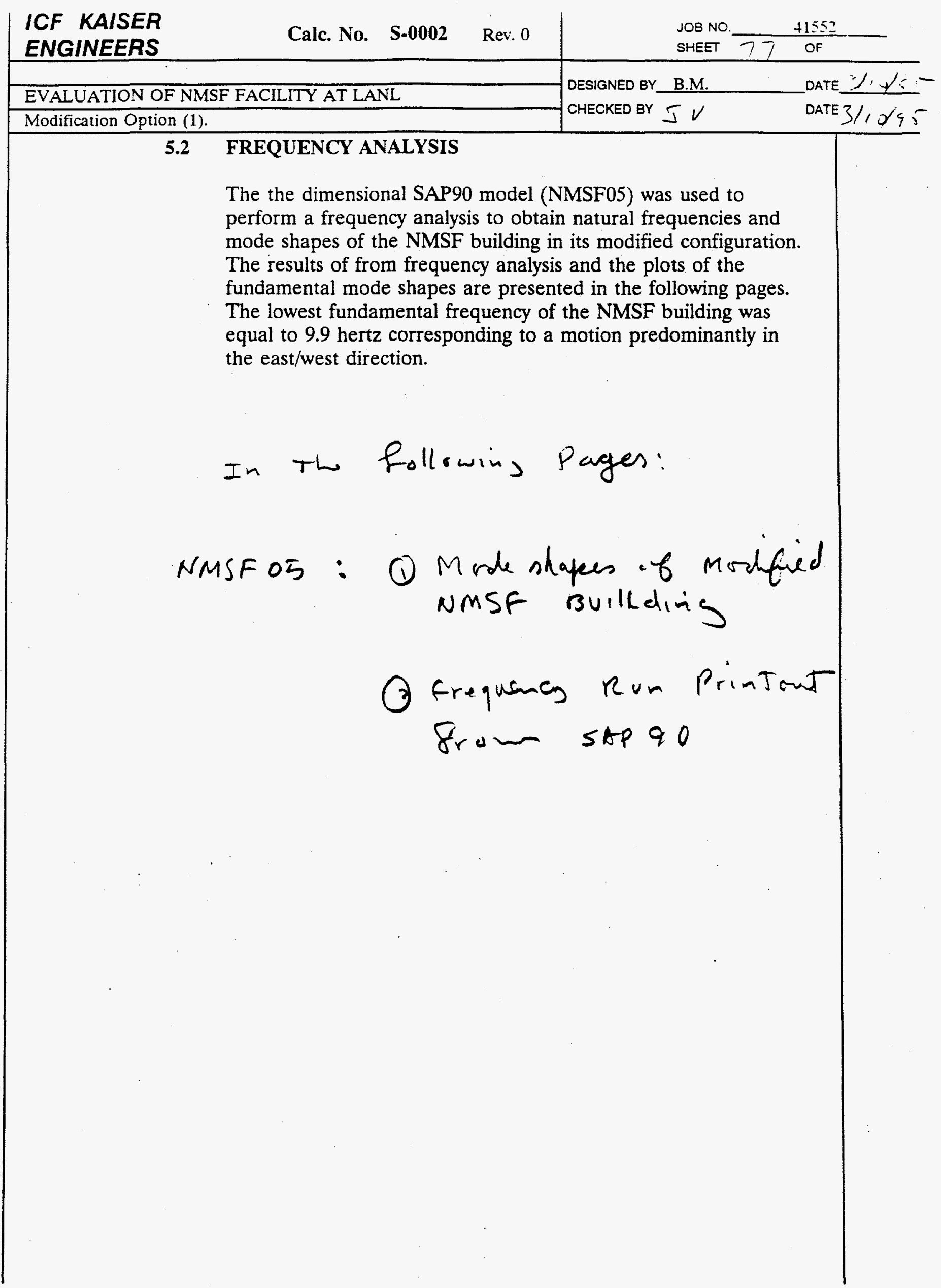




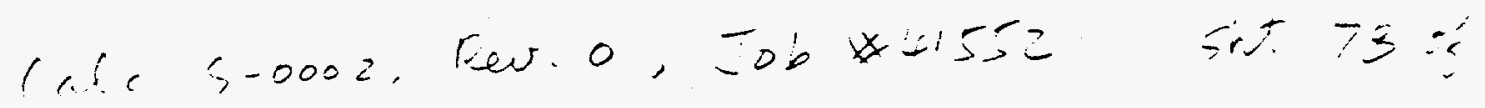

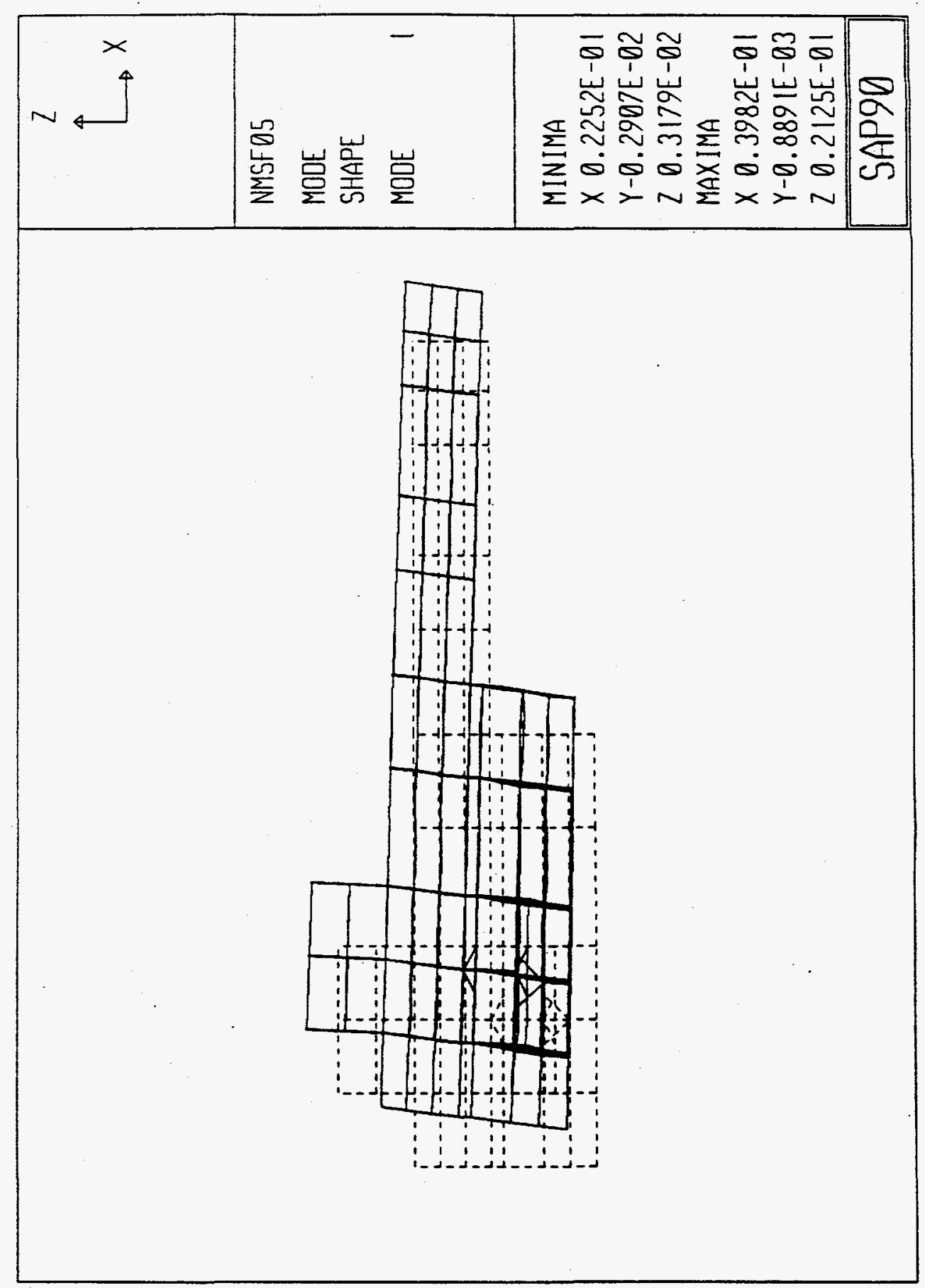




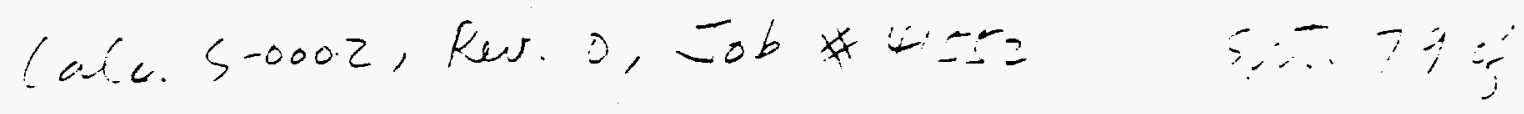

\begin{tabular}{|c|c|c|c|c|}
\hline$N 4$ & 岕崖崖 宾 & $\begin{array}{l}\sim \\
\text { 菅 } \\
\text { 足 }\end{array}$ & 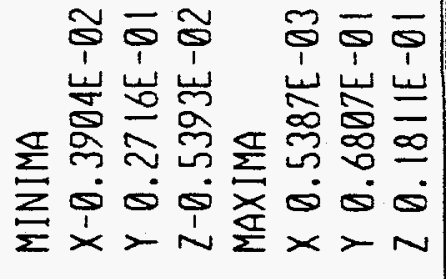 & $\mid \frac{S}{2}$ \\
\hline
\end{tabular}

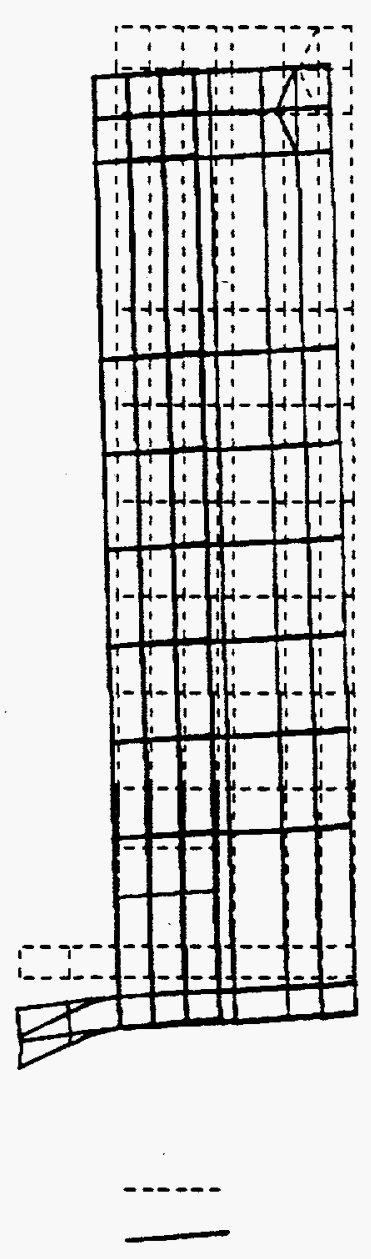


Calc. S-0002, Rev. O, Job 4552 Sut. E00

\begin{tabular}{|c|c|c|c|c|}
\hline$N_{4}^{x}$ & 岕崖崖 & 岂 & 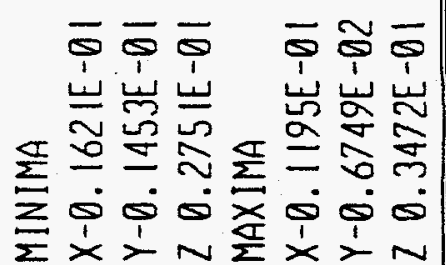 & $\frac{\substack{0 \\
0}}{\sqrt{0}}$ \\
\hline
\end{tabular}

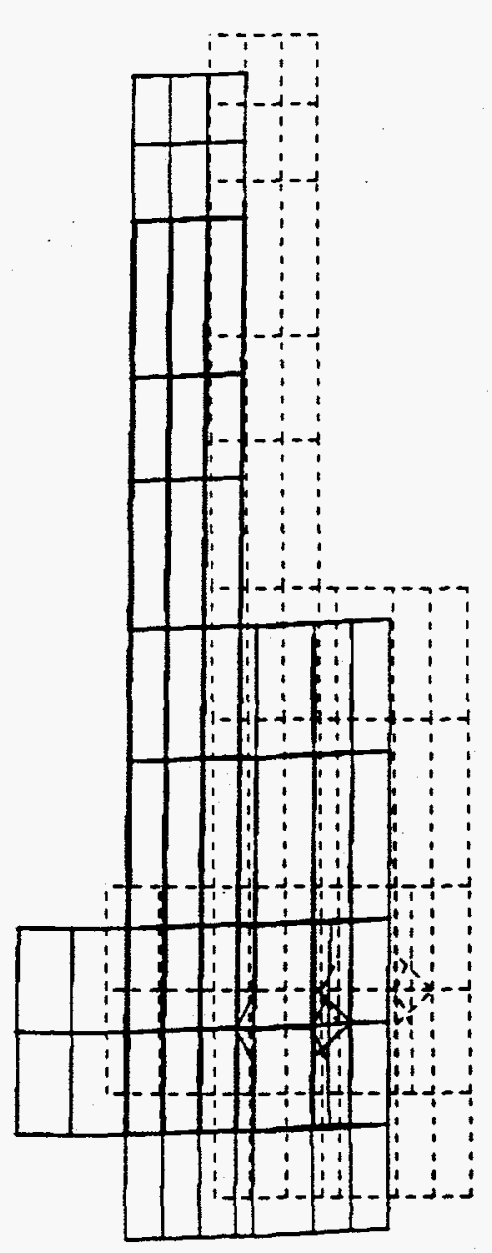




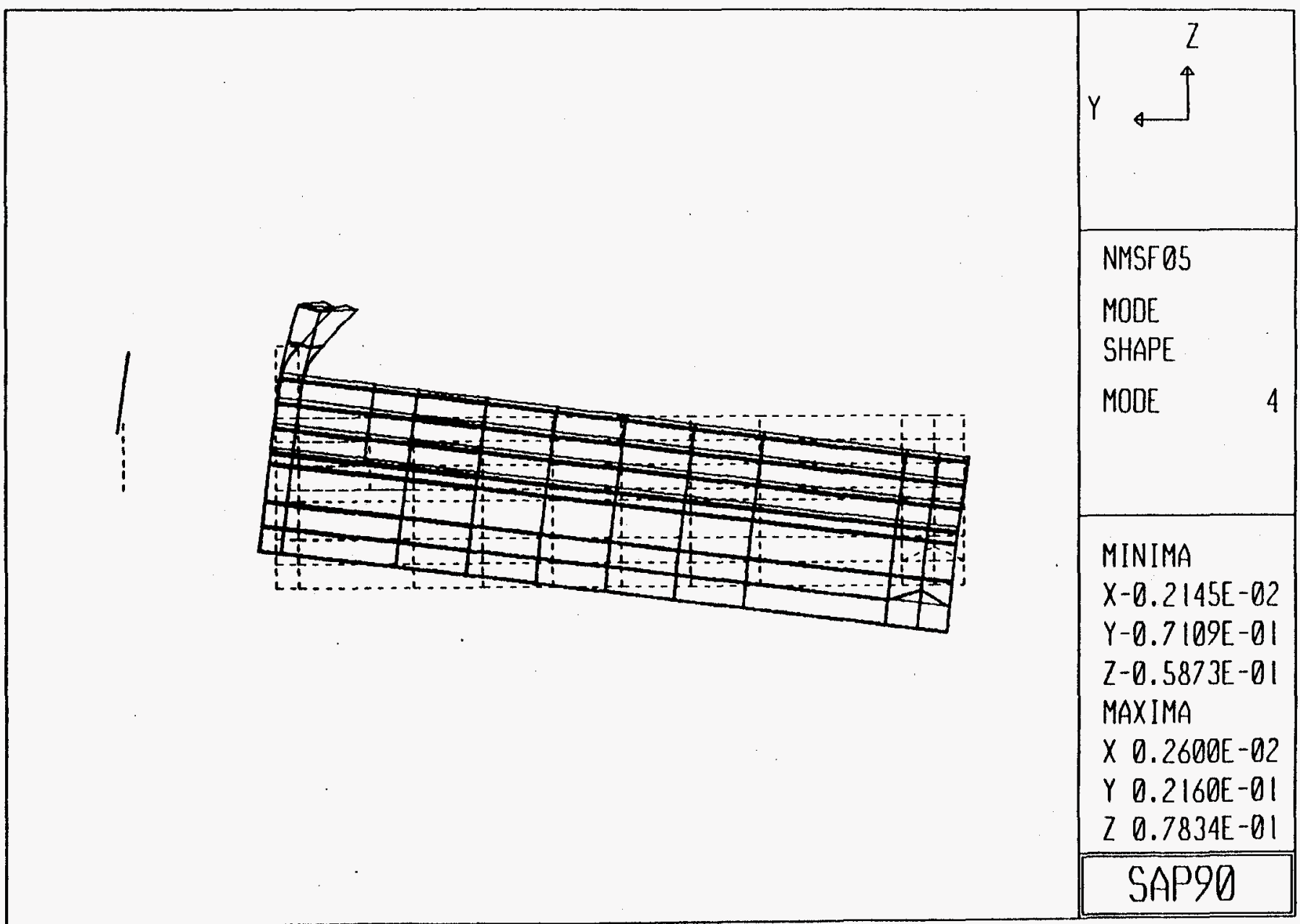




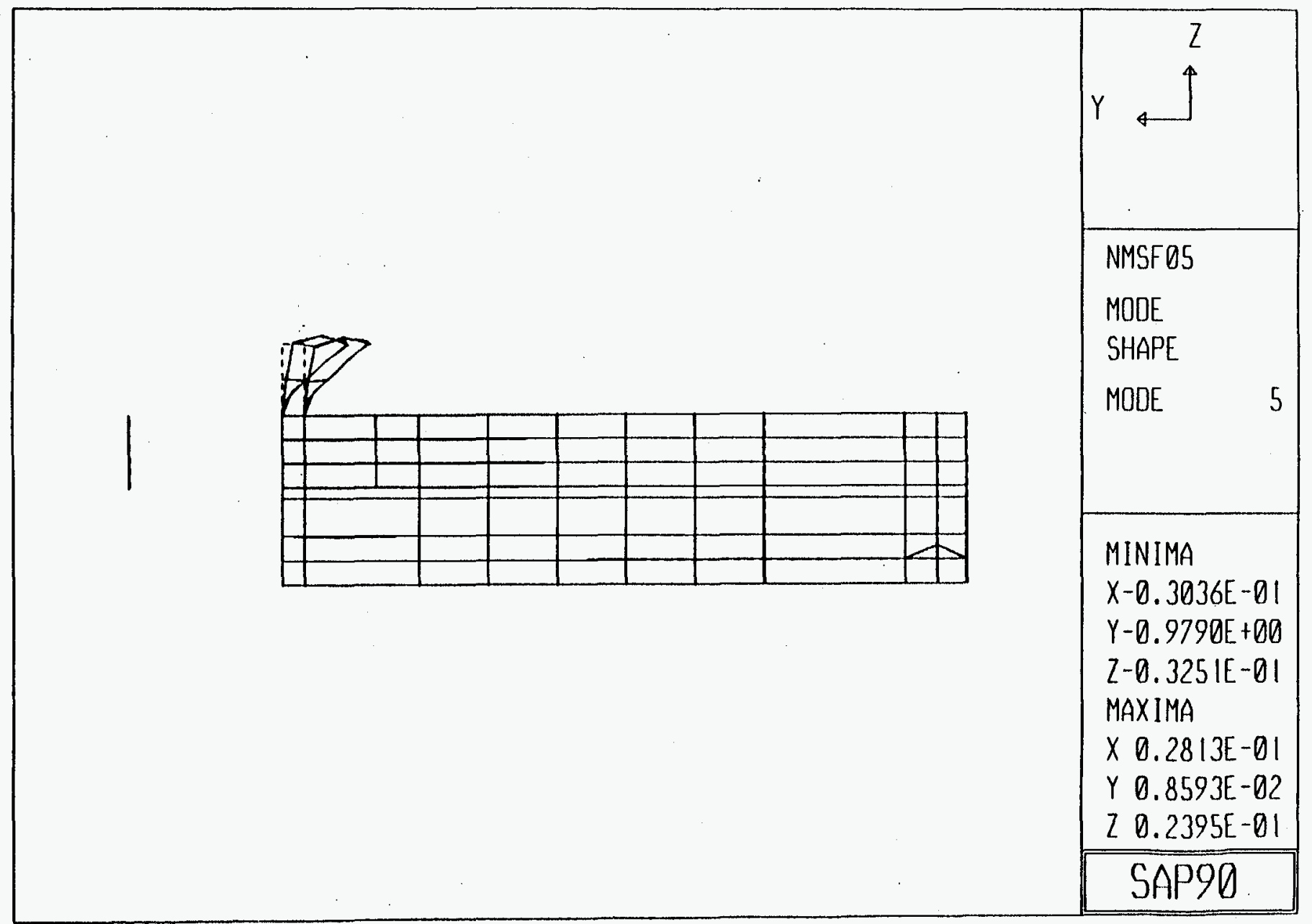




$$
\text { lalc. S-0002, Rev. o, Fob } \$ 41552 \text { Sht. Ė: } ;
$$

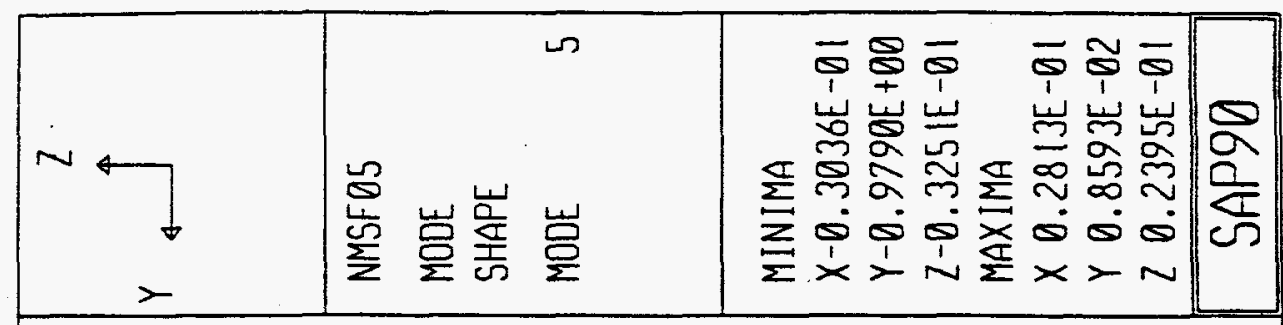

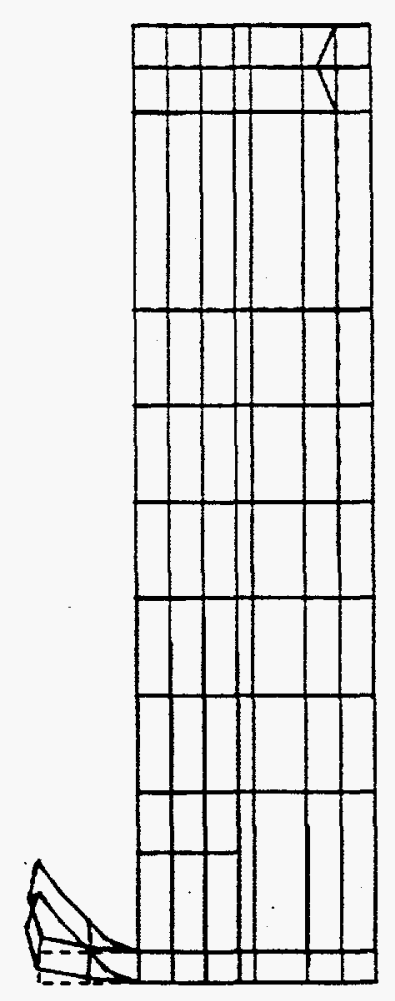




$$
\text { lalc. S-0002, Rev. 0, Job 41552 sht. } 84 \text { of }
$$

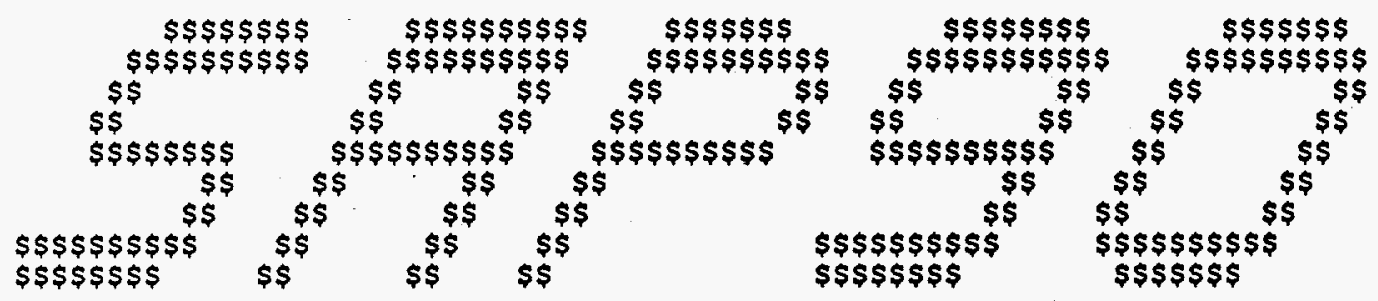

STRUCTURAI ANALYSIS PROGRAMS

VERSION P5.40

Copyright (C) 1978-1992

BDWARD L. WILSON

All rights reserved 


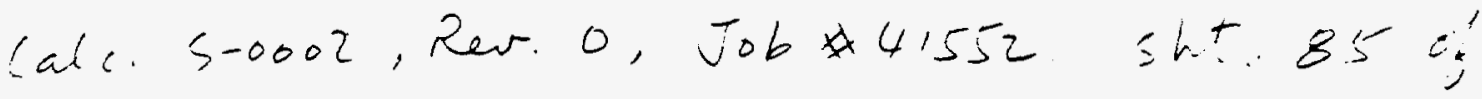

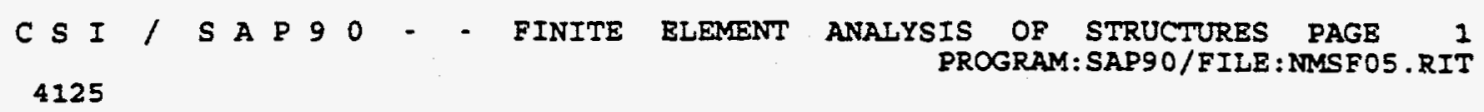

- 2826

$=132$

- 10 
Calc. S-0002, Rev.0, Job $\$ 41552$

sitt. $8 j=\frac{1}{j}$

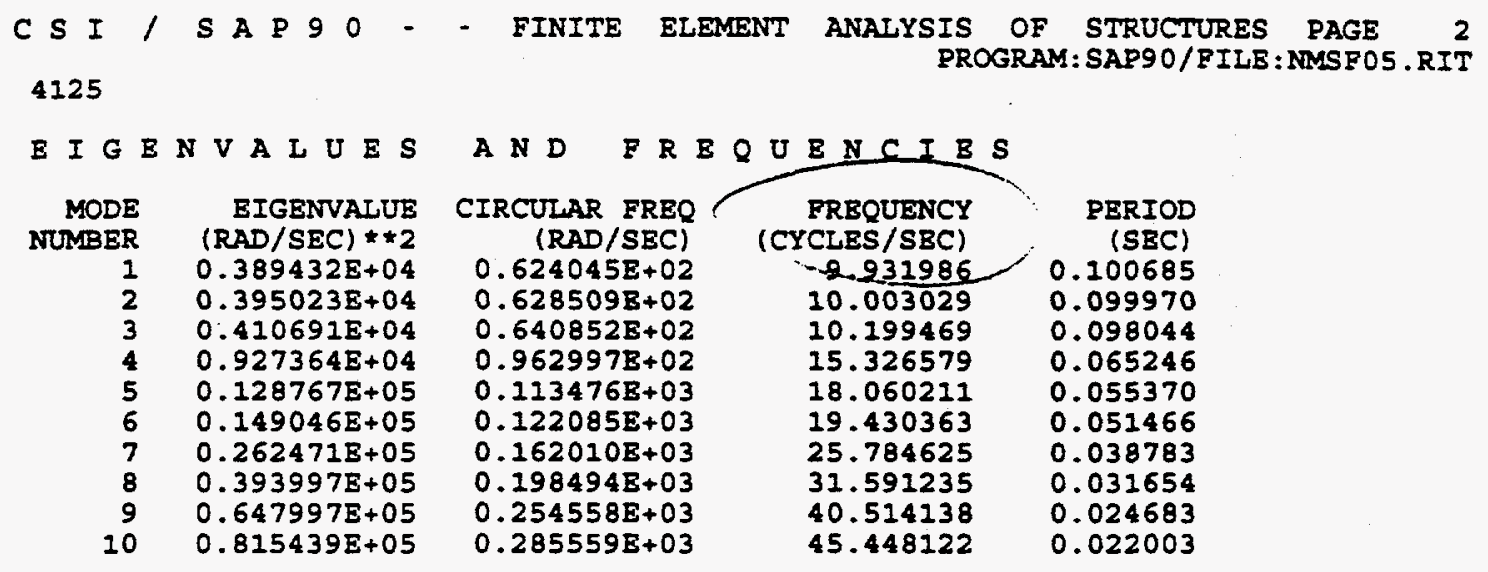




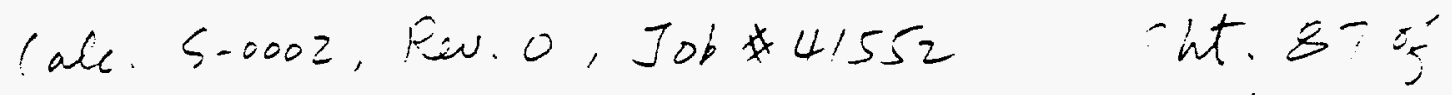

C $S$ I / S A P 90 - - FINITE ELEMENT ANALYSIS OF STRUCTURES PAGB 3

4125

BASETORCETEACTION FACTORS

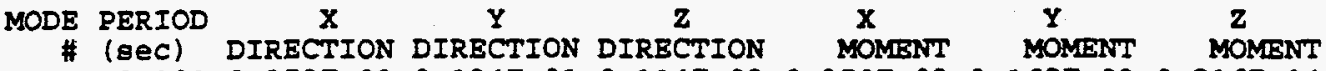

$\begin{array}{lccccc}\text { \# (sec) DIRECTION DIRECTION DIRECTION } & \text { MOMBNT } & \text { MOMENT } & \text { MOMENT } \\ 1 & 0.101 & 0.253 \mathrm{E}+02-0.134 \mathrm{~B}+01 & 0.114 \mathrm{~B}+02 & 0.950 \mathrm{E}+03 & 0.162 \mathrm{~B}+03-0.216 \mathrm{~B}+04\end{array}$

$20.100-0.134 \mathrm{E}+01 \quad 0.269 \mathrm{E}+02 \quad 0.592 \mathrm{E}+01-0.334 \mathrm{E}+03-0.269 \mathrm{E}+03 \quad 0.132 \mathrm{E}+04$

$3 \quad 0.098-0.115 \mathrm{E}+02-0.599 \mathrm{E}+01 \quad 0.249 \mathrm{E}+02 \quad 0.219 \mathrm{~B}+04-0.136 \mathrm{~B}+04 \quad 0.684 \mathrm{E}+03$

$\begin{array}{llllllll}4 & 0.065 & 0.398 E+00 & 0.499 \mathrm{~B}+01 & 0.114 \mathrm{~B}+01 & 0.137 \mathrm{~B}+04-0.221 \mathrm{~B}+03 & 0.790 \mathrm{~B}+02\end{array}$

$5 \quad 0.055-0.994 \mathrm{~B}-01 \quad 0.702 \mathrm{E}+00-0.123 \mathrm{E}+00-0.108 \mathrm{~B}+03 \quad 0.478 \mathrm{~B}+02 \quad 0.186 \mathrm{~B}+03$

$6 \quad 0.0510 .335 E+01-0.293 E+00 \quad 0.207 B+01 \quad 0.770 B+02-0.111 B+04-0.610 E+02$

$7 \quad 0.039-0.303 \mathrm{E}+00-0.34 \mathrm{BB}+00 \quad 0.204 \mathrm{~B}+00-0.247 \mathrm{~B}+02-0.127 \mathrm{~B}+03-0.154 \mathrm{~B}+04$

$8 \quad 0.0320 .272 E+01 \quad 0.162 E+00-0.140 E+00-0.132 E+02 \quad 0.117 E+03-0.375 E+03$

$9 \quad 0.025-0.383 E+00 \quad 0.155 E+01-0.204 E-01-0.477 E+02-0.122 E+02 \quad 0.595 E+03$

$10 \quad 0.0220 .176 \mathrm{E}+00 \quad 0.516 \mathrm{E}+00 \quad 0.192 \mathrm{E}+00-0.399 \mathrm{~B}+01 \quad 0.155 \mathrm{~B}+02-0.242 \mathrm{E}+03$ 
Calc, S-0002, Riw. o, Tob $\$ 41552$ Sitt. 33 of

C S I / S A P 90 - - FINITE ELEMENT ANALYSIS OF STRUCTURES PAGE 4 4125 PROGRAM: SAP9O/FILE : NMSSF 05.RIT

PARTICIPATING MASS - (percent)

$\begin{array}{rrrrrrr}\text { MODE } & \text { X-DIR } & \text { Y-DIR } & \text { Z-DIR } & \text { X-SUM } & \text { Y-SUM } & \text { Z-SUM } \\ 1 & 80.579 & 0.226 & 16.442 & 80.579 & 0.226 & 16.442 \\ 2 & 0.228 & 91.655 & 4.424 & 80.806 & 91.881 & 20.866 \\ 3 & 16.790 & 4.526 & \mathbf{7 8 . 3 6 0} & 97.596 & 96.407 & 99.226 \\ 4 & 0.020 & 3.138 & 0.165 & 97.616 & 99.544 & 99.391 \\ 5 & 0.001 & 0.062 & 0.002 & 97.617 & 99.606 & 99.393 \\ 6 & 1.416 & 0.011 & 0.539 & 99.033 & 99.617 & 99.932 \\ 7 & 0.012 & 0.015 & 0.005 & 99.045 & 99.632 & 99.937 \\ 8 & 0.932 & 0.003 & 0.002 & 99.976 & 99.636 & 99.940 \\ 9 & 0.019 & 0.304 & 0.000 & 99.995 & 99.939 & 99.940 \\ 10 & 0.004 & 0.034 & 0.005 & 99.999 & 99.973 & 99.945 \\ & & & & & \end{array}$




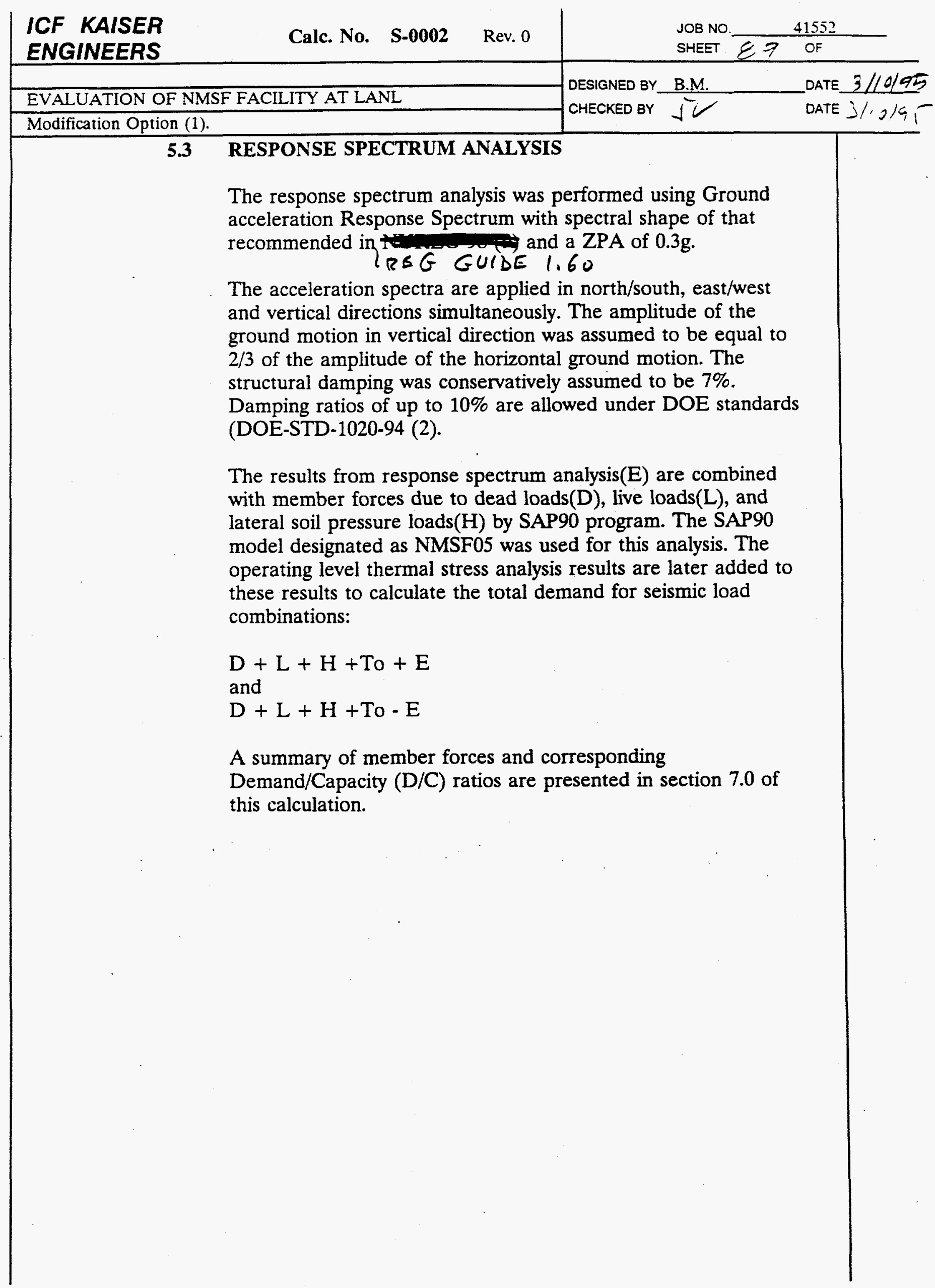




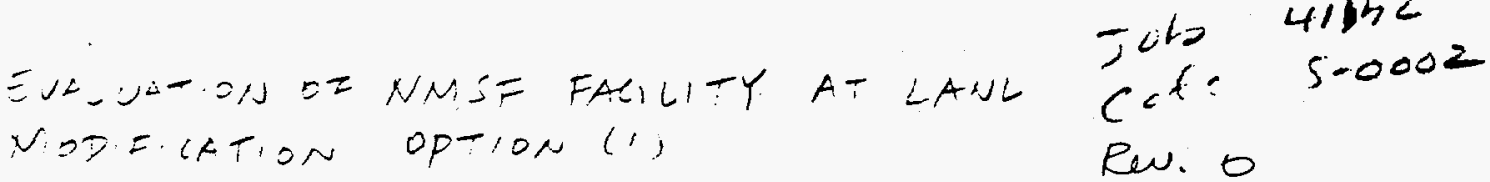

LOS ALAMO / NMSF FACILITY SEISMIC CRITERIA DESIGN GROUND ACCELERATION SPECTRUM (PGA $=0.3 \mathrm{~g}$ )

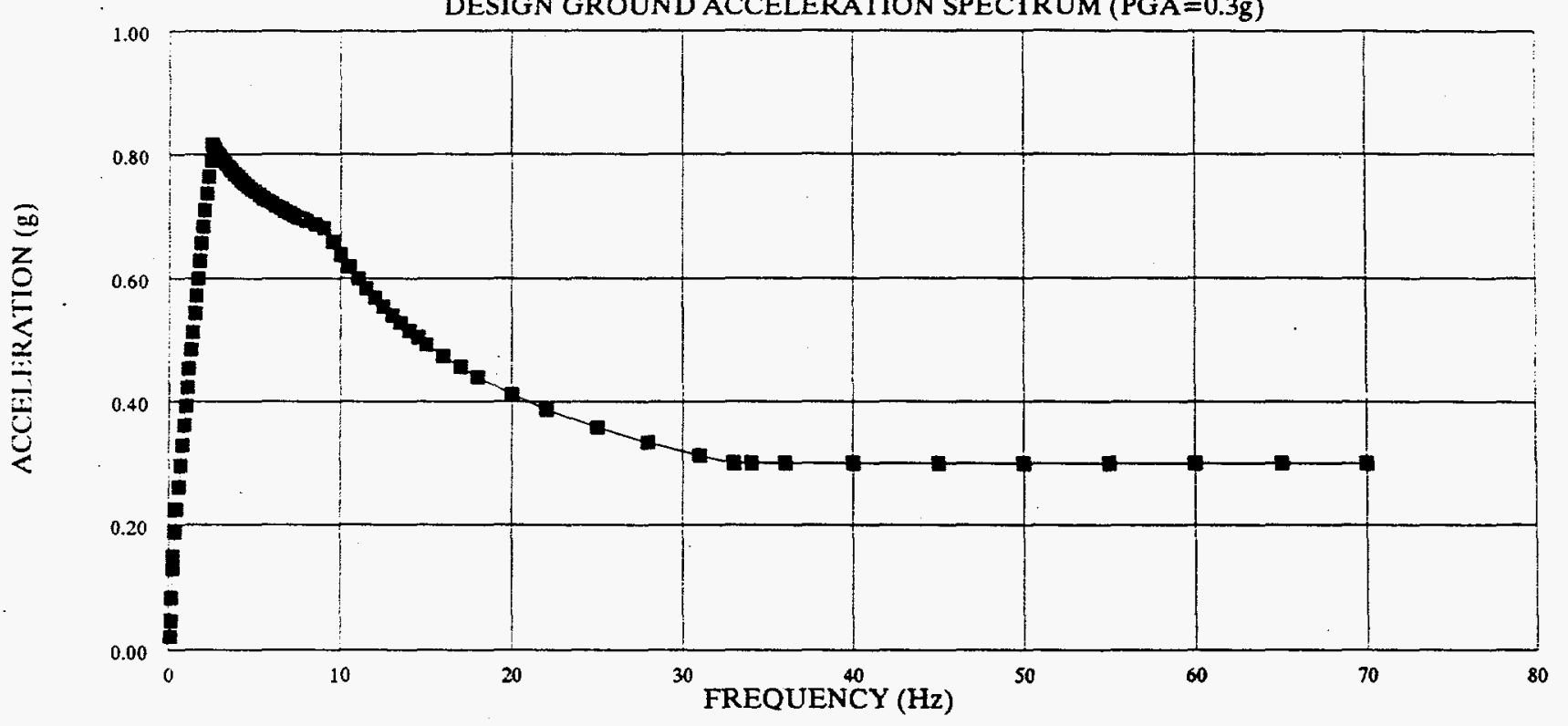

$\rightarrow-5 \%$ DAMPED

REG GUiDE 1.60 


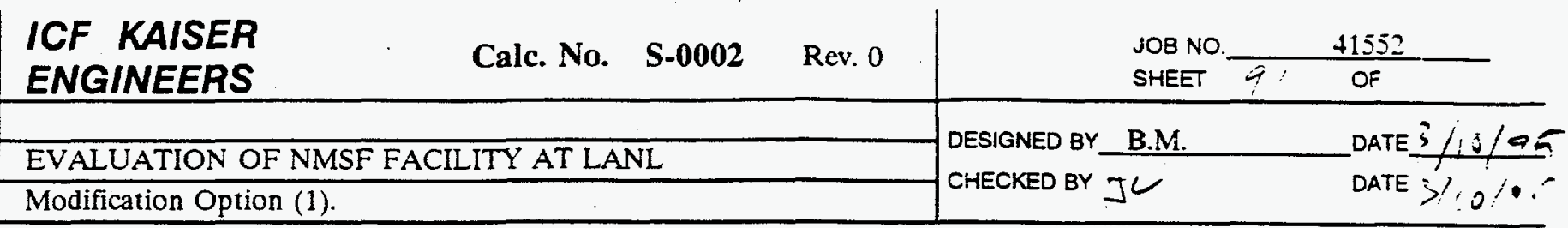

\subsection{UBC STATIC LOAD ANALYSIS}

To address the load criteria requirements for non-seismic load combinations recommended by UBC (4). The 3-dimensional model of the NMSF building was subjected to combinations of the dead loads(D) and live loads(L), static lateral soil pressure (Hs). A static analysis was performed. The SAP90 input data for this analysis is stored in file named NMSF51.

The results from this static analysis in then combined with those from thermal analysis due to accidental (Rare) thermal loads to compute member forces for load combination:

$\mathrm{D}+\mathrm{L}+\mathrm{Hs}+\mathrm{Ta}$

A summary of member forces and corresponding Demand/Capacity $(\mathrm{D} / \mathrm{C})$ ratios are presented in section 7.0 of this calculation. 


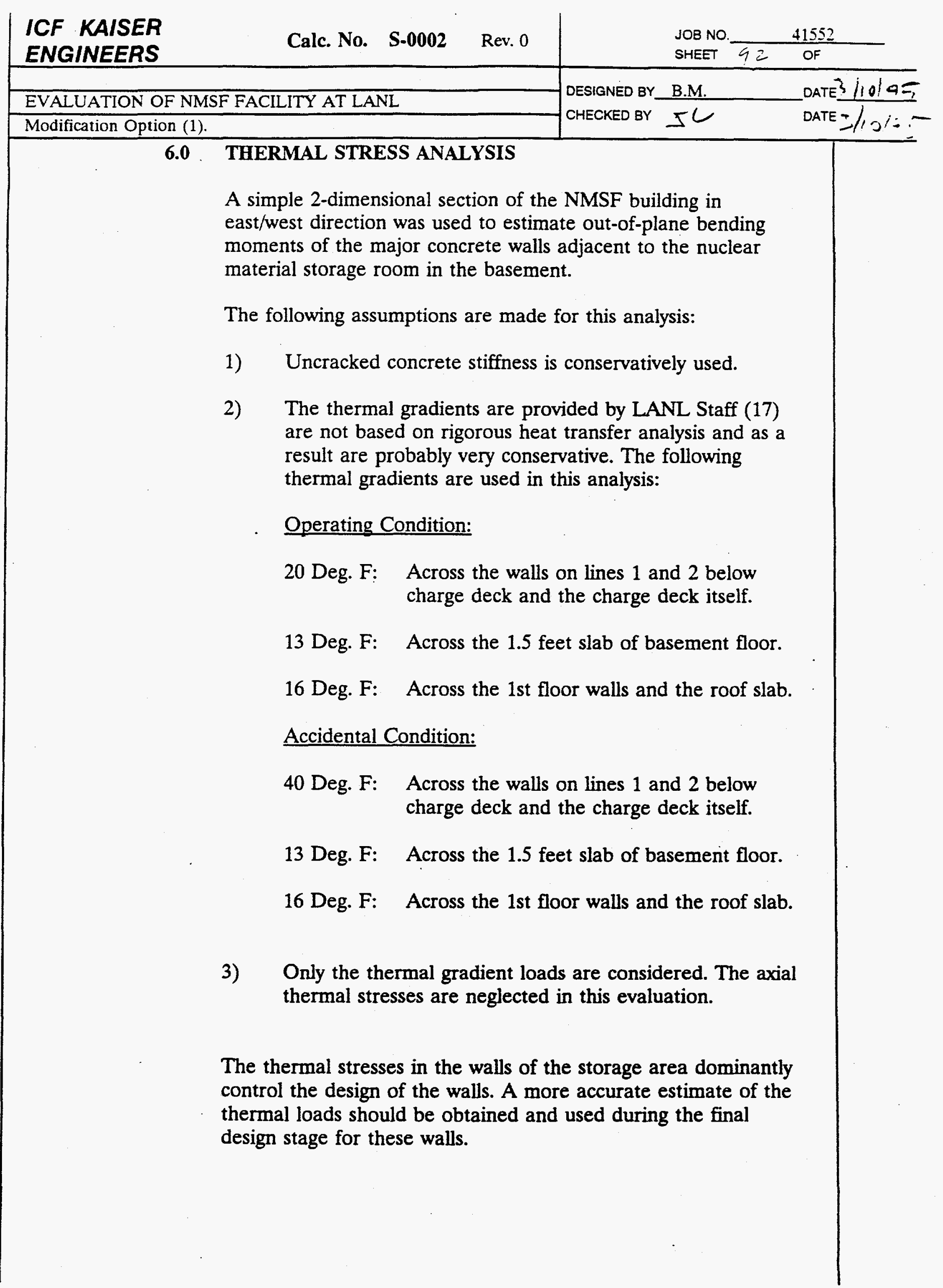




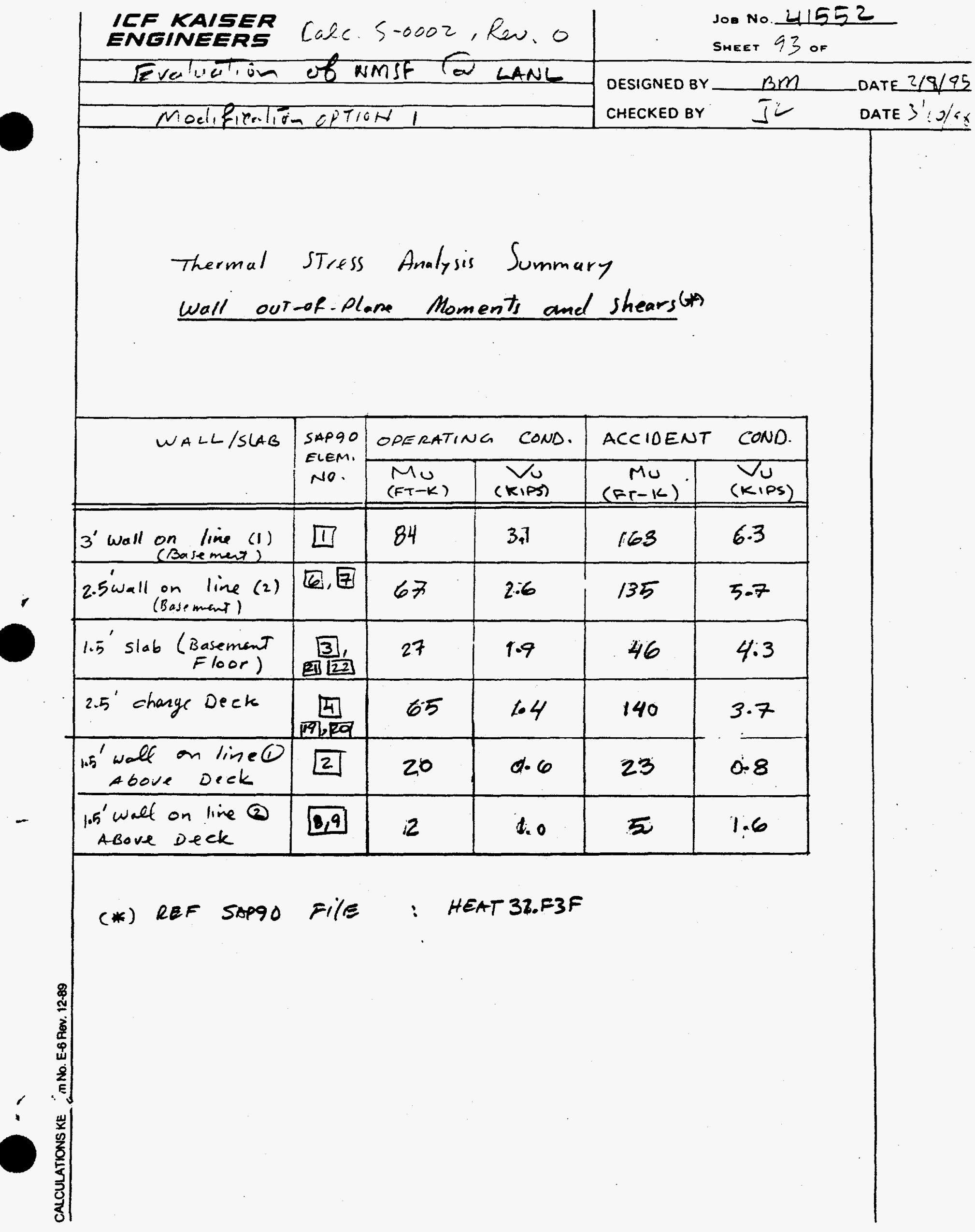




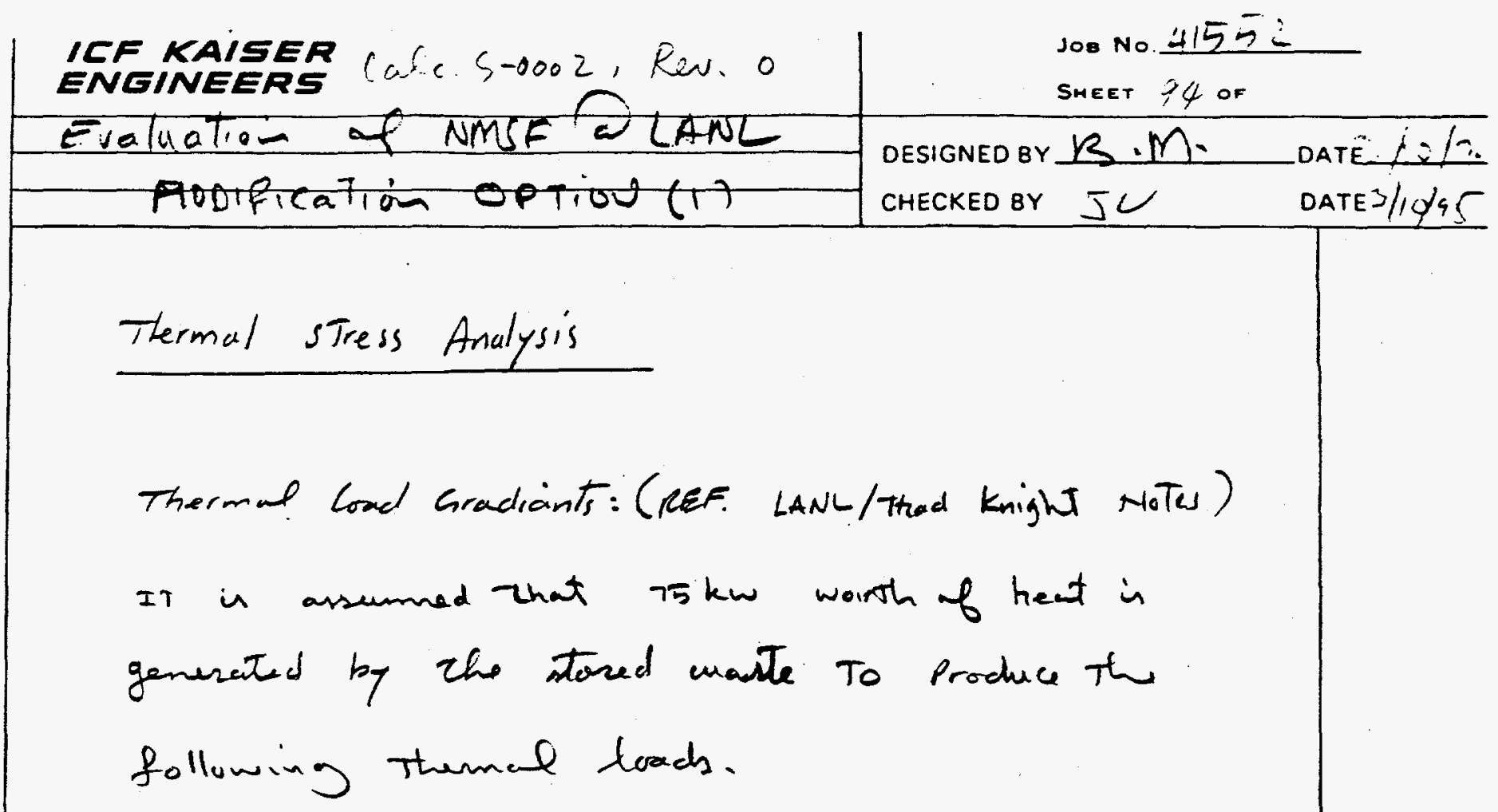

wall

Exterior walls

Basement slab

Change Deck

Roof slab operating Condition

$20^{-F}$

$13^{\circ} F$

$20^{\circ} \mathrm{F}$

$16^{. F}$
Accident Condition

$$
\begin{aligned}
& 40^{\circ} \\
&- 13^{\circ} \\
& 40^{\circ} \mathrm{F} \\
&= 16^{\circ}
\end{aligned}
$$

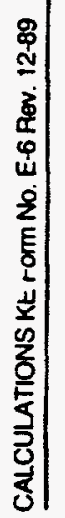




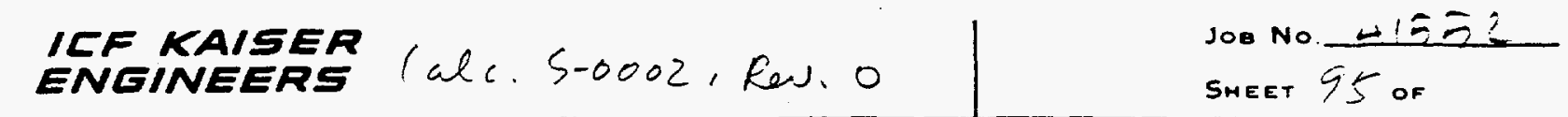

Eval of NMSF W LANL DESIGNEDBY BM DATE
Mupifictron OPTION (1) CHECKED BY JU DATE $3 / 12 /<4$

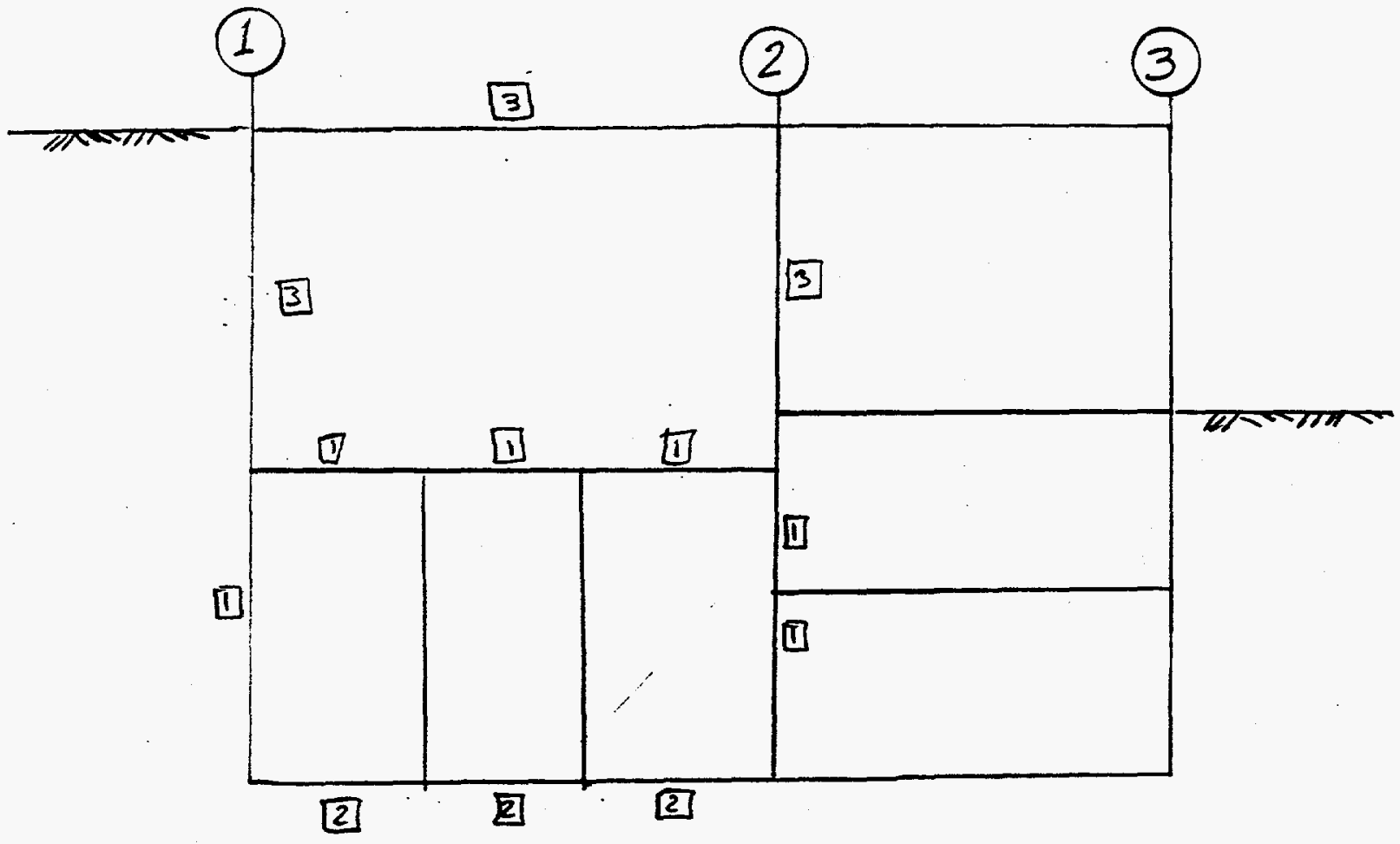

Thermal Gradient

$\Delta T$ Across the Section
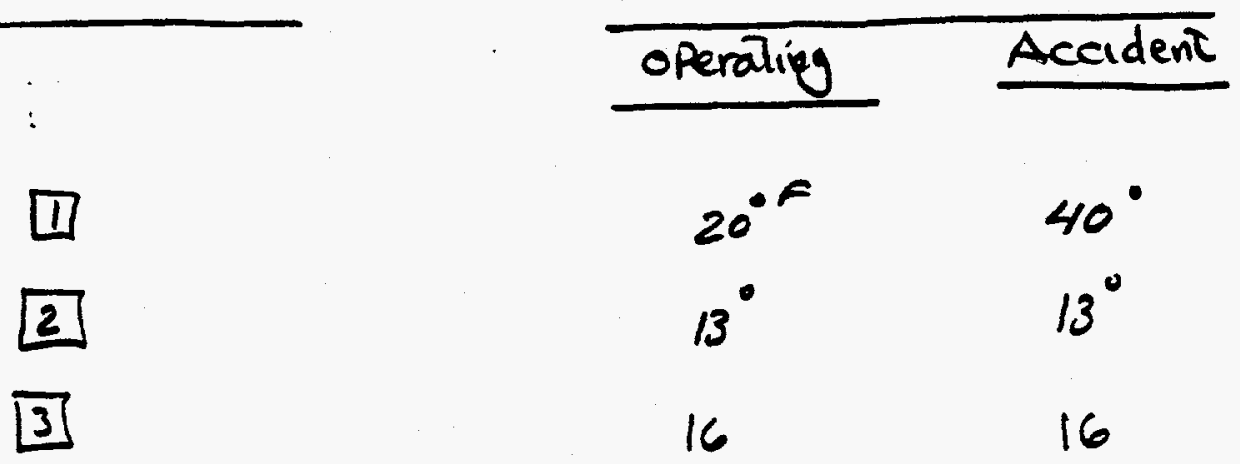


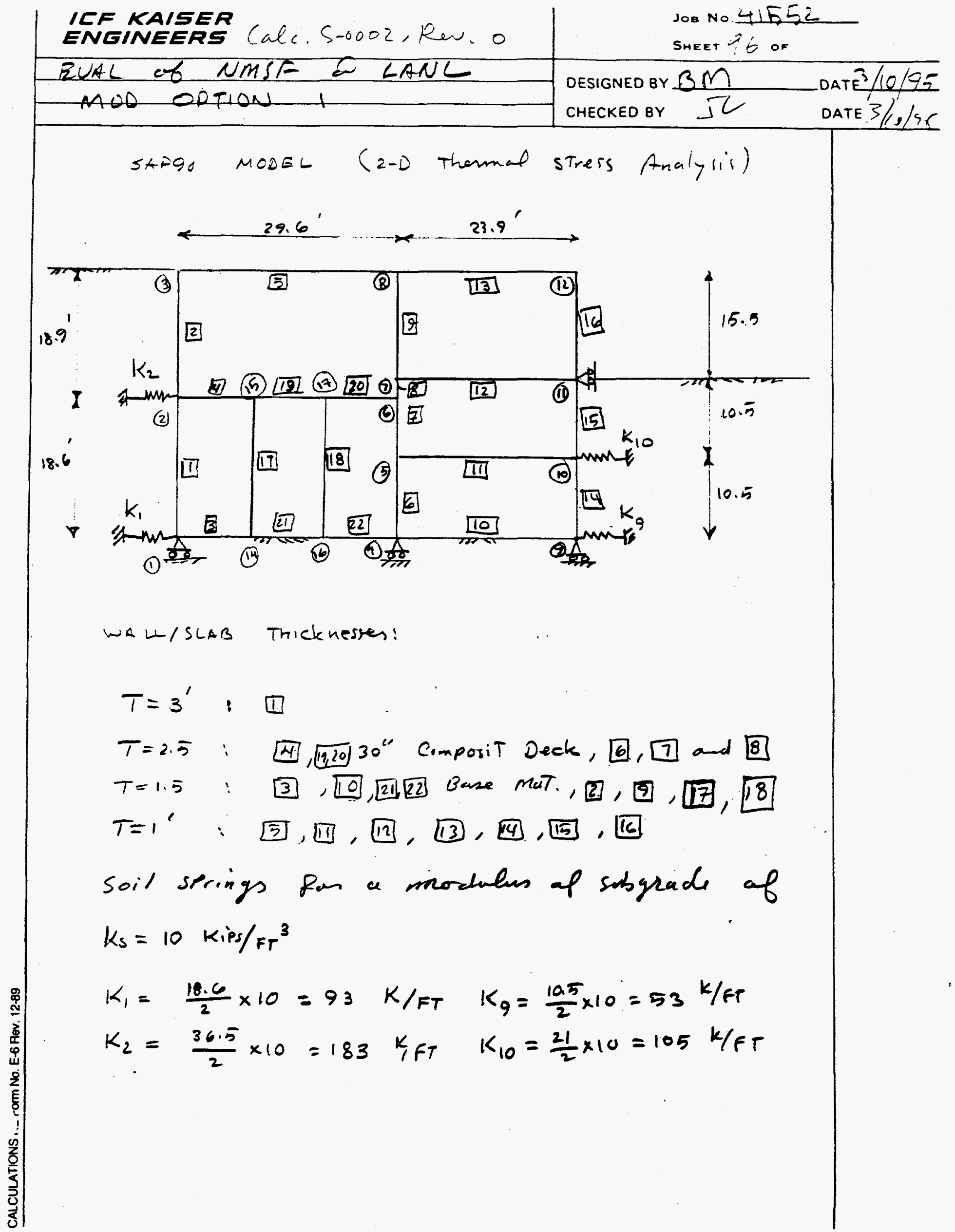




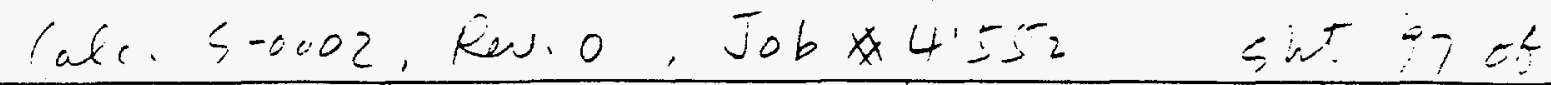

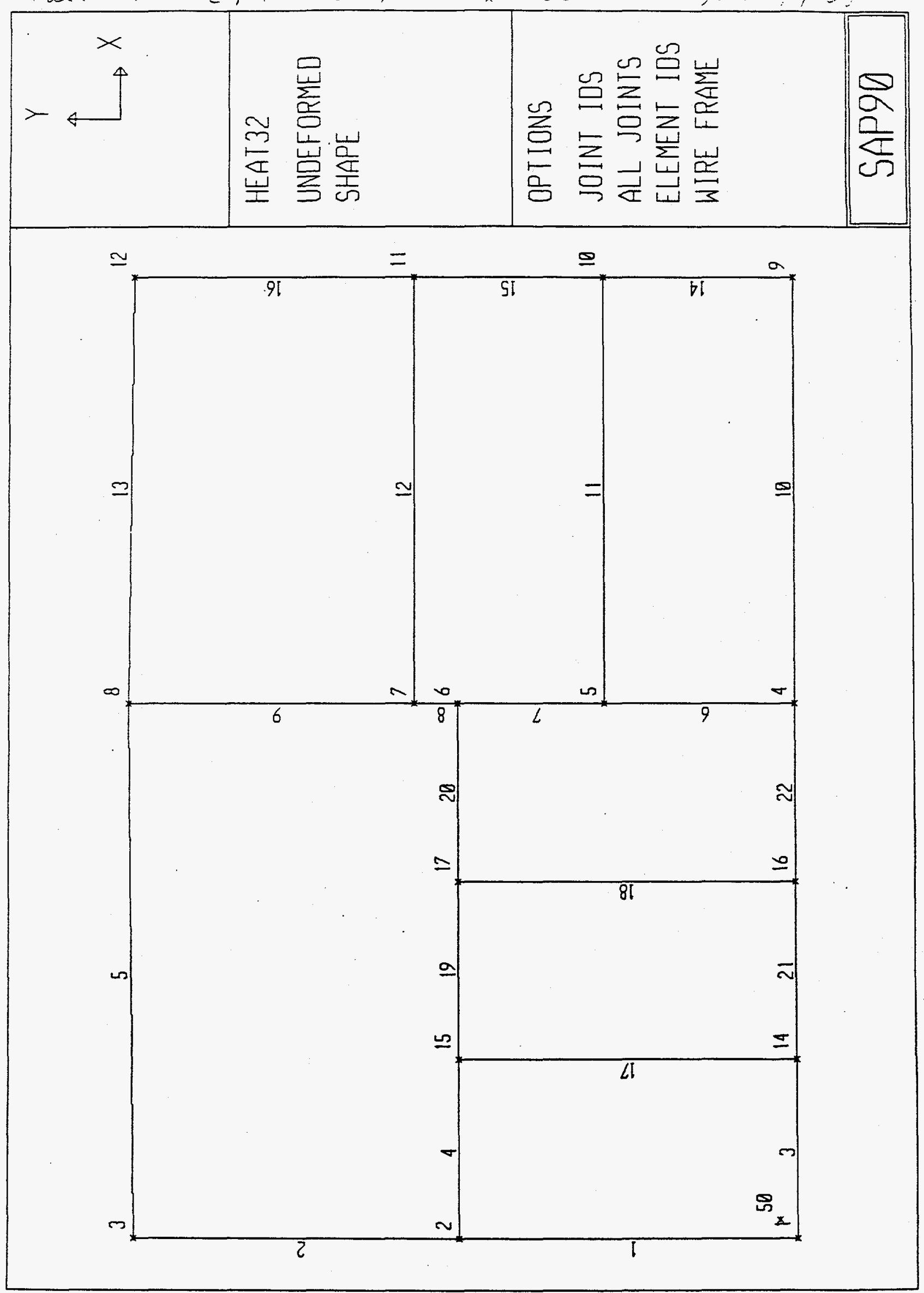


Salc. S-0002, Ees. 0, Tob 441552 Sit ty=

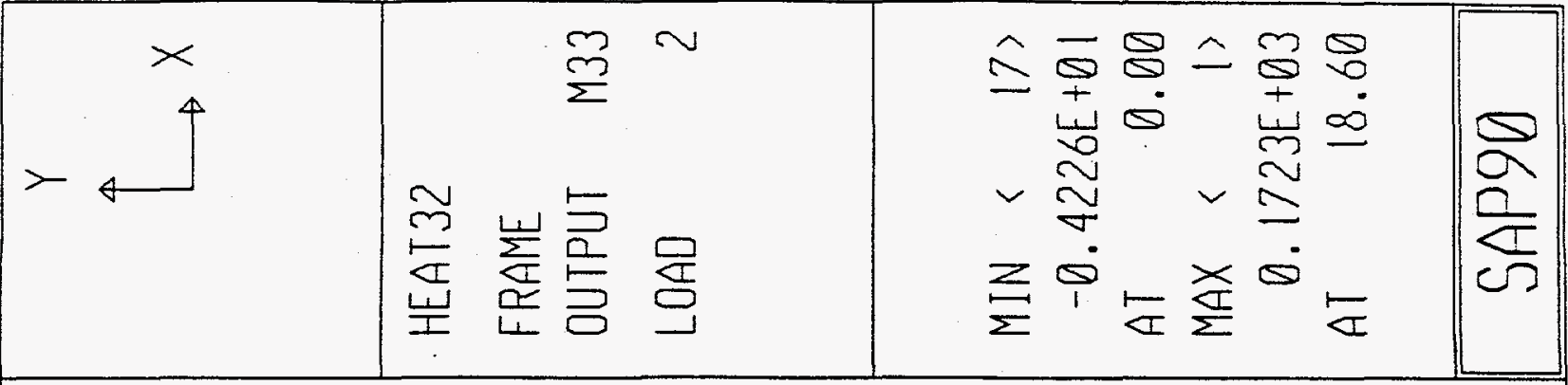

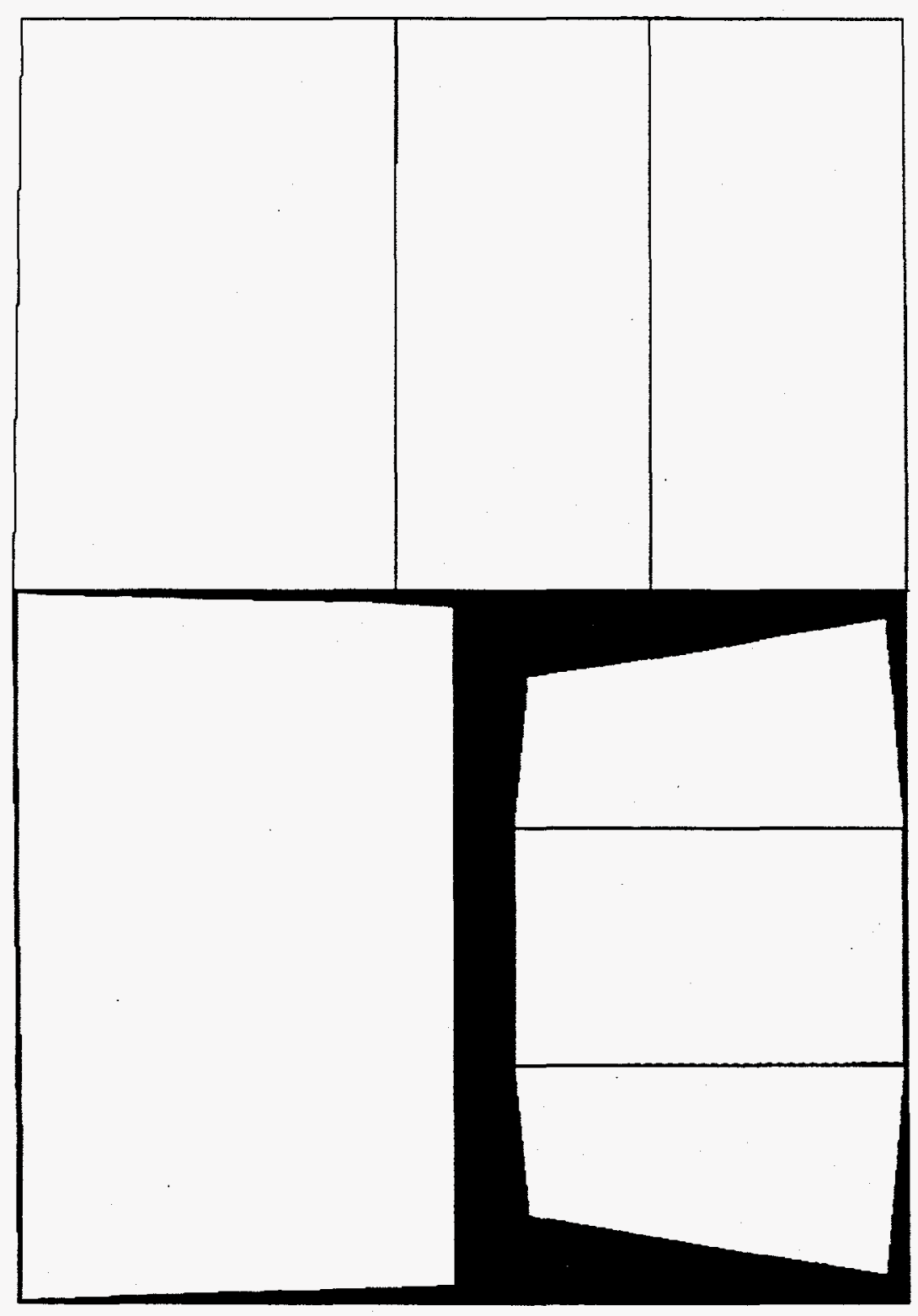




$$
\text { [ }
$$


talc. S-0002, Per.o, Job \&4/552

$\operatorname{sint} 13=-5$

\begin{tabular}{|c|c|c|}
\hline $\begin{array}{r}\times \\
4 \\
+\quad 4\end{array}$ & 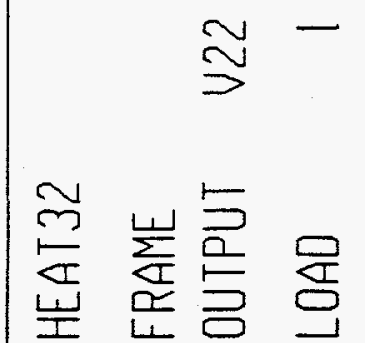 & 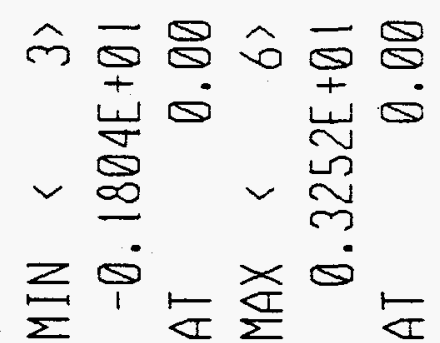 \\
\hline
\end{tabular}

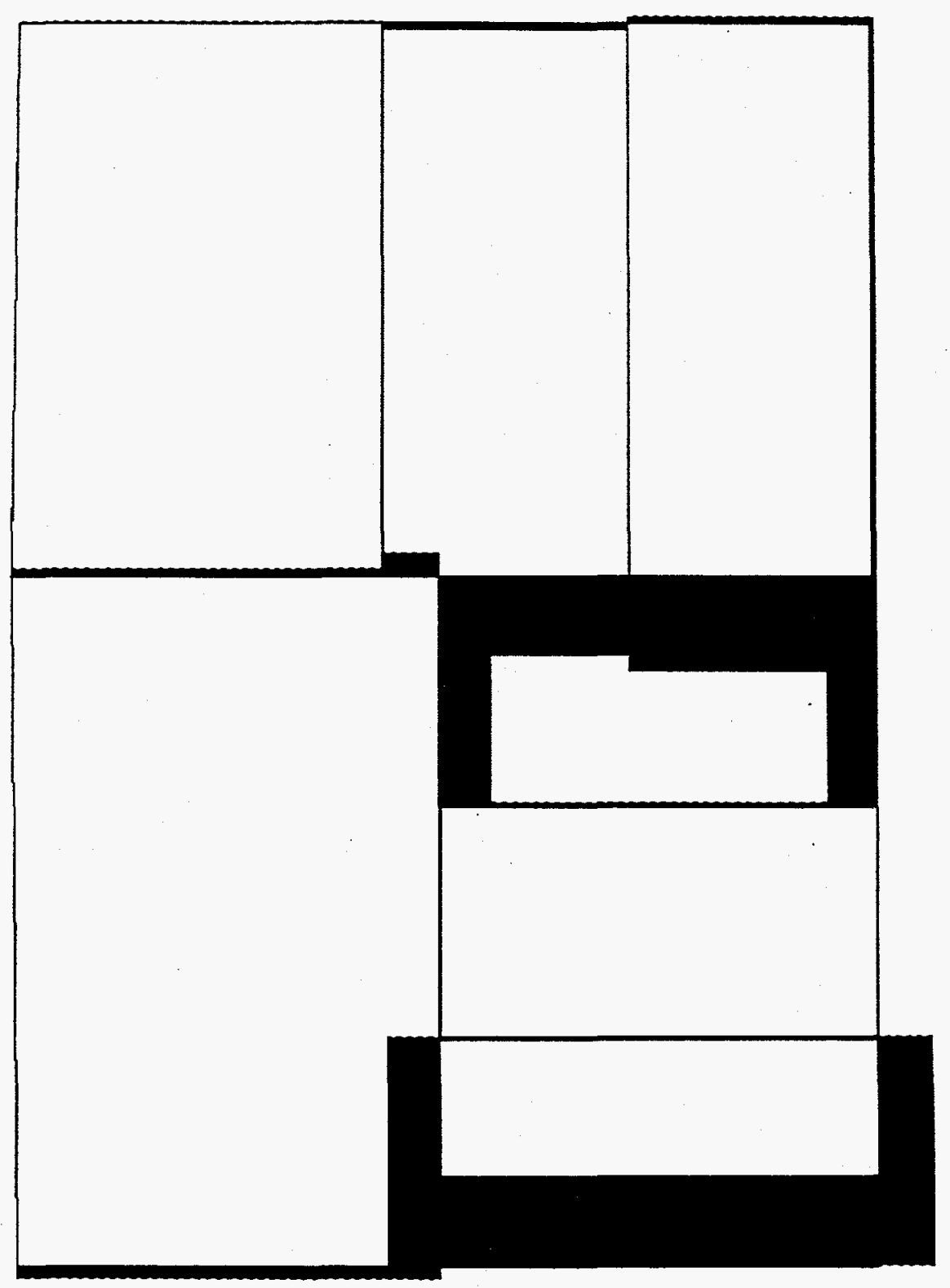




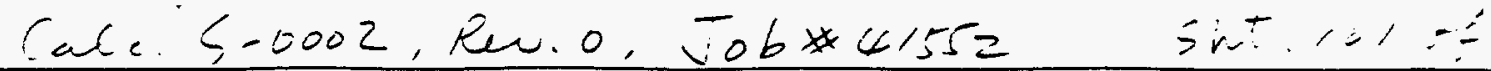

\begin{tabular}{|c|c|c|c|}
\hline $\begin{array}{l}x \\
4 \\
4\end{array}$ & 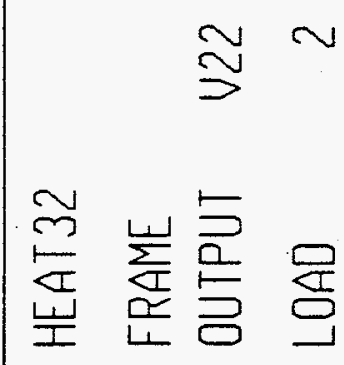 & 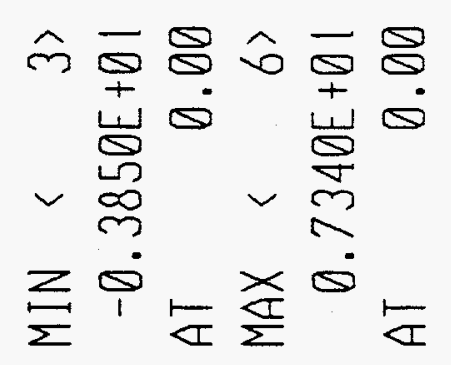 & $\mid \frac{s}{\sigma}$ \\
\hline
\end{tabular}

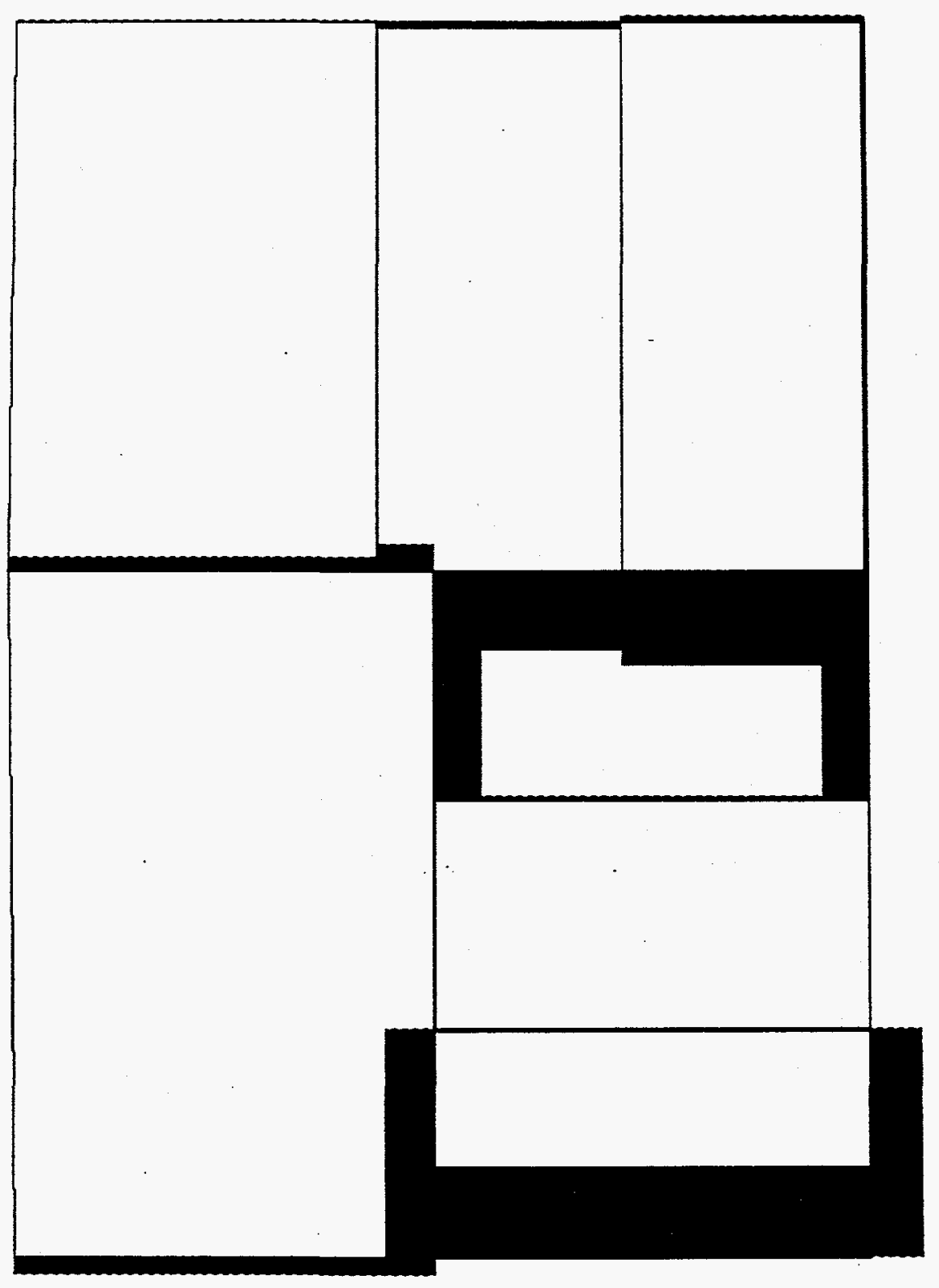


lakc. $=-0002$, Kev. o, job $\$ 41552$ Sit.

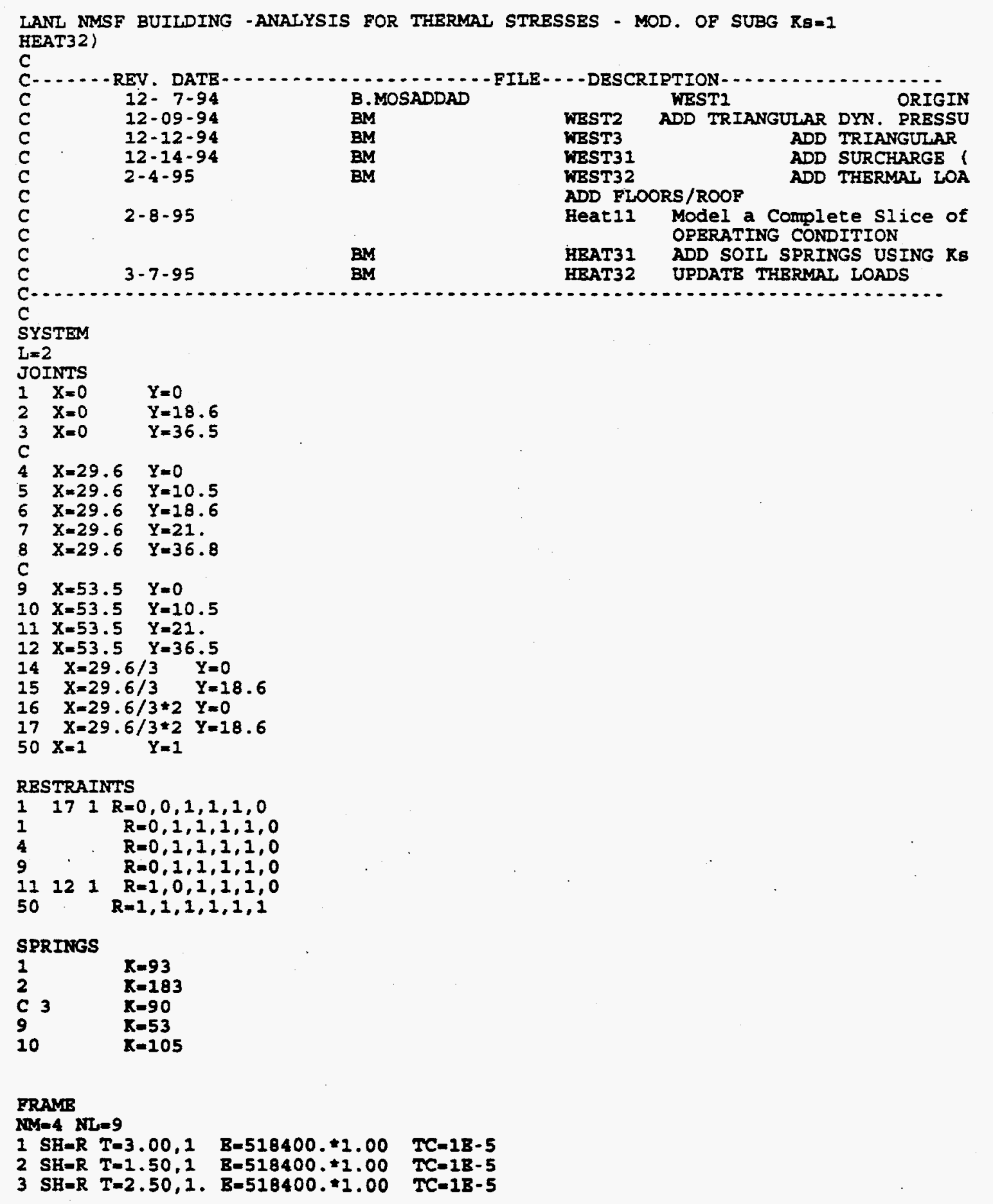


rare S-0002, Kev. o, vob *4.552
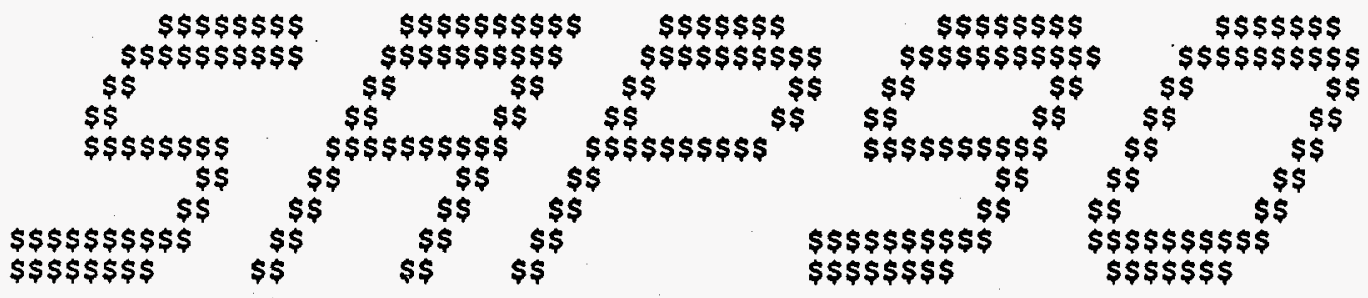

STRUCTURAL ANALYSIS PROGRAMS

VBRSION P5.40

Copyright (C) 1978-1992

BDWARD L. KILSON

All rights reaerved 
Cosc. S.0002, Eev. 0, Job $\$ 41552$ sht. $10.5 \%$

C S I / S A P $90-$ - FINITE ELEMENT ANALYSIS OF STRUCTURES PAGE 1 LANL MMSF BUILDING -ANALYSIS FOR THERMAL STRESSBS - MOD. OF SUBG KS=1

FR A E E LEM E T FOR C B S

\begin{tabular}{|c|c|c|c|c|c|c|c|c|}
\hline $\begin{array}{l}\text { ELT } \\
\text { ID }\end{array}$ & $\begin{array}{l}\text { LOAD } \\
\text { COND }\end{array}$ & $\begin{array}{l}\text { DIST } \\
\text { BNDI }\end{array}$ & SHBAR & $\begin{array}{l}\text { PLANE } \\
\text { MOMENT }\end{array}$ & $\begin{array}{l}\text { AXIAL } \\
\text { BORCE }\end{array}$ & SHEAR & $\begin{array}{l}\text { PLANE } \\
\text { MOMENT }\end{array}$ & $\begin{array}{r}\text { AXIAI } \\
\text { TORQ }\end{array}$ \\
\hline 1 & 1 & $\begin{array}{r}0.000 \\
0.000 \\
18.600 \\
18.600\end{array}$ & $\begin{array}{l}3.069 \\
3.069\end{array}$ & $\begin{array}{l}27.382 \\
84.463\end{array}$ & $\begin{array}{l}-1.452 \\
-1.452\end{array}$ & & & \\
\hline & 2 & $\begin{array}{r}0.000 \\
0.000 \\
18.600 \\
18.600\end{array}$ & $\begin{array}{l}6.284 \\
6.284\end{array}$ & $\begin{array}{r}45.644 \\
162.527\end{array}$ & $\begin{array}{l}-3.788 \\
-3.788\end{array}$ & & & \\
\hline 2 & 1 & $\begin{array}{r}0.000 \\
0.000 \\
17.900 \\
17.900\end{array}$ & $\begin{array}{l}-0.623 \\
-0.623\end{array}$ & $\begin{array}{r}19.726 \\
8.574\end{array}$ & -0.100 & & & \\
\hline & 2 & $\begin{array}{r}0.000 \\
0.000 \\
17.900 \\
17.900\end{array}$ & $\begin{array}{l}-0.792 \\
-0.792\end{array}$ & $\begin{array}{r}22.521 \\
8.351\end{array}$ & $\begin{array}{l}-0.090 \\
-0.090\end{array}$ & & & \\
\hline 3 & $\begin{array}{c}\cdots \\
1\end{array}$ & $\begin{array}{l}0.000 \\
0.000 \\
9.867 \\
9.867\end{array}$ & $\begin{array}{l}-1.921 \\
-1.921\end{array}$ & $\begin{array}{r}27.382 \\
8.426\end{array}$ & 2.979 & & . & \\
\hline & 2 & $\begin{array}{l}0.000 \\
0.000 \\
9.867 \\
9.867\end{array}$ & $\begin{array}{l}-4.341 \\
-4.341\end{array}$ & $\begin{array}{r}45.644 \\
2.817\end{array}$ & $\begin{array}{l}6.126 \\
6.126\end{array}$ & & & \\
\hline 4 & $\begin{array}{c}1 \\
1\end{array}$ & $\begin{array}{l}0.000 \\
0.000 \\
9.867 \\
9.867\end{array}$ & -1.352 & $\begin{array}{l}64.737 \\
51.401\end{array}$ & $\begin{array}{l}-3.684 \\
-3.684\end{array}$ & & & \\
\hline & 2 & $\begin{array}{l}0.000 \\
0.000 \\
9.867 \\
9.867\end{array}$ & $\begin{array}{l}-3.698 \\
-3.698\end{array}$ & $\begin{array}{l}140.006 \\
103.519\end{array}$ & $\begin{array}{l}-7.068 \\
-7.068\end{array}$ & & & \\
\hline 5 & 1 & $\begin{array}{r}0.000 \\
0.000 \\
29.602 \\
29.602\end{array}$ & $\begin{array}{l}-0.107 \\
-0.107\end{array}$ & $\begin{array}{l}8.574 \\
5.419\end{array}$ & 0.622 & & & \\
\hline
\end{tabular}


iate. S-0002, Nev. $0, \sqrt{0} 6 \times 45$

CS I / S A P 90 - - EINITE ELEMENT ANALYSIS OF STRUCTURES PAGE 2 PROGRAM: SAP90/FILE: heat 32. F3F LANL NMSF BUILDING - ANALYSIS FOR THERMAL STRESSES - MOD. OF SUBG KB=1

FRAME ELEMENT FOR C E S

\begin{tabular}{|c|c|c|c|c|c|c|c|c|}
\hline ELT & $\begin{array}{l}\text { LOAD } \\
\text { COND }\end{array}$ & $\begin{array}{l}\text { DIST } \\
\text { ENDI }\end{array}$ & SHEAR & $\begin{array}{l}\text { PLANE } \\
\text { MOMENT }\end{array}$ & $\begin{array}{l}\text { AXIAI } \\
\text { FORCE }\end{array}$ & SHEAR $^{1-3}$ & $\begin{array}{l}\text { PLANE } \\
\text { MOMBNT }\end{array}$ & $\begin{array}{r}\text { AXIAI } \\
\text { TORQ }\end{array}$ \\
\hline & 2 & $\begin{array}{r}0.000 \\
0.000 \\
29.602 \\
29.602\end{array}$ & $\begin{array}{l}-0.098 \\
-0.098\end{array}$ & $\begin{array}{l}8.351 \\
5.451\end{array}$ & $\begin{array}{r}0.791 \\
0.791\end{array}$ & & & \\
\hline 6 & 1 & $\begin{array}{r}0.000 \\
0.000 \\
10.500 \\
10.500\end{array}$ & $\begin{array}{l}2.583 \\
2.583\end{array}$ & $\begin{array}{l}23.338 \\
50.458\end{array}$ & $\begin{array}{l}-1.276 \\
-1.276\end{array}$ & & & \\
\hline & 2 & $\begin{array}{r}0.000 \\
0.000 \\
10.500 \\
10.500\end{array}$ & $\begin{array}{l}5.651 \\
5.651\end{array}$ & $\begin{array}{l}39.627 \\
98.959\end{array}$ & $\begin{array}{l}-2.623 \\
-2.623\end{array}$ & & & \\
\hline 7 & 1 & $\begin{array}{l}0.000 \\
0.000 \\
8.100 \\
8.100\end{array}$ & $\begin{array}{l}2.176 \\
2.176\end{array}$ & $\begin{array}{l}49.662 \\
67.286\end{array}$ & $\begin{array}{l}-1.332 \\
-1.332\end{array}$ & & & \\
\hline & 2 & $\begin{array}{l}0.000 \\
0.000 \\
8.100 \\
8.100\end{array}$ & $\begin{array}{l}4.680 \\
4.680\end{array}$ & $\begin{array}{r}97.456 \\
135.366\end{array}$ & $\begin{array}{l}-2.724 \\
-2.724\end{array}$ & & & \\
\hline 8 & 1 & $\begin{array}{l}0.000 \\
0.000 \\
2.400 \\
2.400\end{array}$ & $\begin{array}{l}-1.029 \\
-1.029\end{array}$ & $\begin{array}{r}2.093 \\
-0.376\end{array}$ & $\begin{array}{l}0.066 \\
0.066\end{array}$ & & & \\
\hline & 2 & $\begin{array}{l}0.000 \\
0.000 \\
2.400 \\
2.400\end{array}$ & $\begin{array}{l}-1.622 \\
-1.622\end{array}$ & $\begin{array}{l}5.188 \\
1.295\end{array}$ & $\begin{array}{l}0.083 \\
0.083\end{array}$ & & & \\
\hline 9 & 1 & $\begin{array}{r}0.000 \\
0.000 \\
15.800 \\
15.800\end{array}$ & $\begin{array}{l}0.330 \\
0.330\end{array}$ & $\begin{array}{r}-0.824 \\
4.397\end{array}$ & $\begin{array}{l}0.028 \\
0.028\end{array}$ & . & & · \\
\hline & 2 & $\begin{array}{r}0.000 \\
0.000 \\
15.800 \\
15.800\end{array}$ & $\begin{array}{l}0.246 \\
0.246\end{array}$ & $\begin{array}{l}0.369 \\
4.262\end{array}$ & $\begin{array}{l}0.004 \\
0.004\end{array}$ & & & \\
\hline
\end{tabular}


calc. 5-0002, hew. 0, Job *41552 Sht. $107 \%$

C S I / S A P 90 - - FINITE ELBMENT ANALYSIS OF STRUCTURES PAGE 3 LANL NMSF BUILDING - ANALYSIS FOR THERMAL STRESSBS - MOD. OF SUBG R8=1

F R A E BLEM E N T FOR C B S

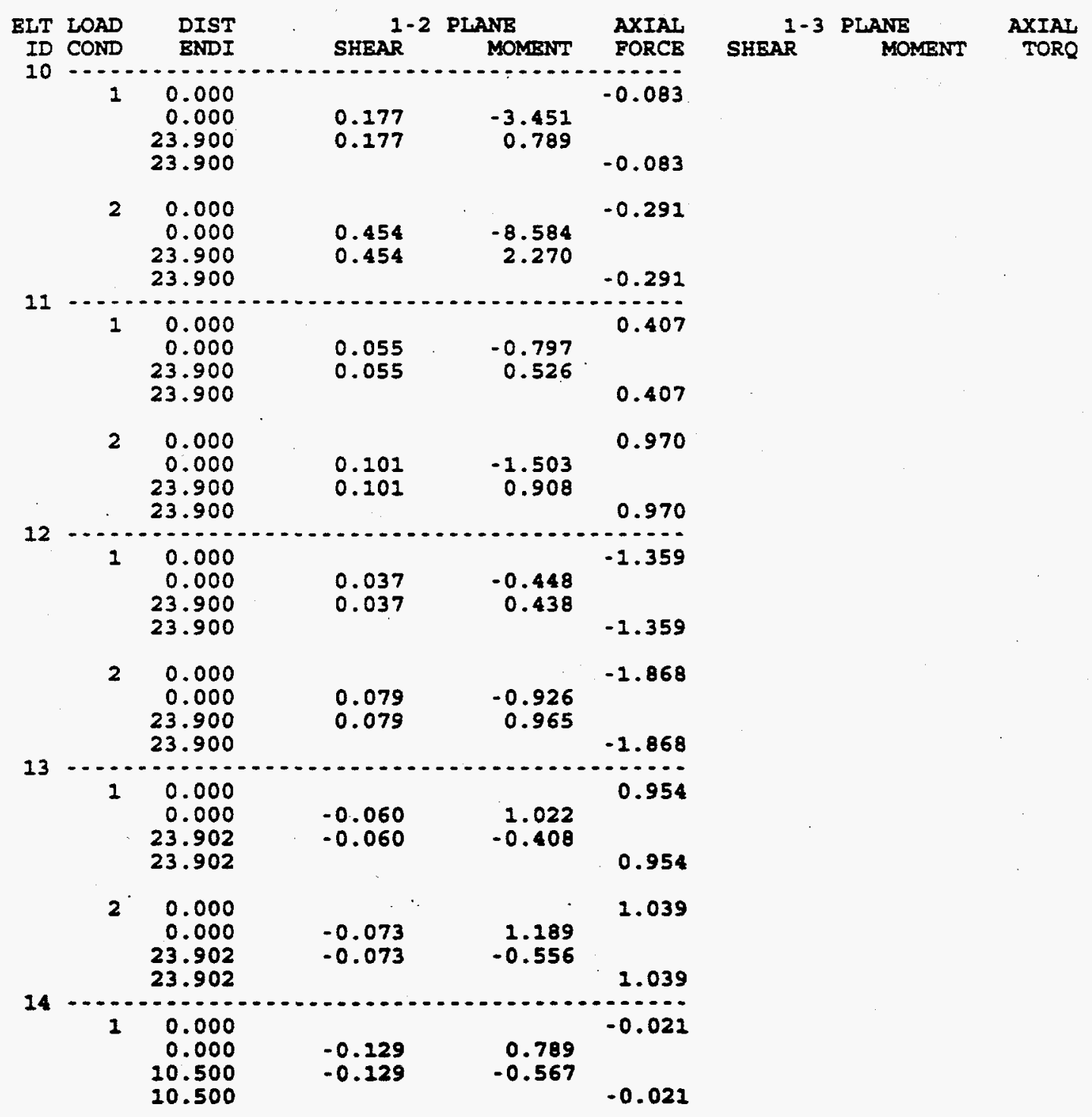


Lale, S-0002, Rev. O, Job *41552, Sirt is =s

C S I $/$ S A P $90-$ - FINITE ELEMINT ANALYSIS OF STRUCTURES PAGE 4 LANL NMSF BUILDING - ANALYSIS FOR THERMAI STRESSBS - MOD. OF SUBG KB=1

F R M E E L E M E N T FOR C B S

\begin{tabular}{|c|c|c|c|c|c|c|c|c|}
\hline $\begin{array}{l}\text { ELT } \\
\text {.ID }\end{array}$ & $\begin{array}{l}\text { LOAD } \\
\text { COND }\end{array}$ & $\begin{array}{l}\text { DIST } \\
\text { ENDI }\end{array}$ & SHEAR & $\begin{array}{l}\text { ANE } \\
\text { MOMENT }\end{array}$ & $\begin{array}{l}\text { AXIAL } \\
\text { PORCE }\end{array}$ & SHEAR ${ }^{1-3}$ & $\begin{array}{l}\text { PLANE } \\
\text { MOMENT }\end{array}$ & $\begin{array}{l}\text { AXIAL } \\
\text { TORQ }\end{array}$ \\
\hline & 2 & $\begin{array}{r}0.000 \\
0.000 \\
10.500 \\
10.500\end{array}$ & $\begin{array}{l}-0.370 \\
-0.370\end{array}$ & $\begin{array}{r}2.270 \\
-1.611\end{array}$ & $\begin{array}{l}-0.094 \\
-0.094\end{array}$ & & & \\
\hline 15 & 1 & $\begin{array}{r}0.000 \\
0.000 \\
10.500 \\
10.500\end{array}$ & $\begin{array}{l}0.196 \\
0.196\end{array}$ & $\begin{array}{r}-1.093 \\
0.966\end{array}$ & 0.035 & & & \\
\hline & 2 & $\begin{array}{r}0.000 \\
0.000 \\
10.500 \\
10.500\end{array}$ & $\begin{array}{l}0.431 \\
0.431\end{array}$ & $\begin{array}{r}-2.520 \\
2.003\end{array}$ & $\begin{array}{l}0.007 \\
0.007\end{array}$ & & & \\
\hline 16 & $\begin{array}{r}\cdots \\
1\end{array}$ & $\begin{array}{r}0.000 \\
0.000 \\
15.500 \\
15.500\end{array}$ & $\begin{array}{l}-0.060 \\
-0.060\end{array}$ & $\begin{array}{r}0.527 \\
-0.408\end{array}$ & 0.072 & & & \\
\hline & 2 & $\begin{array}{r}0.000 \\
0.000 \\
15.500 \\
15.500\end{array}$ & $\begin{array}{l}-0.103 \\
-0.103\end{array}$ & $\begin{array}{r}1.038 \\
-0.556\end{array}$ & $\begin{array}{l}0.086 \\
0.086\end{array}$ & & & \\
\hline 17 & 1 & $\begin{array}{r}0.000 \\
0.000 \\
18.600 \\
18.600\end{array}$ & $\begin{array}{l}0.312 \\
0.312\end{array}$ & $\begin{array}{r}-3.434 \\
2.371\end{array}$ & 1.802 & & & \\
\hline & 2 & $\begin{array}{r}0.000 \\
0.000 \\
18.600 \\
18.600\end{array}$ & $\begin{array}{l}0.579 \\
0.579\end{array}$ & $\begin{array}{r}-6.679 \\
4.083\end{array}$ & $\begin{array}{r}4.297 \\
4.297\end{array}$ & & & \\
\hline 18 & $\begin{array}{r}-\cdots \\
1\end{array}$ & $\begin{array}{r}0.000 \\
0.000 \\
18.600 \\
18.600\end{array}$ & $\begin{array}{l}0.167 \\
0.167\end{array}$ & $\begin{array}{r}-1.042 \\
2.060\end{array}$ & $\begin{array}{l}0.947 \\
0.947\end{array}$ & & & \\
\hline & 2 & $\begin{array}{r}0.000 \\
0.000 \\
18.600 \\
18.600\end{array}$ & $\begin{array}{l}0.187 \\
0.187\end{array}$ & $\begin{array}{r}-0.617 \\
2.866\end{array}$ & $\begin{array}{l}2.209 \\
2.209\end{array}$ & & & \\
\hline
\end{tabular}


laie S-0002, Rev10, Job $* 41552$ Skt. $10 \% 8 \%$

C S I / S A P 90 - - FINITE ELEMENT ANALYSIS OF STRUCTURBS PAGE 5 PROGRAM: SAP9O/FILB: heat 32 . P3F LANL NMSF BUILDING - ANALYSIS FOR THERMAL STRESSBS - MOD. OE SUBG KB=1 F R A E E L E M N T FORCE S

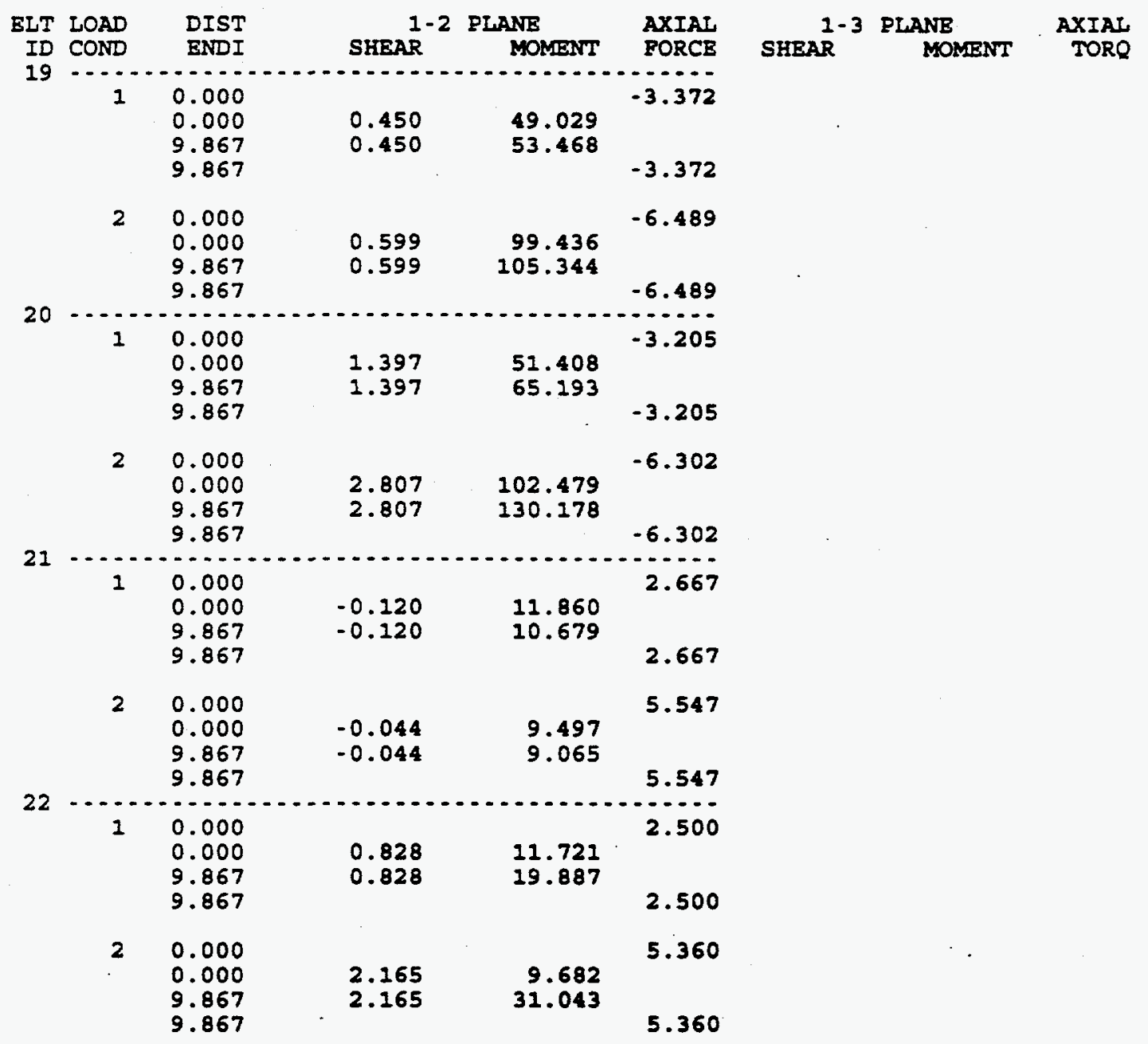




\begin{tabular}{|c|c|c|c|c|}
\hline $\begin{array}{l}\text { ICF KAISER } \\
\text { ENGINEERS }\end{array}$ & Rev. 0 & & $\begin{array}{l}\text { JOBNO. } \\
\text { SHEET } / 10\end{array}$ & $\frac{41552}{\text { OF }}$ \\
\hline FVAIUATION OF NMSF FACIITYY AT I ANI & & \multirow{2}{*}{\multicolumn{2}{|c|}{$\begin{array}{l}\text { DESIGNED BY B.M. } \\
\text { CHECKED BY }\end{array}$}} & \multirow{2}{*}{$\begin{array}{l}\text { DATE } 3 / 10 / 5 \\
\text { DATE } 3 / 10 / 96\end{array}$} \\
\hline Modification Option (1). & & & & \\
\hline
\end{tabular}

7.1 WALL IN-PLANE SHEAR AND BENDING OF SHEAR 


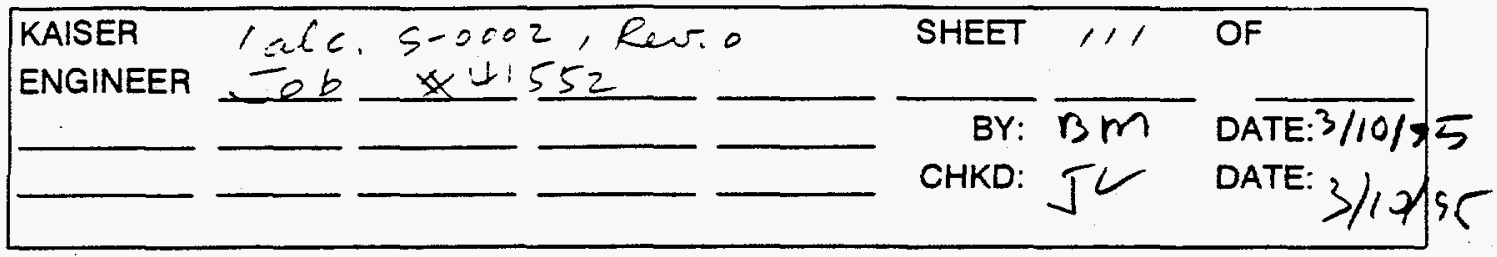

SUMMARY OF IN-PLANE SHEAR D/C RATIOS (OPTION 1)

\begin{tabular}{|c|c|c|c|c|c|c|}
\hline $\begin{array}{l}\text { WALL } \\
\text { SEGME }\end{array}$ & $\begin{array}{r}\text { LENGTH } \\
L \\
\text { (FEET) } \\
\end{array}$ & $\begin{array}{r}\text { WIDTH } \\
\text { Be(*) } \\
\text { (FEET) } \\
\end{array}$ & $\begin{array}{r}\text { CAPACITY } \\
\text { (Vc) } \\
(\mathrm{KIPS}) \\
\end{array}$ & $\begin{array}{r}\text { DEMAND } \\
(\mathrm{Vu}) \\
\text { (KIPS) }\end{array}$ & $\begin{array}{l}(D / C) \\
V u / N c\end{array}$ & \multirow{5}{*}{$0.73 \quad$ (*) } \\
\hline 1 & 130.45 & 3 & 4847 & 1629 & 0.34 & \\
\hline 2 & 18.25 & 1.5 & 339 & 131 & 0.39 & \\
\hline 4 & 18.25 & 1.33 & $\begin{array}{l}301 \\
-424\end{array}$ & 124 & 0.41 & \\
\hline 5 & 130.45 & 2.85 & & 1770 & - & \\
\hline 6 & 148.7 & 1.45 & 2671 & 1080 & 0.40 & \multirow{19}{*}{$o \cdot k$} \\
\hline 9 & 72.5 & 1.38 & 1239 & 1315 & 1.06 & \\
\hline 12 & 53.5 & 1.5 & 994 & 837 & 0.84 & \\
\hline 8 & 44.4 & 1 & 550 & 215 & 0.39 & \\
\hline 10 & 19 & 1 & 235 & 230 & 0.98 & \\
\hline 18 & 29.6 & 1.5 & 550 & 509 & 0.93 & \\
\hline 33 & 23.9 & 1 & 296 & 279 & 0.94 & \\
\hline 11 & 23.9 & 1 & 296 & 227 & 0.77 & \\
\hline 7 & 29.6 & 1.5 & 550 & 274 & 0.50 & \\
\hline 29 & 15 & 1.5 & 279 & 250 & 0.90 & \\
\hline 3 & 29.6 & 1.5 & 550 & 70 & 0.13 & \\
\hline 21 & 148.7 & 1.25 & 2302 & 1072 & 0.47 & \\
\hline 28 & 80 & 1 & 991 & 791 & 0.80 & \\
\hline 30 & 78 & 0.84 & 812 & 578 & 0.71 & \\
\hline 35 & 78 & 1 & 966 & 751 & 0.78 & \\
\hline 22 & 25 & 1 & 310 & 71 & 0.23 & \\
\hline 36 & 19 & 0.67 & 158 & 114 & 0.72 & \\
\hline 25 & 128.7 & 0.86 & 1371 & 826 & 0.60 & \\
\hline 38 & 80 & 1 & 991 & 721 & 0.73 & \\
\hline $\begin{array}{l}V c=.85 * 2 \\
V c=.8 \\
B e=E f f\end{array}$ & $\begin{array}{l}\text { SQRT(f'c) } \\
c \bullet L \bullet B e \\
\text { tive Wall T }\end{array}$ & $=$ & 108 & PSI & & \\
\hline
\end{tabular}

(*) wall 5 is $1.5^{\prime}$ wide $J$ 
lalc. S-0002, Ken, 0, 50b 4i55=

\begin{tabular}{|c|c|c|c|c|c|}
\hline $\begin{array}{r}x \\
4 \quad 4\end{array}$ & $\begin{array}{l}\text { S } \\
\frac{1}{\sum} \\
\sum\end{array}$ & 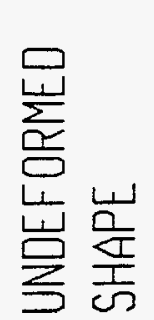 & $\begin{array}{l}\sum^{\infty} \\
\frac{1}{\sigma}\end{array}$ & 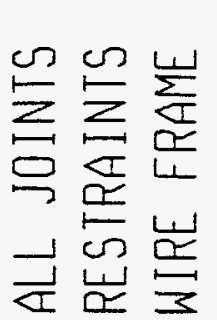 & $\frac{8}{2}$ \\
\hline
\end{tabular}

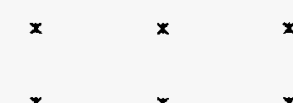

$x \quad x$
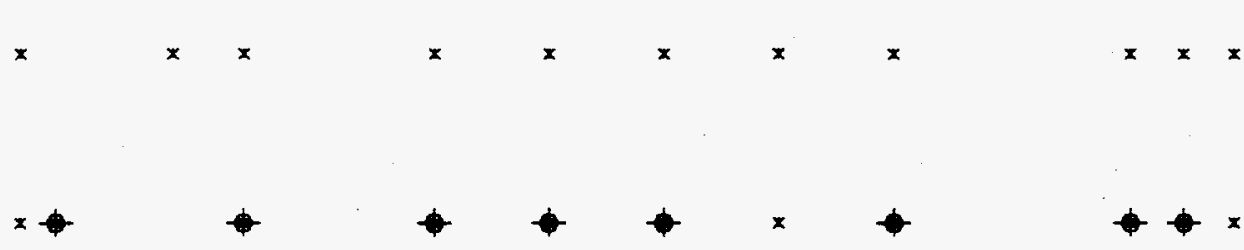

x

$x$
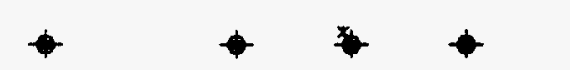

$x$

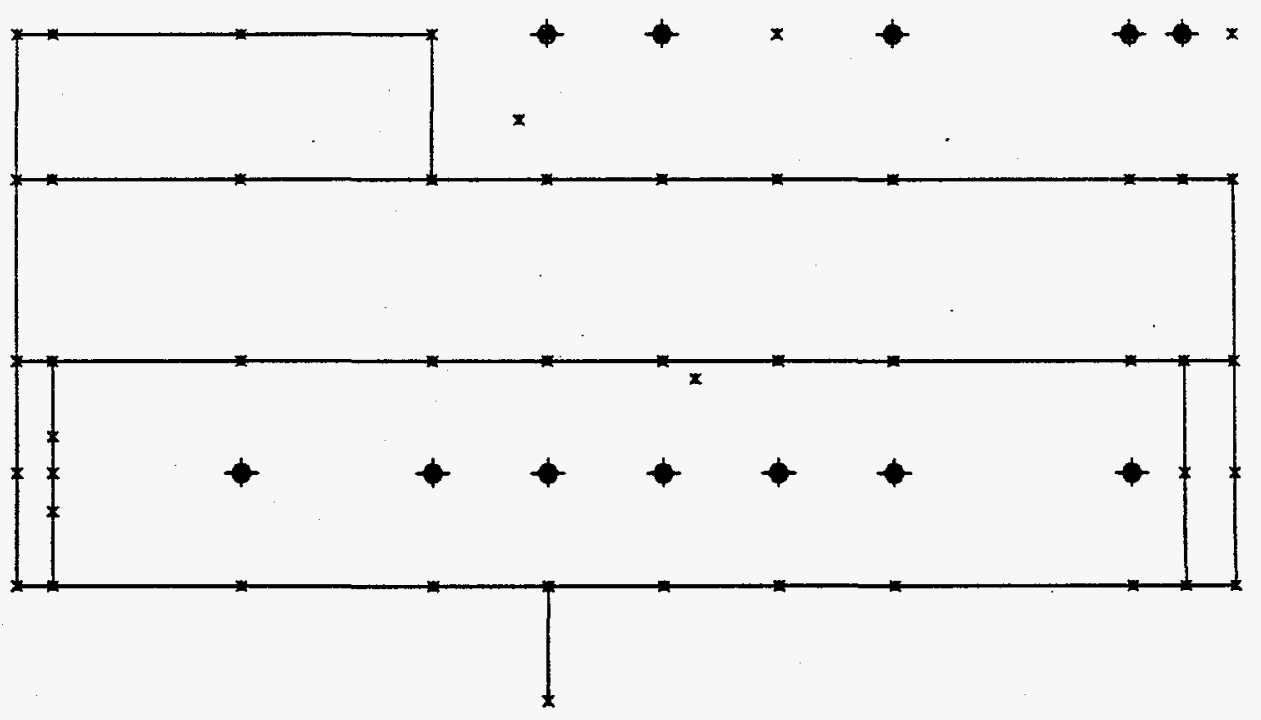


Lale. S-0002, Res. 0, vob $\times 4: 55=$

Sht 1135

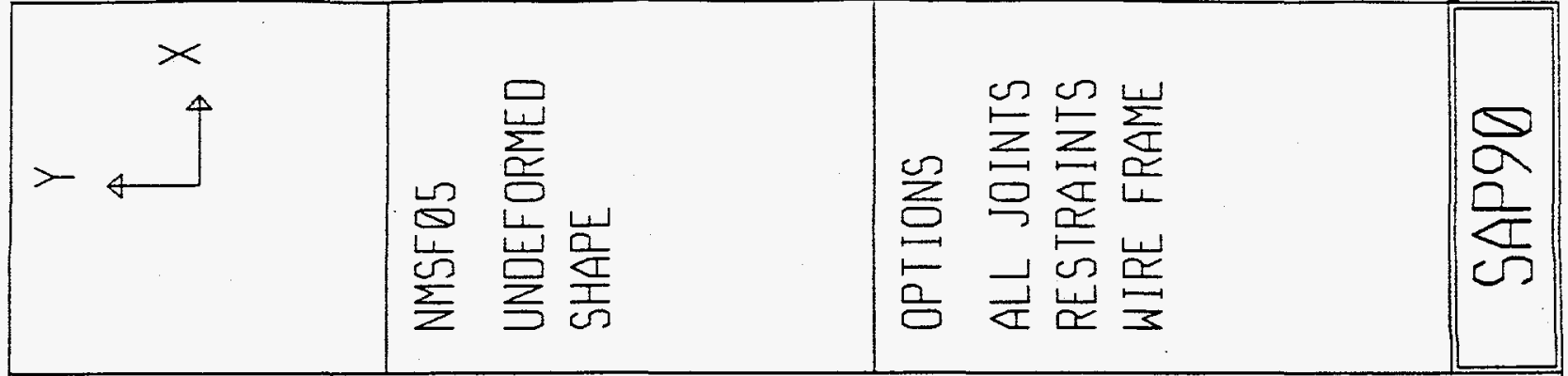

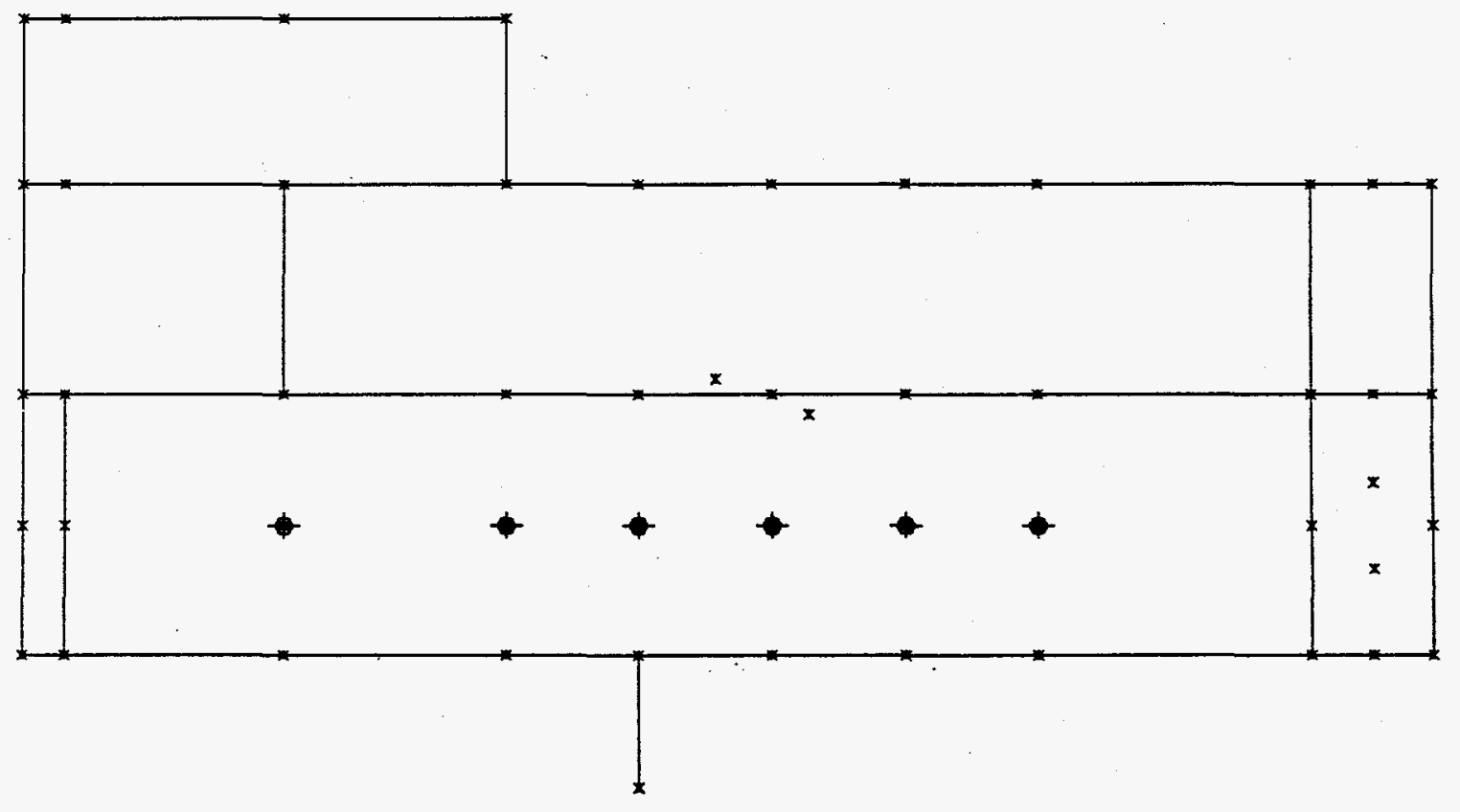


rale. S-0002, Rej.0, Jo6*452 Ert 1:4 50

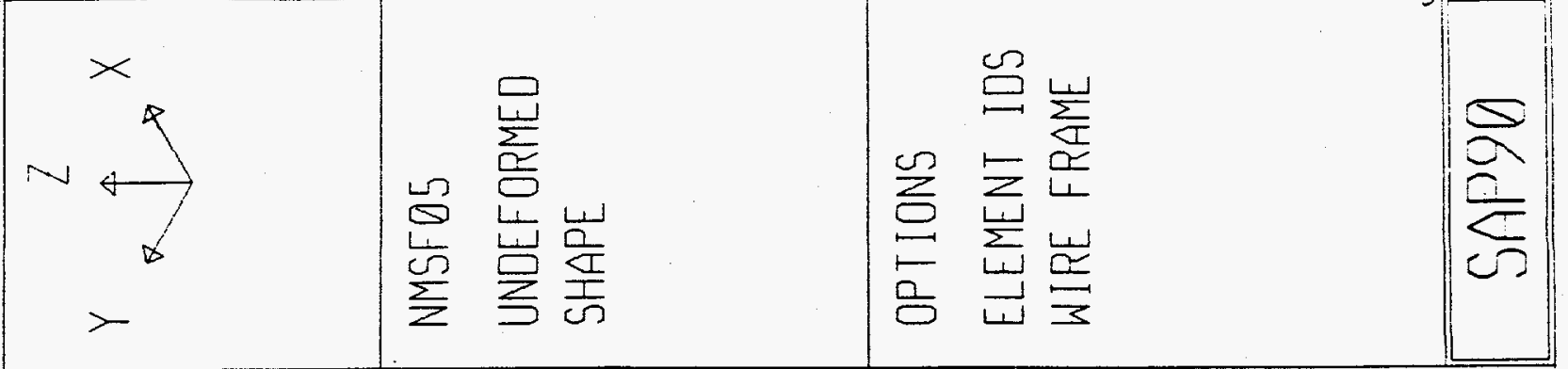

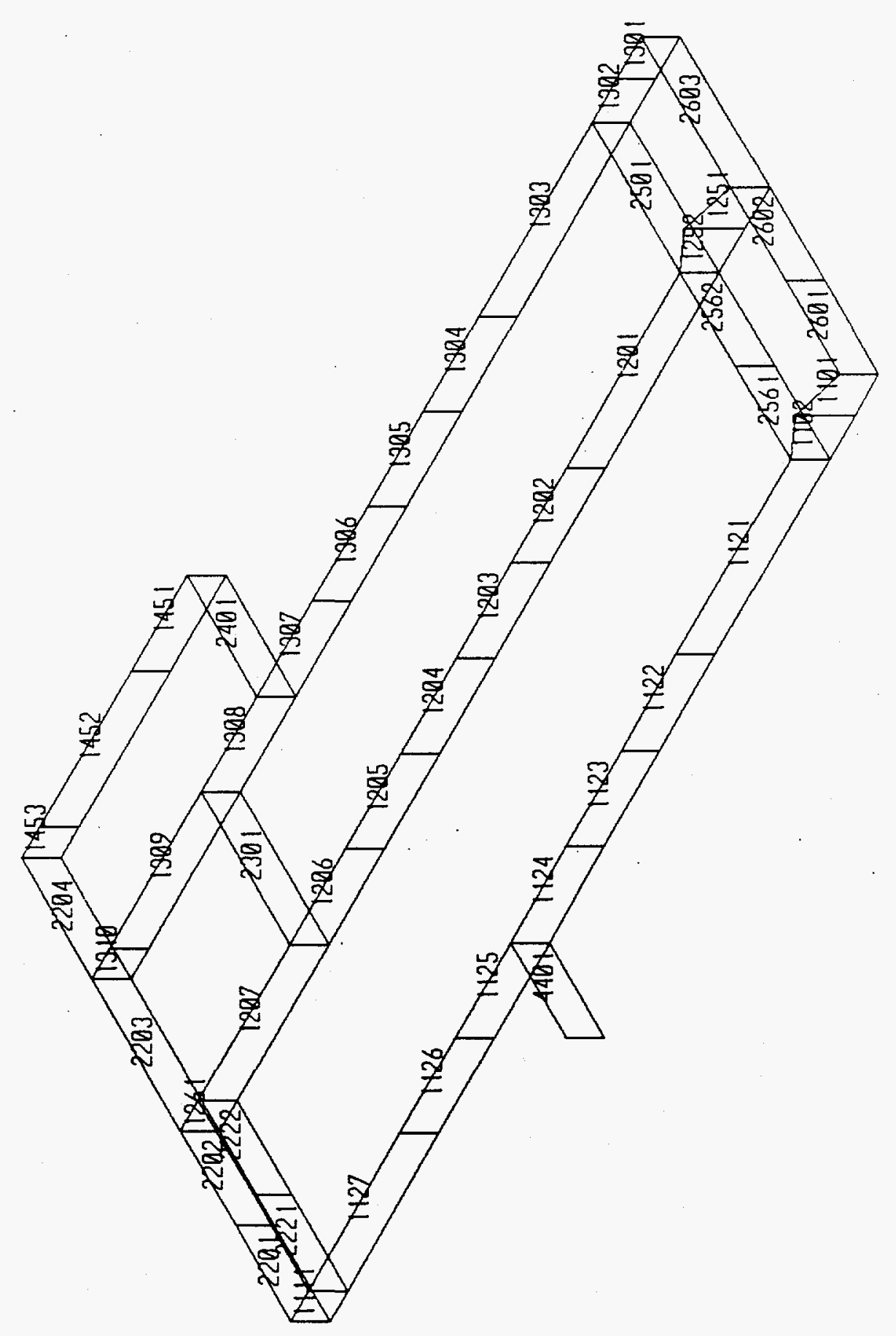




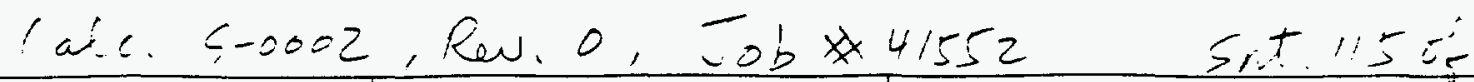

\begin{tabular}{|c|c|c|c|}
\hline $\mid \begin{array}{c}x \\
\infty\end{array}$ & 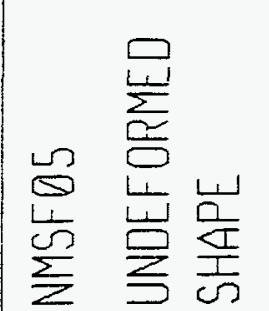 & 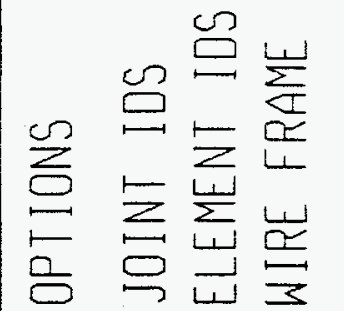 & $\sum_{i=}^{\infty}$ \\
\hline
\end{tabular}

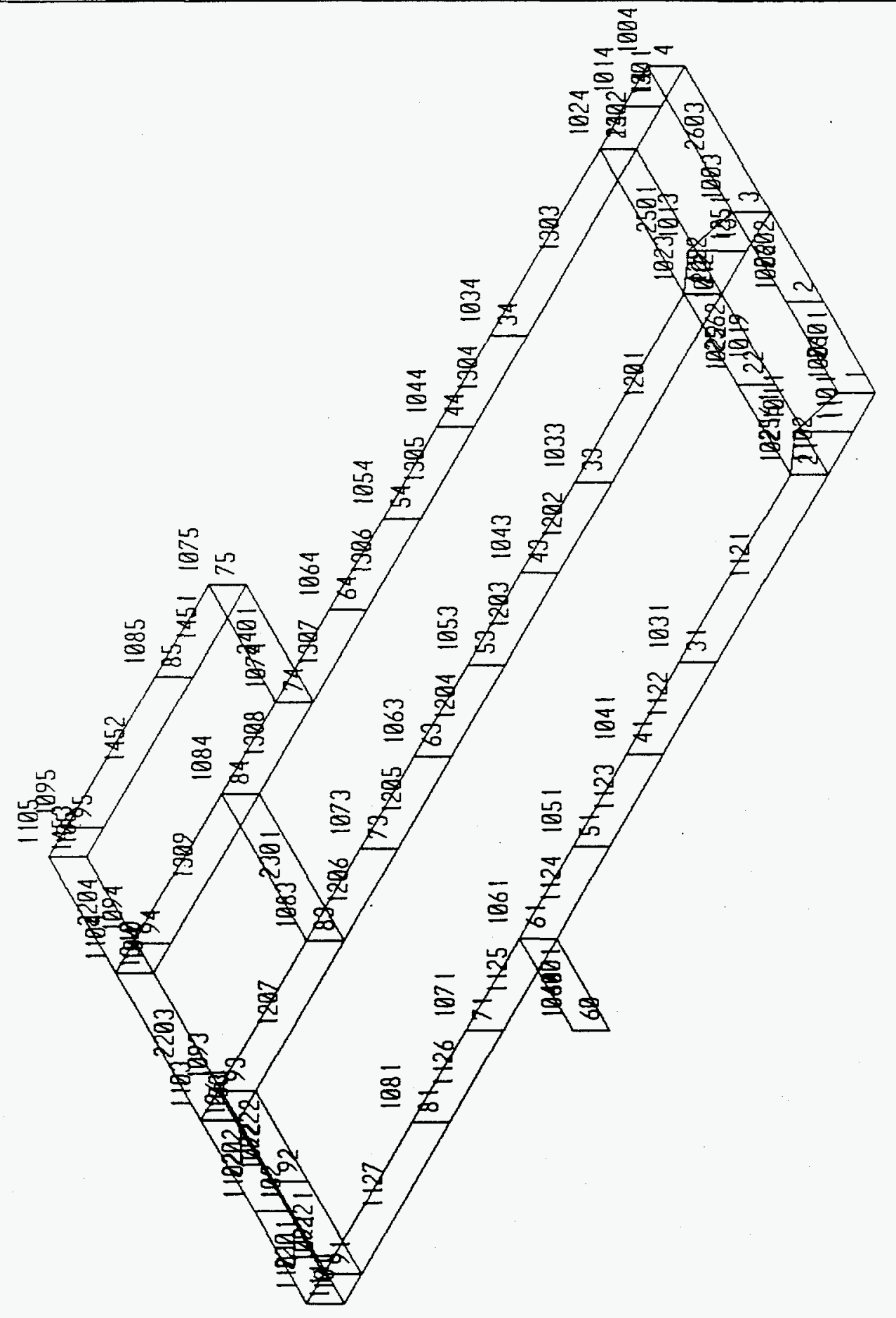


Cale. S-0002, Res. 0, Tob *41552 Sht: 116 of

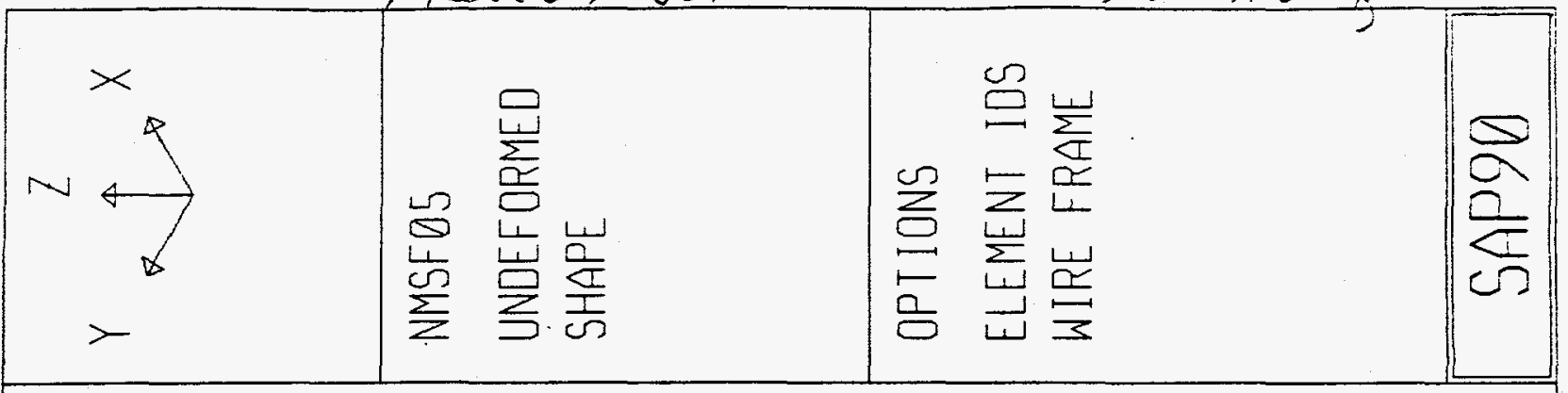

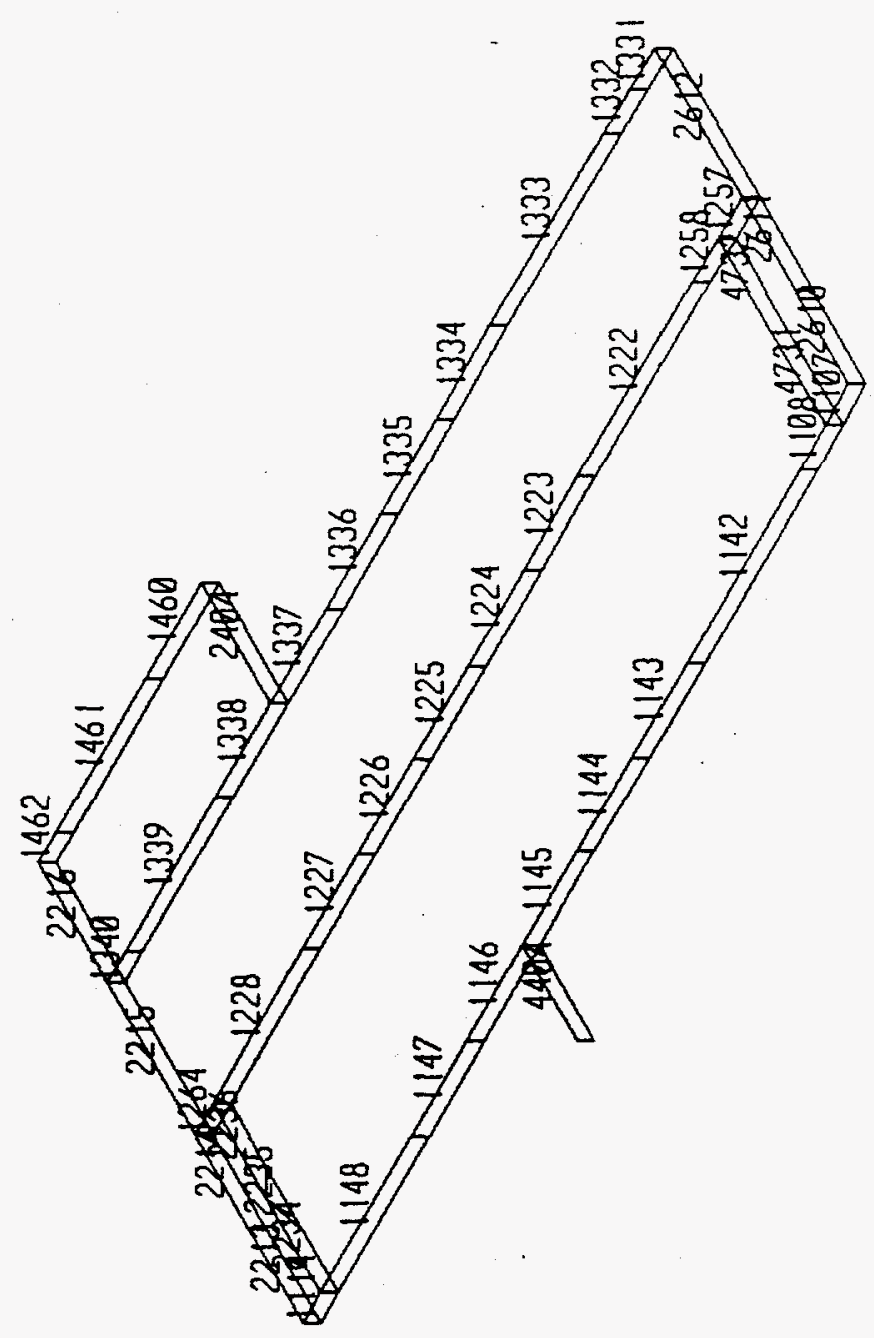


late. 5-0002, Rev. 0, Job $\times 41552$ Sht. 117 ig

\begin{tabular}{|c|c|c|c|}
\hline $\begin{array}{r}x \\
4 \\
\end{array}$ & 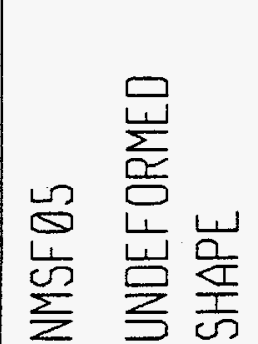 & 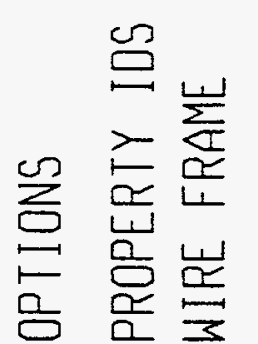 & 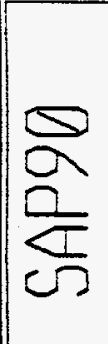 \\
\hline
\end{tabular}
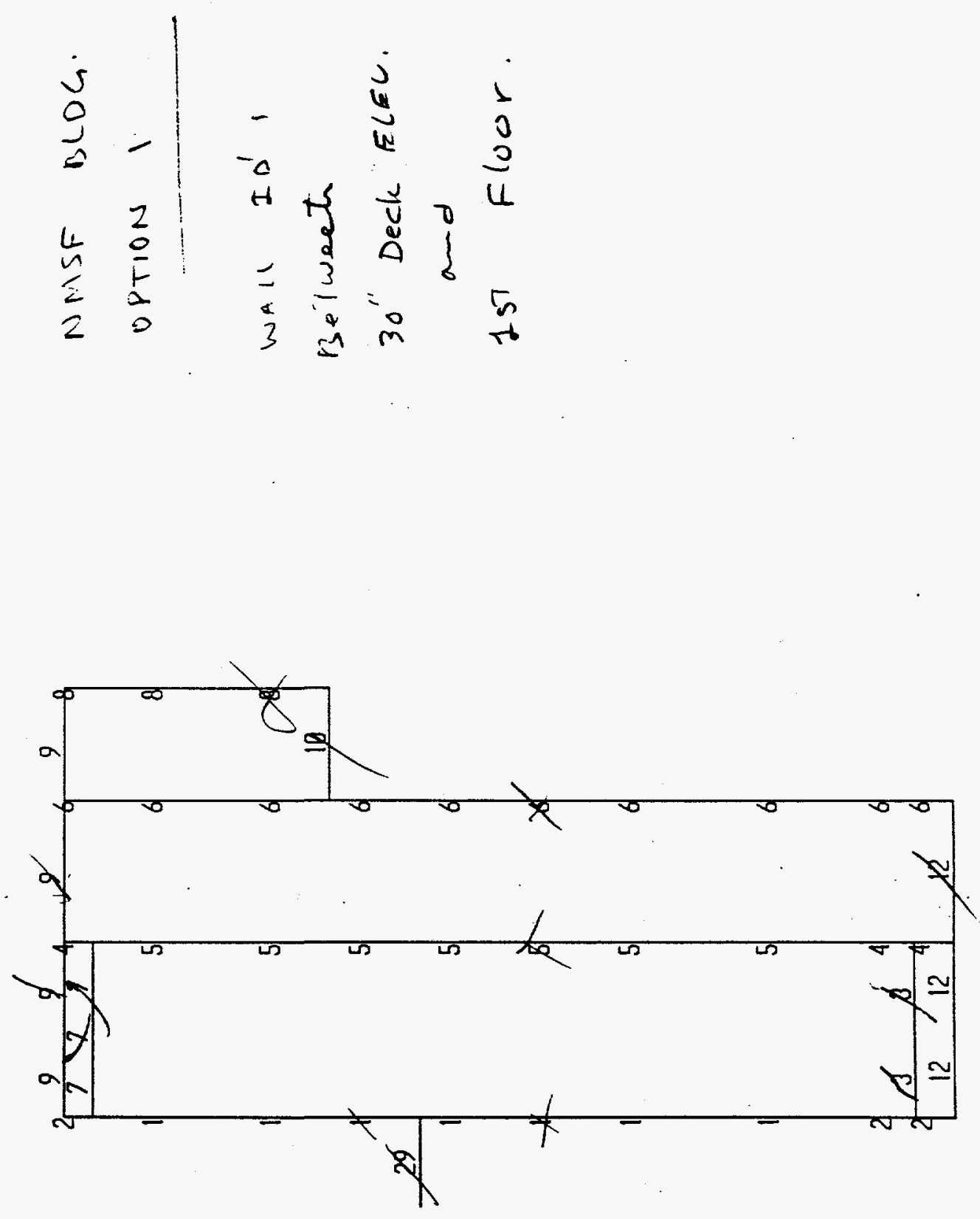


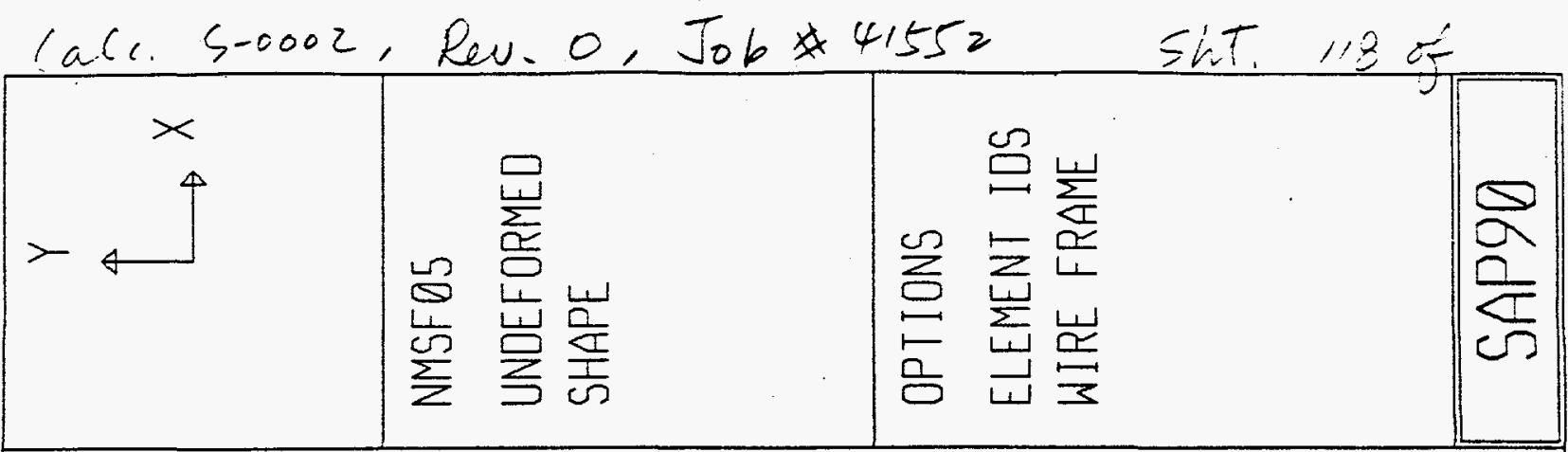
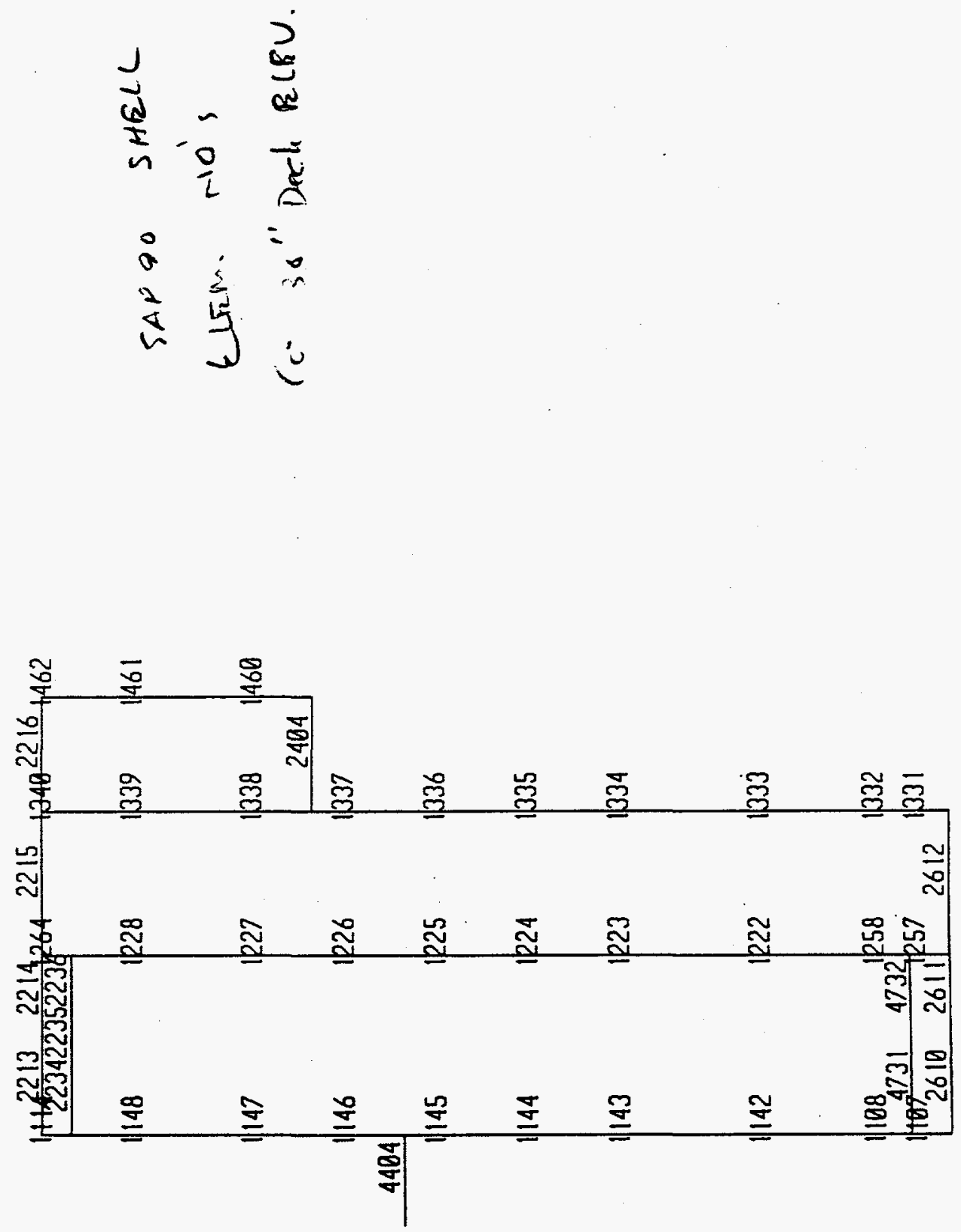
lale. S-0002, Rev. 0, Vob $\times 41552$ Sut : : =

\begin{tabular}{|c|c|c|c|}
\hline $\begin{array}{r}x \\
-4\end{array}$ & 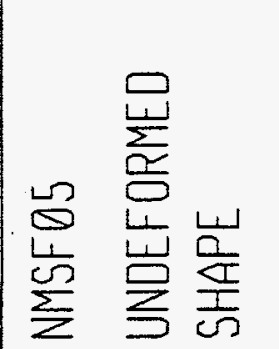 & 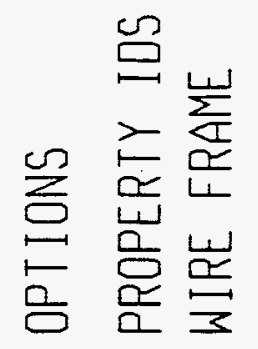 & $\frac{s}{\sqrt{2}}$ \\
\hline
\end{tabular}
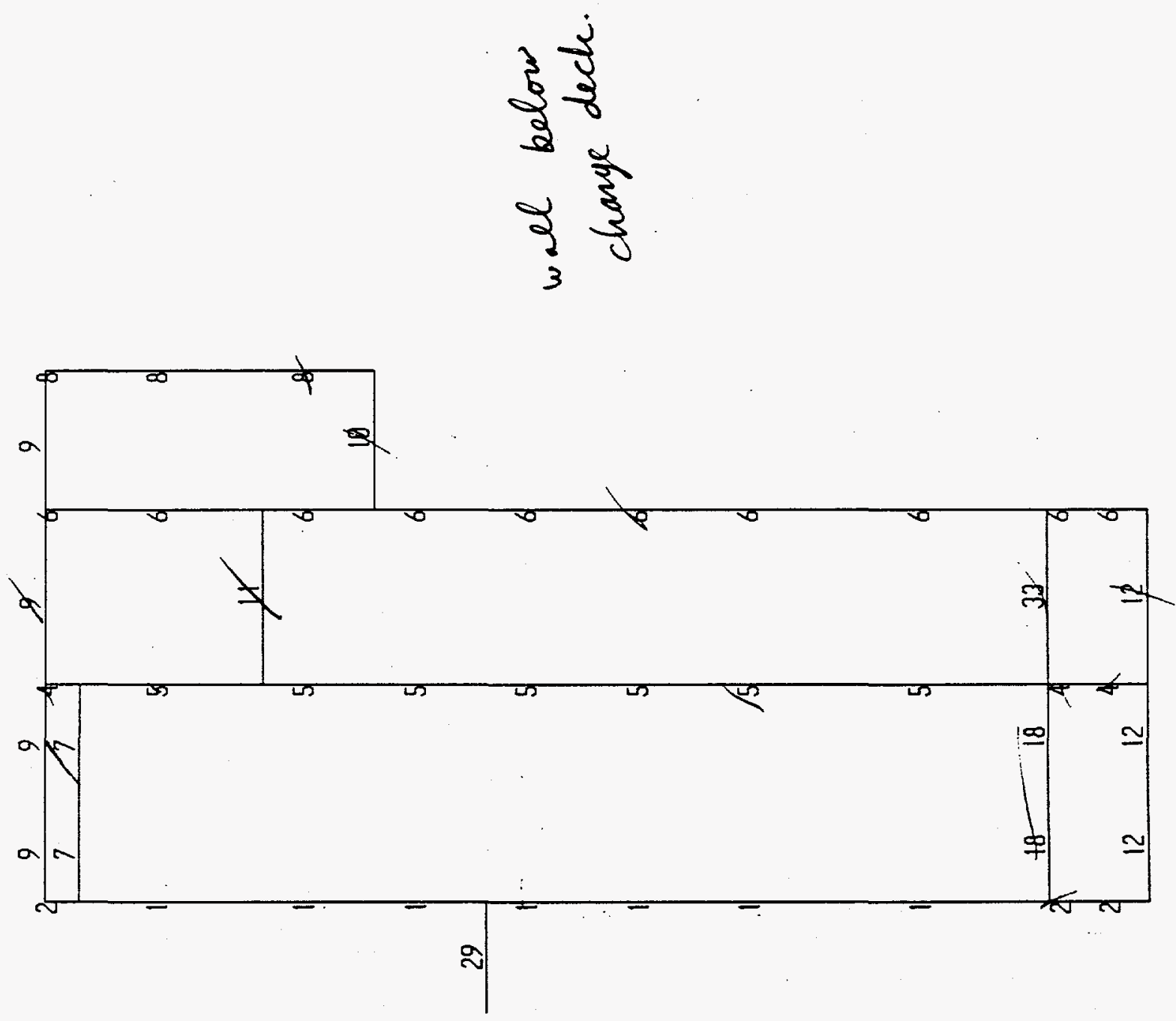


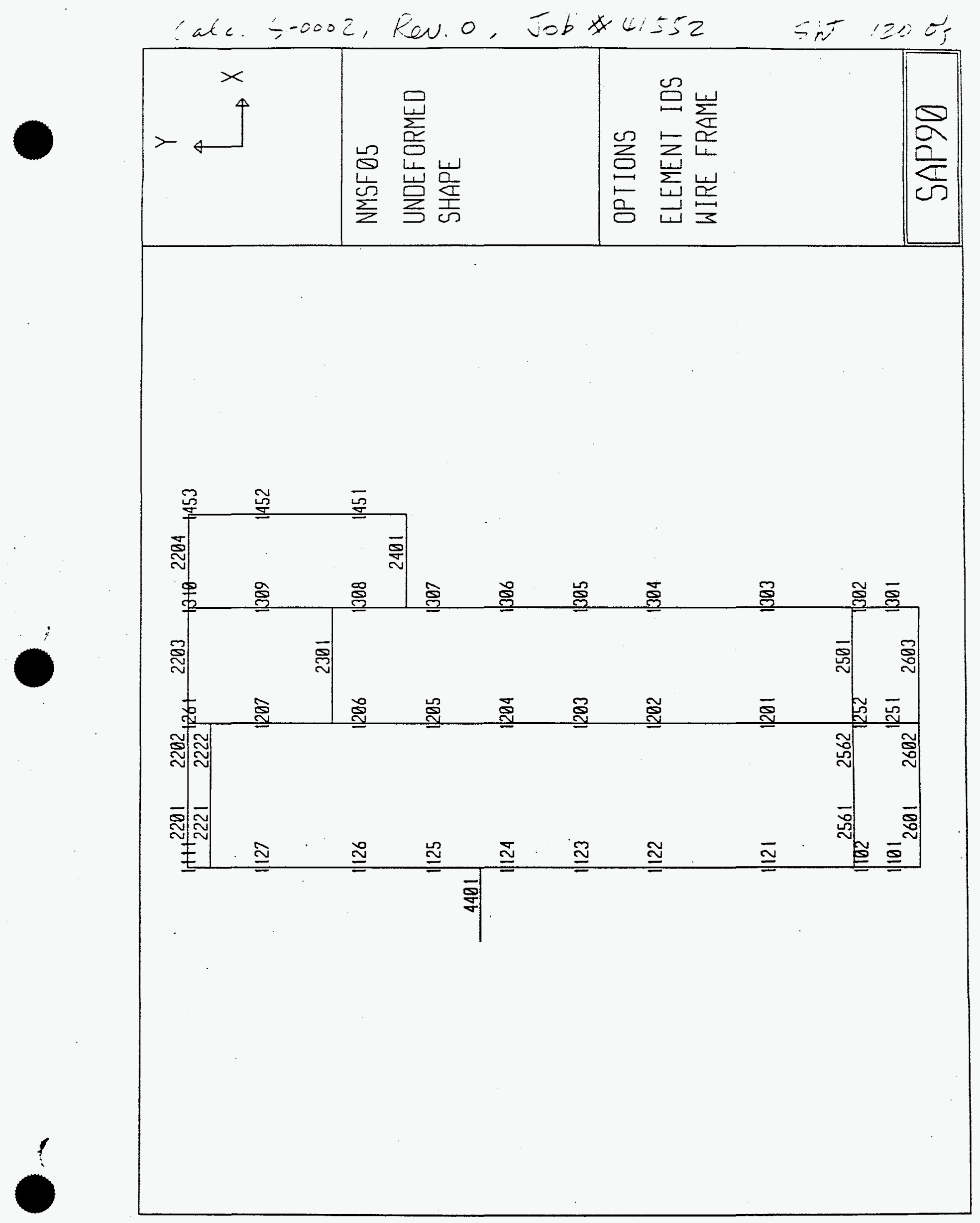


Gale. S-0002, Few. $0,50 b+415=$

int $121 \leq 5$

\begin{tabular}{|c|c|c|c|}
\hline $\begin{array}{r}x \\
4\end{array}$ & 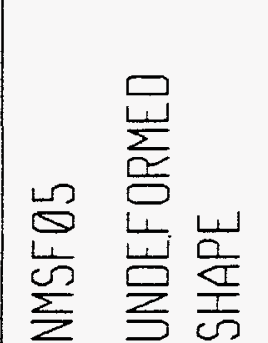 & 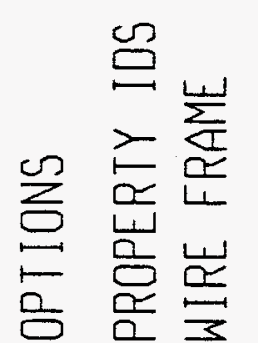 & ${ }_{2}^{\frac{2}{\alpha}}$ \\
\hline
\end{tabular}
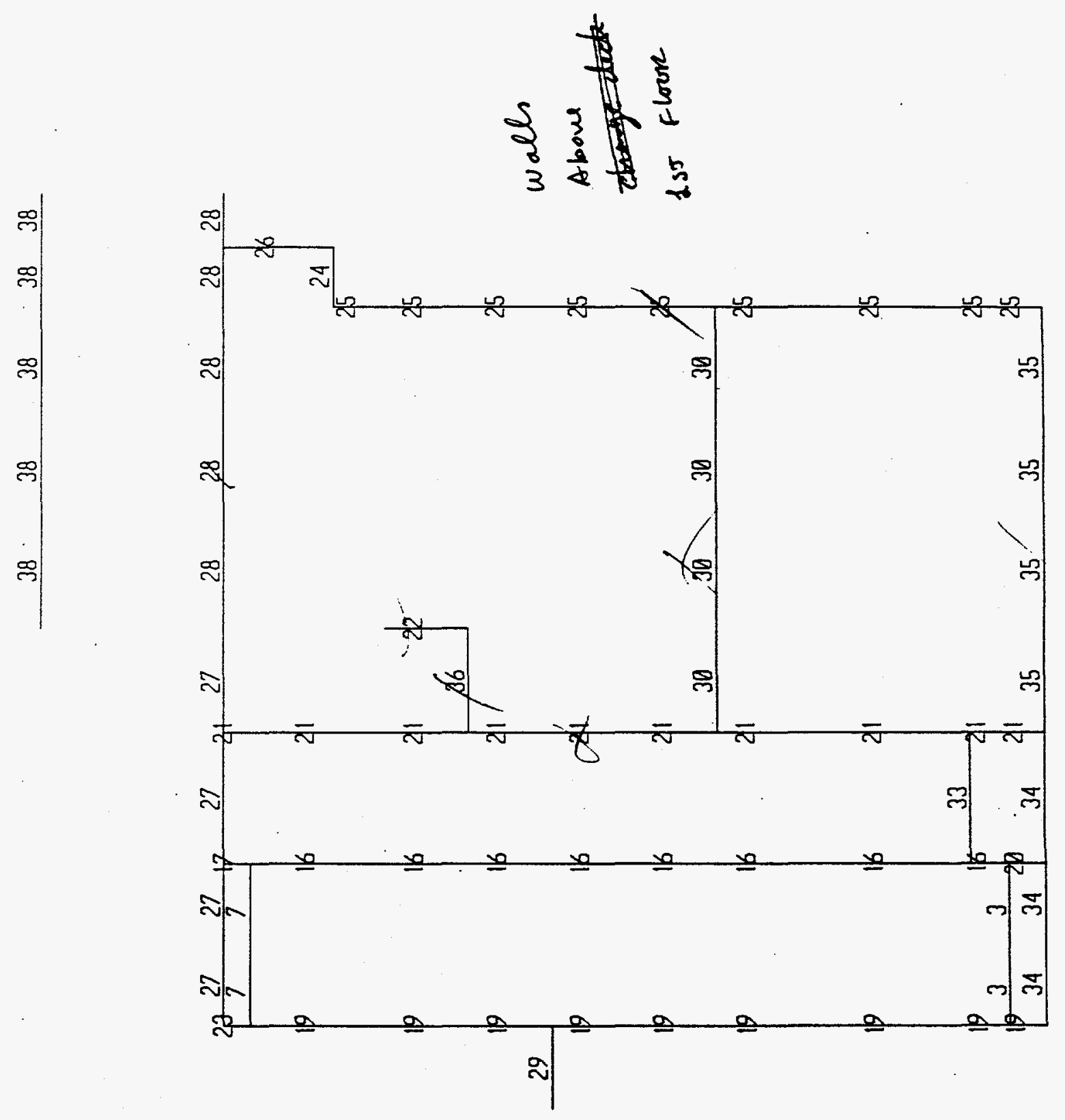


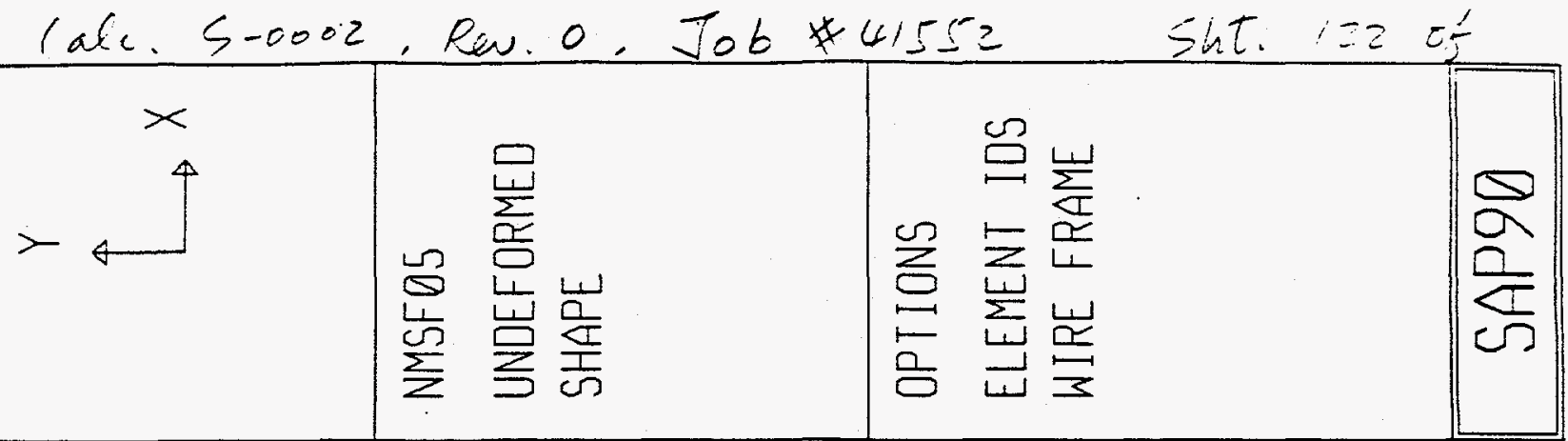

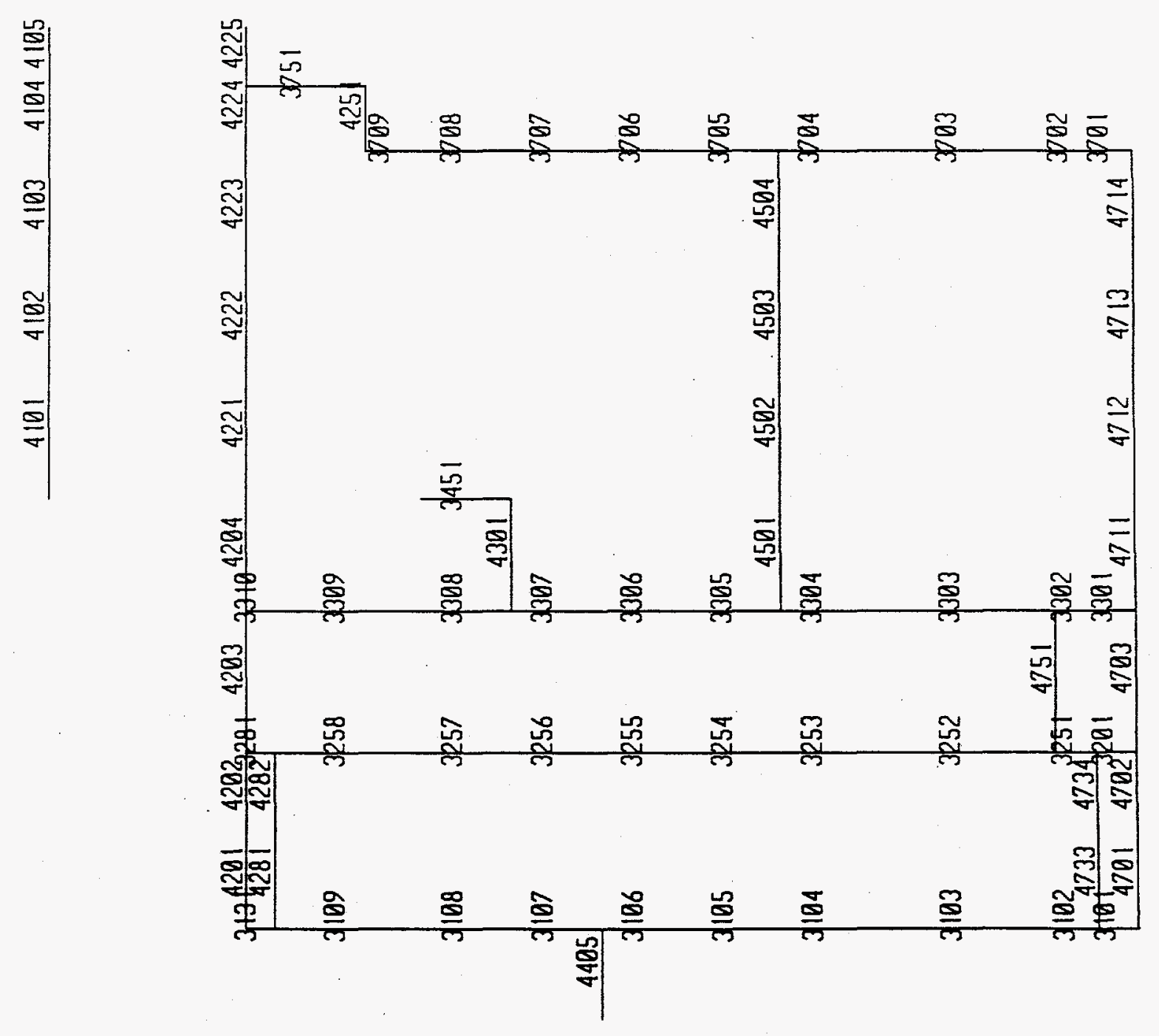




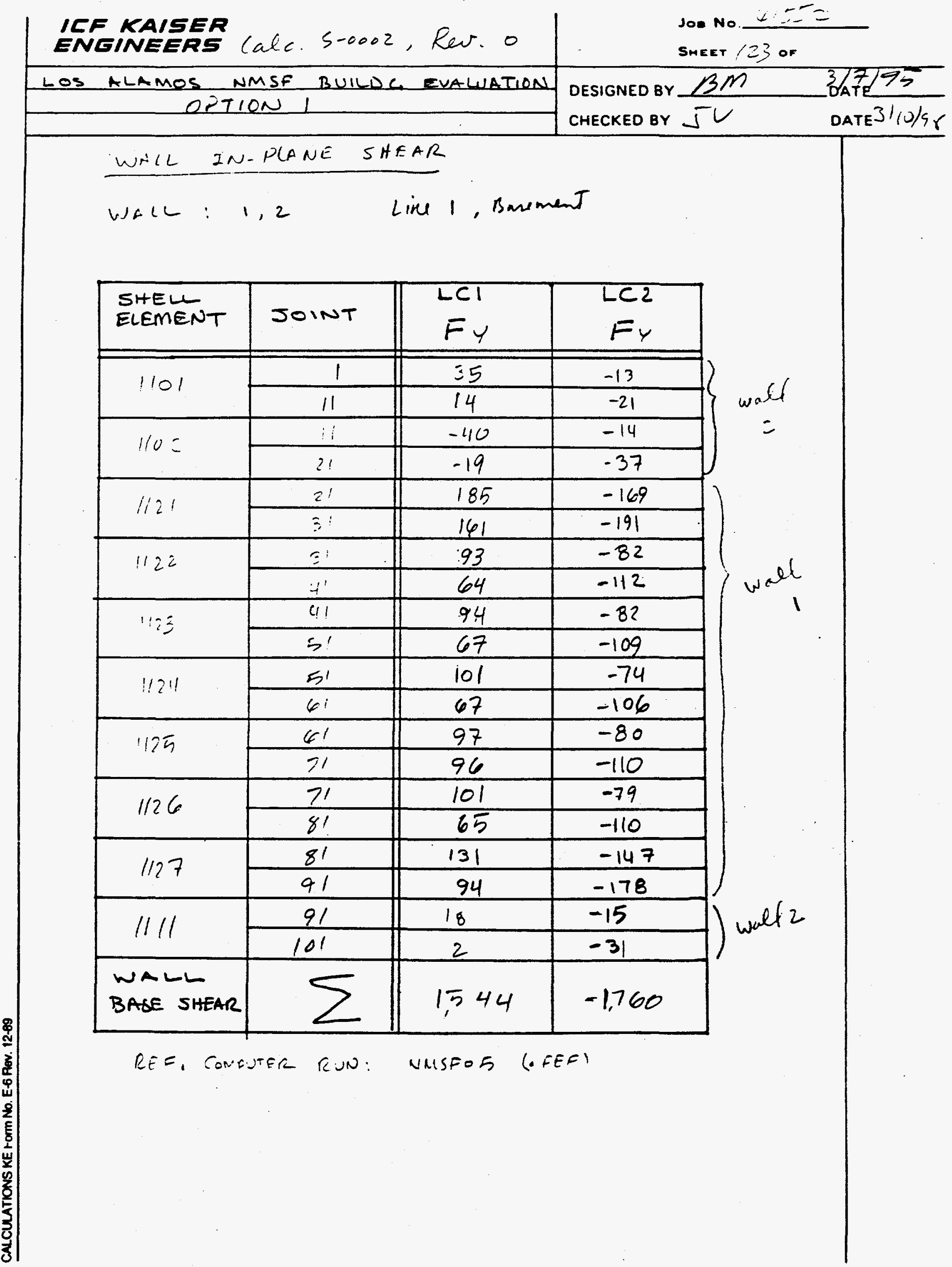


ICF KAISER

ENGINEERS (ate. S-0002, KeN. 0
Joa No.

SHEET / $/ 24$ OF

LOS ALAMOS NMSF BULDC EVALUATION

DESIGNED BY $\beta M$
CHECKED BY DATE $3 / 95$

WALL IN-PLANE SHEAR REF. SAPAO RNN:

WaLL: 1,2 line (1) abore $30^{\prime \prime}$ peck

\begin{tabular}{|c|c|c|c|}
\hline $\begin{array}{l}\text { SHELUL } \\
\text { ELEMENT }\end{array}$ & SOINT & $\begin{array}{c}\text { LCI } \\
\text { FY }\end{array}$ & $\begin{array}{l}L C^{2} \\
F Y\end{array}$ \\
\hline \multirow{2}{*}{1142} & 3021 & & -27 \\
\hline & 31 & & -29 \\
\hline \multirow{2}{*}{3} & 31 & & -12 \\
\hline & 41 & & -16 \\
\hline \multirow{2}{*}{4} & 41 & & -11 \\
\hline & 51 & & -17 \\
\hline \multirow{2}{*}{5} & 51 & & -11 \\
\hline & 61 & & -17 \\
\hline \multirow{2}{*}{6} & 61 & & -10 \\
\hline & 71 & & -18 \\
\hline \multirow{2}{*}{7} & 71 & & -9 \\
\hline & 81 & & -19 \\
\hline \multirow{2}{*}{8} & 61 & & -17 \\
\hline & 91 & & -29 \\
\hline \multirow{2}{*}{1107} & 3001 & & -4 \\
\hline & $30 b 1$ & & -4 \\
\hline \multirow{2}{*}{8} & 11 & & -4 \\
\hline & 21 & & -5 \\
\hline \multirow{2}{*}{1114} & 3091 & & 1 \\
\hline & 3101 & & -7 \\
\hline WALL & - & & \\
\hline BASE SHEAR & & & \\
\hline
\end{tabular}


ENEINEERS (all. S.0002, Rev. O Jos No. $e_{1}-\cdots=$ LOS ALAMOS NMSF BUILDC EVALUATION OPTION

DESIGNEDBY $B M$ CHECKED \&Y TC

WALL IN-PLANE SHEAR

WALL: 4,5

line 2 rsavent?

\begin{tabular}{|c|c|c|c|}
\hline $\begin{array}{l}\text { SHEL } \\
\text { ELEMENT }\end{array}$ & SOINT & $\begin{array}{l}\text { LCI } \\
F_{Y}\end{array}$ & $\begin{array}{l}\text { LC2 } \\
F_{Y}\end{array}$ \\
\hline \multirow[t]{2}{*}{$:: \equiv$} & $\vdots$ & 19 & -10 \\
\hline & 15 & .7 & $.7-1$ \\
\hline \multirow{2}{*}{$17 \%$} & $\vdots \vdots$ & 26 & -15 \\
\hline & 23 & 9 & .54 \\
\hline \multirow{2}{*}{$\therefore$} & $i$ & 183 & -194 \\
\hline & $\vdots \vdots$ & 163 & -217 \\
\hline \multirow{2}{*}{ E } & 3 & 97 & -90 \\
\hline & $4 \vdots$ & 77 & -112 \\
\hline \multirow{2}{*}{$\ddot{z}$} & $4 ;$ & 99 & -89 \\
\hline & 53 & 76 & -112 \\
\hline \multirow{2}{*}{$2 i$} & $=3$ & 100 & -88 \\
\hline & $D^{2}$ & 76 & -112 \\
\hline \multirow{2}{*}{$\because$} & 63 & 101 & -88 \\
\hline & 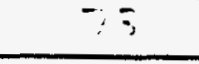 & 76 & -112 \\
\hline \multirow{2}{*}{6} & 73 & 102 & -88 \\
\hline & 83 & 75 & -112 \\
\hline \multirow{2}{*}{7} & 83 & 140 & -159 \\
\hline & 93 & 112 & -187 \\
\hline \multirow{2}{*}{1261} & 93 & 12 & -9 \\
\hline & 103 & 5 & -23 \\
\hline $\begin{array}{l}\text { WALL } \\
\text { BASE SHEAR }\end{array}$ & & $1555^{k}$ & $1894^{k}$ \\
\hline
\end{tabular}

DAT $3 \longdiv { 7 / 9 5 }$ DATE $3 / / 0 / 45$ 


\section{IEF KAISER}

ENGINEERS (alc. S-0002, Rev. o LOS NLAMOS NMSF BUILDC EVALUATION

DESIGNED BY BM

REF. SAPQO RUN:

WALL IN-PLANE SHEAR AT MEZZ RLEV.

\begin{tabular}{|c|c|c|c|}
\hline $\begin{array}{l}\text { SHELL } \\
\text { ELEMENT }\end{array}$ & SOINT & $\begin{array}{l}\text { LCI } \\
F_{Y}\end{array}$ & $\begin{array}{l}\text { LC2 } \\
F_{Y}\end{array}$ \\
\hline \multirow{2}{*}{1215} & 2023 & & -202 \\
\hline & 33 & & -226 \\
\hline \multirow{2}{*}{16} & 33 & & -82 \\
\hline & 43 & & -119 \\
\hline \multirow{2}{*}{17} & 43 & & -83 \\
\hline & 53 & & -120 \\
\hline \multirow{2}{*}{18} & 53 & & -82 \\
\hline & 63 & & -122 \\
\hline \multirow{2}{*}{19} & 63 & & -82 \\
\hline & 73 & & -124 \\
\hline \multirow{2}{*}{20} & 73 & & -82 \\
\hline & 83 & & -125 \\
\hline \multirow{2}{*}{21} & 83 & & -123 \\
\hline & 93 & & -116 \\
\hline \multirow{2}{*}{1255} & 2003 & & -13 \\
\hline & 13 & & -26 \\
\hline \multirow{2}{*}{1256} & 13 & & -19 \\
\hline & 23 & & -30 \\
\hline \multirow{2}{*}{1263} & 2093 & & -50 \\
\hline & 2103 & & +57 \\
\hline $\begin{array}{l}\text { WALL } \\
\text { BASE SHEAR }\end{array}$ & $\angle$ & & $3^{k}$ \\
\hline
\end{tabular}


ICF KAISER

ENEINEERS ( W C. S-0002, ReN. O
LOS ALAMOS NMSF BUILDC EVALUATION
WALC IN-PLANE SHEAR WALL: 5,4
DESIGNEO BY $B M$ CHECKED BY JU

$\frac{\text { SOE NOL }}{\text { SHEET/27 }} \frac{B M}{5 V}$

REF. SAPOO RNN: $30 "$ beck Level

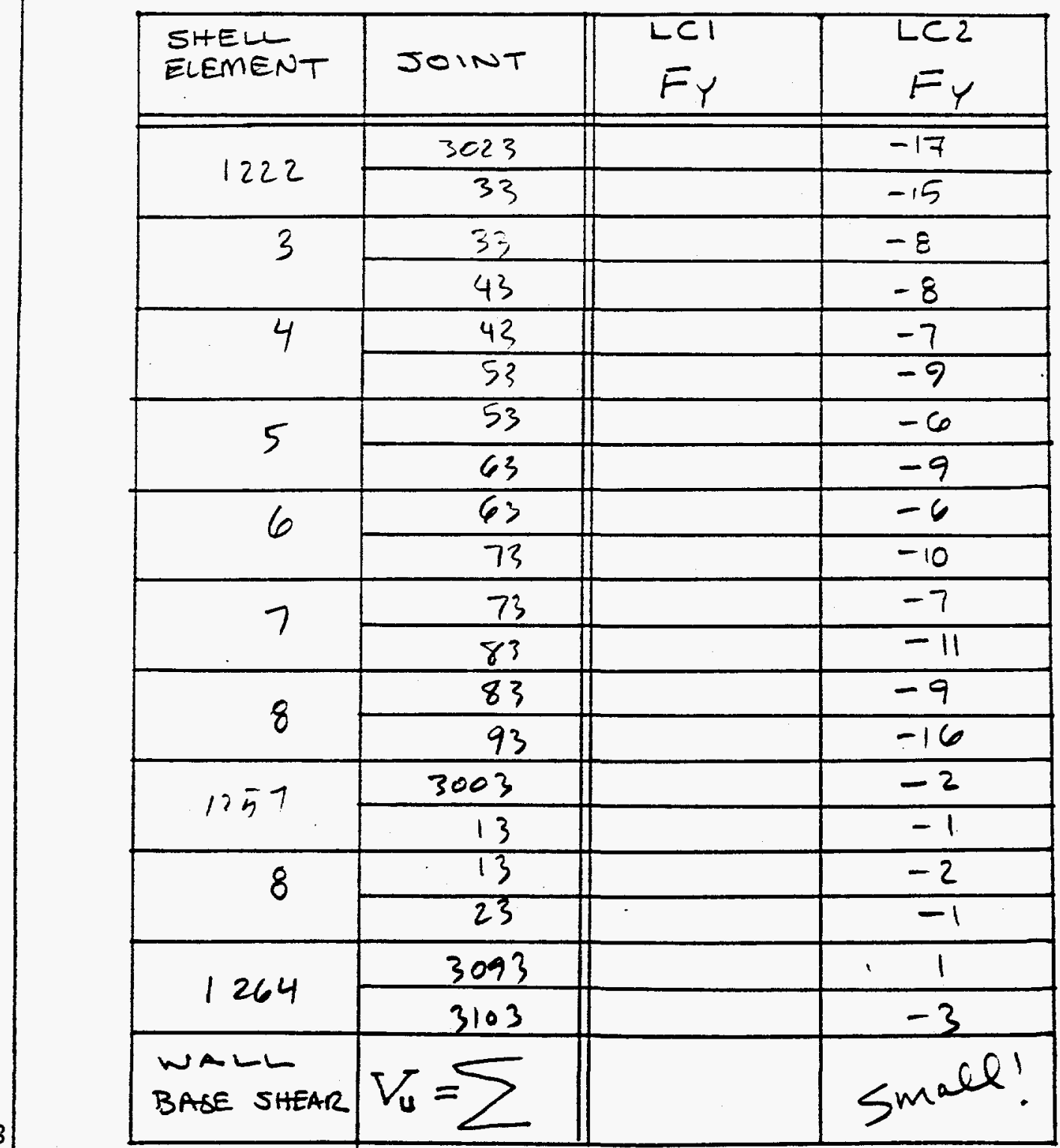


ICF KAISER Lalc. S-0.002, ReU. O
ENGINEERS Los alamos nMSF BUILDC Evaluation

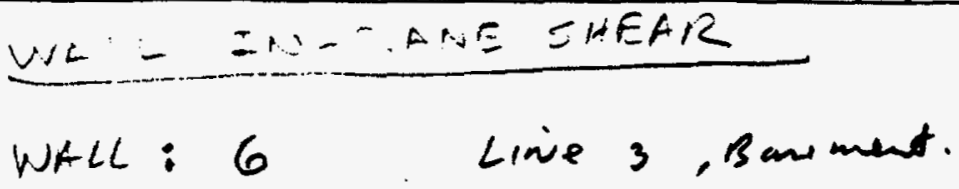

DESIGNEDBY $\operatorname{BM}$ CHECKEO BY Ј
Jo. No. $3: 55=$

SMEET/28 OF

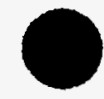

\begin{tabular}{|c|c|c|c|}
\hline $\begin{array}{l}\text { SHEL } \\
\text { ELEMENT }\end{array}$ & SOINT & $\begin{array}{c}\text { LCI } \\
F_{Y}\end{array}$ & $\begin{array}{l}L C_{2} \\
F_{Y}\end{array}$ \\
\hline \multirow{2}{*}{$1 \leq 01$} & 4 & 20 & -20 \\
\hline & 14 & 6 & -31 \\
\hline \multirow{2}{*}{$30=$} & 1.1 & 24 & -34 \\
\hline & 24 & 12 & -34 \\
\hline \multirow{2}{*}{150} & 211 & 97 & -107 \\
\hline & 34 & 89 & -118 \\
\hline \multirow{2}{*}{ : } & 34 & 53 & -49 \\
\hline & 44 & 44 & -58 \\
\hline \multirow{2}{*}{$1 \equiv \sigma^{-}$} & CHA & $\approx 4$ & -49 \\
\hline & $b, 14$ & 43 & -59 \\
\hline & $3 \therefore$ & 54 & -48 \\
\hline & 64 & 42 & .59 \\
\hline \multirow[t]{2}{*}{ - } & 601 & $=2$ & -49 \\
\hline & -11 & 41 & -59 \\
\hline \multirow{2}{*}{-} & $7 ! 1$ & 54 & -47 \\
\hline & 80 & 42 & -58 \\
\hline \multirow{2}{*}{9} & 84 & 79 & -82 \\
\hline & 94 & 65 & -96 \\
\hline \multirow{2}{*}{1310} & 94 & 14 & -10 \\
\hline & 104 & 7 & -23 \\
\hline $\begin{array}{l}\text { WALL } \\
\text { BASE SHEAR }\end{array}$ & & $892^{k}$ & $.080^{k}$ \\
\hline
\end{tabular}


ICF KAISER

ENGINEERS Calc. S-0002, RQN. LOS ALAMOS NMSF BUILDC EVALUATION

WALL IN-PLANE SHEAR

WALL: $\mathscr{C}$

line 3

\begin{tabular}{|c|c|c|c|}
\hline $\begin{array}{l}\text { SHELU } \\
\text { ELEMENT }\end{array}$ & SOINT & $\begin{array}{l}\text { LCI } \\
F_{Y}\end{array}$ & $\begin{array}{c}L C^{2} \\
F_{Y}\end{array}$ \\
\hline \multirow{2}{*}{1321} & 20041 & & -19 \\
\hline & 14 & & -25 \\
\hline \multirow[t]{2}{*}{2} & 161 & & -8 \\
\hline & 24 & & -27 \\
\hline \multirow{2}{*}{3} & 24 & & -104 \\
\hline & 34 & & -117 \\
\hline \multirow{2}{*}{4} & 34 & & -47 \\
\hline & 44 & & -60 \\
\hline \multirow{2}{*}{5} & 44 & & -45 \\
\hline & 54 & & -61 \\
\hline \multirow{2}{*}{6} & 50 & & -45 \\
\hline & 64 & & -61 \\
\hline \multirow{2}{*}{7} & 64 & & -44 \\
\hline & 74 & & -61 \\
\hline \multirow[b]{2}{*}{8} & 74 & & -44 \\
\hline & 84 & & -61 \\
\hline \multirow[b]{2}{*}{9} & 84 & & -61 \\
\hline & 94 & & -50 \\
\hline \multirow{2}{*}{1340} & 994 & & -45 \\
\hline & 100 & & 54 \\
\hline WAUL & & & 949 \\
\hline
\end{tabular}

Jor No. $\div-\div=$

SHEET, 29 OF OESIGNED BY 11 DATE/7/95 CHECKED BY Jw DATE $\$ / 1 / \%$ :

REF. SAPGO RNN: AT PIEZZ RLEV. 
IEF KAISER

ENEINEERS (alc. S-0002 T ReN. 0

soe No. $-5=$

SHEET: $S$ OF LOS ALAMOS NMSF BUILDC EVALUATION ofirive

DESIGNED BY

$\sin$ DATE/7/9i CHECKED BY 5 DATE $\rangle / 1 \% / 8$

WAL IN-PIANE SHEAR

WHLL: 9

Line $B$

(Barement)

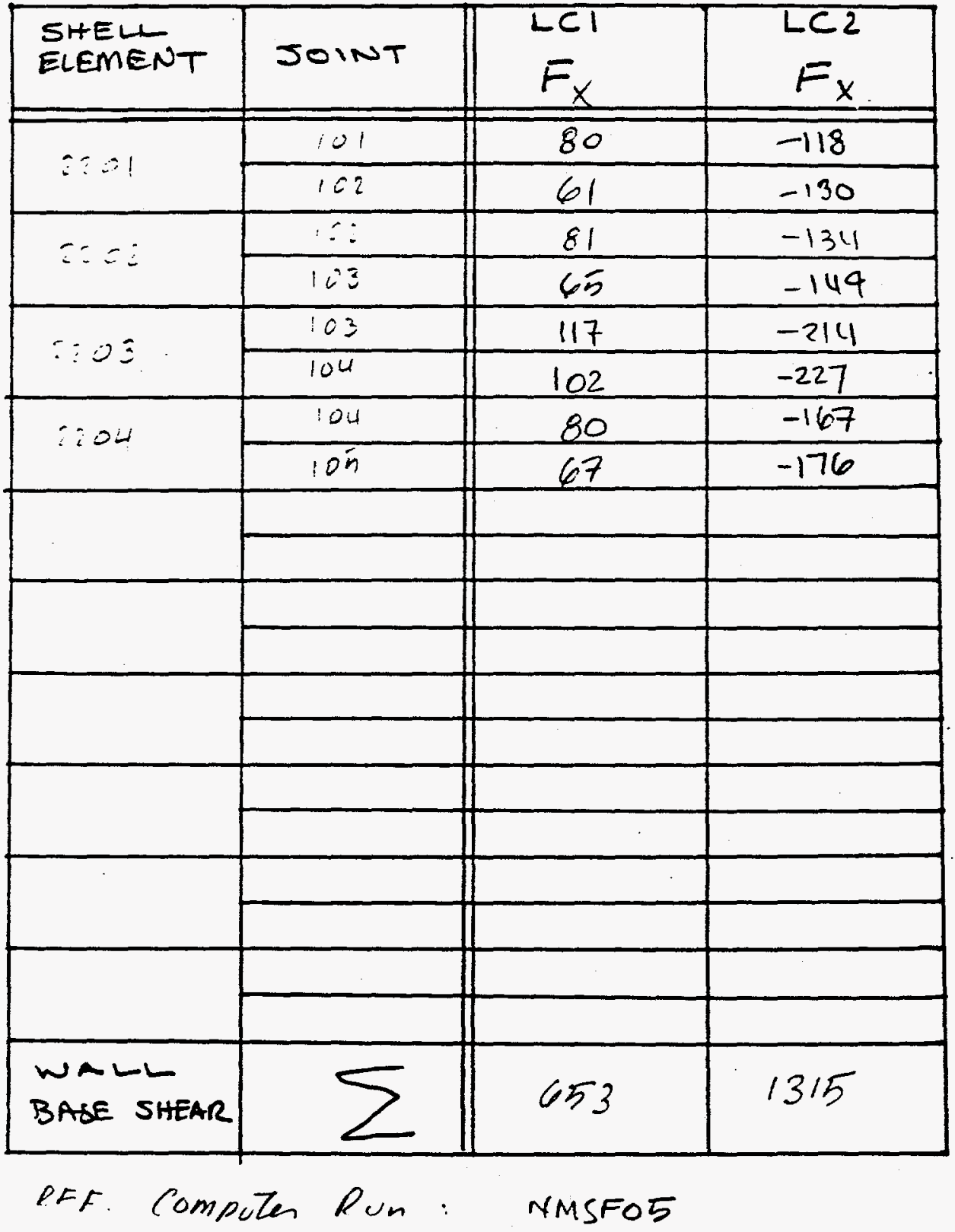


IEF KAISER

ENEINEERS Cale. S-0002, KN.O
Joe No. $\angle-\frac{1}{2}=$

SHEET/3/ of LOS ALAMOS NMSF BUILDC EVALUATION

DESIGNED BY $B M$ $3 \sqrt{19} / 9$

WALL IN-PLANE SHEAR REF. SARGO RNN: WALL: 9 line B 30" Dech FLKV.

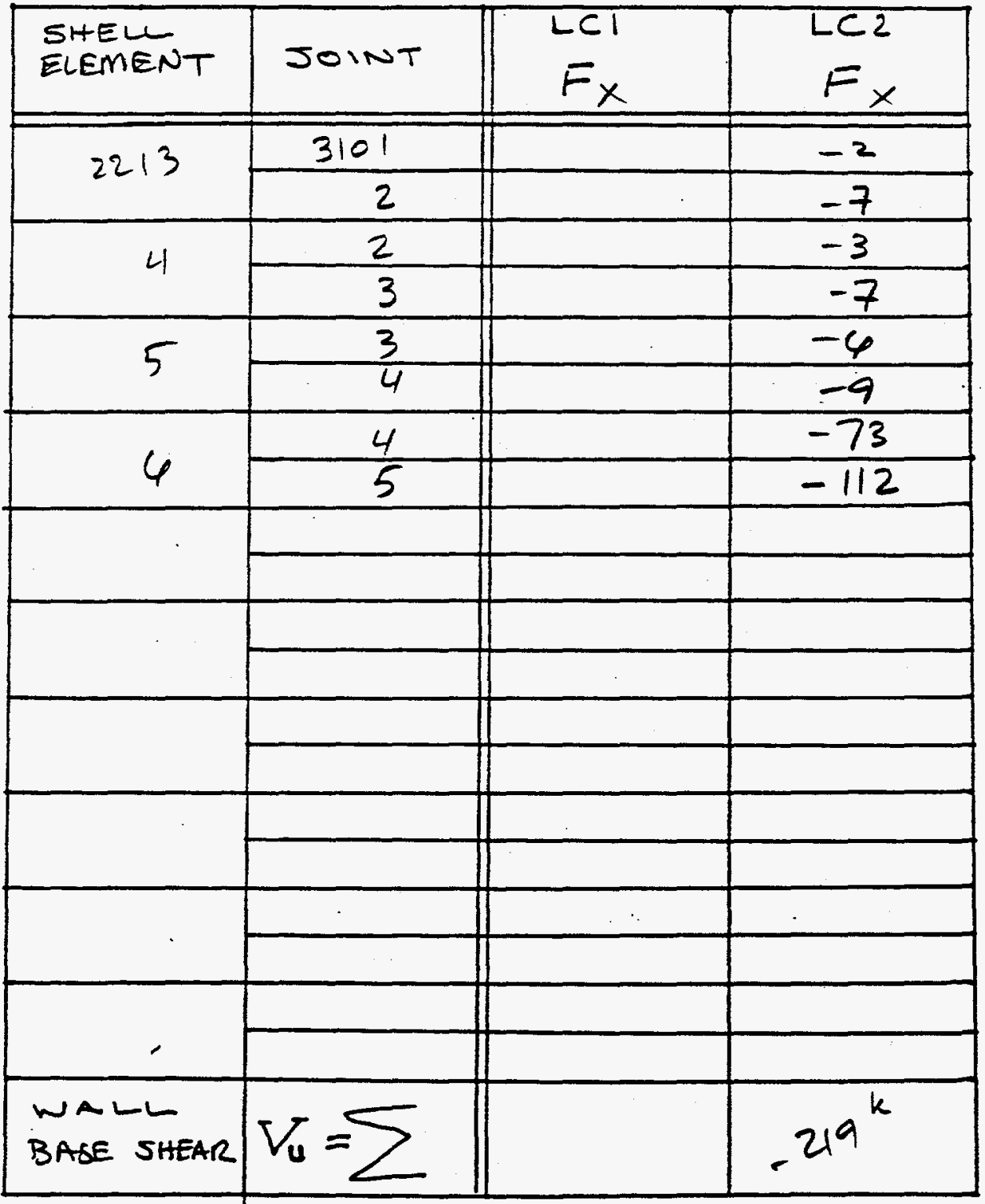




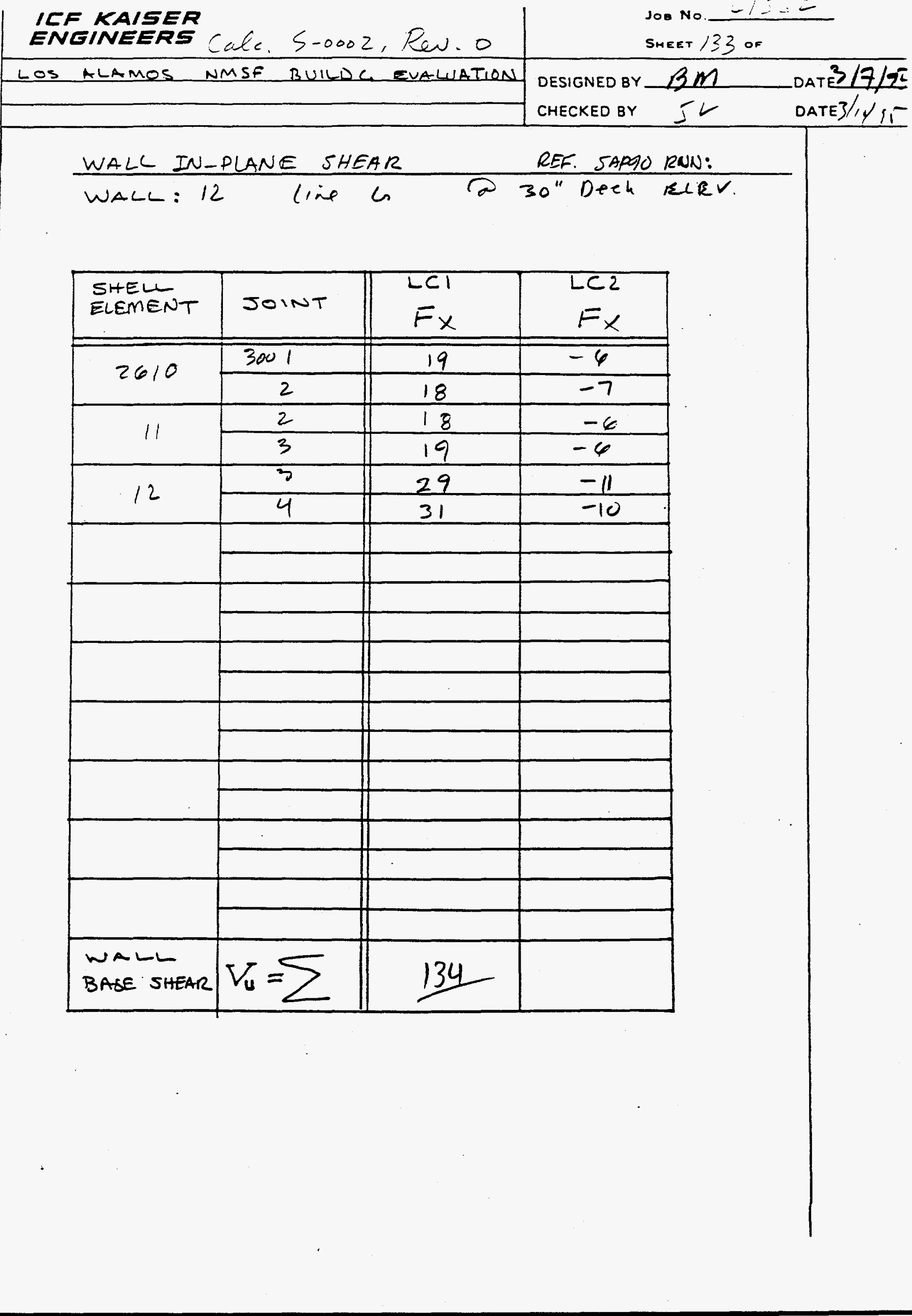


ICF KAISER

ENGINEERS Calc. S-0002, Rev. 0

$\operatorname{Sos} \mathrm{NO}_{0}-\frac{-5}{5}=$

SHEET, 34 OF

LOS ALAMOS NMSF BUILDC EVALUATION

DESIGNED BY

$B M$

$3 / 7 / 95$

CHECKED $8 Y$.

JV

DATE $/ 1 \%<5$

WALL IN-PLANE SHEAR

REF. SAPOO RNN:

WALL: 8

line 5

(Barement)

\begin{tabular}{|c|c|c|c|}
\hline $\begin{array}{l}\text { SHEL } \\
\text { ELEMENT }\end{array}$ & SOINT & $\begin{array}{c}L C I \\
F_{Y}\end{array}$ & $\begin{array}{c}L_{C 2} \\
F_{Y}\end{array}$ \\
\hline \multirow{2}{*}{1451} & 75 & 40 & -24 \\
\hline & 85 & 30 & -32 \\
\hline \multirow{2}{*}{2} & 85 & 49 & -61 \\
\hline & 95 & 43 & -69 \\
\hline \multirow{2}{*}{3} & 25 & 9 & -13 \\
\hline & 105 & 2 & -16 \\
\hline & & & \\
\hline & & & \\
\hline & & & \\
\hline & & & \\
\hline & & . & \\
\hline & & & \\
\hline & & & \\
\hline & & & \\
\hline & & & \\
\hline & & & \\
\hline & & & \\
\hline & & & \\
\hline WALL & & 173 & 21 \\
\hline BASE SHEAR & & 11 & \\
\hline
\end{tabular}


IEF KAISER

ENGINEERS latc. S-0002, Rew.o

LOS ALAMOS NMSF BUILDC EVALUATION

WALL IN-PLANE SHEAR

WALL: 10 line $\mathrm{Ct}$

\begin{tabular}{|c|c|c|c|}
\hline SHEL & SOINT & $\begin{array}{l}L_{C 1} \\
F_{X}\end{array}$ & $\begin{array}{l}L C_{2} \\
F_{x}\end{array}$ \\
\hline \multirow{2}{*}{2401} & 74 & 57 & -102 \\
\hline & 75 & 60 & -128 \\
\hline & & & \\
\hline & & & \\
\hline & & & \\
\hline & & & \\
\hline & & & \\
\hline & & & \\
\hline & & & \\
\hline & & & \\
\hline & & & \\
\hline & & & \\
\hline & & & \\
\hline & & & \\
\hline & & & \\
\hline & & & \\
\hline & & & \\
\hline $\begin{array}{l}\text { WALLL } \\
\text { BABE SHEAR }\end{array}$ & & 117 & \\
\hline
\end{tabular}

Jos No. $-\cdots=$

SHEET! 3 S OF

DESIGNED BY BMl DATE/7/45 CHECKED BY JU DATE $\$, Y, S$

REF. SAPAO RNN: 


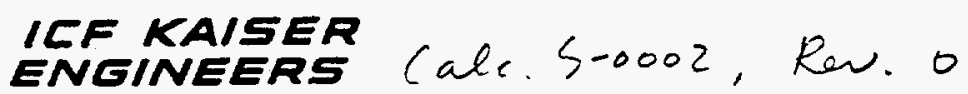

Joe No.

SHEET/36 of

LOS ALAMOS NMSF BUILDC EVALUATION

DESIGNED BY $\beta M$

DATE $3 / 7 / 5$

CHECKED BY $\triangle 0$

DATE $/ 19 / 91$

WALL IN-PLANE SHEAR

REF. SAPAO RNN:

WALL: 18 ine (FT) Banemed

\begin{tabular}{|c|c|c|c|}
\hline $\begin{array}{l}\text { SHELL } \\
\text { ELEMENT }\end{array}$ & SOINT & $\begin{array}{l}L_{C I} \\
F_{x}\end{array}$ & $\begin{array}{c}L C^{2} \\
F_{x}\end{array}$ \\
\hline \multirow{2}{*}{2561} & 21 & & -109 \\
\hline & 22 & & -120 \\
\hline \multirow{2}{*}{2562} & 22 & & -135 \\
\hline & 23 & & -125 \\
\hline & & & \\
\hline & & & \\
\hline & & & \\
\hline & & & \\
\hline & & & \\
\hline & & & \\
\hline & & & \\
\hline & & & \\
\hline & & & \\
\hline & & & \\
\hline$\cdot$ & & & \\
\hline & & & \\
\hline & & & \\
\hline WALL & & & \\
\hline BASE SHEAR & & & \\
\hline
\end{tabular}


ILF KAISER

ENGINEERS lalc. S-0002, Rew. 0

Jos No.

LOS ALAMOS NMSF BUILDC EVALUATION

$$
\text { OPTIONI }
$$

CHECKED BY ГC

REF. SAPAO RNN:

WALL IN-PLANE SHEAR

WALL: 33 line(F+) Barenents

\begin{tabular}{|c|c|c|c|}
\hline $\begin{array}{l}\text { SHELU } \\
\text { ELEMENT }\end{array}$ & JOINT & $\begin{array}{c}\text { LCI } \\
F_{x}\end{array}$ & $\begin{array}{l}L C 2 \\
F{ }_{x}\end{array}$ \\
\hline \multirow{2}{*}{2501} & 23 & & -131 \\
\hline & 24 & & -148 \\
\hline & & & \\
\hline & & & \\
\hline & & & \\
\hline & & & \\
\hline & & & \\
\hline & & & \\
\hline & & & \\
\hline & & & \\
\hline & & & \\
\hline & & & \\
\hline & & & \\
\hline & & & \\
\hline & & & \\
\hline & . & & \\
\hline & & & \\
\hline & & & \\
\hline & & & \\
\hline WALL & T & & \\
\hline BASE SHEAR & & & -279 \\
\hline
\end{tabular}


ICF KAISER

ENGINEERS

Calc. S-0002, Rev. 0

Joe NO $-1, T=$

SMEET/39or

LOS aLAMOS NMSF BUILDC EVALUATION DESIGNEDBY $\beta M$

DATे $/ 7 / 9 \overline{2}$

OPTION 1

CHECKED BY I $\mathrm{V}$

DATE $Y / 1 / 95$

WALL IN-PLANE SHEAR

REF. SAPAO RNN:

WALL: 11

line $C_{-}$, Basemet

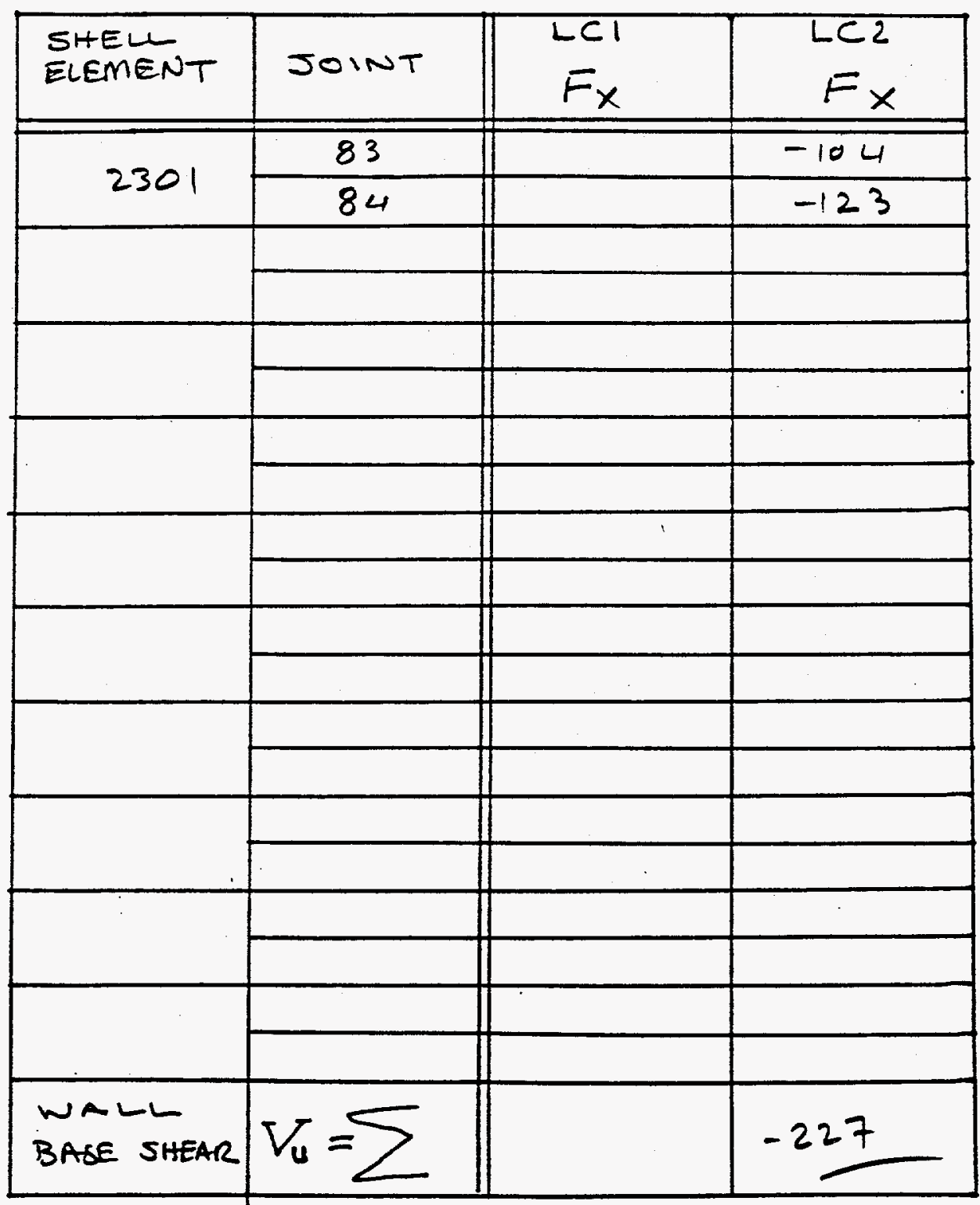


IEF KAISER

ENGINEERS LaCC. S-0002, Rew.

Jos No.

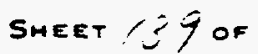

LOS ALAMOS NMSF BUILDC EVALUATION

DESIGNED BY $\beta, n$

$\operatorname{DAT} \hat{\bar{E}} / 7 / \bar{\varepsilon}$

CHECKED BY IV DATE $3 / 1 / 2 / 4)^{-}$

WALL IN-PLANE SHEAR

REF. SAPGO RUN:

WALL: 7 worth Comcate Buffle (Baremet)

\begin{tabular}{|c|c|c|}
\hline $\begin{array}{l}\text { SHEU } \\
\text { ELEMENT }\end{array}$ & SOINT & $\begin{array}{c}L_{C I} \\
F_{x}\end{array}$ \\
\hline
\end{tabular}

2221

\begin{tabular}{|l|l|l|l|}
\hline \multirow{2}{*}{2222} & 92 & & -84 \\
\cline { 2 - 4 } & 93 & & -77 \\
\hline
\end{tabular}

WALL

bASE SHEAR

$$
V_{u}=\sum
$$

274 


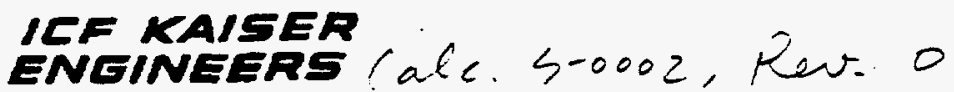

Jos No

SMEET $\% 0$ OF

LOS ALAMOS NMSF BUILDC EVALUATION

DESIGNED BY $B m$

DATE $=I \perp 9=$

CHECKED BY. T

DATE $\gamma / 1 \% 45$

WALL IN-PLANE SHEAR

REF. SAPOO RUN:

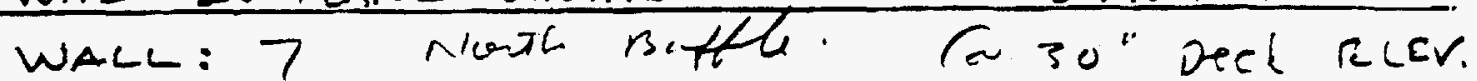

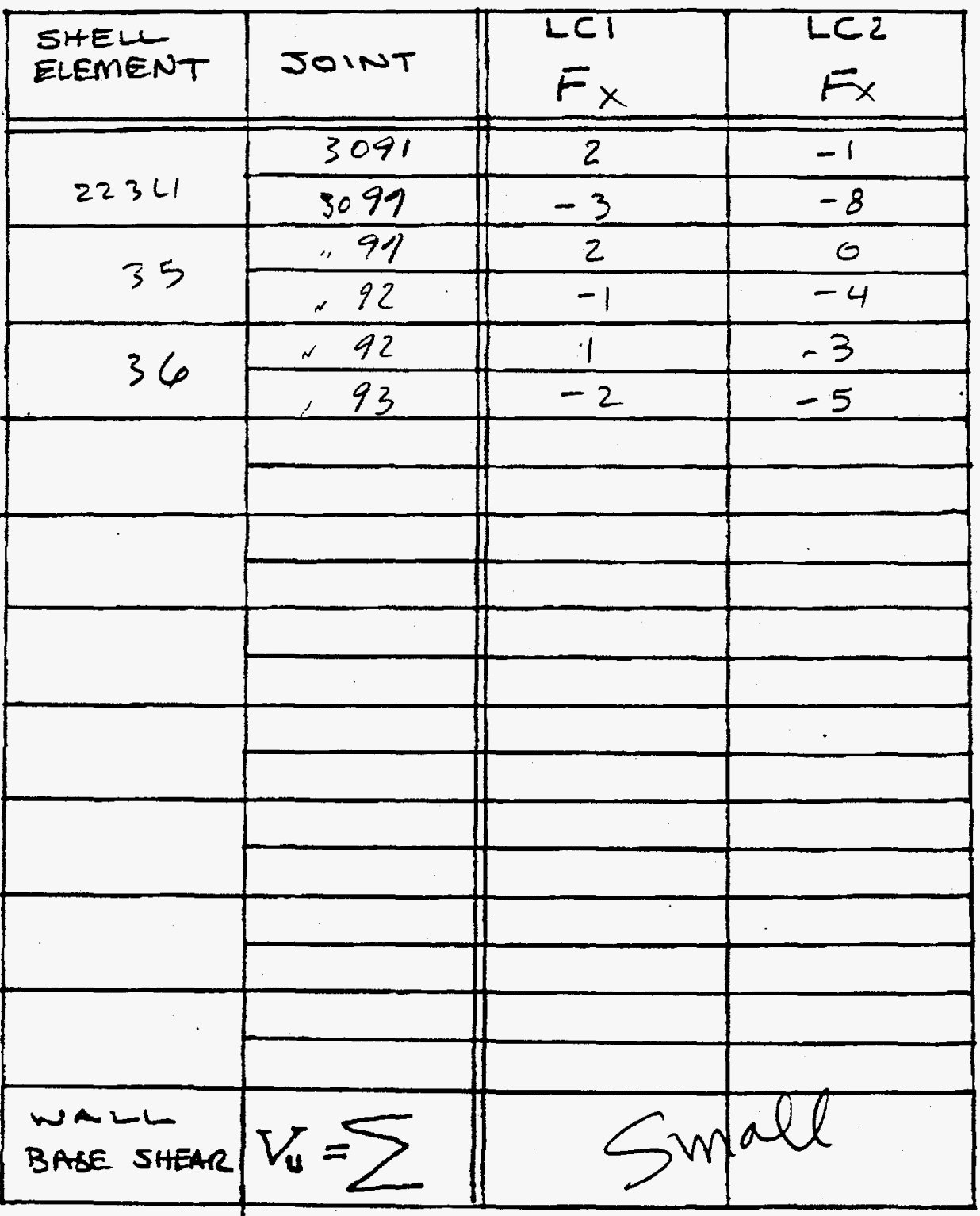


ICF KAISER

ENGINEERS la l,$S-0002$, Rew. 0

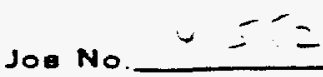

SHEET $/ \psi /$ OF

LOS ALAMOS NMSF BUILDC EVALWTION EPTION I

DESIGNED BY

BMA DATE $3 / 7 / 95$ CHECKED BY DATE $Y 1 \times 45$

WALL IN-PLANE SHEAR REF. SAPGO RNN:

WALL: 29 BUTKESS (BMency)

\begin{tabular}{|c|c|c|c|}
\hline $\begin{array}{l}\text { SHELL } \\
\text { ELEMENT }\end{array}$ & SOINT & $\begin{array}{l}L C_{1} \\
F_{x}\end{array}$ & $\begin{array}{l}\angle C_{2} \\
F_{x}\end{array}$ \\
\hline \multirow{2}{*}{4401} & 60 & & -134 \\
\hline & $Q 1$ & & -116 \\
\hline & & & \\
\hline & & & \\
\hline & & & \\
\hline & & & \\
\hline & & & \\
\hline & & & \\
\hline & & & \\
\hline & & & \\
\hline & & & \\
\hline & & & \\
\hline & & & \\
\hline & & & \\
\hline & & & \\
\hline & & & \\
\hline & & & \\
\hline & & & \\
\hline & & & \\
\hline WALL & & & -250 \\
\hline BASE SHEAR & & & $-\angle n$ \\
\hline
\end{tabular}




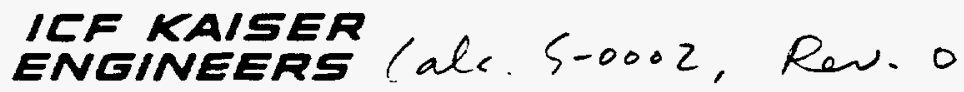

ENGINEERS (ale. S-0002, Rew.

LOS ALAMOS NMSF BUILDC EVALUATION

WALC IN-PLANE SHEAR

WALL: 3

SOUTh BAFFLE
Joe No. 4 :

SHEET/ $/ 42$ OF
DESIGNED BY $A M$ CHECKED BY 5 REF. SAPAO RNN:

( $630^{\circ}$ Dech RL.)

\begin{tabular}{|c|c|c|c|}
\hline $\begin{array}{l}\text { SHELL } \\
\text { ELEMENT }\end{array}$ & JOINT & $\begin{array}{l}L C I \\
F_{x}\end{array}$ & $\begin{array}{c}L C_{2} \\
F_{x}\end{array}$ \\
\hline \multirow{2}{*}{4731} & 3011 & 18 & -6 \\
\hline & 12 & 17 & -7 \\
\hline \multirow{2}{*}{4732} & 3012 & 17 & -6 \\
\hline & 13 & 18 & -6 \\
\hline & & & \\
\hline & & & \\
\hline & & & \\
\hline & & & \\
\hline & & & \\
\hline & & & \\
\hline & & & \\
\hline & & & \\
\hline & & & \\
\hline & & & \\
\hline & & & \\
\hline & & & \\
\hline & & & \\
\hline $\begin{array}{l}\text { WALL } \\
\text { BASE SHEAR }\end{array}$ & & & \\
\hline
\end{tabular}


ENEINEERS ( a C . S-0002, Rev. 0

WALL IN-PLANE SHEAR

REF. SAPIO RUN:

WALL: 21 live 3 first flour

\begin{tabular}{|c|c|c|c|}
\hline $\begin{array}{l}\text { SHELL } \\
\text { ELEMENT }\end{array}$ & SOINT & $\begin{array}{l}\text { LCI } \\
\text { FY }\end{array}$ & $\begin{array}{l}L C^{2} \\
F_{Y}\end{array}$ \\
\hline \multirow{2}{*}{3301} & 4004 & 20 & -18 \\
\hline & 14 & 16 & -19 \\
\hline \multirow{2}{*}{2} & 14 & 24 & -21 \\
\hline & 24 & 18 & -24 \\
\hline \multirow[b]{2}{*}{3} & 24 & 90 & -98 \\
\hline & 34 & 86 & 104 \\
\hline \multirow{2}{*}{4} & 34 & 46 & -48 \\
\hline & पप & 40 & -51 \\
\hline \multirow[b]{2}{*}{5} & 44 & 46 & -46 \\
\hline & 54 & 41 & -51 \\
\hline \multirow{2}{*}{6} & 54 & 46 & -47 \\
\hline & 64 & 40 & -52 \\
\hline \multirow[b]{2}{*}{7} & 64 & & -46 \\
\hline & 74 & & -51 \\
\hline \multirow[t]{2}{*}{ : } & 74 & & -44 \\
\hline & 84 & & -53 \\
\hline \multirow{2}{*}{9} & 84 & & -107 \\
\hline & 94 & & -118 \\
\hline \multirow{2}{*}{10} & 74 & & -48 \\
\hline & 104 & & -26 \\
\hline WALL & - & & 1.72 \\
\hline
\end{tabular}


WALL IN-PLANE SHEAR

REF. SAPGO RUN:

WALL: 28

line

$B$

1 st Flar

\begin{tabular}{|c|c|c|c|}
\hline $\begin{array}{l}\text { SHELL } \\
\text { ELEMENT }\end{array}$ & SOINT & $\begin{array}{l}\text { LCI } \\
F_{x}\end{array}$ & $\begin{array}{l}\text { LC2 } \\
F_{x}\end{array}$ \\
\hline \multirow{2}{*}{4221} & 4105 & & -105 \\
\hline & 6 & & -111 \\
\hline \multirow{2}{*}{2} & 6 & & -72 \\
\hline & 7 & & -78 \\
\hline \multirow{2}{*}{3} & 7 & & -109 \\
\hline & 8 & & -118 \\
\hline \multirow{2}{*}{4} & 8 & & -53 \\
\hline & 9 & & -58 \\
\hline \multirow{2}{*}{5} & 9 & & -38 \\
\hline & 4110 & & -49 \\
\hline & & & \\
\hline & & & \\
\hline & & & \\
\hline & & & \\
\hline & & & \\
\hline & & & \\
\hline & & & \\
\hline & & & \\
\hline WALL & & & $k$ \\
\hline BASE SHEAR & & & \\
\hline
\end{tabular}


IEF KAISER

ENEINEERS Ca.C.S-0002, Rew. O LOS ALAMOS NMSF BUILDC EVALUATION (शThen)

WALL IN-PLANE SHEAR 137 Fluor

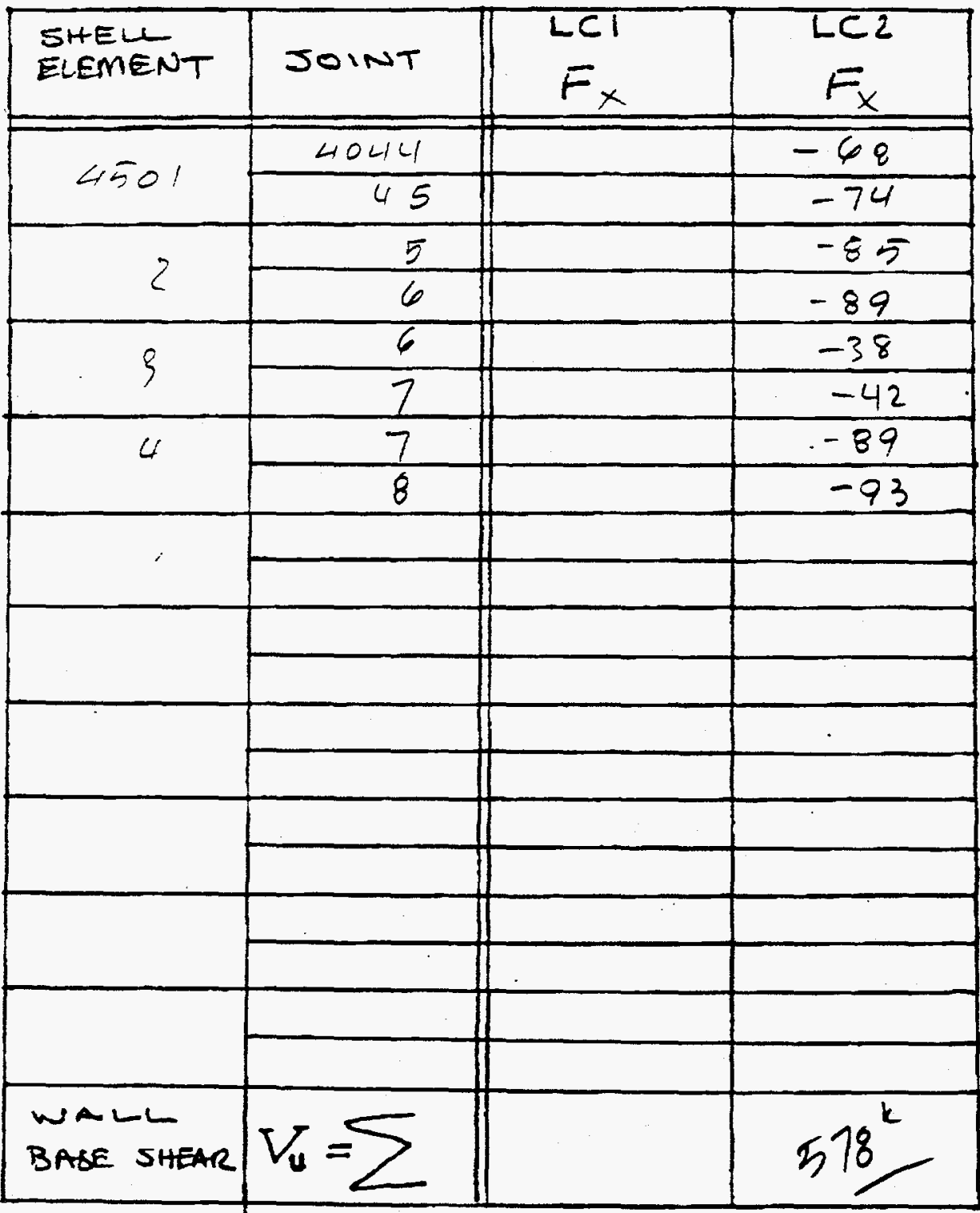

WALL: 30 1 7 Fluor

Jop No.

SHEET//; OF

DESIGNED BY 13217 DATE $3 / 7 / 95$ CHECKED BY TU DATE $3 / 10 / 4$ S

REF. SAPAO RUN: 


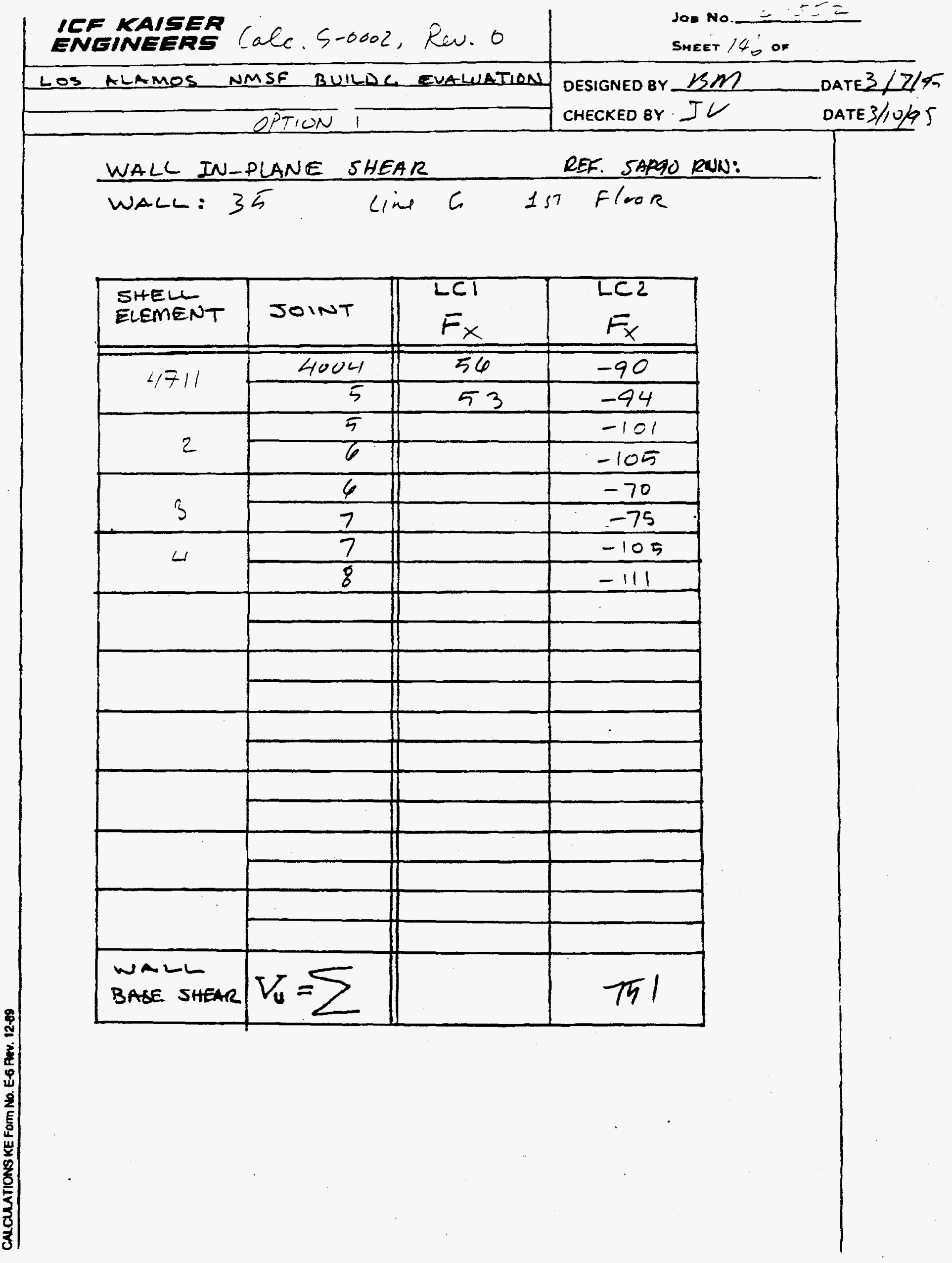


SHEET $\angle 7$ OF

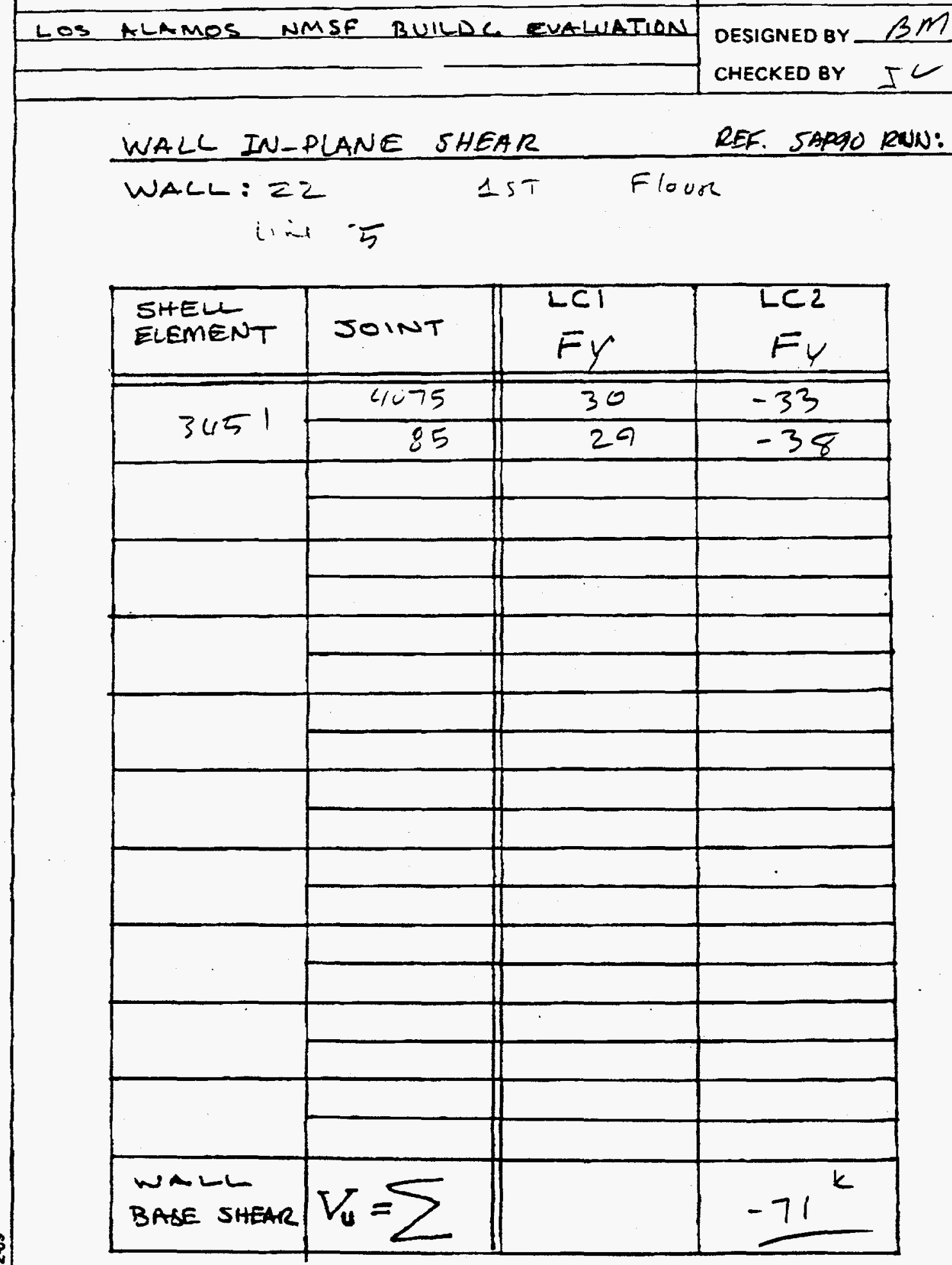

WALL IN-PLANE SHEAR

-


REF KAIEER (aC. S-000Z, RU. o

Joe No.L

SHEET' 3 oF

LOS NLAMOS NMSF BUILDC EVALUATION

OESIGNED BY $\beta M$

DATE $3 / 1 / 95$

CHECKED BY IV

DATE $3 / 245$

WALL IN-PLANE SHEAR

REF. SAPOO RUN:

WALL: 36 line $C$ - IST floor

\begin{tabular}{|c|c|c|c|}
\hline $\begin{array}{l}\text { SHEL } \\
\text { ELEMENT }\end{array}$ & SOINT & $\begin{array}{l}\mathrm{LCI} \\
F_{x}\end{array}$ & $\begin{array}{c}\text { LC2 } \\
F_{x}\end{array}$ \\
\hline \multirow{2}{*}{4301} & 4074 & 39 & -54 \\
\hline & 4075 & 39 & -60 \\
\hline & & & \\
\hline & & & \\
\hline & & & \\
\hline & & & \\
\hline & & & \\
\hline & & & \\
\hline & & & \\
\hline & & & \\
\hline & & & \\
\hline & & & . \\
\hline & & & \\
\hline & & & \\
\hline & & & \\
\hline & & & \\
\hline & & & \\
\hline & & & \\
\hline WALL & & & \\
\hline BASE SHEAR & & & \\
\hline
\end{tabular}




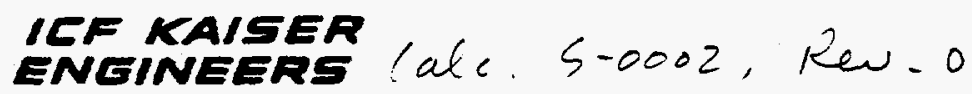

Joe No.

$=$

LOS ALAMOS NMSF BUILDC EVALUATION

DESIGNED BY $3 \mathrm{~B}$

DATE

CHECKEO $6 Y$

$T 2$

DATE $3 \% 0 / 45$

WALL IN-PLANE SHEAR

REF. SAPAO RUN:

WALL: 25 Ling 7 Ist fler

\begin{tabular}{|c|c|c|c|}
\hline $\begin{array}{l}\text { SHEU } \\
\text { ELEMENT }\end{array}$ & SOINT & $\begin{array}{l}L C I \\
F_{Y}\end{array}$ & $\begin{array}{l}L_{2} \\
F_{Y}\end{array}$ \\
\hline \multirow{2}{*}{3701} & 4008 & 19 & -18 \\
\hline & 18 & 14 & -20 \\
\hline \multirow{2}{*}{2} & 18 & & -21 \\
\hline & 28 & & -24 \\
\hline \multirow{2}{*}{3} & 28 & & -98 \\
\hline & 38 & & -101 \\
\hline \multirow{2}{*}{4} & 38 & & -46 \\
\hline & 48 & & -50 \\
\hline \multirow{2}{*}{5} & 48 & & -46 \\
\hline & 98 & & $-5 !$ \\
\hline \multirow{2}{*}{6} & 58 & & -46 \\
\hline & 68 & & -51 \\
\hline \multirow{2}{*}{7} & 68 & & -46 \\
\hline & 98 & & -51 \\
\hline \multirow{2}{*}{8} & 78 & & -47 \\
\hline & 88 & & -52 \\
\hline \multirow{4}{*}{9} & 88 & & -26 \\
\hline & 98 & & -32 \\
\hline & & & $\cdot$ \\
\hline & & & - \\
\hline$w \sim 2 L$ & & & \\
\hline BAEE SHEAR & & & 826 \\
\hline
\end{tabular}




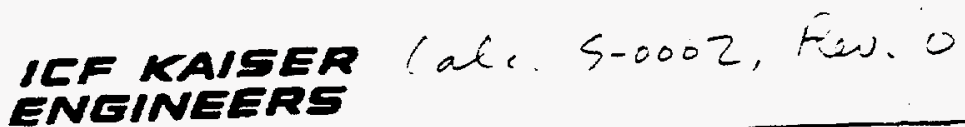
LOS NLAMOS NMSF BUILDC EVALUATION

DESIGNED BY

SMEET/, SOOF

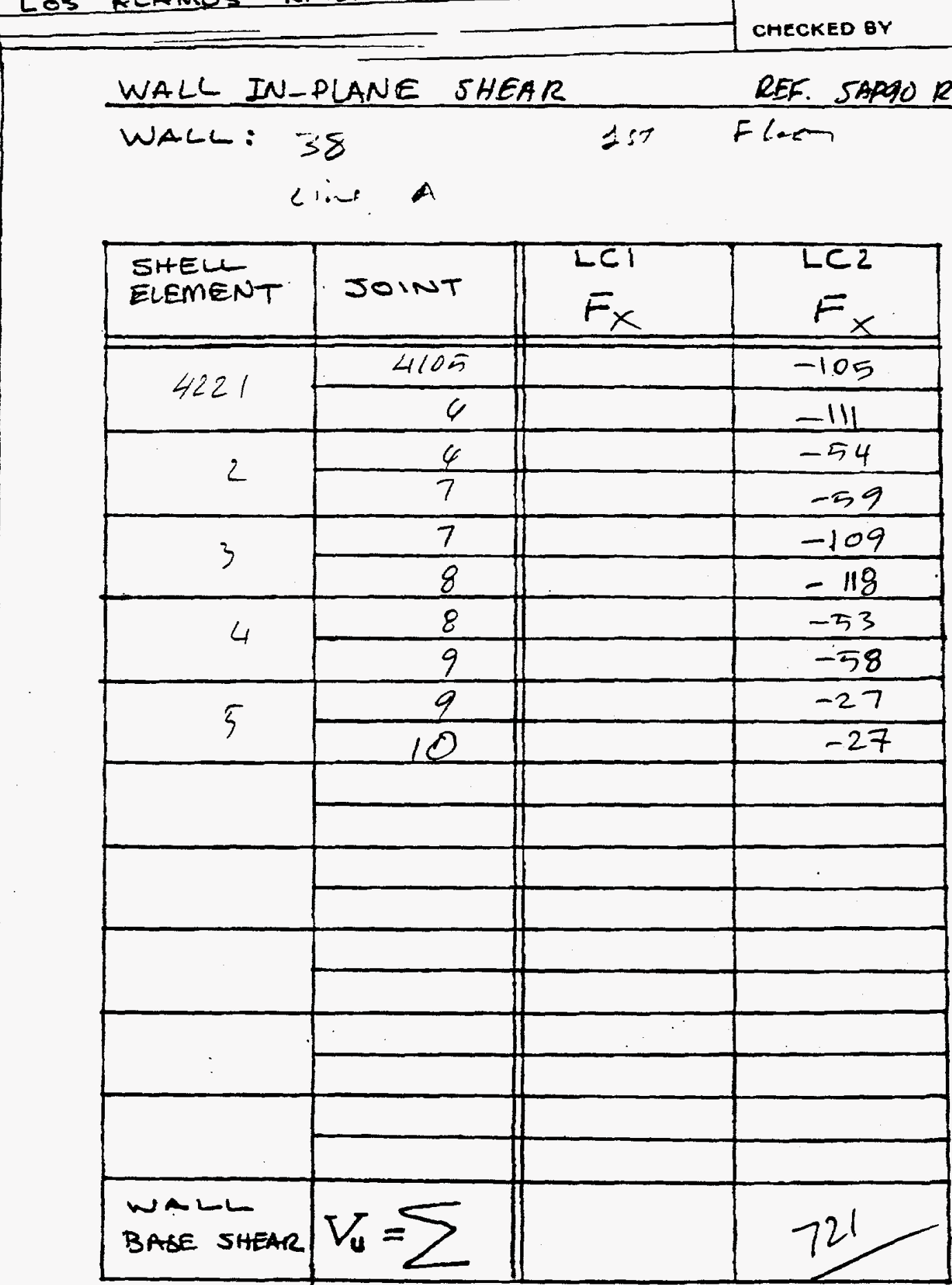
DATE $3 / 10 / 05$ DATE $\% 0 / 96$ 
EVALUATION OF. NMSF FACILITY AT LANL

DESIGNED BY B.M. DATE $3 / 4 / 95$

Modification Option (1). CHECKED BY

7.2 WALL OUT-of-PLANE SHEAR AND BENDING 


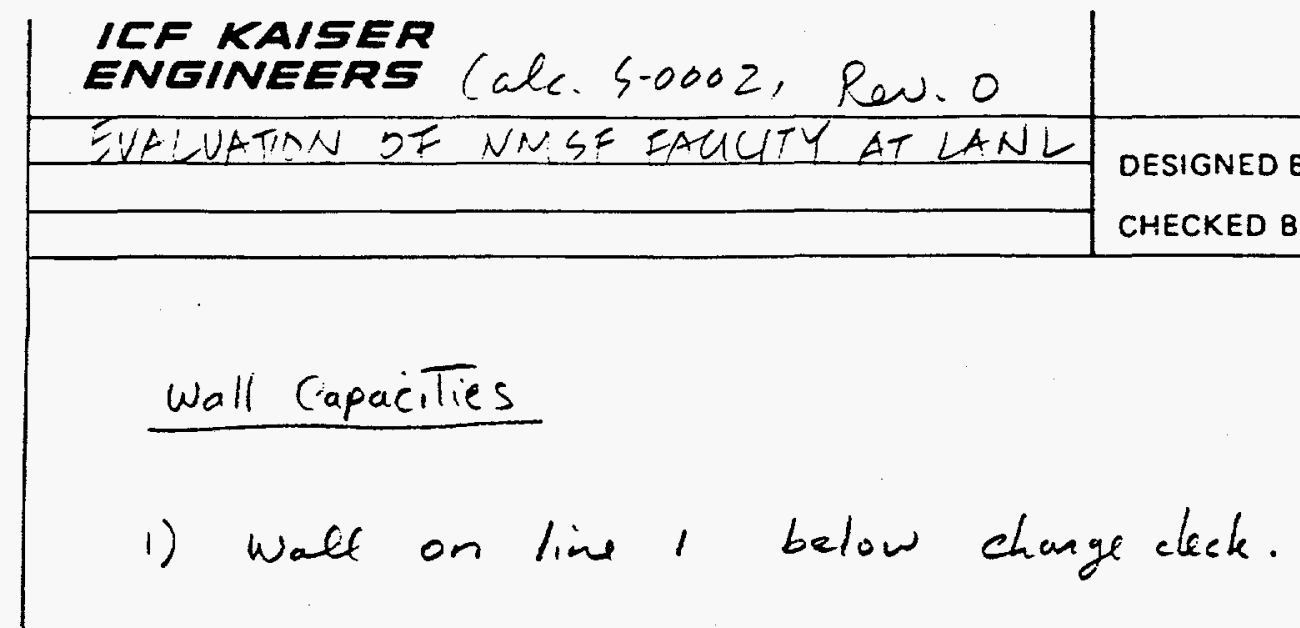

Soo No__ :

Exisling wall $1.5^{\prime}$

New wall 1.5

To7.. Wull Thicknem 3-0"

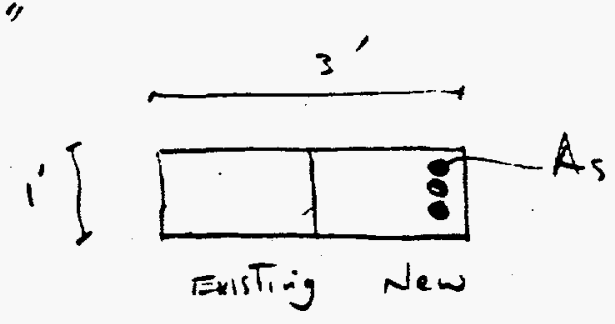

USE \#9D5 in New Concuete

$$
\begin{aligned}
\rightarrow A_{s} & =1 \times 12 / 5=2.4 \mathrm{in}^{2} \\
a & =A_{s} \cdot f_{7} /\left(.85 f_{c}^{\prime} \times b\right) \\
& =2.4 \times 60 /(.85 \times 4 \times 12)=3.53^{\prime \prime} \\
d & =36^{\prime \prime}-3=33^{\circ}
\end{aligned}
$$$$
M_{u}=.95 \cdot A_{s} \cdot f_{y} \cdot(d-a / 2)
$$$$
=.95 \times 2.4 \times 60(33-3.53 / 2)
$$$$
=4273 \mathrm{k} \text {-in }
$$$$
=356 \quad K-F T
$$

$$
\text { * } D / c=279 / 356=0.78<1.0 \text { o.k. }
$$

Notice that dexign of wall i Controlled by Prelim Therual Anabyis. A mose accurate Themal Anolyis 


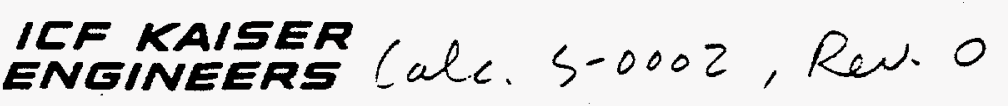

Joe No $r-\ldots$

EVALUATIONS OF NOSE FACILITY LT DANU

DESIGNED BY BM

$\operatorname{DAT} 3 / 9 / 95$

CHECKED BY

DATES 1 $10 / 45$

based on future Want Transfer analysis of th concrete well of NMSF storage area in basement shall change Amount of steed needed. Current design is based or Preliminary thermal gradients across the concrete wall, furnished by LANL STaff.

2) walk on. line 2 below change deck

Existing wall Theremin $120 "$

New

$1-6^{n}$

Total wall thickness $z$-c"

$$
\begin{aligned}
& \text { USE } \# 925 \rightarrow a_{5}=2.4 \mathrm{in}^{2} \\
& a=3.53^{\prime \prime} \\
& d=30-3=27^{4} \\
& M_{u}=.95 \times 2.4 \times 60(27-3.53 / 2) \\
& =3452 \mathrm{k}-\mathrm{in} \\
& =287.7 \mathrm{~K} F T \text {. } \\
& D / C=201 / 287.7=0.70<1 . \quad 0 . k \text {. }
\end{aligned}
$$


IVF KAISER

0

EvaLUATION OF NMSF FAMLTY AT LANL

DESIGNED BY $3 M$

CHECKED BY TL

DATE $3 / 4 / 9=$

DATE $3 / 12 / 4$

check wall on line (2) Above change Deck

Thin is a new wall 1-4" Thick infilled between existing Columns on line (2).

Local moment due to Crane:

$$
M=30 \quad K-F T \text {. }
$$

Load Combinations

$$
\begin{aligned}
& M=30+38=68 \cdot k-F T \\
& M=8+1.7 \times 38=73 k-F T \\
& M=Z+73=75 k-F T \\
& M=73+1.4 \times 5=80 k-9 T
\end{aligned}
$$

(LCD)

(LC)

(Demand 1)

(Demand 2)

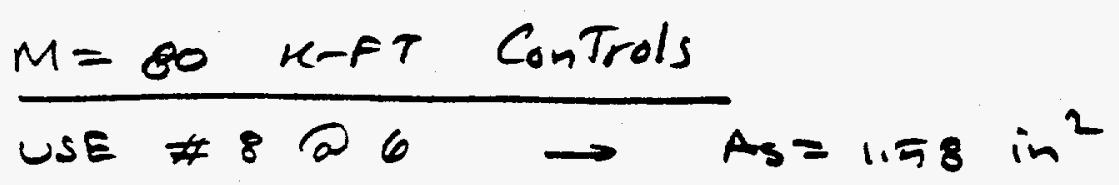

$$
\begin{aligned}
& a=1.78 \times 60 /(.85 \times 4 \times 12)=2.32^{\prime \prime} \\
& d=16-2=14^{\prime \prime} \\
& M_{u}=.95 \times 1.58 \times 60(14-227 / 2) \\
& =96 \mathrm{~K}-\mathrm{PT} \text {. }
\end{aligned}
$$

$$
D / C=80 / 96=0.83<1.0 \quad \text {...k. }
$$




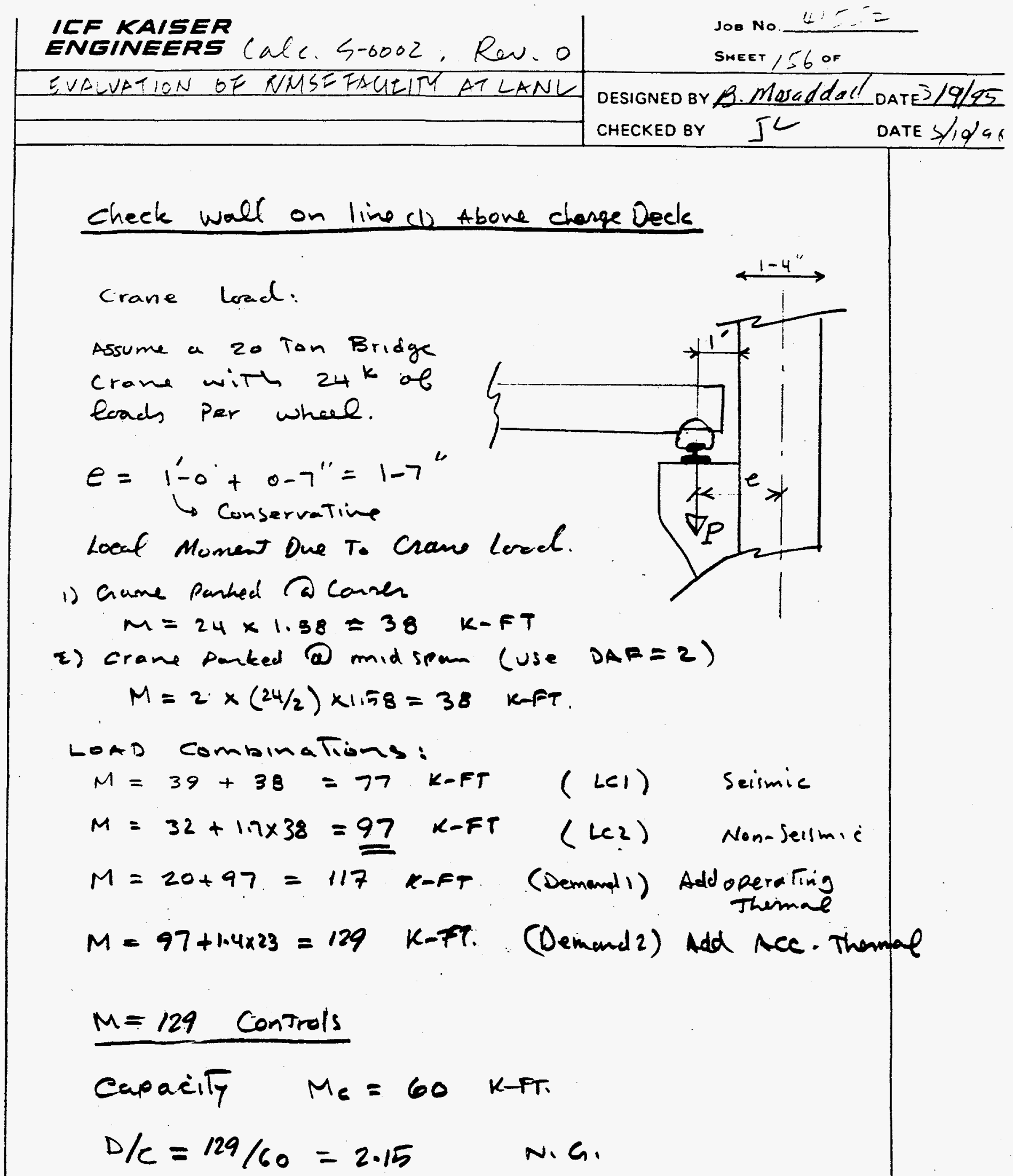

Need to strengthen the wall or use a lighter crane!

See calculation for a Conceptual nodbication to this wall in sec. 8.4 of thin ells. 


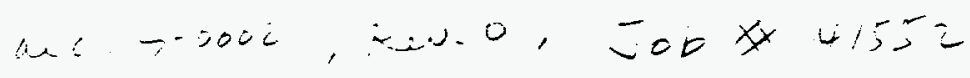

six. 157of

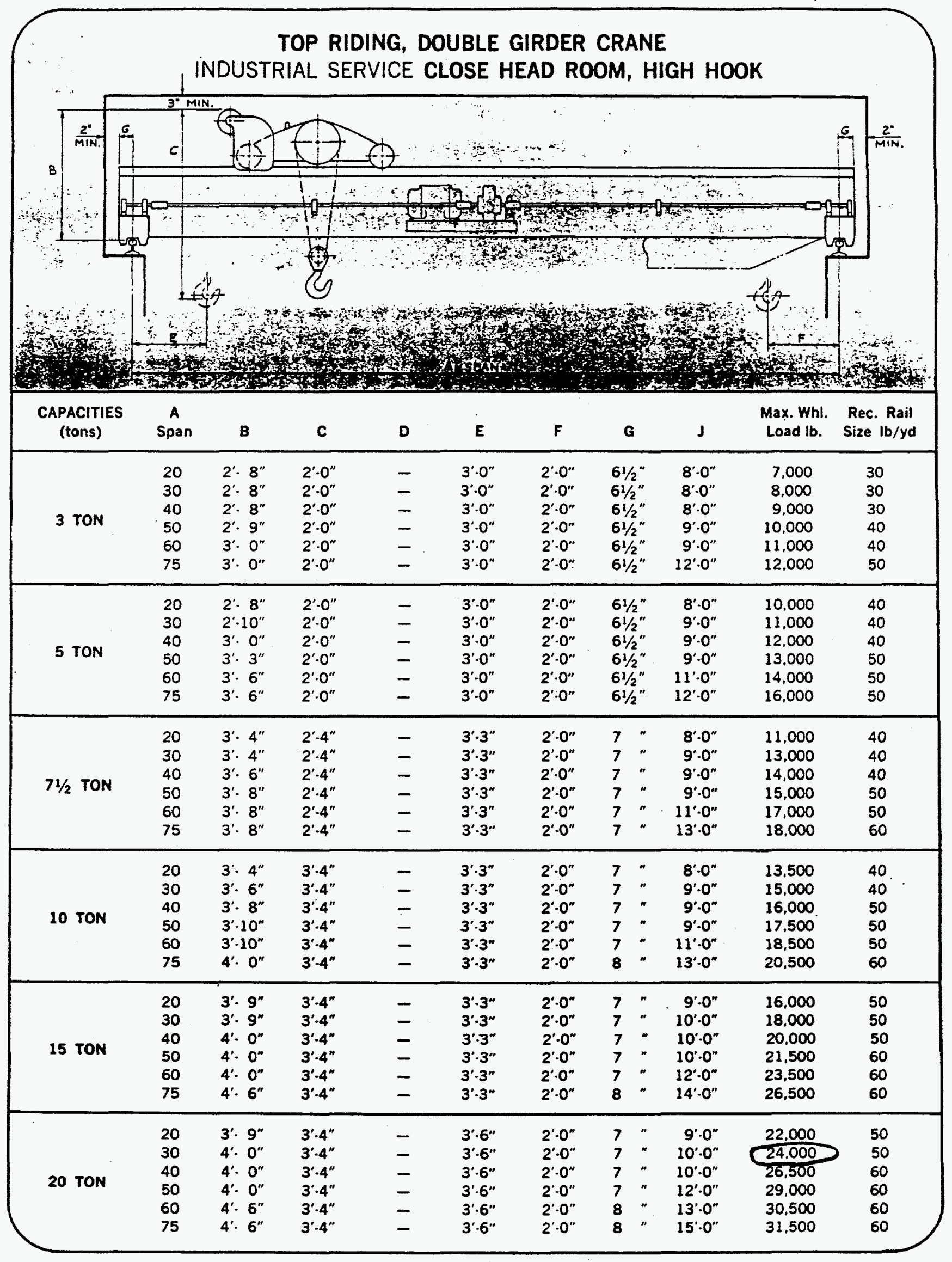




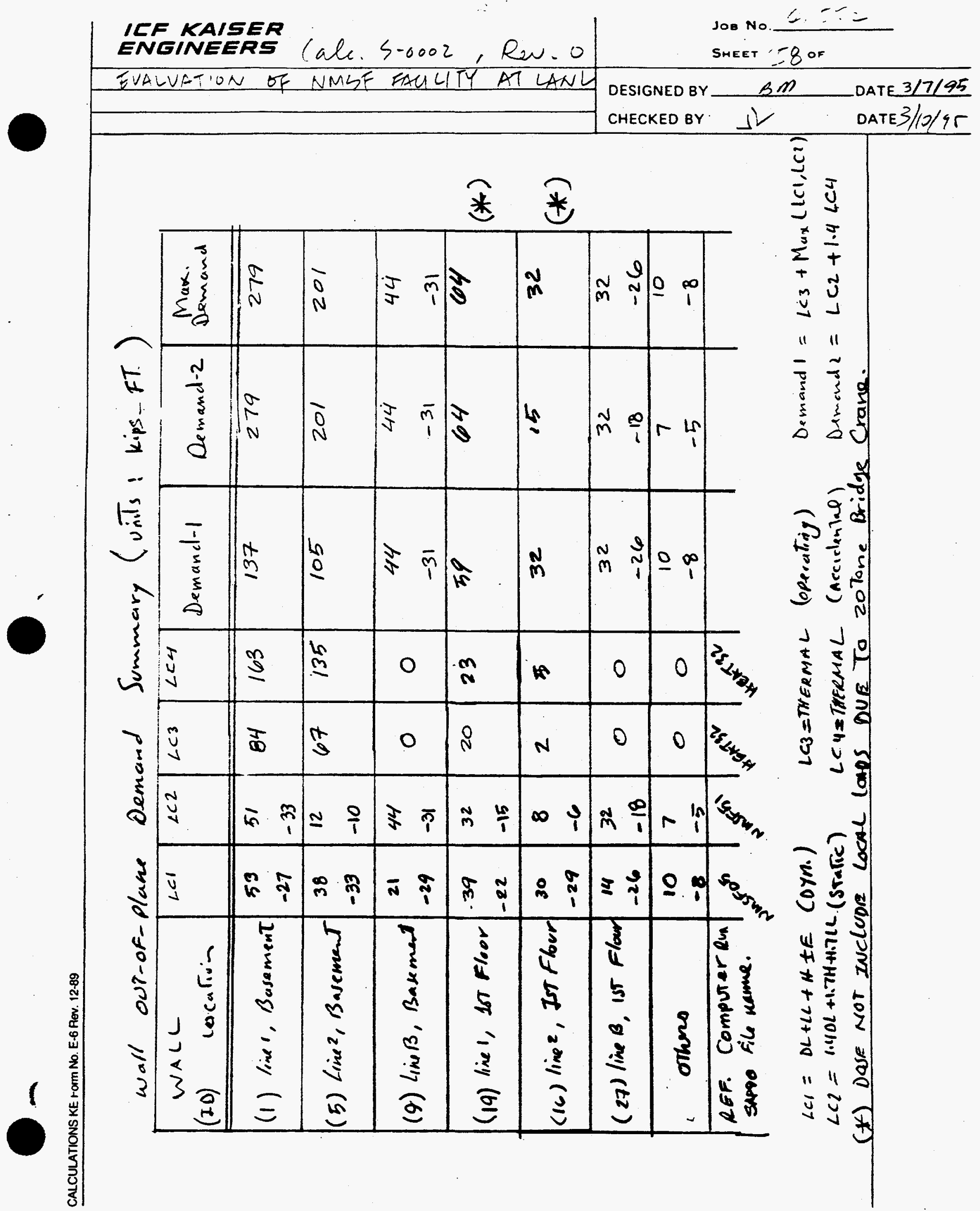


(alc. S-00o2, Rev.0, Job *41552 sht.159 of

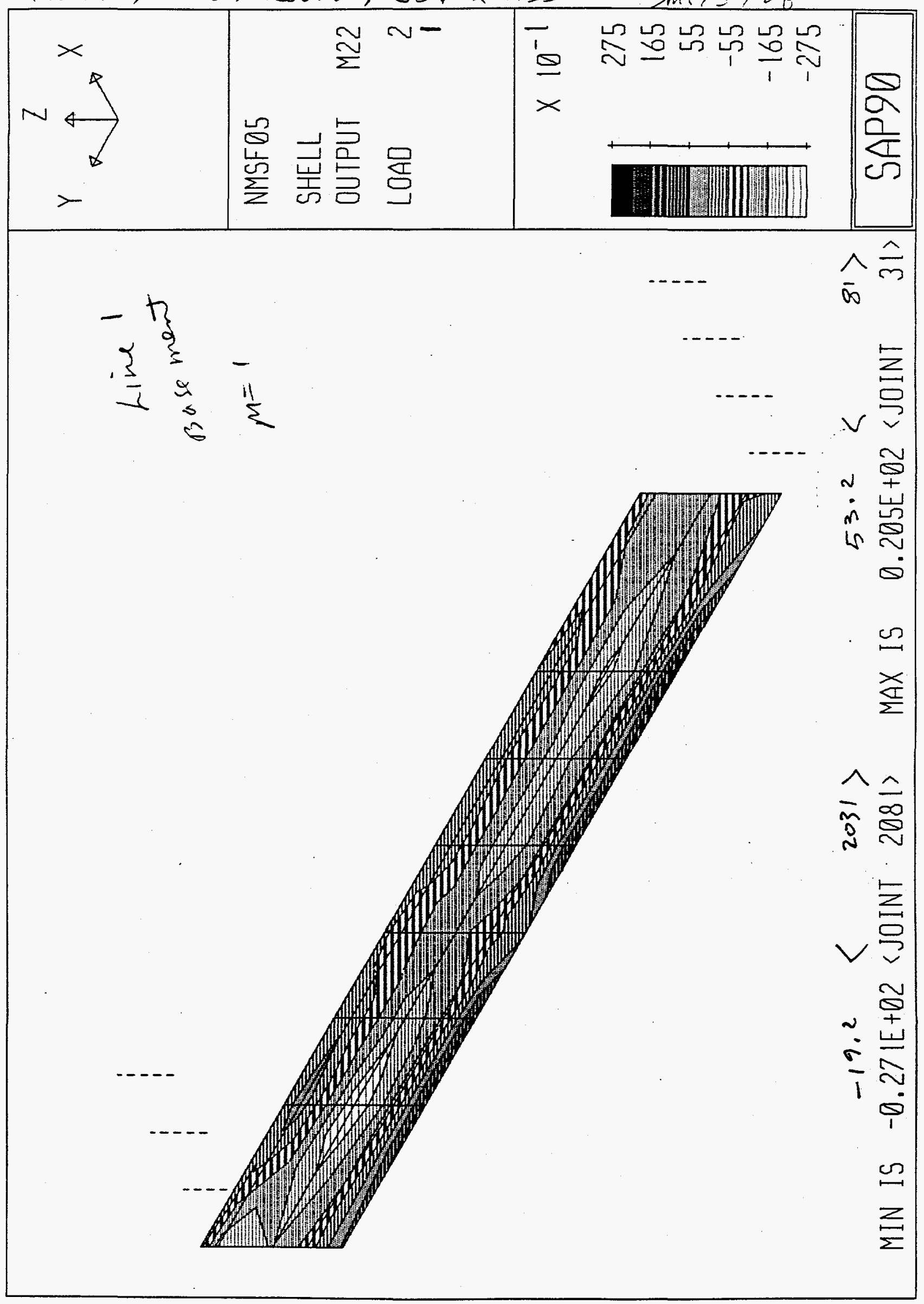


(alc, S-0002, Rew 0, Job $\times 41552$ sht $160=1$

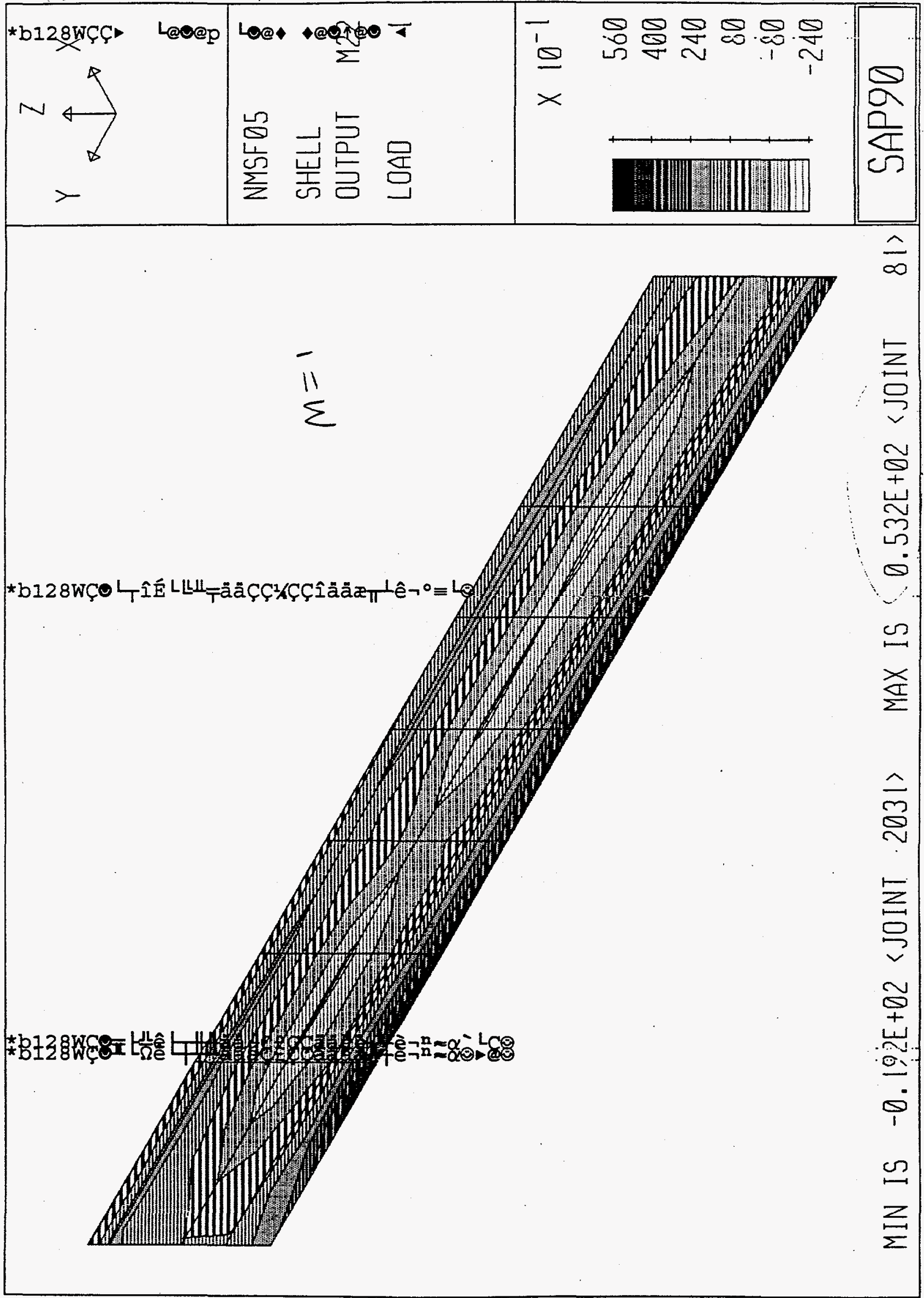


lalc.s-0002, Rev. O, Job * 41552 Sht. 161 of

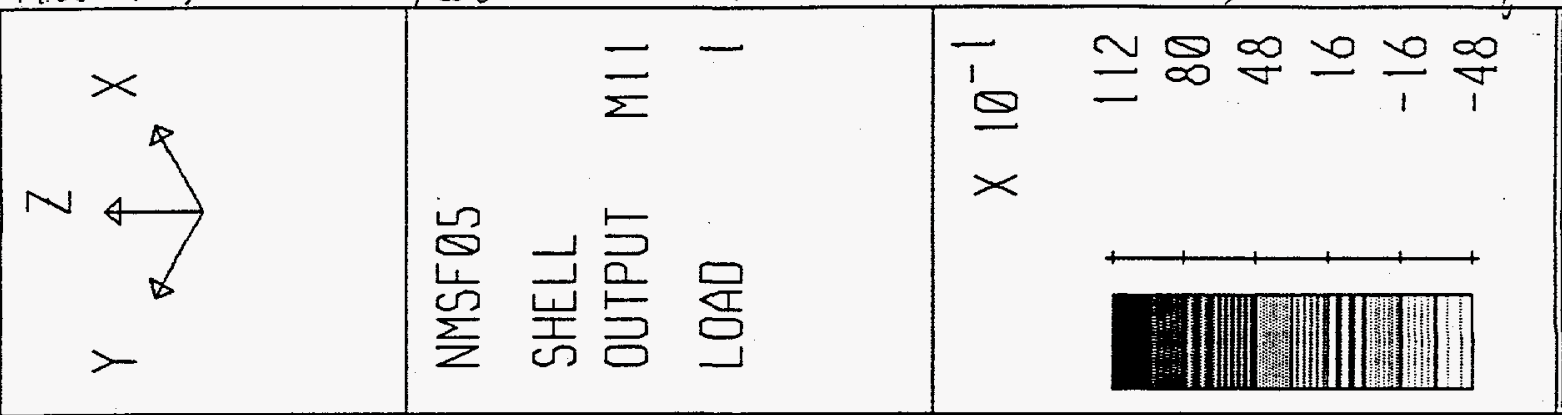

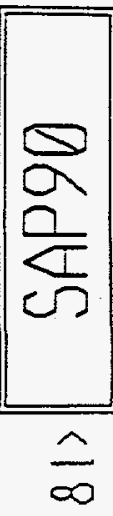

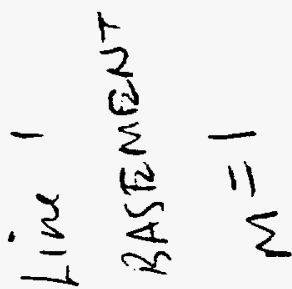

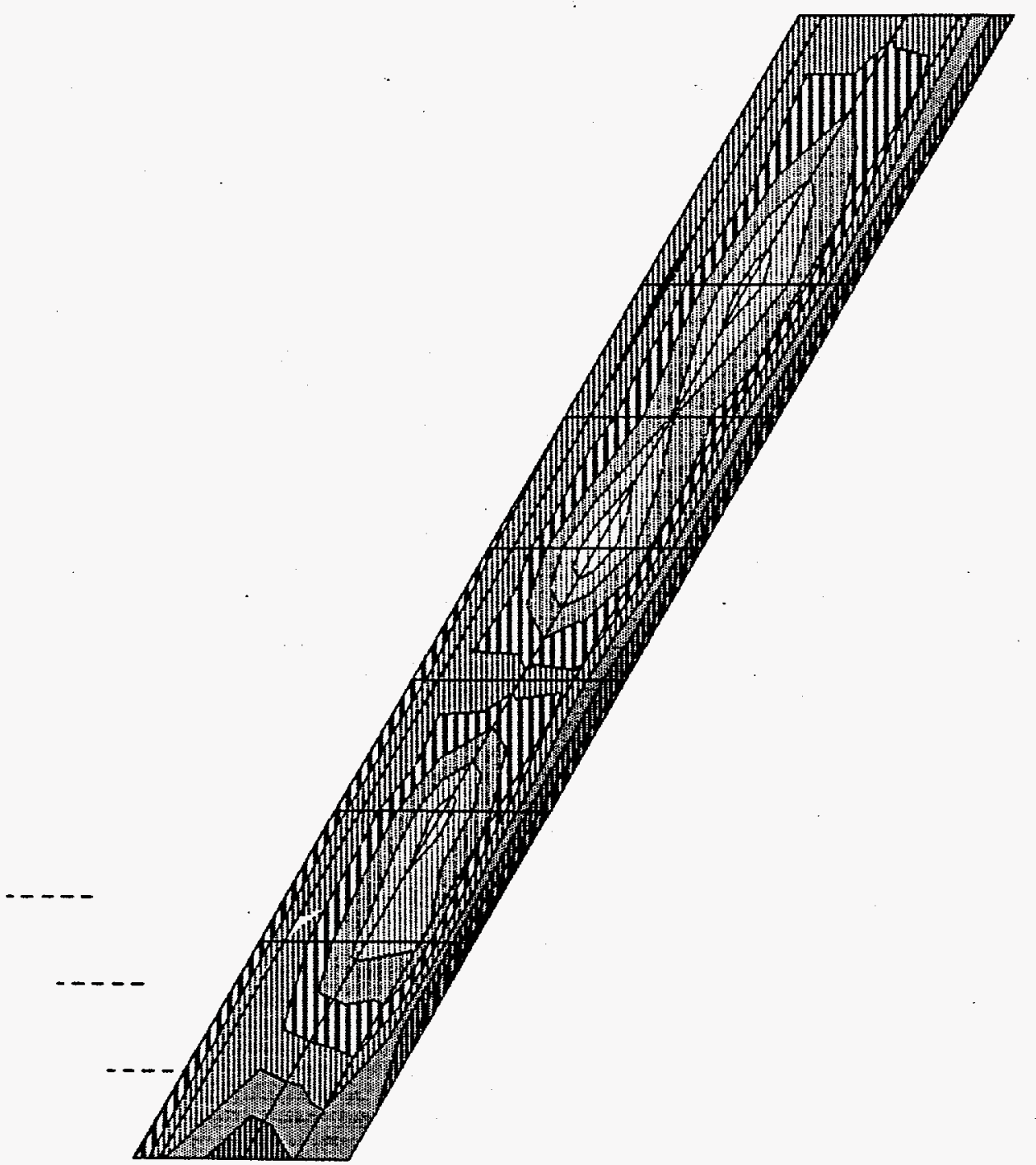

孚

$\stackrel{\hat{\sim}}{\stackrel{\Xi}{\sim}}$

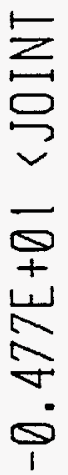

s

$\underset{\Sigma}{\rightleftarrows}$ 
(alc. S-0002, Rew. 0, Job*41552 Sht. 1t2 of

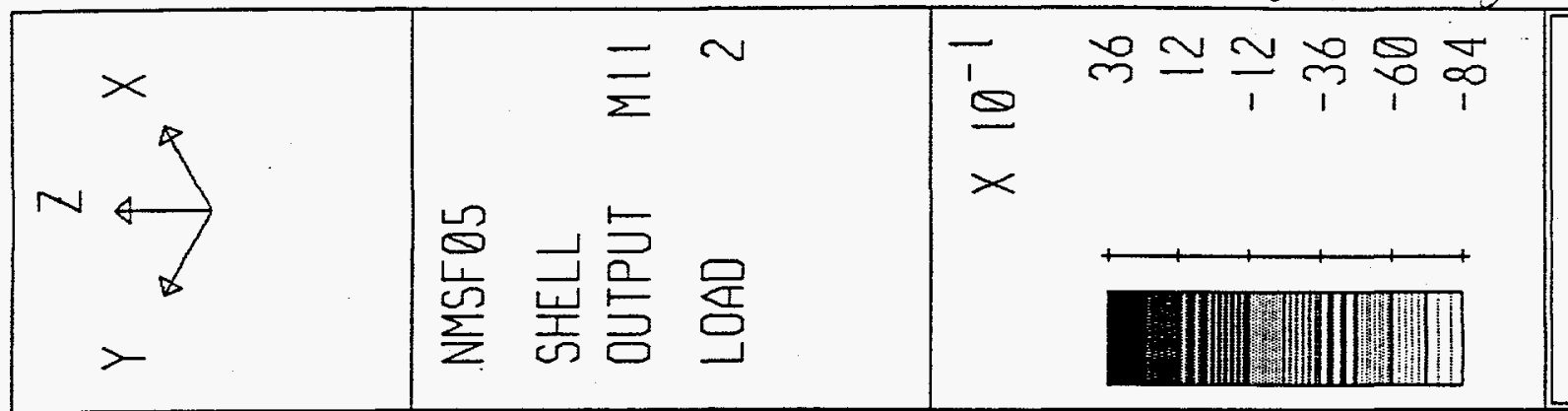

衰

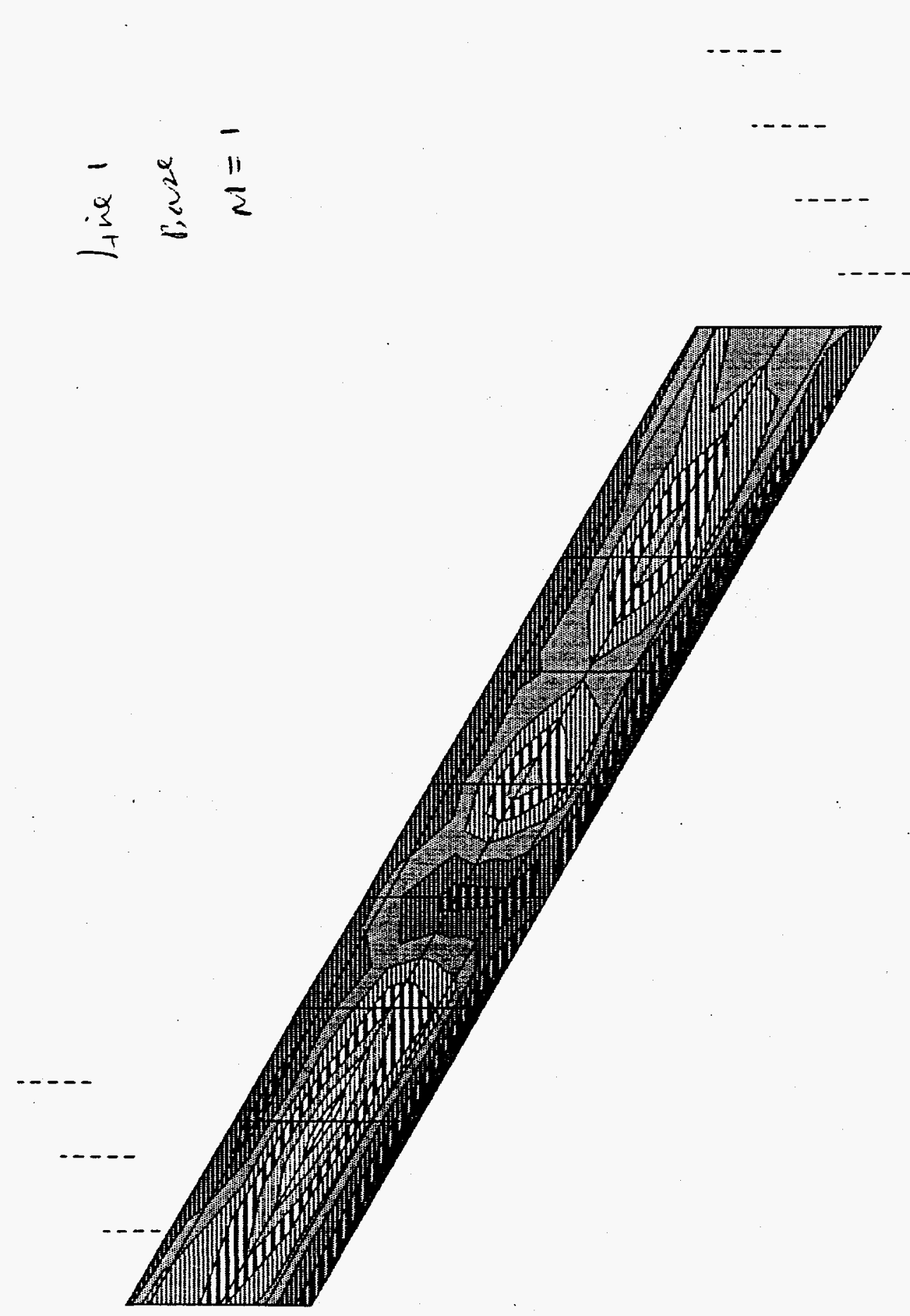

$\hat{n}$

$\underset{⿱}{\varrho}$

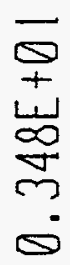

s

$\underset{⿱ 亠 乂}{\Sigma}$

$\stackrel{\infty}{\infty}$

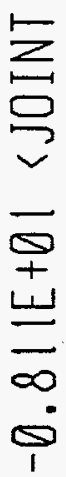

$\stackrel{2}{\longrightarrow}$

$\sum$ 


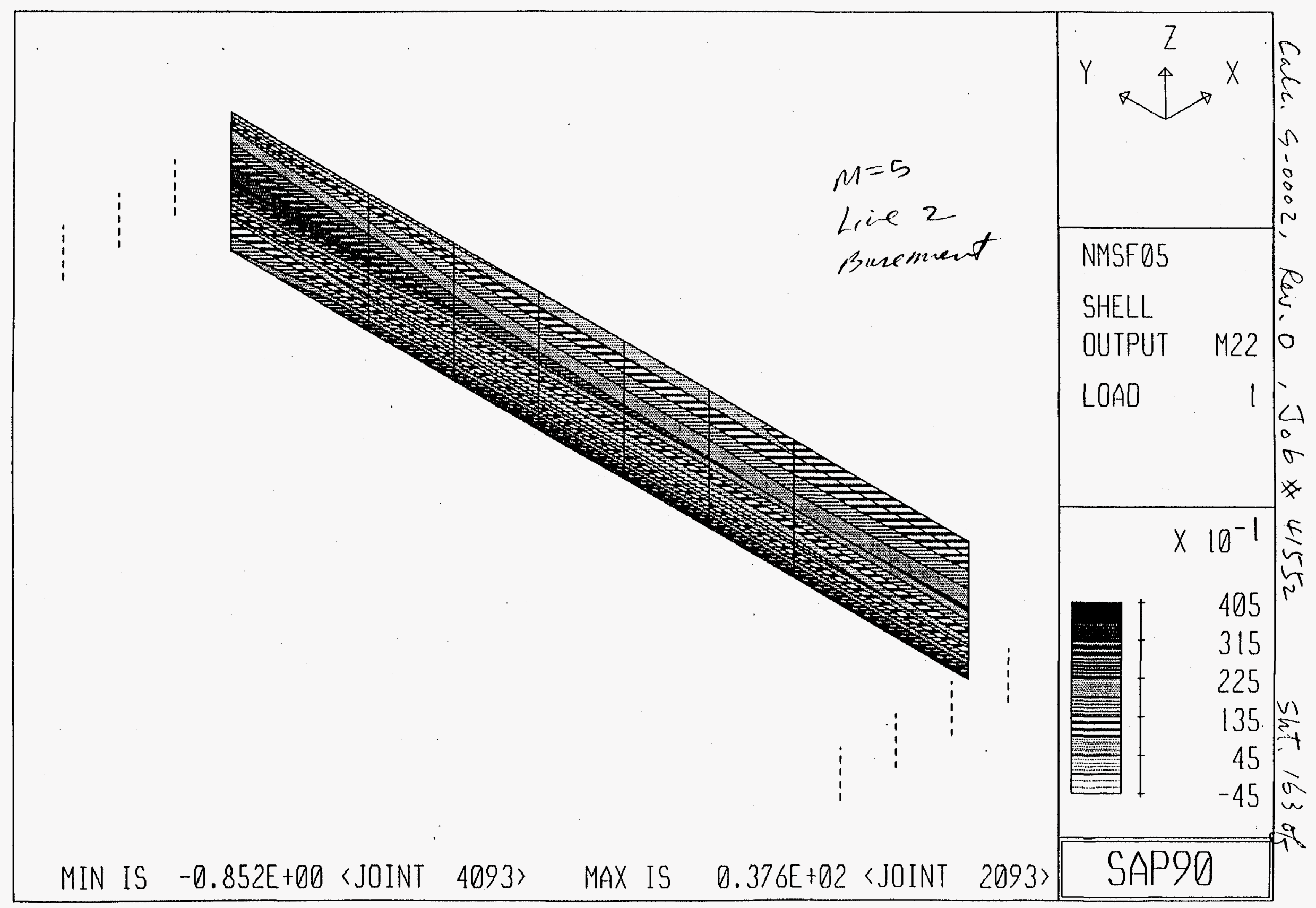


Calc. S-0002, Piv.0, Job $\$ 41552 \quad 5 h^{+} 164$ of

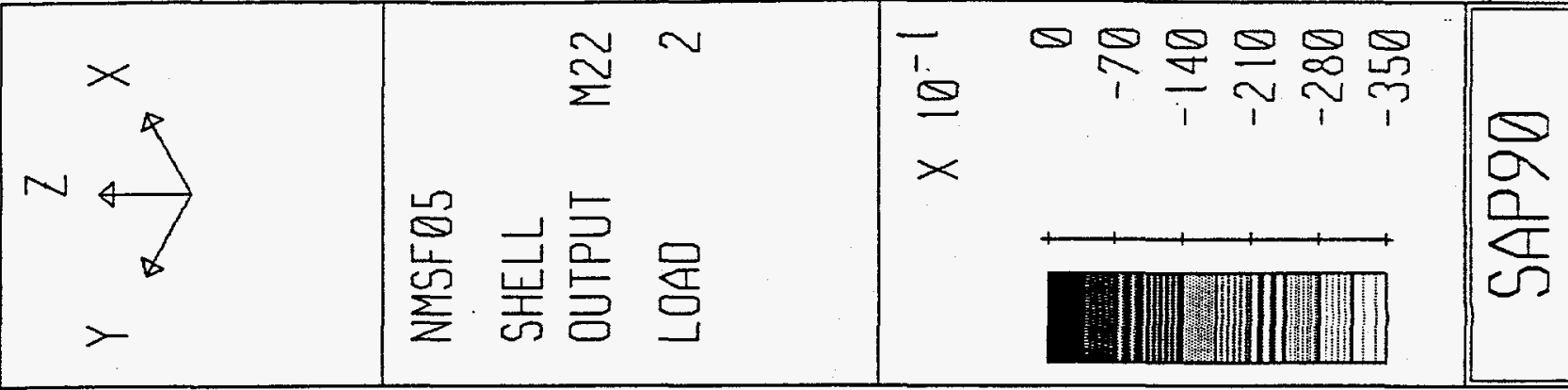

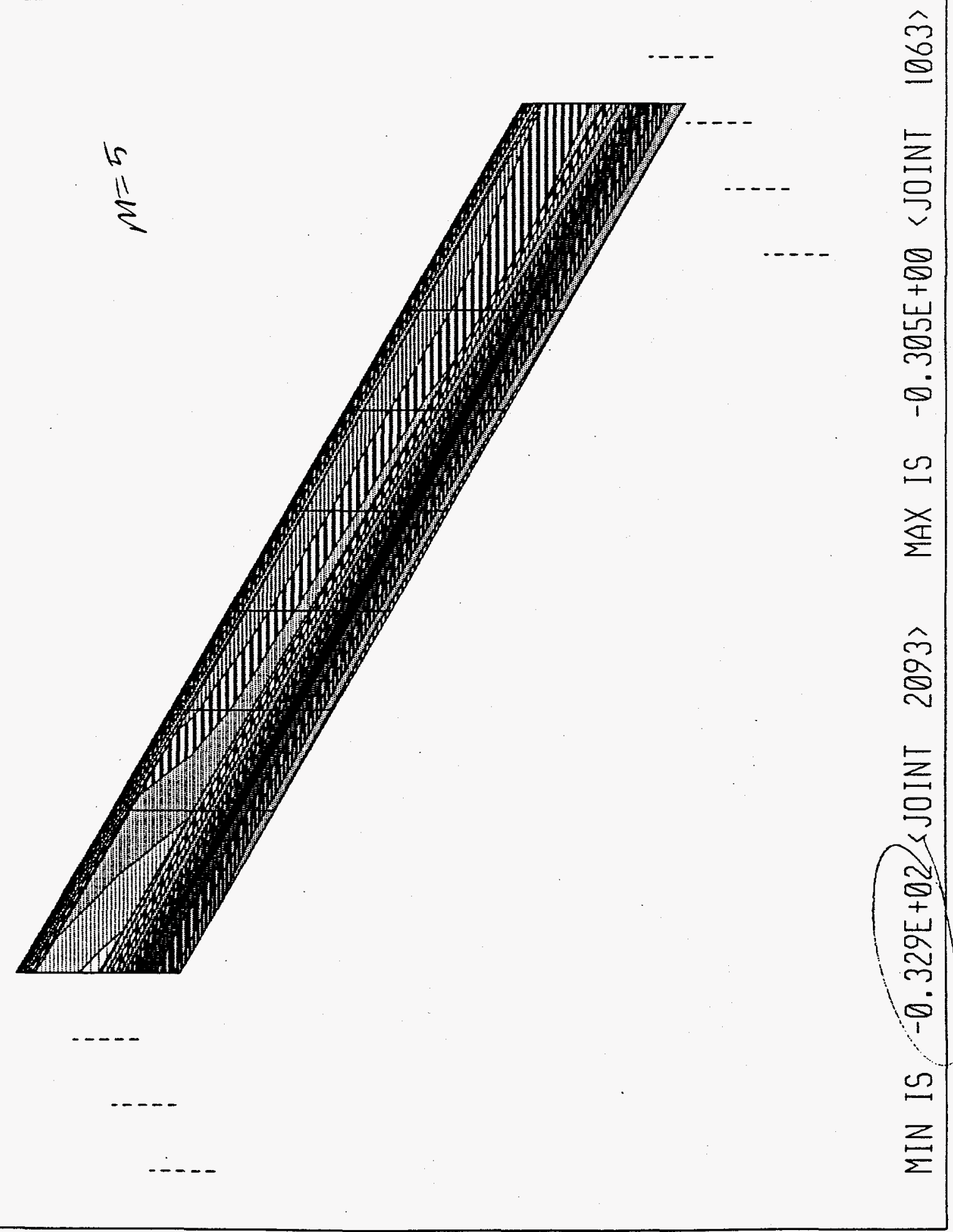


lale 4-0002, Res.0, Job 441552 Sht. 165 of

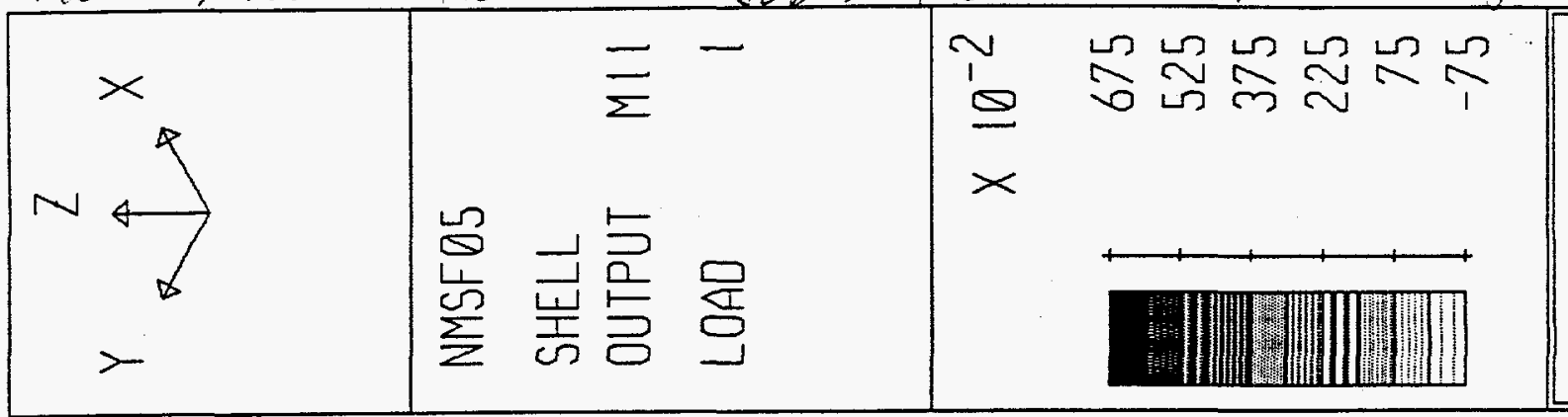

迹

n) $\frac{2}{2} \frac{1}{2} \frac{11}{2} \frac{11}{2}$

$\widehat{\text { ถิ }}$

$$
\text { (n) }
$$


Cale. S.0002, Rev.0, Tob *41552. Sint.166 of

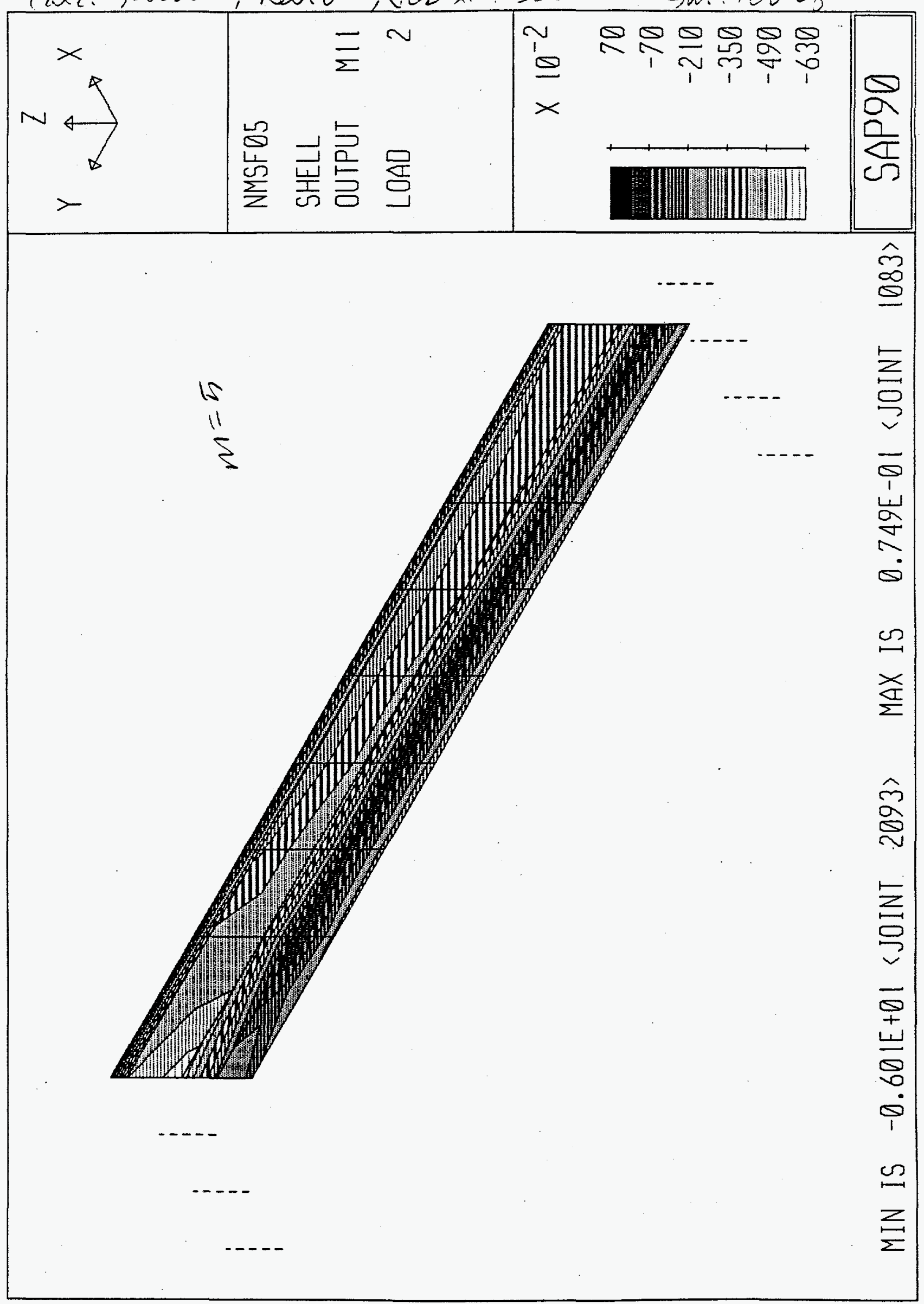




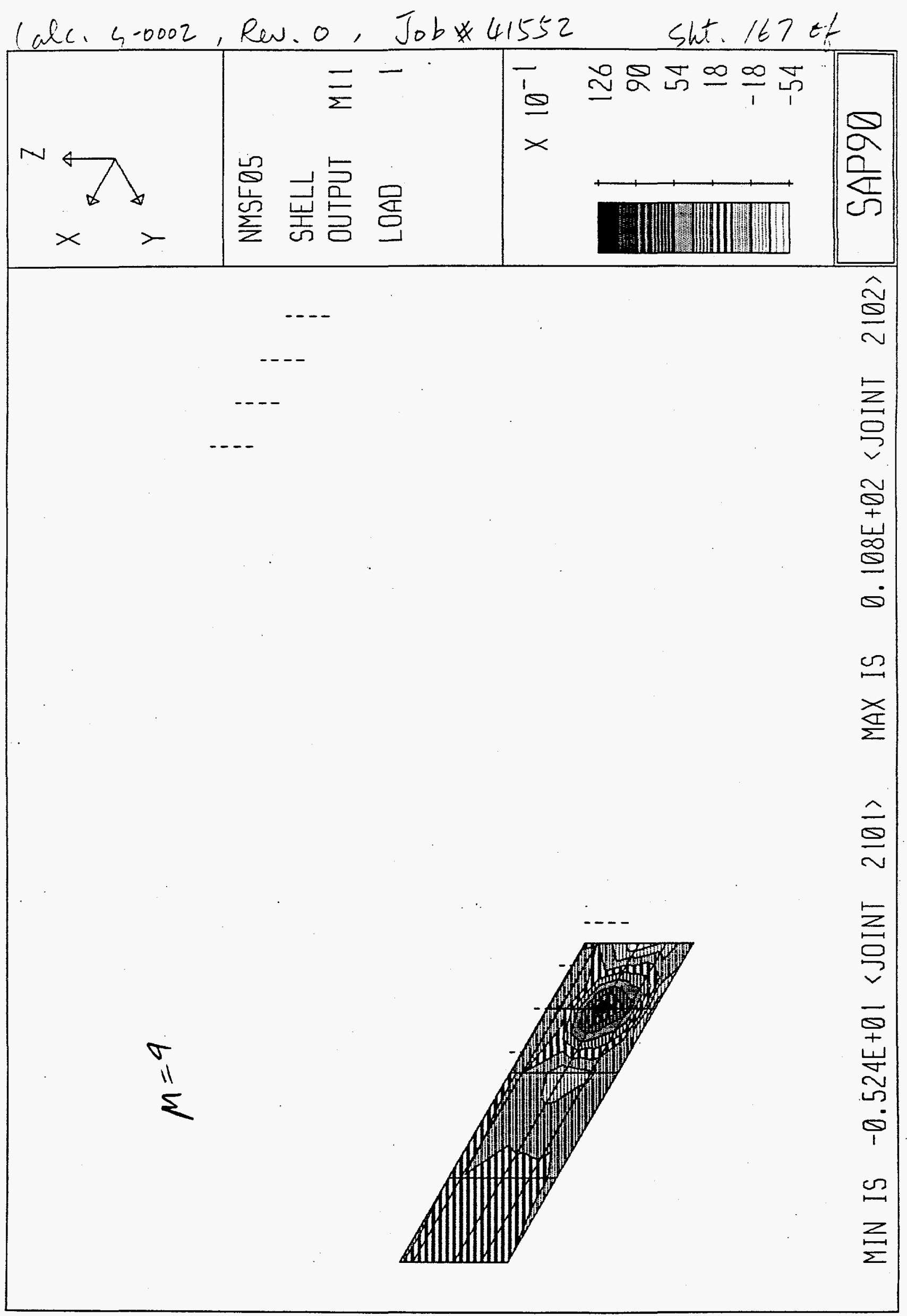




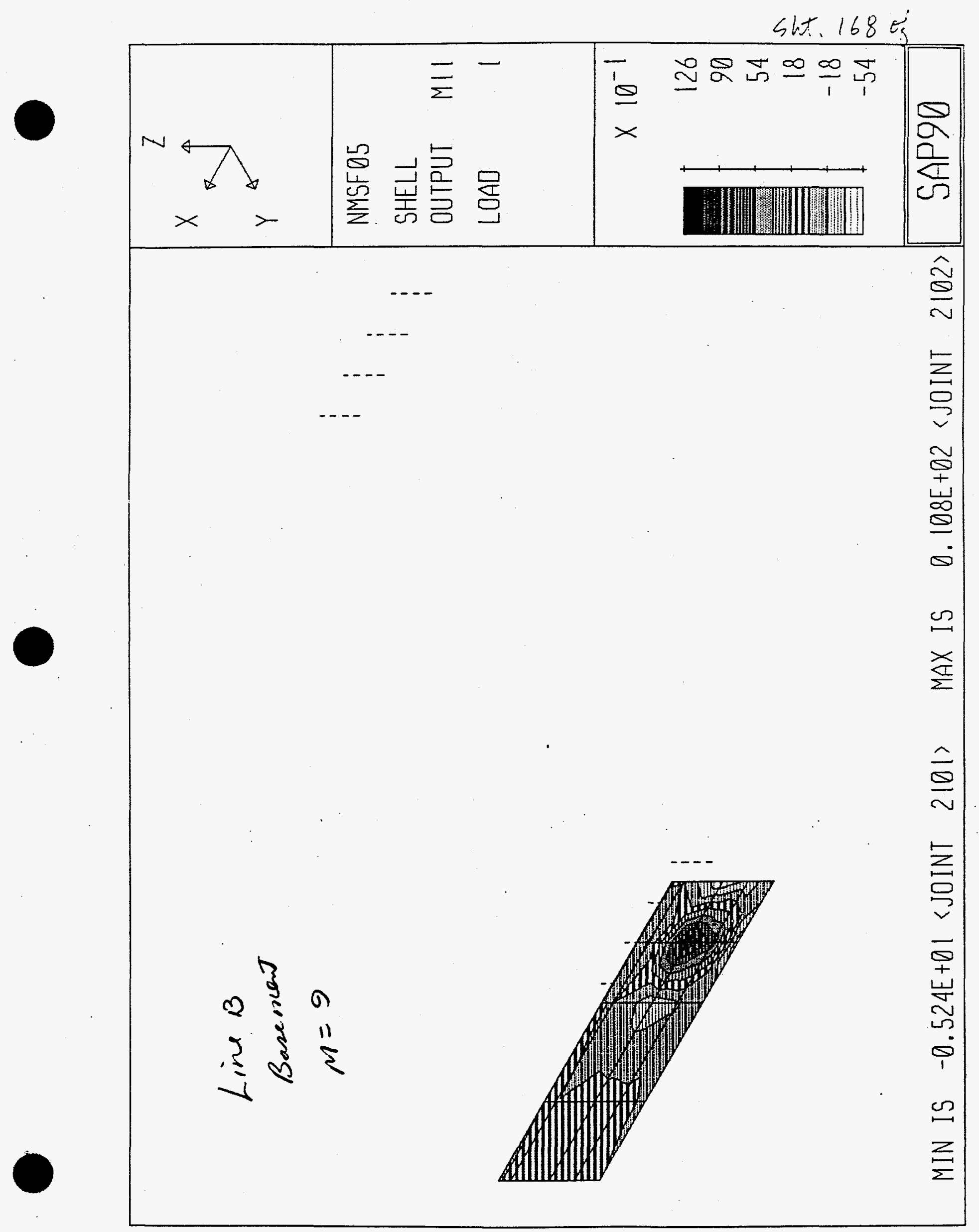




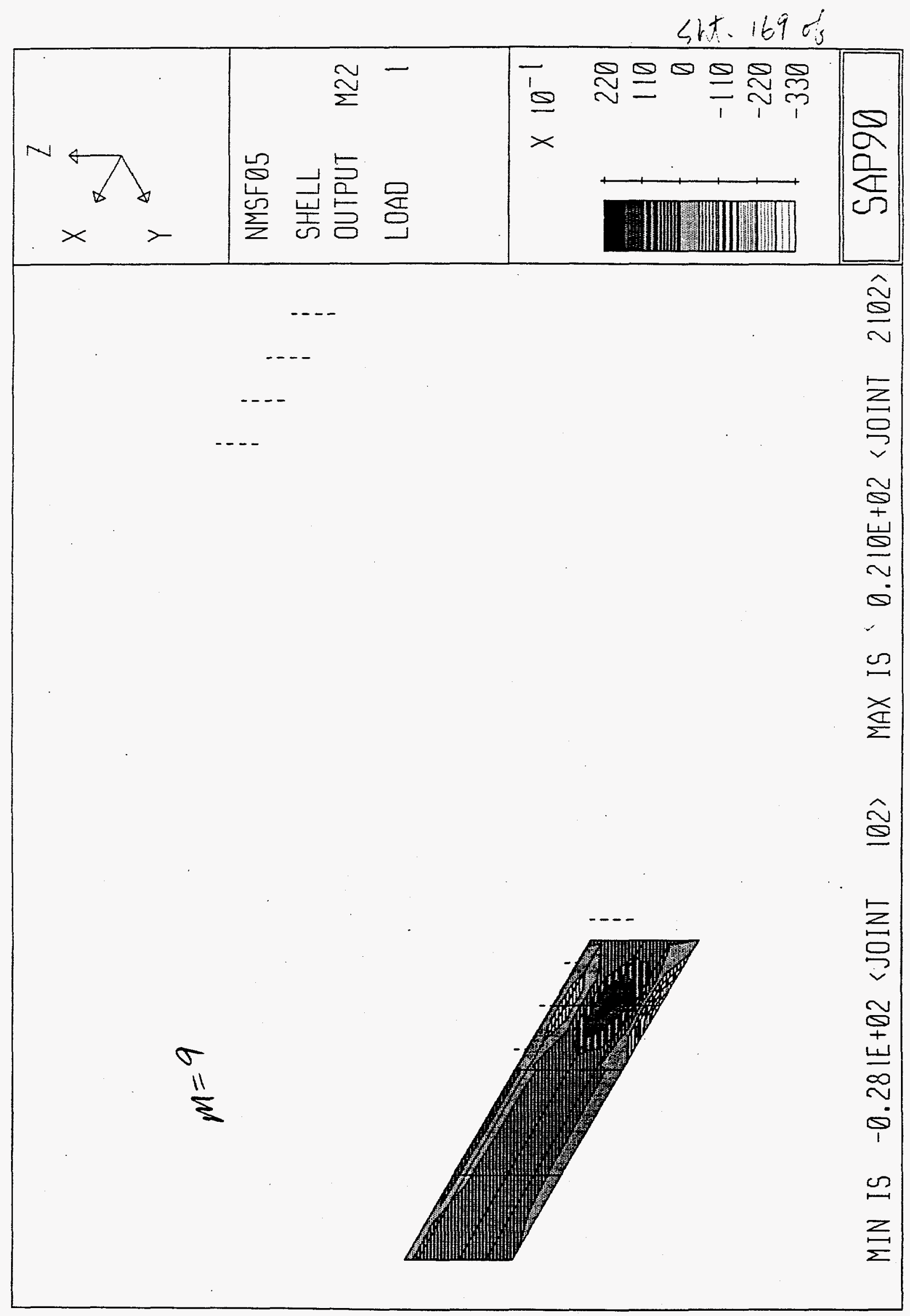




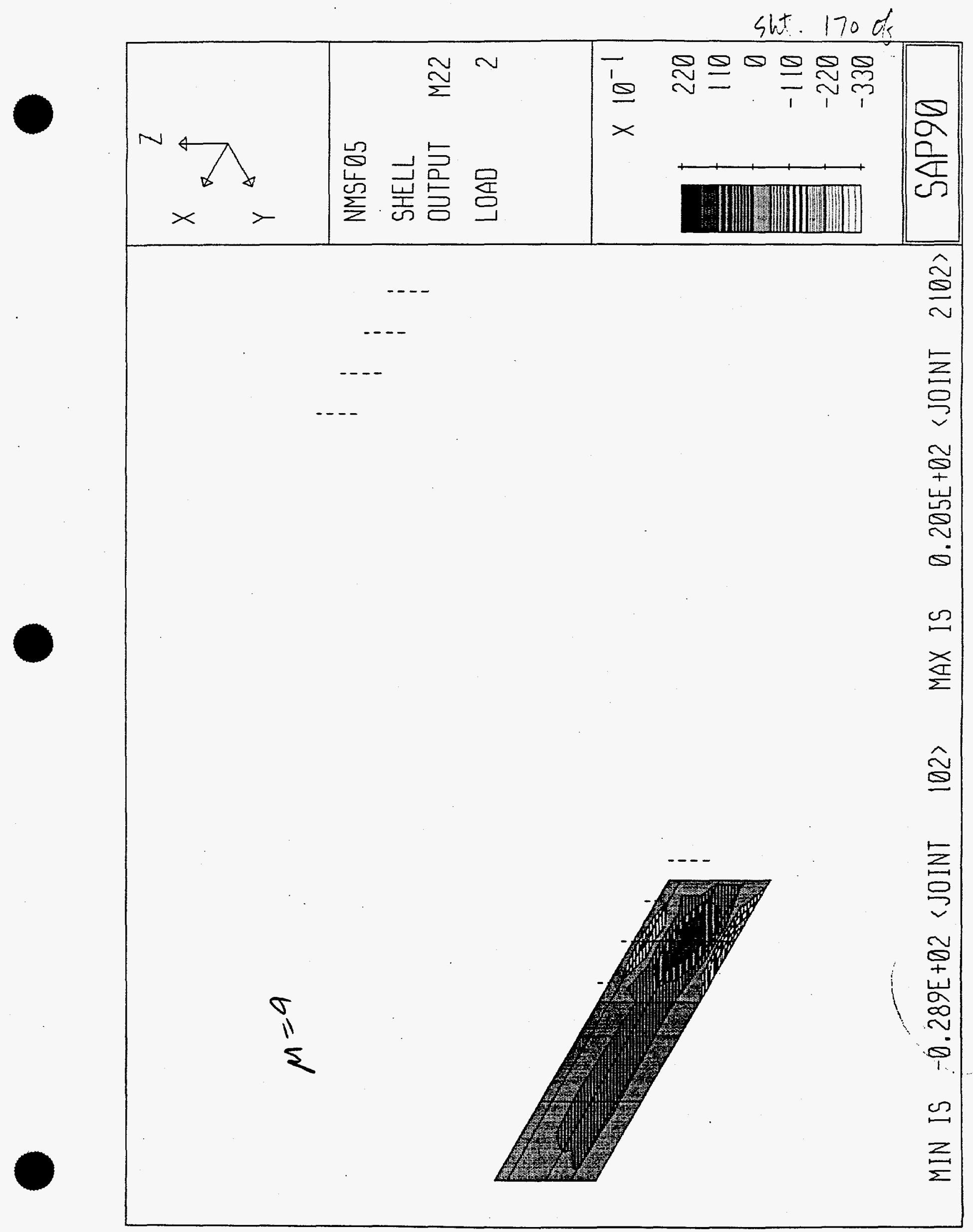




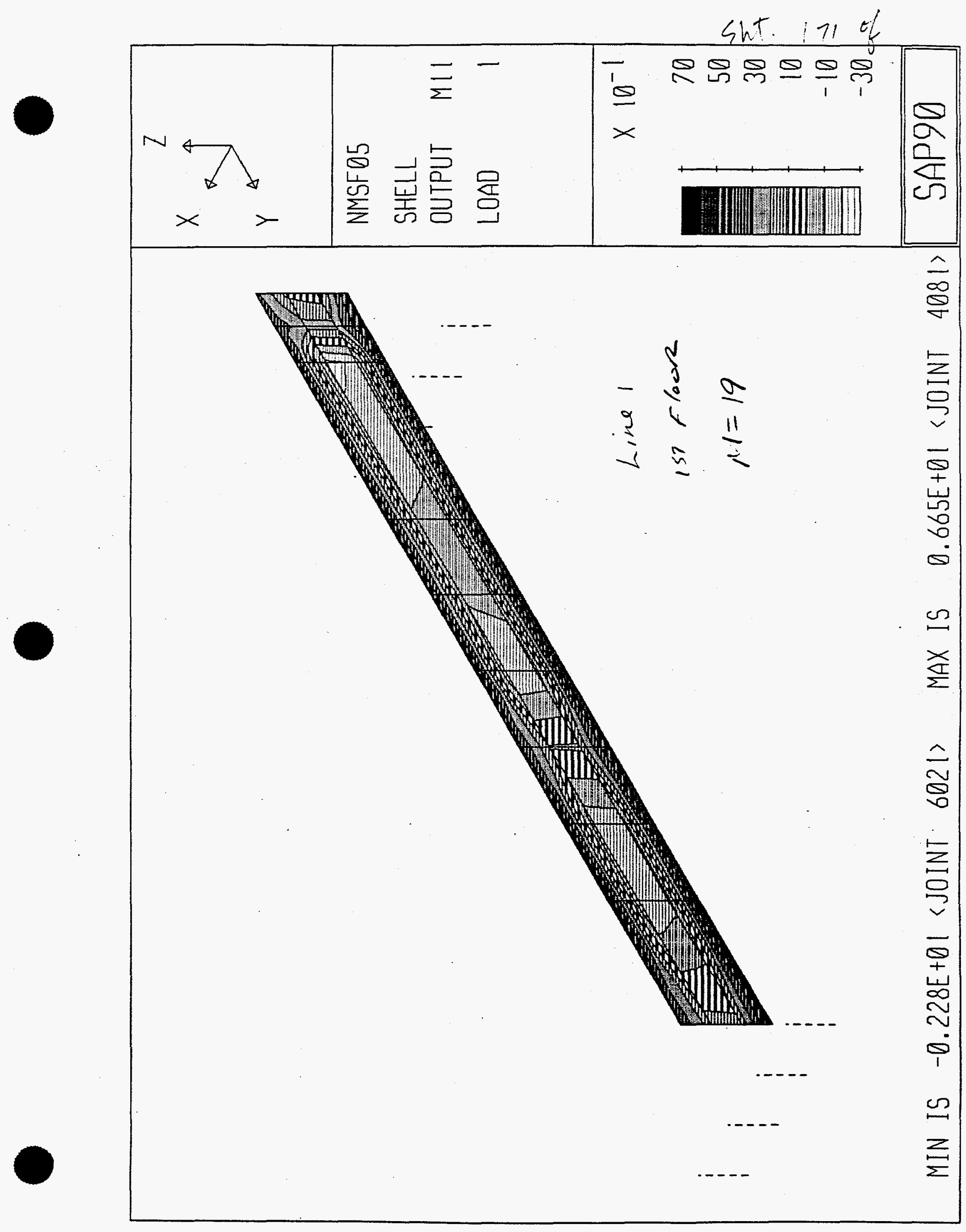




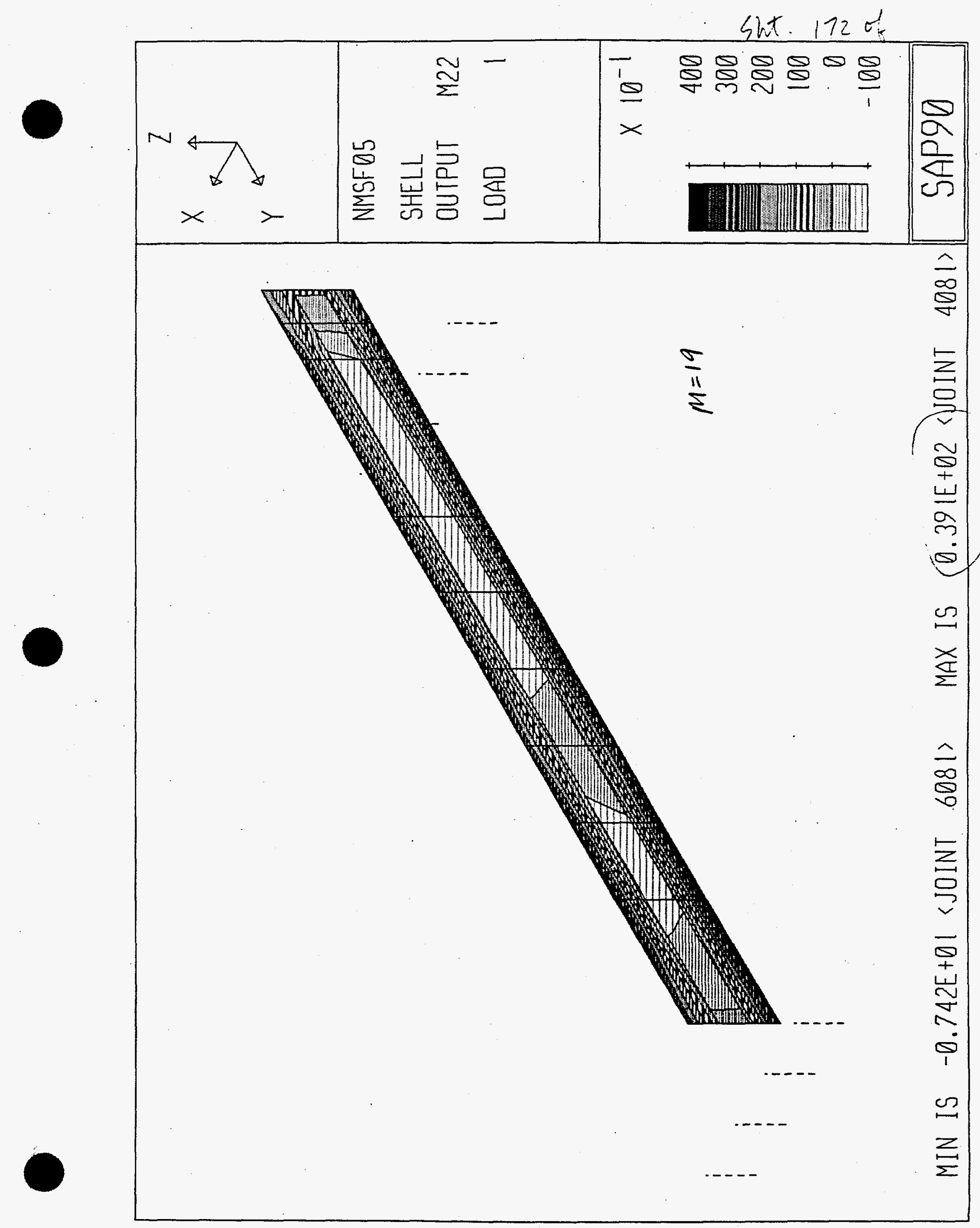




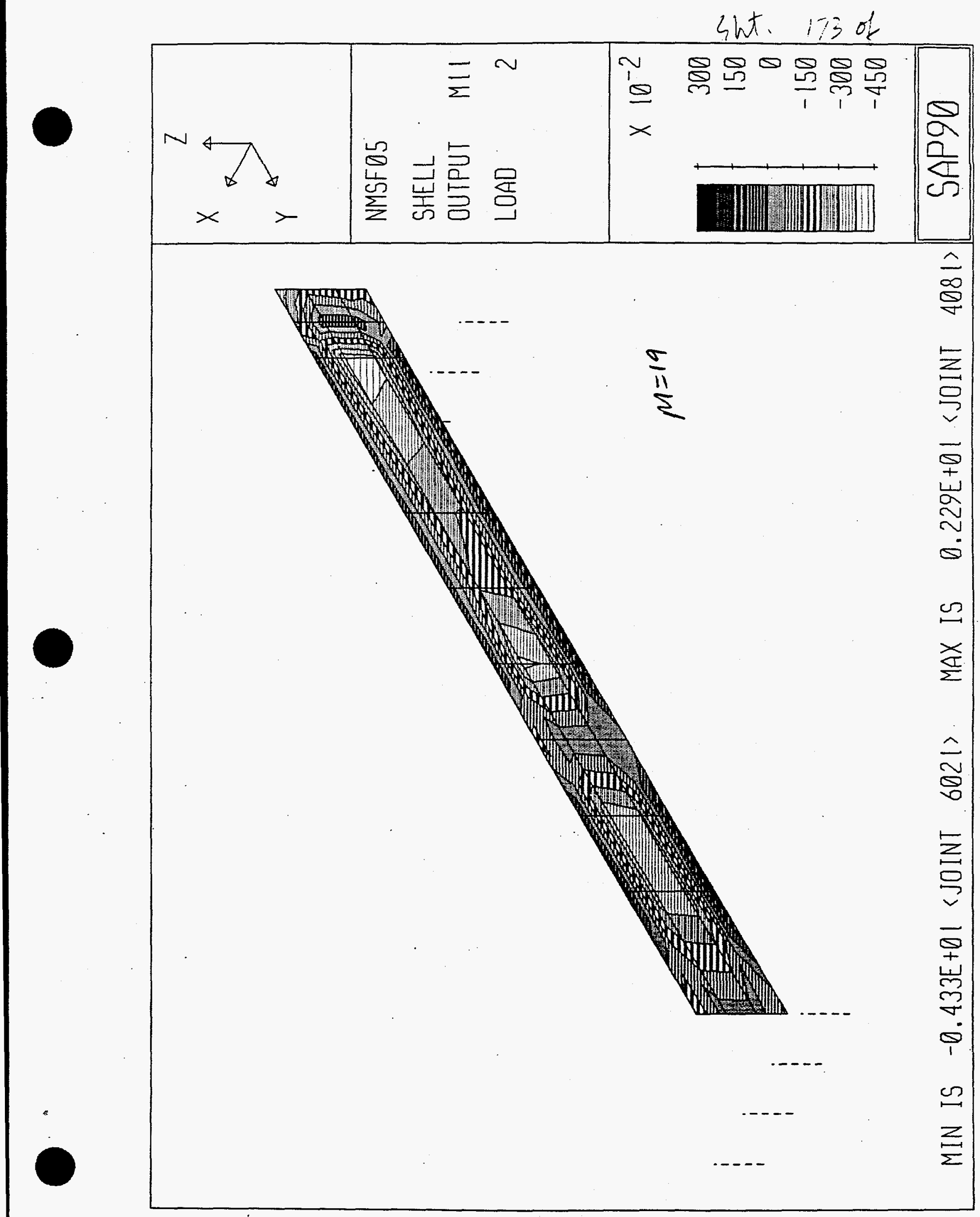




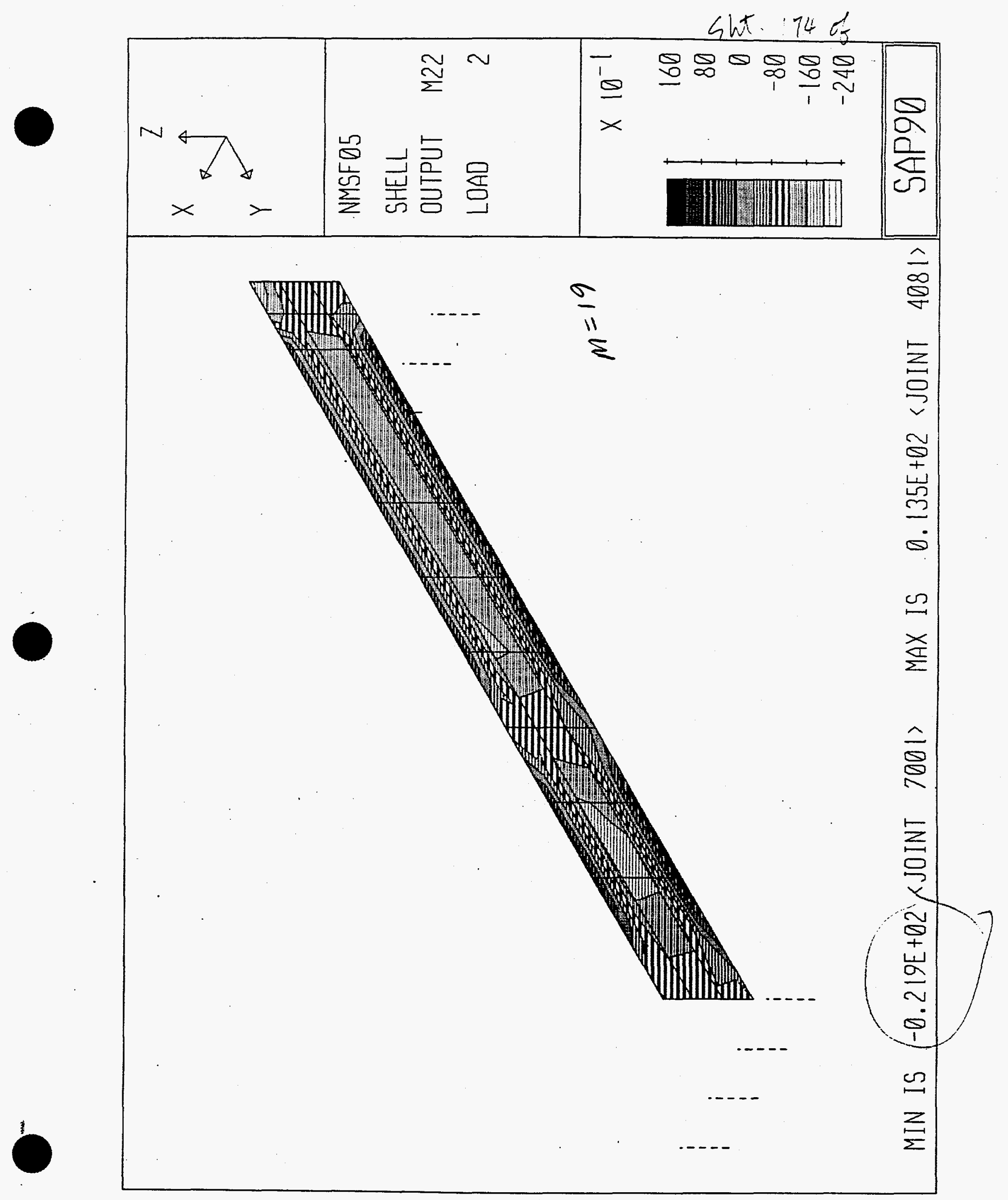




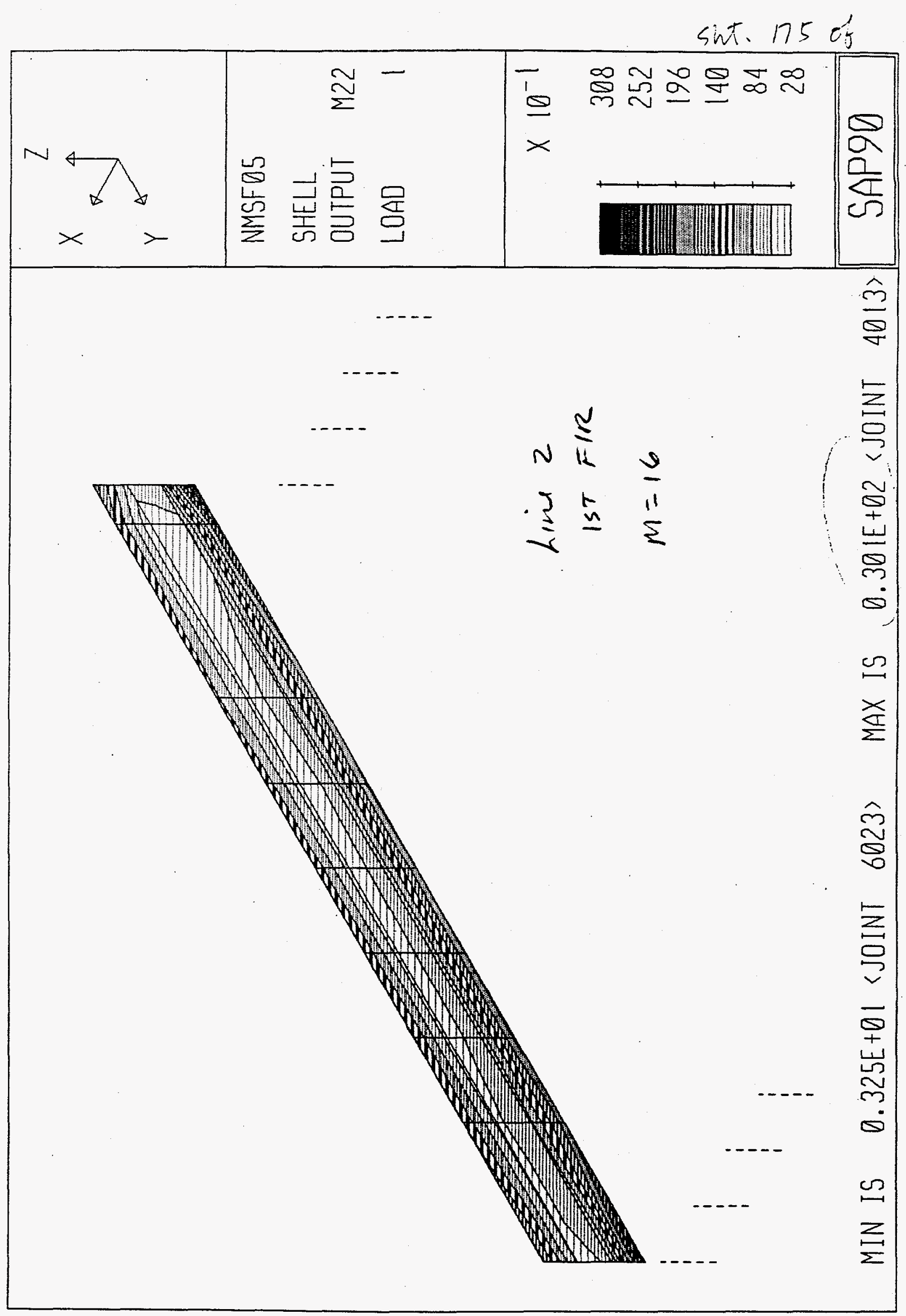




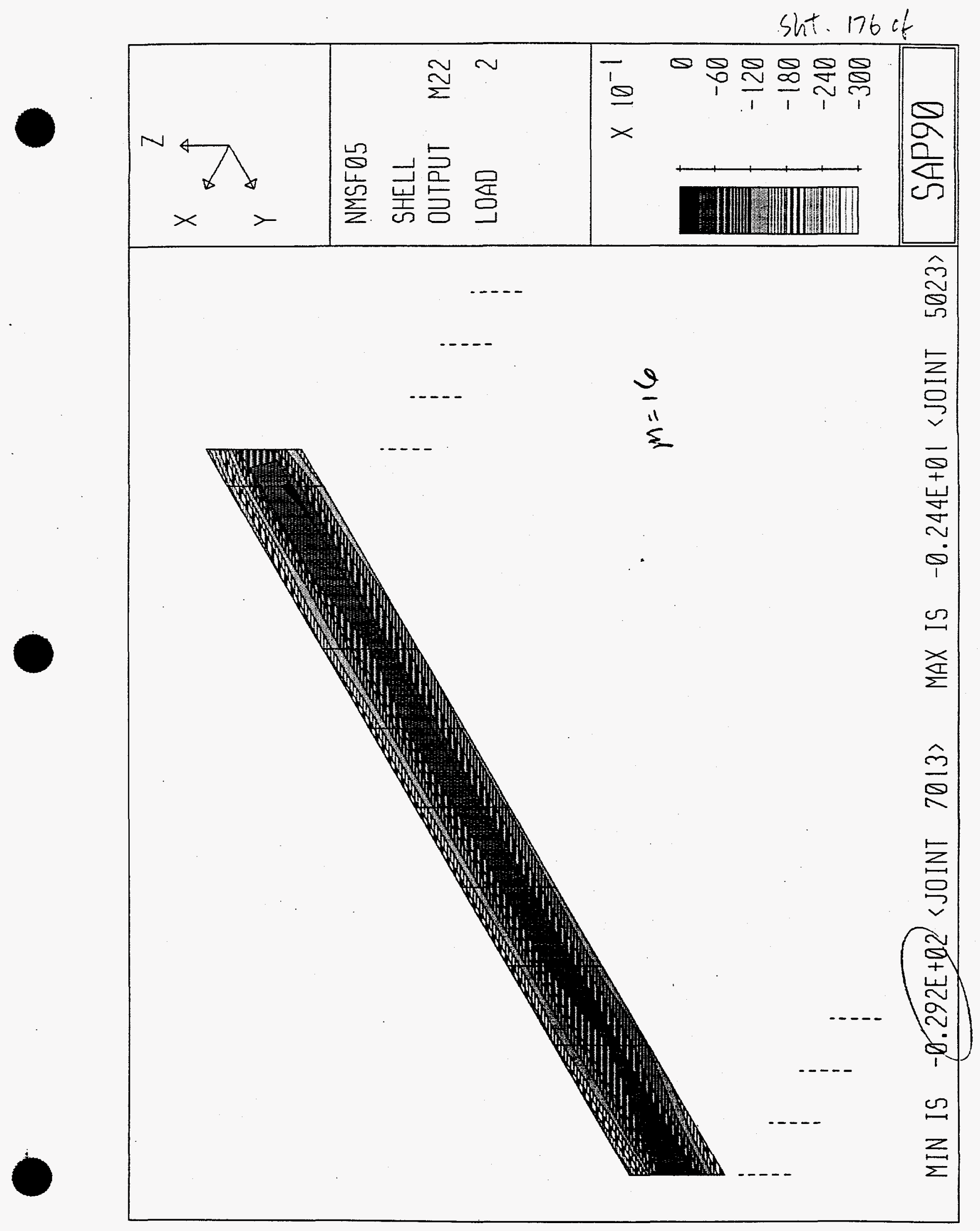




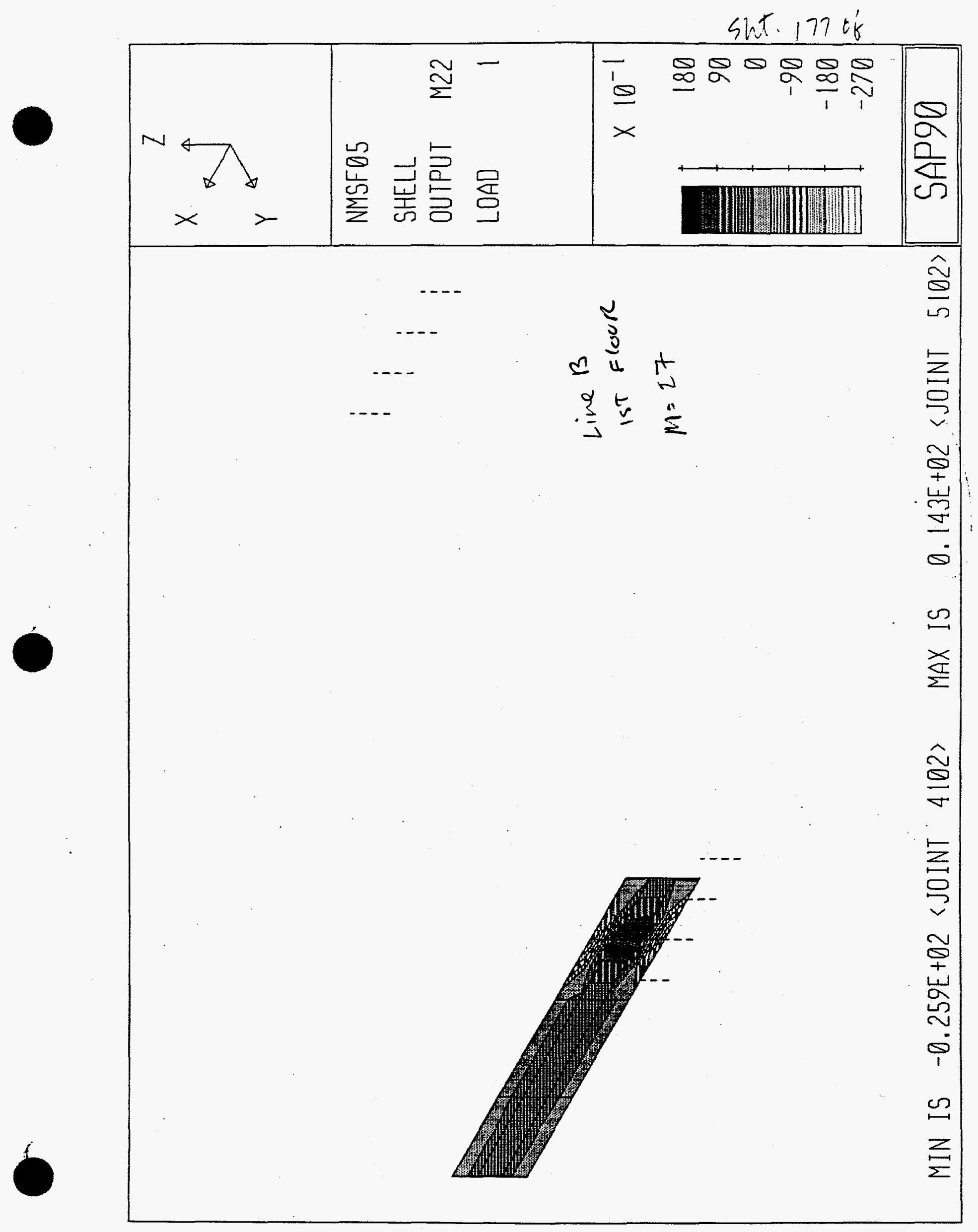




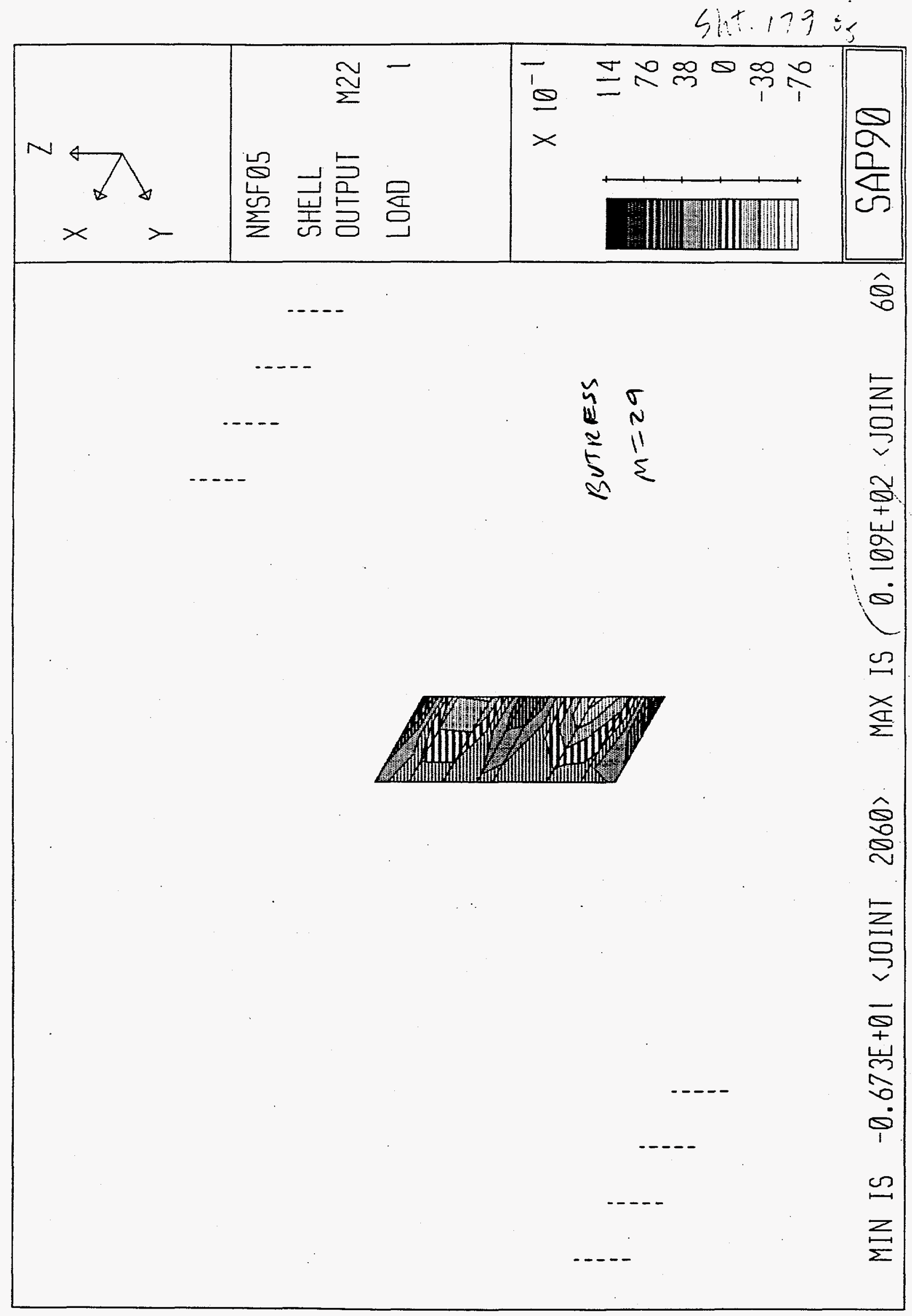


Sht 1800 o's

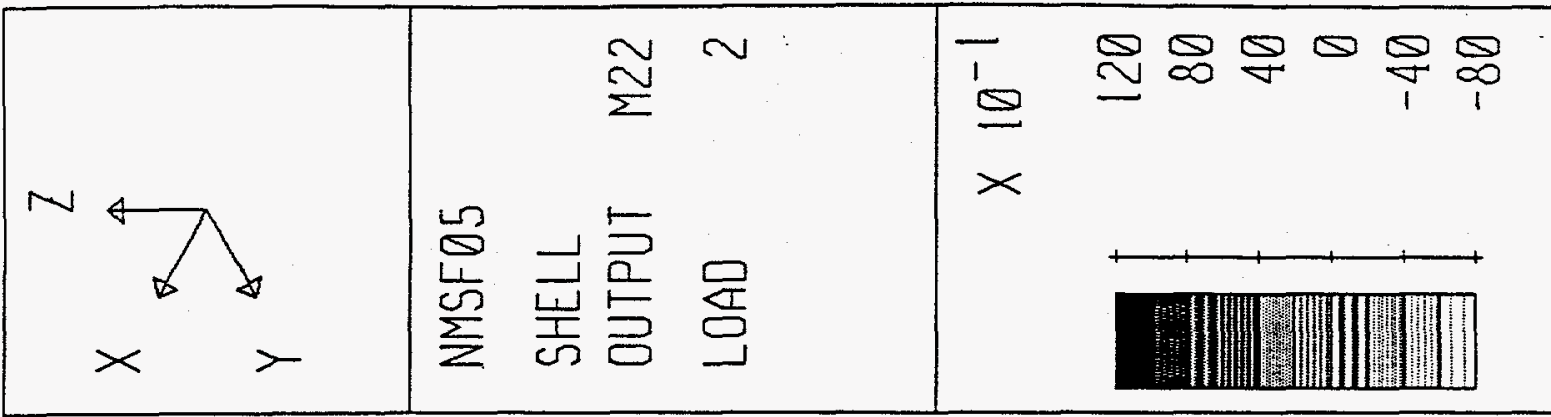

$\frac{D}{a}$

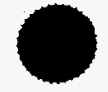

-..-

$\frac{\Omega}{2 \pi}$

$\widehat{\vartheta}$

$\frac{5}{11}$

$\ldots$

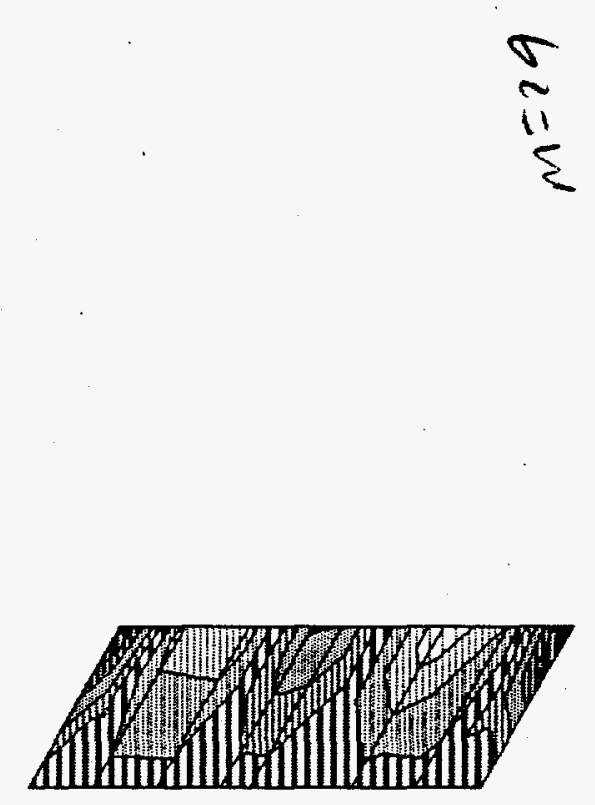

$\underset{z}{z}$

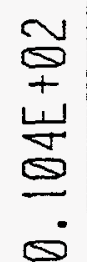

$\frac{X}{\Sigma}$

Allninim
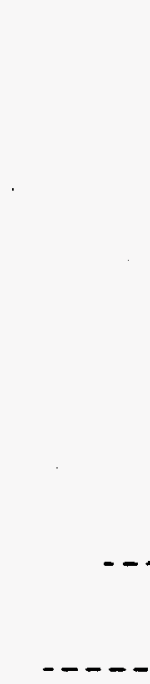


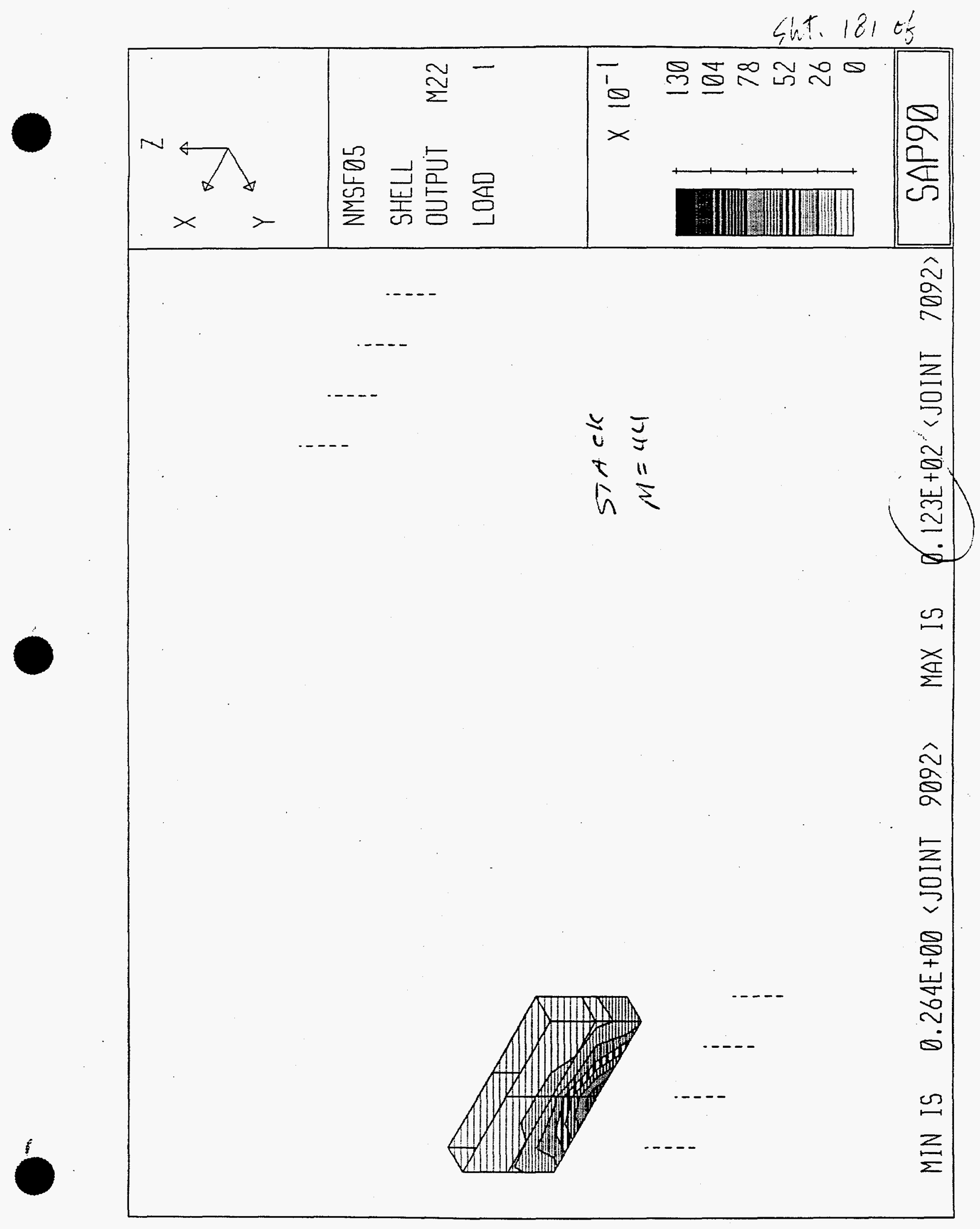


Sint. 182 of

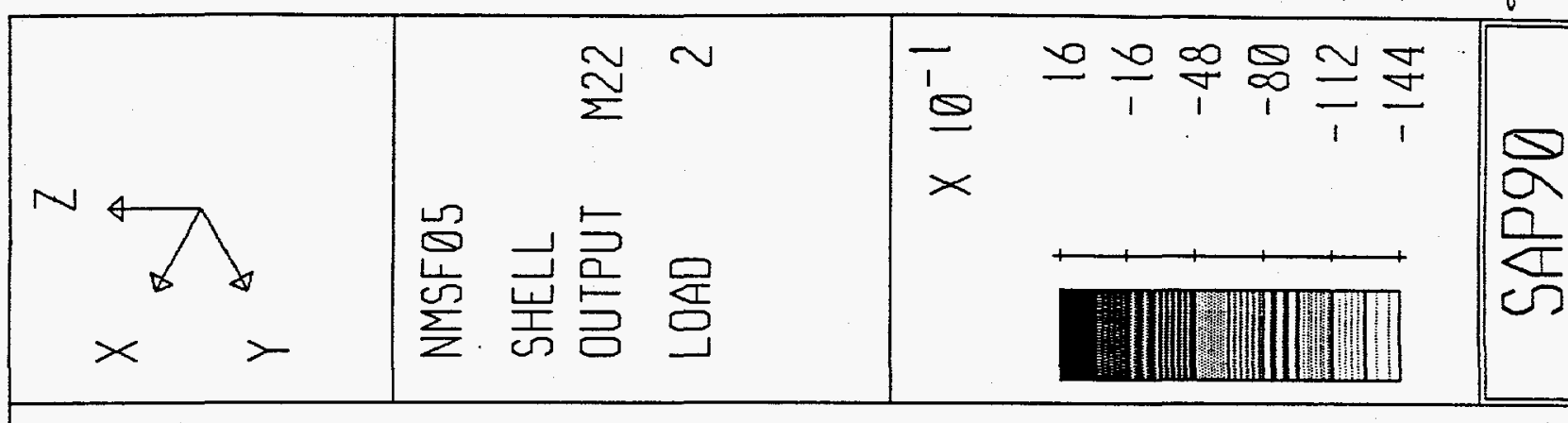

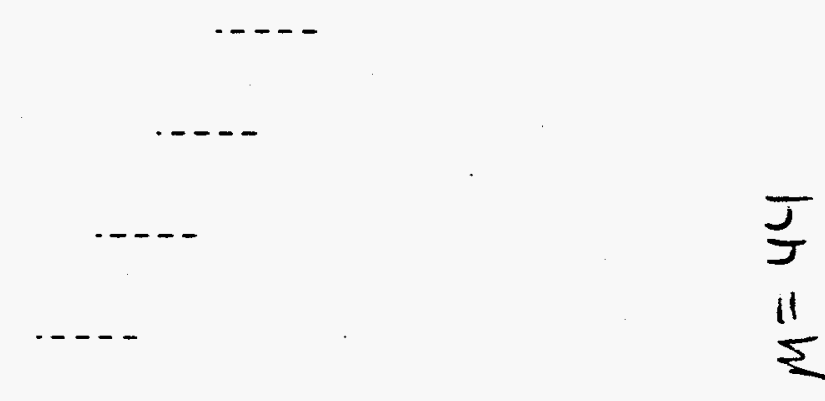

$\Sigma$

$\widehat{\infty}$

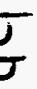

$\underset{\Xi}{\Xi}$

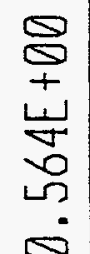

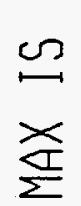

$\widehat{\vartheta}$

E
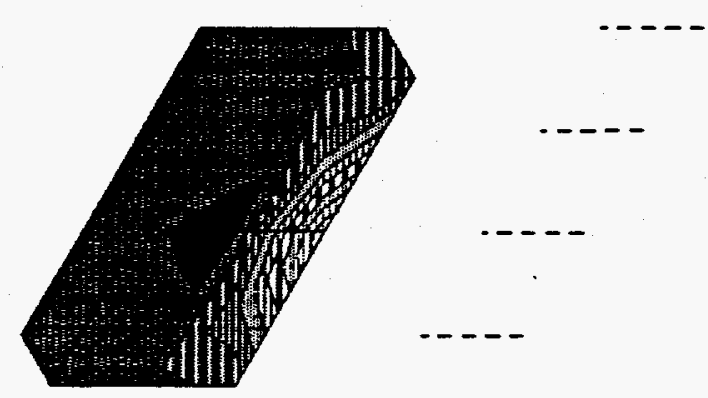

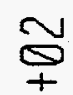

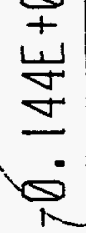

o

$\sum$ 
Sht. 18: $=$

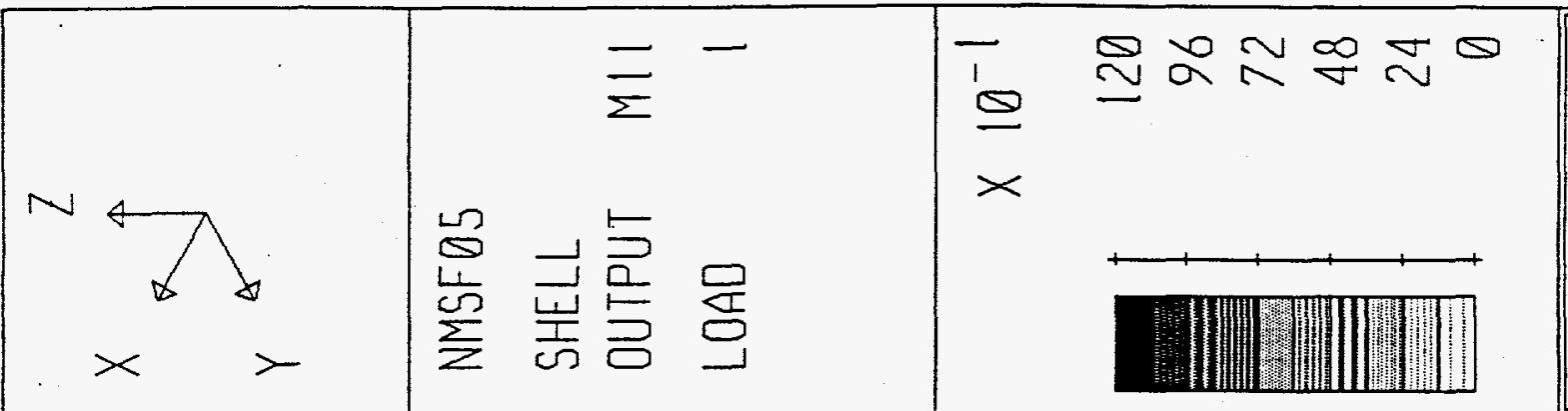

$\frac{\pi}{2 \pi}$

$\hat{\sigma}$

-..-

3

1

$\ldots$

亯

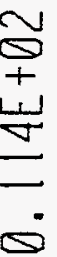

ת

$\frac{x}{\Sigma}$

ले

$\underline{\nabla}$

点

8

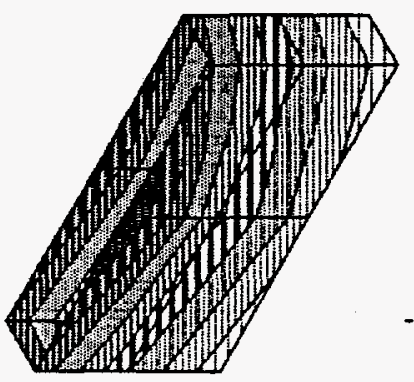

ए

$\Delta$

$\stackrel{n}{z}$ 
$\sin 184 \%$

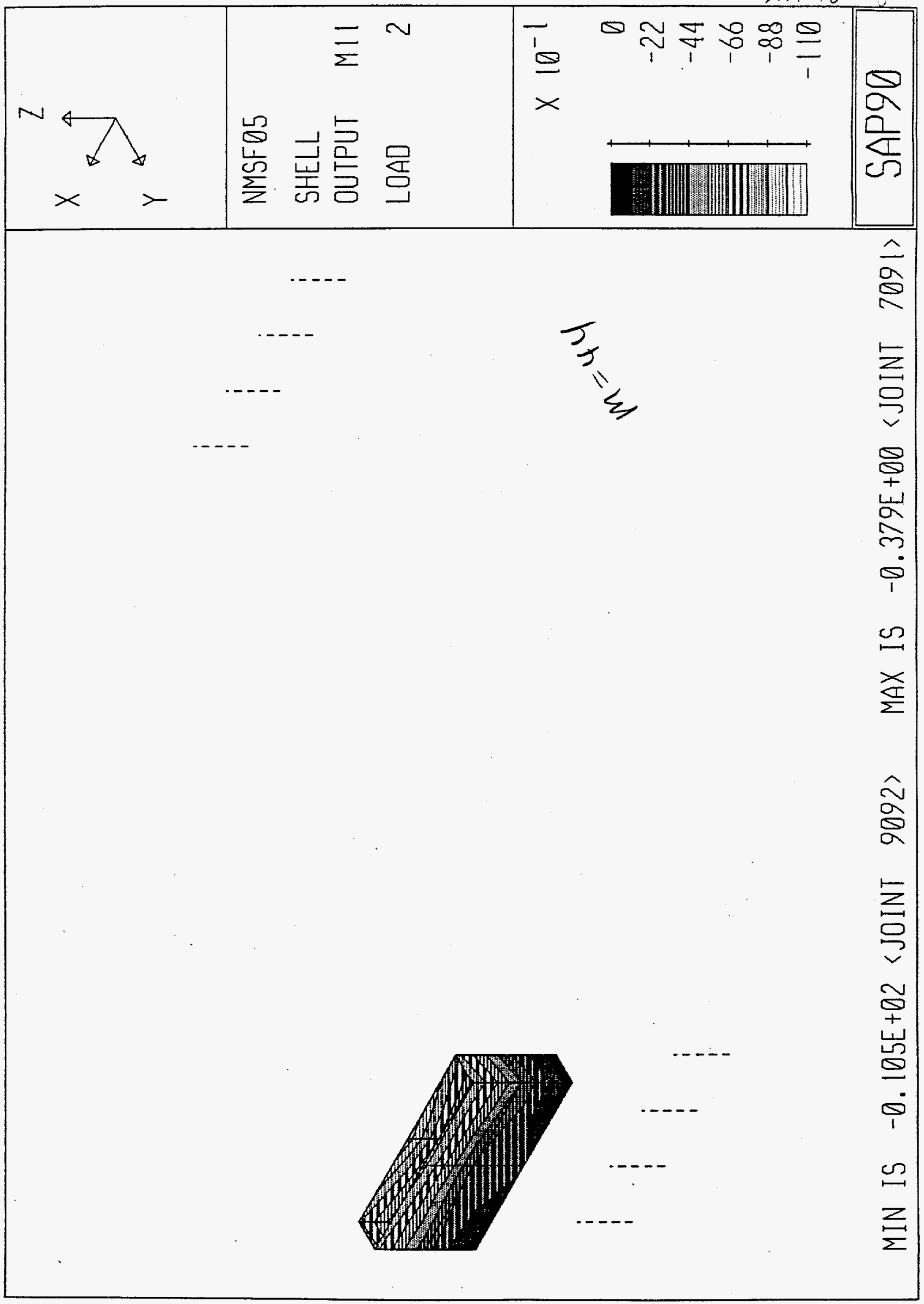




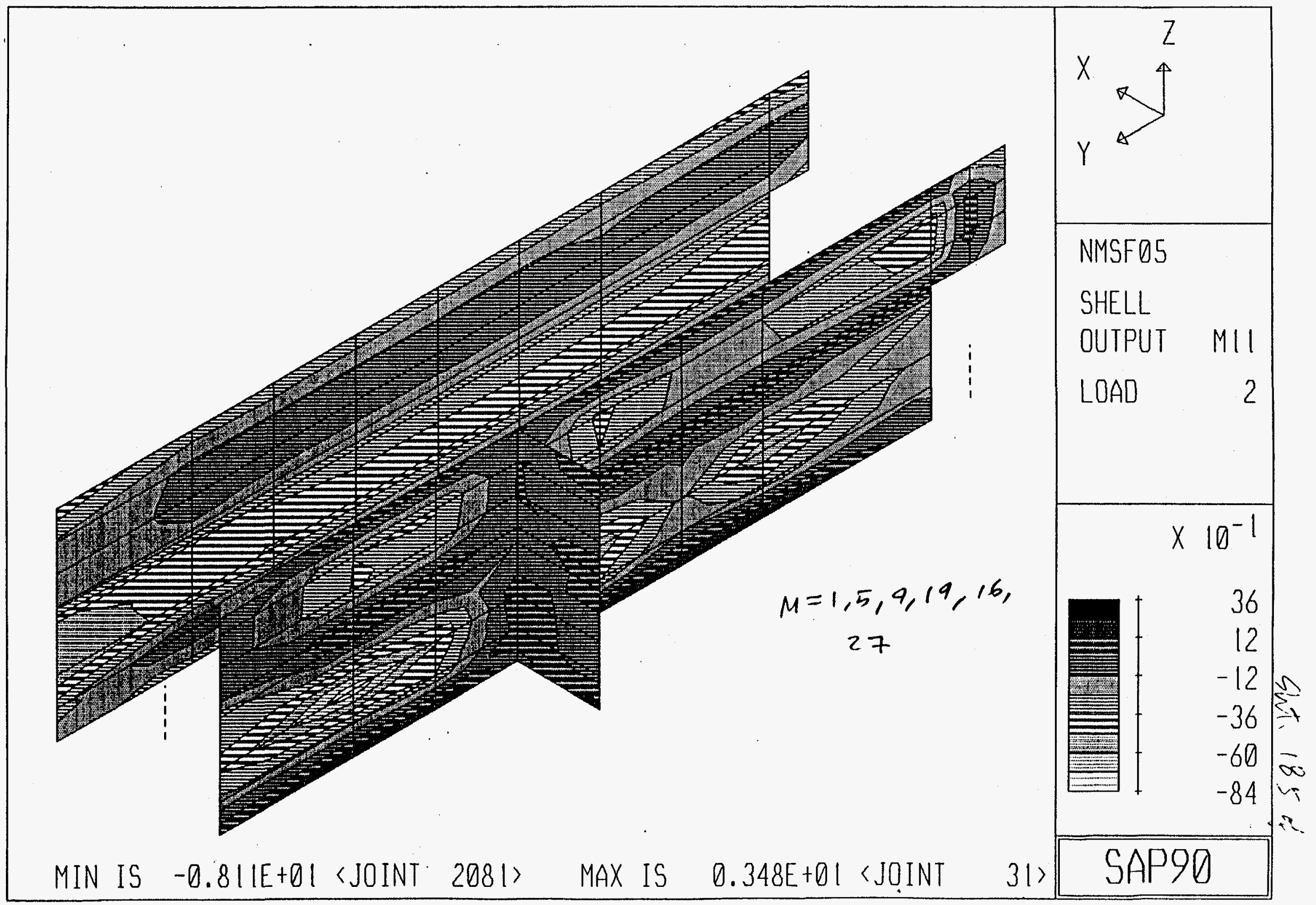




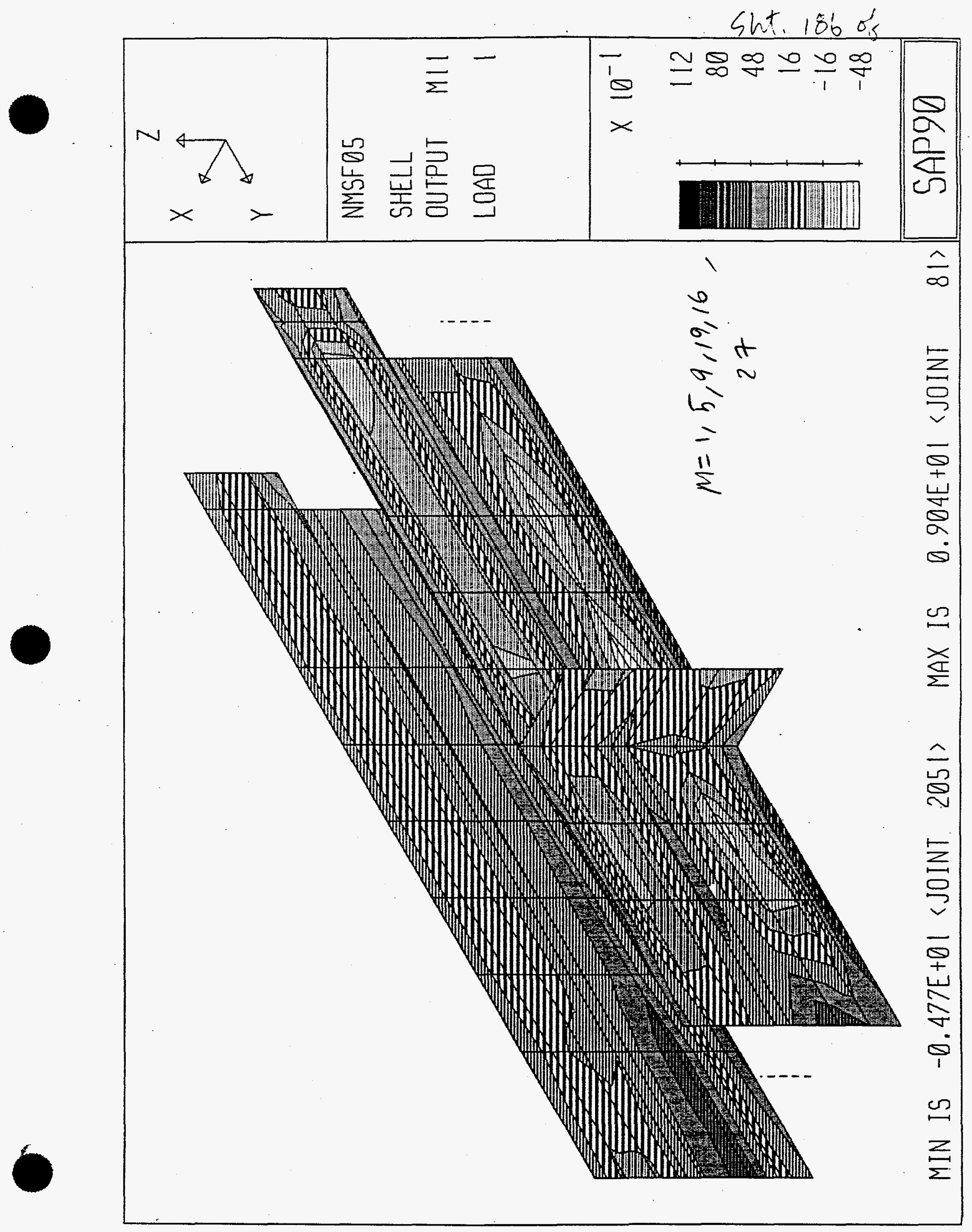




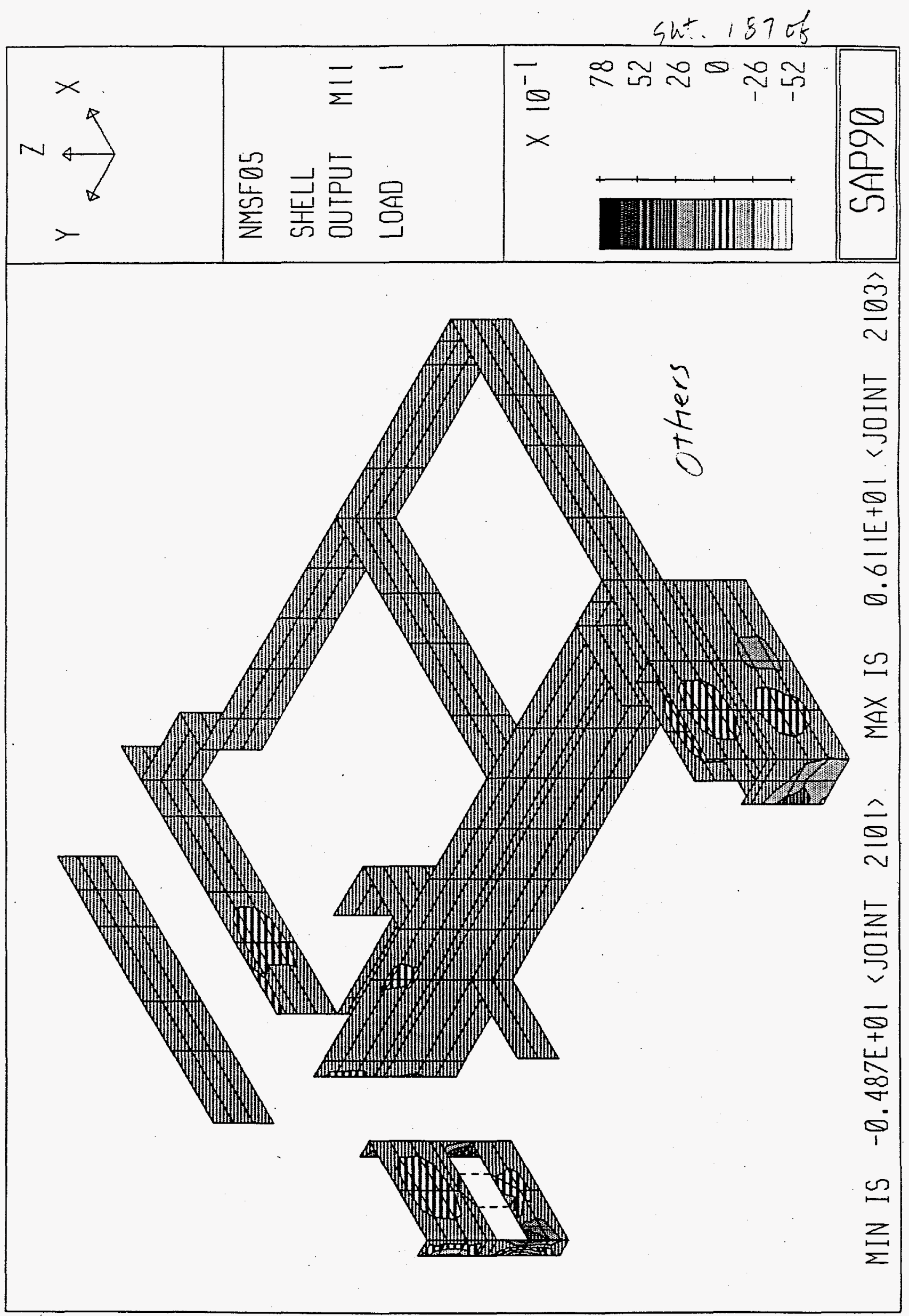


Sht $188 \%$

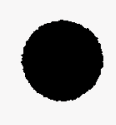

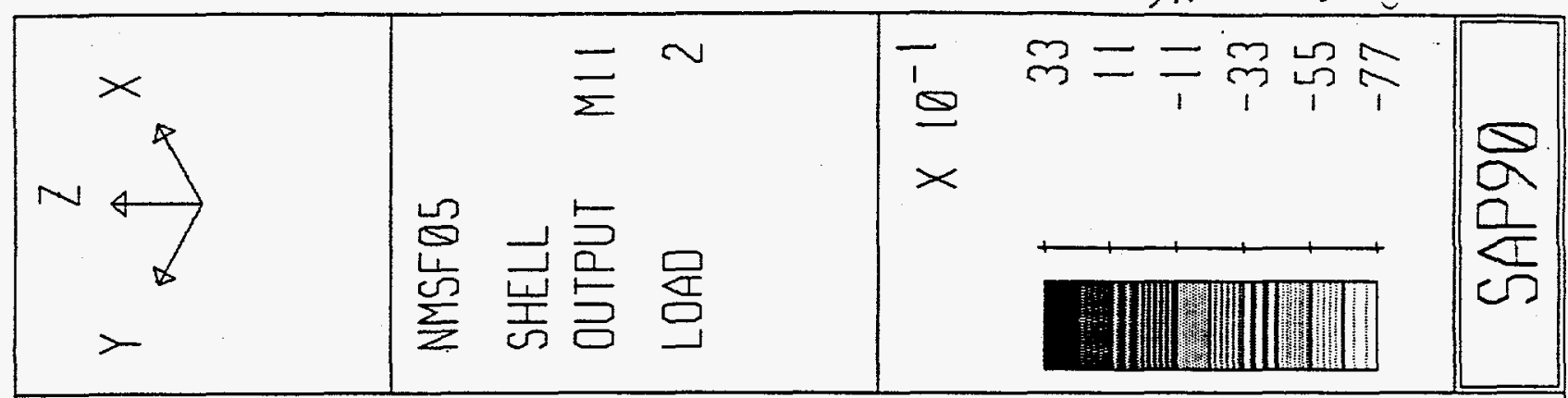

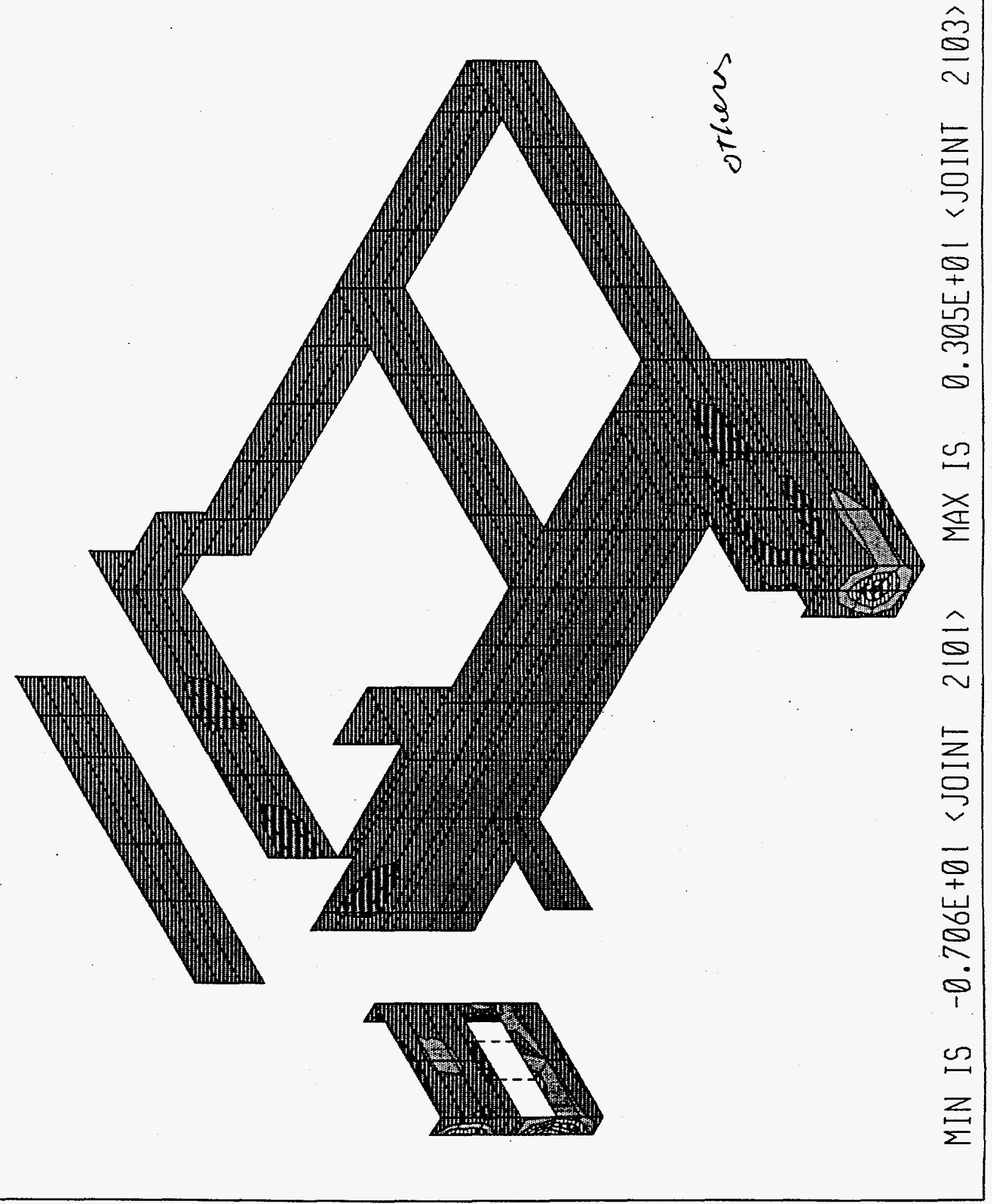




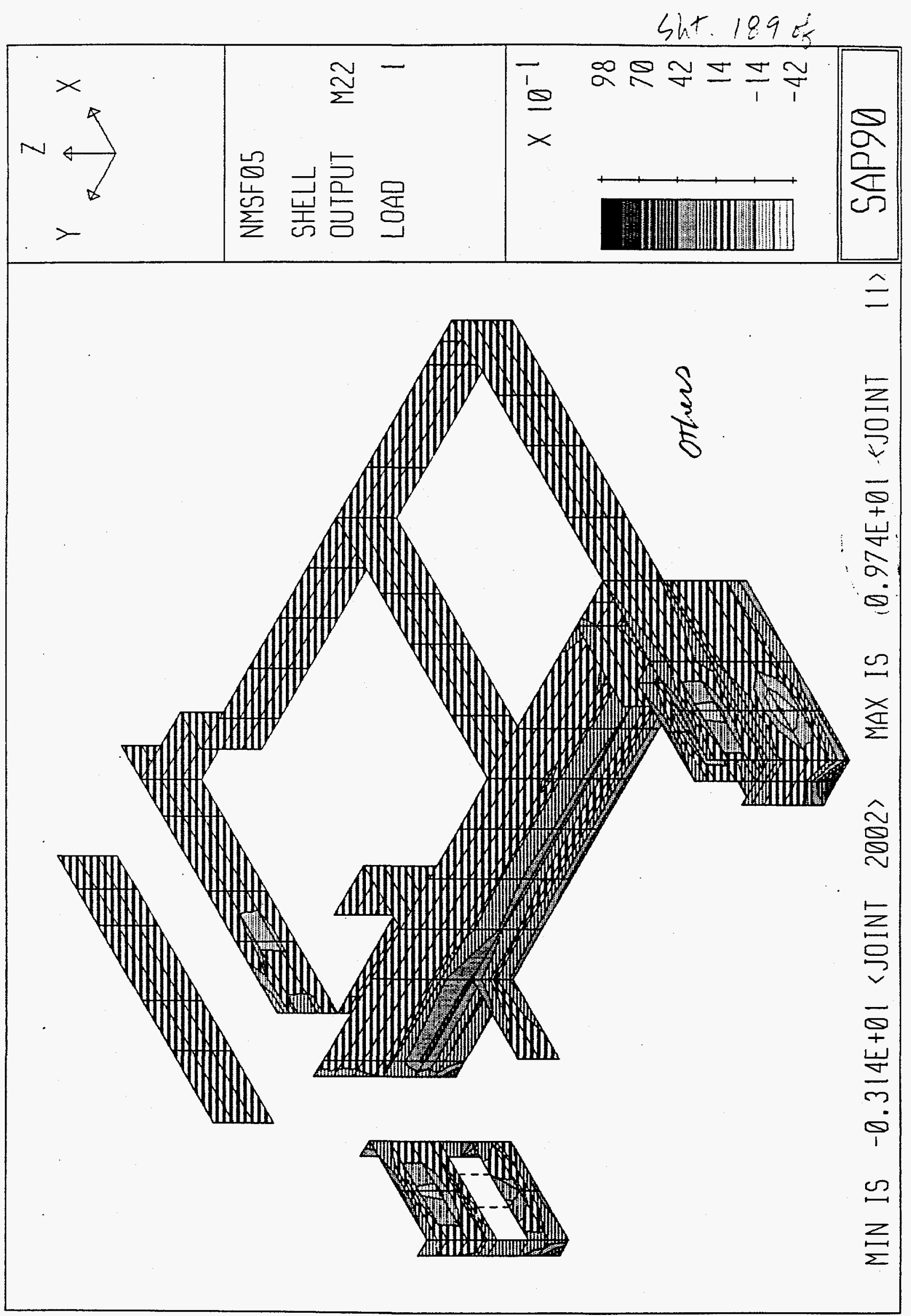




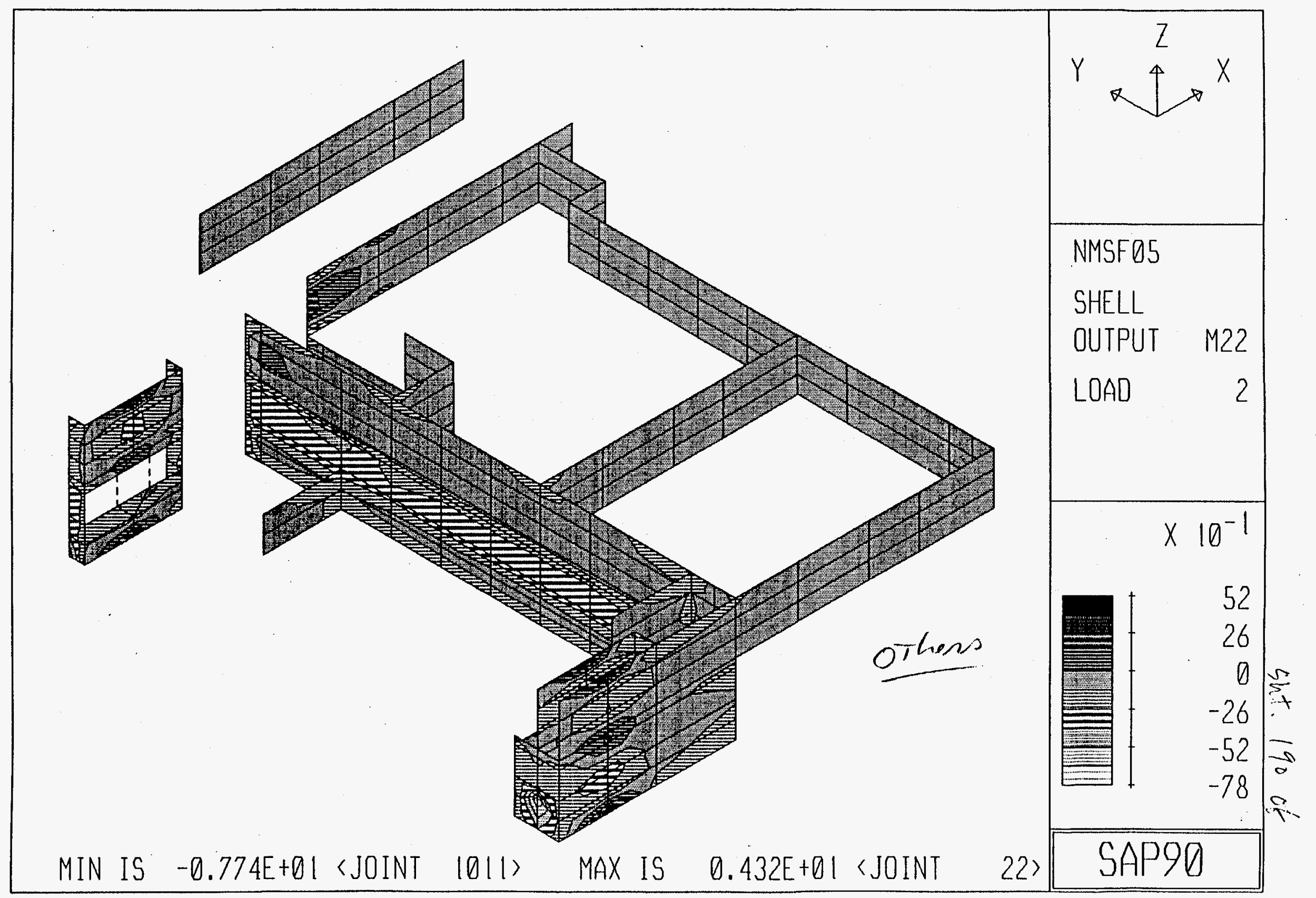




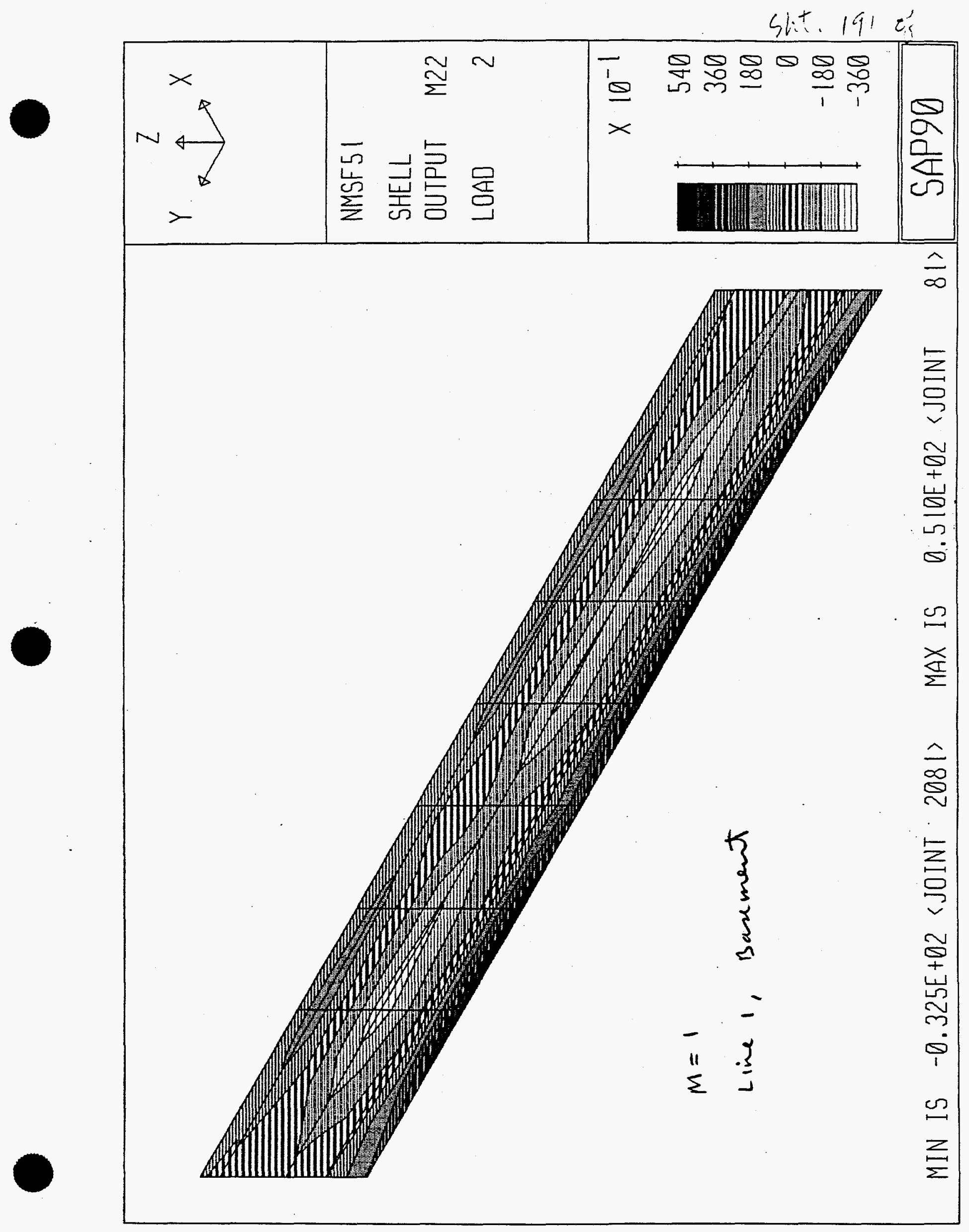



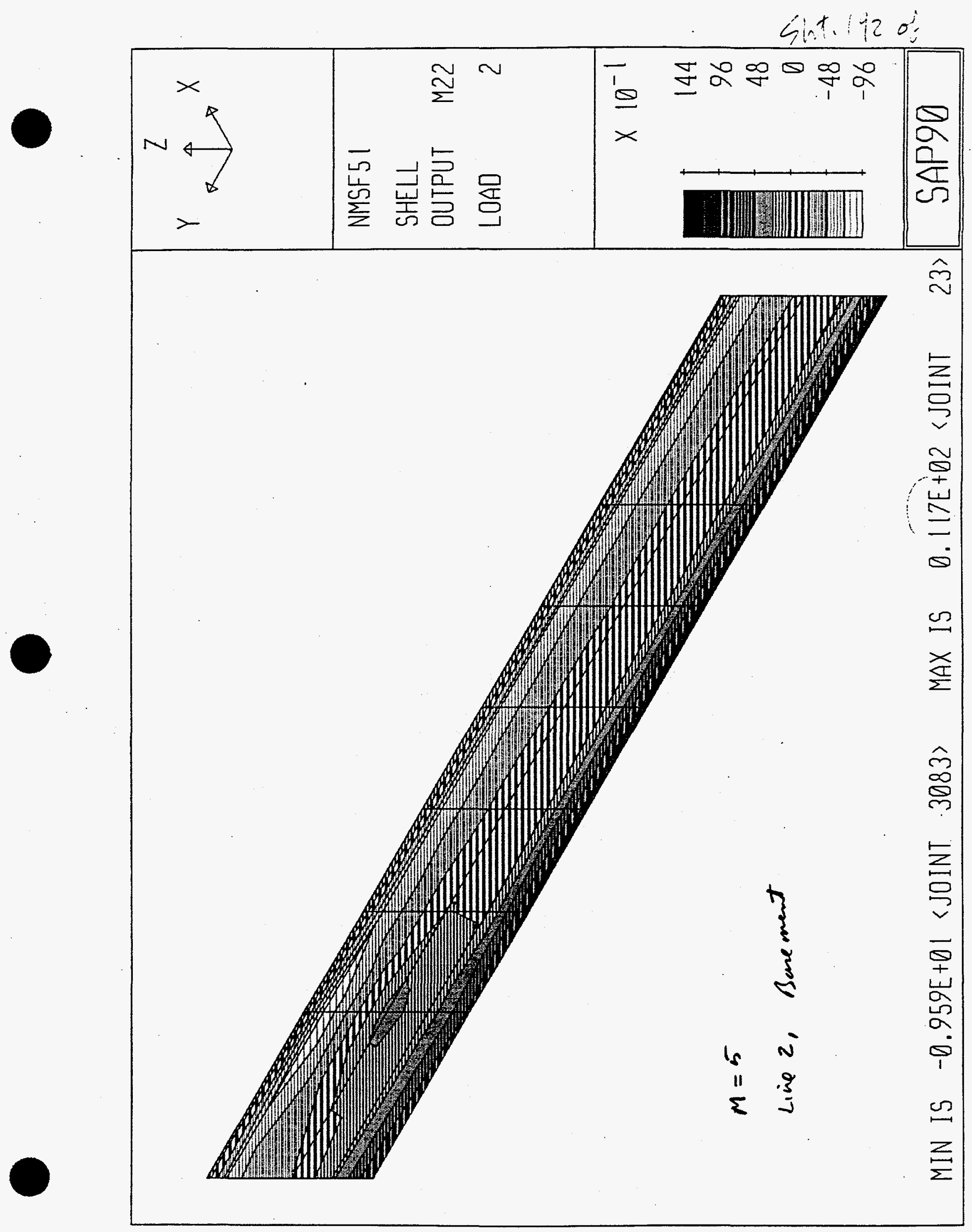
Sint. 1938

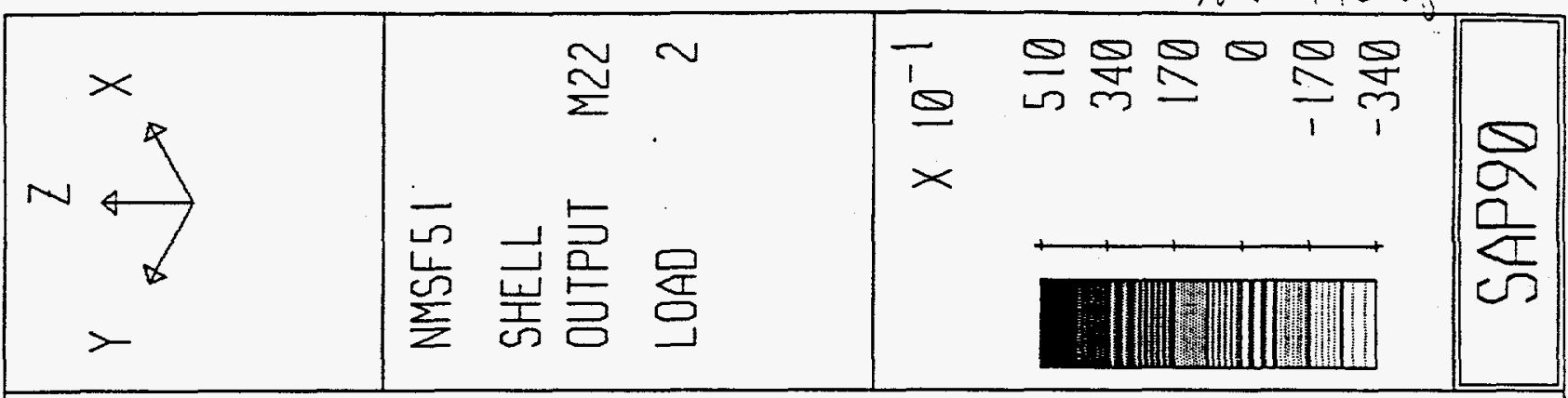
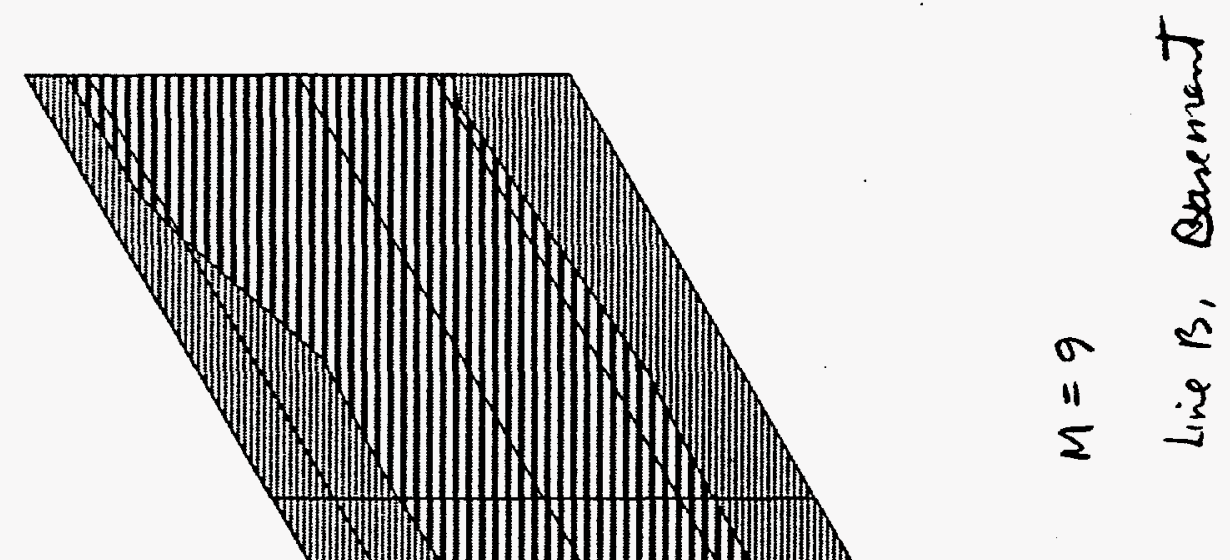

$\widehat{\otimes}$
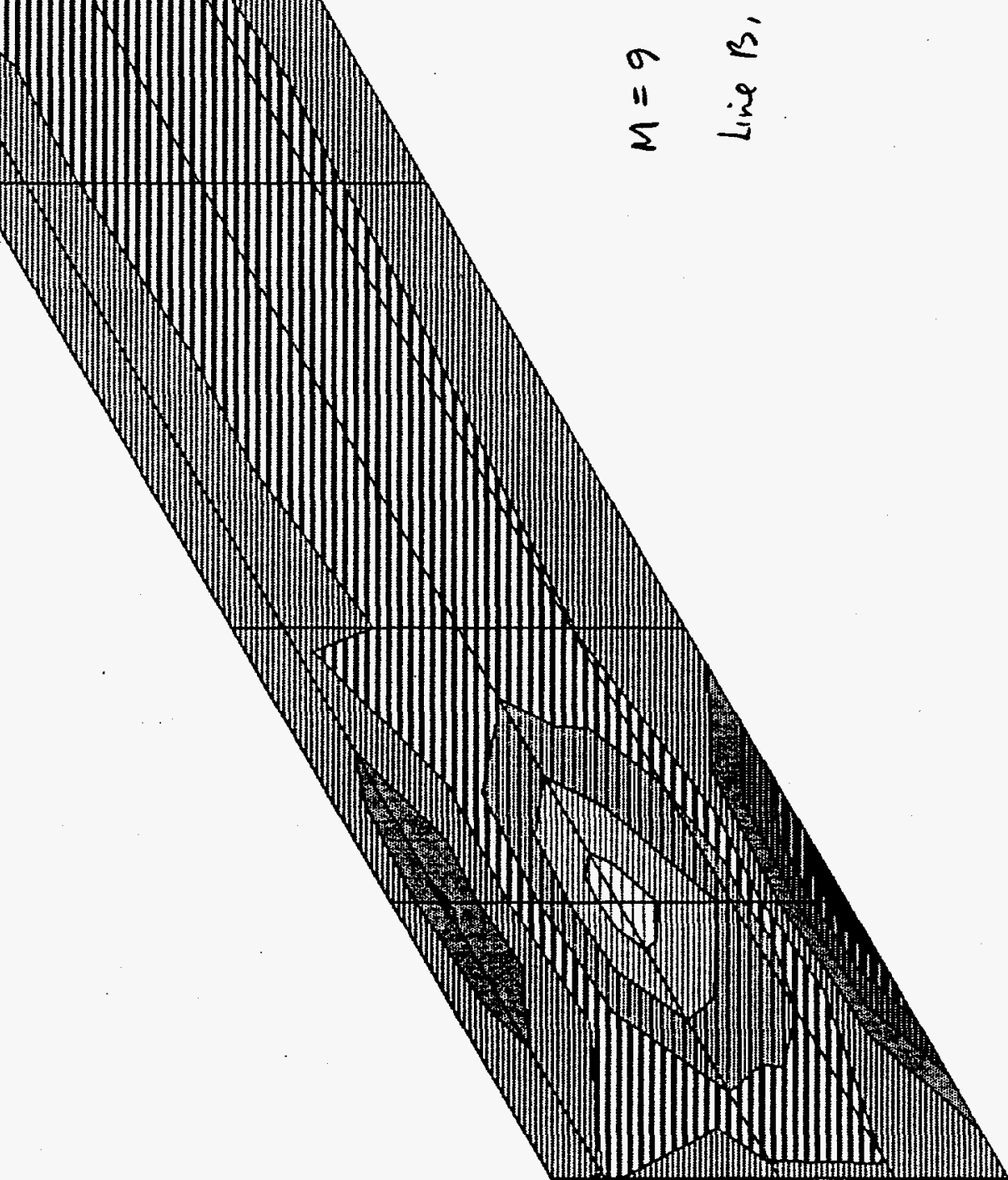

$\hat{\Omega}$ 


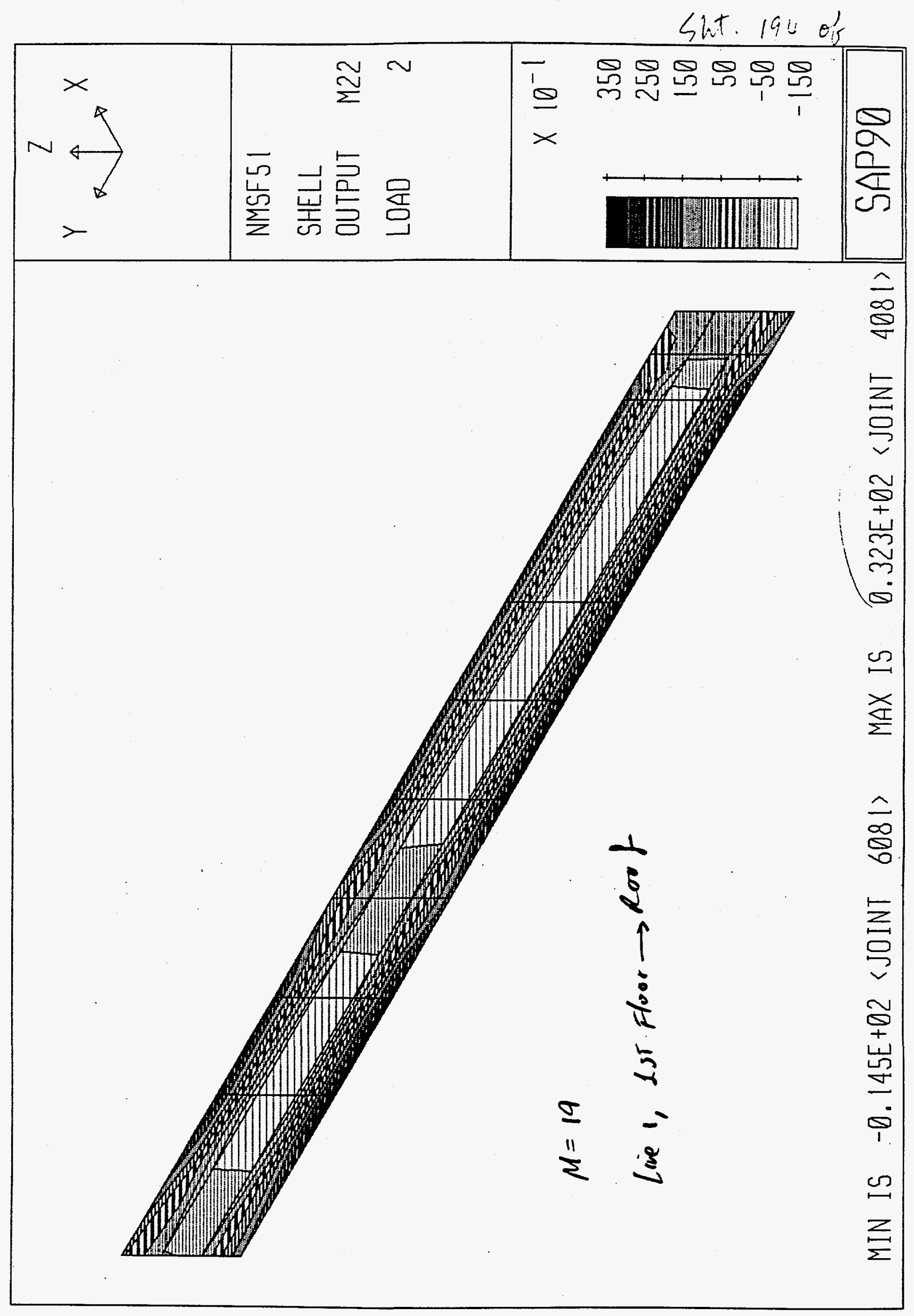




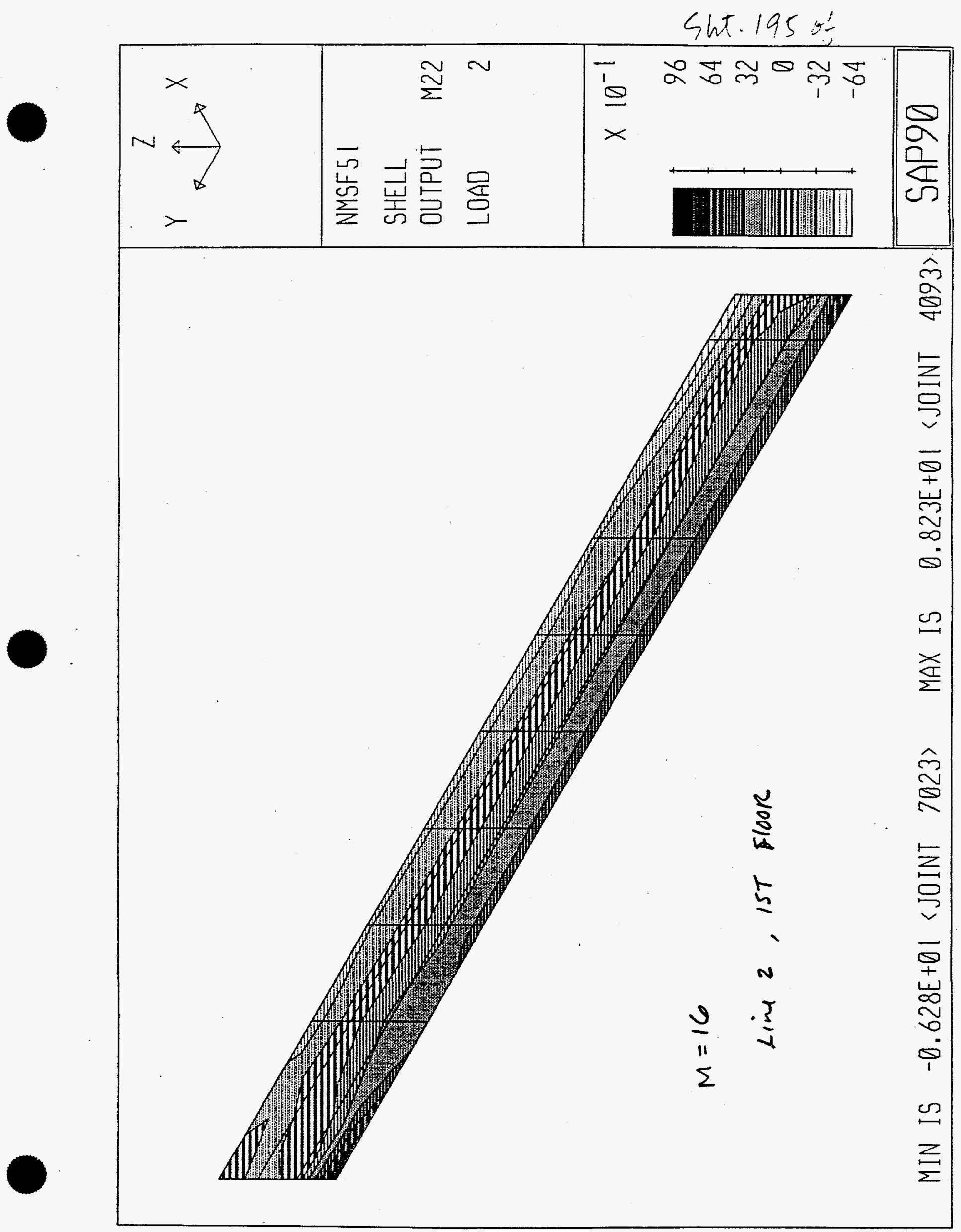




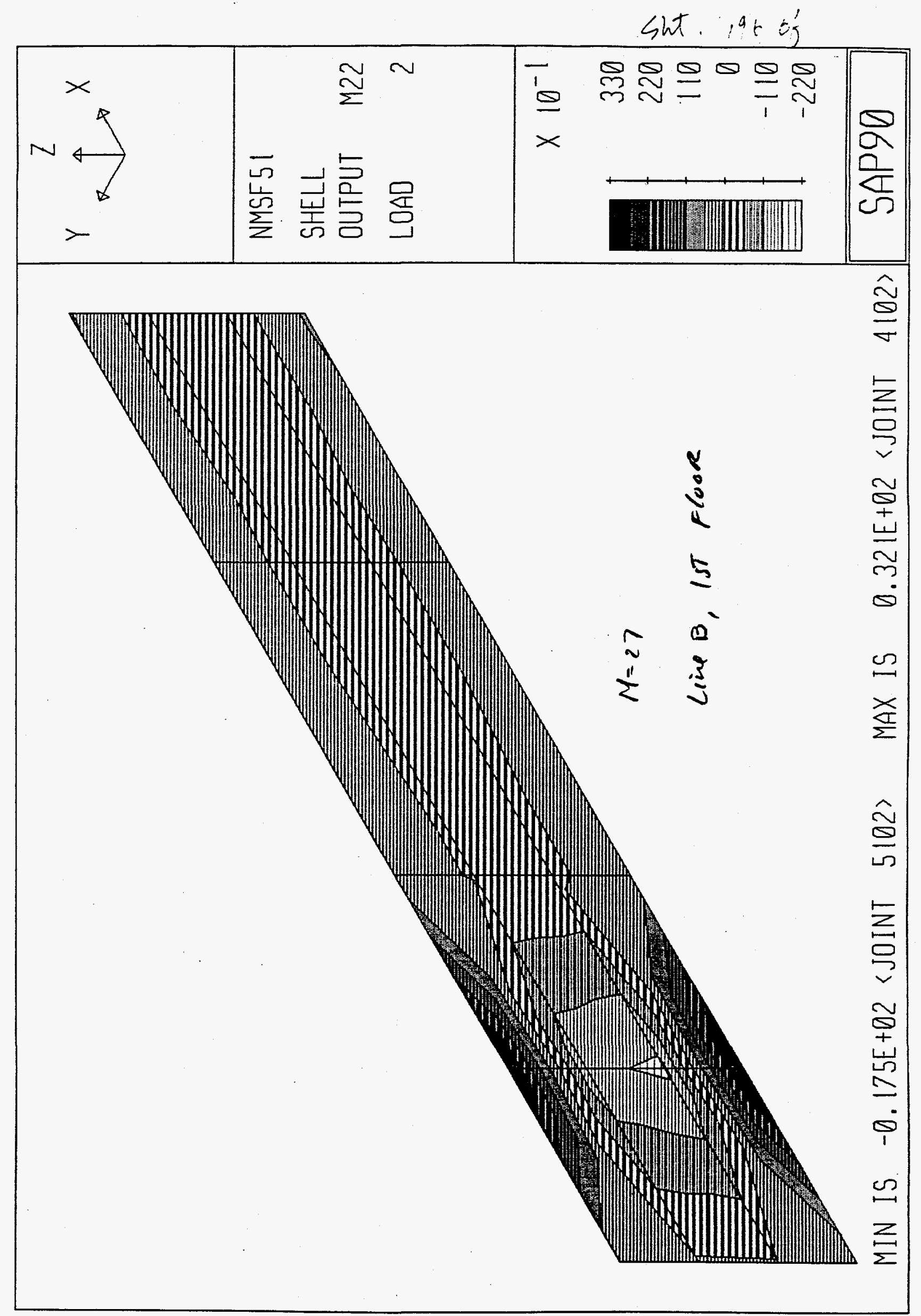


Sht. 197 o'

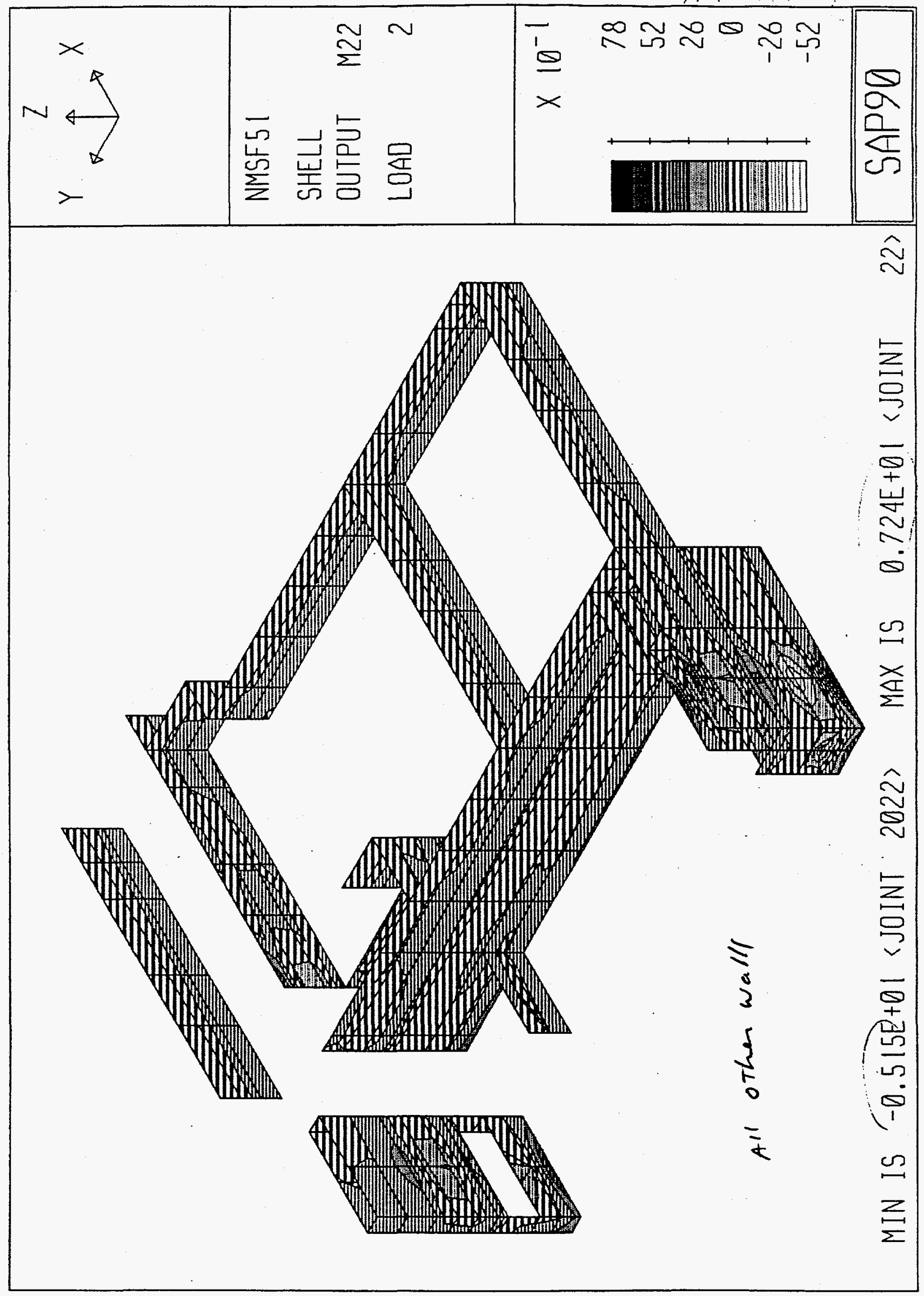




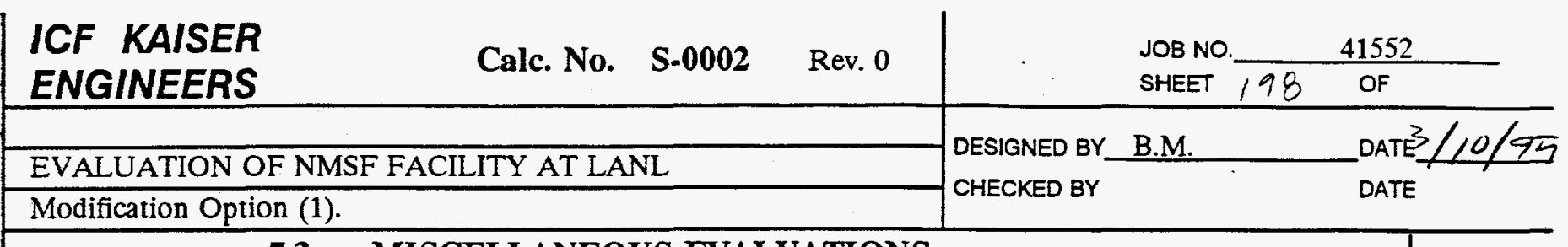

\subsection{MISCELLANEOUS EVALUATIONS}




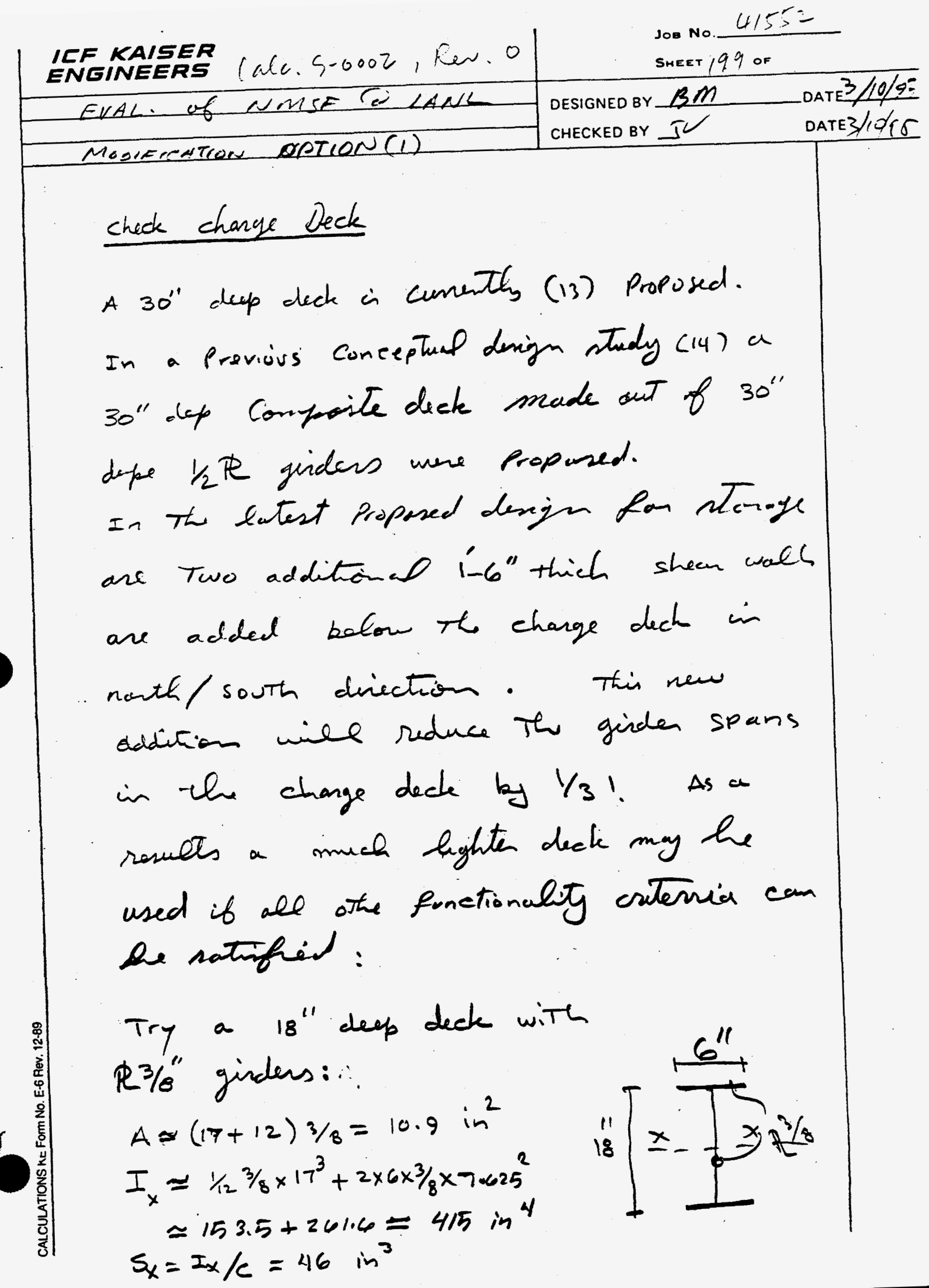




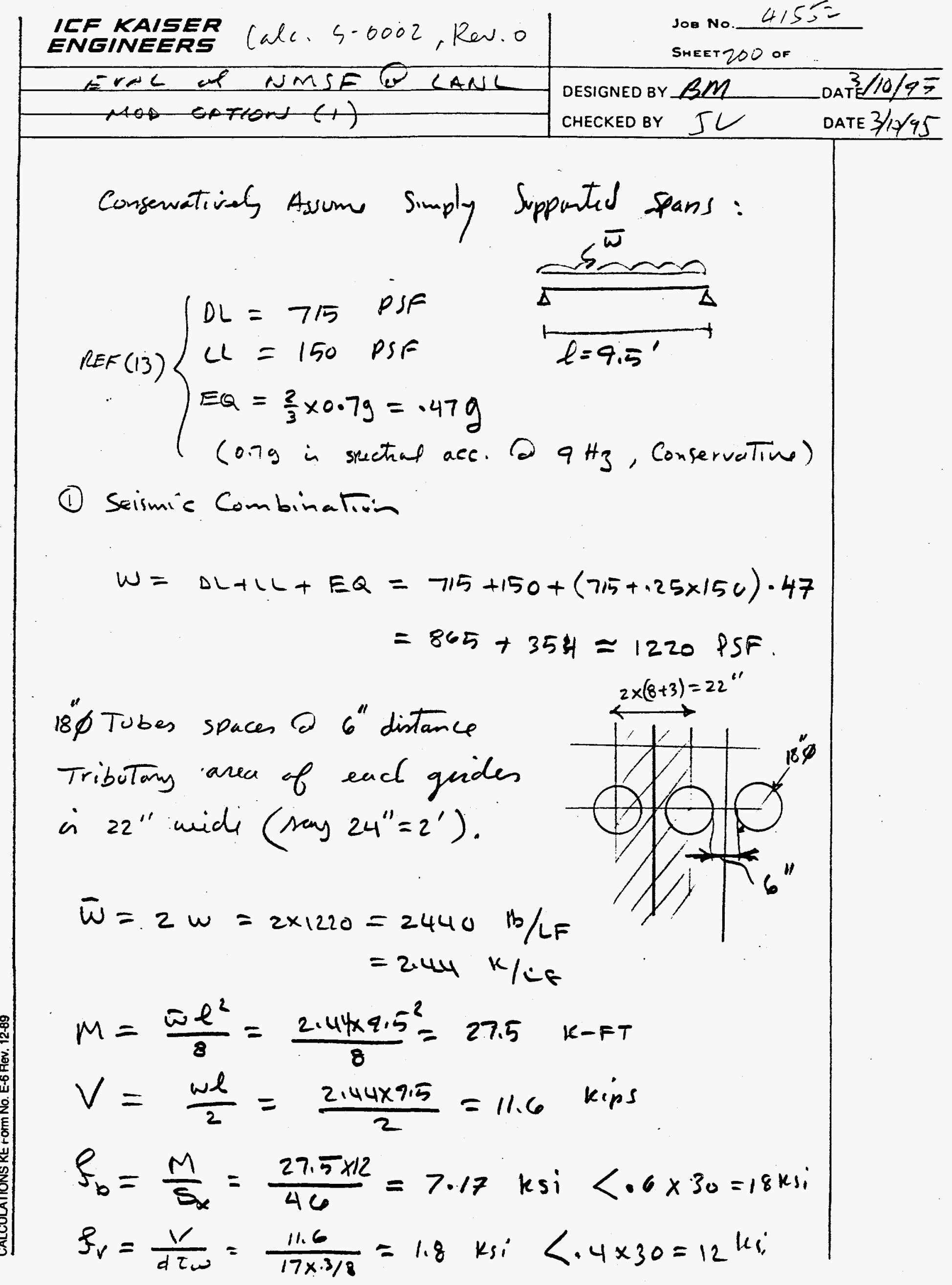




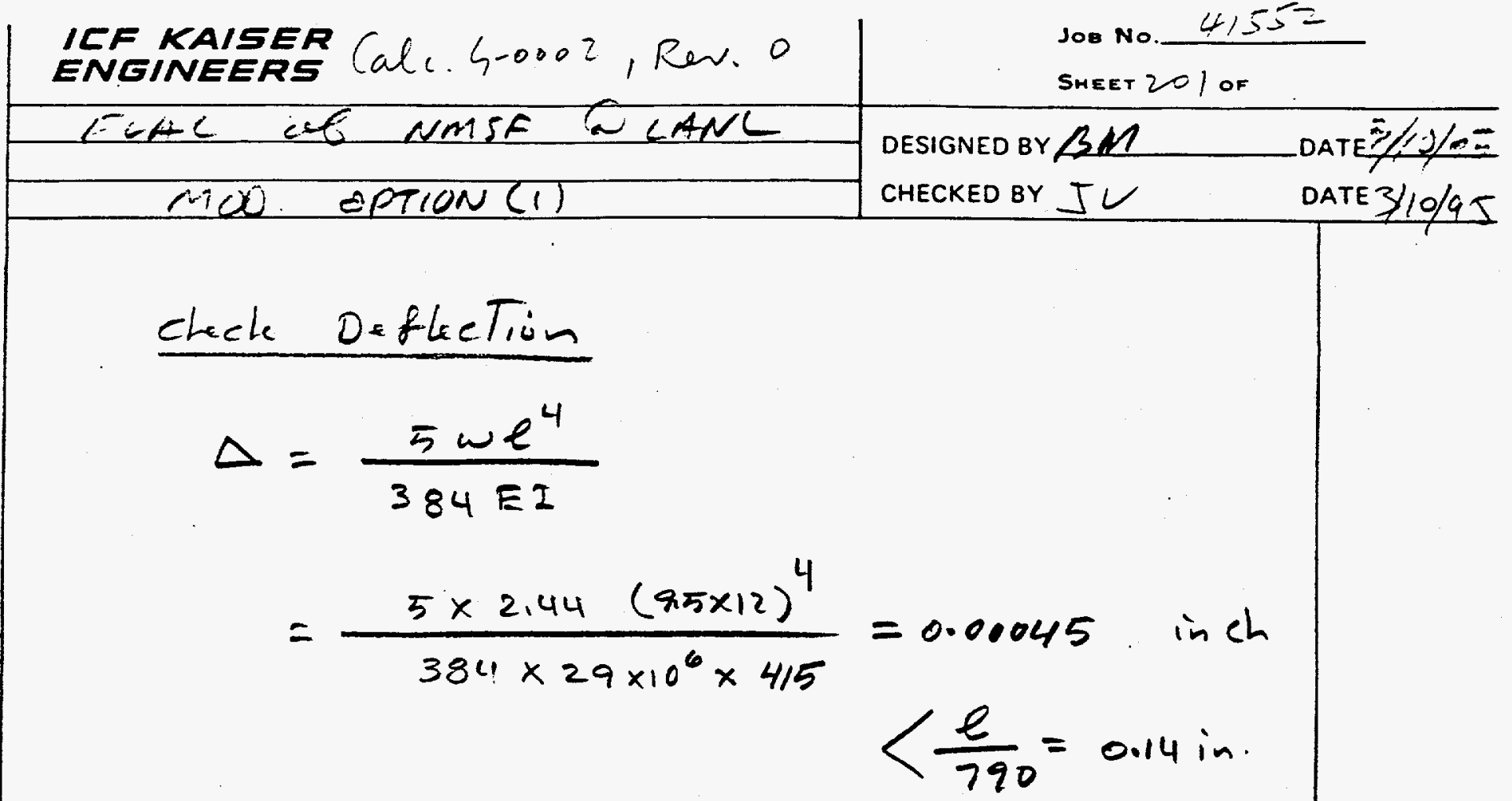

Very 5Tiff! o.k.

$$
\begin{aligned}
& \text { D/c Ratios (for 18"Deck) } \\
& \text { Bending }=\frac{7.17}{18}=0.40 \\
& \text { shean }=\frac{1.8}{12}=0.15
\end{aligned}
$$




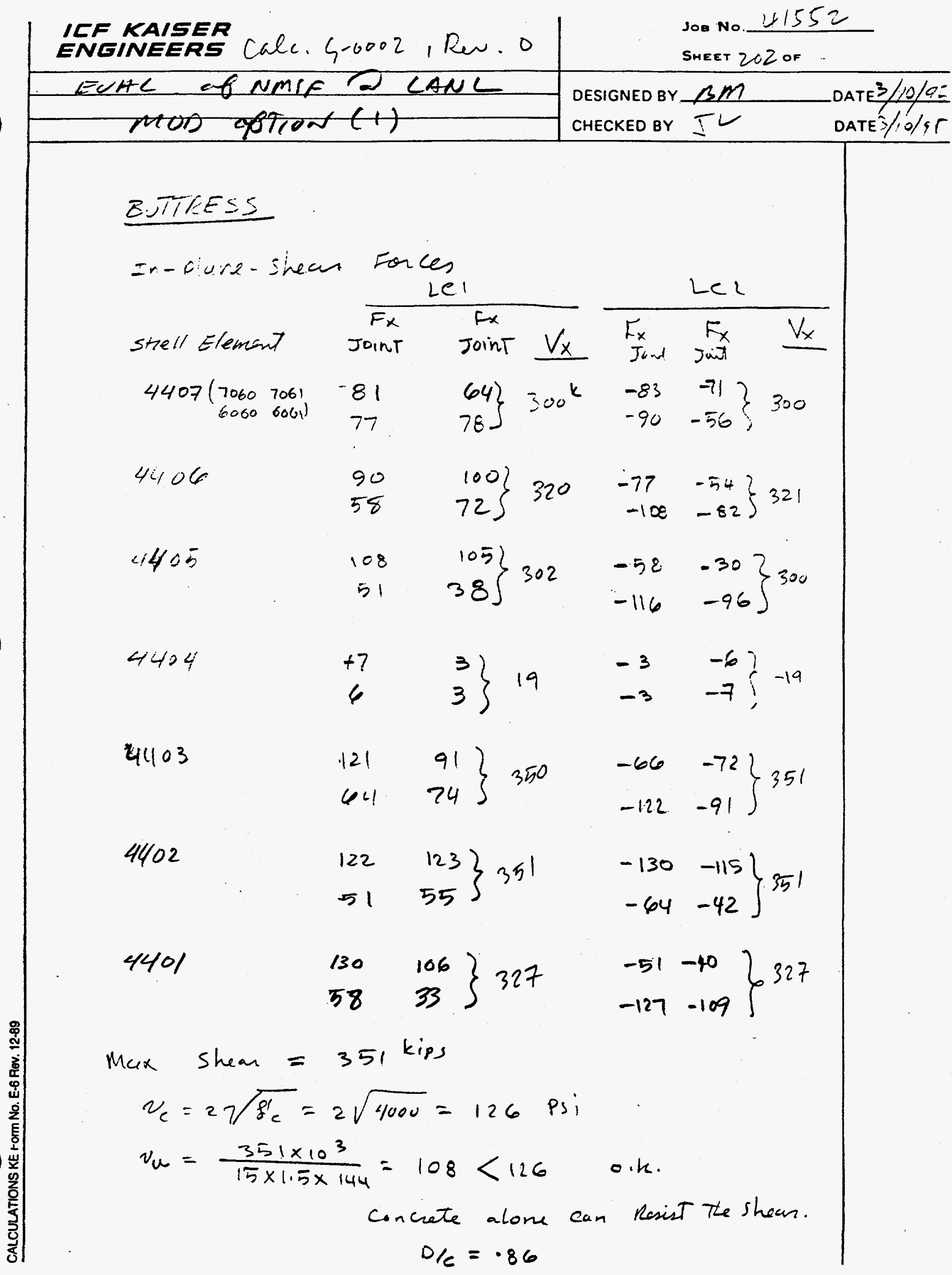




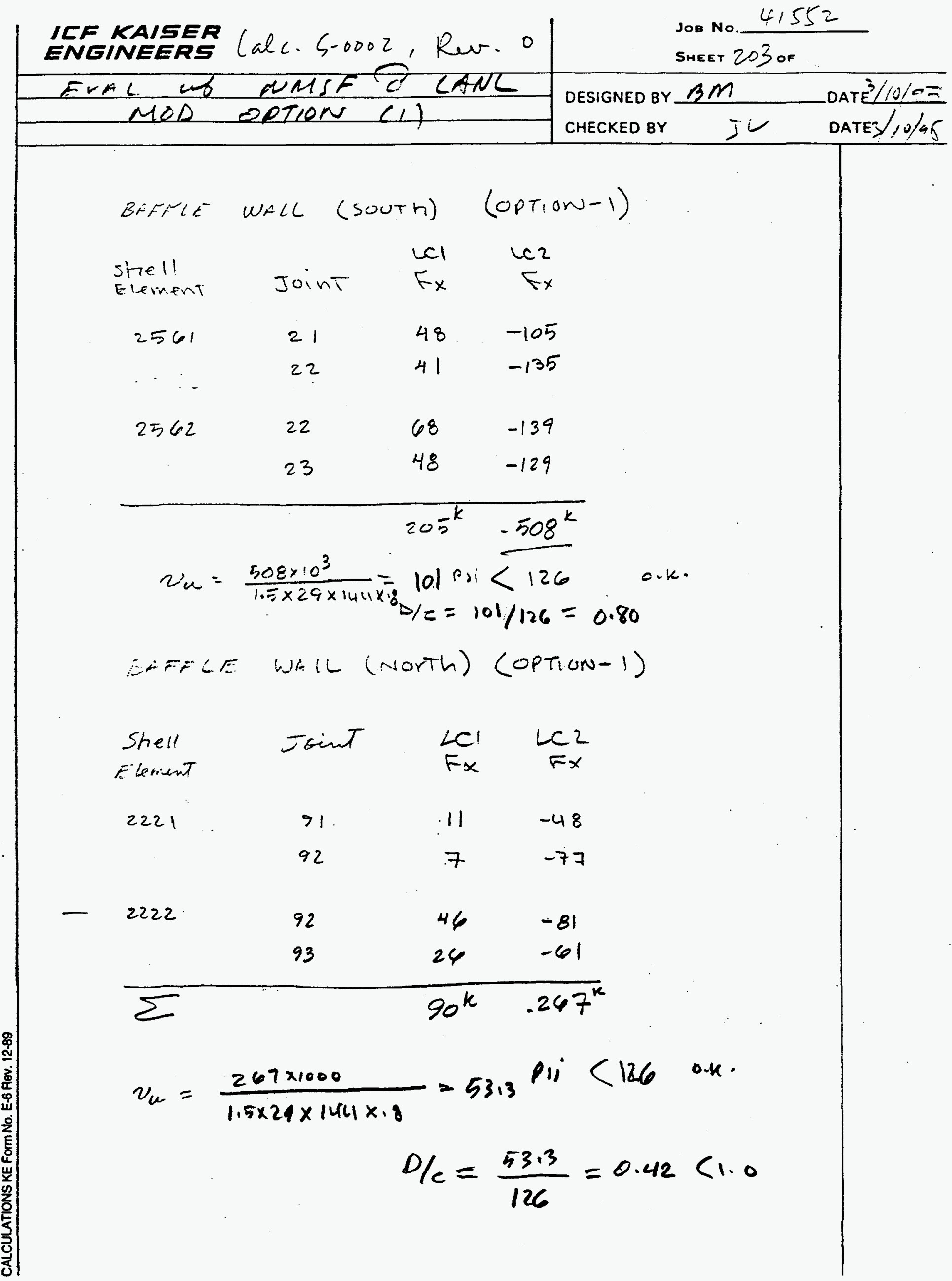




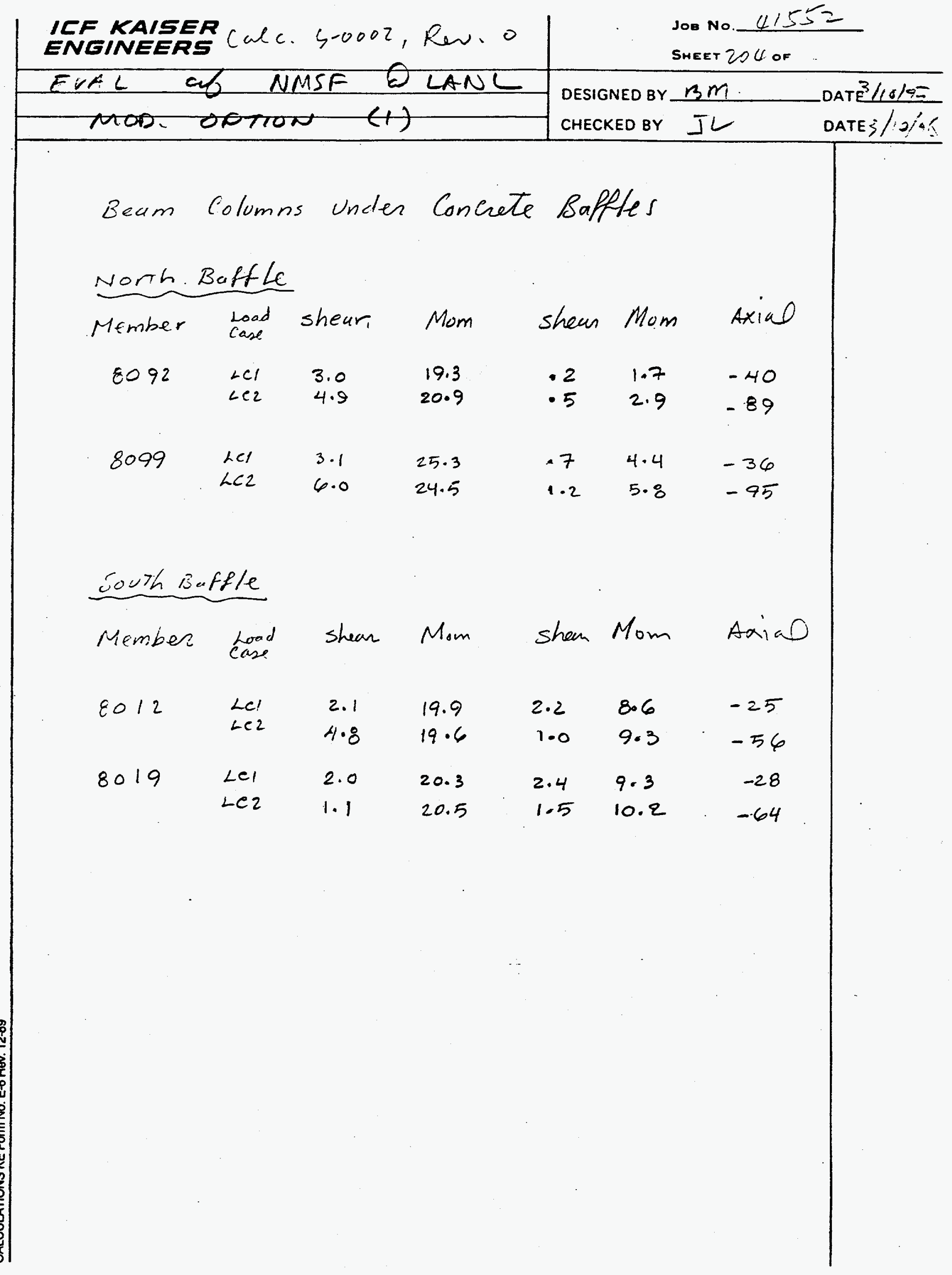




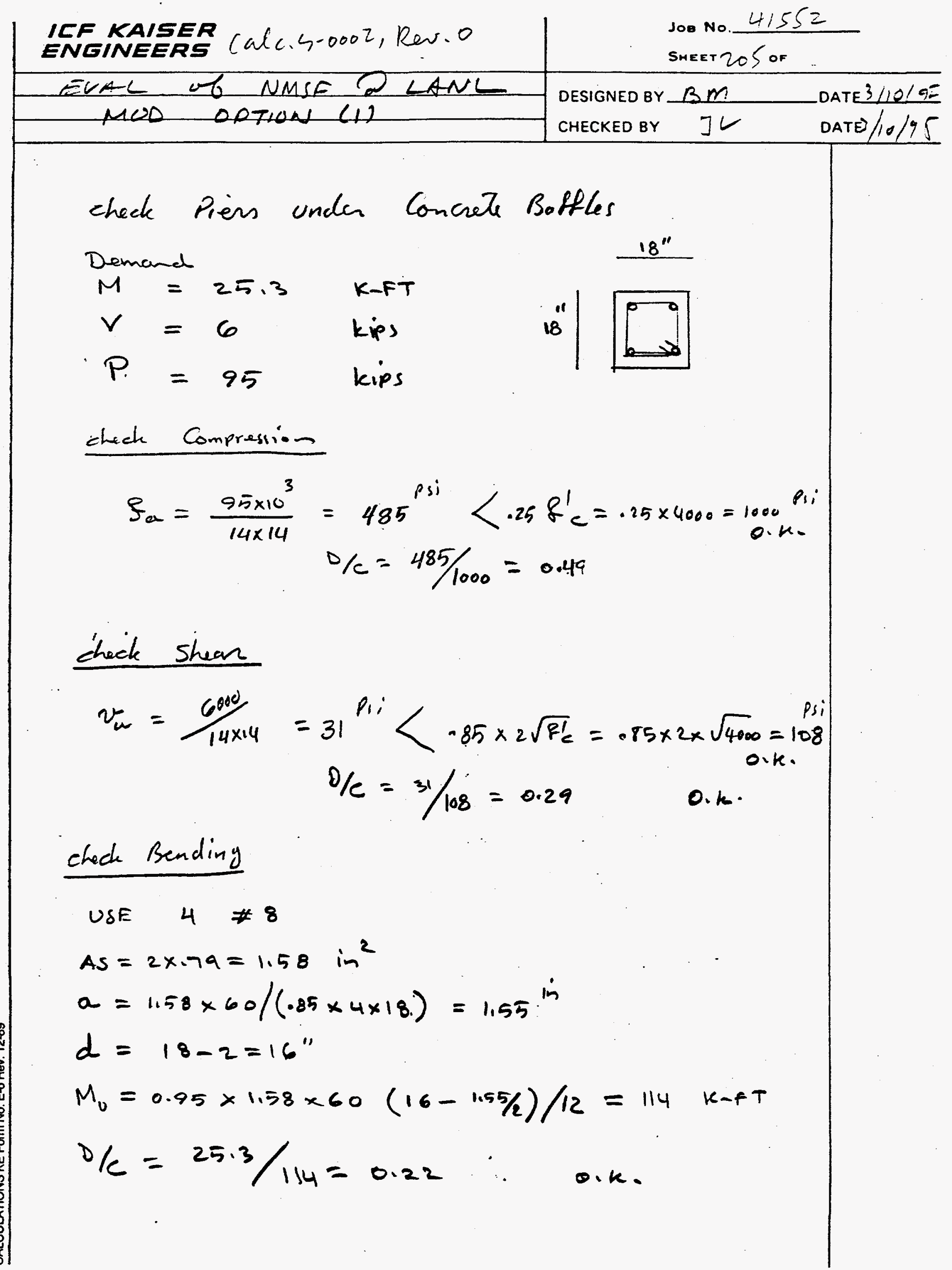




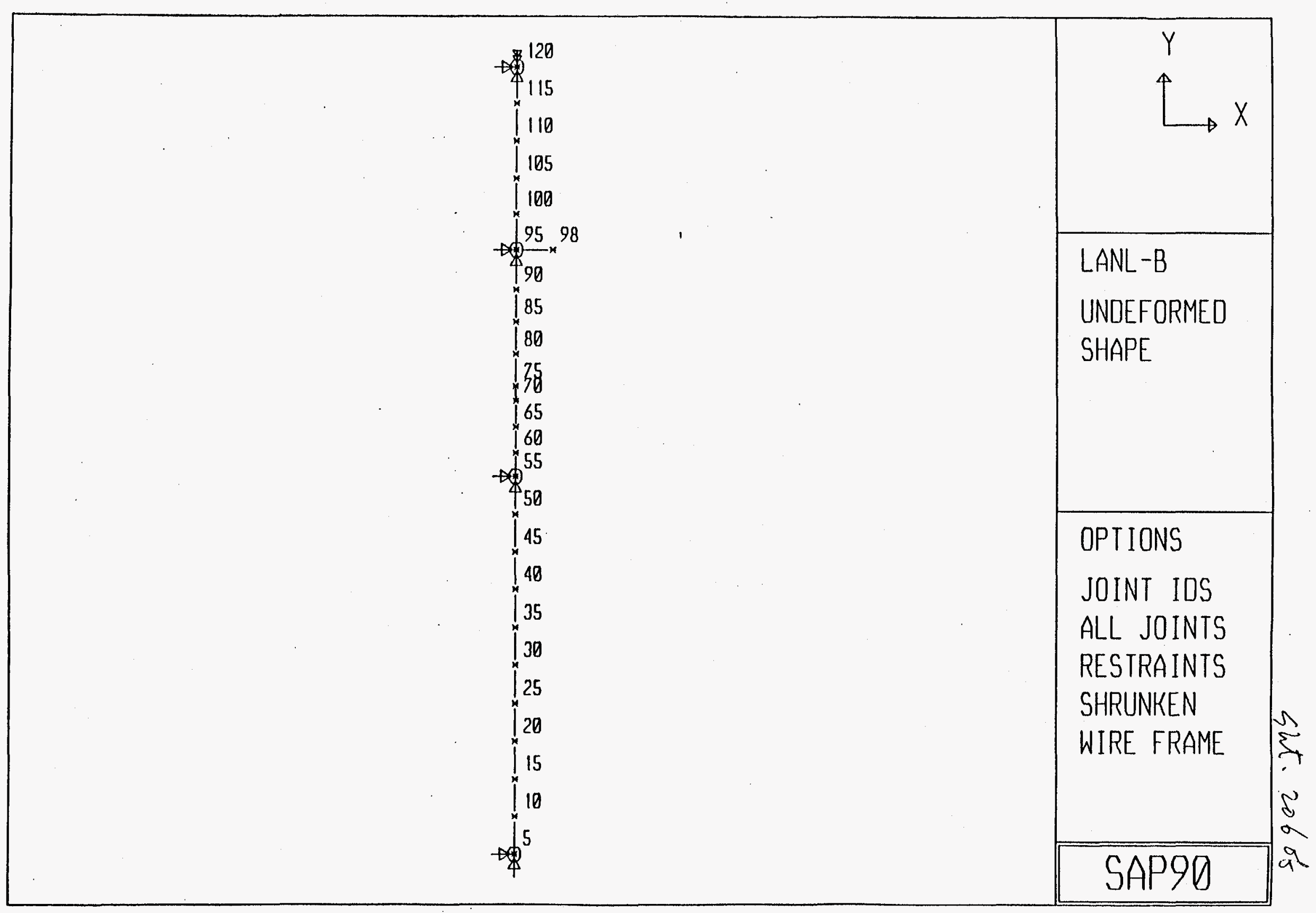



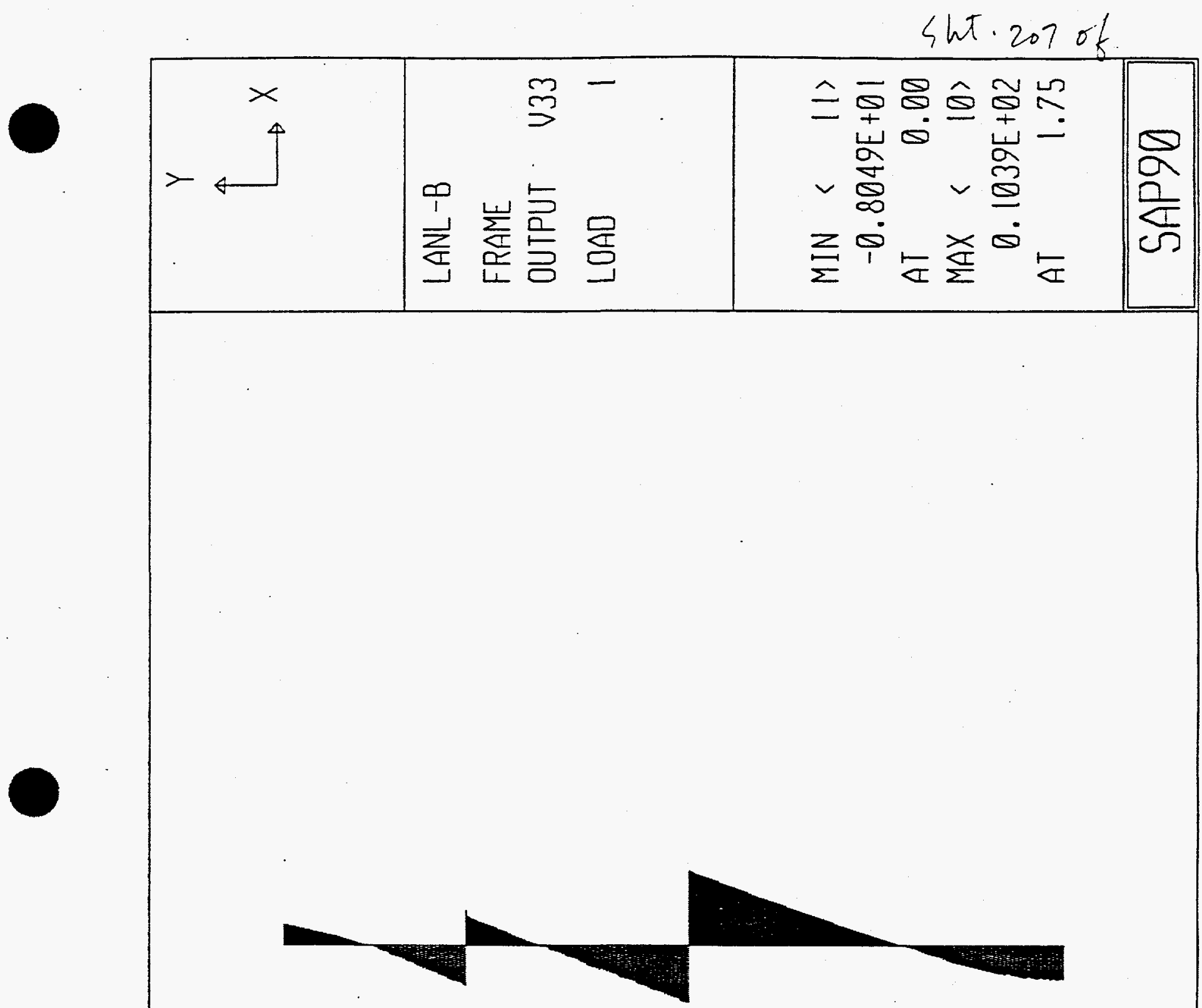


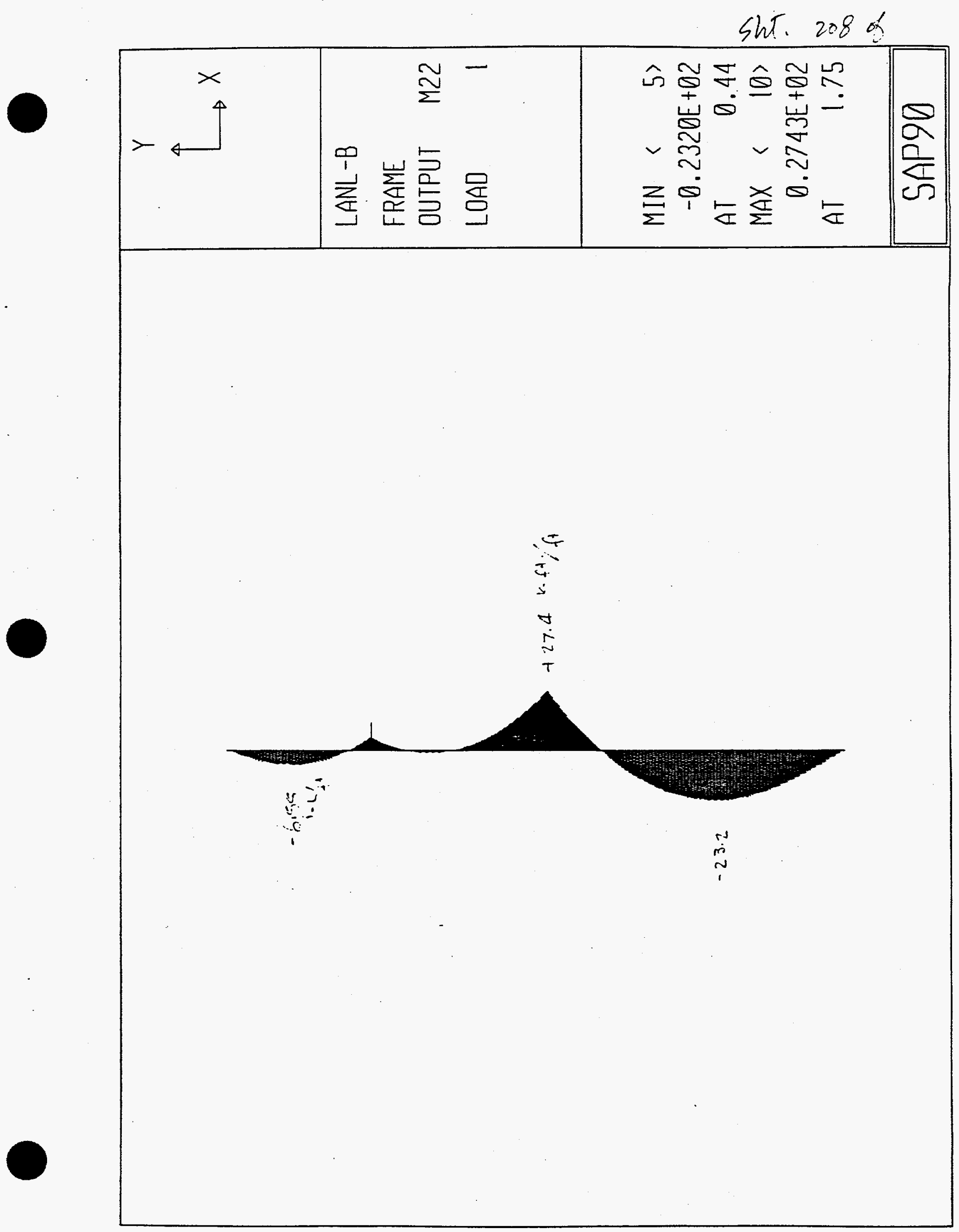


Sut. 209 of

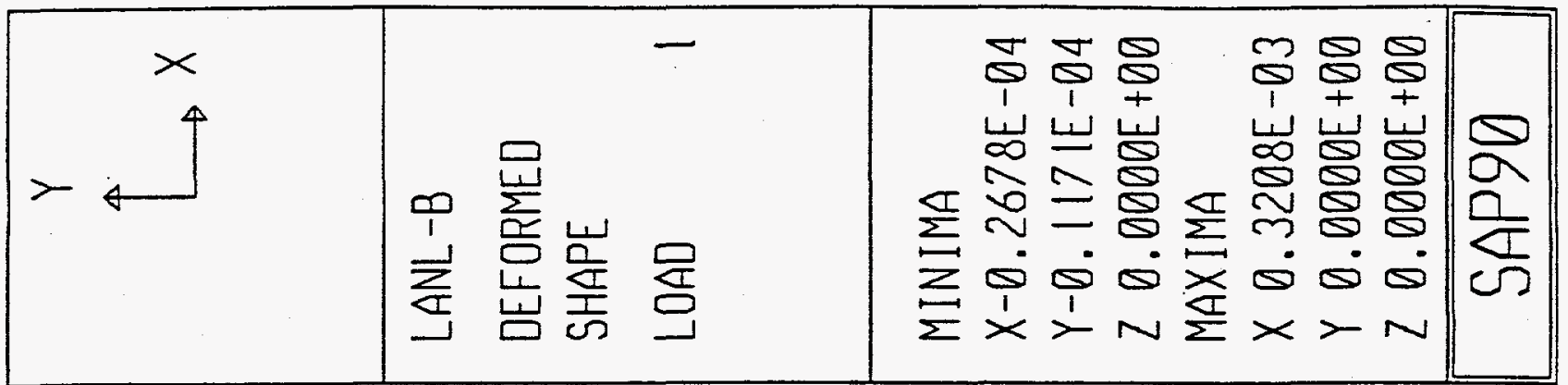

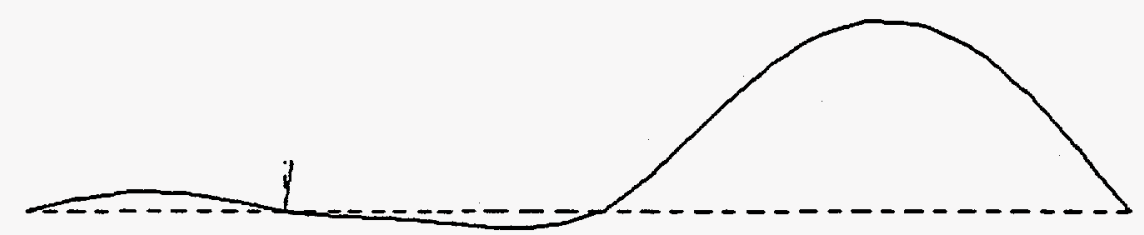



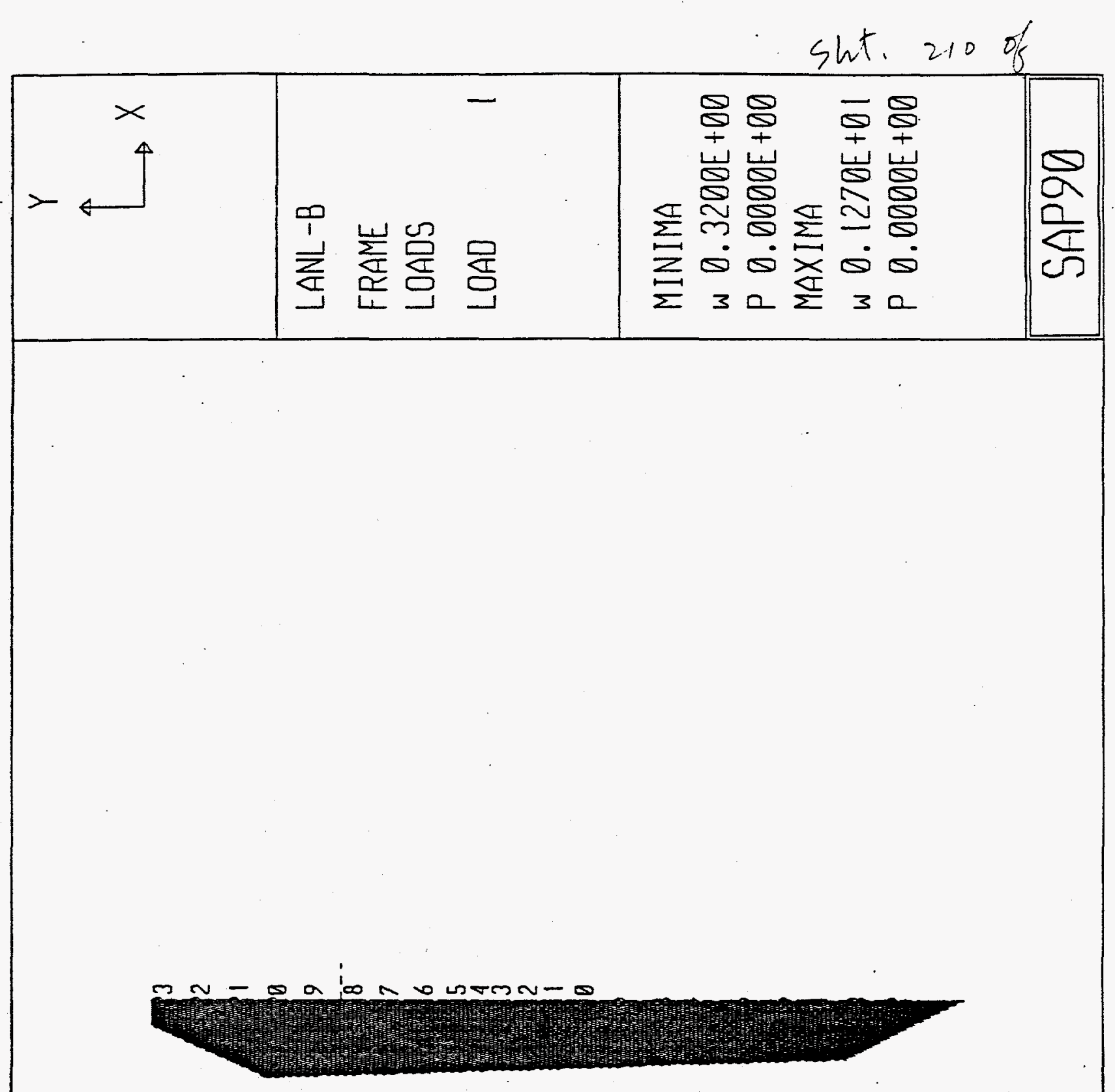


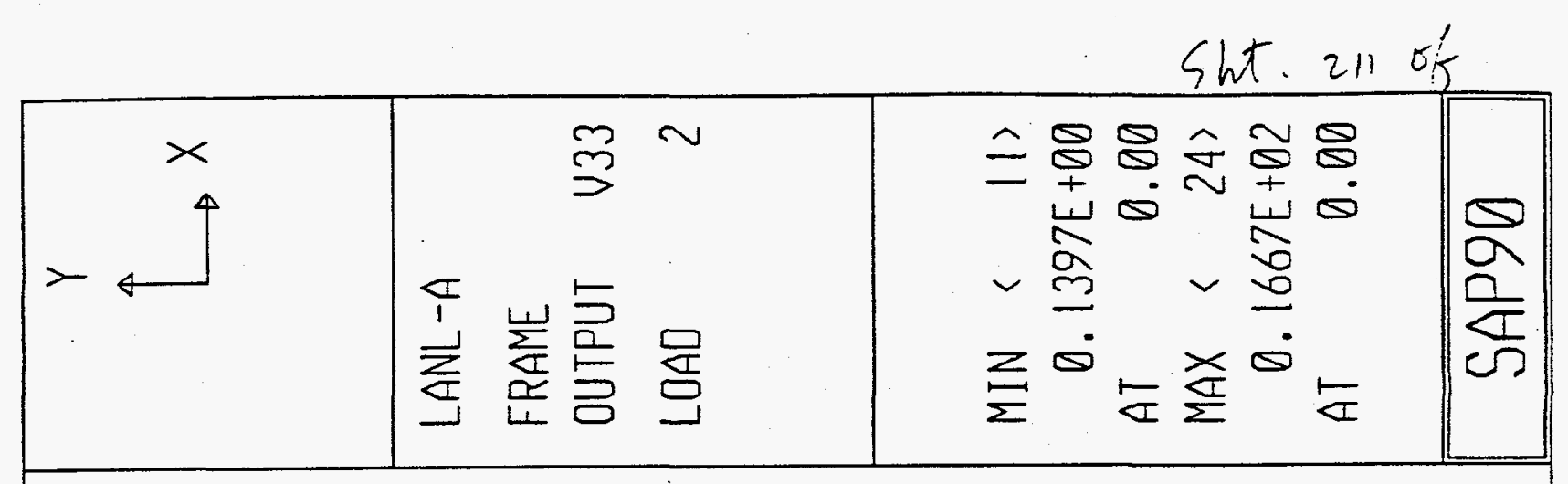




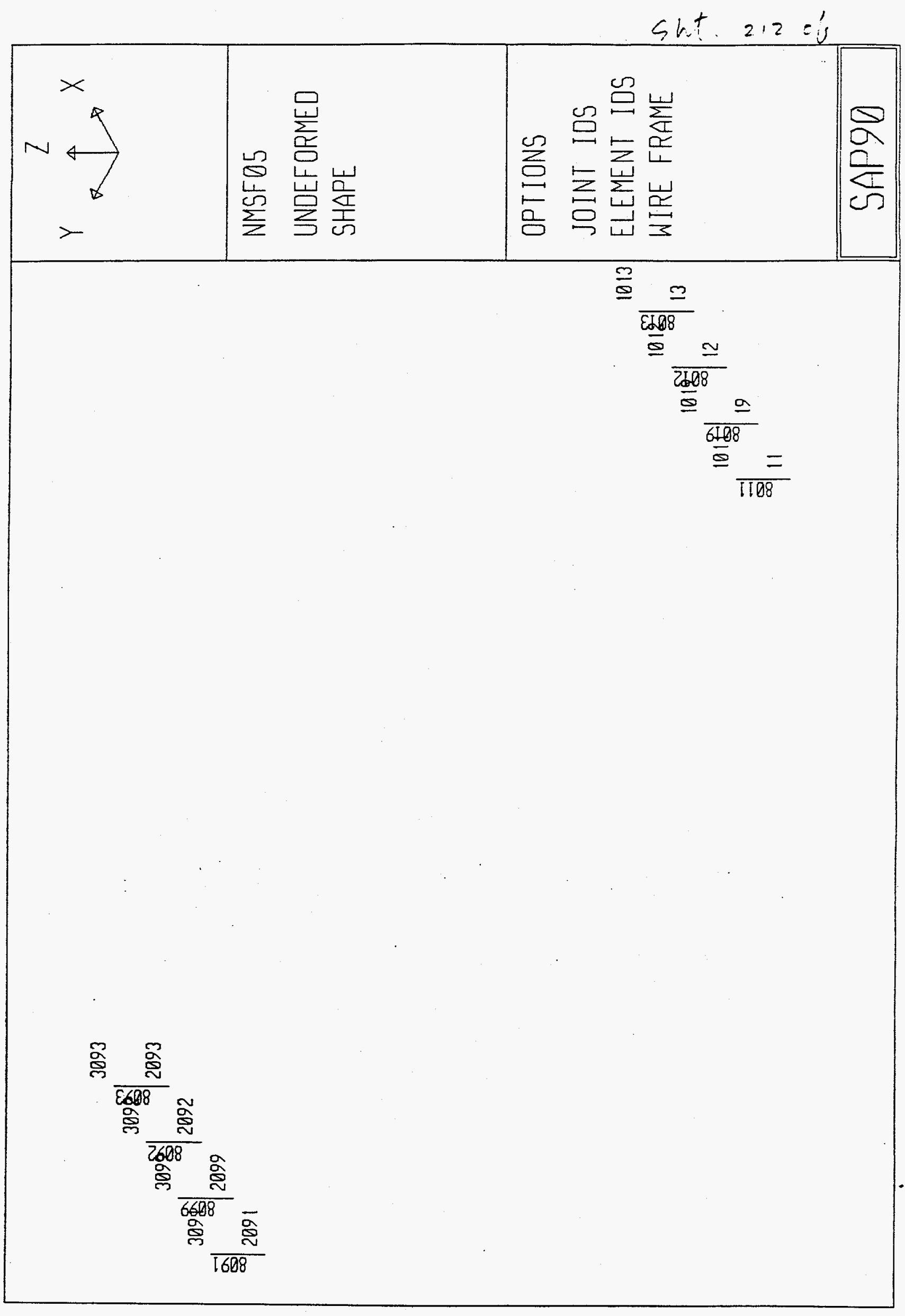


sint. 213 of

\begin{tabular}{|c|c|c|c|}
\hline$\underset{\infty}{\stackrel{x}{\longrightarrow}}$ & 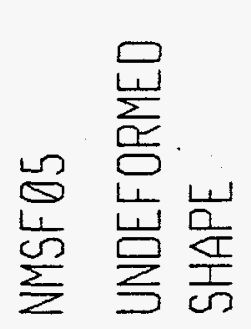 & 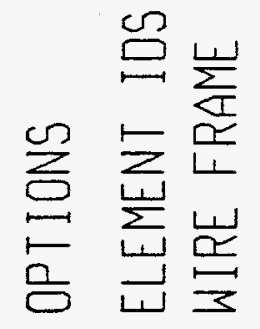 & $\frac{\sqrt{2}}{2}$ \\
\hline
\end{tabular}

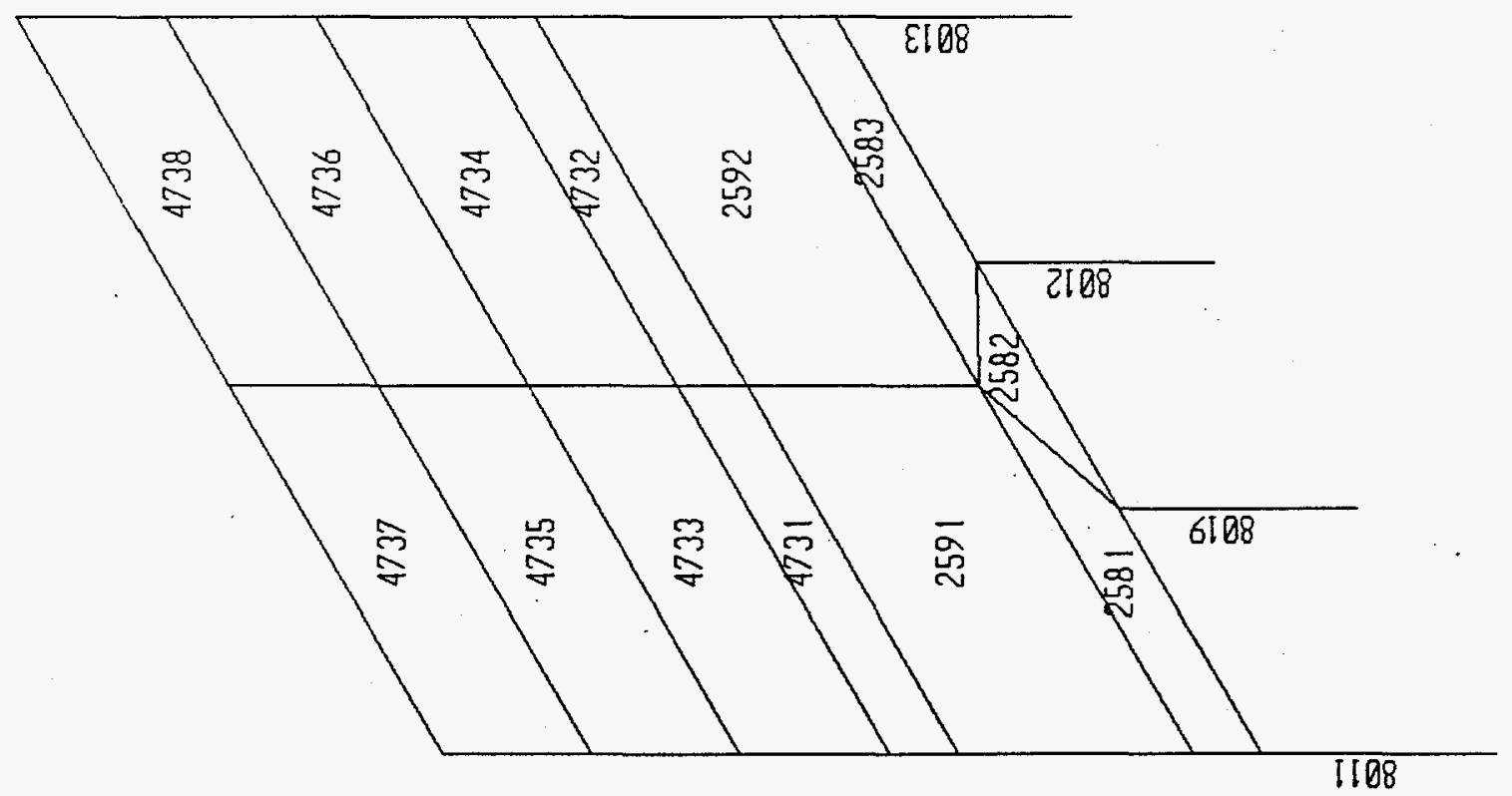


Sht 214 of

\begin{tabular}{|c|c|c|}
\hline$N \underset{\infty}{<}$ & 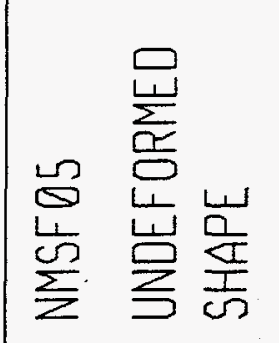 & 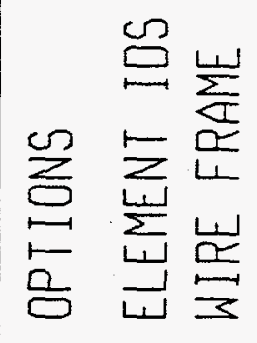 \\
\hline
\end{tabular}

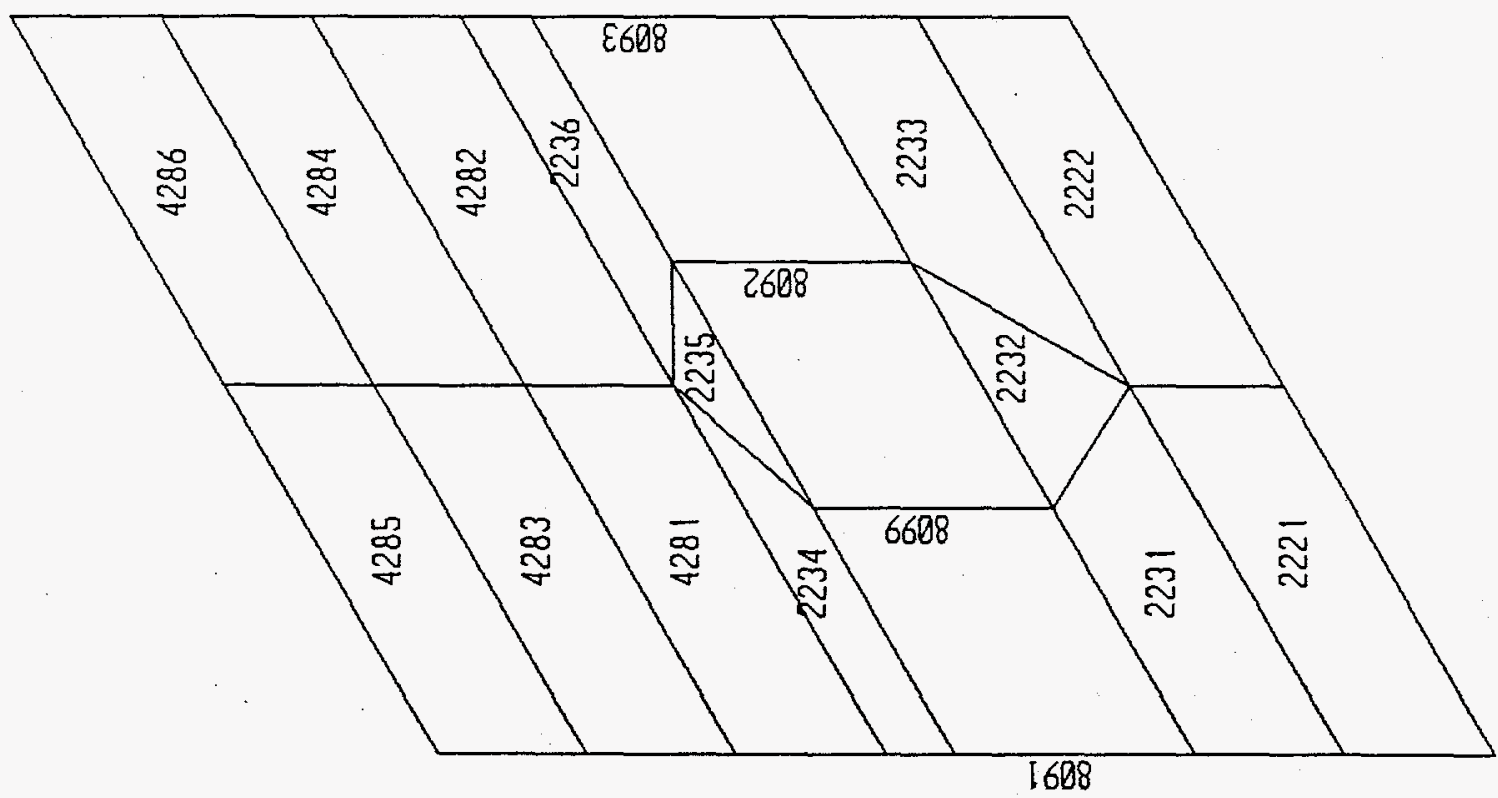




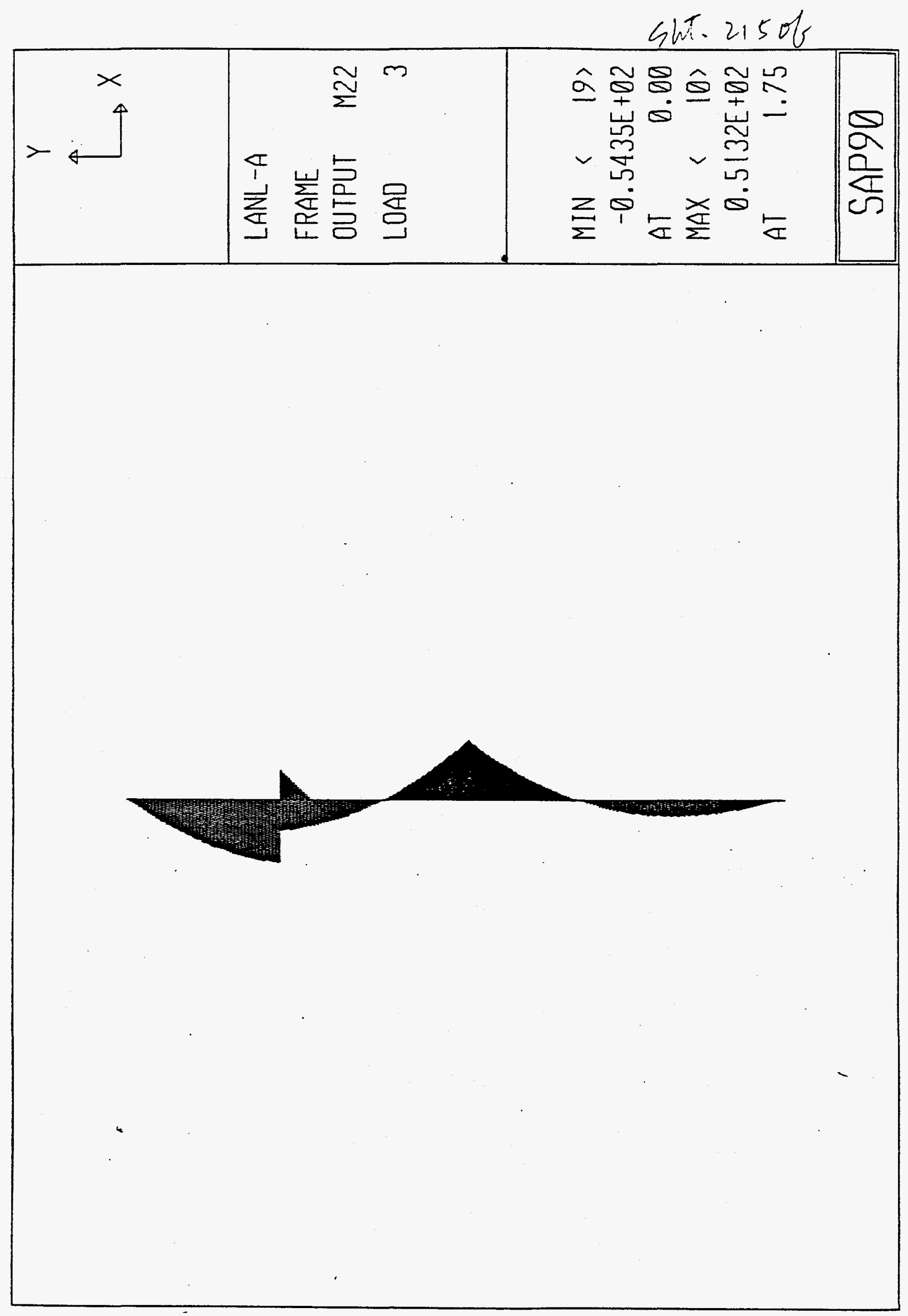




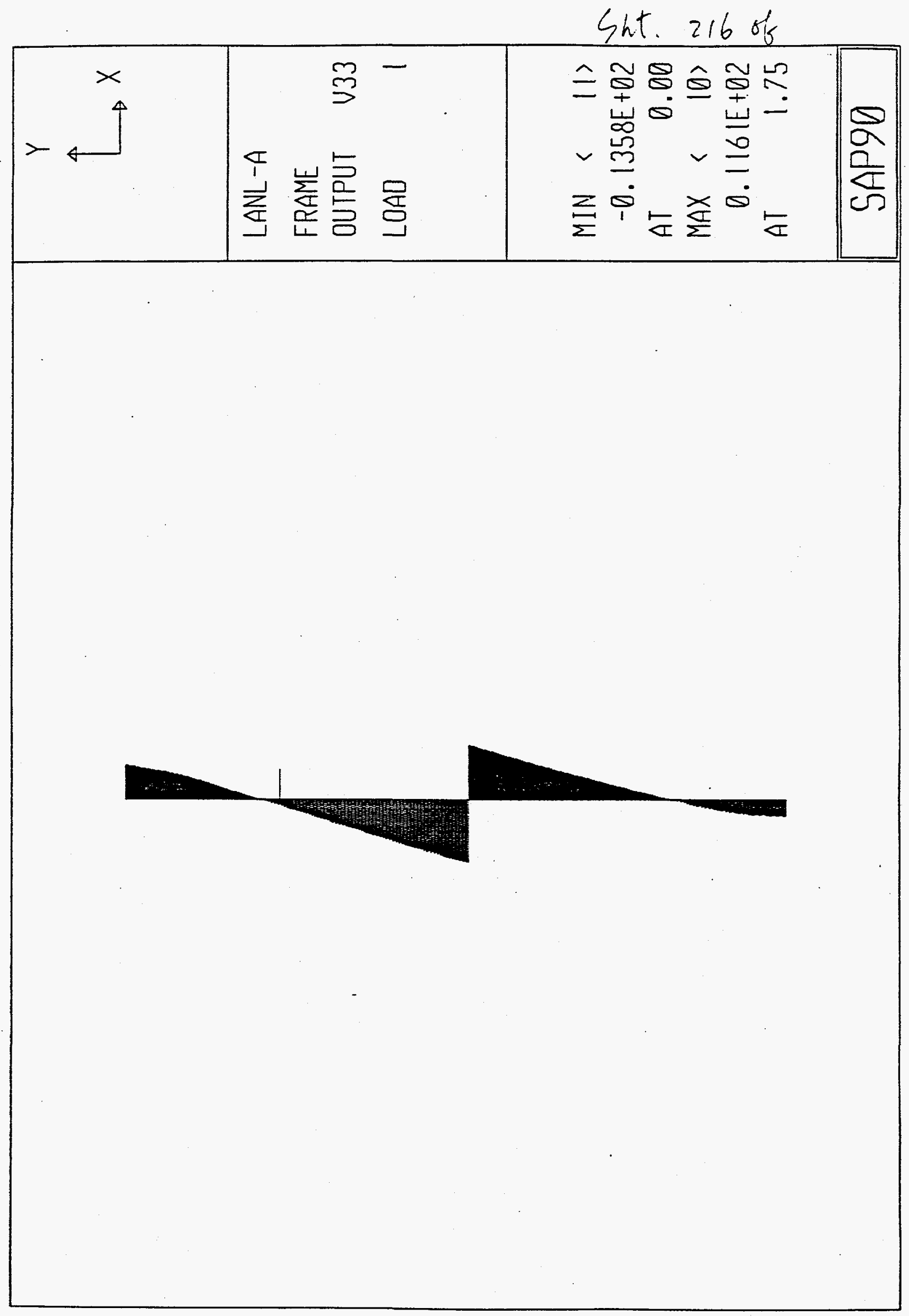


-

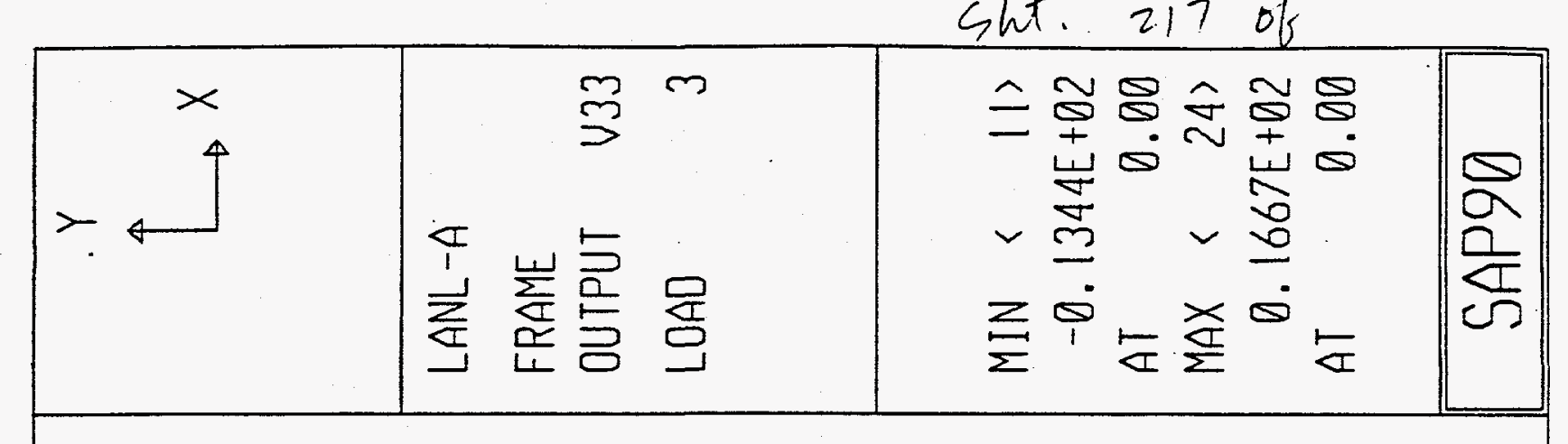




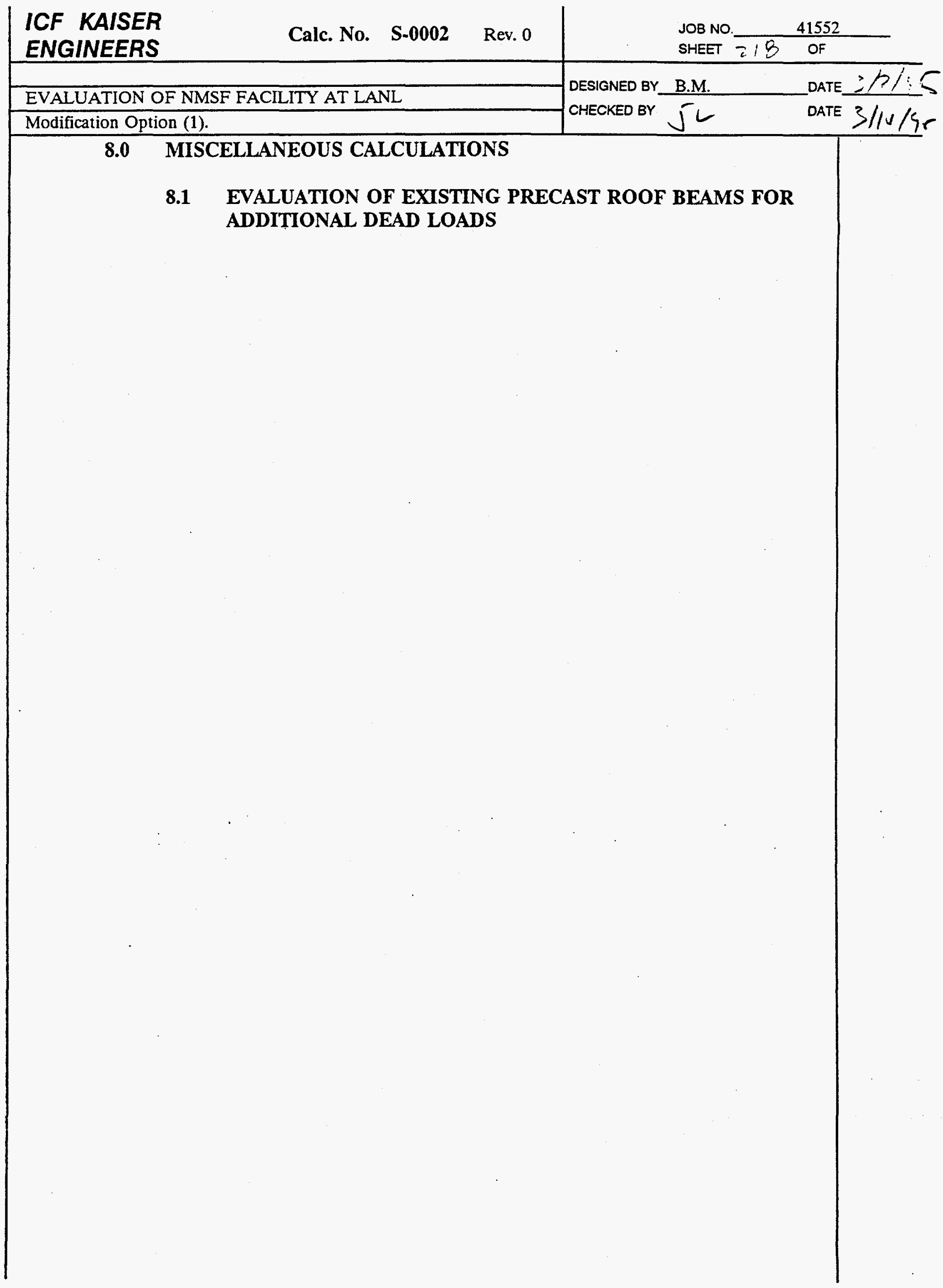




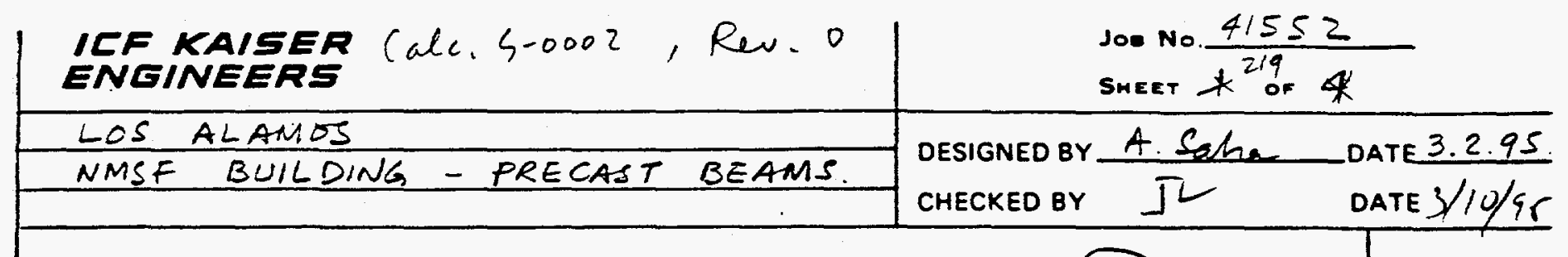

Existing precast roof beams over (B) to (E.1) and

Beam span $=10^{\prime}+59^{\prime}=69^{\prime}-0$

(3) to (7).

Design load from ding. S.3, Design Criteria Note's superimposed L.L. $=50$ psf.

11 $\quad$ D.L. $=40$ psf (in addition to self wt).

Seismic acceleration $=0.22 \mathrm{~g}$.

vendor data about precast beam is not available.

The following has been assumed (measured from detail (25) Ding. $(5-10)$.

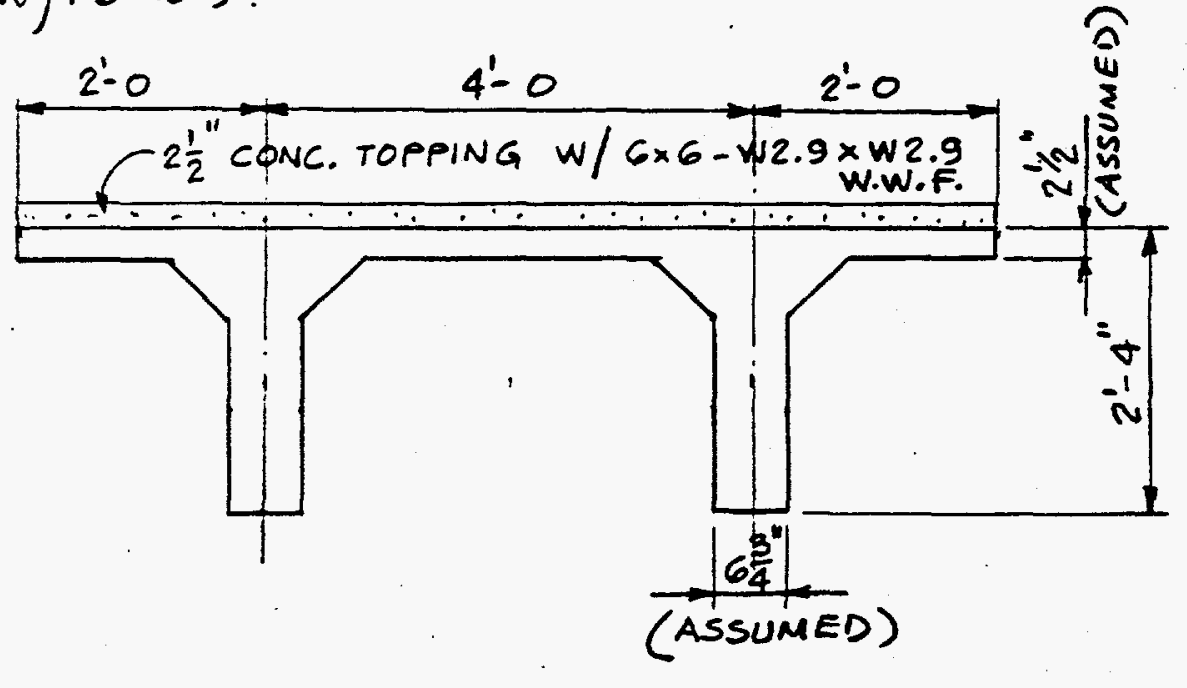

Required: Total $8^{\text {"thick }}$ slab

Existing $=2 \frac{1 "}{2}+2 \frac{1}{2}=5$ " Thick

Additional $8-5^{\prime \prime}=3^{\prime \prime}$ thick conc. is required.

Existing $2 \frac{11}{2}$ thick cone. weighs $=2.5 \times \frac{150}{12} \approx 31^{16} / \mathrm{ft}^{2}$

Additional $3^{n}$ thick conc. will weigh $=3 \times \frac{150}{12} \approx 37^{16} / \mathrm{ft}^{2}$

Weight of existing precast units $\frac{150}{8^{\prime}}\left[8^{\prime} \times 2.33^{\prime}-\left(8-\frac{6.75 \times 2}{12}\right)\left(2.33^{\prime}-\frac{2.5}{12}\right)\right]$

$$
\begin{aligned}
=\frac{150}{8^{\prime}}\left[18.667^{t^{2}}-14.609\right] & =76.1^{16 / \mathrm{ft}^{2}} \\
& \approx 76^{16} / \mathrm{ft}^{2} .
\end{aligned}
$$


IF KAISER

JOE NO. $\frac{41552}{220}$

LOS ALAMOS

NOSE BUILDING - PRECAST BEAMS.

DESIGNED BY A. Sate

DATE 3.6 .75

CHECKED BY DATE $3 / 10 / 45$

Area of steel in precast beams is not available.

Assume $14-7 / 6$ ia.

$270^{k}$ strand.

$$
\left.\begin{array}{l}
f_{c}^{\prime}=5000 \text { psi } \\
f_{c}^{\prime}=3000 \text { precast units }
\end{array}\right\} \begin{aligned}
& \text { Fop ping. } \\
& \mathrm{s}-3
\end{aligned}
$$

$$
\begin{aligned}
& A_{p s}=14 \times 0.115=1.61 \mathrm{in}^{2} . \\
& \bar{\omega}_{p}=\frac{A_{p s} f_{p u}}{b d f_{C}^{\prime}}=\frac{1.61}{8 \times 12 \times 24} \times \frac{270}{5}=0.0377 \approx 0.04
\end{aligned}
$$

From Table 3.9 .2

$$
\begin{aligned}
& c / d=0.055 ; f_{p s} / f_{p u}=0.981 ; \bar{j}_{n}=0.959 \\
& c=0.055 \times d=0.055 \times 24=1.32 \mathrm{in} .<2.5 \mathrm{in} .
\end{aligned}
$$

Neutral axis lies within the flange, Rectangular Section

$$
\begin{aligned}
& \phi M_{n}=\phi A_{p s} f_{p u} \overline{j u} d \\
& =0.9 \times 1.61 \times 270 \times 0.959 \times 24 / 12=750.4^{\mathrm{kft}} \\
& f_{p s}=0.981 \times 270=265^{\circ} \mathrm{ks} \text {; } \\
& \omega_{u}=1.4(76+10)+1.7 \times 20^{\text {assumed }}=154.4^{16} / \mathrm{ft} \approx 0.16^{\mathrm{K}} / \mathrm{ft} \\
& M_{n}=\frac{0.16 \times(69)^{2}}{8}=95.2^{\mathrm{kft}} / \mathrm{ft} . \times 8^{\prime}=762^{\mathrm{kft}} \text { for } 8^{\prime} \text { midi } \\
& x 750^{\text {kt allowable. }}
\end{aligned}
$$

Assume 16-7/16" din. $270^{k}$ strand with $d x 24$ in.

$$
A_{p s}=16 \times 0.115=1.84 \mathrm{in}^{2} \text {. }
$$

Existing composite section:

$$
\begin{aligned}
& \bar{\omega}_{p}=\frac{1.84}{8 \times 12 \times 26.5} \times \frac{270}{3}=0.065 \\
& c / d=0.087 ; f_{p s} / f_{p n}=0.973 ; \bar{j}_{n}=0.932 \\
& c=0.087 \times 26.5=2.30 \mathrm{in} .<2.5 \mathrm{in}
\end{aligned}
$$




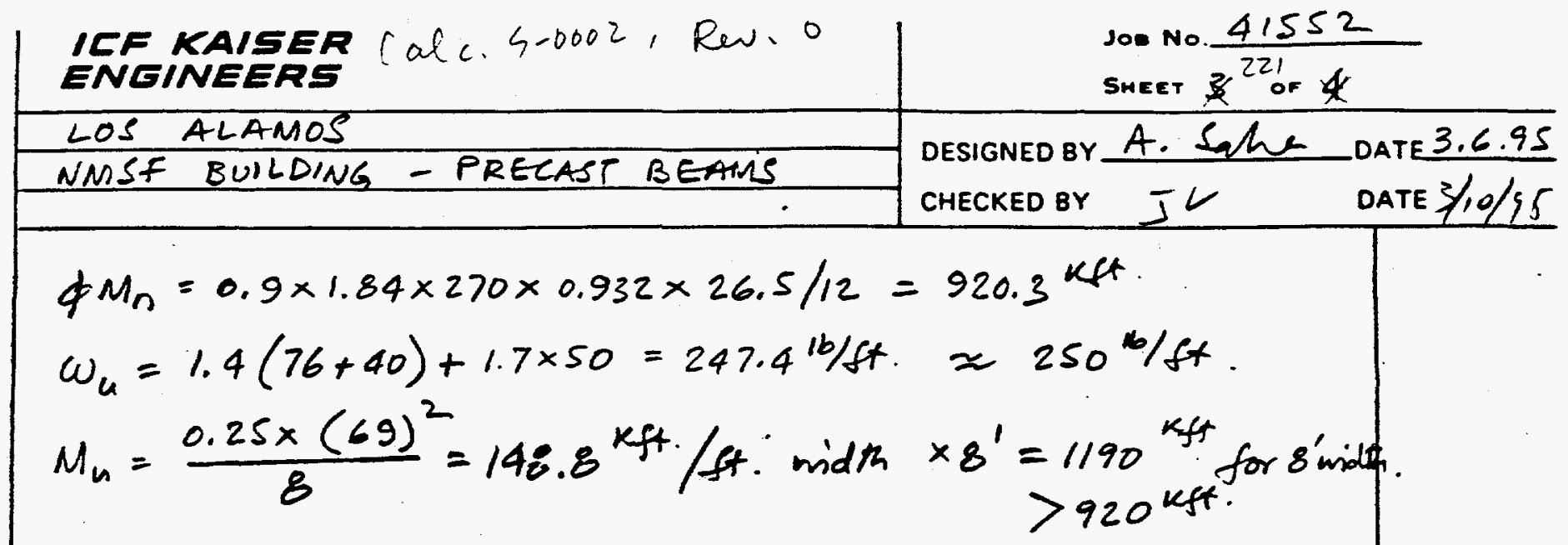

Reduction in L.L. due to tribe. area

$$
R=0.08\left(8^{\prime} \times 69^{\prime}-150\right)=32.2 \longleftarrow \text { governs. }
$$

$$
\begin{aligned}
& \text { or } R=23.1\left(1+\frac{116}{50}\right)=76.7>32.2 \\
& \therefore \quad l . L_{.}=(1-0.32) \times 50=34 \mathrm{k} / \mathrm{ft}^{2} . \\
& \omega_{u}=1.4(76+40)+1.7 \times 34=220 \mathrm{Psf} \\
& M_{n}=1190 \times \frac{22}{25}=1047 \mathrm{kft}>920 \mathrm{kft} \text { Say } 0 . \mathrm{K} .
\end{aligned}
$$

New composite Section:

$$
\begin{aligned}
\bar{\omega}_{p} & =\frac{1.84}{8 \times 12 \times 29.5} \times \frac{270}{3}=0.0585 \\
c / d & =0.079 ; f_{p s} / f_{p n}=0.971 ; \bar{j}_{n}=0.939 . \\
c & =0.079 \times 29.5=2.33 \mathrm{in} . \\
\phi M_{n} & =0.9 \times 1.34 \times 270 \times 0.939 \times 29.5 / 12=1032 \mathrm{kgt} .
\end{aligned}
$$

Increase in capacity due to increase in depth

$$
=1032-920=112^{\mathrm{kst}} \text {. or } \frac{112}{920} \times 100=12.2 \% \text {. }
$$

Increase in load due to addition of $3^{\prime \prime}$ of cone.

$$
=37^{16} / 8 t^{2} \text { or } \frac{37}{(76+40+34)}=\frac{37}{150} \times 100=24.67 \% \text {. }
$$

Increase in load is about $12.5 \%$ more than increase in flexure capacity. 


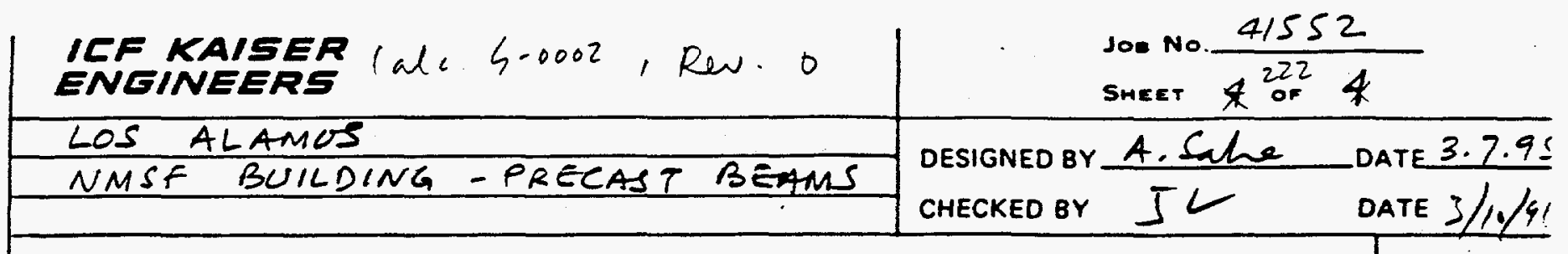

Shear Capacity:

shear reinforcement in beam web is not available Existing Condition:

$$
V_{u}=(1.4 \times 116+1.7 \times 34) \times \frac{69}{2}=7590^{16 .}
$$

New Condition:

$$
v_{u}=(220+1.4 \times 37) \times \frac{69}{2}=9377 \frac{16}{-}
$$

Increase in shear load $=\frac{9377-7590}{7590} \times 100=23.5 \%$

Modification to existing roo beams may be required unless:

(i) Existing beams have flexural capacity to carry additional $13 \%$ load.

(ii) Existing beams have shear capacity to carry additional $24 \%$ load.

Possible solutions:

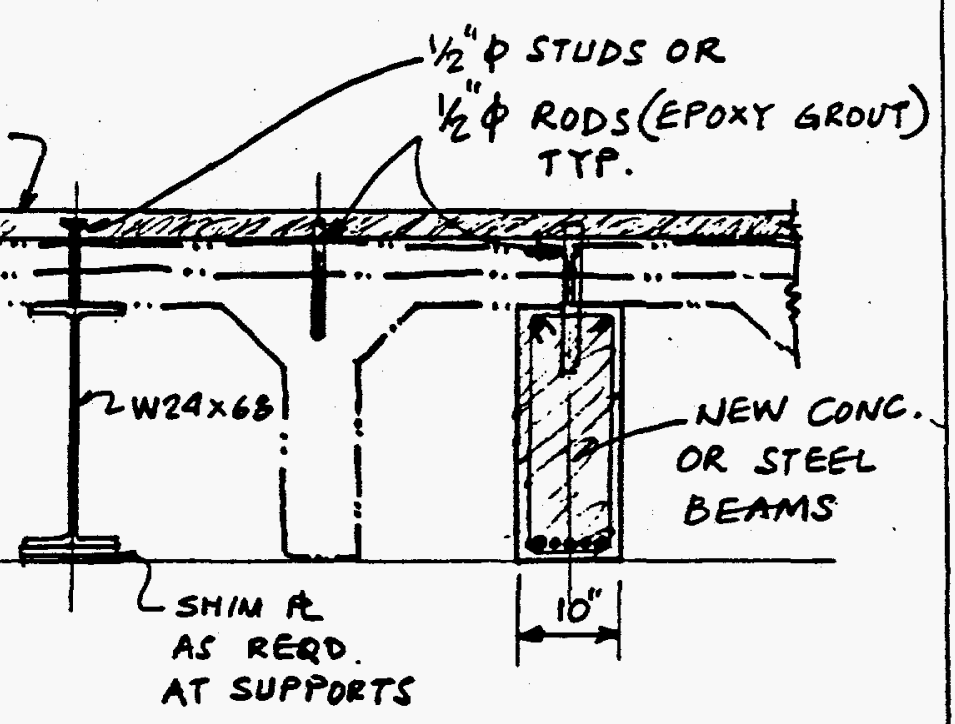

NEW CONCRETE OR STEEL BEAMS WILL BE 4'-O OR B'-O OC. SPACING WILL BE DETERMINED DURING FINAL DESIGN. 


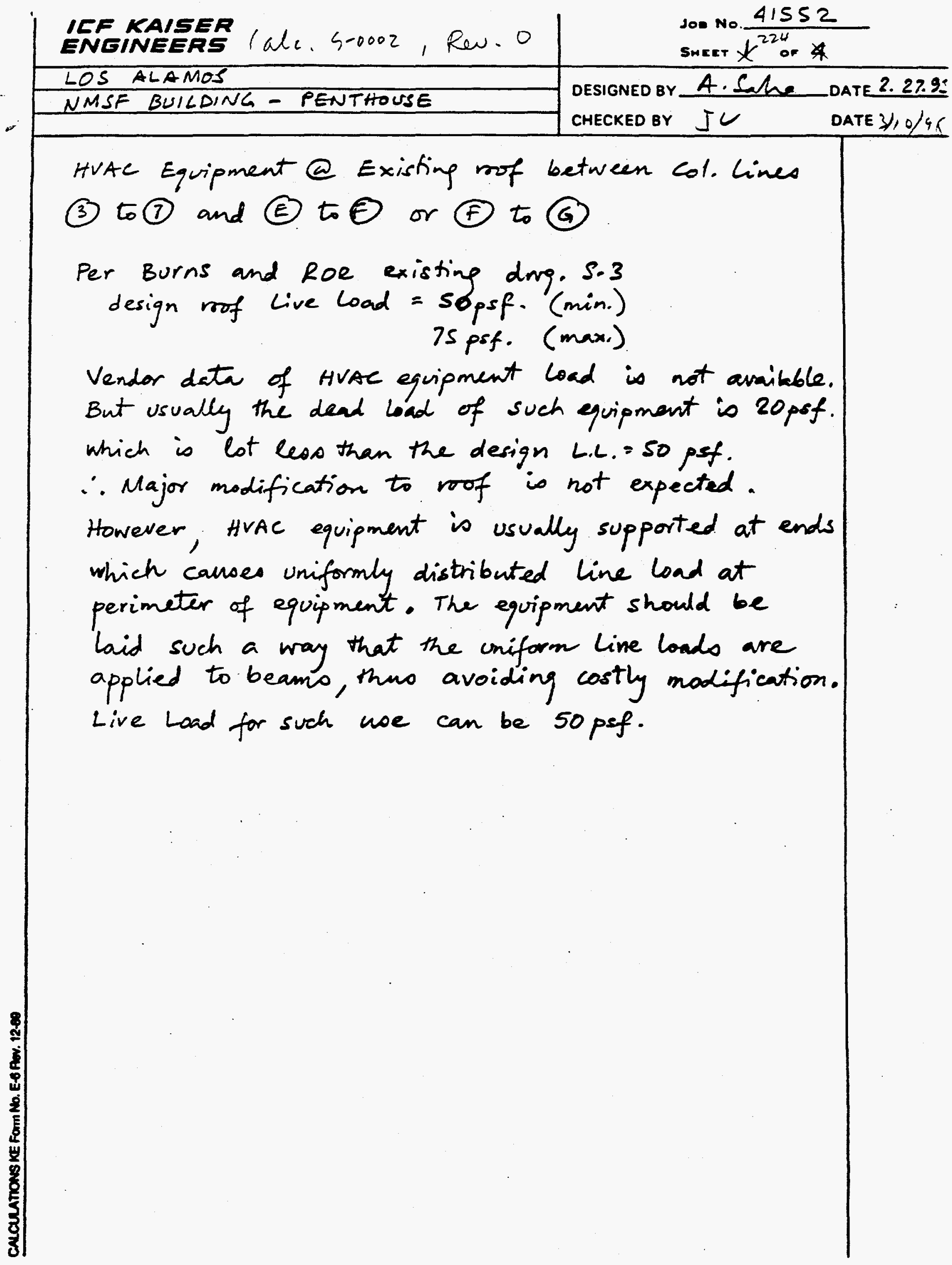




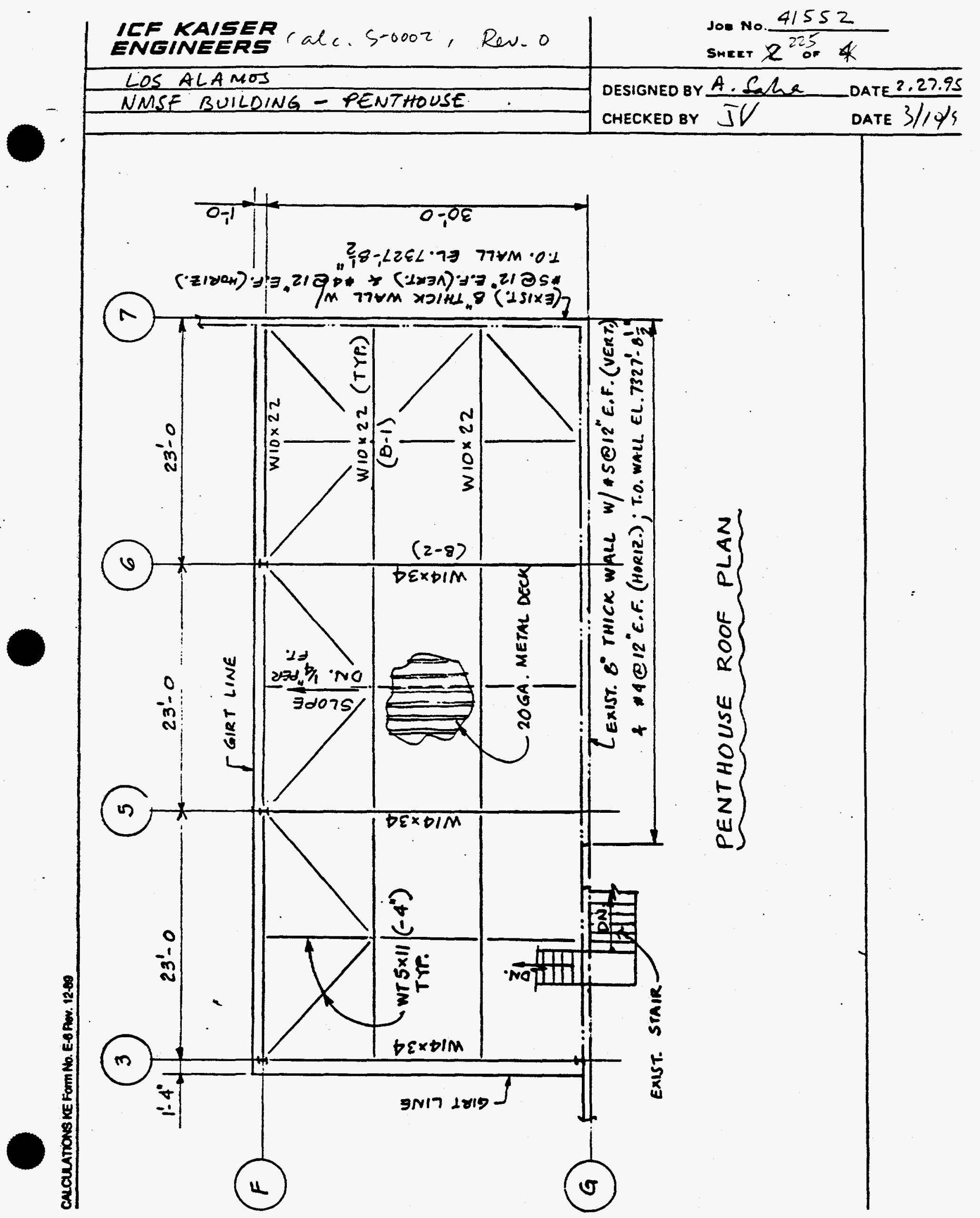




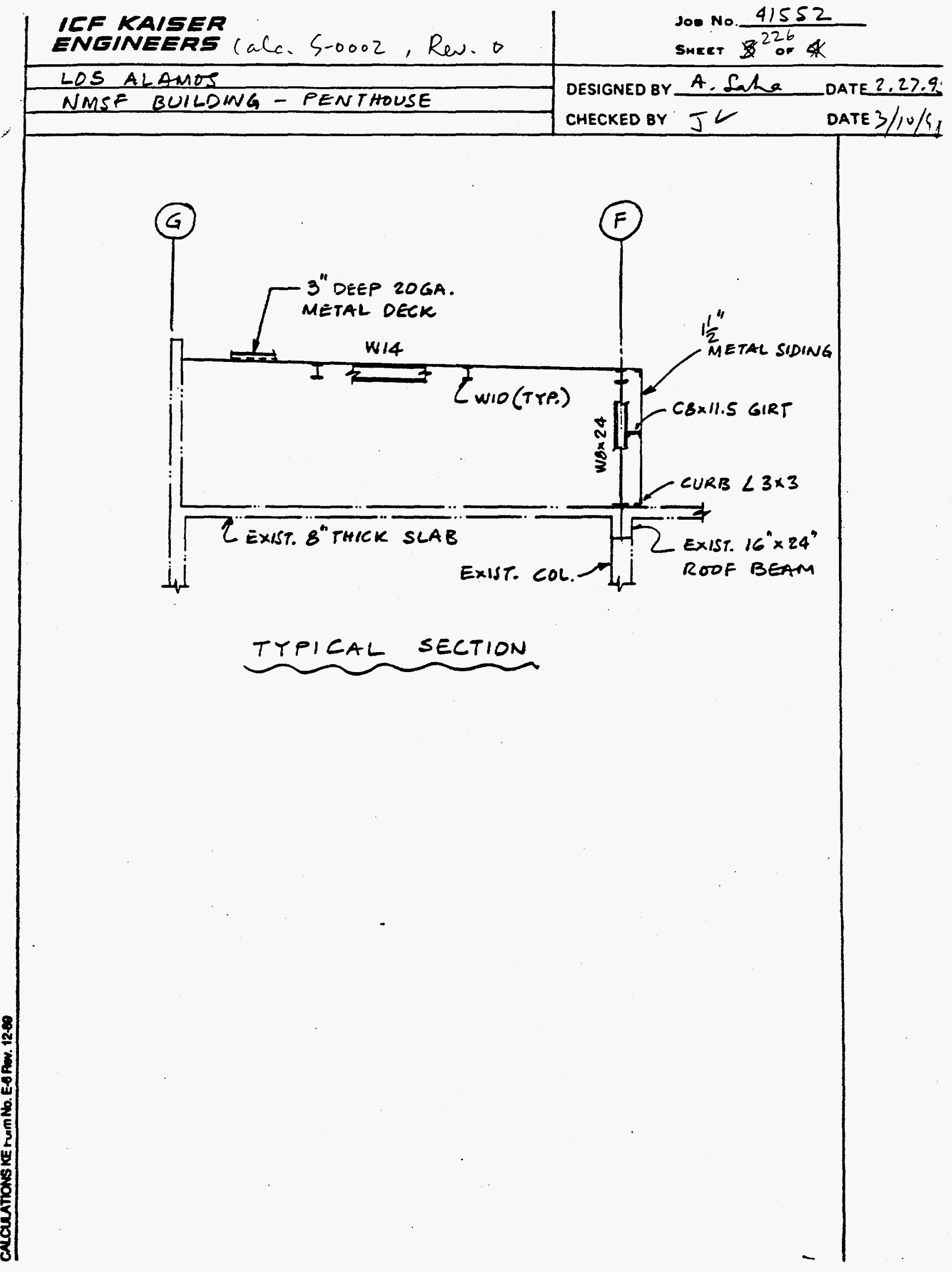




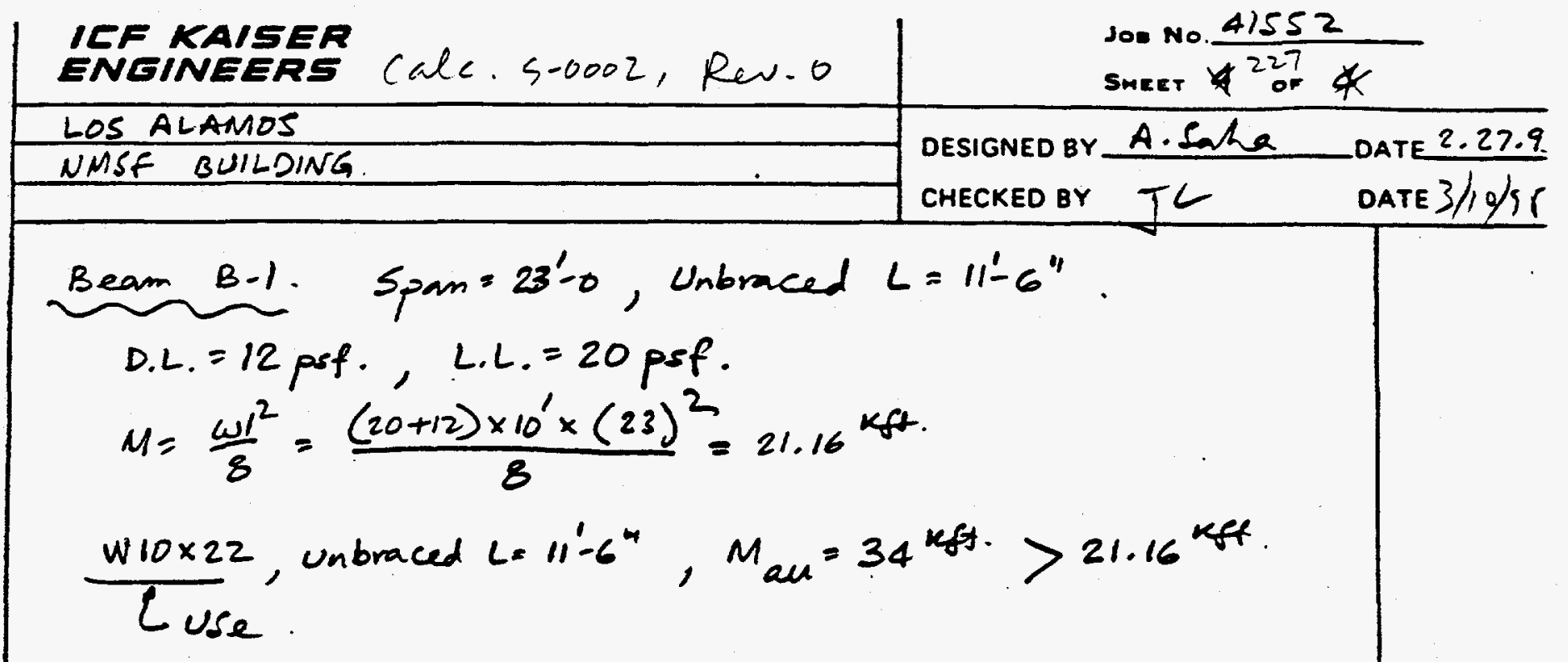

Beam B.2

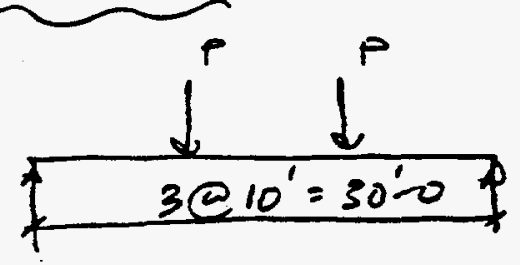

$$
\begin{aligned}
& P=320^{\mathrm{dk/k} \times 23^{\prime}}=7.4^{k} \\
& M_{\text {max }}=7.4^{k} \times 10^{\prime}=74^{\mathrm{kft}}
\end{aligned}
$$

Unbraced $L=10^{\prime}-0$

wi $\times 34$ good for $3 g^{\text {kt. }}>74^{\mathrm{kft}}$.

Col. Lond $=7.4^{k}+$ Wind Load.

Provide $W 8 \times 24$

Lines (6) \& (7), existing $8^{n}$ thick wall will also carry lateral load.

line $\Theta \longrightarrow$ New bracing

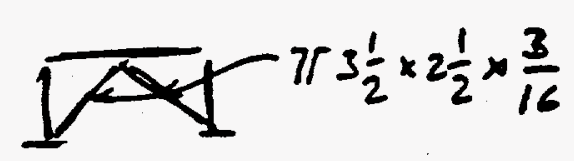
Line (3) $\longrightarrow$ New bracing. 


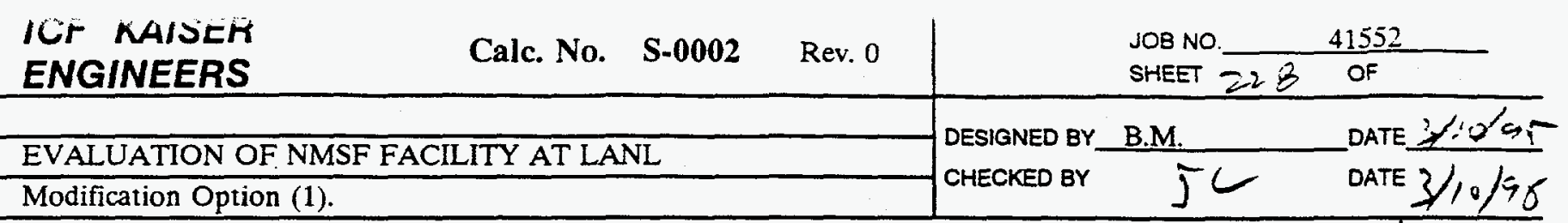

8.3 ANALYSIS OF NMSF BUILDING INTERACTION WTTH THE BURIED TUNNEL 
ICF KAISER

JOQ No. $\frac{41552-0.5 t-13}{2230}$

Les Alamos Natirnel 0.6 (LANL)

DESIGNED BY S.k.

DATE $2 / 60 / 95$

CHECKED BY BYP

DATE $=$ I 7 ?

1.0 9ntrobuction

Buried Tuanel Analysis

A simglified approach to evaluate the seismic Vulaterabitity of buried $R / C$ tunnels is presented. The procedure $\therefore$ abopked Grom the buried pipe analysin, ot two types of liadings ars inveskigated:

i. Benking atid bating of buried turad due to wave propagation

2. Benting axil bating dae to relative movemat btwa ta zeiltingt th soil $@$ peratanti- of a Guried tuand to Guilting-Seisaric Ancha movemeat (SAM).

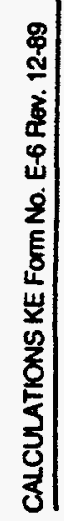


ICF KAISER

Jos No. $\frac{4: 552-056-03}{3^{1}}$

SHEET 2 of 20

$\angle A N L$

DESIGNED BY

s.k.

DATE $\frac{2 / 10 / 49}{\text { DATE } 2 / 7 / 92}$

2.0 References

1. C.K. Goen, "Soil-Structure Puthaction Analysis"

$$
\text { PF4-ESA13-26-001, Rev2, May 10, 1994 }
$$

2. N.M. Newame, J.A. Blume, \& K.K. Ka pue, "Design Respoure Speedre for Nuclear Power Plants", ASCE Struetural Eagigersing meeting, San Fracisce, CA, Adid 1993

3. The Commitice o.. Seismic Analys.is of the ASCE Stractumal Division Conmitte on Nuclear Structuras o Mateids, "Seiric Responss of Buried Pipes a Stmatmal Congunors" 1983, ASCE, N.Y.

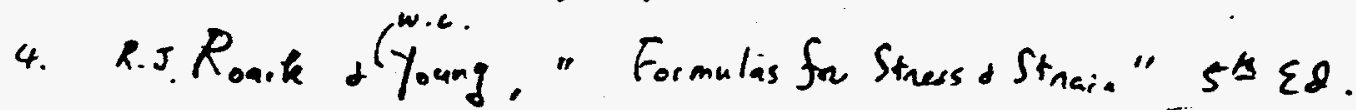

1905, MCGnav-ltill, Tac. N.Y.

4a. SAKURA1 \& TAKAHSHI', "DYNTAMC STRES OF UNDORGROUDO PIPQU 5.

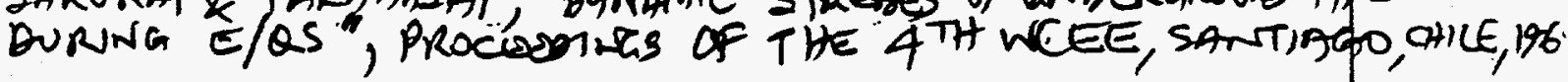
M. B. Atalay," Seimic Amalgsis + Desig. of Buried Piping" Lifelia Earagare Engineoring, the third U.S. Conferewce, Lo' Angeles, CA, Aug. 22-23, 199,

6. ASME, ASME $B_{31.1}$ Aypendix VII

D. C.H. Trautmana + T.O. Q'Rourke,"Laterel Fna-Diop/ant Response of Buied Pipe", ASCE Journal of Feoterhirel Enginany, $\forall .1111, M_{0.9}, S_{e p} .1985$

8. Drawings LA-ZJ.B.S.1.1 -

II. 1 


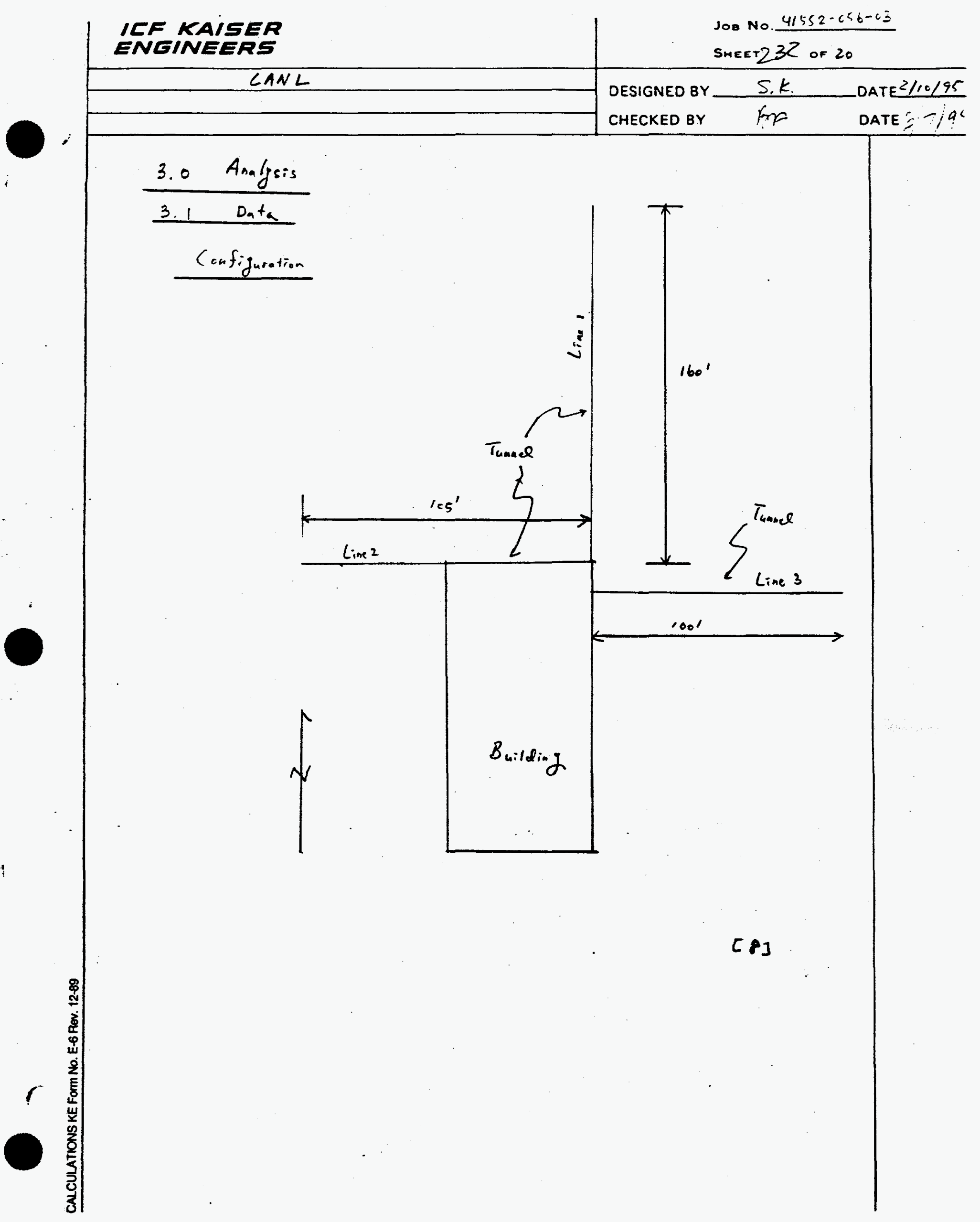




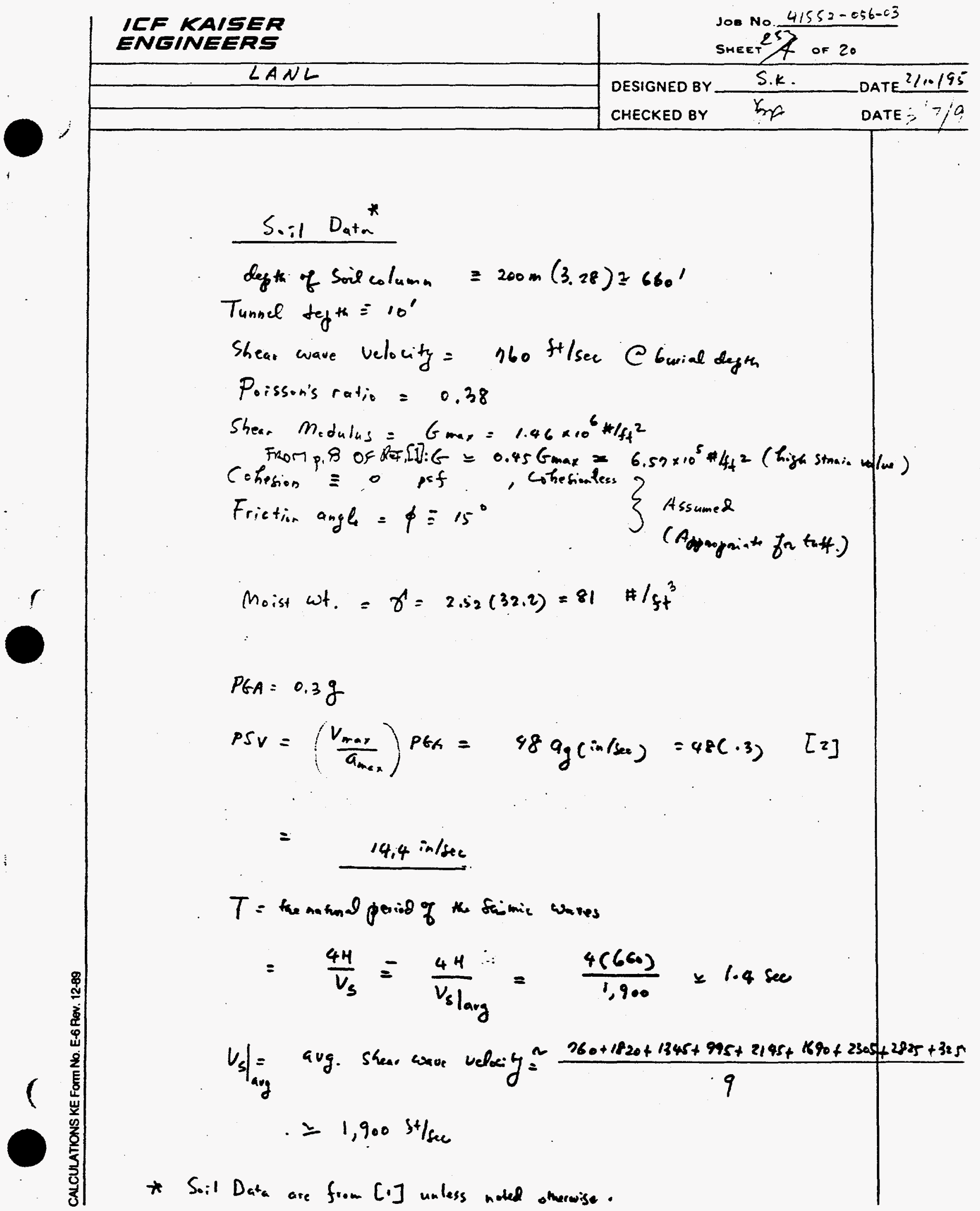




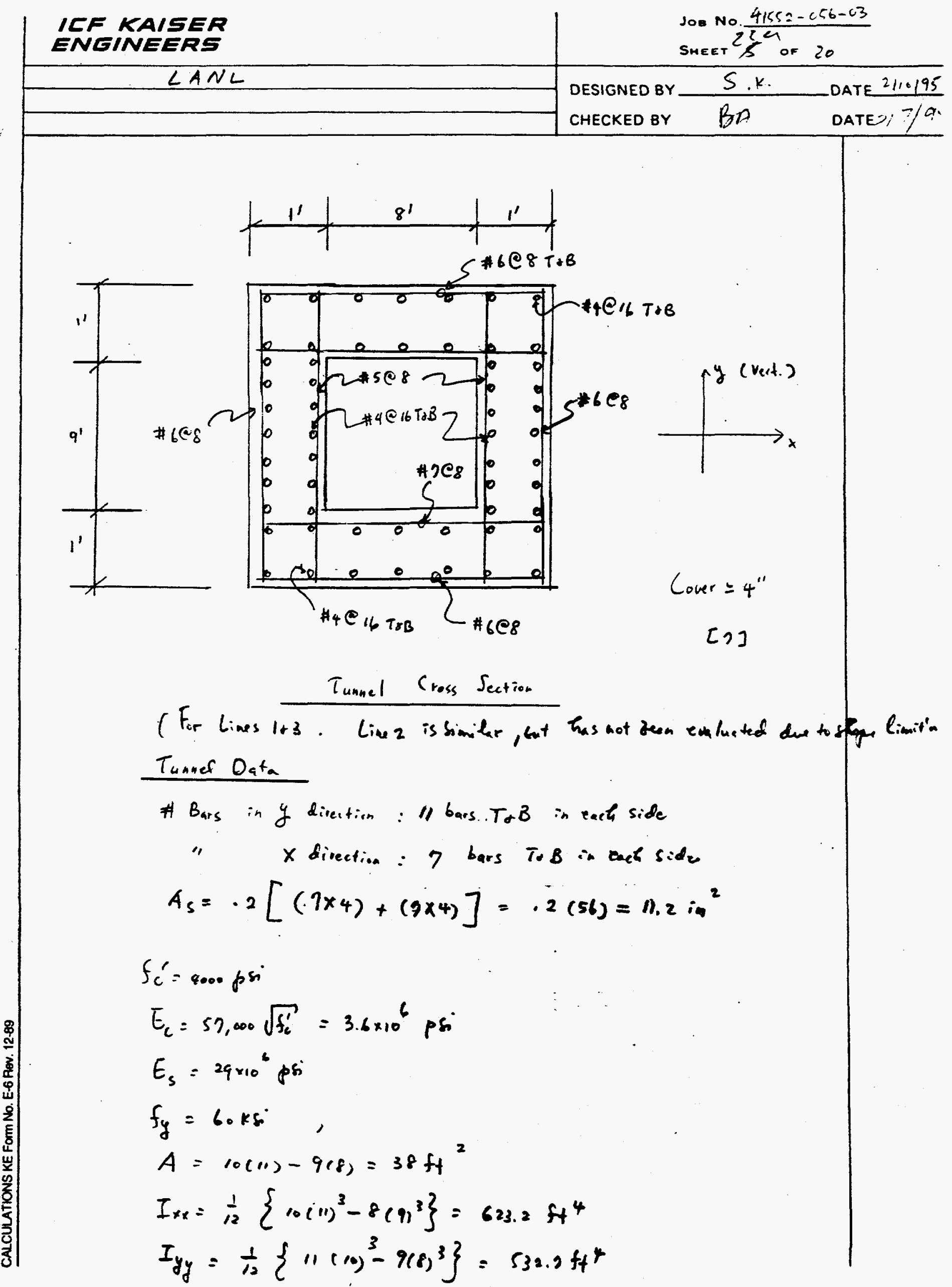




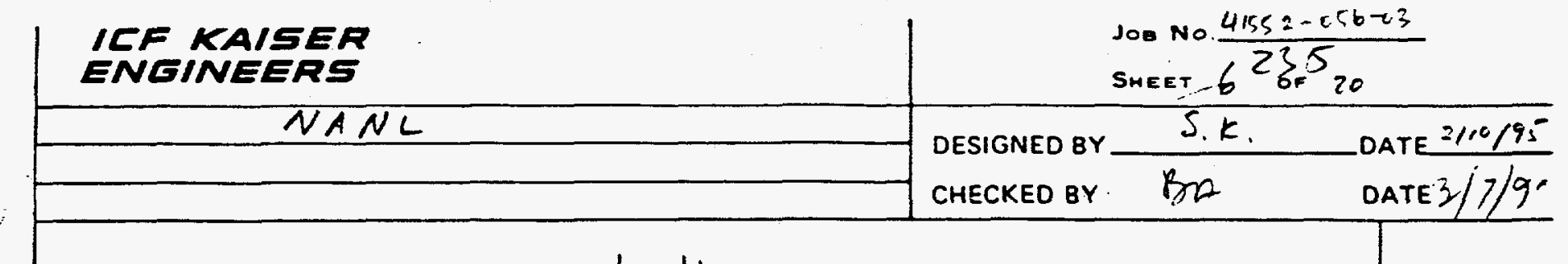

3.2 Wave Propagation Loading

(i) Axial Loads:

$$
\varepsilon_{a}= \pm \frac{b}{c}=\frac{14.4}{360(12)}=1.6 \times 10^{-3} \mathrm{in} / \mathrm{in} \cdot[3]
$$

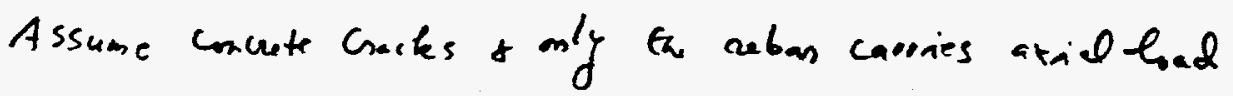

$$
F_{a}=1.6 \times 10^{-3}\left(29 \times 10^{3}\right)\left(11.2 \mathrm{in}^{2}\right)=520^{\mathrm{k}}
$$

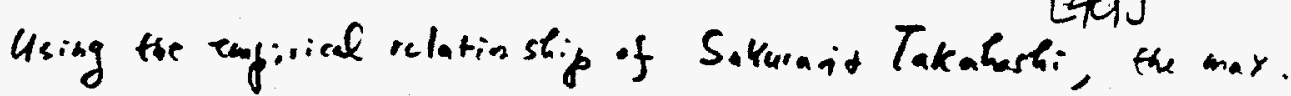
axial load from the ax al strain cabot exceed

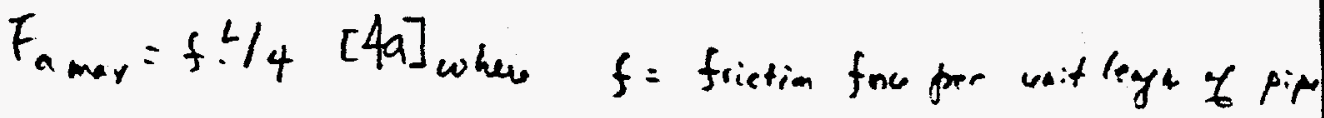
$L=$ ware length of seismic waves

Assuming tor friction met. of 0.5 ,

$$
\begin{aligned}
& \sigma=\gamma l=81(10)=810 \text { psf } \\
& f=810(.5)(11+10)(2)=17,010 \# / 5 t .
\end{aligned}
$$

Checking siik-sid failure just away from the pipe,

$$
\begin{aligned}
& \tau=\sigma \tan \phi \\
&=810 \tan 15^{\circ}=217 \text { psf } \\
& f=217(11+10)(2)=-9,114 \| / 5 t \\
& \therefore u_{s_{e}} f=9,114 H / 5 t
\end{aligned}
$$




$$
\begin{aligned}
& \text { | InF KAISER } \\
& \begin{array}{l}
\text { JOE NO. } \frac{41562-\operatorname{cs} 6-c j}{2 ? C} 20 \\
\text { SHEET }]_{\text {OF } 20}
\end{array} \\
& \text { NAN } \\
& \text { DESIGNED BY } \\
& \text { Sk. } \\
& \text { DATE } 2 / 10 / 9 \text { ! } \\
& L=T C=1.4(360) \\
& \text { [3] } \\
& =1,064^{\prime} \\
& F_{a \text { mar }}=f L / 4=(9,114)(1,064) / 4 \cong 2,420^{K} \\
& \text { Note that } F_{\text {max }}>F_{a} \\
& \therefore \text { Use } F_{a}=520^{k}
\end{aligned}
$$

Assume resultant seismic wave is Causing only honizontel motions

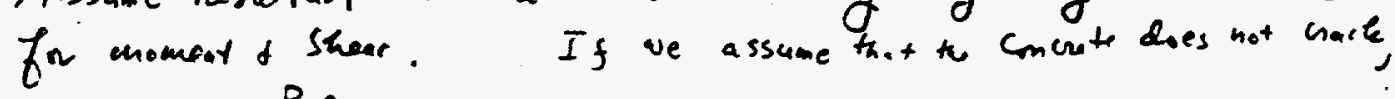

$$
\begin{aligned}
\varepsilon_{b} & = \pm \frac{R_{a}}{c^{2}}[3] \\
& = \pm \frac{12(5)(.3)(386.9)}{\{n 60(12)\}^{2}}=8.36 \times 10^{-5} \mathrm{ia} / \mathrm{in}
\end{aligned}
$$

Strain Compatich Determinate- of $M+V$ :

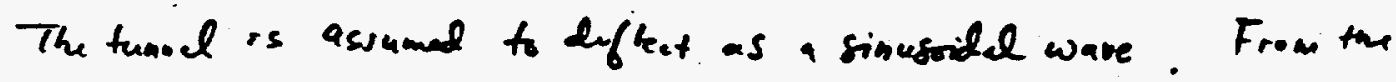
wave of, we get:

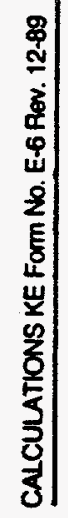

$$
\begin{aligned}
& y=\Delta^{\prime} \sin \left(\frac{\pi x}{42}\right) \quad[3] \\
& y^{\prime \prime}=-\Delta^{\prime}\left(\frac{2 \pi}{2}\right)^{2} \sin \left(\frac{\pi x}{42}\right), y^{\prime \prime}=-\Delta^{\prime}\left(\frac{2 \pi}{4}\right)^{3} \cos \left(\frac{\pi x}{42}\right)
\end{aligned}
$$

$$
M=E I y^{\prime \prime}+V=E I y^{\prime \prime \prime}
$$




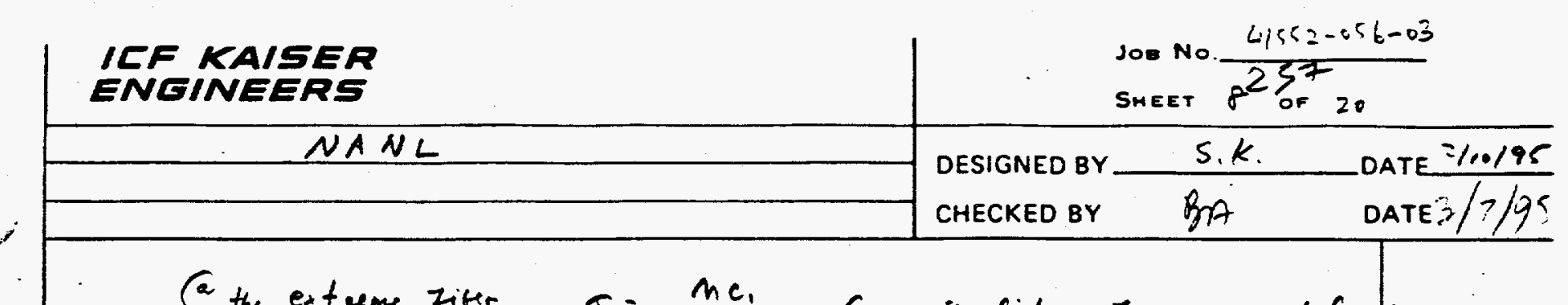

Ca the extreme fiver, $\sigma=\frac{M c_{1}}{I}, c_{1}=$ the vintage from ton neut rel ax is

Then, $E \varepsilon=E f y^{\prime \prime} c_{1} / \Psi^{\prime},+\varepsilon=y^{\prime \prime} c_{1}$

$$
\begin{aligned}
\Delta^{\prime} & =y^{\prime \prime} /\left(\frac{2 \pi}{2}\right)^{2}, y^{\prime \prime}=\frac{M}{E_{I}}=\frac{\varepsilon}{c_{1}} \\
\Delta^{\prime} & =\frac{M}{E_{I}\left(\frac{2 \pi}{L}\right)^{2}}=\frac{\varepsilon}{C_{1}\left(\frac{2 \pi}{L}\right)^{2}} \\
& =\frac{8.36 \times 10^{-5}}{S(12)\left\{\frac{2 \pi}{1064(12)}\right\}^{2}} \cong 5.75 "
\end{aligned}
$$

Then $y_{\text {max }}^{\prime \prime}=\Delta^{\prime}\left(\frac{2 \pi}{2}\right)^{2}=(5.75 / 12)\left(\frac{2 \pi}{1064}\right)^{2}=1.692 \times 10^{-5} 1 / \mathrm{ft}$.

$$
\begin{gathered}
\varepsilon_{m a x}=y_{m a,}^{\prime \prime} c_{1}=1.672 \times 10^{-5}(5)= \\
\varepsilon_{\max } \ll \varepsilon_{a 110, \text { mamas }}=.003
\end{gathered}
$$

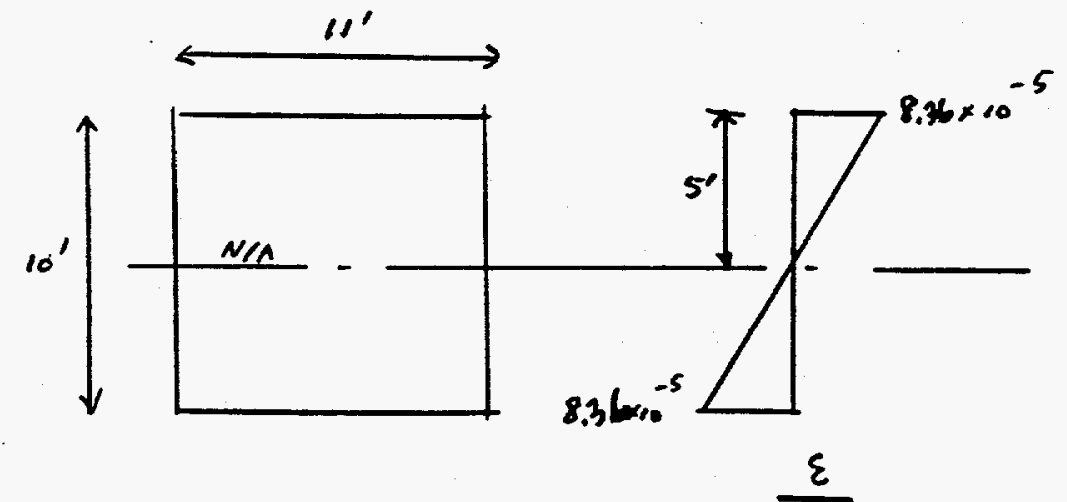

$\varepsilon$ 


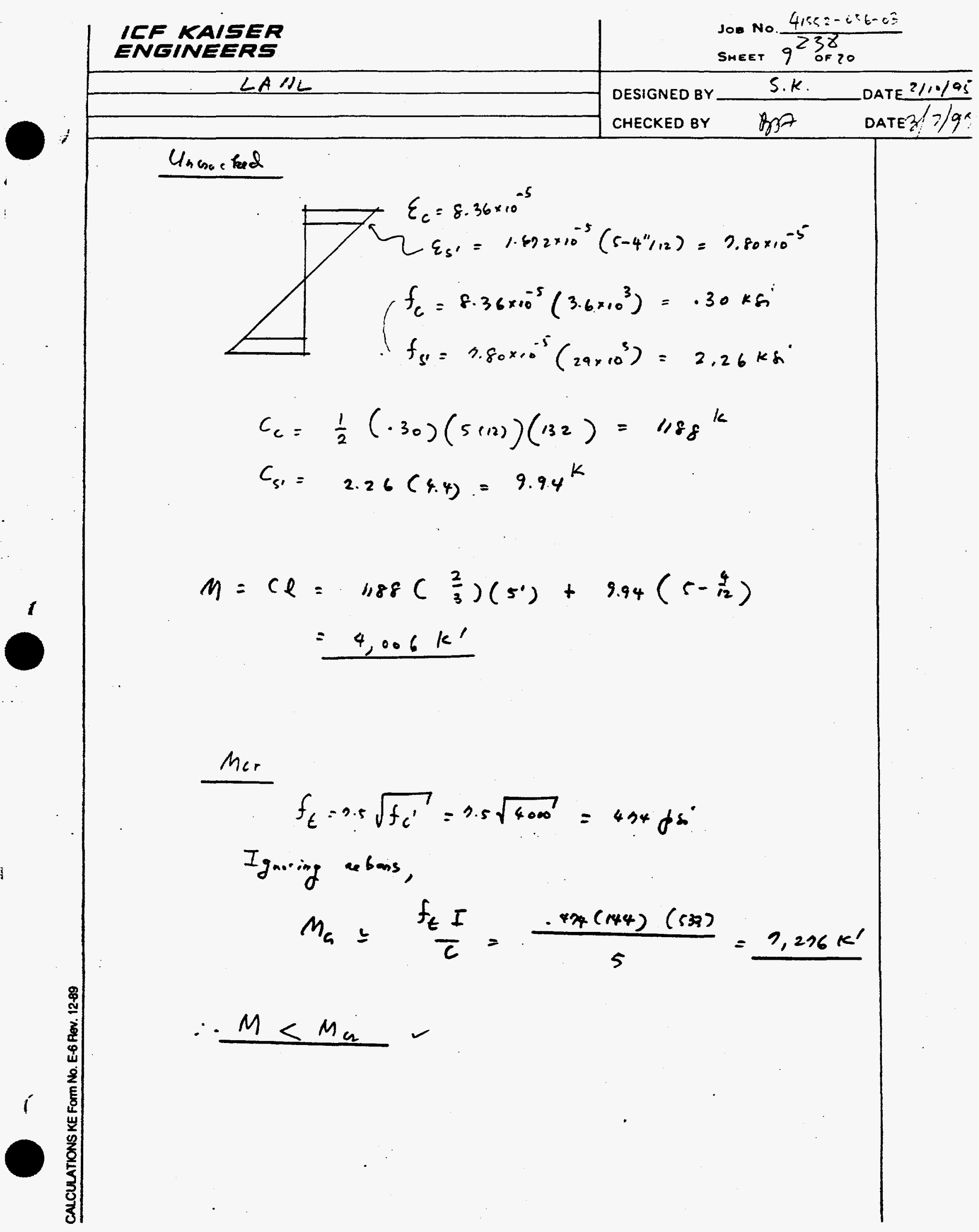




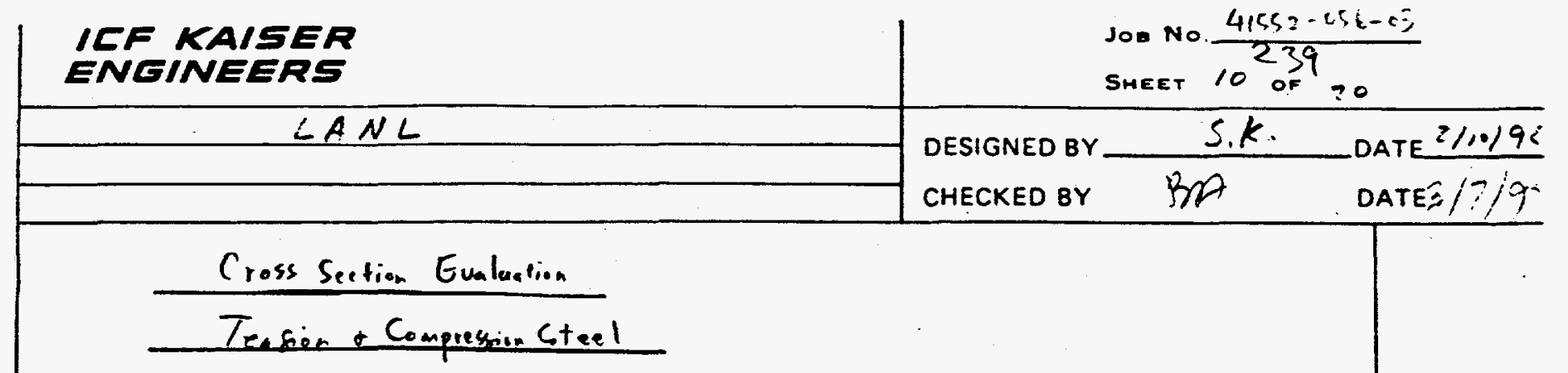

The strain is $\quad 7.80 \times 10^{-5}=\varepsilon_{\text {s }}$

$$
\varepsilon_{y}=F_{s} / E_{s}=.002, \quad \varepsilon_{y}>\varepsilon_{s}
$$

$\therefore$ Rebus do not yield.

$$
\sigma_{A_{S}}=\varepsilon E_{s}=\left(0.80 \times 10^{-5}\right)\left(29 \times 10^{3}\right)=2.3 \mathrm{ks}
$$

Adding the stress the to th axil bad,

$$
\sigma_{A, \text { total }}=2.3+\frac{520}{1.2}=46.9 \mathrm{kfi}<\sigma_{y}=60 \mathrm{~kg} .
$$

Concrete

$$
\begin{aligned}
& f_{c}^{\prime}=4 \mathrm{~kg} d o \text { assume lines, } \sigma-\varepsilon \text { up to } \sigma=.45 f_{c}^{\prime} \\
& \left.\varepsilon_{c}\right|_{\text {linear } \text { limit }}=.45(4) / 3.6 \times 10^{3}=.0005 \mathrm{i} / \mathrm{m} \\
& \left.\varepsilon_{c}\right|_{\text {le. }}>\varepsilon_{c \text { ma, }}=8.36 \times 10^{-5}
\end{aligned}
$$

Adding the stress due to the axil load,

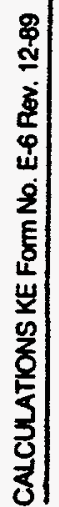

$$
\begin{aligned}
\varepsilon_{c, t+l} & =8.36 \times 10^{-5}+\frac{520}{10(4)(144)\left(3.6 \times 0^{3}\right)} \\
& =9.27 \times 10^{-5} \ll \varepsilon_{c a l l o w}=.03
\end{aligned}
$$

$\therefore$ If we assume that the concrete does not coal, concrete strain a steel stresses are whin the allowath. 


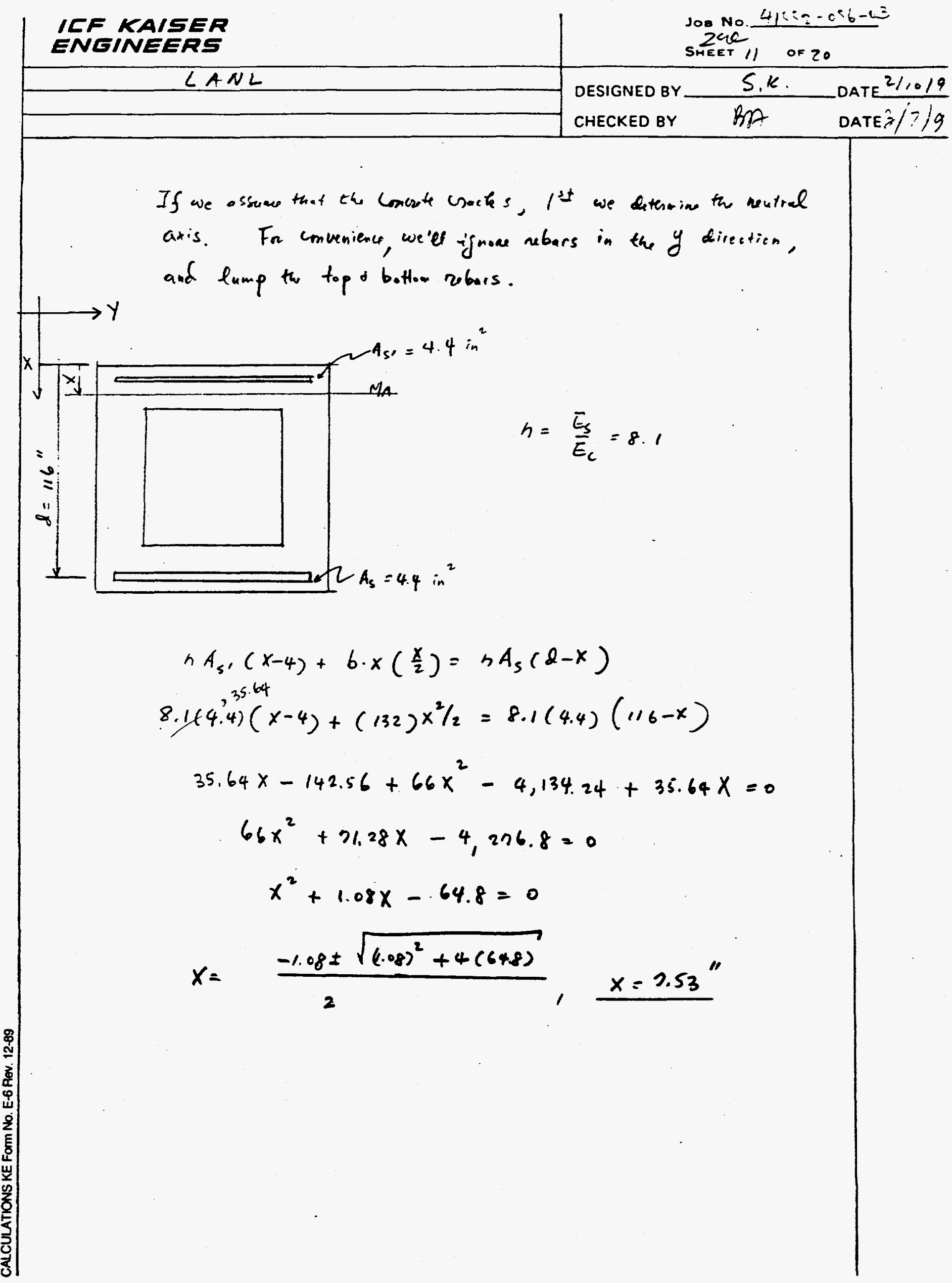




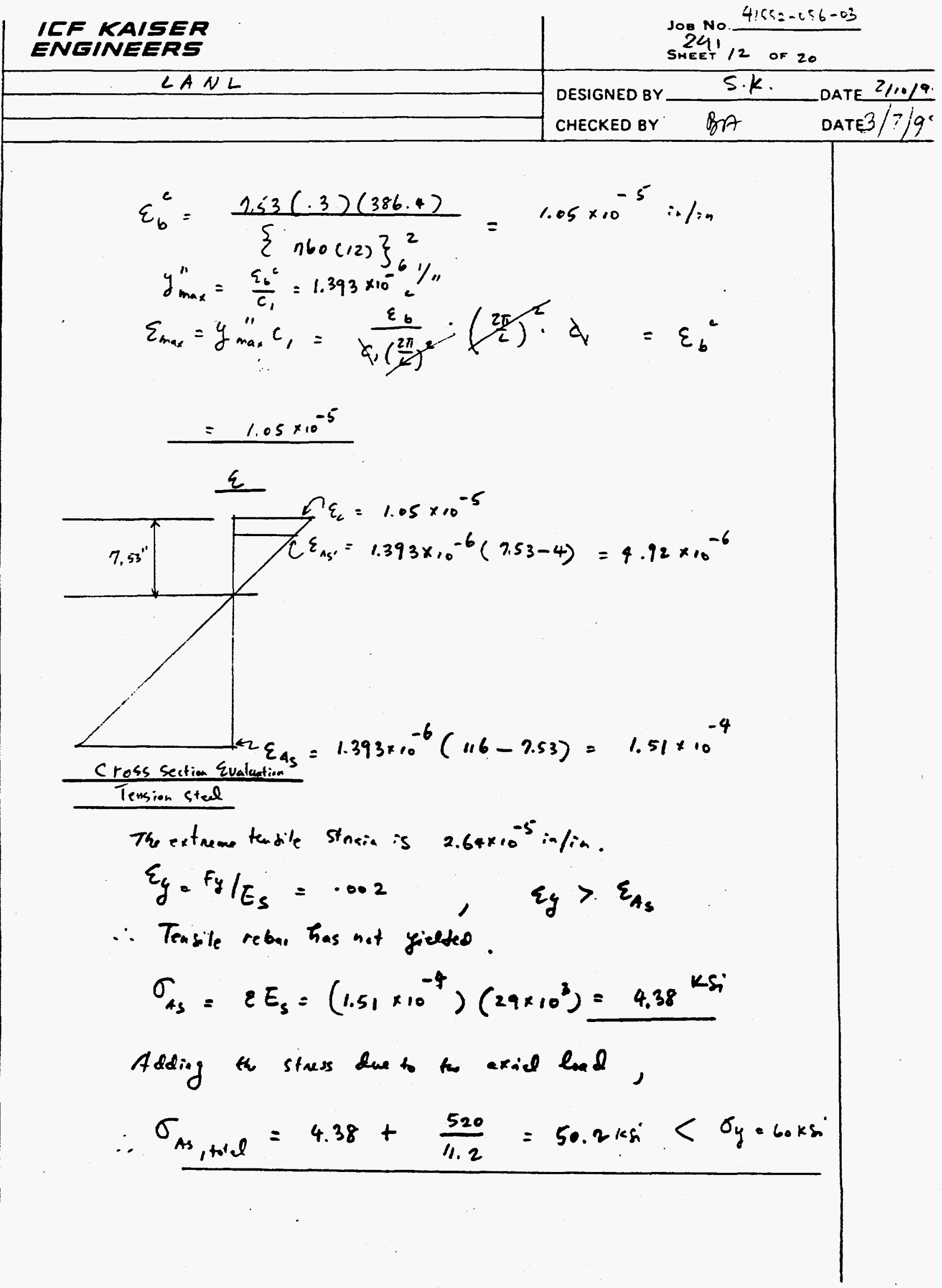




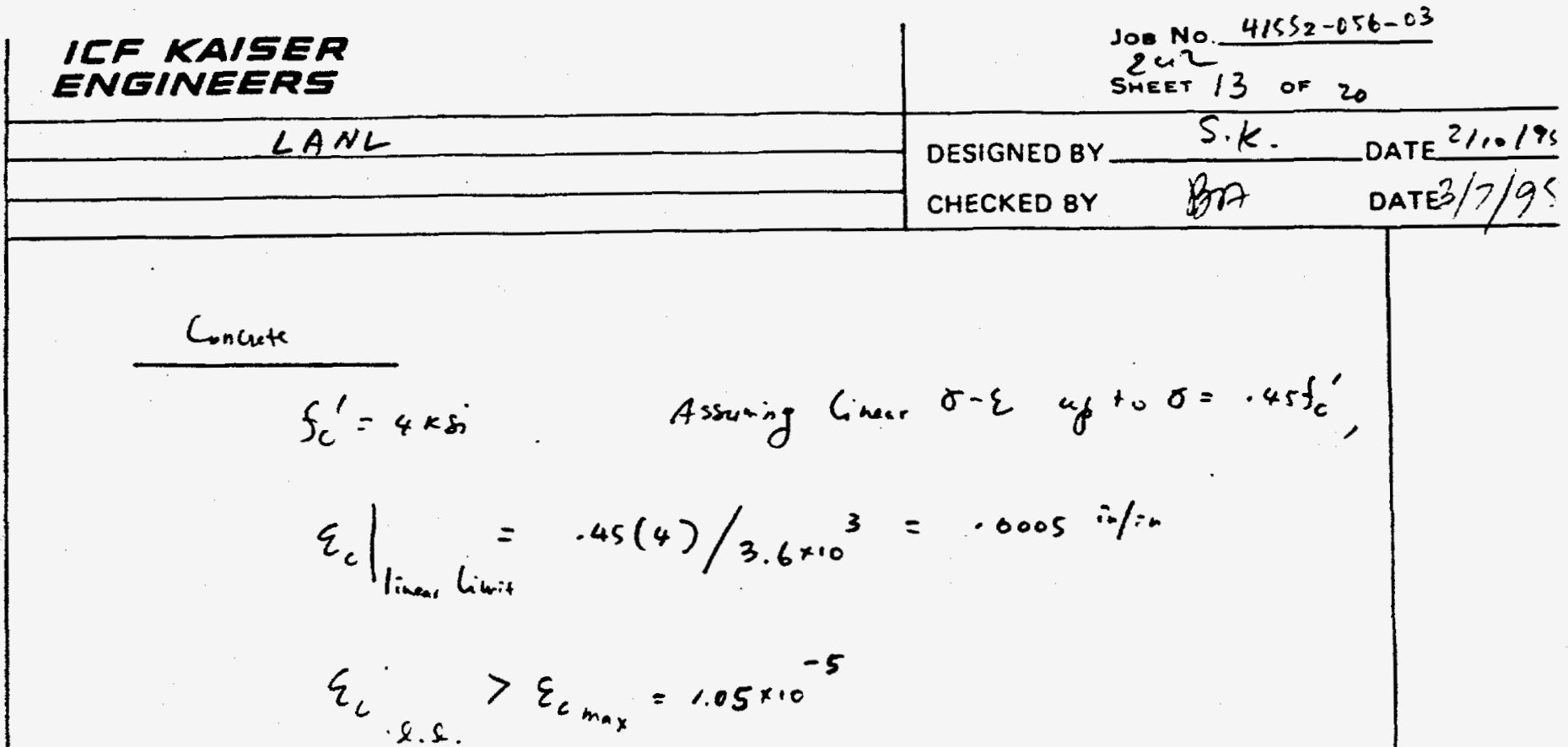

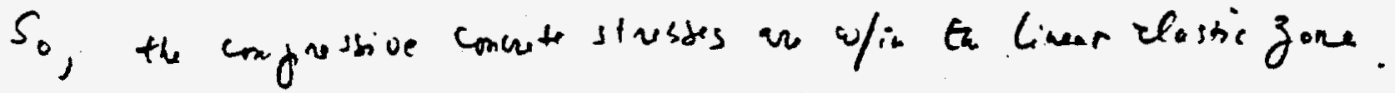
A2ding the stus der to the arial lo.e,

$$
\begin{aligned}
\varepsilon_{c, \text { tets }} & =1.05 \times 10^{-6}+\frac{520^{K}}{10(11)(144)\left(3.6 \times 10^{3}\right)} \\
& =1.02 \times 10^{-5} \ll \varepsilon_{c} \text { elleres }
\end{aligned}
$$

$\therefore$ If we assund that the conceate cracts, Concute compressioe straint tensile stal stress are w/in the aldowable. 


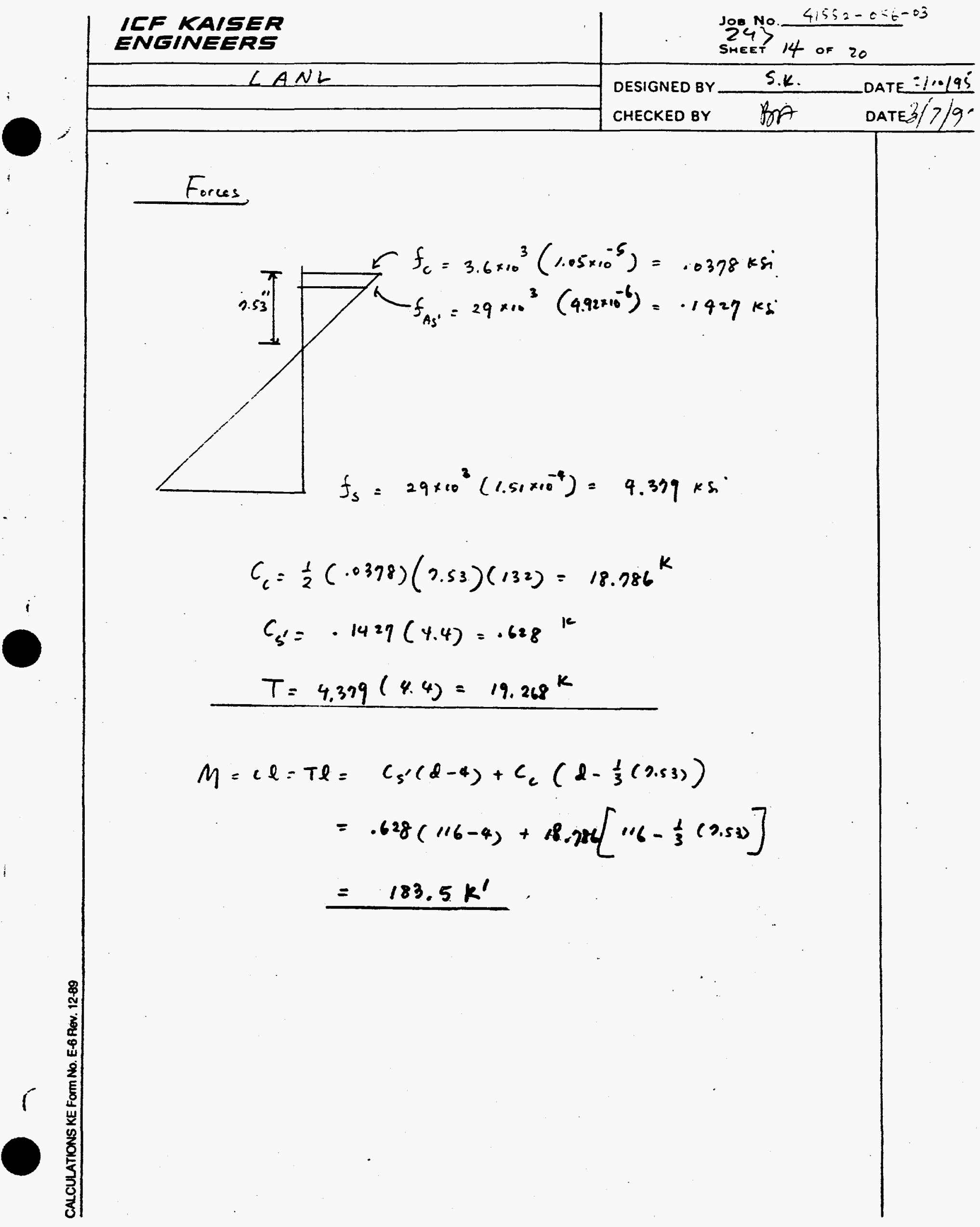




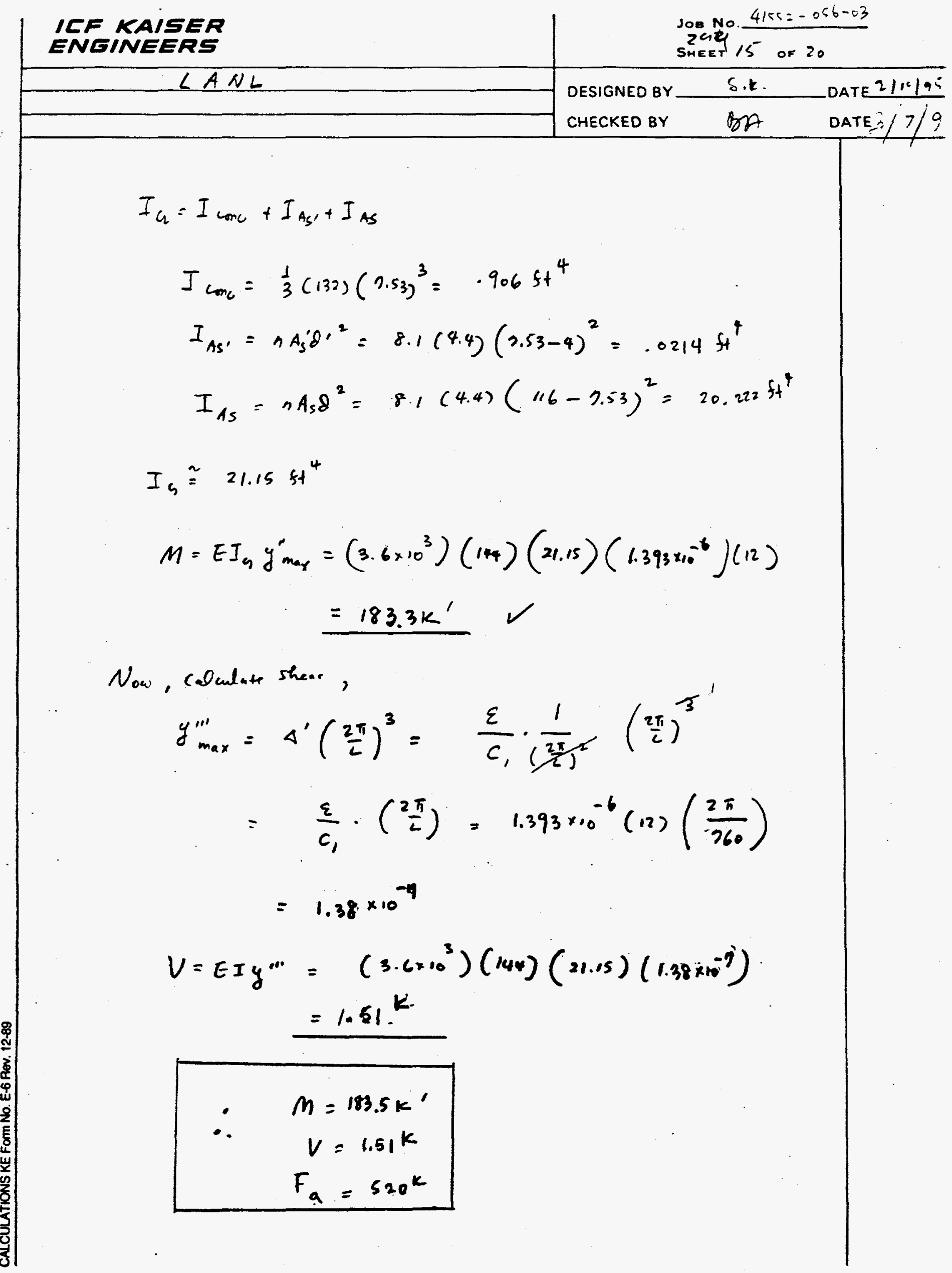




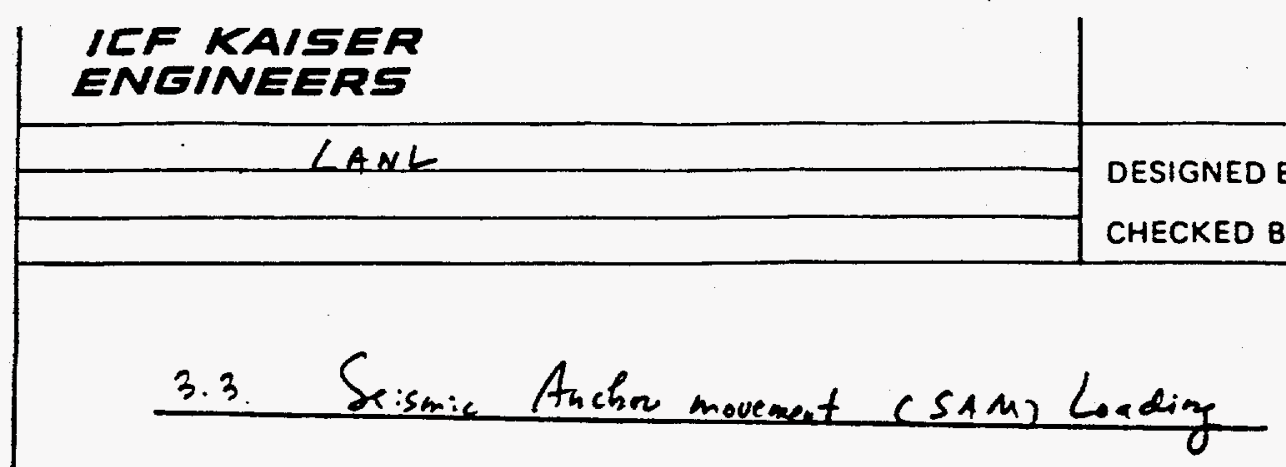

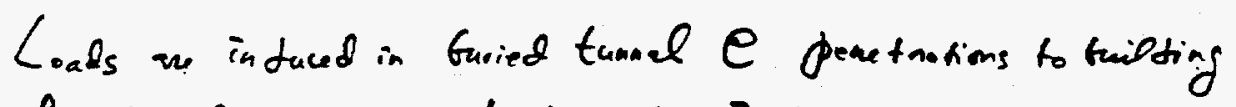
tue to relative movement btw. the building the soil.

(i) Arid Direction

$$
\begin{aligned}
P & =\sqrt{2 E A f \delta_{\text {ariel }}} \quad \text { Assume } \delta_{\text {axil }}=.1^{\prime \prime} \\
& {[5] } \\
& =2\left(3.6 \times 10^{6}\right)(38.144)\left(9, \frac{114}{2}\right)(0.1) \\
l_{p} & =\frac{1929}{9.114}=189^{\prime}
\end{aligned}
$$$$
\text { So, } \quad P_{\text {max }}=f l_{\text {max }}
$$

$$
=(9.114)(160) \cong 1,460^{k}
$$




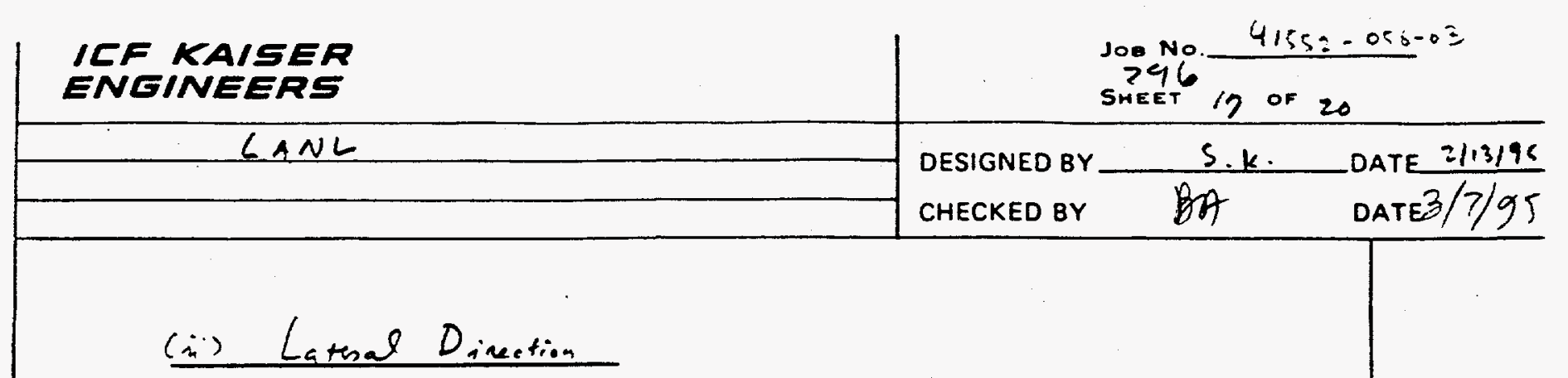

In the case of alatioe movint btuas. to builting + sail in the finuction transuese to tas tunnel, bats as determinad assuming tar tuncel os a bean supponted on laski fountation w/ a fixed end $P$ the conmetion to the Guldling.

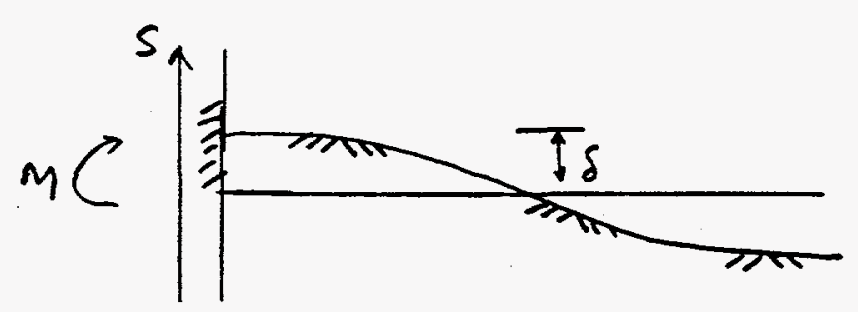

$$
\begin{aligned}
& \text { Caluntaing } k \text { [6] }
\end{aligned}
$$

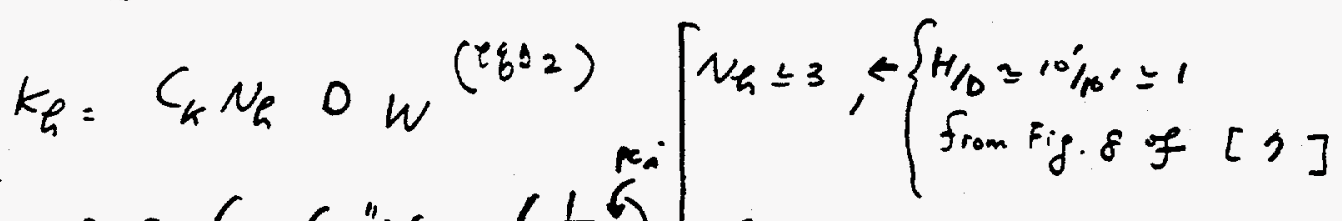

$$
\begin{aligned}
& =50(3)(120)(81)\left(\frac{1}{1928}\right)\left[C_{k}=50\right. \\
& \simeq 844 \text { ps: } \\
& \beta=\left(k_{h} / 4 E I\right)^{\frac{1}{4}}=\left[\frac{(844)}{4\left(3.6 \times 10^{6}\right)(21.15)(R)^{4}}\right]^{\frac{1}{4}} \\
& \leq .0341 / 12 \quad[4] \\
& \beta l=0.034(100)(12) \leq 4.08<6 ; F_{\text {iaite logh }} \text {. }
\end{aligned}
$$

Use a finite beam formula. 
IVF KAISER

Joe No. $4+52-056-0=$

SHEET $r 8$ of 20

LAN

DESIGNED BY

s.k.

DATE $2 / 13 / 95$

CHECKED BY. MOA

DATE $3 / 2 / 9^{\circ}$

Table 7 Case 6 of fixed end byte + gee night [4]

$$
a=0=x, \quad y_{A}=\theta_{A}=0
$$

$C_{1}=\cosh \beta l \cos \beta l=\cosh (4.1) \cos (9.1)=-10.35$

$C_{a 2}=\cosh \beta(l-a) \sin \beta(l-a)+\sinh \beta(l-a) \cos \beta(l-a)$

$=\cosh (4.1) \sin (4.1)+\sinh (4.1) \cos (4.1)$

$\leq-24.694-17.338=-42.03$

$C_{a_{3}}=\sinh \beta(l-a) \sin \beta(l-a)=\sinh (4.1) \sin (9.1)$

$\leq-24.68$

$c_{11}=\sinh ^{2} \beta l-\sin ^{2} \beta l=\sinh ^{2}(4.1)-\sin ^{2}(4.1)$ $=-909.1$

$2+C_{11}=911 \cdot 1$

$c_{4}=\cosh \beta l \sin \beta l-\sin h \beta l \cos \beta l$

$=\cosh (4.1) \sin (4.1)-\sinh (4.1) \cos (4.1)$

$=-24.69 t+17.338=-7.36$

$C_{2}=\cos b \beta l \sin \beta l+\sin \beta \beta l \cos \beta l=-24.694-19.338=-42.03$

$k_{1}=\frac{c_{1} c_{a_{2}}+c_{4} c_{a_{3}}}{2+c_{n}}=\frac{117,35(-42.03)+(-0.36)(-24.68)}{911.1}=1,0$.

$k_{\frac{1}{4}}=\frac{2 C_{1} C_{a_{3}}-C_{2} C_{a_{2}}}{2+C_{11}}=\frac{2(-12.35)(-24.68)-(-42.03)(-42.03)}{911.1}$

$\therefore \quad 1.0$ 


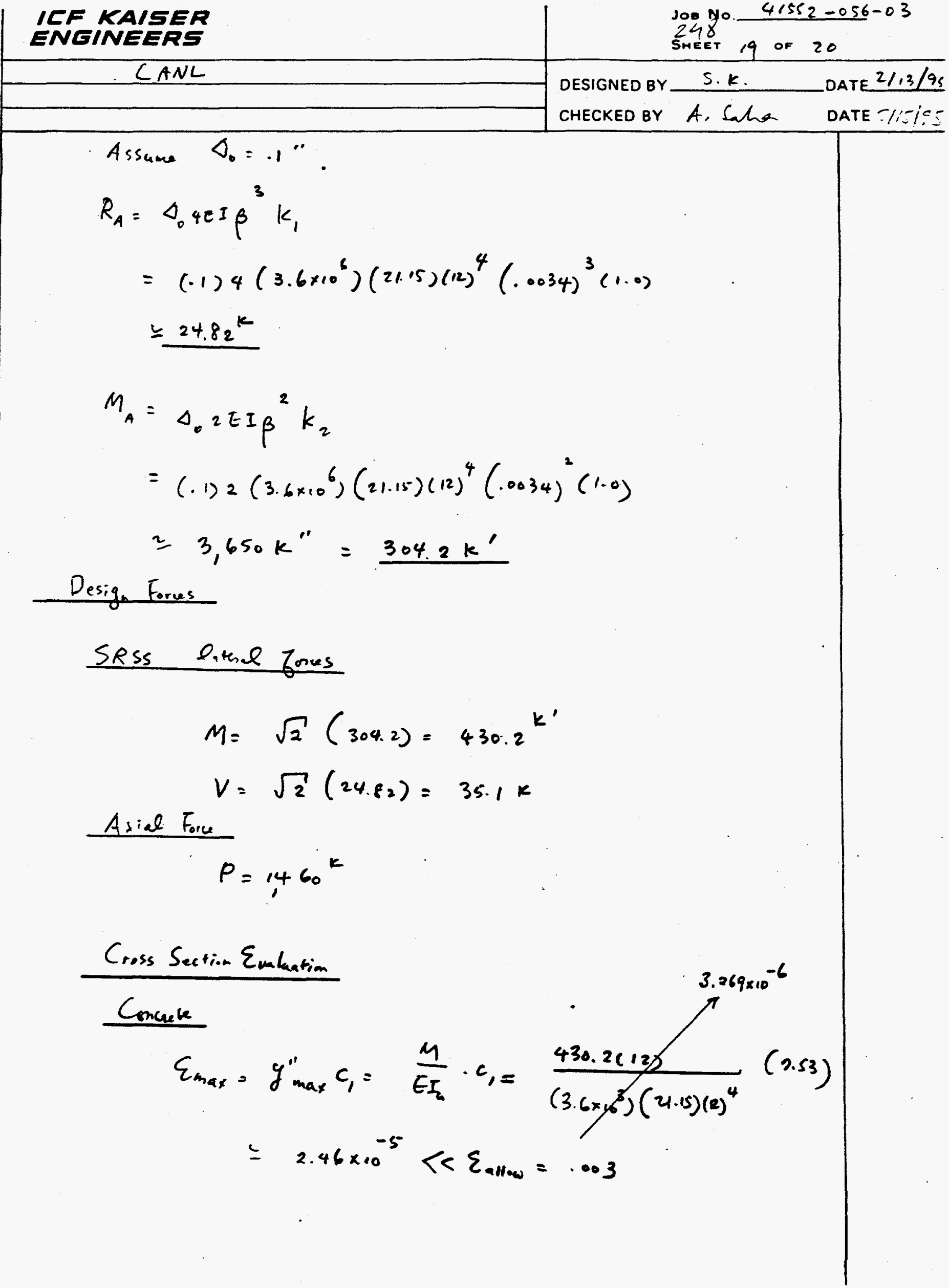




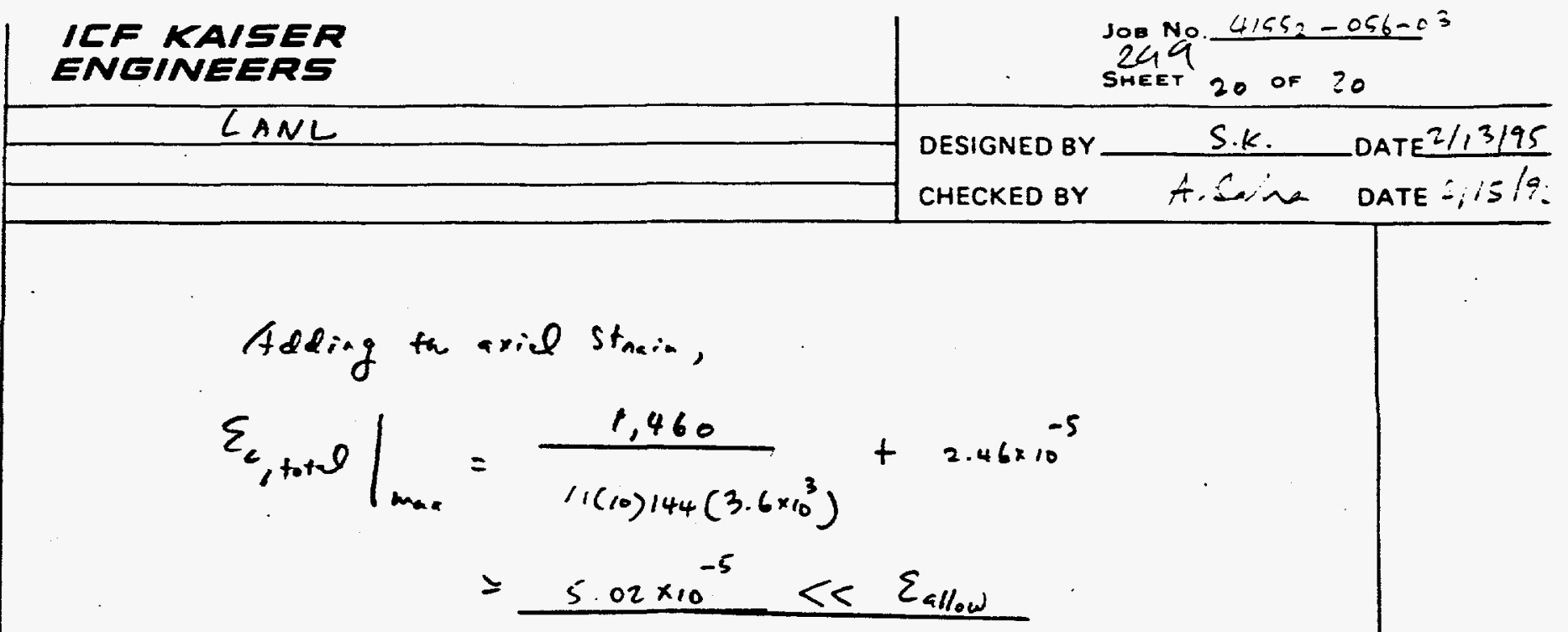

Stael

$$
\varepsilon_{A S}=3.20 \times 10^{-6}(116-0.53)=3.55 \times 10^{-4}<\varepsilon_{y}=.002
$$

Tensile nebar has a.t yilted.

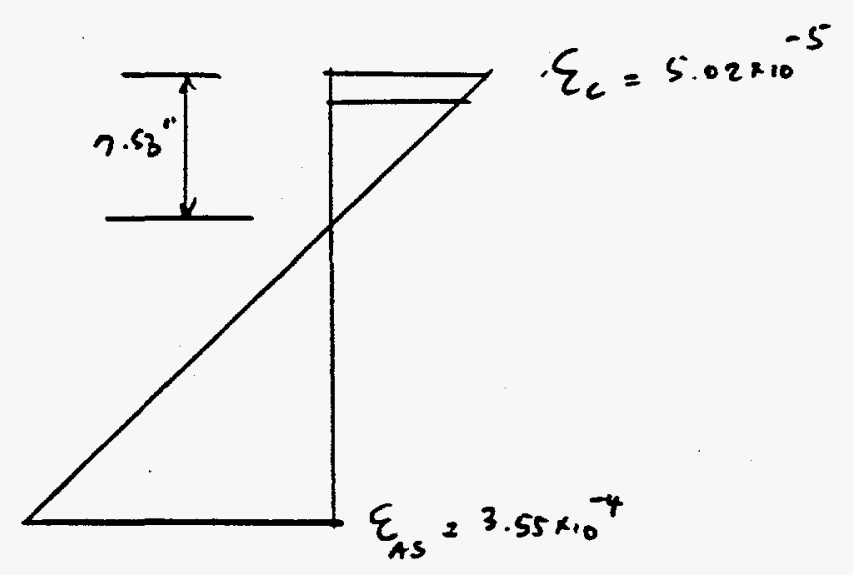

$\therefore$ Due to SAM Loating, Conoute inpresive strair it temile

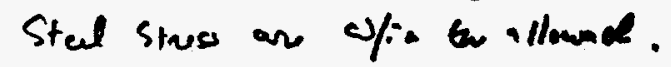


Rev. 0 SHEET $=51$

OF

EVALUATЮN OF NMSF FACILITY AT LANL

DESIGNED BY B.M. DATE: $/ 2 / 45$

Modification Option (1).

CHECKED BY 5u
DATE $3 / / \% 5:$

\subsection{EVALUATION OF INTERIOR WALLS IN ADMIN AREA}




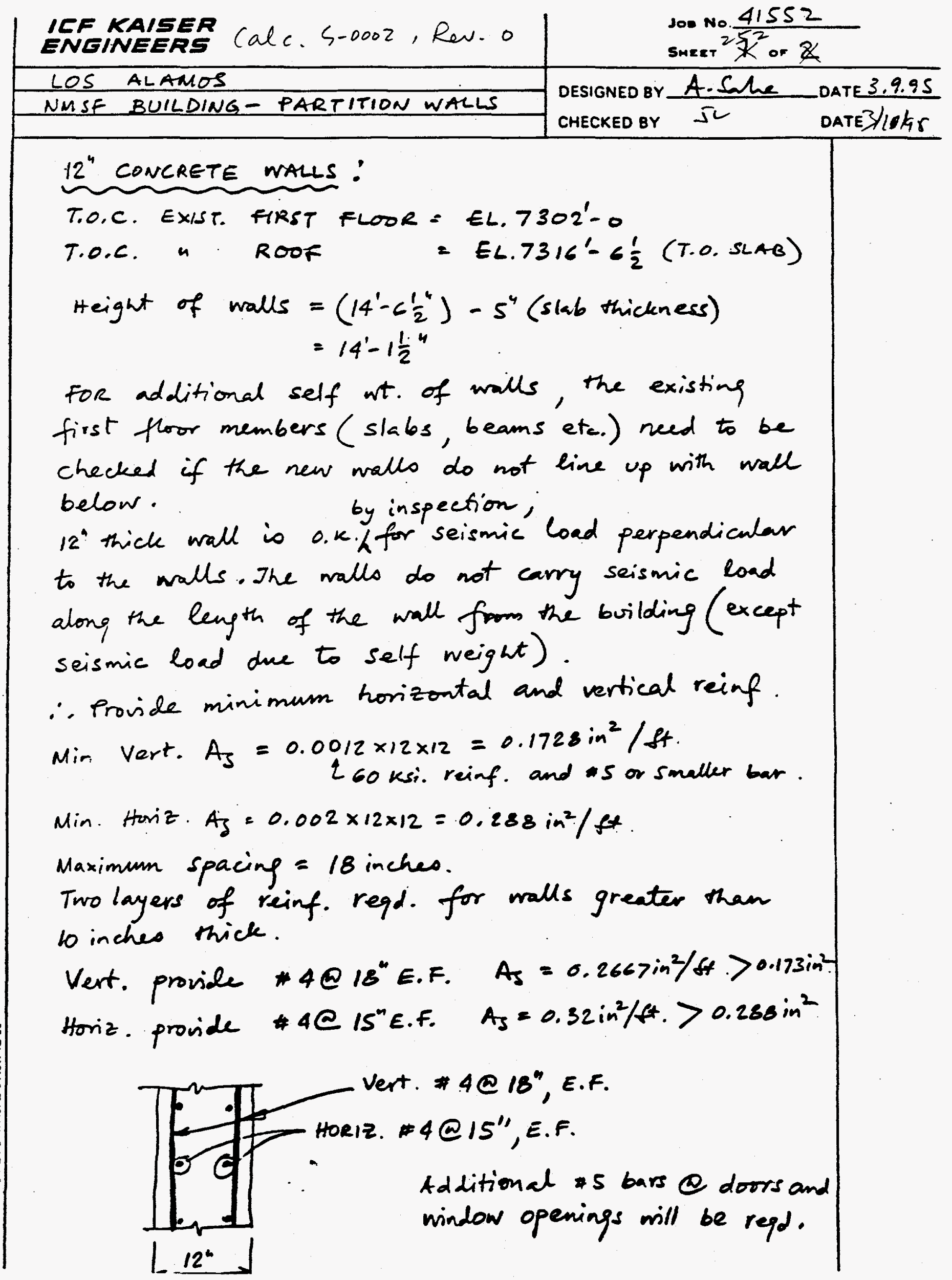




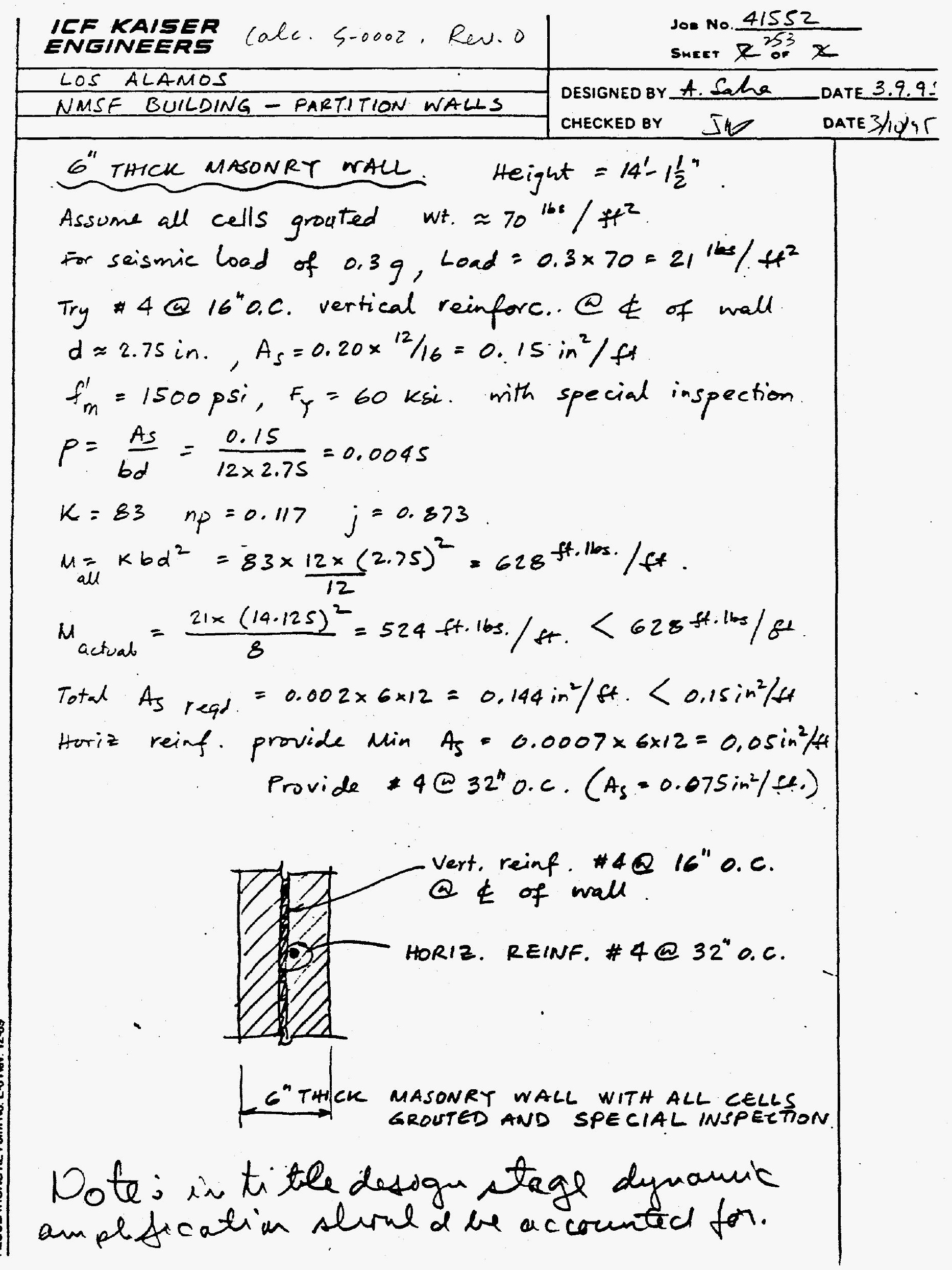




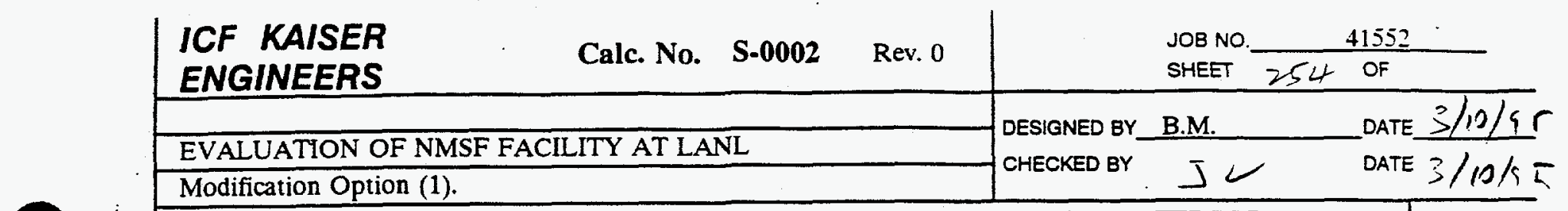

8.5 CONCEPTUAL RETROFIT DESIGN, WEST EXTERIOR WALL 


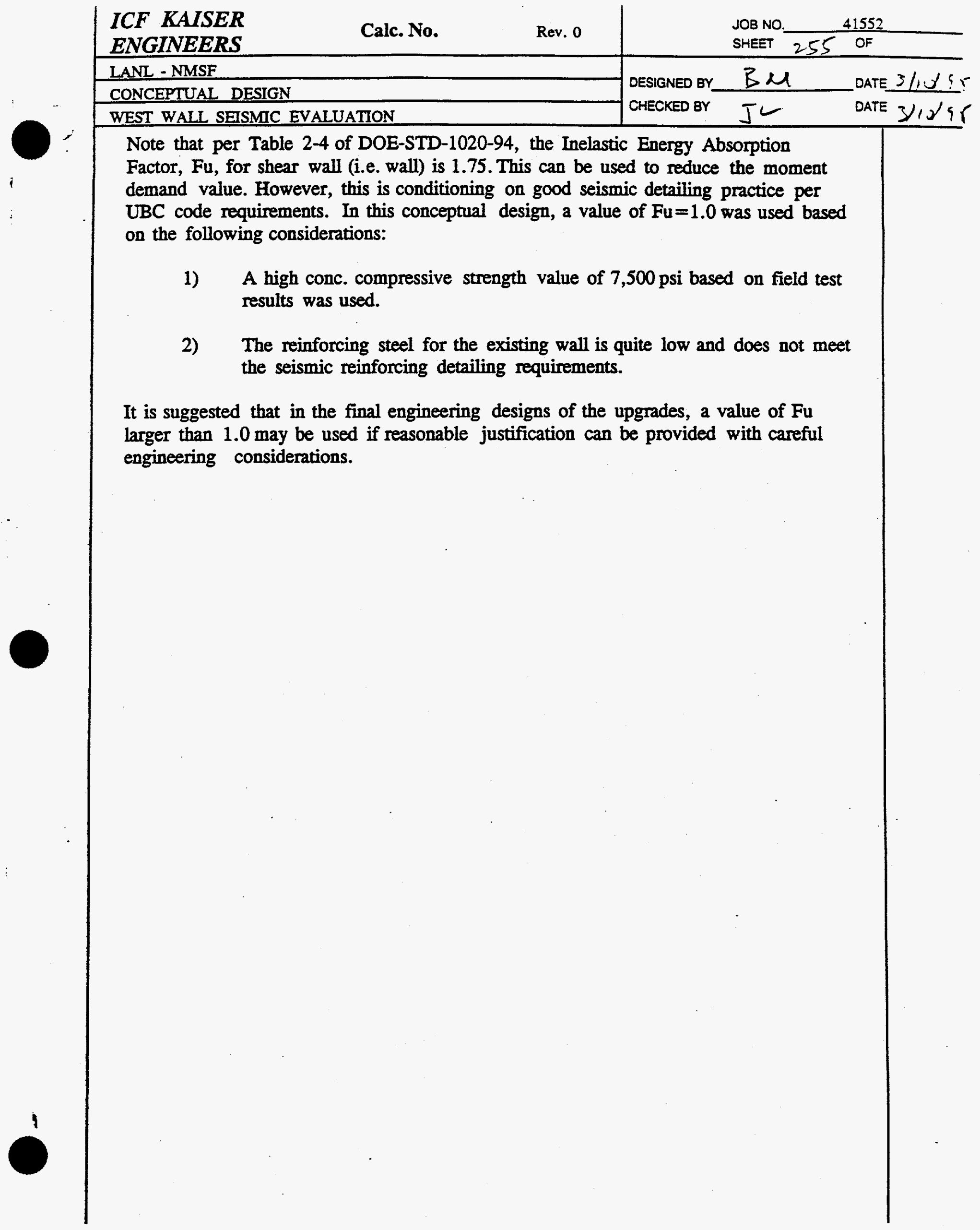




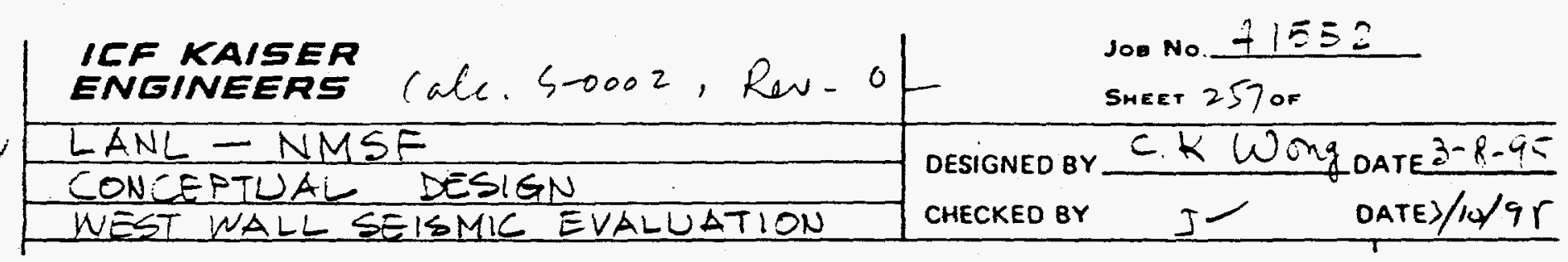

$\prod_{1}^{116+1}$

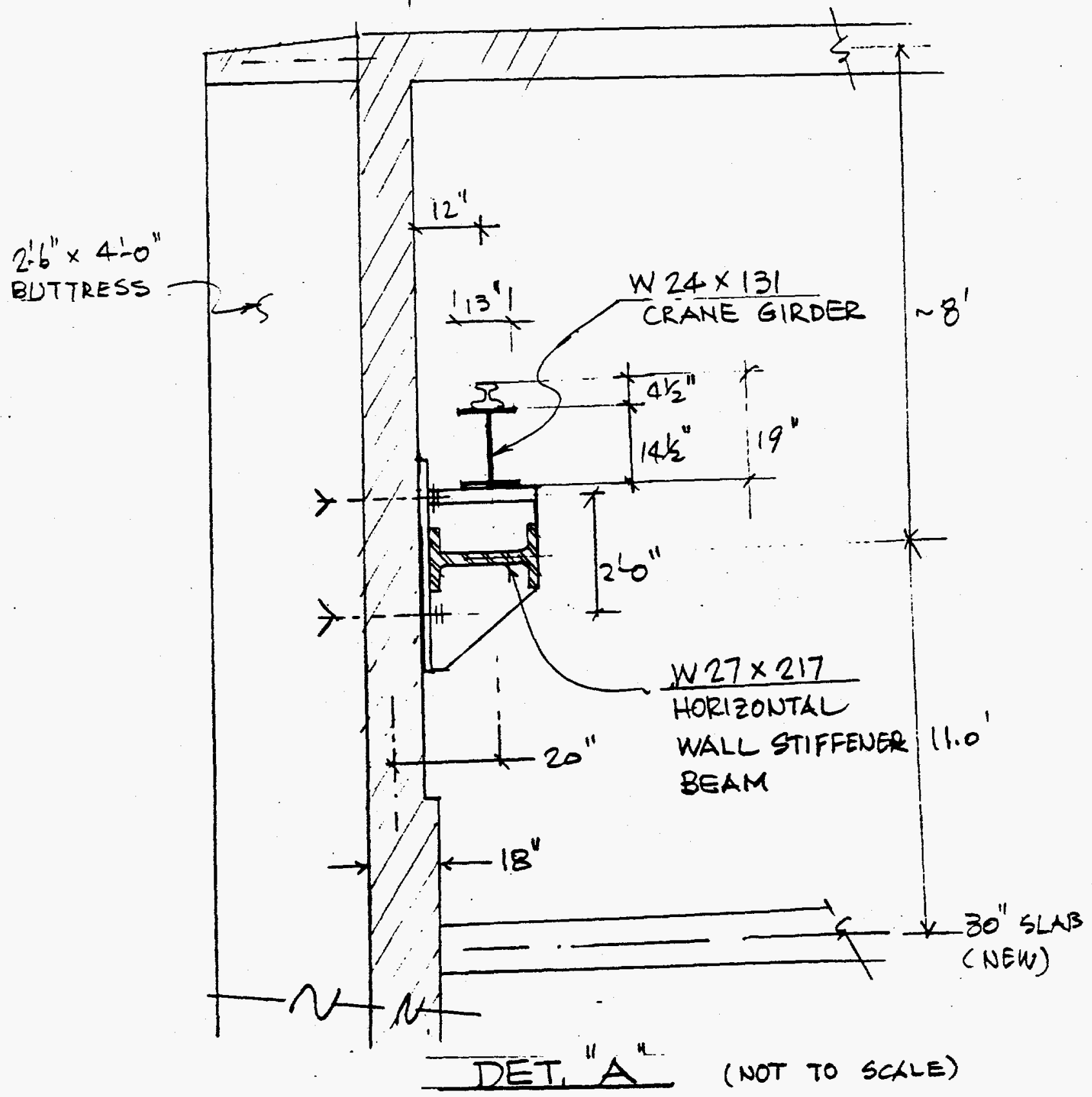




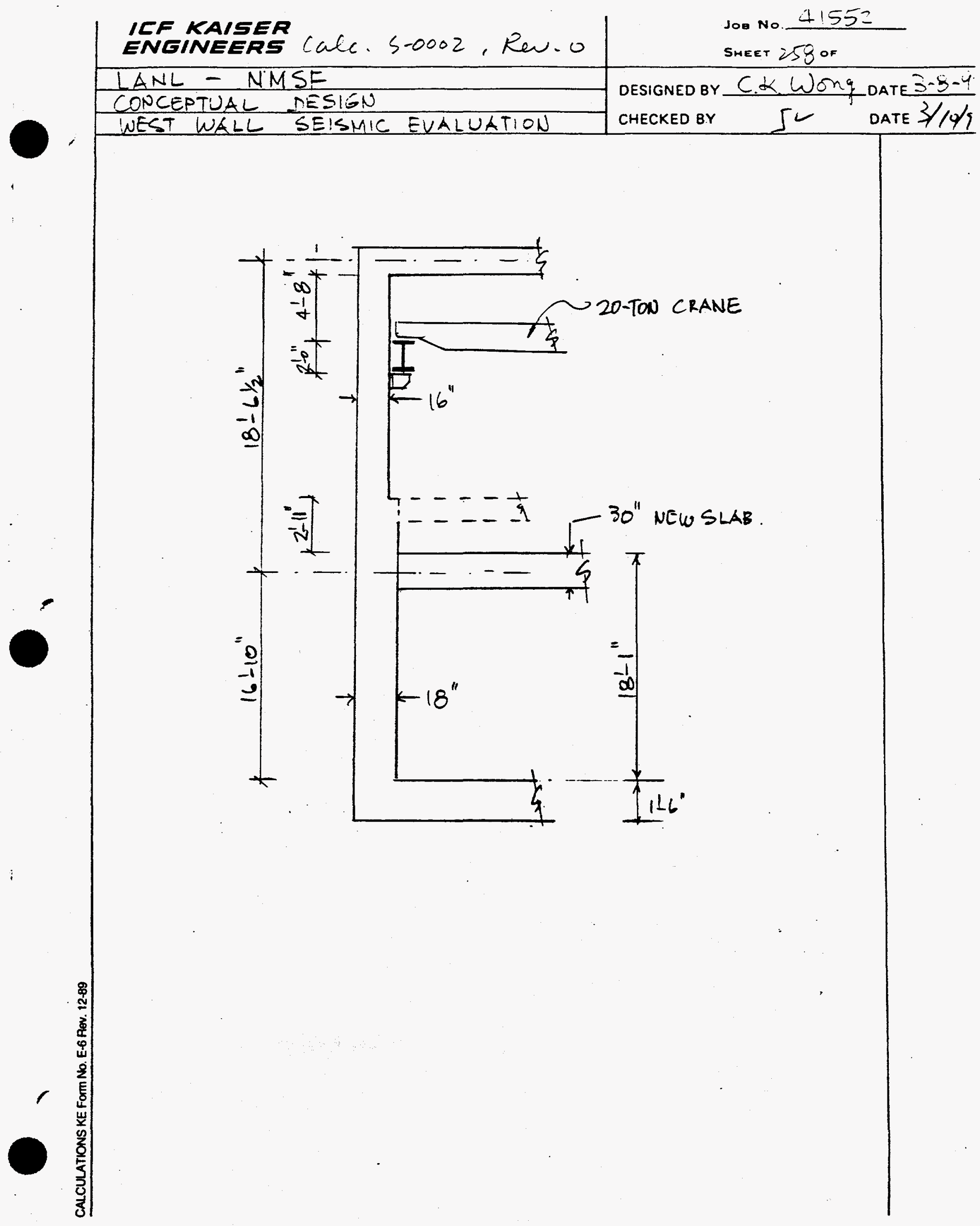




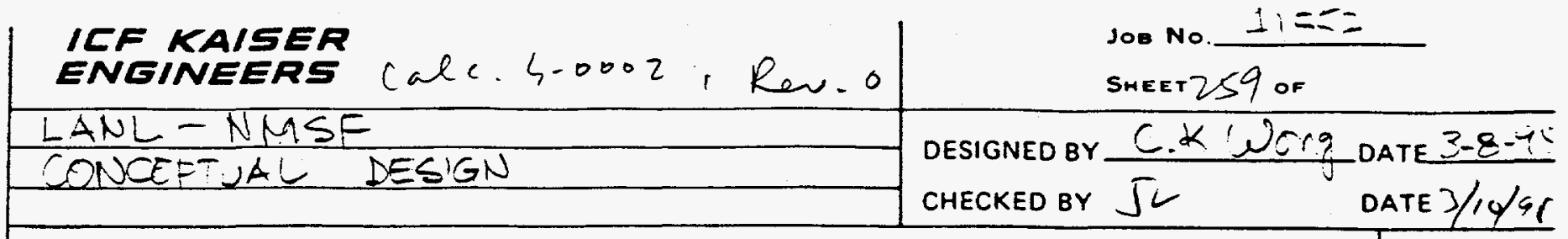

WEST WALL

The wall between the $1^{\text {st }}$ floor and the roof (spanning vertically, will be critical and controlling the design:

using the following approx. evaluation

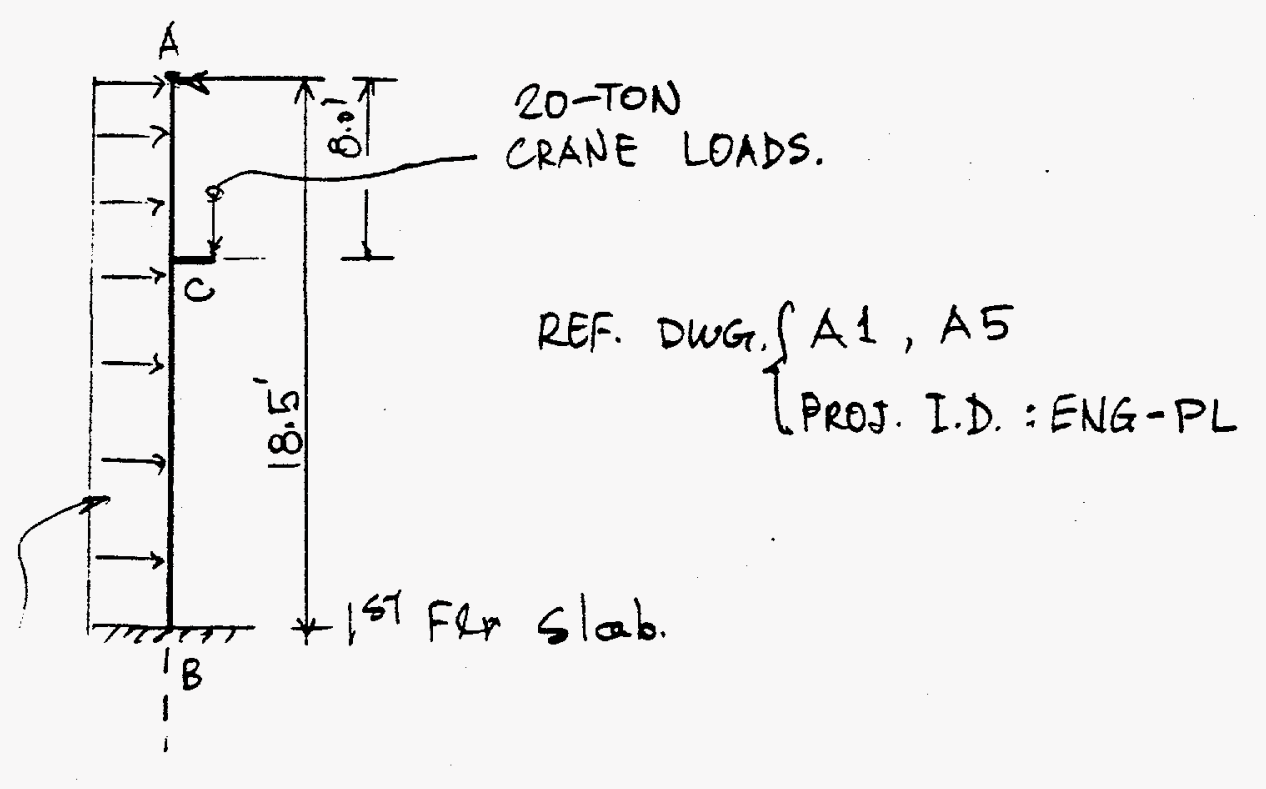

conservatively use approx uniform load.

Dynamic + Static soil pressure (See attached sit.).

$$
P_{T}(A V G)=\frac{1.27+1.21+1.15+1.12}{4}=\frac{4.75}{4} \cong 1.20 \mathrm{KSF} .
$$

Seismic selfuright effect of wall.

Peak o value from NUREG/CA-9800 spectrum

( $=0.81 \mathrm{~g}$.

equiv. static factor

$$
\begin{aligned}
\omega & =1.33^{\prime}(.150) \times 0.81 \times 1.5 \\
& =0.24 \mathrm{k} / \mathrm{ft} .
\end{aligned}
$$




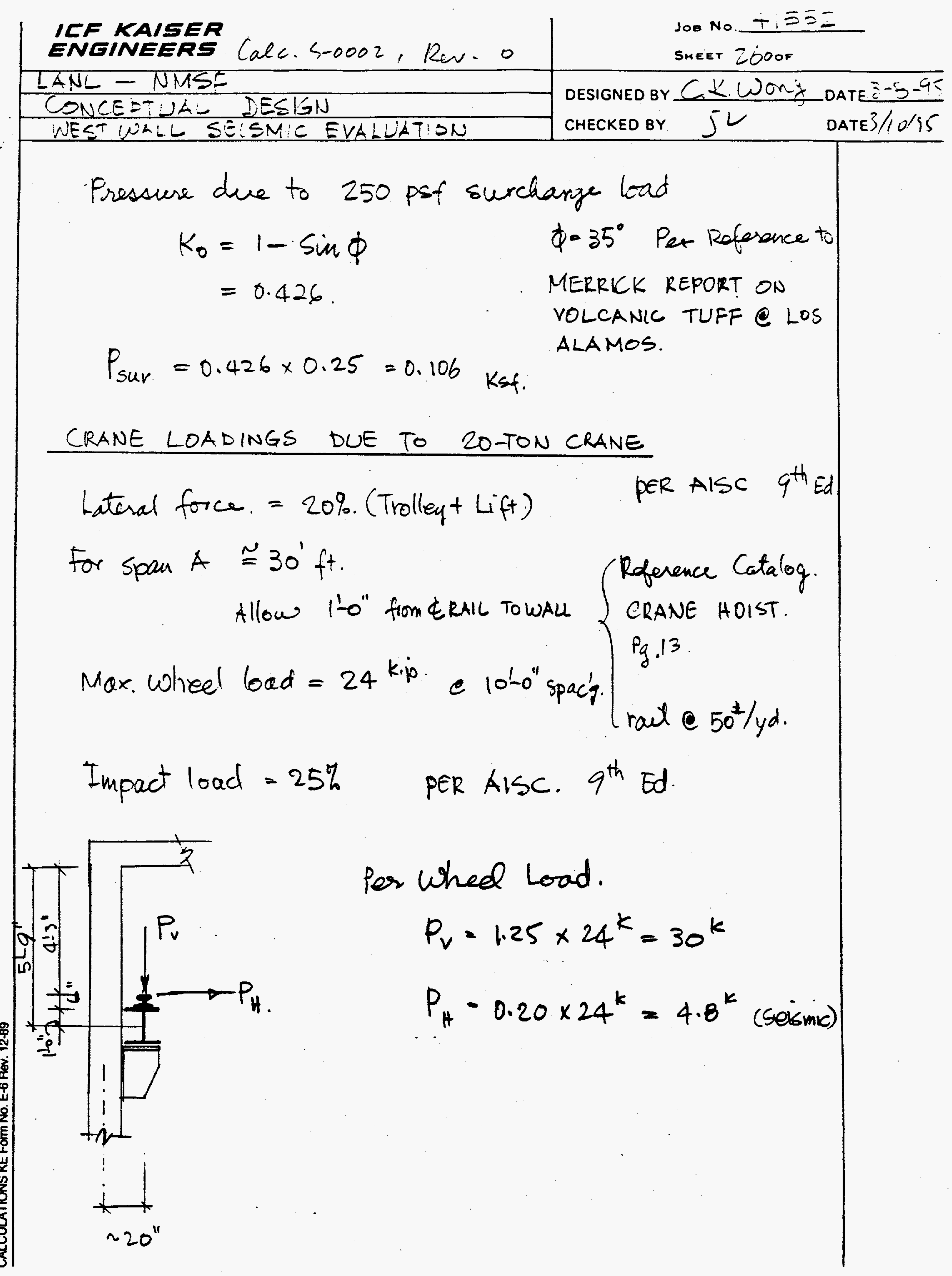




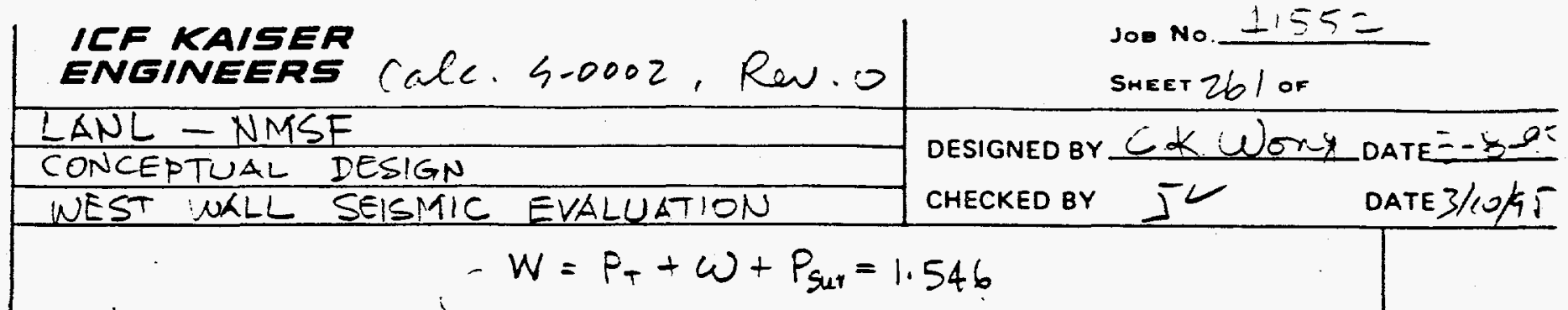

B.

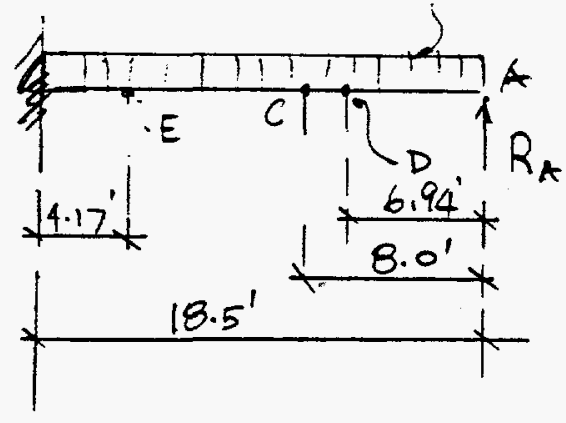

Under soil pressure + seismic Selfie.

$$
\begin{aligned}
& R_{A}=\frac{3 \omega l}{8}=\frac{1.546(3)\left(18.5^{\prime}\right)}{8}=10.7^{\mathrm{k}} \\
& V_{B}=\frac{5 \omega l}{8}=\frac{5(1.546) 18.5}{8}=17.9^{\mathrm{k}}
\end{aligned}
$$

MAX.

$$
-M_{B}=\frac{\omega l^{2}}{8}=\frac{1.546(18.5)^{2}}{8}=66.1 \mathrm{k-ft} / \mathrm{ft} .
$$

InTERIOR SIDE:

$$
+M_{D}=\frac{q}{128} \omega l^{2}=37.2^{k-f t / f t}>21.6 \mathrm{k-ft} / \mathrm{ft} .
$$

Check shear.

$$
\begin{aligned}
V=R_{A}=10.7^{k}< & 20.8^{k \mid p s .} \\
& \text { OIK. CONSERVATIVE }
\end{aligned}
$$

Exterior Side:

$$
\begin{aligned}
-M_{B}=66.1^{\mathrm{k}-\mathrm{ft} / \mathrm{ft} .}<97.27^{\mathrm{k}-\mathrm{ft} / \mathrm{ft} .} 0 . \mathrm{k} \\
V_{B}=17.9^{\mathrm{k}}<23.7^{\mathrm{kips}} \quad \text { (Consenvatuie) } \\
-M_{E}=10.7^{\mathrm{k}}\left(14.33^{\prime}\right)-\frac{1}{2}(1.546)(14.33)^{2} \\
=153.33-158.73-5.40 \mathrm{k-1} / \mathrm{ft}<52.87 \mathrm{k-ft} / \mathrm{ft}
\end{aligned}
$$$$
\text { ok. }
$$

Shear 0.1 by Inspection. 
IEF KAISER

ENEINEERS CaCc.S-0002, Rev.O

Jo. No. $4 ! 5=$

LANL - NAMSF

CONCEPTLLL DESIGN

WEST WALL SEISM1IC

EVALUATION

DESIGNED BY

SHEET 26 ? OF

WESI WALL

REF: $\begin{aligned} & \text { DOE } D R G N O . \\ & \angle A-Z J-B . S .8 .1\end{aligned}$

REF: $\begin{aligned} & \text { DOE } D R G N O . \\ & \angle A-Z J-B . S .8 .1\end{aligned}$

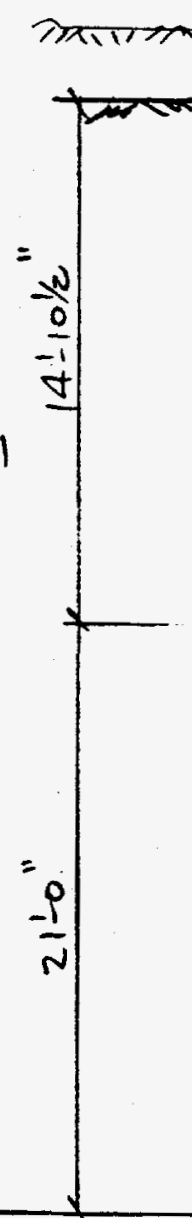

EL. $7316^{\prime}-10^{\prime} \leqslant$

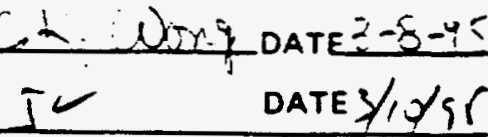

フフז

- ato"

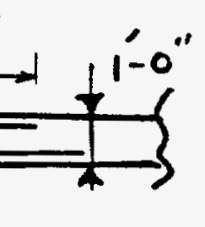

$1-4^{4}$

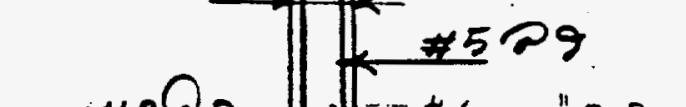

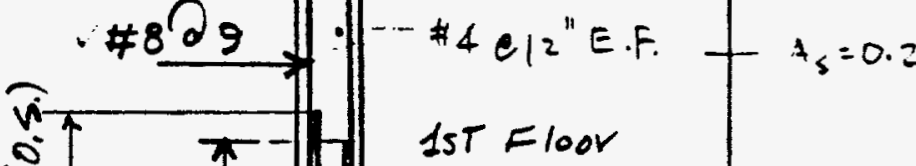




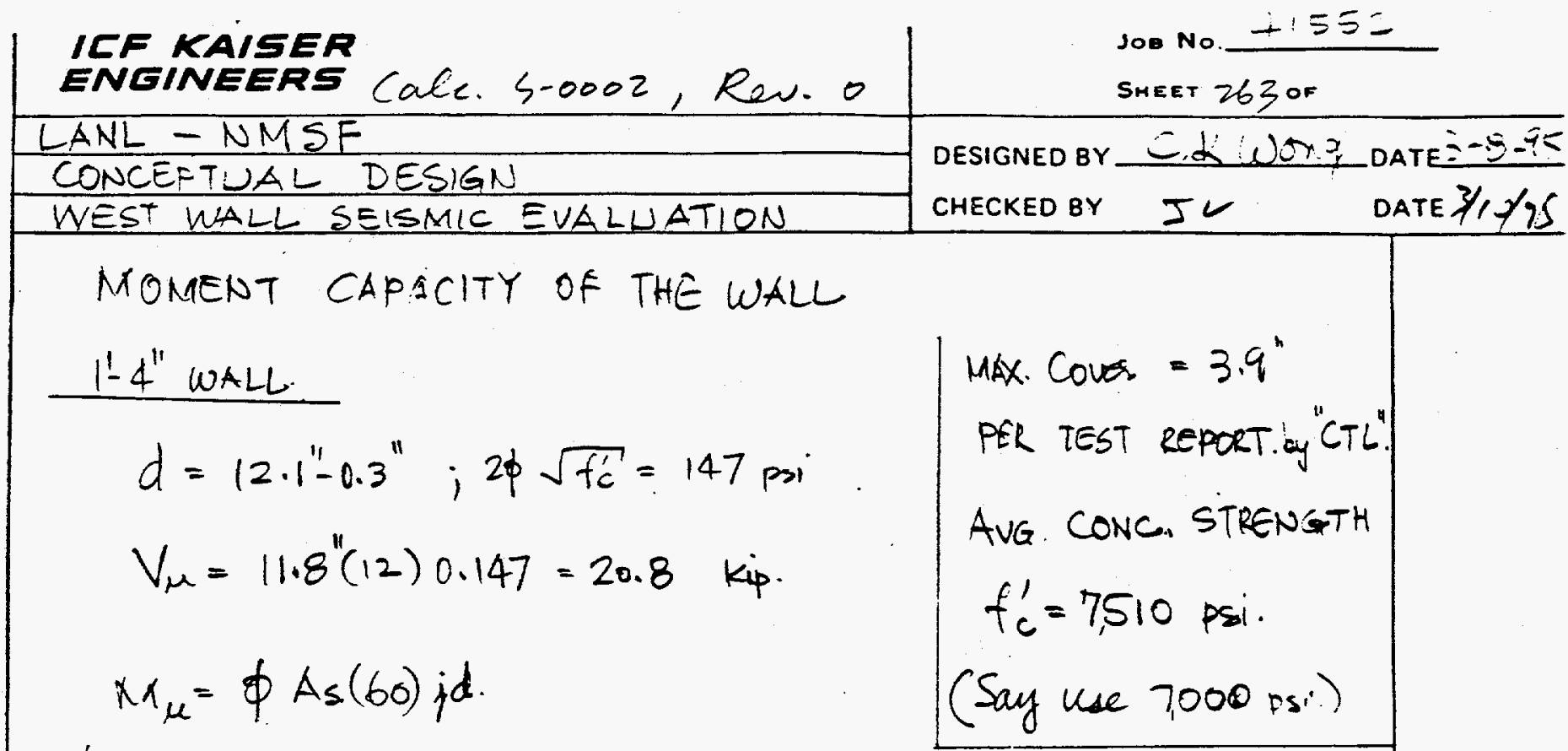

INTR SIDE:

$$
\begin{array}{rlrl}
Q & =\frac{A_{s}(60)}{0.85(7.5)(12)}=0.324 " & \text { For } & +M \\
\text { of } & \# 5 e q^{\prime \prime} \\
M_{\mu} & =0.9(0.413)(60)\left(11.8-\frac{0.324}{2}\right) / 12 & A_{s} & =\frac{12}{9}(0.31) \mathrm{m}^{2} \\
& =21.63 \mathrm{ktt} / \mathrm{ft} . & & =0.413 \mathrm{~m}^{2}
\end{array}
$$

Exterior Side:-

$$
\begin{aligned}
a & =\frac{1.05(60)}{0.85(7.5)(12)}=0.82^{\prime \prime} \\
M_{u} & =0.9(1.05)(60)\left(11.6^{\prime \prime}-0.41^{\prime \prime}\right) / 12 \\
& =52.87 \mathrm{k}-\mathrm{ft} / \mathrm{ft} .
\end{aligned}
$$

$$
\begin{aligned}
w / \neq 8 & =q^{\prime \prime} \text { c.c. } \\
A_{s} & =\frac{12}{9}(0.79) \\
& =1.05 \mathrm{~m}^{2}
\end{aligned}
$$

For. $116^{\prime \prime}$ WALL

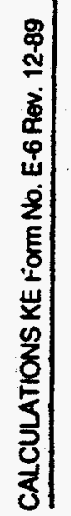

$$
\begin{aligned}
a & =1.33^{n} \\
M_{\mu} & =0.9 .(1.69)(60)\left(14.1-0.64^{\prime}-0.67^{\prime \prime}\right) / 12 \\
& =97.27 \mathrm{k-ft} / \mathrm{ft} . \\
V_{u} & =13.46^{\prime \prime}\left(12^{\prime \prime}\right)(0.147)=23.7^{\mathrm{kjps} .}
\end{aligned}
$$$$
\text { c) * } 10 \text { e } q^{\prime c} \text { cc. }
$$$$
A_{s}=\frac{12}{9}\left(1.27^{\mathrm{m}^{2}}\right)
$$$$
=1.69 \mathrm{im}^{2}
$$ 


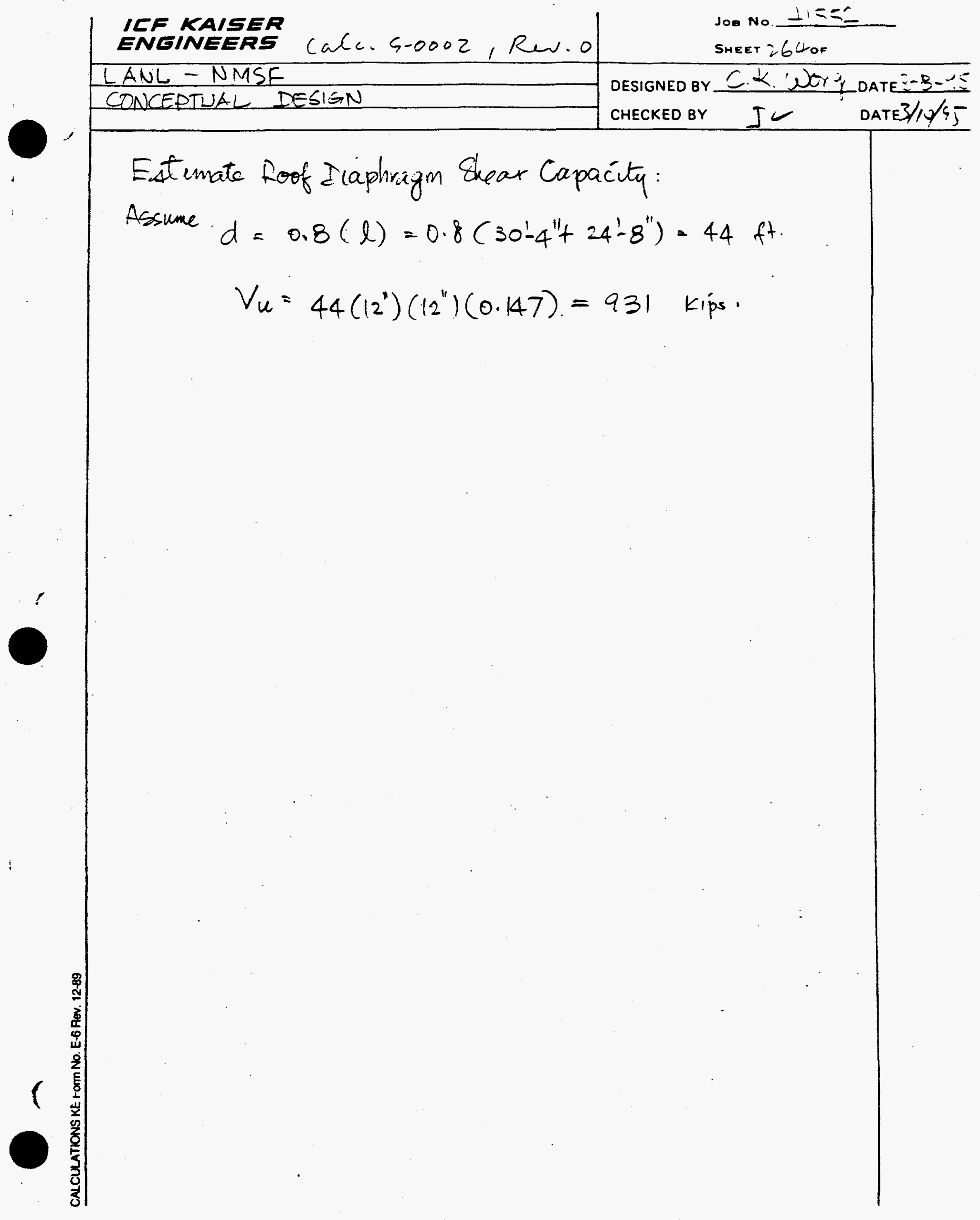




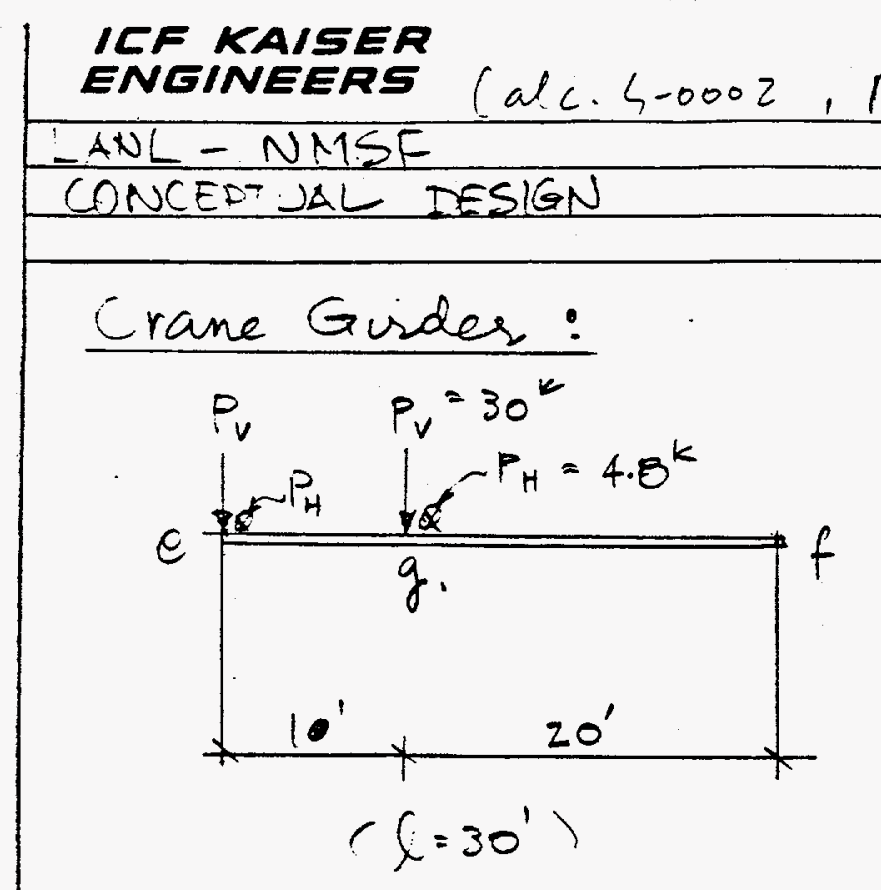

For 20-TON TRAVELLING CRANE

Try 30'0" span

Max.reaction@support

$$
\begin{aligned}
& R_{e v}=30^{k}+\frac{20}{30} \times 30=50^{k i p s} \\
& R_{e H}=4.8^{k}+\frac{4.8}{30} \times 20=8^{k i p s}
\end{aligned}
$$

Distribute over 3'0" width on wall.

$$
\begin{aligned}
& \frac{R_{e V}}{3}=\frac{50}{3}=16.67^{k} \\
& \frac{R_{E H}}{3}=\frac{8}{3}=2.67^{k}
\end{aligned}
$$

Girder max. moment.

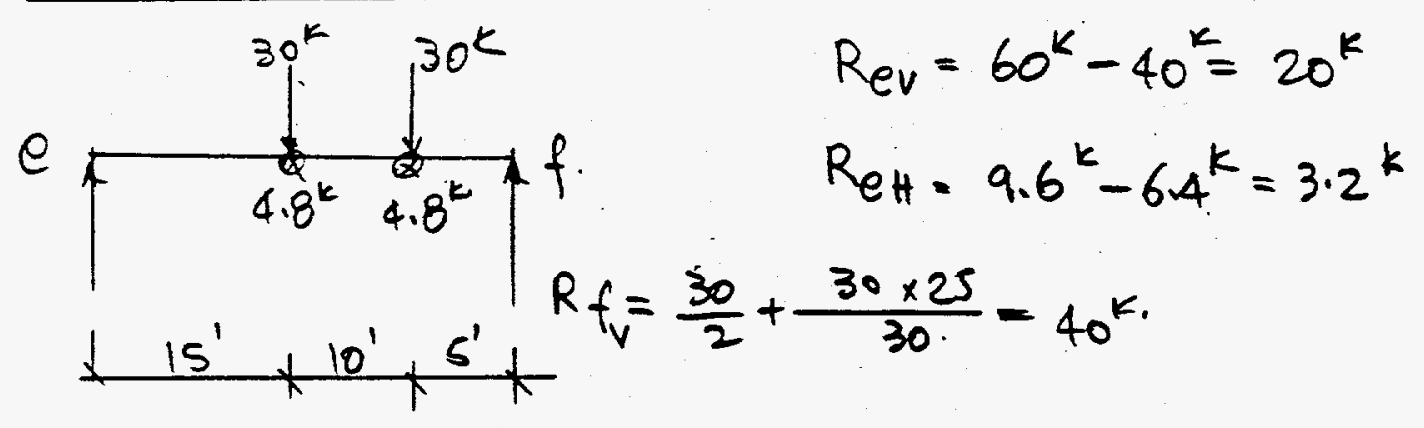

$$
\begin{gathered}
R_{f_{H}}=\frac{4.8}{30} \times 40=6.4^{k .} \\
M_{A_{\text {(max })}}=40^{k} \times 15-30 \times 10=300^{k-f t .} \\
M_{B \text { (max })}=6.4^{k} \times 15-4.8 \times 10=48^{k-f+.}
\end{gathered}
$$




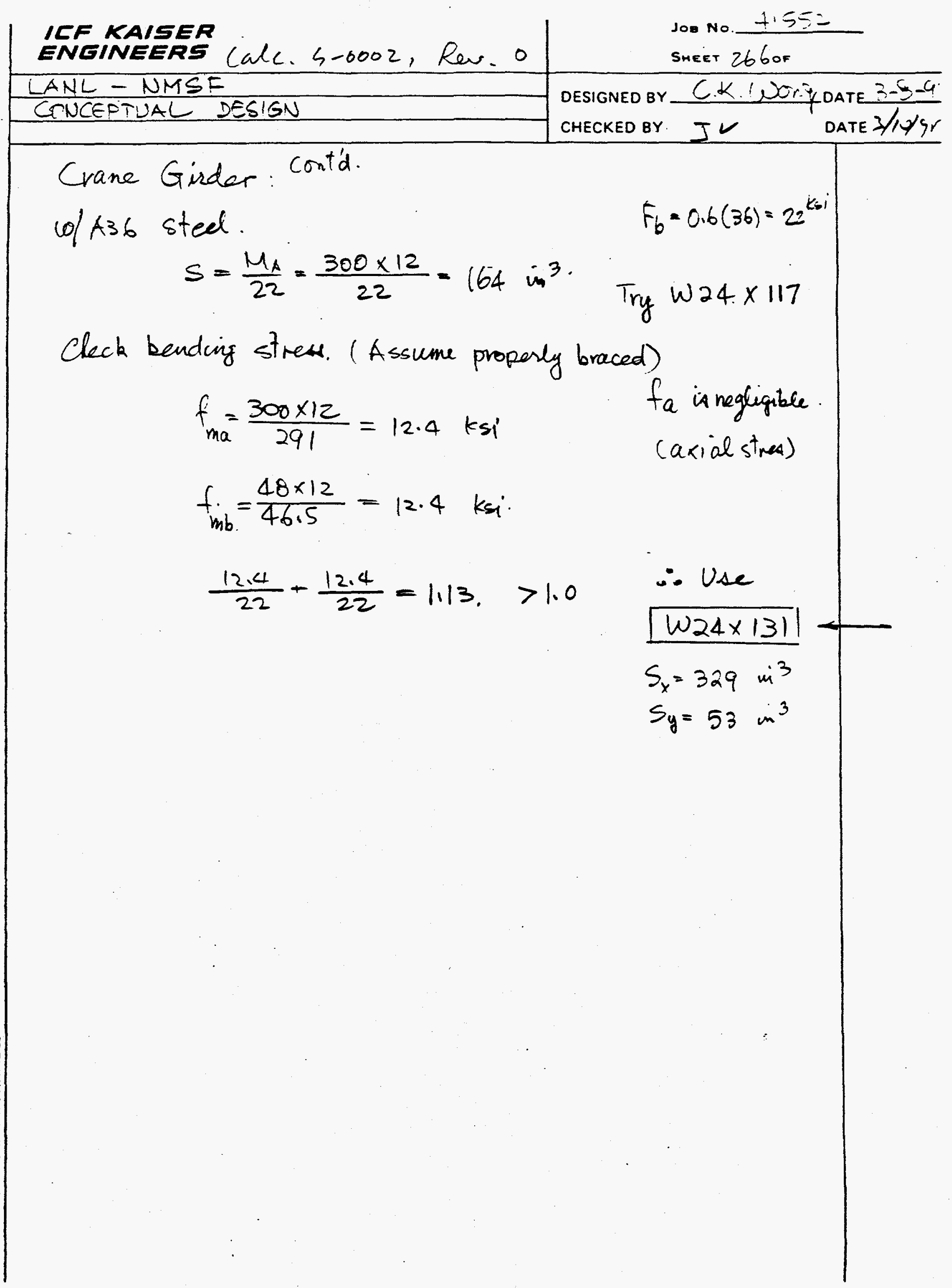




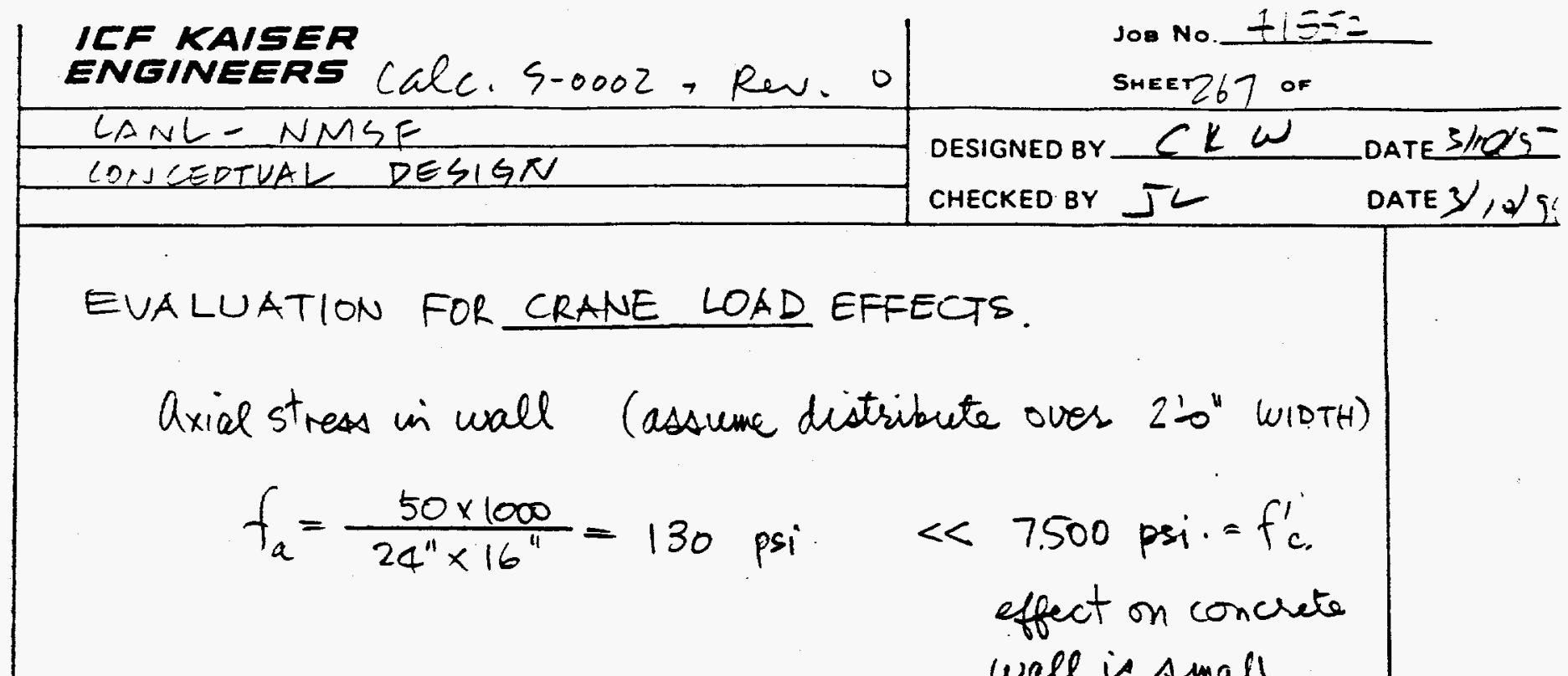

Moment transmits to buttress. effect on concrete wall is small $\left(1.7 \% f_{c}^{\prime}\right)$

$$
\begin{aligned}
M & =50^{k} \times \frac{20^{\prime \prime}}{12 \prime}+\left(2.0^{\prime}+\frac{20^{\prime \prime}}{12^{\prime \prime}}\right) \times 8^{k} \\
& =83.3^{k-1}+29.3^{k-1}=112.7^{k-f+}
\end{aligned}
$$




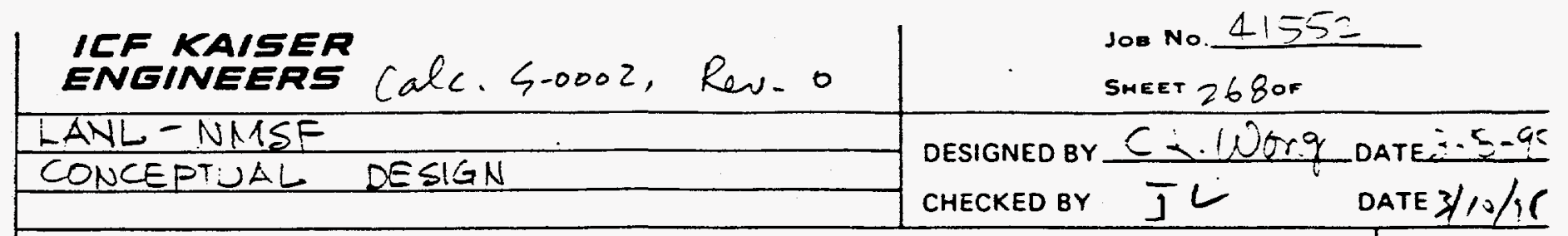

In this cualuaters, it is shown that the existing design of the west wall is not adequate. It is overstressed in moment when subjected to soil pressure (static + seismic) load along.

For the interior side, the moment capacity is $21.6 \mathrm{k-t} / \mathrm{t}$ (based on $f_{c}^{\prime}=7500$ psi, from test results). Whereas, the moment demand is $37.2 \mathrm{k-ft} / \mathrm{ft}$.

$$
\frac{\text { Demand }}{\text { Capacity }}=\frac{D}{C}=\frac{37.2}{21.6}=1.72>1.0 \text {. }
$$

Thermal grade nt loading will further increase the overstressed condition: Therefore, provide horizontal beam to stiffen the wall at the crane bracket support location. Based on the 2-D SAP computer aualypi result. (Run LAN L-B), the moment reduced to $6.55 \mathrm{k}-\mathrm{ft} / \mathrm{ft}$. This will provide adequate moment margin. 


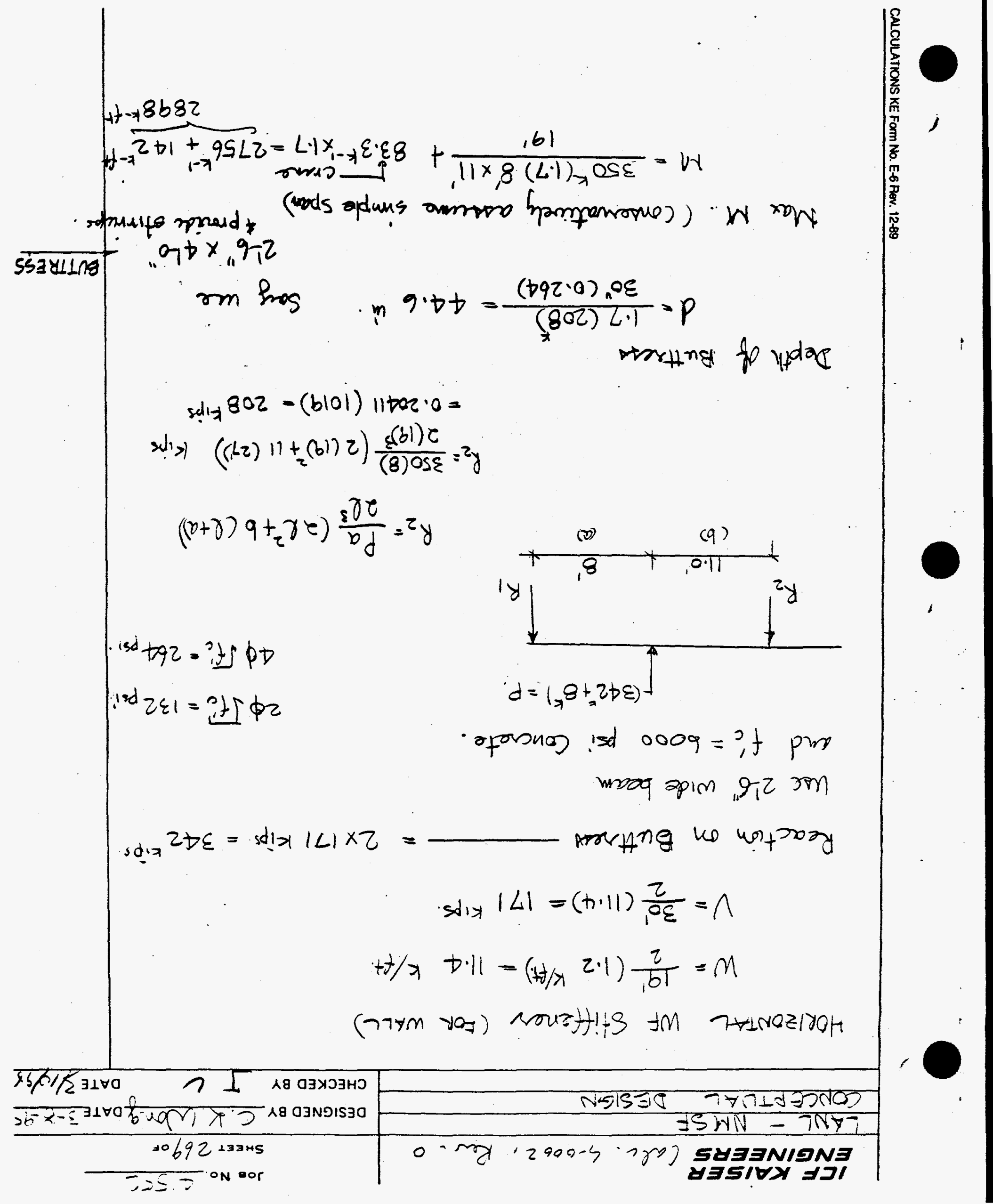




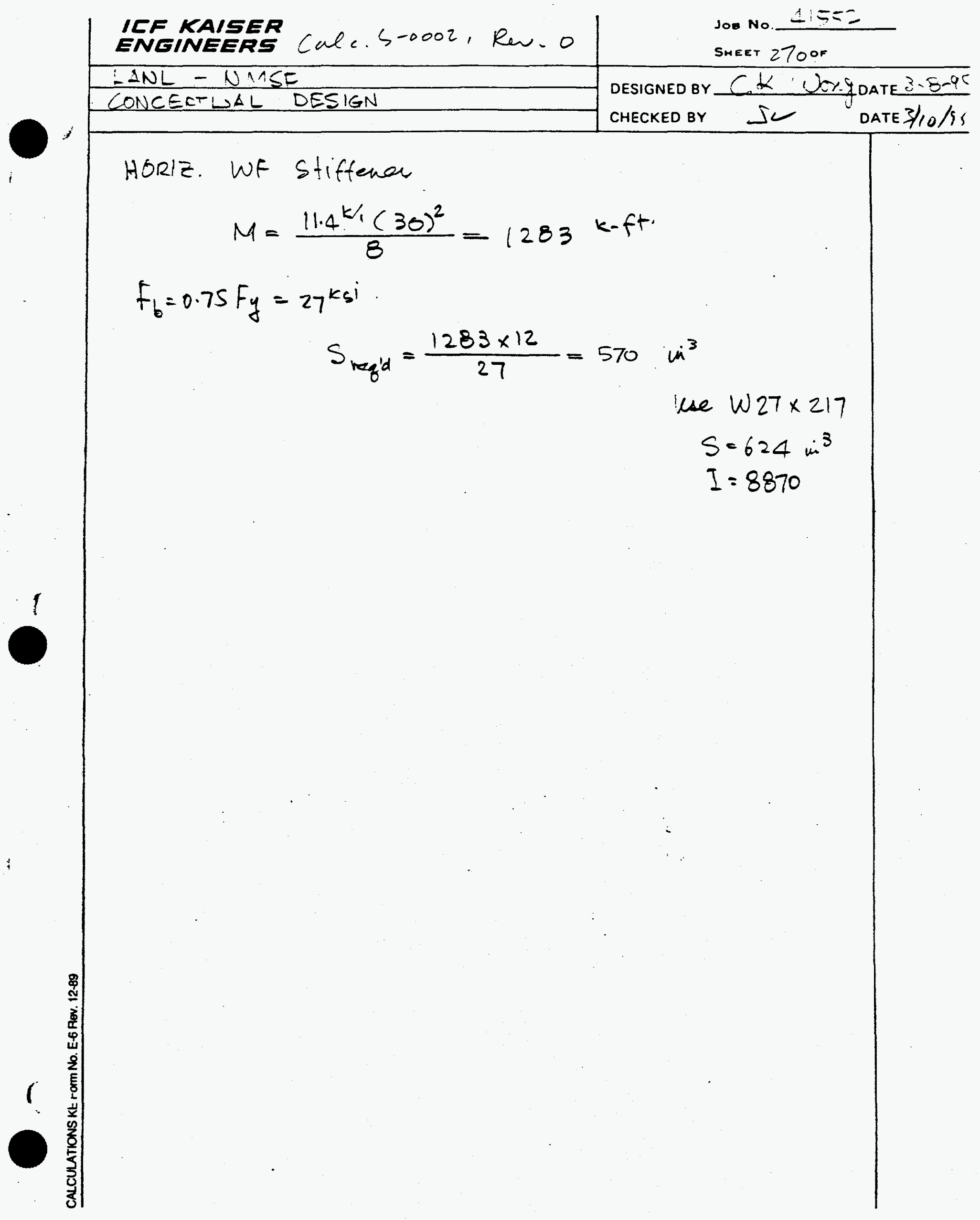




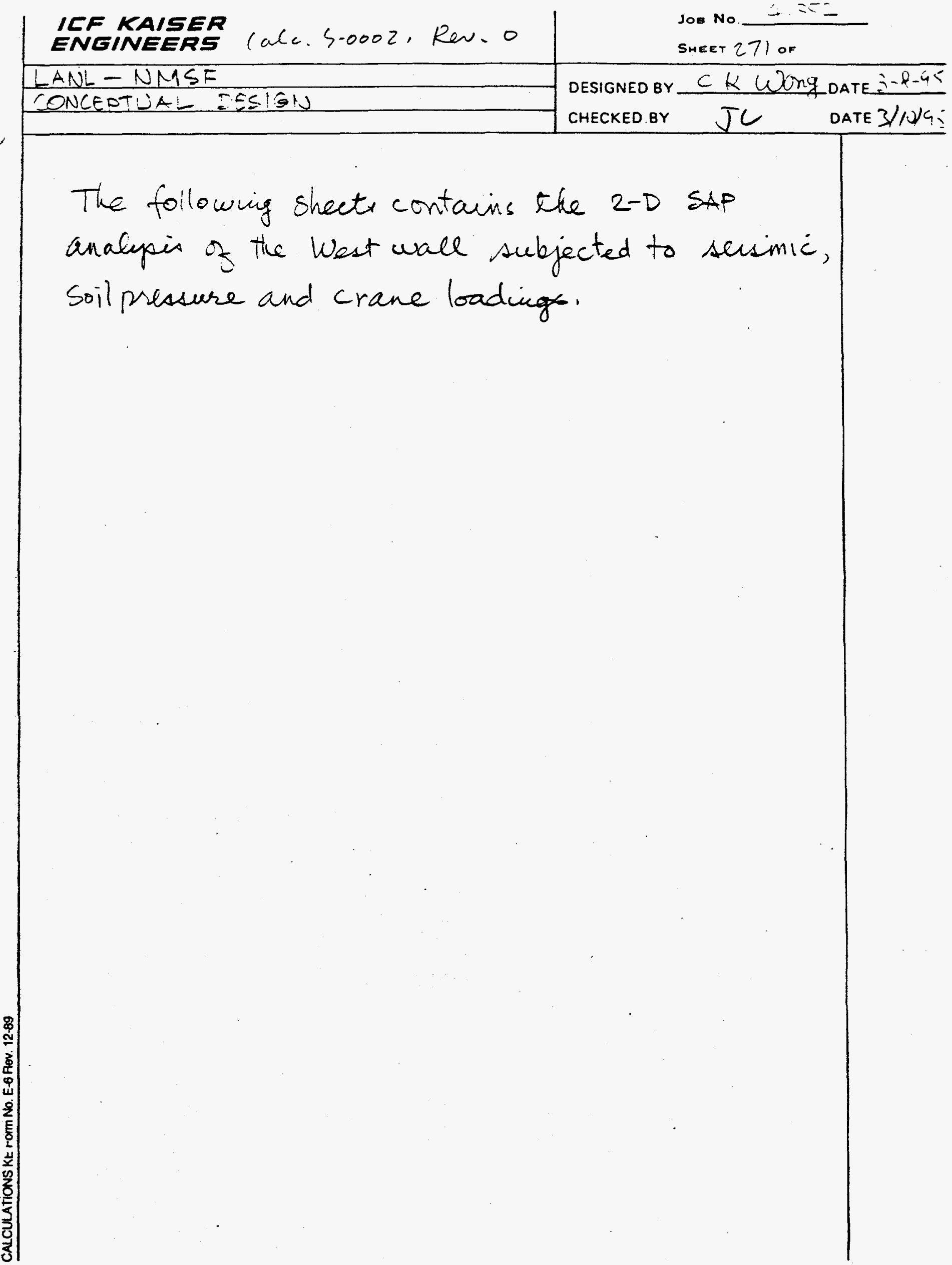




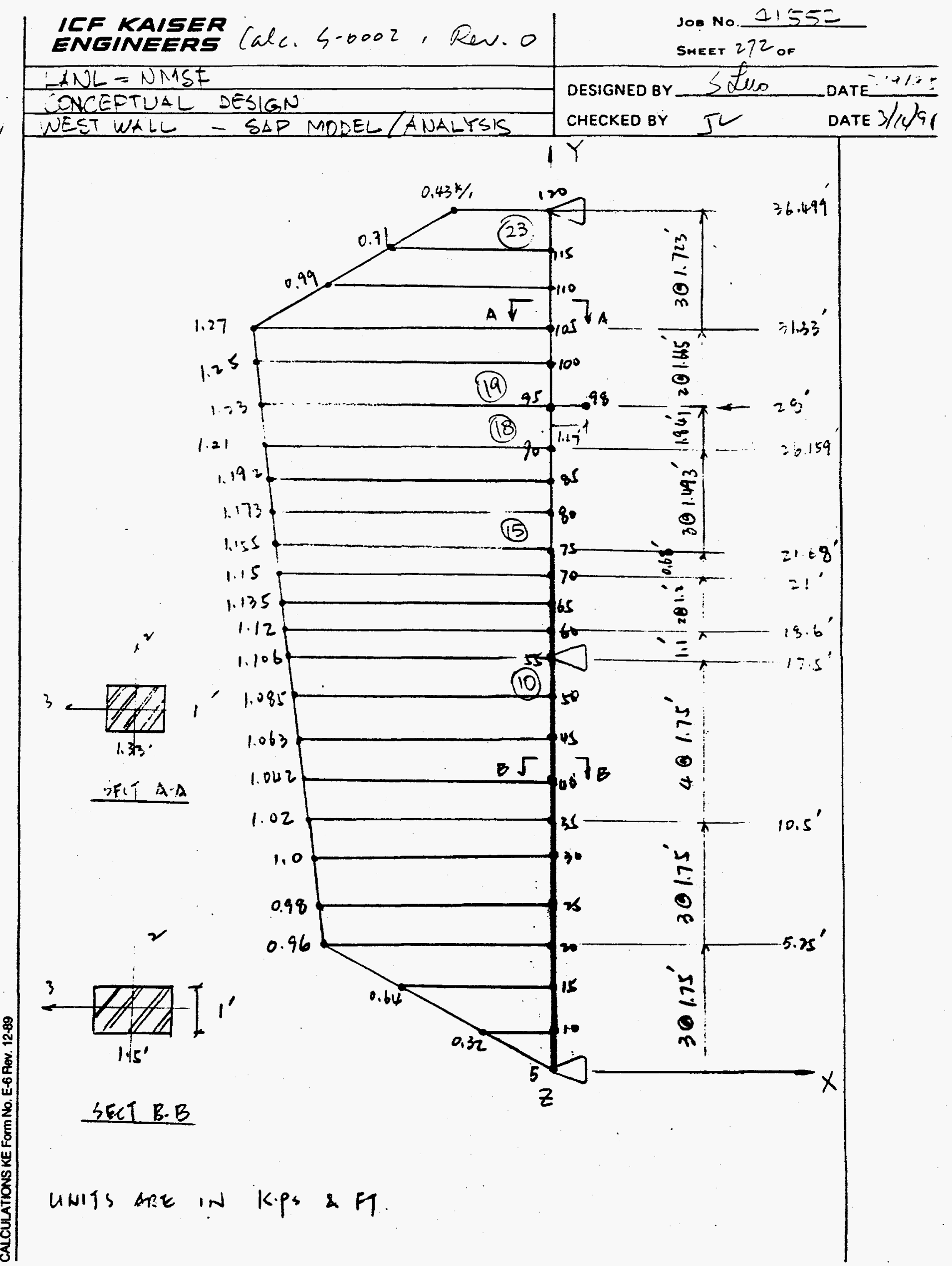


Ght. 273 ois

\begin{tabular}{|c|c|c|c|}
\hline $\begin{array}{r}x \\
-4\end{array}$ & 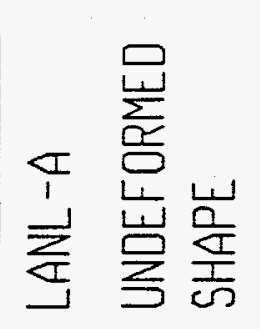 & 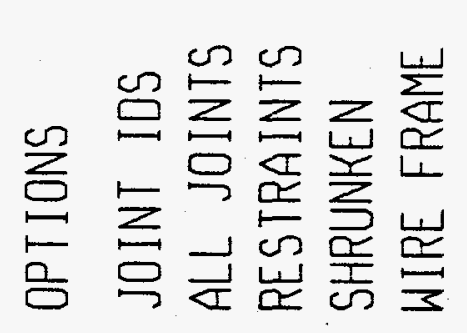 & $\frac{\sqrt{2}}{\frac{1}{2}}$ \\
\hline
\end{tabular}

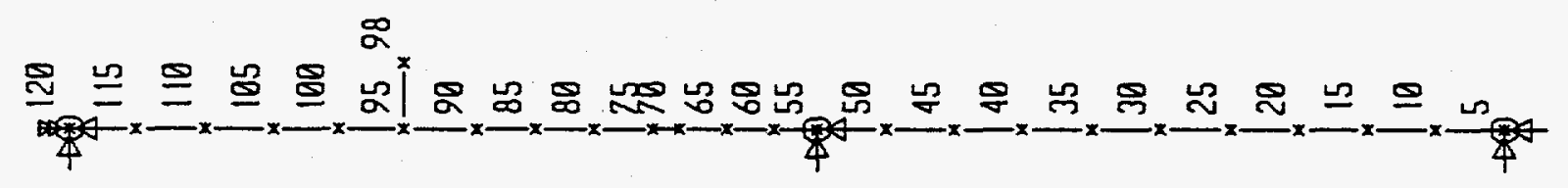




$$
-
$$


Sht. $275 \%$

\begin{tabular}{|c|c|c|c|c|c|}
\hline$>\leftarrow$ & $\sum_{i}^{\frac{T}{I}}$ & 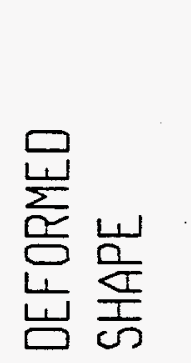 & $\underset{\square}{巳 寸}$ & 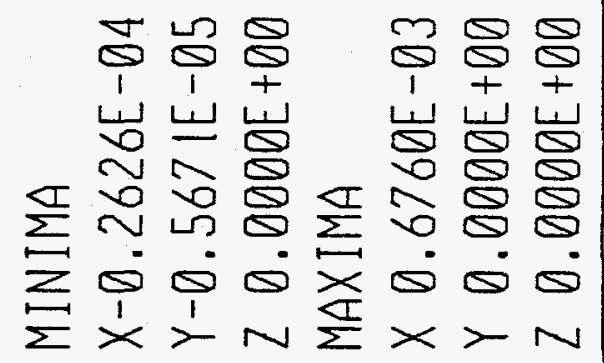 & $\frac{\overparen{S}}{\frac{1}{2}}$ \\
\hline
\end{tabular}

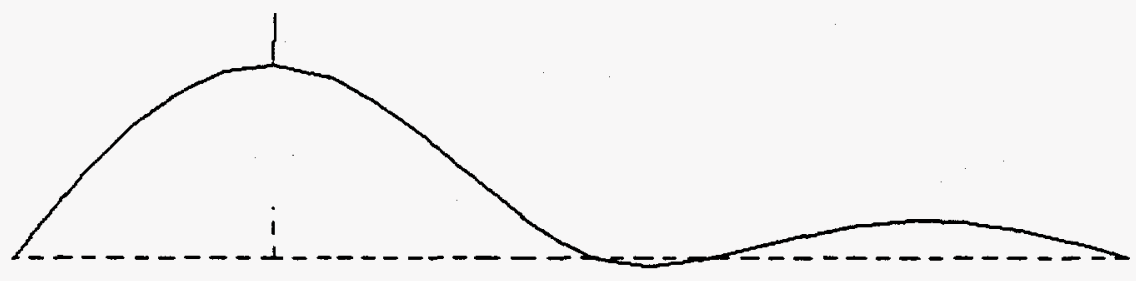


Sht $2760 \%$

\begin{tabular}{|c|c|c|c|c|c|c|c|c|c|c|c|c|}
\hline$\times$ & $\underset{I}{I}$ & 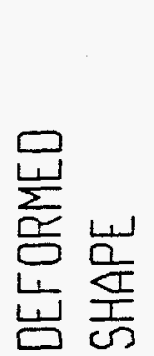 & $\underset{\Xi}{\Xi}$ & $\underset{\sum}{\sum} \underset{\sum}{\sum}$ & 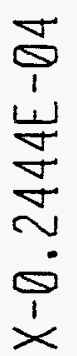 & 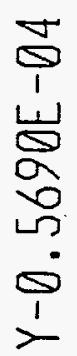 & 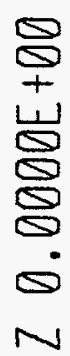 & $\underset{\sum}{\sum}$ & 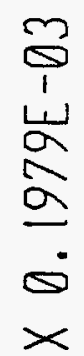 & 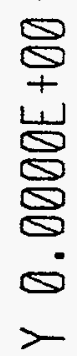 & 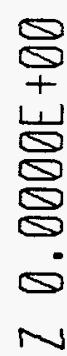 & $\frac{1}{\infty}$ \\
\hline
\end{tabular}

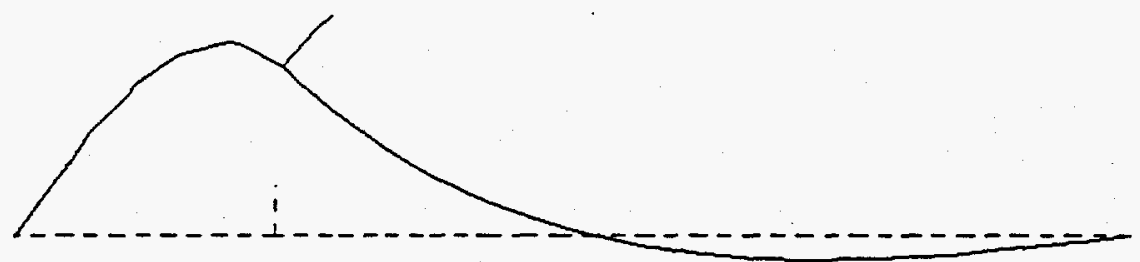


sht. 277 ef

\begin{tabular}{|c|c|c|c|c|c|c|c|c|c|c|c|c|}
\hline $\begin{array}{r}x \\
-4\end{array}$ & $\stackrel{I}{i}$ & 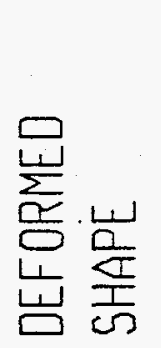 & $\begin{array}{l}m \\
\Xi \\
\Xi\end{array}$ & $\sum_{\sum}^{\sum}$ & 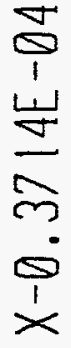 & 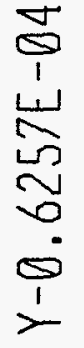 & 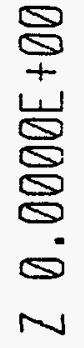 & $\underset{\sum}{\sum}$ & $\begin{array}{c}\infty \\
\infty \\
\infty \\
\infty \\
\infty \\
\infty \\
\infty \\
\infty\end{array}$ & 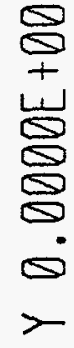 & $\begin{array}{l}\Phi \\
\stackrel{S}{+} \\
\dot{S} \\
\stackrel{S}{S} \\
\stackrel{S}{S} \\
N\end{array}$ & $\frac{1}{\sigma}$ \\
\hline
\end{tabular}

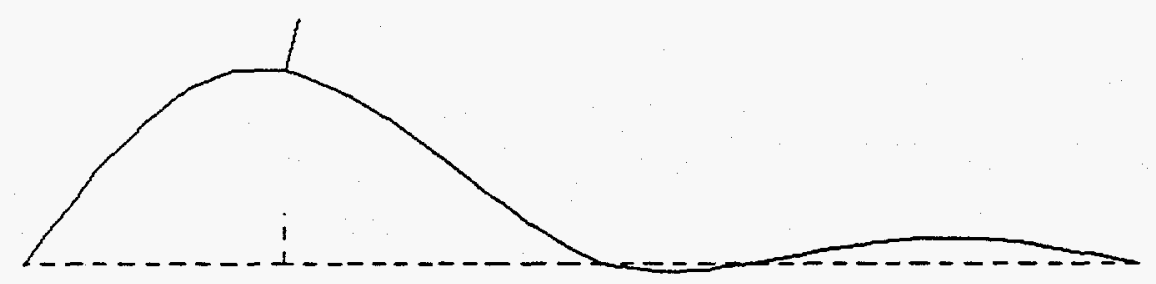




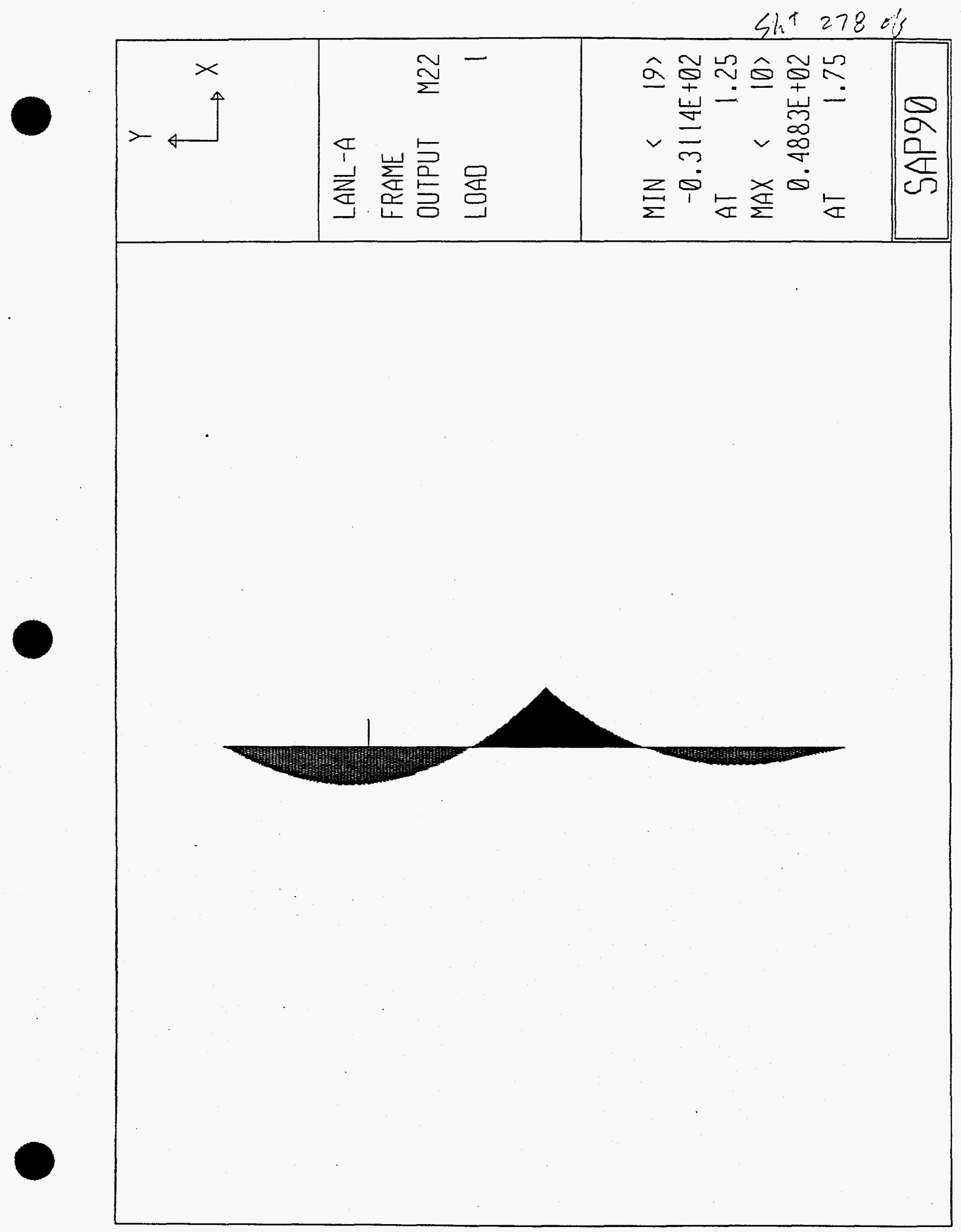


Sht $2700 \%$

\begin{tabular}{|c|c|c|c|c|c|c|c|c|c|c|c|}
\hline $\begin{array}{r}x \\
4\end{array}$ & $\begin{array}{l}I \\
\sum \\
\sum \\
\end{array}$ & $\frac{w}{\frac{1}{L}}$ & $\begin{array}{l}\frac{N}{2} \\
\equiv \\
\equiv \\
\equiv\end{array}$ & $\begin{array}{l}\curvearrowright \\
\Xi \\
\Xi\end{array}$ & $\begin{array}{l}\underset{\sim}{\sim} \\
\underset{\sum}{Z}\end{array}$ & 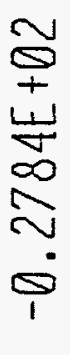 & $\begin{array}{c}\mathbb{S} \\
\dot{\sigma} \\
\stackrel{\mathbb{I}}{\mathscr{E}}\end{array}$ & $\begin{array}{l}\widehat{\infty} \\
\sim \\
\underset{\Sigma}{\Sigma}\end{array}$ & 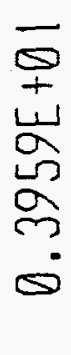 & $\begin{array}{l}\infty \\
- \\
\\
\leftarrow\end{array}$ & $\frac{1}{0}$ \\
\hline
\end{tabular}

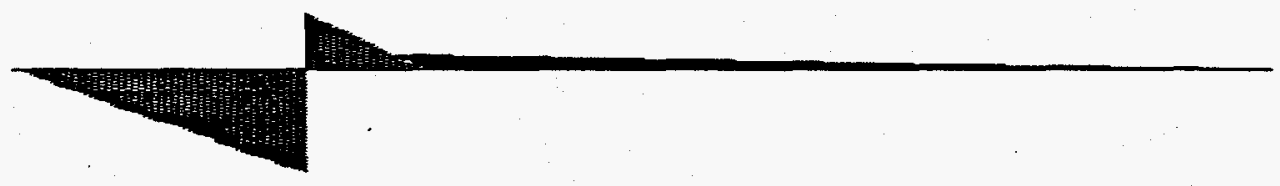




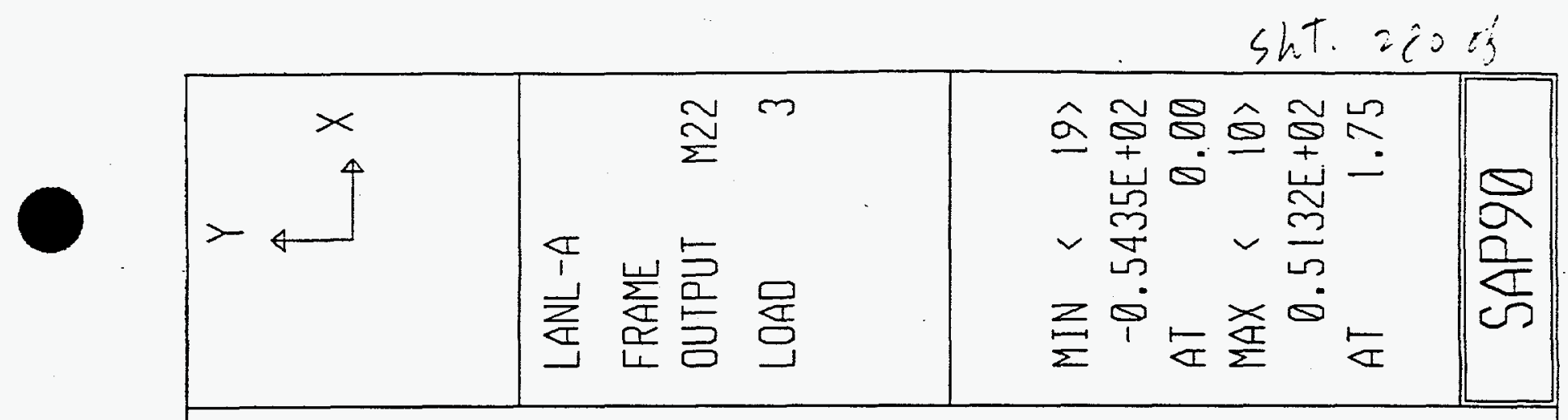


Slet. 2810

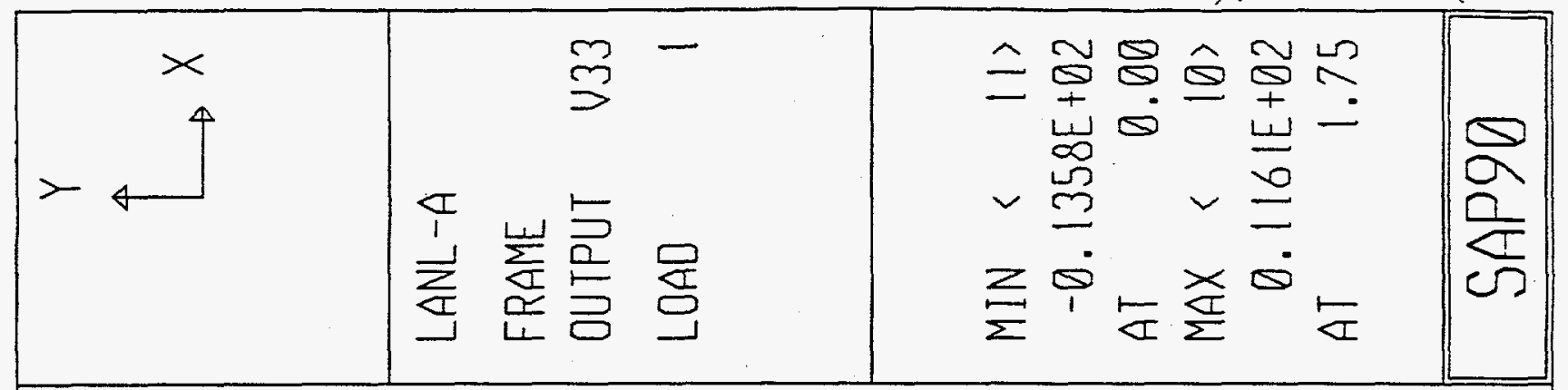

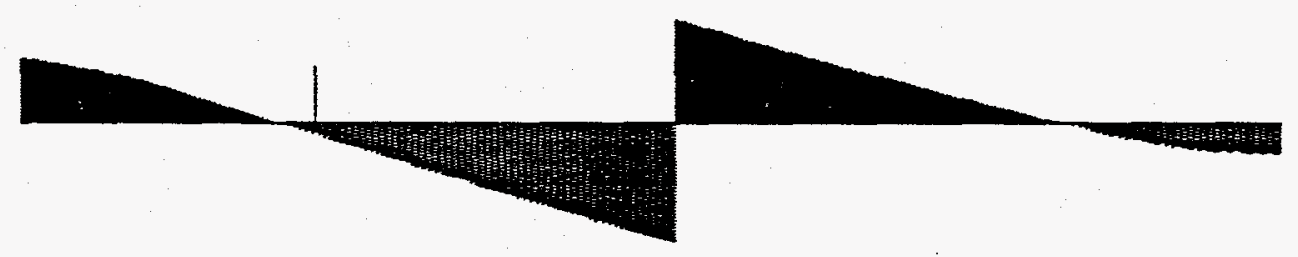




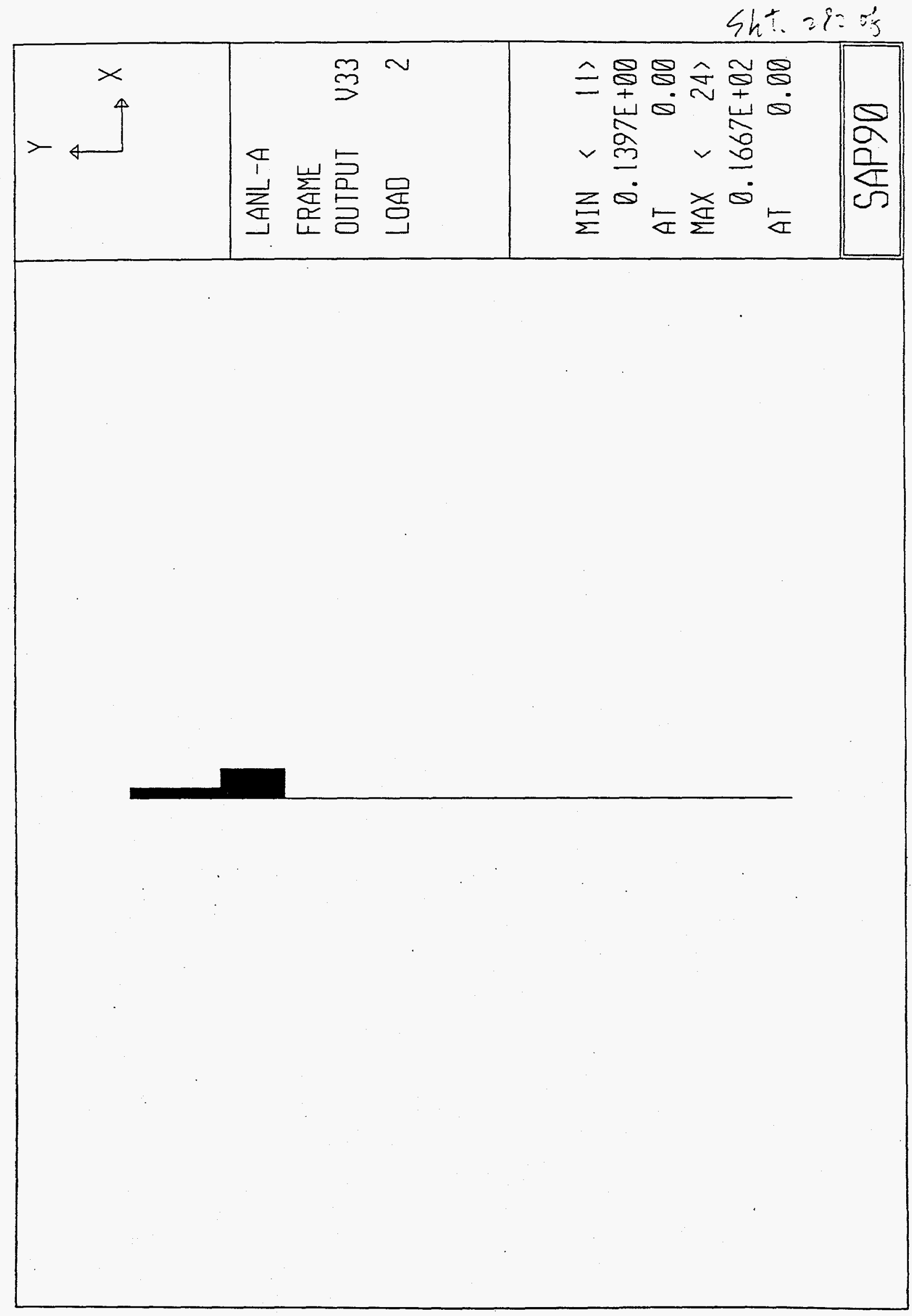




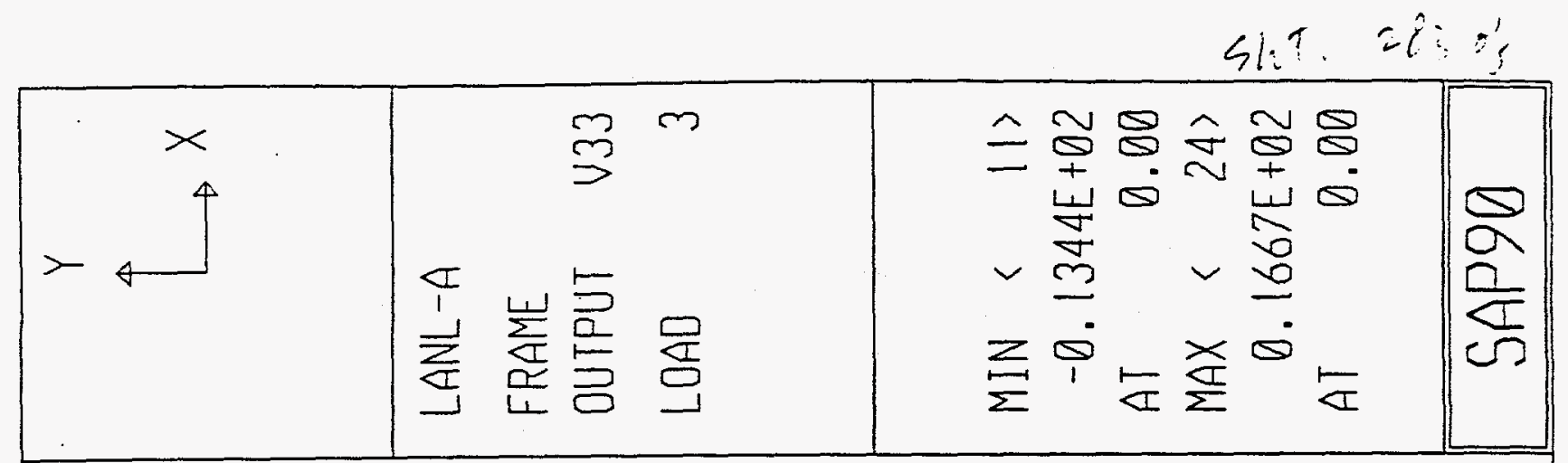

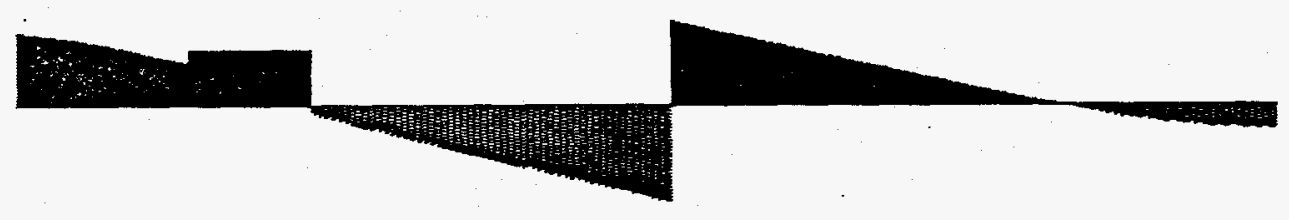


Sint 284 of

\begin{tabular}{|c|c|c|c|c|c|c|}
\hline $\begin{array}{r}x \\
4\end{array}$ & 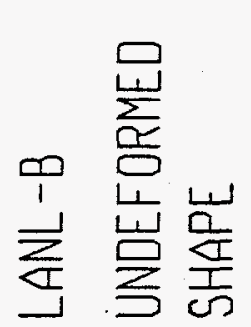 & $\begin{array}{l}\frac{0}{\Xi} \\
\frac{5}{5}\end{array}$ & 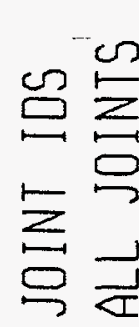 & 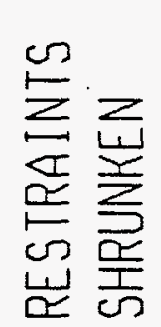 & 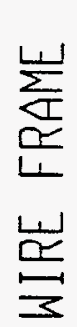 & 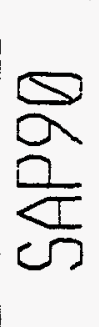 \\
\hline
\end{tabular}

은 트 
Sint. 2850

\begin{tabular}{|c|c|c|c|c|c|c|c|c|c|c|}
\hline $\begin{array}{r}x \\
-4\end{array}$ & $\frac{\infty}{1}$ & 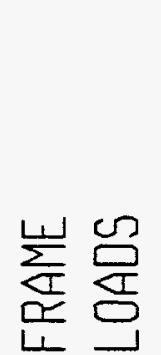 & $\underset{\Xi}{\Xi}$ & $\underset{\sum}{\sum}$ & 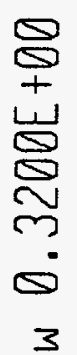 & 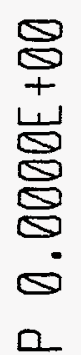 & $\sum_{\sum}^{\frac{I}{I}}$ & $\begin{array}{l}\bar{S} \\
+ \\
5 \\
5 \\
5 \\
5\end{array}$ & 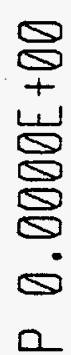 & $\frac{a}{2 \pi}$ \\
\hline
\end{tabular}

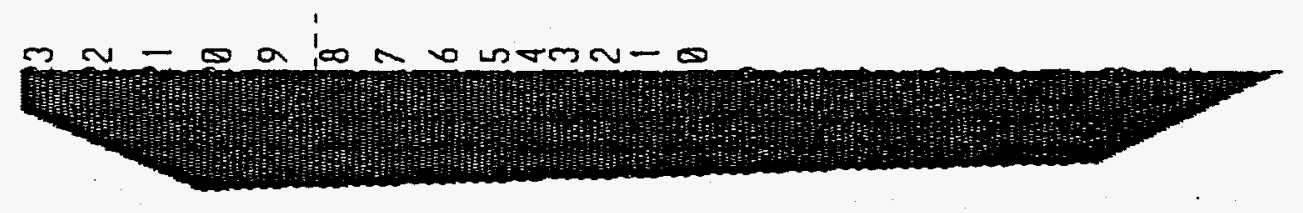



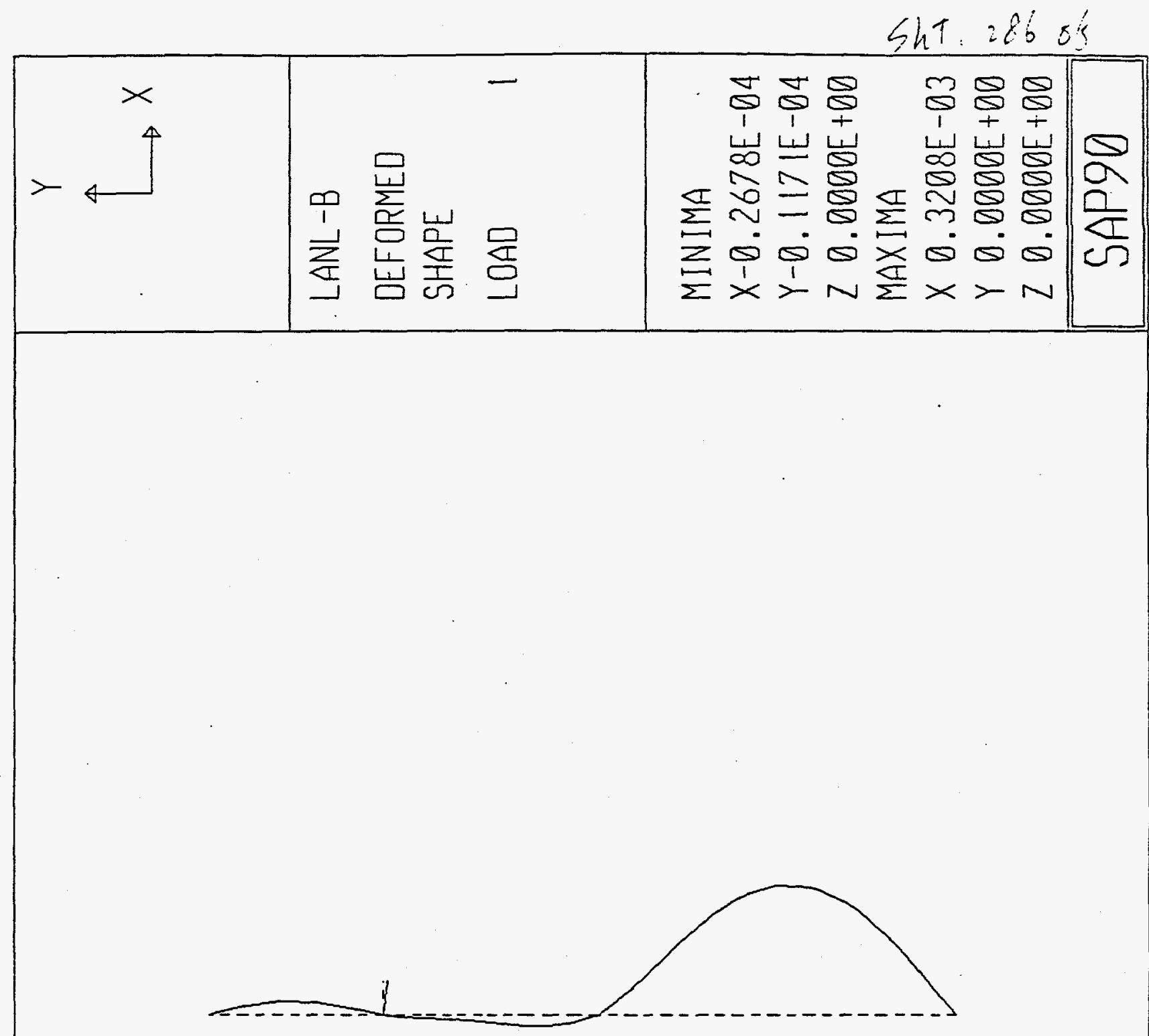
Sht. 2870 ,

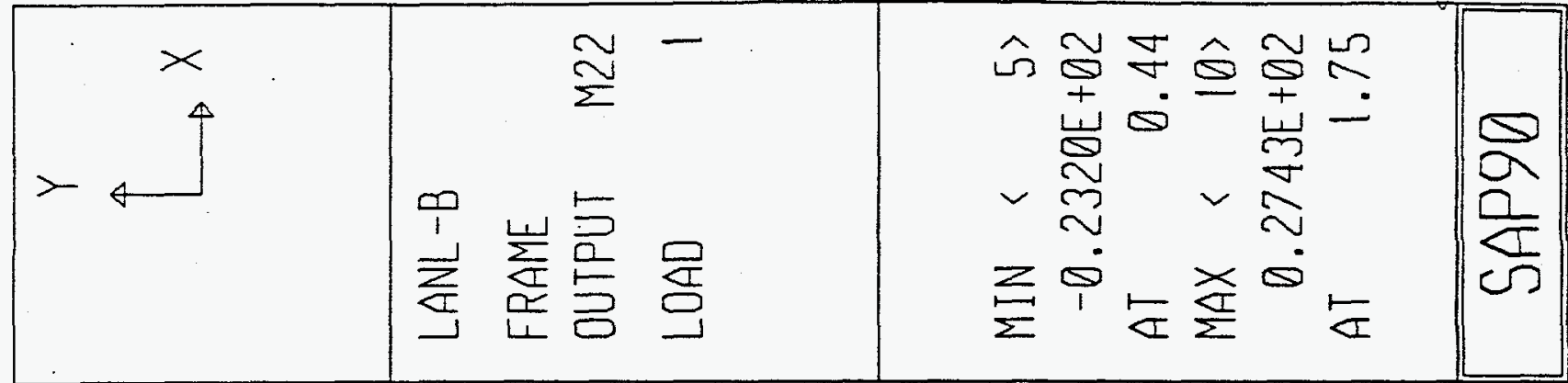

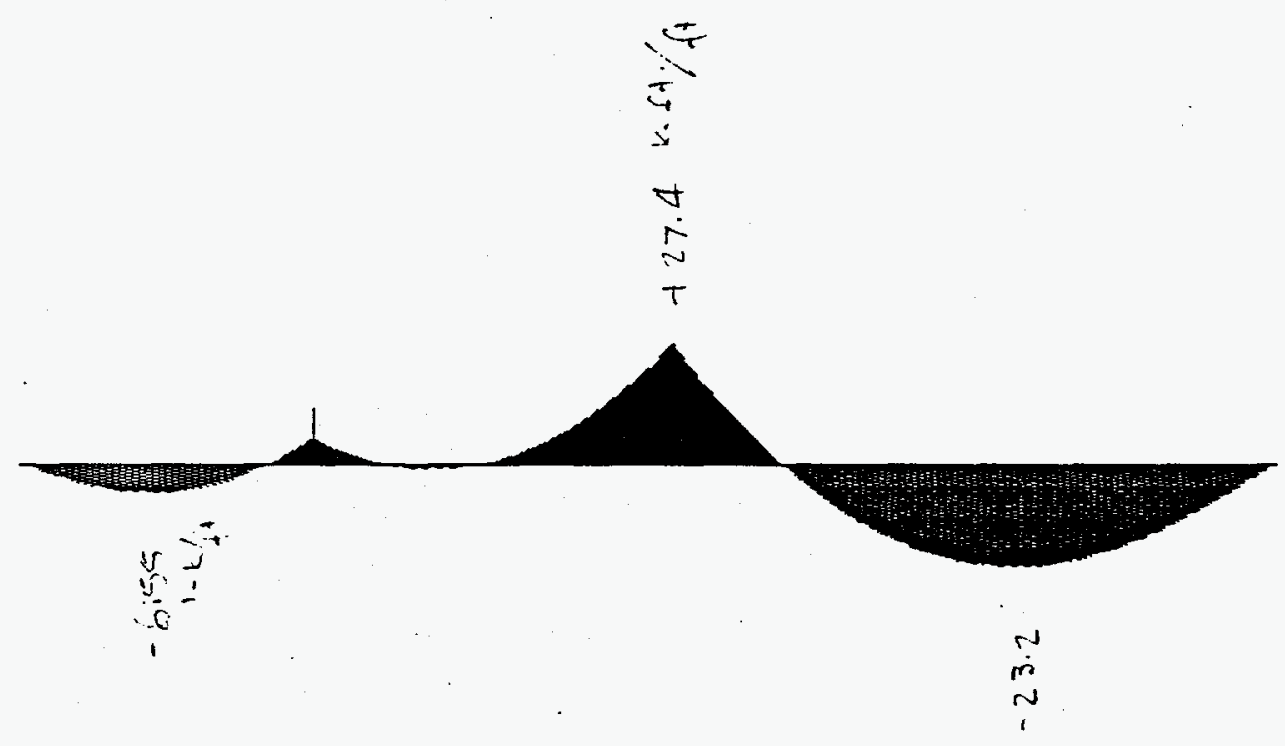



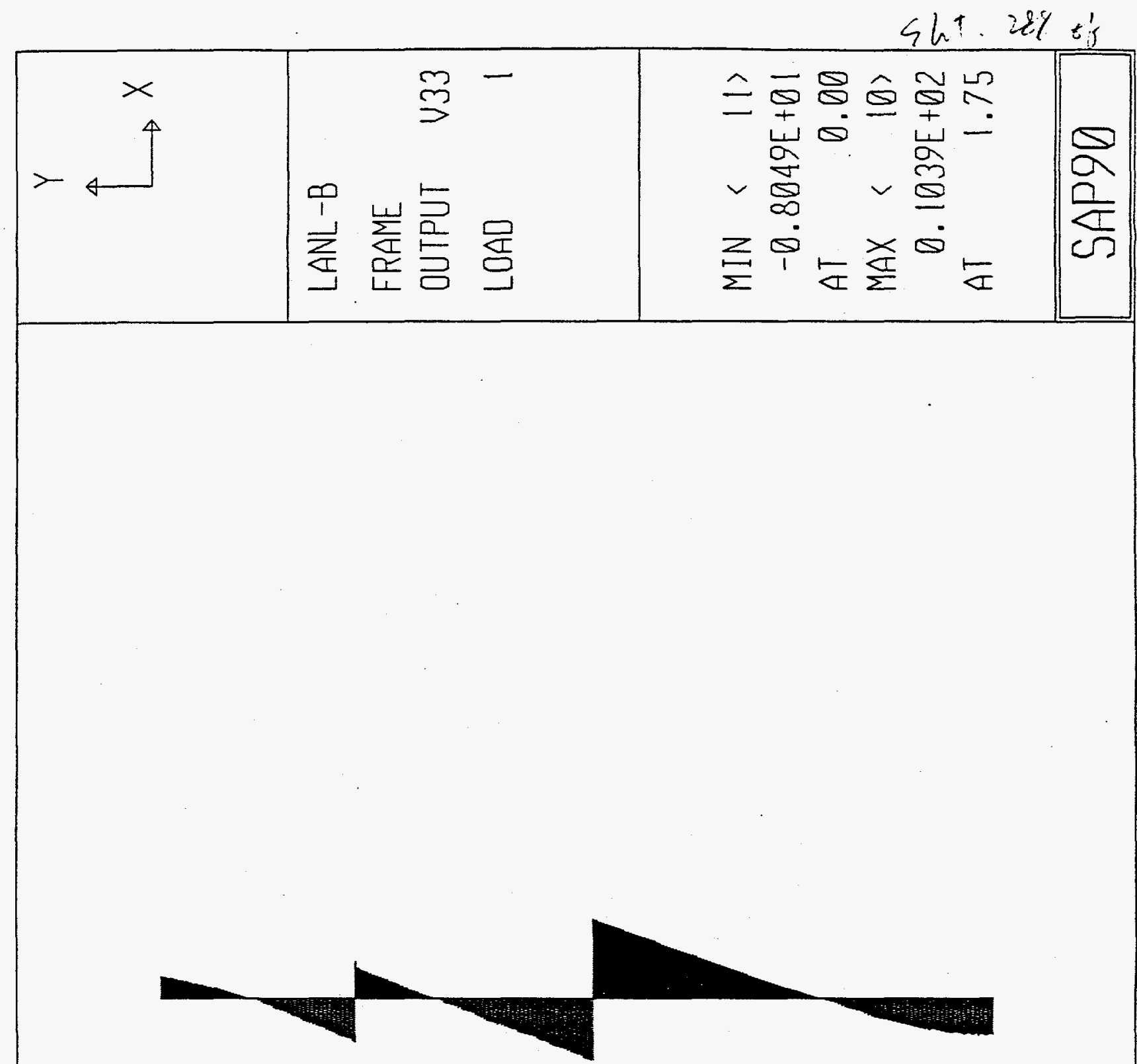
LANL - NMSF CONCEPTUAL DESIGN

C WEST WALL SEISMIC EVALUATION

C UNITS: ft, kips

C FILE: LANL-A

SYSTEM

$\mathrm{L}=2$

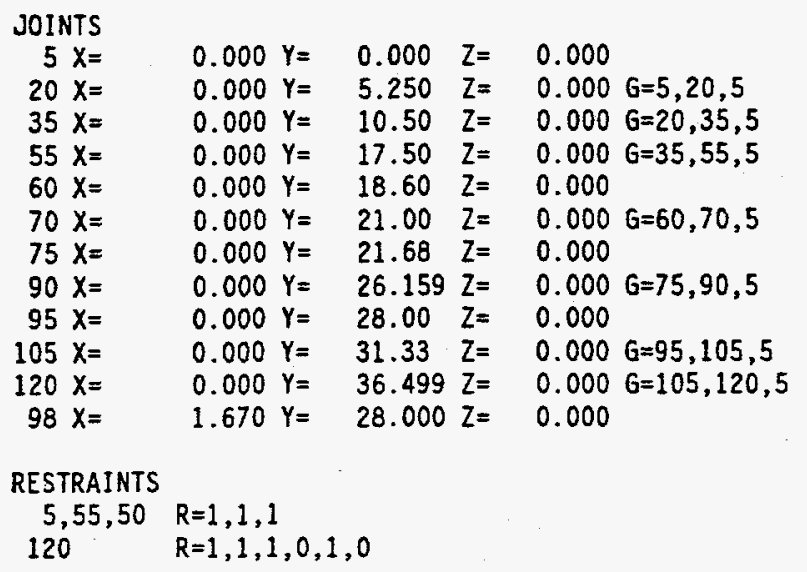

\section{MATERIALS}

FRAME

$N M=3 \quad N L=23$

$1 \quad S H=R \quad T=1.1 .5 \quad E=7108340 \quad W=0.216 \quad M=0.216 / 32.2: 1.5^{\prime} \times 1$, SECTION

SH=R $T=1.1 .33 \quad E=7108340 \quad W=0.192 \quad M=0.192 / 32.2: 1.33^{\prime} \times 1$, SECTION

$3 \quad S H=R \quad T=8,8 \quad E=7108340 \quad W=0.0 \quad M=0.0 / 32.2 \quad$ :RIGID MEMBER

1 TRAP $=0,0, \quad 0,1.749,0,0.320$

TRAP $=0,0,0.320,1.749,0,0.640$

TRAP $=0,0,0.640,1.749,0,0.960$

TRAP $=0,0,0.960,1.749,0,0.980$

TRAP $=0,0,0.980,1.749,0,1.000$

TRAP $=0,0,1.000,1.749,0,1.020$

TRAP $=0,0,1.020,1.749,0,1.042$

TRAP $=0,0,1.042,1.749,0,1.063$

TRAP $=0,0,1.063,1.749,0,1.085$

10 TRAP $=0,0,1.085,1.749,0,1.106$

11 TRAP $=0,0,1.106,1.099,0,1.120$

12 TRAP $=0,0,1.120,1.199,0,1.135$

13 TRAP $=0,0,1.135,1.199,0,1.150$

14 TRAP $=0,0,1.150,0.679,0,1.155$

- 15 TRAP $=0,0,1.155,1.492,0,1.173$

16 TRAP $=0,0,1.173,1.492,0,1.192$

17 TRAP $=0,0,1.192,1.492,0,1.210$

18 TRAP $=0,0,1.210,1.840,0,1.230$

19 TRAP $=0,0,1.230,1.664,0,1.250$

20 TRAP $=0,0,1.250,1.664,0,1.270$

21 TRAP $=0,0,1.270,1.722,0,0.990$

22 TRAP $=0,0,0.990,1.722,0,0.710$

23 TRAP $=0,0,0.710,1.722,0,0.430$

$\begin{array}{rrrlllll}1 & 5 & 10 & M= & 1 & L P= & 3,0 & N S L=1 \\ 2 & 10 & 15 & M= & 1 & L P= & 3,0 & N S L=2 \\ 3 & 15 & 20 & M= & 1 & L P= & 3,0 & N S L=3 \\ 4 & 20 & 25 & M= & 1 & L P= & 3,0 & N S L=4 \\ 5 & 25 & 30 & M= & 1 & L P= & 3,0 & N S L=5 \\ 6 & 30 & 35 & M= & 1 & L P= & 3,0 & N S L=6 \\ 7 & 35 & 40 & M= & 1 & L P= & 3,0 & N S L=7 \\ 8 & 40 & 45 & M= & 1 & L P= & 3,0 & N S L=8 \\ 9 & 45 & 50 & M= & 1 & L P= & 3,0 & N S L=9 \\ 10 & 50 & 55 & M= & 1 & L P= & 3,0 & N S L=10 \\ 11 & 55 & 60 & M= & 1 & L P= & 3,0 & N S L=11 \\ 12 & 60 & 65 & M= & 1 & L P= & 3,0 & N S L=12 \\ 13 & 65 & 70 & M= & 1 & L P= & 3,0 & N S L=13 \\ 14 & 70 & 75 & M= & 1 & L P= & 3,0 & N S L=14\end{array}$

File: LANL-A

Page 1 of 2 
sht. 290 if

$\begin{array}{rrrrlllll}15 & 75 & 80 & M= & 2 & L P= & 3,0 & N S L=15 \\ 16 & 80 & 85 & M= & 2 & L P= & 3,0 & N S L=16 \\ 17 & 85 & 90 & M= & 2 & L P= & 3,0 & N S L=17 \\ 18 & 90 & 95 & M= & 2 & L P= & 3,0 & N S L=18 \\ 19 & 95 & 100 & M= & 2 & L P= & 3,0 & N S L=19 \\ 20 & 100 & 105 & M= & 2 & L P= & 3,0 & N S L=20 \\ 21 & 105 & 110 & M= & 2 & L P= & 3,0 & N S L=21 \\ 22 & 110 & 115 & M= & 2 & L P= & 3,0 & N S L=22 \\ 23 & 115 & 120 & M= & 2 & L P= & 3,0 & N S L=23 \\ 24 & 95 & 98 & M= & 3 & L P= & 2,0 & \end{array}$

LOADS

$98 \quad L=2 \quad F=2.67,-16.67$

COMBO

$1 \mathrm{C}=1,0,0$;

$2 \mathrm{C}=0.0,1$

$3 C=1 \cdot 0,1 \cdot 0$

SELECT

$N T=1 \quad I D=5,120,5$

$N T=2 \quad I D=5,120,5$

$N T=5 \quad I D=1,24,1$ 
Sht. 291 of 


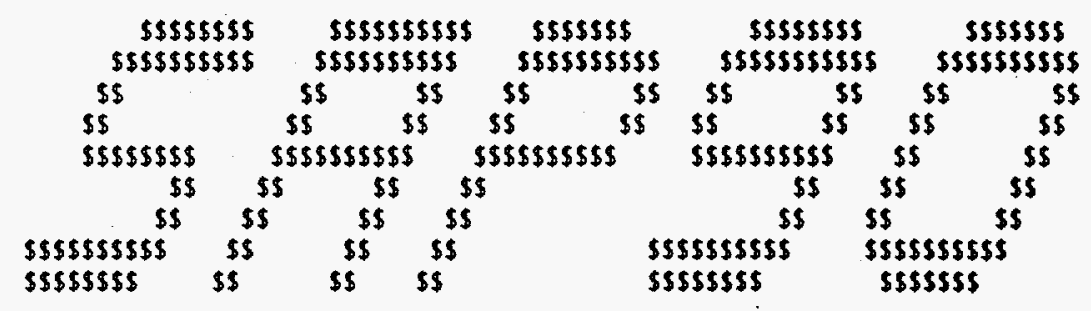

STRUCTURAL ANALYSIS PROGRAMS

VERSION P5.40

Copyright (C) 1978-1992

EDWARD L. WILSON

All rights reserved 
CSI I SAP 90 ... FINITE ELEMENT ANALYSIS OF STRUCTURES PAGE 1 LANL - NMSF CONCEPTUAL DESIGN PROGRAM:SAP90/FILE:lanl-a.F3F

FRAME ELEMENT FORCES

\begin{tabular}{|c|c|c|c|c|c|c|c|}
\hline 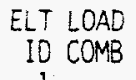 & $\begin{array}{l}\text { DIST } \\
\text { ENDI }\end{array}$ & SHEAR & $\begin{array}{l}\text { ANE } \\
\text { MOMENT }\end{array}$ & $\begin{array}{l}\text { AXIAL } \\
\text { FORCE }\end{array}$ & SHEAR & $\begin{array}{l}\text { LANE } \\
\text { MOMENT }\end{array}$ & $\begin{array}{l}\text { AXIAL } \\
\text { TORQ }\end{array}$ \\
\hline . & $\begin{array}{l}0.000 \\
0.000 \\
1.749 \\
1.750 \\
1.750\end{array}$ & $\begin{array}{l}0.000 \\
0.000 \\
0.000\end{array}$ & $\begin{array}{l}0.000 \\
0.000 \\
0.000\end{array}$ & $\begin{array}{l}0.000 \\
0.000\end{array}$ & $\begin{array}{l}-3.542 \\
-3.262 \\
-3.262\end{array}$ & $\begin{array}{r}0.000 \\
-6.032 \\
-6.035\end{array}$ & \\
\hline 2 & $\begin{array}{l}0.000 \\
0.000 \\
1.750 \\
1.750\end{array}$ & $\begin{array}{l}0.000 \\
0.000\end{array}$ & $\begin{array}{l}0.000 \\
0.000\end{array}$ & $\begin{array}{l}0.000 \\
0.000\end{array}$ & $\begin{array}{l}0.142 \\
0.142\end{array}$ & $\begin{array}{l}0.000 \\
0.249\end{array}$ & \\
\hline 3 & $\begin{array}{l}0.000 \\
0.000 \\
1.749 \\
1.750 \\
1.750\end{array}$ & $\begin{array}{l}0.000 \\
0.000 \\
0.000\end{array}$ & $\begin{array}{l}0.000 \\
0.000 \\
0.000\end{array}$ & $\begin{array}{l}0.000 \\
0.000\end{array}$ & $\begin{array}{l}-3.400 \\
-3.120 \\
-3.120\end{array}$ & $\begin{array}{r}0.000 \\
-5.783 \\
-5.786\end{array}$ & \\
\hline 1 & $\begin{array}{l}0.000 \\
0.000 \\
1.749 \\
1.750 \\
1.750\end{array}$ & $\begin{array}{l}0.000 \\
0.000 \\
0.000\end{array}$ & $\begin{array}{l}0.000 \\
0.000 \\
0.000\end{array}$ & 0.000 & $\begin{array}{l}-3.262 \\
-2.423 \\
-2.423\end{array}$ & $\begin{array}{r}-6.035 \\
-11.088 \\
-11.091\end{array}$ & \\
\hline 2 & $\begin{array}{l}0.000 \\
0.000 \\
1.750 \\
1.750\end{array}$ & $\begin{array}{l}0.000 \\
0.000\end{array}$ & $\begin{array}{l}0.000 \\
0.000\end{array}$ & $\begin{array}{l}0.000 \\
0.000\end{array}$ & $\begin{array}{l}0.142 \\
0.142\end{array}$ & $\begin{array}{l}0.249 \\
0.499\end{array}$ & \\
\hline 3 & $\begin{array}{l}0.000 \\
0.000 \\
1.749 \\
1.750 \\
1.750\end{array}$ & $\begin{array}{l}0.000 \\
0.000 \\
0.000\end{array}$ & $\begin{array}{l}0.000 \\
0.000 \\
0.000\end{array}$ & 0.000 & $\begin{array}{l}-3.120 \\
-2.280 \\
-2.280\end{array}$ & $\begin{array}{r}-5.786 \\
-10.590 \\
-10.592\end{array}$ & \\
\hline 1 & $\begin{array}{l}0.000 \\
0.000 \\
1.749 \\
1.750 \\
1.750\end{array}$ & $\begin{array}{l}0.000 \\
0.000 \\
0.000\end{array}$ & $\begin{array}{l}0.000 \\
0.000 \\
0.000\end{array}$ & 0.000 & $\begin{array}{l}-2.423 \\
-1.023 \\
-1.023\end{array}$ & $\begin{array}{l}-11.091 \\
-14.186 \\
-14.187\end{array}$ & \\
\hline 2 & $\begin{array}{l}0.000 \\
0.000 \\
1.750 \\
1.750\end{array}$ & $\begin{array}{l}0.000 \\
0.000\end{array}$ & $\begin{array}{l}0.000 \\
0.000\end{array}$ & $\begin{array}{l}0.000 \\
0.000\end{array}$ & $\begin{array}{l}0.142 \\
0.142\end{array}$ & $\begin{array}{l}0.499 \\
0.748\end{array}$ & \\
\hline 3 & $\begin{array}{l}0.000 \\
0.000\end{array}$ & 0.000 & 0.000 & 0.000 & -2.280 & -10.592 & \\
\hline
\end{tabular}


CSI / SAPGO - FINITE ELEMENT ANALYSIS OF STRUCTURES PAGE 2

LANL - NMSF CONCEPTUAL DESIGN

FRAME ELEMENT FORCES

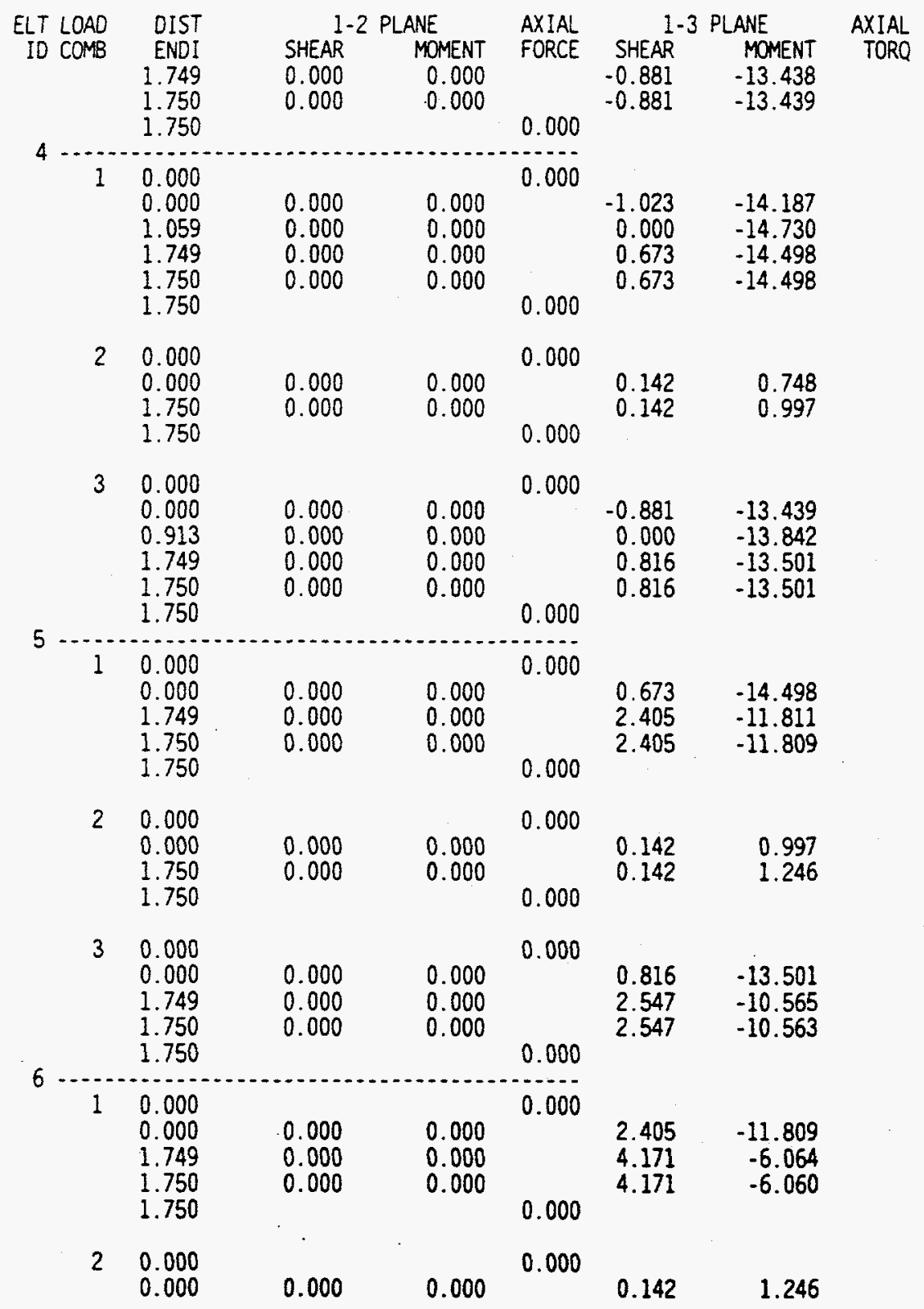




\section{Sht $255 \%$}

CSI / SAP 90 - FINITE ELEMENT ANALYSIS OF STRUCTURES PAGE 3

LANL - NMSF CONCEPTUAL OESIGN

PROGRAM:SAP9O/FILE: lanl-a.F3F

FRAME. ELEMENT FORCES

\begin{tabular}{|c|c|c|c|c|c|c|c|}
\hline & \multirow{3}{*}{$\begin{array}{r}\text { DIST } \\
\text { ENDI } \\
1.750 \\
1.750\end{array}$} & \multicolumn{2}{|c|}{ 1-2 PLANE } & \multirow{2}{*}{$\begin{array}{l}\text { AXIAL } \\
\text { FORCE }\end{array}$} & \multicolumn{2}{|c|}{ 1-3 PLANE } & \multirow{3}{*}{$\begin{array}{c}\text { AXIAL } \\
\text { TORQ }\end{array}$} \\
\hline & & SHEAR & MOMENT & & \multirow{2}{*}{$\begin{array}{l}\text { SHEAR } \\
0.142\end{array}$} & \multirow{2}{*}{$\begin{array}{r}\text { MOMENT } \\
1.496\end{array}$} & \\
\hline & & & & 0.000 & & & \\
\hline \multirow[t]{4}{*}{3} & 0.000 & & & 0.000 & & \multirow{4}{*}{$\begin{array}{r}-10.563 \\
-4.568 \\
-4.564\end{array}$} & \\
\hline & $\begin{array}{l}0.000 \\
1.749\end{array}$ & $\begin{array}{l}0.000 \\
0.000\end{array}$ & $\begin{array}{l}0.000 \\
0.000\end{array}$ & & \multirow{3}{*}{$\begin{array}{l}2.547 \\
4.314 \\
4.314\end{array}$} & & \\
\hline & 1.750 & & & & & & \\
\hline & & & & 0.000 & & & \\
\hline \multirow{4}{*}{1} & 0.000 & & & 0.000 & & \multirow{4}{*}{$\begin{array}{r}-6.060 \\
2.807 \\
2.813\end{array}$} & \\
\hline & 0.000 & & & & \multirow{3}{*}{$\begin{array}{l}4.171 \\
5.974 \\
5.974\end{array}$} & & \\
\hline & $\begin{array}{l}1.749 \\
1.750\end{array}$ & 0.000 & 0.000 & & & & \\
\hline & $\begin{array}{l}1.750 \\
\end{array}$ & & & 0.000 & & & \\
\hline \multirow[t]{3}{*}{2} & & & & 0.000 & \multirow{3}{*}{$\begin{array}{l}0.142 \\
0.142\end{array}$} & \multirow{3}{*}{$\begin{array}{l}1.496 \\
1.745\end{array}$} & \\
\hline & 0.000 & 0.000 & 0.000 & & & & \\
\hline & 1.750 & & & 0.000 & & & \\
\hline \multirow[t]{4}{*}{3} & 0.000 & & & 0.000 & \multirow{4}{*}{$\begin{array}{l}4.314 \\
6.117 \\
6.117\end{array}$} & \multirow{4}{*}{$\begin{array}{r}-4.564 \\
4.552 \\
4.558\end{array}$} & \\
\hline & 0.000 & & & & & & \\
\hline & $\begin{array}{l}1.749 \\
1.750\end{array}$ & $\begin{array}{l}0.000 \\
0.000\end{array}$ & $\begin{array}{l}0.000 \\
0.000\end{array}$ & & & & \\
\hline & 1.750 & & & 0.000 & & & \\
\hline \multirow{4}{*}{8} & 0.000 & & & 0.000 & \multirow{4}{*}{$\begin{array}{l}5.974 \\
7.815 \\
7.815\end{array}$} & \multirow{4}{*}{$\begin{array}{r}2.813 \\
14.866 \\
14.874\end{array}$} & \\
\hline & 0.000 & 0.000 & 0.000 & & & & \\
\hline & 1.749 & 0.000 & 0.000 & & & & \\
\hline & $\begin{array}{l}1.750 \\
1.750\end{array}$ & & & 0.000 & & & \\
\hline \multirow{3}{*}{2} & 0.000 & & & 0.000 & \multirow{3}{*}{$\begin{array}{l}0.142 \\
0.142\end{array}$} & \multirow{3}{*}{$\begin{array}{l}1.745 \\
1.994\end{array}$} & \\
\hline & 0.000 & 0.000 & 0.000 & & & & \\
\hline & $\begin{array}{l}1.750 \\
1.750\end{array}$ & & & 0.000 & & & \\
\hline \multirow{4}{*}{3} & 0.000 & & & 0.000 & \multirow{5}{*}{$\begin{array}{l}6.117 \\
7.958 \\
7.958\end{array}$} & \multirow{4}{*}{$\begin{array}{r}4.558 \\
16.861 \\
16.868\end{array}$} & \\
\hline & 0.000 & & & & & & \\
\hline & 1.749 & 0.000 & 0.000 & & & & \\
\hline & $\begin{array}{l}1.750 \\
1.750\end{array}$ & & & 0.000 & & & \\
\hline 9 & & & & & & & \\
\hline 1 & & & & 0.000 & & & \\
\hline & $\begin{array}{l}0.000 \\
1.749\end{array}$ & $\begin{array}{l}0.000 \\
0.000\end{array}$ & $\begin{array}{l}0.000 \\
0.000\end{array}$ & & $\begin{array}{l}7.815 \\
9.694\end{array}$ & $\begin{array}{l}14.874 \\
30.180\end{array}$ & \\
\hline & 1.750 & 0.000 & 0.000 & & $\begin{array}{l}9.6944 \\
9.694\end{array}$ & $\begin{array}{l}30.180 \\
30.190\end{array}$ & \\
\hline & 1.750 & & & 0.000 & & & \\
\hline
\end{tabular}


$\operatorname{sht.}=0 ;$

CSI / SAP 90 - - FINITE ELEMENT ANALYSIS OF STRUCTURES PAGE 4

LANL - NMSF CONCEPTUAL DESIGN

FRAME ELEMENT FORCES

\begin{tabular}{|c|c|c|c|c|c|c|c|}
\hline $\begin{array}{l}\text { ELT LOAD } \\
\text { ID COMB }\end{array}$ & $\begin{array}{l}\text { DIST } \\
\text { ENDI }\end{array}$ & SHEAR & $\begin{array}{l}\text { ANE } \\
\text { MOMENT }\end{array}$ & $\begin{array}{l}\text { AXIAL } \\
\text { FORCE }\end{array}$ & SHEAR & $\begin{array}{l}\text { LANE } \\
\text { MOMENT }\end{array}$ & $\begin{array}{c}\text { AXIAL } \\
\text { TORO }\end{array}$ \\
\hline 2 & $\begin{array}{l}0.000 \\
0.000 \\
1.750 \\
1.750\end{array}$ & $\begin{array}{l}0.000 \\
0.000\end{array}$ & $\begin{array}{l}0.000 \\
0.000\end{array}$ & $\begin{array}{l}0.000 \\
0.000\end{array}$ & $\begin{array}{l}0.142 \\
0.142\end{array}$ & $\begin{array}{l}1.994 \\
2.244\end{array}$ & \\
\hline 3 & $\begin{array}{l}0.000 \\
0.000 \\
1.749 \\
1.750 \\
1.750\end{array}$ & $\begin{array}{l}0.000 \\
0.000 \\
0.000\end{array}$ & $\begin{array}{l}0.000 \\
0.000 \\
0.000\end{array}$ & $\begin{array}{l}0.000 \\
0.000\end{array}$ & $\begin{array}{l}7.958 \\
9.836 \\
9.836\end{array}$ & $\begin{array}{l}16.868 \\
32.423 \\
32.433\end{array}$ & \\
\hline 1 & $\begin{array}{l}0.000 \\
0.000 \\
1.749 \\
1.750 \\
1.750\end{array}$ & $\begin{array}{l}0.000 \\
0.000 \\
0.000\end{array}$ & $\begin{array}{l}0.000 \\
0.000 \\
0.000\end{array}$ & 0.000 & $\begin{array}{r}9.694 \\
11.610 \\
11.610\end{array}$ & $\begin{array}{l}30.190 \\
48.814 \\
48.825\end{array}$ & \\
\hline 2 & $\begin{array}{l}0.000 \\
0.000 \\
1.750 \\
1.750\end{array}$ & $\begin{array}{l}0.000 \\
0.000\end{array}$ & $\begin{array}{l}0.000 \\
0.000\end{array}$ & $\begin{array}{l}0.000 \\
0.000\end{array}$ & $\begin{array}{l}0.142 \\
0.142\end{array}$ & $\begin{array}{l}2.244 \\
2.493\end{array}$ & \\
\hline 3 & $\begin{array}{l}0.000 \\
0.000 \\
1.749 \\
1.750 \\
1.750\end{array}$ & $\begin{array}{l}0.000 \\
0.000 \\
0.000\end{array}$ & $\begin{array}{l}0.000 \\
0.000 \\
0.000\end{array}$ & $\begin{array}{l}0.000 \\
0.000\end{array}$ & $\begin{array}{r}9.836 \\
11.752 \\
11.752\end{array}$ & $\begin{array}{l}32.433 \\
51.306 \\
51.318\end{array}$ & \\
\hline 1 & $\begin{array}{l}0.000 \\
0.000 \\
1.099 \\
1.100 \\
1.100\end{array}$ & $\begin{array}{l}0.000 \\
0.000 \\
0.000\end{array}$ & $\begin{array}{l}0.000 \\
0.000 \\
0.000\end{array}$ & $\begin{array}{l}0.000 \\
0.000\end{array}$ & $\begin{array}{l}-13.576 \\
-12.353 \\
-12.353\end{array}$ & $\begin{array}{l}48.825 \\
34.576 \\
34.563\end{array}$ & \\
\hline 2 & $\begin{array}{l}0.000 \\
0.000 \\
1.100 \\
1.100\end{array}$ & $\begin{array}{l}0.000 \\
0.000\end{array}$ & $\begin{array}{l}0.000 \\
0.000\end{array}$ & $\begin{array}{l}-7.648 \\
-7.648\end{array}$ & $\begin{array}{l}0.140 \\
0.140\end{array}$ & $\begin{array}{l}2.493 \\
2.646\end{array}$ & \\
\hline 3 & $\begin{array}{l}0.000 \\
0.000 \\
1.099 \\
1.100 \\
1.100\end{array}$ & $\begin{array}{l}0.000 \\
0.000 \\
0.000\end{array}$ & $\begin{array}{l}0.000 \\
0.000 \\
0.000\end{array}$ & -7.648 & $\begin{array}{r}-13.437 \\
-12.214 \\
-12.214\end{array}$ & $\begin{array}{l}51.318 \\
37.222 \\
37.210\end{array}$ & \\
\hline 1 & $\begin{array}{l}0.000 \\
0.000\end{array}$ & 0.000 & 0.000 & 0.000 & -12.353 & 34.563 & \\
\hline
\end{tabular}


CSI / SAPQO - FINITE ELEMENT ANALYSIS OF STRUCTURES PAGE 5 LANL - NMSF CONCEPTUAL DESIGN

FRAME ELEMENT FOR.CES

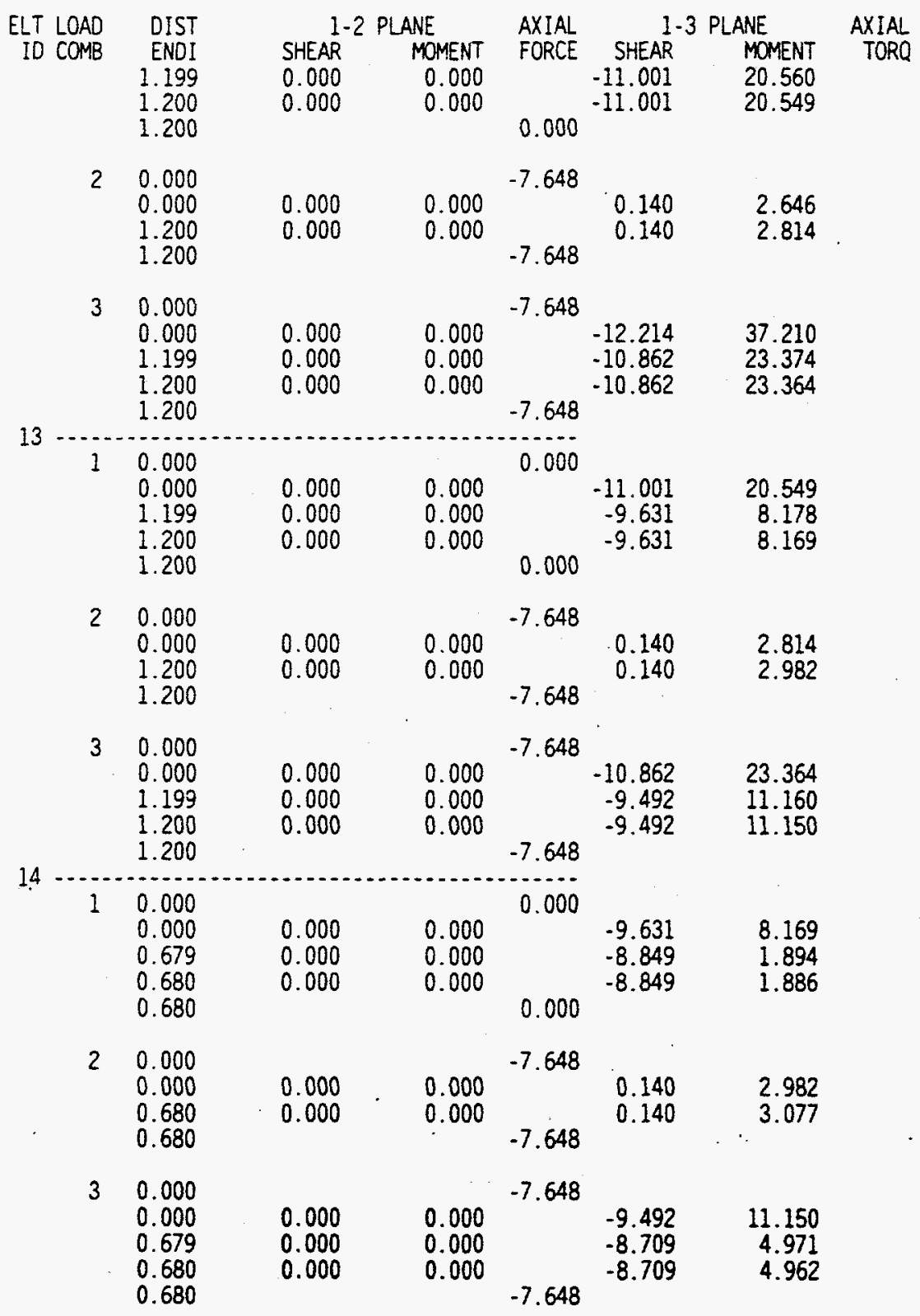


CS I / SAP9O- - FINITE ELEMENT ANALYSIS OF STRUCTURES PAGE 6

LANL - NMSF CONCEPTUAL DESIGN

FRAME ELEMENT FORCES

\begin{tabular}{|c|c|c|c|c|c|c|c|}
\hline $\begin{array}{l}\text { ELT LOAD } \\
\text { ID COMB }\end{array}$ & $\begin{array}{l}\text { DIST } \\
\text { ENDI }\end{array}$ & $\begin{array}{l}1 \\
\text { SHEAR }\end{array}$ & $\begin{array}{l}\text { ANE } \\
\text { MOMENT }\end{array}$ & $\begin{array}{l}\text { AXIAL } \\
\text { FORCE }\end{array}$ & SHEAR & $\begin{array}{l}\text { LANE } \\
\text { MOMENT }\end{array}$ & $\begin{array}{r}\text { AXIAL } \\
\text { TORQ }\end{array}$ \\
\hline 1 & $\begin{array}{l}0.000 \\
0.000 \\
1.492 \\
1.493 \\
1.493\end{array}$ & $\begin{array}{l}0.000 \\
0.000 \\
0.000\end{array}$ & $\begin{array}{l}0.000 \\
0.000 \\
0.000\end{array}$ & $\begin{array}{l}0.000 \\
0.000\end{array}$ & $\begin{array}{l}-8.849 \\
-7.112 \\
-7.112\end{array}$ & $\begin{array}{r}1.886 \\
-10.025 \\
-10.032\end{array}$ & \\
\hline 2 & $\begin{array}{l}0.000 \\
0.000 \\
1.493 \\
1.493\end{array}$ & $\begin{array}{l}0.000 \\
0.000\end{array}$ & $\begin{array}{l}0.000 \\
0.000\end{array}$ & $\begin{array}{l}-7.648 \\
-7.648\end{array}$ & $\begin{array}{l}0.140 \\
0.140\end{array}$ & $\begin{array}{l}3.077 \\
3.285\end{array}$ & \\
\hline 3 & $\begin{array}{l}0.000 \\
0.000 \\
1.492 \\
1.493 \\
1.493\end{array}$ & $\begin{array}{l}0.000 \\
0.000 \\
0.000\end{array}$ & $\begin{array}{l}0.000 \\
0.000 \\
0.000\end{array}$ & -7.648 & $\begin{array}{l}-8.709 \\
-6.973 \\
-6.973\end{array}$ & $\begin{array}{r}4.962 \\
-6.740 \\
-6.747\end{array}$ & \\
\hline 1 & $\begin{array}{l}0.000 \\
0.000 \\
1.492 \\
1.493 \\
1.493\end{array}$ & $\begin{array}{l}0.000 \\
0.000 \\
0.000\end{array}$ & $\begin{array}{l}0.000 \\
0.000 \\
0.000\end{array}$ & 0.000 & $\begin{array}{l}-7.112 \\
-5.348 \\
-5.348\end{array}$ & $\begin{array}{l}-10.032 \\
-19.331 \\
-19.336\end{array}$ & \\
\hline 2 & $\begin{array}{l}0.000 \\
0.000 \\
1.493 \\
1.493\end{array}$ & $\begin{array}{l}0.000 \\
0.000\end{array}$ & $\begin{array}{l}0.000 \\
0.000\end{array}$ & $\begin{array}{l}-7.648 \\
-7.648\end{array}$ & $\begin{array}{l}0.140 \\
0.140\end{array}$ & $\begin{array}{l}3.285 \\
3.494\end{array}$ & \\
\hline 3 & $\begin{array}{l}0.000 \\
0.000 \\
1.492 \\
1.493 \\
1.493\end{array}$ & $\begin{array}{l}0.000 \\
0.000 \\
0.000\end{array}$ & $\begin{array}{l}0.000 \\
0.000 \\
0.000\end{array}$ & -7.648 & $\begin{array}{l}-6.973 \\
-5.208 \\
-5.208\end{array}$ & $\begin{array}{r}-6.747 \\
-15.837 \\
-15.842\end{array}$ & \\
\hline 1 & $\begin{array}{l}0.000 \\
0.000 \\
1.492 \\
1.493 \\
1.493\end{array}$ & $\begin{array}{l}0.000 \\
0.000 \\
0.000\end{array}$ & $\begin{array}{l}0.000 \\
0.000 \\
0.000\end{array}$ & 0.000 & $\begin{array}{l}-5.348 \\
-3.556 \\
-3.556\end{array}$ & $\begin{array}{l}-19.336 \\
-25.982 \\
-25.985\end{array}$ & \\
\hline 2 & $\begin{array}{l}0.000 \\
0.000 \\
1.493 \\
1.493\end{array}$ & $\begin{array}{l}0.000 \\
0.000\end{array}$ & $\begin{array}{l}0.000 \\
0.000\end{array}$ & $\begin{array}{l}-7.648 \\
-7.648\end{array}$ & $\begin{array}{l}0.140 \\
0.140\end{array}$ & $\begin{array}{l}3.494 \\
3.702\end{array}$ & \\
\hline 3 & $\begin{array}{l}0.000 \\
0.000\end{array}$ & 0.000 & 0.000 & -7.648 & -5.208 & -15.842 & \\
\hline
\end{tabular}


CS 1 / SAPGO -. FINITE ELEMENT ANALYSIS OF STRUCTURES PAGE 7

LANL - NMSF CONCEPTUAL DESIGN

PROGRAM: SAP9O/FILE: : ani-a. F3F

FRAME ELEMENT FORCES

\begin{tabular}{|c|c|c|c|c|c|c|c|}
\hline $\begin{array}{r}E L T \text { LOAD } \\
\text { ID COMB }\end{array}$ & $\begin{array}{r}\text { DIST } \\
\text { ENDI } \\
1.492 \\
1.493 \\
1.493\end{array}$ & $\begin{array}{l}1- \\
\text { SHEAR } \\
0.000 \\
0.000\end{array}$ & $\begin{array}{l}\text { ANE } \\
\text { MOMENT } \\
0.000 \\
0.000\end{array}$ & $\begin{array}{l}\text { AXIAL } \\
\text { FORCE } \\
-7.648\end{array}$ & $\begin{array}{r}\text { SHEAR } \\
-3.416 \\
-3.416\end{array}$ & $\begin{array}{l}\text { LANE } \\
\text { MOMENT } \\
-22.280 \\
-22.283\end{array}$ & $\begin{array}{l}\text { AXIAL } \\
\text { TORQ }\end{array}$ \\
\hline 1 & $\begin{array}{l}0.000 \\
0.000 \\
1.840 \\
1.841 \\
1.841\end{array}$ & $\begin{array}{l}0.000 \\
0.000 \\
0.000\end{array}$ & $\begin{array}{l}0.000 \\
0.000 \\
0.000\end{array}$ & $\begin{array}{l}0.000 \\
0.000\end{array}$ & $\begin{array}{l}-3.556 \\
-1.311 \\
-1.311\end{array}$ & $\begin{array}{l}-25.985 \\
-30.469 \\
-30.470\end{array}$ & \\
\hline 2 & $\begin{array}{l}0.000 \\
0.000 \\
1.841 \\
1.841\end{array}$ & $\begin{array}{l}0.000 \\
0.000\end{array}$ & $\begin{array}{l}0.000 \\
0.000\end{array}$ & $\begin{array}{l}-7.548 \\
-7.648\end{array}$ & $\begin{array}{l}0.140 \\
0.140\end{array}$ & $\begin{array}{l}3.702 \\
3.959\end{array}$ & \\
\hline 3 & $\begin{array}{l}0.000 \\
0.000 \\
1.840 \\
1.841 \\
1.841\end{array}$ & $\begin{array}{l}0.000 \\
0.000 \\
0.000\end{array}$ & $\begin{array}{l}0.000 \\
0.000 \\
0.000\end{array}$ & $\begin{array}{l}-7.648 \\
-7.648\end{array}$ & $\begin{array}{l}-3.416 \\
-1.172 \\
-1.172\end{array}$ & $\begin{array}{l}-22.283 \\
-26.510 \\
-26.511\end{array}$ & \\
\hline 1 & $\begin{array}{l}0.000 \\
0.000 \\
1.061 \\
1.664 \\
1.665 \\
1.665\end{array}$ & $\begin{array}{l}0.000 \\
0.000 \\
0.000 \\
0.000\end{array}$ & $\begin{array}{l}0.000 \\
0.000 \\
0.000 \\
0.000\end{array}$ & 0.000 & $\begin{array}{r}-1.311 \\
0.000 \\
0.752 \\
0.752\end{array}$ & $\begin{array}{r}-30.470 \\
-31.167 \\
-30.940 \\
-30.939\end{array}$ & \\
\hline 2 & $\begin{array}{l}0.000 \\
0.000 \\
1.665 \\
1.665\end{array}$ & $\begin{array}{l}0.000 \\
0.000\end{array}$ & $\begin{array}{l}0.000 \\
0.000\end{array}$ & $\begin{array}{l}9.022 \\
9.022\end{array}$ & $\begin{array}{l}2.810 \\
2.810\end{array}$ & $\begin{array}{l}-23.879 \\
-19.201\end{array}$ & \\
\hline 3 & $\begin{array}{l}0.000 \\
0.000 \\
1.664 \\
1.665 \\
1.665\end{array}$ & $\begin{array}{l}0.000 \\
0.000 \\
0.000\end{array}$ & $\begin{array}{l}0.000 \\
0.000 \\
0.000\end{array}$ & 9.022 & $\begin{array}{l}1.498 \\
3.562 \\
3.562\end{array}$ & $\begin{array}{l}-54.350 \\
-50.144 \\
-50.141\end{array}$ & \\
\hline 1 & $\begin{array}{l}0.000 \\
0.000 \\
1.664 \\
1.665 \\
1.665\end{array}$ & $\begin{array}{l}0.000 \\
0.000 \\
0.000\end{array}$ & $\begin{array}{l}0.000 \\
0.000 \\
0.000\end{array}$ & 0.000 & $\begin{array}{l}0.752 \\
2.849 \\
2.849\end{array}$ & $\begin{array}{l}-30.939 \\
-27.948 \\
-27.945\end{array}$ & \\
\hline 2 & $\begin{array}{l}0.000 \\
0.000 \\
1.665\end{array}$ & $\begin{array}{l}0.000 \\
0.000\end{array}$ & $\begin{array}{l}0.000 \\
0.000\end{array}$ & 9.022 & $\begin{array}{l}2.810 \\
2.810\end{array}$ & $\begin{array}{l}-19.201 \\
-14.523\end{array}$ & \\
\hline
\end{tabular}


CSI / SAPGO - FINITE ELEMENT ANALYSIS OF STRUCTURES PAGE 8 LANL - NMSF CONCEPTUAL DESIGN

PROGRAM:SAP90/FILE: lanl-a. F3F

FRAME ELEMENT FORCES

\begin{tabular}{|c|c|c|c|c|c|c|c|}
\hline \multirow{2}{*}{$\begin{array}{l}\text { ELT LOAD } \\
\text { IO COMB }\end{array}$} & \multirow{2}{*}{$\begin{array}{r}\text { DIST } \\
\text { ENDI } \\
1.665\end{array}$} & \multicolumn{2}{|c|}{ 1-2 PLANE } & \multirow{2}{*}{$\begin{array}{l}\text { AXIAL } \\
\text { FORCE } \\
9.022\end{array}$} & \multicolumn{2}{|c|}{ 1-3 PLAI } & \multirow{2}{*}{$\begin{array}{l}\text { AXIAL } \\
\text { TORQ }\end{array}$} \\
\hline & & SHEAR & MOMENT & & SHEAR & MOMENT & \\
\hline 3 & $\begin{array}{l}0.000 \\
0.000 \\
1.664 \\
1.665 \\
1.665\end{array}$ & $\begin{array}{l}0.000 \\
0.000 \\
0.000\end{array}$ & $\begin{array}{l}0.000 \\
0.000 \\
0.000\end{array}$ & $\begin{array}{l}9.022 \\
9.022\end{array}$ & $\begin{array}{l}3.562 \\
5.658 \\
5.658\end{array}$ & $\begin{array}{l}-50.141 \\
-42.474 \\
-42.468\end{array}$ & \\
\hline 1 & $\begin{array}{l}0.000 \\
0.000 \\
1.722 \\
1.723 \\
1.723\end{array}$ & $\begin{array}{l}0.000 \\
0.000 \\
0.000\end{array}$ & $\begin{array}{l}0.000 \\
0.000 \\
0.000\end{array}$ & 0.000 & $\begin{array}{l}2.849 \\
4.795 \\
4.795\end{array}$ & $\begin{array}{l}-27.945 \\
-21.295 \\
-21.290\end{array}$ & \\
\hline 2 & $\begin{array}{l}0.000 \\
0.000 \\
1.723 \\
1.723\end{array}$ & $\begin{array}{l}0.000 \\
0.000\end{array}$ & $\begin{array}{l}0.000 \\
0.000\end{array}$ & $\begin{array}{l}9.022 \\
9.022\end{array}$ & $\begin{array}{l}2.810 \\
2.810\end{array}$ & $\begin{array}{r}-14.523 \\
-9.682\end{array}$ & \\
\hline 3 & $\begin{array}{l}0.000 \\
0.000 \\
1.722 \\
1.723 \\
1.723\end{array}$ & $\begin{array}{l}0.000 \\
0.000 \\
0.000\end{array}$ & $\begin{array}{l}0.000 \\
0.000 \\
0.000\end{array}$ & 9.022 & $\begin{array}{l}5.658 \\
7.604 \\
7.604\end{array}$ & $\begin{array}{l}-42.468 \\
-30.980 \\
-30.972\end{array}$ & \\
\hline 1 & $\begin{array}{l}0.000 \\
0.000 \\
1.722 \\
1.723 \\
1.723\end{array}$ & $\begin{array}{l}0.000 \\
0.000 \\
0.000\end{array}$ & $\begin{array}{l}0.000 \\
0.000 \\
0.000\end{array}$ & 0.000 & $\begin{array}{l}4.795 \\
6.258 \\
6.258\end{array}$ & $\begin{array}{l}-21.290 \\
-11.705 \\
-11.698\end{array}$ & \\
\hline 2 & $\begin{array}{l}0.000 \\
0.000 \\
1.723 \\
1.723\end{array}$ & $\begin{array}{l}0.000 \\
0.000\end{array}$ & $\begin{array}{l}0.000 \\
0.000\end{array}$ & $\begin{array}{l}9.022 \\
9.022\end{array}$ & $\begin{array}{l}2.810 \\
2.810\end{array}$ & $\begin{array}{l}-9.682 \\
-4.841\end{array}$ & \\
\hline 3 & $\begin{array}{l}0.000 \\
0.000 \\
1.722 \\
1.723 \\
1.723\end{array}$ & $\begin{array}{l}0.000 \\
0.000 \\
0.000\end{array}$ & $\begin{array}{l}0.000 \\
0.000 \\
0.000\end{array}$ & 9.022 & $\begin{array}{l}7.604 \\
9.068 \\
9.068\end{array}$ & $\begin{array}{l}-30.972 \\
-16.548 \\
-16.539\end{array}$ & \\
\hline 1 & $\begin{array}{l}0.000 \\
0.000 \\
1.722 \\
1.723 \\
1.723\end{array}$ & $\begin{array}{l}0.000 \\
0.000 \\
0.000\end{array}$ & $\begin{array}{l}0.000 \\
0.000 \\
0.000\end{array}$ & 0.000 & $\begin{array}{l}6.258 \\
7.240 \\
7.240\end{array}$ & $\begin{array}{r}-11.698 \\
-0.007 \\
0.000\end{array}$ & \\
\hline
\end{tabular}


CSI / SAP9O - FINITE ELEMENT ANALYSIS OF STRUCTURES PAGE 99

LANL - NMSF CONCEPTUAL DESIGN

FRAME ELEMENT FORCES

\begin{tabular}{|c|c|c|c|c|c|c|c|}
\hline $\begin{array}{l}\text { ELT LOAD } \\
\text { ID COMB }\end{array}$ & OIST & $1-$ & ANE & AXIAL & SHE & LANE & AXIAL \\
\hline 2 & $\begin{array}{l}0.000 \\
0.000 \\
1.723 \\
1.723\end{array}$ & $\begin{array}{l}0.000 \\
0.000\end{array}$ & $\begin{array}{l}0.000 \\
0.000\end{array}$ & $\begin{array}{l}9.022 \\
9.022\end{array}$ & $\begin{array}{l}2.810 \\
2.810\end{array}$ & $\begin{array}{r}-4.841 \\
0.0000\end{array}$ & \\
\hline 3 & $\begin{array}{l}0.000 \\
0.000 \\
1.722 \\
1.723 \\
1.723\end{array}$ & $\begin{array}{l}0.000 \\
0.000 \\
0.000\end{array}$ & $\begin{array}{l}0.000 \\
0.000 \\
0.000\end{array}$ & $\begin{array}{l}9.022 \\
9.022\end{array}$ & $\begin{array}{r}9.068 \\
10.050 \\
10.050\end{array}$ & $\begin{array}{r}-16.539 \\
-0.010 \\
0.000\end{array}$ & \\
\hline 1 & $\begin{array}{l}0.000 \\
0.000 \\
1.670 \\
1.670\end{array}$ & $\begin{array}{l}0.000 \\
0.000\end{array}$ & $\begin{array}{l}0.000 \\
0.000\end{array}$ & $\begin{array}{l}0.000 \\
0.000\end{array}$ & $\begin{array}{l}0.000 \\
0.000\end{array}$ & $\begin{array}{l}0.000 \\
0.000\end{array}$ & \\
\hline 2 & $\begin{array}{l}0.000 \\
0.000 \\
1.670 \\
1.670\end{array}$ & $\begin{array}{l}0.000 \\
0.000\end{array}$ & $\begin{array}{l}0.000 \\
0.000\end{array}$ & $\begin{array}{l}2.670 \\
2.670\end{array}$ & $\begin{array}{l}16.670 \\
16.670\end{array}$ & $\begin{array}{r}-27.839 \\
0.000\end{array}$ & \\
\hline 3 & $\begin{array}{l}0.000 \\
0.000 \\
1.670 \\
1.670\end{array}$ & $\begin{array}{l}0.000 \\
0.000\end{array}$ & $\begin{array}{l}0.000 \\
0.000\end{array}$ & $\begin{array}{r}2.670 \\
2.670\end{array}$ & $\begin{array}{l}16.670 \\
16.670\end{array}$ & $\begin{array}{r}-27.839 \\
0.000\end{array}$ & \\
\hline
\end{tabular}


LANL - NMSF CONCEPTUAL DESIGN

C WEST WALL SEISMIC EVALUATION (with modificaiton - add support at node 95)

C UNITS: $\mathrm{ft}$, kips

C FILE: LANL-B

SYSTEM

$L=2$

JOINTS

$5 x=$

$20 x=$

$35 x=$

$0.000 Y=5.250 \quad Z=0.000 \mathrm{G}=5,20,5$

$55 x=$

$60 x=$

$10.50 \quad Z=$

$0.000 \mathrm{G}=20,35,5$

$70 x=$

$75 x=$

$0.000 Y=$

17.50. $Z=$

$0.000 \quad G=35,55,5$

$0.000 Y=$

$18.60 \quad z=$

0.000

$90 x=$
$95 x=$

$0.000 Y=$

$21.00 \quad Z=$

$0.000 \mathrm{G}=60,70,5$

$0.000 Y=$

$21.68 \mathrm{Z}=$

0.000

$0.000 Y=28.00 \quad Z=$

$0.000 \mathrm{G}=75,90,5$

$105 x=$

$0.000 Y=$

$31.33 \quad Z=$

0.000

$120 x=$

$0.000 Y=36.499 Z=$

$0.000 \mathrm{G}=95,105,5$

$98 x=$

$1.670 Y=$

$28.000 \mathrm{Z}=$

$0.000 \mathrm{G}=105,120,5$

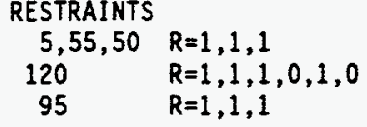

C MATERIALS

\section{FRAME}

$N M=3 \quad N L=23$

$1 \quad S H=R \quad T=1,1.5 \quad E=7108340 \quad W=0.216$

2 $S H=R \quad T=1,1.33 \quad E=7108340 \quad W=0.192$

$3 \quad S H=R \quad T=8,8 \quad E=7108340 \quad W=0.0$

1 TRAP $=0,0, \quad 0,1.749,0,0.320$

2 TRAP $=0,0,0.320,1.749,0,0.640$

3 TRAP $=0,0,0.640,1.749,0,0.960$

TRAP $=0,0,0.960,1.749,0,0.980$

TRAP $=0,0,0.980,1.749,0,1.000$

TRAP $=0,0,1.000,1.749,0,1.020$

TRAP $=0,0,1.020,1.749,0,1.042$

TRAP $=0,0,1.042,1.749,0,1.063$

TRAP $=0,0,1.063,1.749,0,1.085$

10 TRAP $=0,0,1.085,1.749,0,1.106$

11 TRAP $=0,0,1.106,1.099,0,1.120$

12 TRAP $=0,0,1.120,1.199,0,1.135$

13 TRAP $=0,0,1.135,1.199,0,1.150$

14 TRAP $=0,0,1.150,0.679,0,1.155$

15 TRAP $=0,0,1.155,1.492,0,1.173$

16 TRAP $=0,0,1.173,1.492,0,1.192$

17 TRAP $=0,0,1.192,1.492,0,1.210$

18 TRAP $=0,0,1.210,1.840,0,1.230$

19 TRAP $=0,0,1.230,1.664,0,1.250$

20 TRAP $=0,0,1.250,1.664,0,1.270$

21 TRAP $=0,0,1.270,1.722,0,0.990$

22 TRAP $=0,0,0.990,1.722,0,0.710$

23 TRAP $=0,0,0.710,1.722,0,0.430$

$\begin{array}{rrrlllll}1 & 5 & 10 & M= & 1 & L P= & 3,0 & N S L=1 \\ 2 & 10 & 15 & M= & 1 & L P= & 3,0 & N S L=2 \\ 3 & 15 & 20 & M= & 1 & L P= & 3,0 & N S L=3 \\ 4 & 20 & 25 & M= & 1 & L P= & 3,0 & N S L=4 \\ 5 & 25 & 30 & M= & 1 & L P= & 3,0 & N S L=5 \\ 6 & 30 & 35 & M= & 1 & L P= & 3,0 & N S L=6 \\ 7 & 35 & 40 & M= & 1 & L P= & 3,0 & M S L=7 \\ 8 & 40 & 45 & M= & 1 & L P= & 3,0 & N S L=8 \\ 9 & 45 & 50 & M= & 1 & L P= & 3,0 & N S L=9 \\ 10 & 50 & 55 & M= & 1 & L P= & 3,0 & N S L=10 \\ 11 & 55 & 60 & M= & 1 & L P= & 3,0 & N S L=11 \\ 12 & 60 & 65 & M= & 1 & L P= & 3,0 & N S L=12 \\ 13 & 65 & 70 & M= & 1 & L P= & 3,0 & N S L=13\end{array}$


NT $=1 \quad I D=5,120,5$

$N T=2 \quad I D=5,120,5$

$N T=5 \quad I D=1,24,1$ 



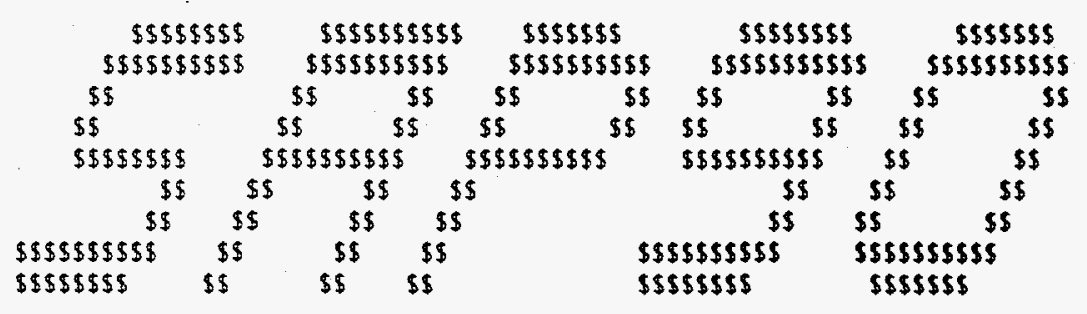

STRUCTURAL ANALYSIS PROGRAMS

VERSION P5. 40

Copyright (C) 1978-1992

EOWARD L. WILSON

All rights reserved 
CSI / SAP9O- - FINITE ELEMENT ANALYSIS OF STRUCTURES PAGE 1 LANL - NMSF CONCEPTUAL DESIGN

FRAME ELEMENT FORCES

\begin{tabular}{|c|c|c|c|c|c|c|c|}
\hline \multirow[t]{2}{*}{011} & $\begin{array}{l}\text { DIST } \\
\text { ENDI }\end{array}$ & SHEAR & 1.2 PLANE & $\begin{array}{l}\text { AXIAL } \\
\text { FORCE }\end{array}$ & SHEAR & $\begin{array}{l}\text { LANE } \\
\text { MOMENT }\end{array}$ & \multirow[t]{2}{*}{$\begin{array}{l}\text { AXIAL } \\
\text { TORQ }\end{array}$} \\
\hline & $\begin{array}{l}0.000 \\
0.000 \\
1.749 \\
1.750 \\
1.750\end{array}$ & $\begin{array}{l}0.000 \\
0.000 \\
0.000\end{array}$ & $\begin{array}{l}0.000 \\
0.000 \\
0.000\end{array}$ & 0.000 & $\begin{array}{l}-4.765 \\
-4.485 \\
-4.485\end{array}$ & $\begin{array}{r}0.000 \\
-8.170 \\
-8.175\end{array}$ & \\
\hline 2 & $\begin{array}{l}0.000 \\
0.000 \\
1.749 \\
1.750 \\
1.750\end{array}$ & $\begin{array}{l}0.000 \\
0.000 \\
0.000\end{array}$ & $\begin{array}{l}0.000 \\
0.000 \\
0.000\end{array}$ & 0.000 & $\begin{array}{l}-4.485 \\
-3.645 \\
-3.645\end{array}$ & $\begin{array}{r}-8.175 \\
-15.366 \\
-15.369\end{array}$ & \\
\hline $\begin{array}{c}3 \cdots \\
1\end{array}$ & $\begin{array}{l}0.000 \\
0.000 \\
1.749 \\
1.750 \\
1.750\end{array}$ & $\begin{array}{l}0.000 \\
0.000 \\
0.000\end{array}$ & $\begin{array}{l}0.000 \\
0.000 \\
0.000\end{array}$ & 0.000 & $\begin{array}{l}-3.645 \\
-2.246 \\
-2.246\end{array}$ & $\begin{array}{l}-15.369 \\
-20.603 \\
-20.605\end{array}$ & \\
\hline 1 & $\begin{array}{l}0.000 \\
0.000 \\
1.749 \\
1.750 \\
1.750\end{array}$ & $\begin{array}{l}0.000 \\
0.000 \\
0.000\end{array}$ & $\begin{array}{l}0.000 \\
0.000 \\
0.000\end{array}$ & 0.000 & $\begin{array}{l}-2.246 \\
-0.549 \\
-0.549\end{array}$ & $\begin{array}{l}-20.605 \\
-23.055 \\
-23.056\end{array}$ & \\
\hline 1 & $\begin{array}{l}0.000 \\
0.000 \\
0.559 \\
1.749 \\
1.750 \\
1.750\end{array}$ & $\begin{array}{l}0.000 \\
0.000 \\
0.000 \\
0.000\end{array}$ & $\begin{array}{l}0.000 \\
0.000 \\
0.000 \\
0.000\end{array}$ & 0.000 & $\begin{array}{r}-0.549 \\
0.000 \\
1.182 \\
1.182\end{array}$ & $\begin{array}{l}-23.056 \\
-23.209 \\
-22.507 \\
-22.506\end{array}$ & \\
\hline $\begin{array}{c}6 \\
1\end{array}$ & $\begin{array}{l}0.000 \\
0.000 \\
1.749 \\
1.750 \\
1.750\end{array}$ & $\begin{array}{l}0.000 \\
0.000 \\
0.000\end{array}$ & $\begin{array}{l}0.000 \\
0.000 \\
0.000\end{array}$ & 0.000 & $\begin{array}{l}1.182 \\
2.949 \\
2.949\end{array}$ & $\begin{array}{l}-22.506 \\
-18.899 \\
-18.896\end{array}$ & \\
\hline 1 & $\begin{array}{l}0.000 \\
0.000 \\
1.749 \\
1.750 \\
1.750\end{array}$ & $\begin{array}{l}0.000 \\
0.000 \\
0.000\end{array}$ & $\begin{array}{l}0.000 \\
0.000 \\
0.000\end{array}$ & 0.000 & $\begin{array}{l}2.949 \\
4.752 \\
4.752\end{array}$ & $\begin{array}{l}-18.896 \\
-12.168 \\
-12.163\end{array}$ & \\
\hline
\end{tabular}


CSI / SAP 0 - - FINITE ELEMENT ANALYSIS OF STRUCTURES PAGE 2

LANL - NMSF CONCEPTUAL DESIGN

FRAME ELEMENT FORCES

\begin{tabular}{|c|c|c|c|c|c|c|c|}
\hline $\begin{array}{l}\text { ELT } L O A D \\
\text { ID COMB }\end{array}$ & $\begin{array}{l}\text { OIST } \\
\text { ENDI }\end{array}$ & SHEAR & $\begin{array}{l}\text { ANE } \\
\text { MOMENT }\end{array}$ & $\begin{array}{l}\text { AXIAL } \\
\text { FORCE }\end{array}$ & SHEAR & $\begin{array}{l}\text { LANE } \\
\text { MOMENT }\end{array}$ & $\begin{array}{l}\text { AXIAL } \\
\text { TORQ }\end{array}$ \\
\hline 1 & $\begin{array}{l}0.000 \\
0.000 \\
1.749 \\
1.750 \\
1.750\end{array}$ & $\begin{array}{l}0.000 \\
0.000 \\
0.000\end{array}$ & $\begin{array}{l}0.000 \\
0.000 \\
0.000\end{array}$ & $\begin{array}{l}0.000 \\
0.000\end{array}$ & $\begin{array}{l}4.752 \\
6.593 \\
6.593\end{array}$ & $\begin{array}{r}-12.163 \\
-2.248 \\
-2.242\end{array}$ & \\
\hline 1 & $\begin{array}{l}0.000 \\
0.000 \\
1.749 \\
1.750 \\
1.750\end{array}$ & $\begin{array}{l}0.000 \\
0.000 \\
0.000\end{array}$ & $\begin{array}{l}0.000 \\
0.000 \\
0.000\end{array}$ & $\begin{array}{l}0.000 \\
0.000\end{array}$ & $\begin{array}{l}6.593 \\
8.471 \\
8.471\end{array}$ & $\begin{array}{l}-2.242 \\
10.926 \\
10.934\end{array}$ & \\
\hline 1 & $\begin{array}{l}0.000 \\
0.000 \\
1.749 \\
1.750 \\
1.750\end{array}$ & $\begin{array}{l}0.000 \\
0.000 \\
0.000\end{array}$ & $\begin{array}{l}0.000 \\
0.000 \\
0.000\end{array}$ & $\begin{array}{l}0.000 \\
0.000\end{array}$ & $\begin{array}{r}8.471 \\
10.387 \\
10.387\end{array}$ & $\begin{array}{l}10.934 \\
27.420 \\
27.431\end{array}$ & \\
\hline 1 & $\begin{array}{l}0.000 \\
0.000 \\
1.099 \\
1.100 \\
1.100\end{array}$ & $\begin{array}{l}0.000 \\
0.000 \\
0.000\end{array}$ & $\begin{array}{l}0.000 \\
0.000 \\
0.000\end{array}$ & $\begin{array}{l}0.000 \\
0.000\end{array}$ & $\begin{array}{l}-8.049 \\
-6.825 \\
-6.825\end{array}$ & $\begin{array}{l}27.431 \\
19.256 \\
19.249\end{array}$ & \\
\hline 1 & $\begin{array}{l}0.000 \\
0.000 \\
1.199 \\
1.200 \\
1.200\end{array}$ & $\begin{array}{l}0.000 \\
0.000 \\
0.000\end{array}$ & $\begin{array}{l}0.000 \\
0.000 \\
0.000\end{array}$ & $\begin{array}{l}0.000 \\
0.000\end{array}$ & $\begin{array}{l}-6.825 \\
-5.474 \\
-5.474\end{array}$ & $\begin{array}{l}19.249 \\
11.874 \\
11.869\end{array}$ & \\
\hline 1 & $\begin{array}{l}0.000 \\
0.000 \\
1.199 \\
1.200 \\
1.200\end{array}$ & $\begin{array}{l}0.000 \\
0.000 \\
0.000\end{array}$ & $\begin{array}{l}0.000 \\
0.000 \\
0.000\end{array}$ & 0.000 & $\begin{array}{l}-5.474 \\
-4.104 \\
-4.104\end{array}$ & $\begin{array}{r}11.869 \\
6.125 \\
6.121\end{array}$ & \\
\hline 1 & $\begin{array}{l}0.000 \\
0.000 \\
0.679 \\
0.680 \\
0.680\end{array}$ & $\begin{array}{l}0.000 \\
0.000 \\
0.000\end{array}$ & $\begin{array}{l}0.000 \\
0.000 \\
0.000\end{array}$ & 0.000 & $\begin{array}{l}-4.104 \\
-3.321 \\
-3.321\end{array}$ & $\begin{array}{l}6.121 \\
3.600 \\
3.597\end{array}$ & \\
\hline
\end{tabular}


$C \leq 1$ / SAP 90 - FINITE ELEMENT ANALYSIS OF STRUCTURES PAGE 3 LANL - NMSF CONCEPTUAL DESIGN

FRAME ELEMENT FORCES

\begin{tabular}{|c|c|c|c|c|c|c|c|}
\hline ELT LOAD & DIST & $\stackrel{1-}{1-}$ & ANE & AXIAL & SHEAD & ANE & AXIAL \\
\hline & ENO: & & & & SHEAR & MOMENT & \\
\hline 1 & $\begin{array}{l}0.000 \\
0.000 \\
1.492 \\
1.493 \\
1.493\end{array}$ & $\begin{array}{l}0.000 \\
0.000 \\
0.000\end{array}$ & $\begin{array}{l}0.000 \\
0.000 \\
0.000\end{array}$ & $\begin{array}{l}0.000 \\
0.000\end{array}$ & $\begin{array}{l}-3.321 \\
-1.584 \\
-1.584\end{array}$ & $\begin{array}{r}3.597 \\
-0.066 \\
-0.068\end{array}$ & \\
\hline 1 & $\begin{array}{l}0.000 \\
0.000 \\
1.341 \\
1.492 \\
1.493 \\
1.493\end{array}$ & $\begin{array}{l}0.000 \\
0.000 \\
0.000 \\
0.000\end{array}$ & $\begin{array}{l}0.000 \\
0.000 \\
0.000 \\
0.000\end{array}$ & 0.000 & $\begin{array}{r}-1.584 \\
0.000 \\
0.180 \\
0.180\end{array}$ & $\begin{array}{l}-0.068 \\
-1.133 \\
-1.119 \\
-1.119\end{array}$ & \\
\hline 1 & $\begin{array}{l}0.000 \\
0.000 \\
1.492 \\
1.493 \\
1.493\end{array}$ & $\begin{array}{l}0.000 \\
0.000 \\
0.000\end{array}$ & $\begin{array}{l}0.000 \\
0.000 \\
0.000\end{array}$ & 0.000 & $\begin{array}{l}0.180 \\
1.972 \\
1.972\end{array}$ & $\begin{array}{r}-1.119 \\
0.483 \\
0.485\end{array}$ & \\
\hline 1 & $\begin{array}{l}0.000 \\
0.000 \\
1.840 \\
1.841 \\
1.841\end{array}$ & $\begin{array}{l}0.000 \\
0.000 \\
0.000\end{array}$ & $\begin{array}{l}0.000 \\
0.000 \\
0.000\end{array}$ & 0.000 & $\begin{array}{l}1.972 \\
4.217 \\
4.217\end{array}$ & $\begin{array}{l}0.485 \\
6.172 \\
6.177\end{array}$ & \\
\hline 1 & $\begin{array}{l}0.000 \\
0.000 \\
1.654 \\
1.665 \\
1.665\end{array}$ & $\begin{array}{l}0.000 \\
0.000 \\
0.000\end{array}$ & $\begin{array}{l}0.000 \\
0.000 \\
0.000\end{array}$ & 0.000 & $\begin{array}{l}-5.623 \\
-3.560 \\
-3.560\end{array}$ & $\begin{array}{r}6.177 \\
-1.468 \\
-1.472\end{array}$ & \\
\hline 1 & $\begin{array}{l}0.000 \\
0.000 \\
1.664 \\
1.665 \\
1.665\end{array}$ & $\begin{array}{l}0.000 \\
0.000 \\
0.000\end{array}$ & $\begin{array}{l}0.000 \\
0.000 \\
0.000\end{array}$ & 0.000 & $\begin{array}{l}-3.560 \\
-1.463 \\
-1.463\end{array}$ & $\begin{array}{l}-1.472 \\
-5.656 \\
-5.657\end{array}$ & \\
\hline 1 & $\begin{array}{l}0.000 \\
0.000 \\
1.253 \\
1.722 \\
1.723 \\
1.723\end{array}$ & $\begin{array}{l}0.000 \\
0.000 \\
0.000 \\
0.000\end{array}$ & $\begin{array}{l}0.000 \\
0.000 \\
0.000 \\
0.000\end{array}$ & 0.000 & $\begin{array}{r}-1.463 \\
0.000 \\
0.483 \\
0.483\end{array}$ & $\begin{array}{l}-5.657 \\
-6.547 \\
-6.432 \\
-6.431\end{array}$ & \\
\hline
\end{tabular}


CSI / SAPGO - FINITE ELEMENT ANALYSIS OF STRUCTURES PAGE 4 LANL - NMSF CONCEPTUAL DESIGN

PROGRAM:SAFGO/FILE: IanI-D. F JF

FRAME ELEMENT FORCES

\begin{tabular}{|c|c|c|c|c|c|c|c|}
\hline $\begin{array}{l}\text { ELT LOAD } \\
\text { ID COMB }\end{array}$ & $\begin{array}{l}\text { DIST } \\
\text { ENDI }\end{array}$ & SHEAR & $\begin{array}{l}\text { ANE } \\
\text { MOMENT }\end{array}$ & $\begin{array}{l}\text { AXIAL } \\
\text { FORCE }\end{array}$ & SHEAR & $\begin{array}{l}\text { ANE } \\
\text { MOMENT }\end{array}$ & $\begin{array}{l}\text { AXIA } \\
\text { TOR }\end{array}$ \\
\hline 1 & $\begin{array}{l}0.000 \\
0.000 \\
1.722 \\
1.723 \\
1.723\end{array}$ & $\begin{array}{l}0.000 \\
0.000 \\
0.000\end{array}$ & $\begin{array}{l}0.000 \\
0.000 \\
0.000\end{array}$ & 0.000 & $\begin{array}{l}0.483 \\
1.946 \\
1.946\end{array}$ & $\begin{array}{l}-6.431 \\
-4.271 \\
-4.269\end{array}$ & \\
\hline 1 & $\begin{array}{l}0.000 \\
0.000 \\
1.722 \\
1.723 \\
1.723\end{array}$ & $\begin{array}{l}0.000 \\
0.000 \\
0.000\end{array}$ & $\begin{array}{l}0.000 \\
0.000 \\
0.000\end{array}$ & 0.000 & $\begin{array}{l}1.946 \\
2.928 \\
2.928\end{array}$ & $\begin{array}{r}-4.269 \\
-0.003 \\
0.000\end{array}$ & \\
\hline 1 & $\begin{array}{l}0.000 \\
0.000 \\
1.670 \\
1.670\end{array}$ & $\begin{array}{l}0.000 \\
0.000\end{array}$ & $\begin{array}{l}0.000 \\
0.000\end{array}$ & $\begin{array}{l}0.000 \\
0.000\end{array}$ & $\begin{array}{l}0.000 \\
0.000\end{array}$ & $\begin{array}{l}0.000 \\
0.000\end{array}$ & \\
\hline
\end{tabular}




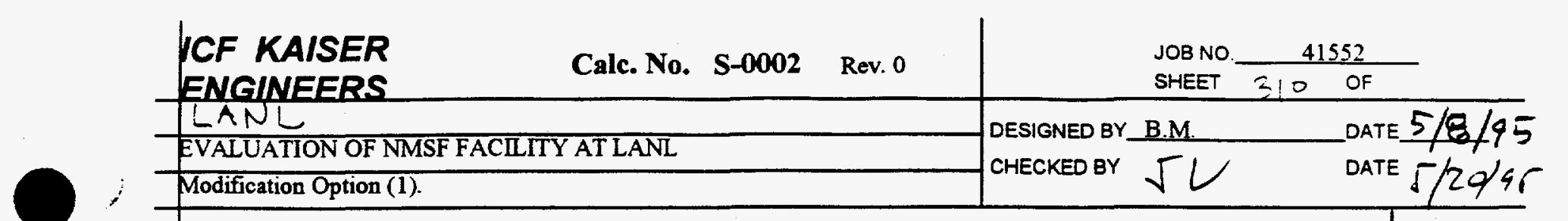

\subsection{Additional Analyses and Evaluations of NMSF Structure}

\subsubsection{References}

1) LANL Review Comments on the submittal of $90 \%$ documents for seismic evaluation of as-built and modified NMSF facility.

\subsubsection{Re-Analysis using 3-D Model}

This additional Analysis was performed to study the impact of new structural components that were added to the scope of modifications. these new components consist of two 1.5 foot thick reinforced concrete walls under the proposed charge deck in north/south and four buttresses, $2.5^{\prime} \mathrm{X} 4^{\prime}$ in cross section, at the west exterior wall. These wall $s$ and buttresses were not included in the SAP90 model of the structure used for analysis of the modified structure. The objective of this analysis was to study the impact of including these walls and buttresses in the model on the structural evaluation results for NMSF building. Also as part of this analysis effort the floor mass calculations were revised to reflect the followings:

1) The live load of the charge deck was increased to $250 \mathrm{psf}$ from $150 \mathrm{psf}$

2) The dead loads of the charge deck and roof slabs were revised to reflect the latest drawings including the weight of the new rooftop equipment penthouse.

The Following summarizes the result of this study. The detail of calculations are presented in the following pages.

1) As expected the impact on the dynamic characteristics of the structure was not significant. The rigid box like structure of the NMSF building primarily goes through rigid body translational and rocking motion with slight change in the fundamental frequencies due to addition of new masses and rigidities of the additional walls and buttresses.

2) Addition of new walls and buttresses adds to the load carrying capacity of the lateral load resisting system of the building. The addition of buttresses reduces the out-of-plane demand on the west wall and additional new walls help reduce the demand on adjacent walls which already had a comfortable margin of safety against reaching their ultimate capacity under the postulated DBE criteria. 
IVF KAISER

ENGINEERS

Joe No.

SHeEt

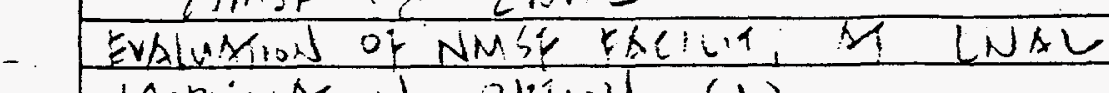

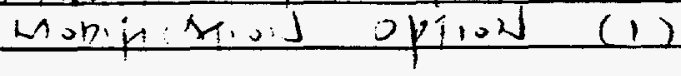

DESIGNED BY $B M$ DATE $4 / 24 / 96$ CHECKED BY TL DATE $\widetilde{T} / 1 / 96$

modification to sago model

1) Addition of Two í-6" Basement walls in N/S Dir. [REF: ICE KAISER $50 \%$ Drawing sheet $A 7$, March 16, 95] 2) Addition of Maid is Buttresses on the wat wall.

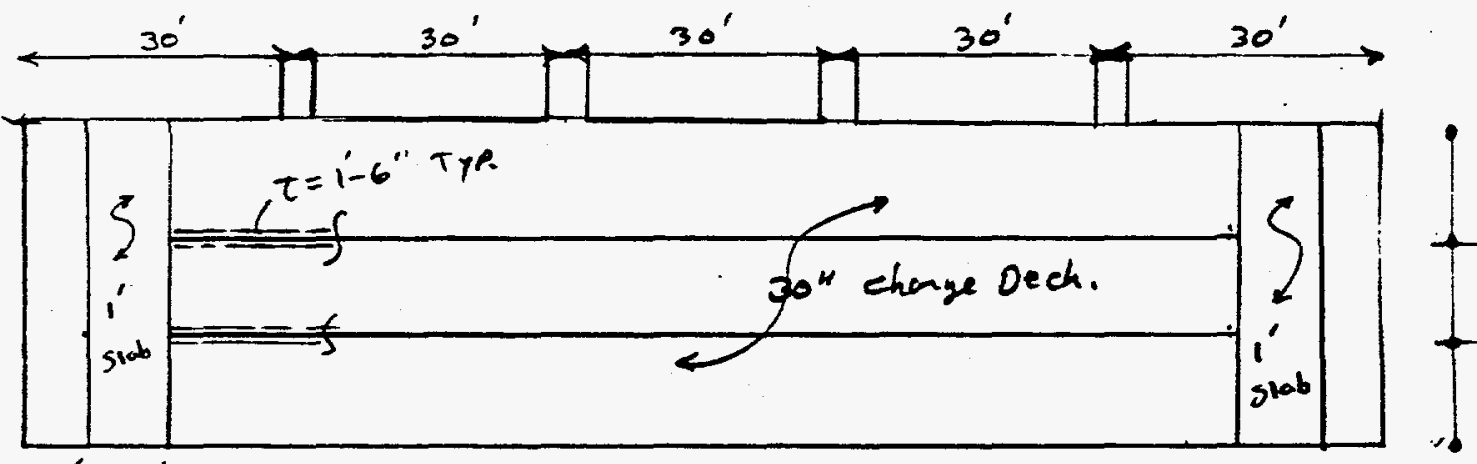

6.6.8.8.

$6.8^{\circ} .5 .5^{\prime}$

PLAN - Change Decd ELEV.

3) Remove Buttress on line (D)

shell Elements $4401 \rightarrow 4407$

$\mid$ 
6.5. $312 \div$

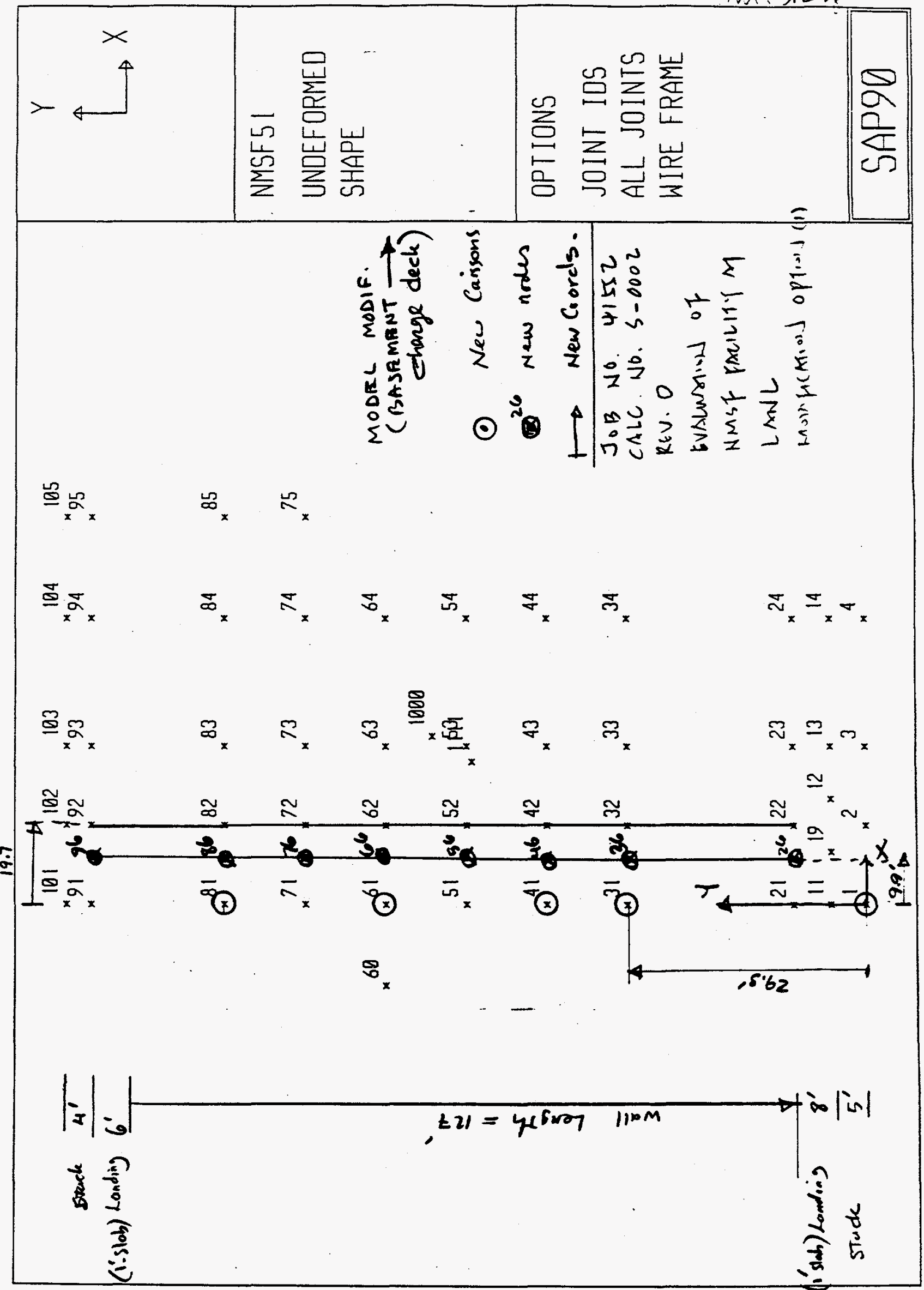


EVAL:MA,I 2; NMLF EALILIT, M UNL LA>pi!rij optiol (1)

New Basencin walls (Flement/Numbering)
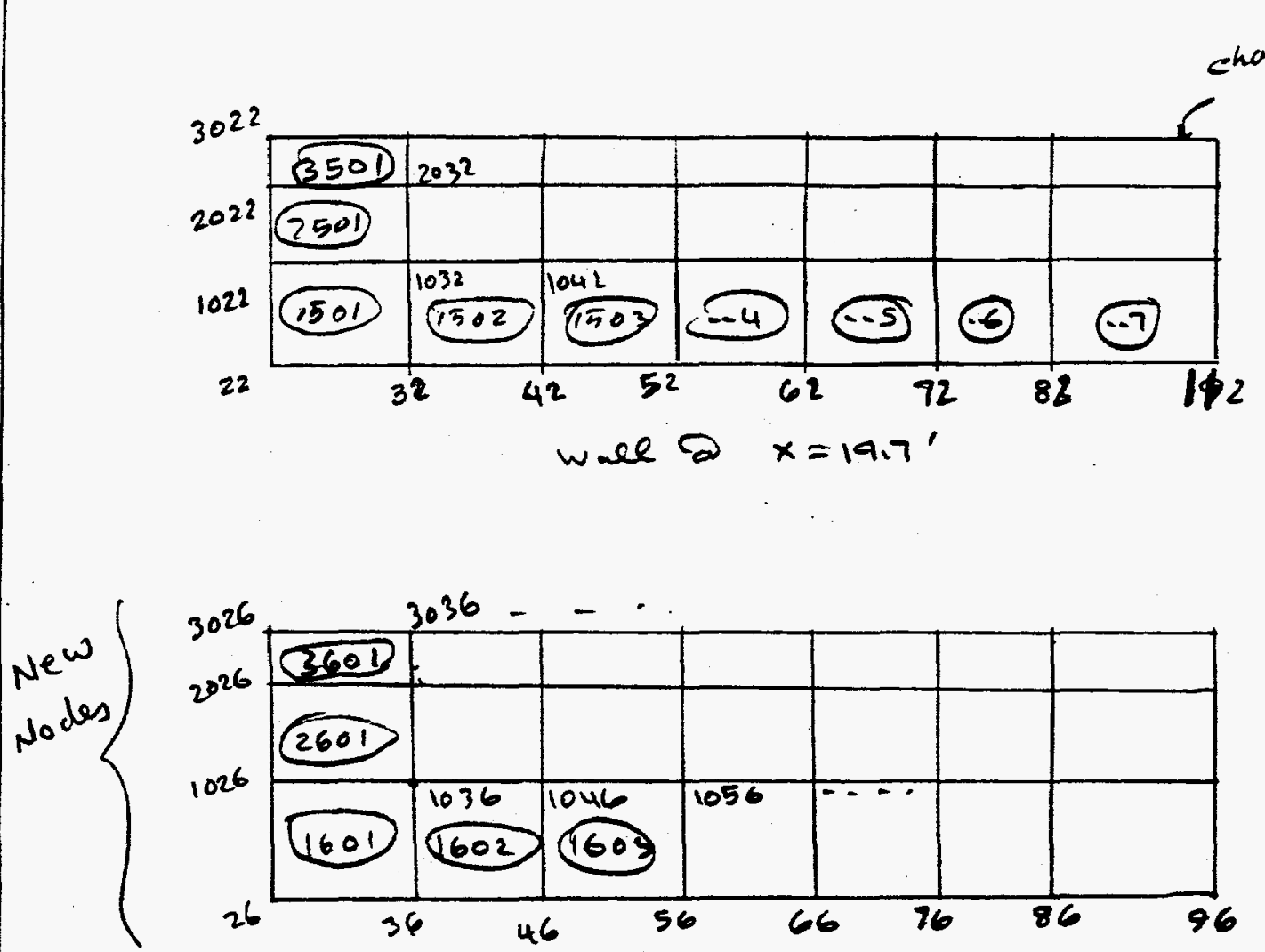

wale $2 x=9.9^{\prime}$

New Cuisions
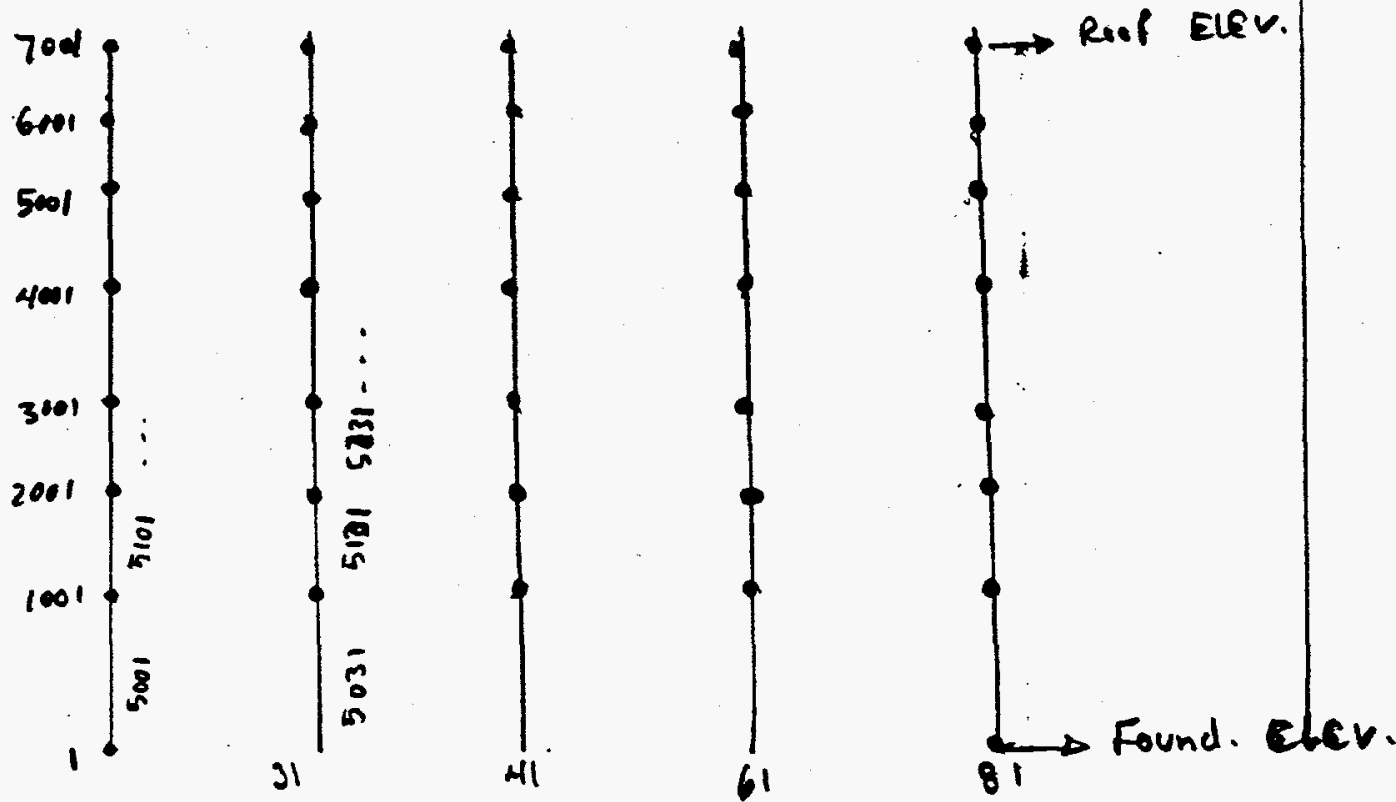


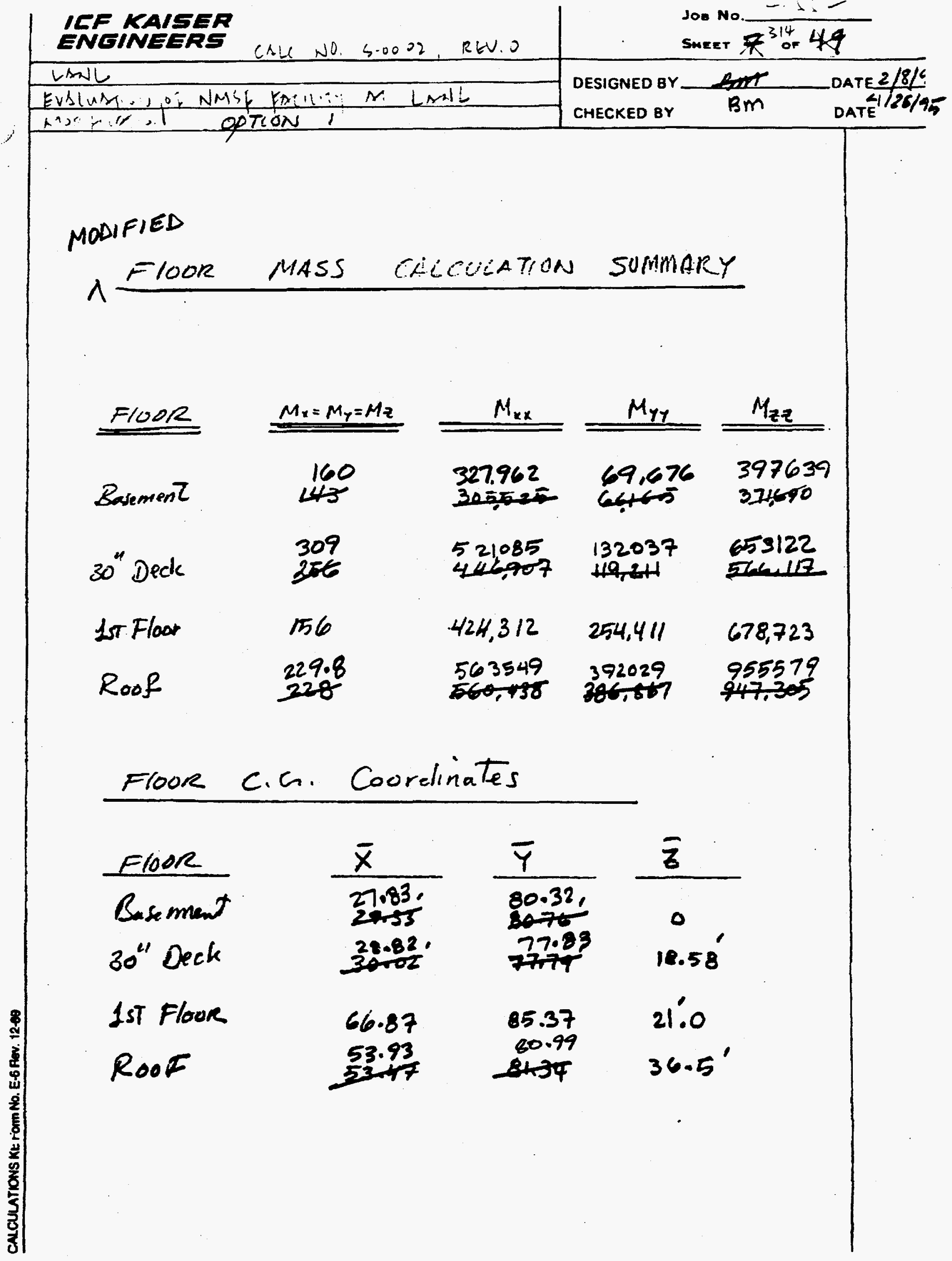




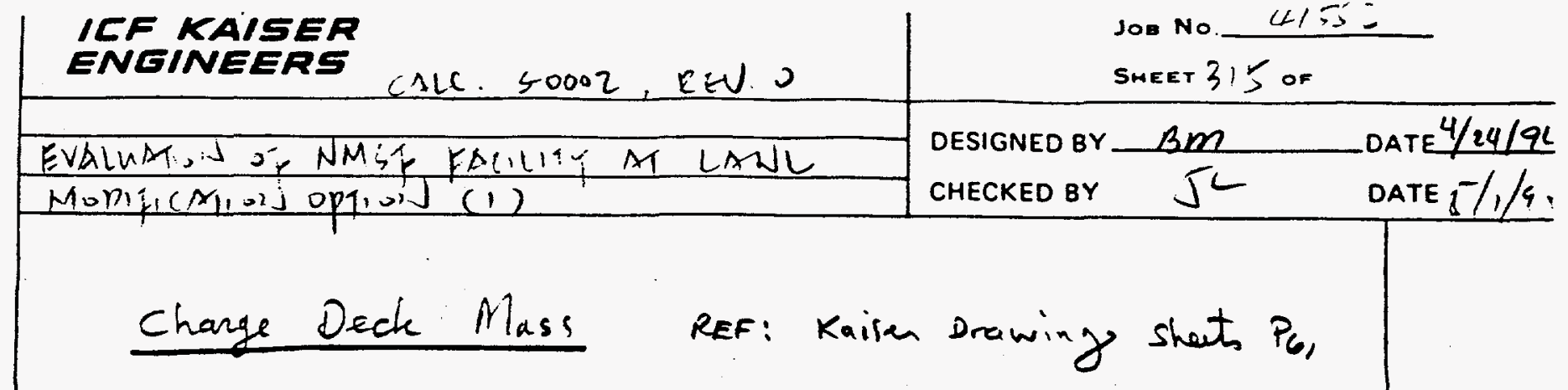

$$
\begin{aligned}
& \begin{aligned}
\text { Total Area } & =\left(150-427^{\prime}\right. \\
& =1830 \mathrm{FT}^{2}
\end{aligned} \\
& \text { concrete }=\frac{30}{12} \times 1830 \times 0.15 \quad=686^{k} \\
& \text { STee11/2t Tonkin }=\left(\frac{5}{12} \times 1830 \times 0.490\right) \times 2=75^{k} \\
& \text { steel IR, Web }=\left(\frac{30 \times 1}{144} \times 127 \times .490\right)(3 \times 4)=136^{k} \\
& \text { Dry wells }(522)=\left(\pi .1 .5 \times \frac{.5}{12} \times 17 \times .49\right)(522)=854^{k}
\end{aligned}
$$

zndary Tob o Contents ( $z^{k}$ Per wall).

$$
=(2)(522) \quad=1044^{k}
$$

Concrete shad Blocks $=\left(\left(\frac{22.5^{2}}{4} \times 7 \times \cdot 8+\frac{17^{2}}{4} \times 7 \times 26^{\prime \prime}\right) \frac{0.15}{12^{3}}\right)(522)$

$$
=\left(0,788^{k}\right)(522)=412^{k}
$$

TOTAL Dead Loads

Eq. Unit. Load

$$
\begin{aligned}
& =3227^{k} \\
& =3227 /(127 \times 29.6)- \\
& =0.865 / F T^{2}
\end{aligned}
$$

Live loads ( 250 PSF) $=1 . \mathrm{TT}=0.25 \mathrm{~K} / \mathrm{FT}^{2}$ USE $25 \%$ of L.L. In Masses: $=0.25 \times .25=0.0625^{k} / \mathrm{FT}^{2}$ Equiv unit mass $=(.860+.0625) / 32.2=0.0286\left(K-E^{-1}-\xi^{2}\right)$ 


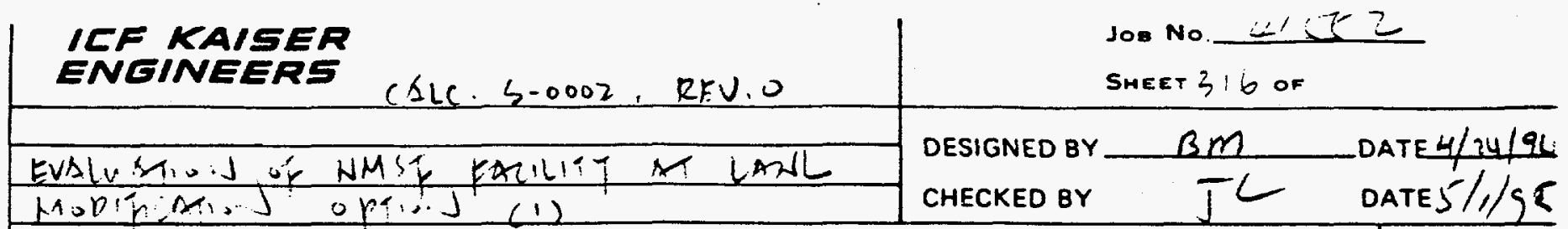

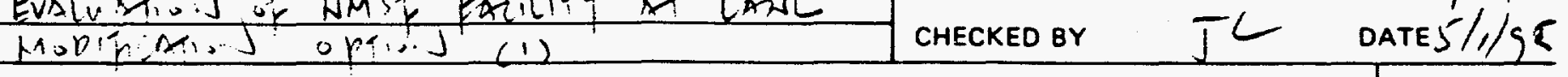

charge Deck Mass/D.L. Cafculation Summary:

$$
\begin{aligned}
& \text { D.L. } \\
& =0.860 \\
& K \cdot F^{-2} \\
& \text { L.L. } \\
& =0.250 \\
& k \cdot E^{2} \\
& \left(w_{1}\right) \quad D . c+C L \\
& =1.110 \\
& K-F^{-2} \\
& =1.629 \\
& k-f^{-2} \\
& =(0.86+.25 x .25) / 32.2 \\
& \text { (m) Mass } \\
& \text { (Unif Areal Mars) } \\
& =0.02865 \\
& K \cdot F^{-3} \cdot 8^{2}
\end{aligned}
$$$$
\left(w_{2}\right) 1.4 D L+1.7 L L
$$

Dead wi./Mass of Equpment Penthous:

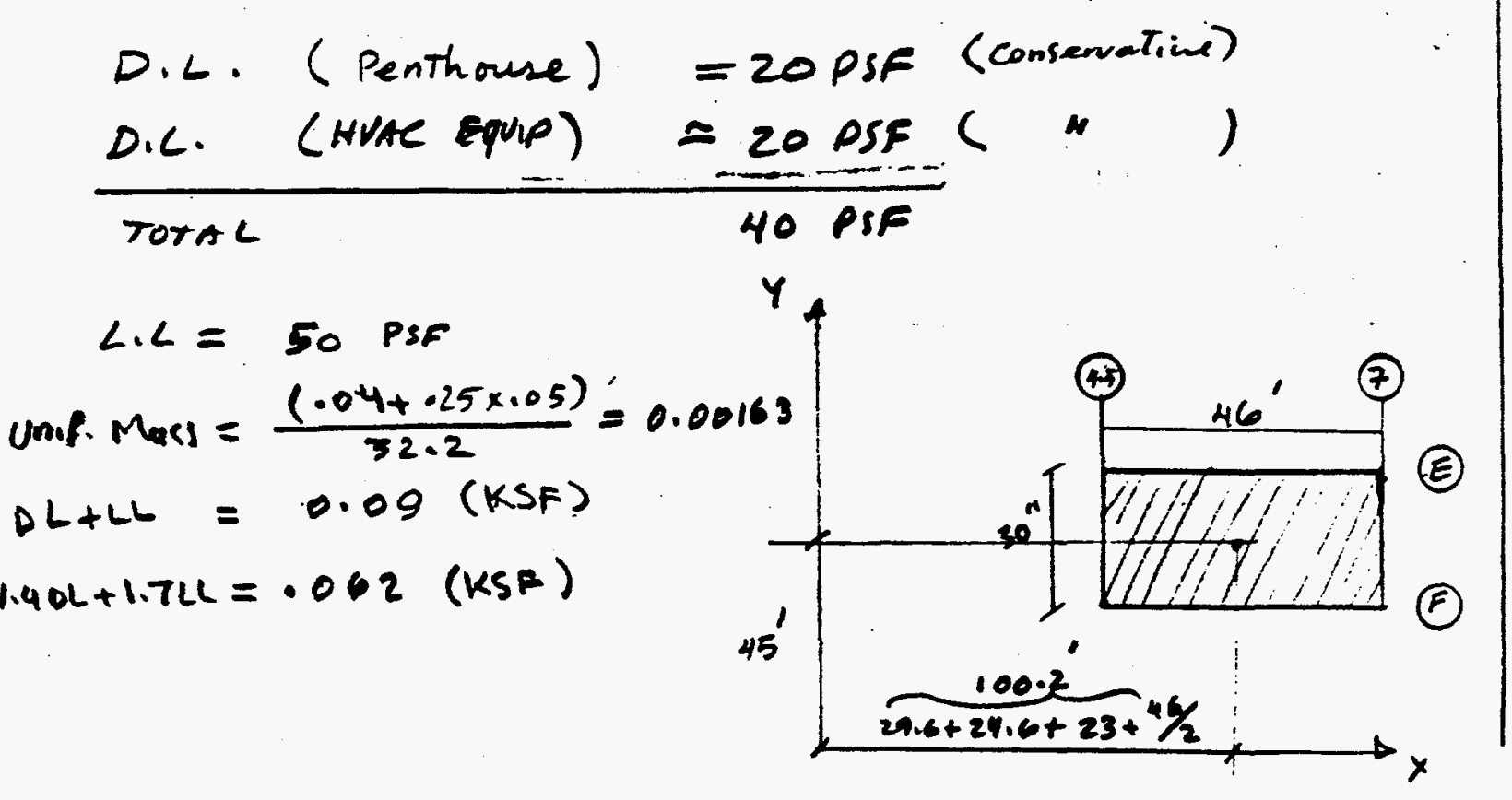




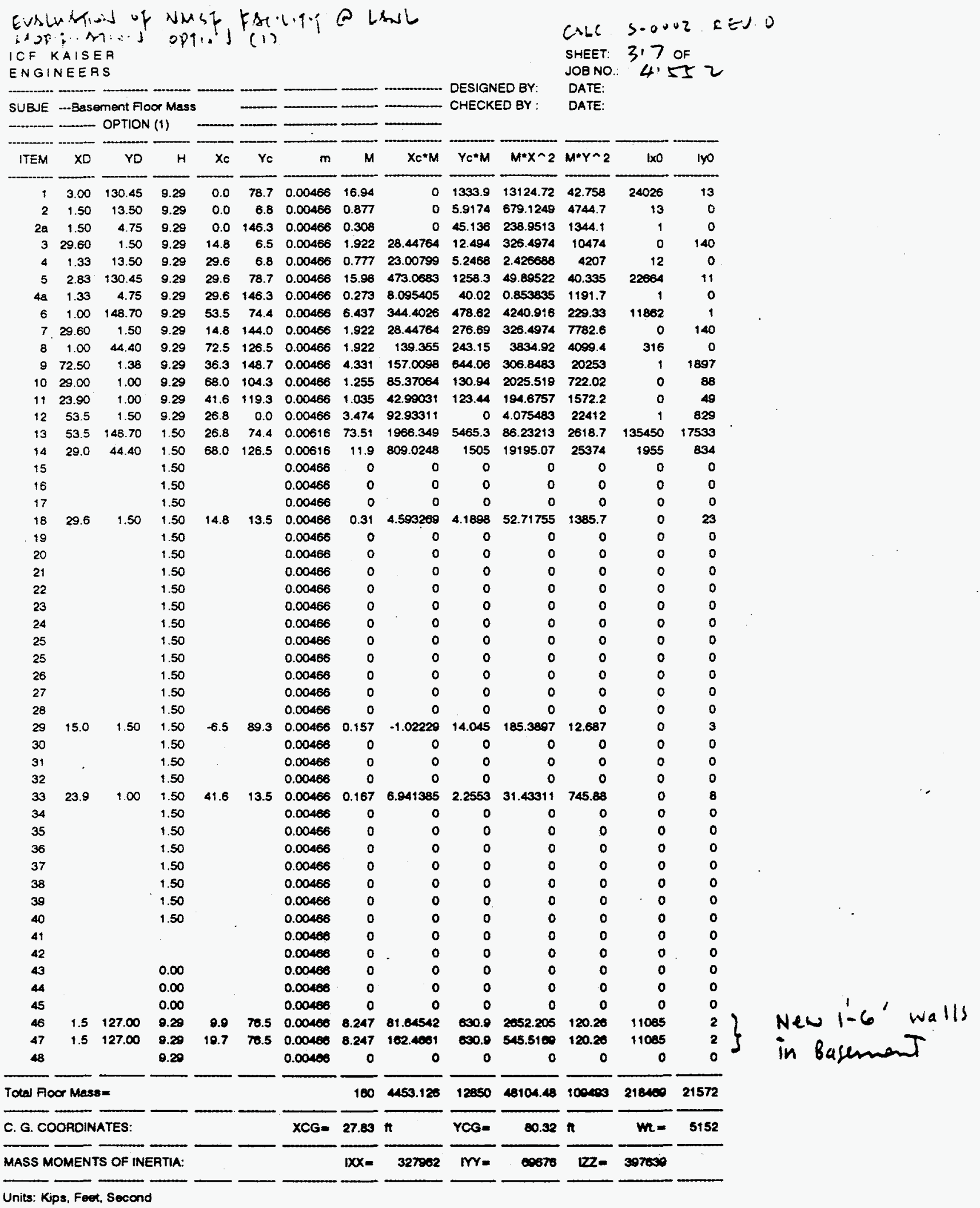


csu s-0002, KEJ O

ICF KAISER

ENGINEERS

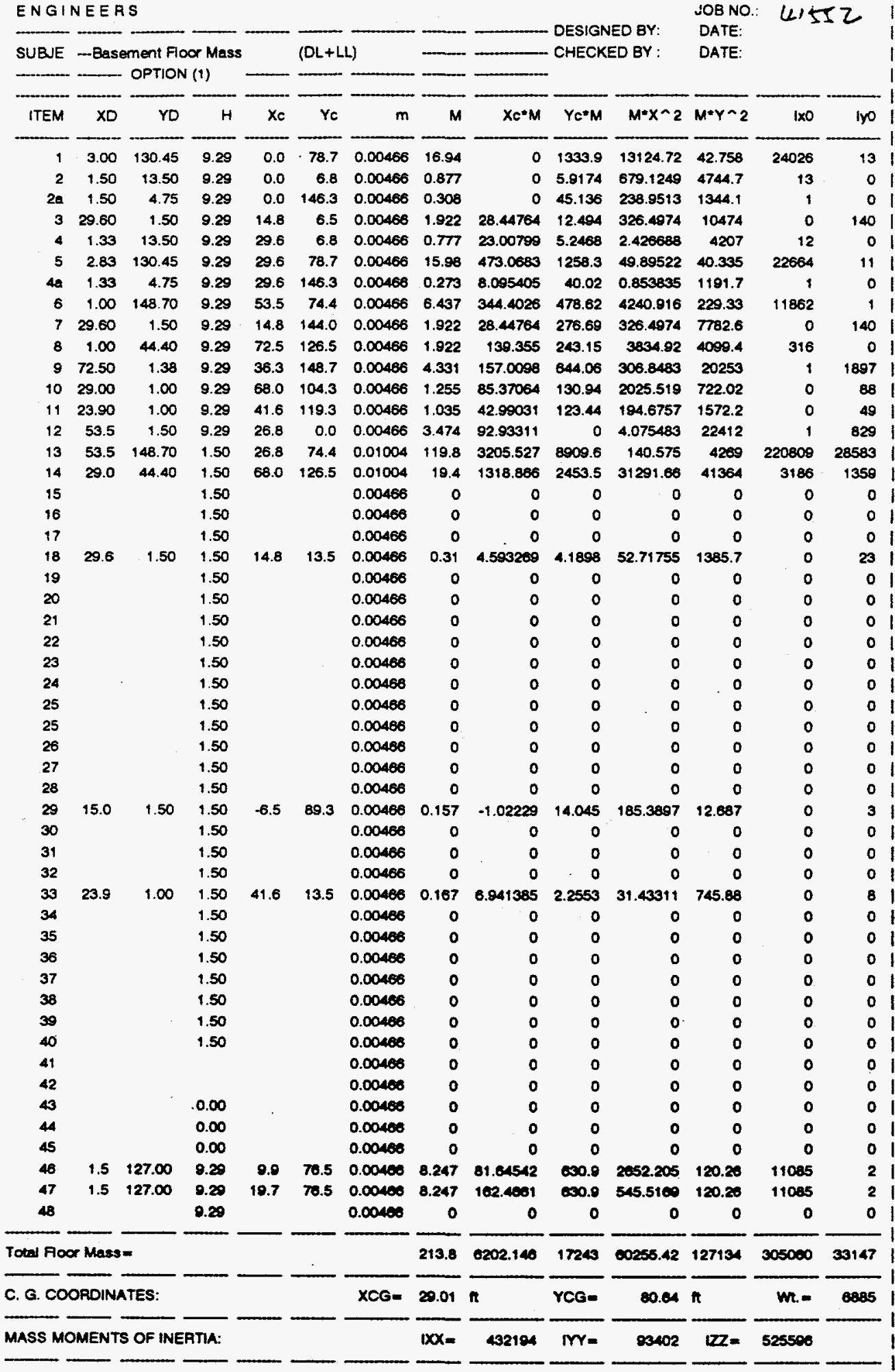




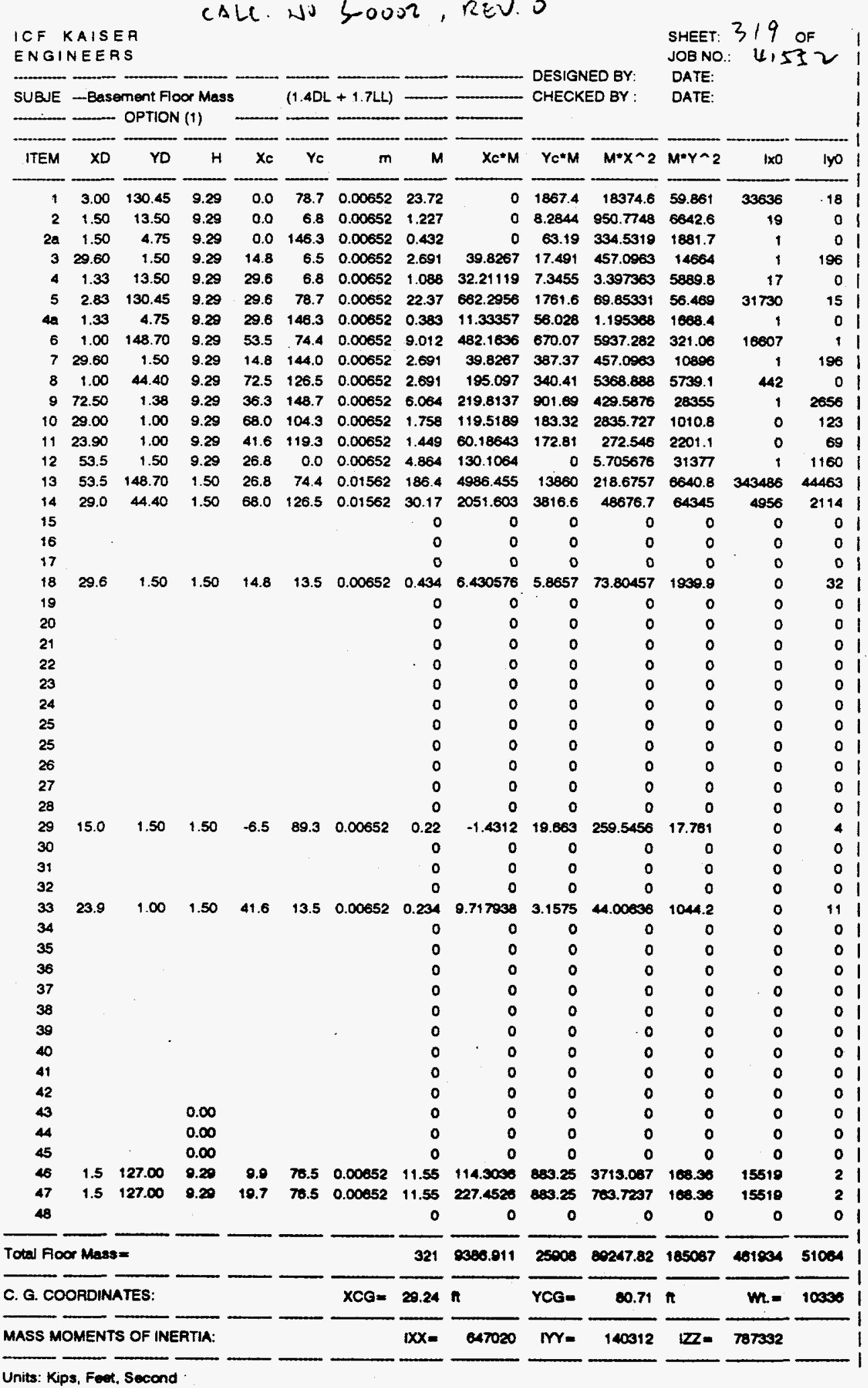


CALC. NO S0002, REU. O

ICF KAISEA

ENGINEERS

DESIGNED BY:

SUBE - CHARGE DECK ELEV. CHECKED BY:

SHEET: 320 OF JOB NO: 4 III 2

DATE:

DATE:

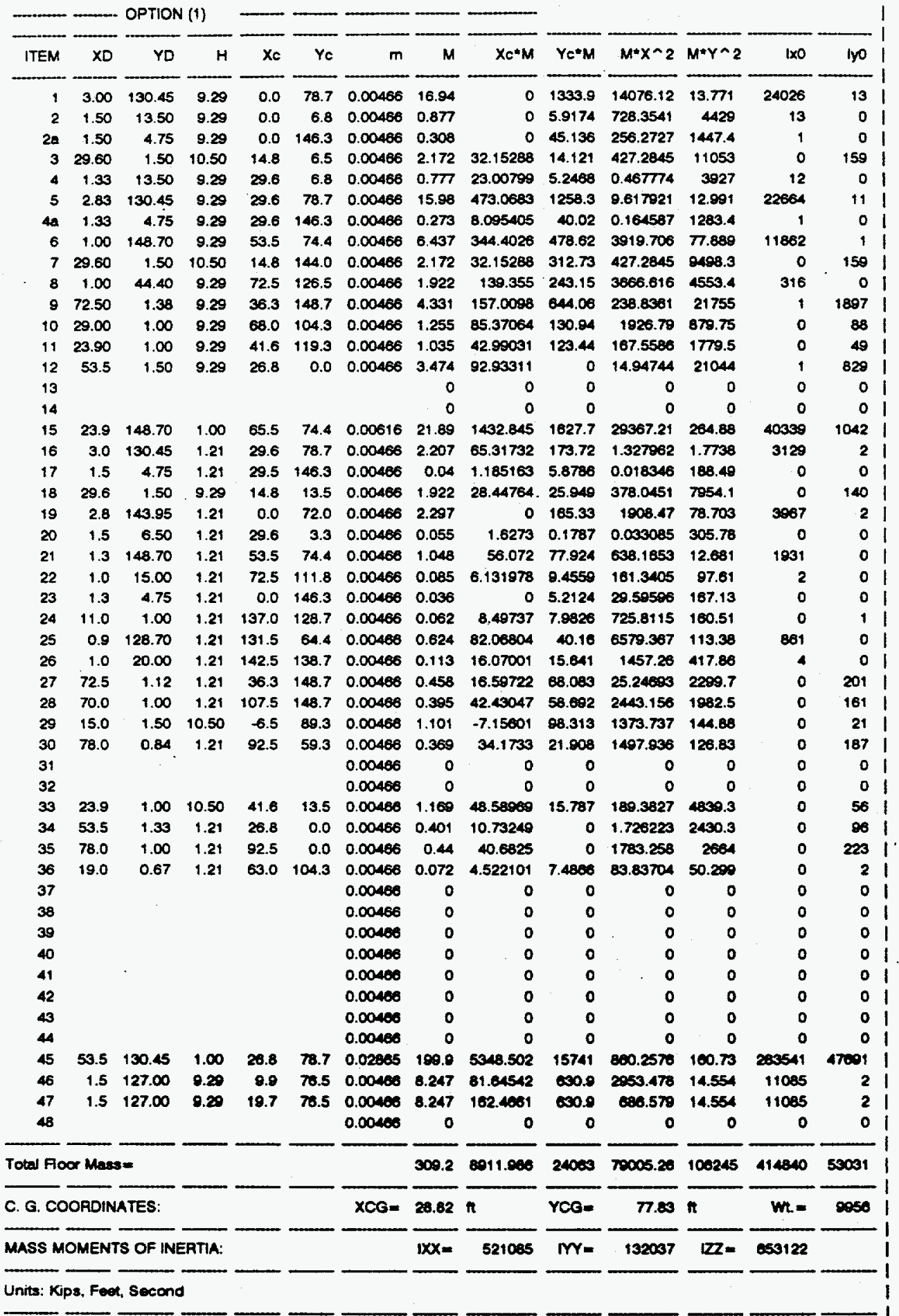




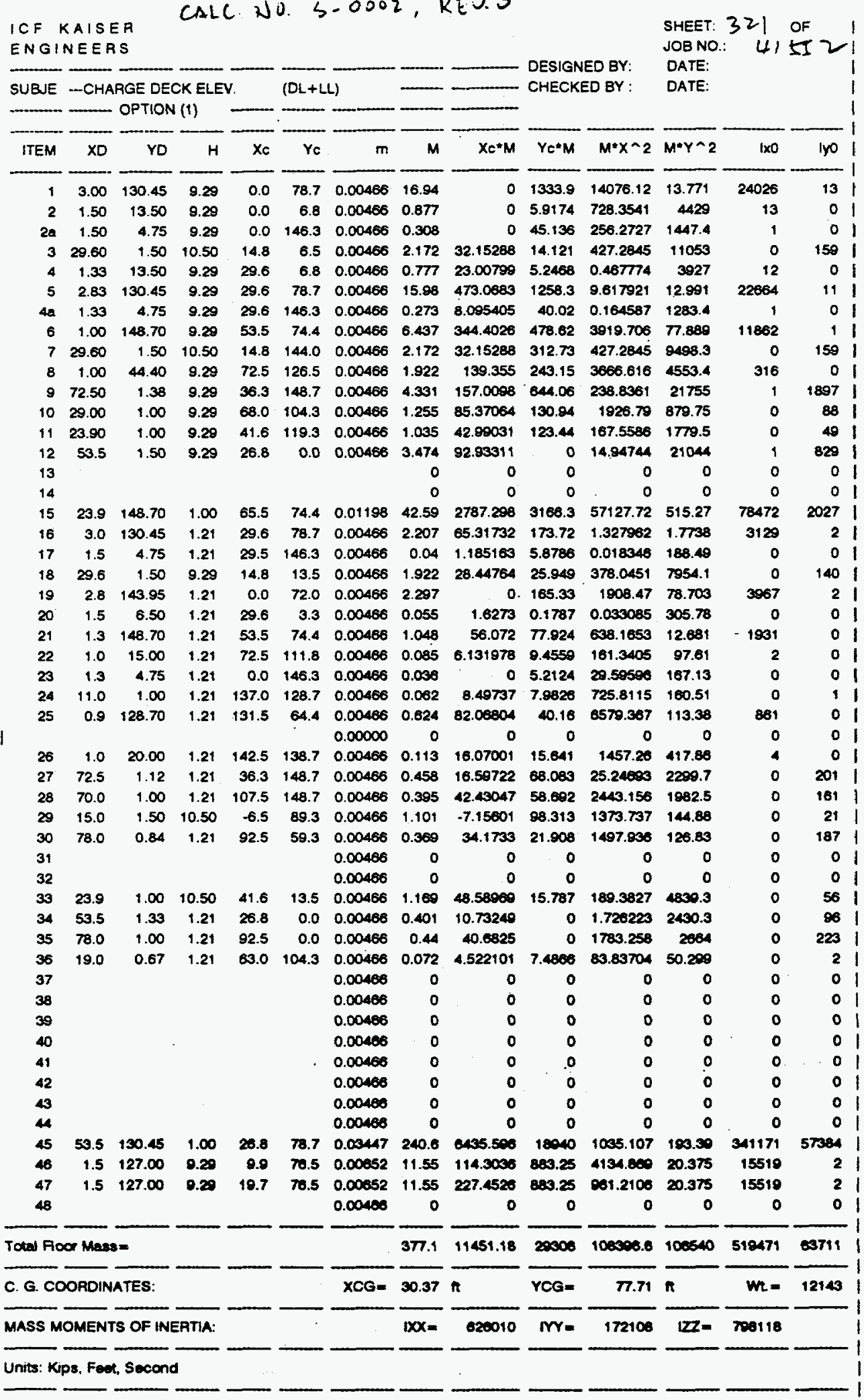


CALC NU. S-0002, REU. O

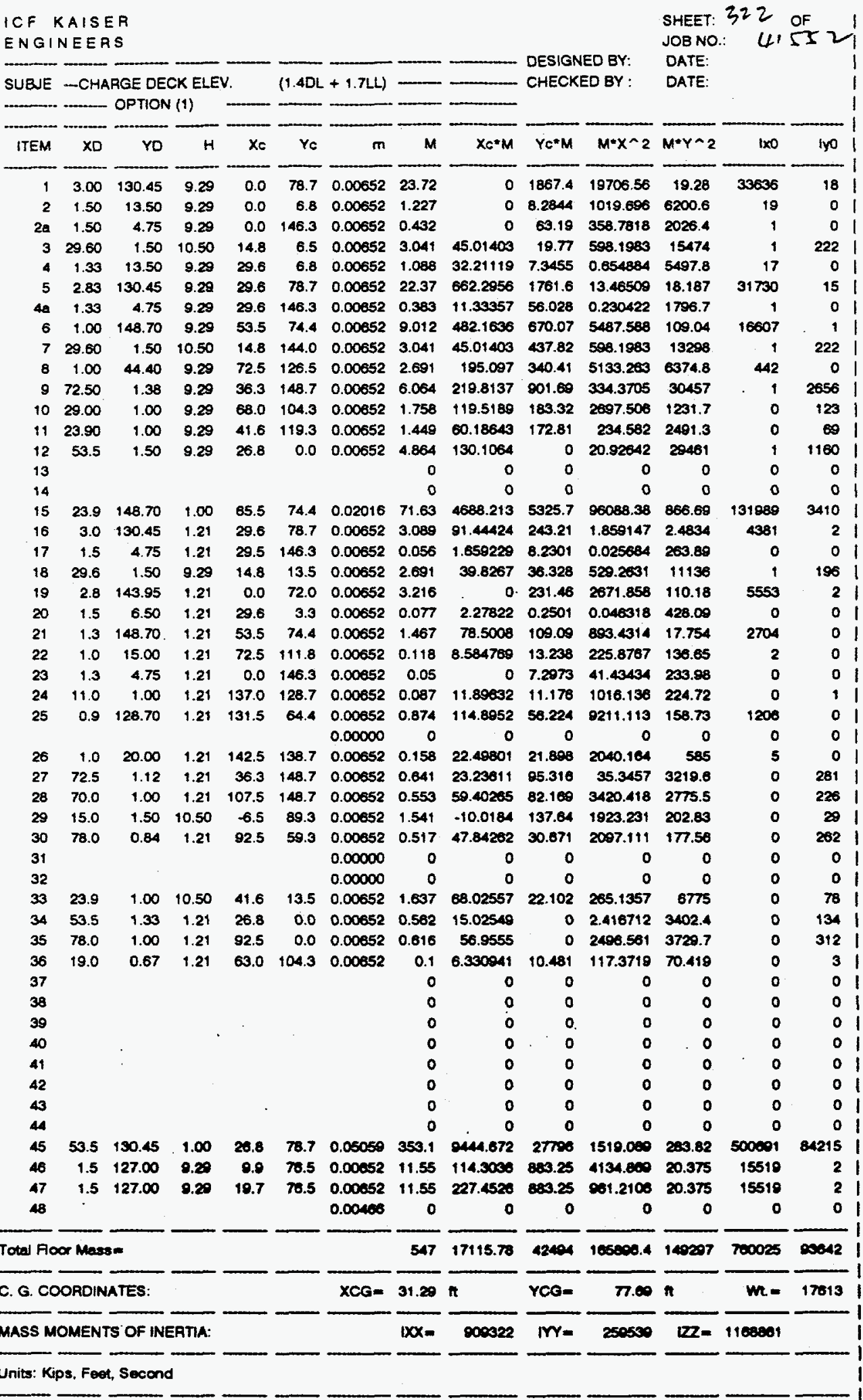




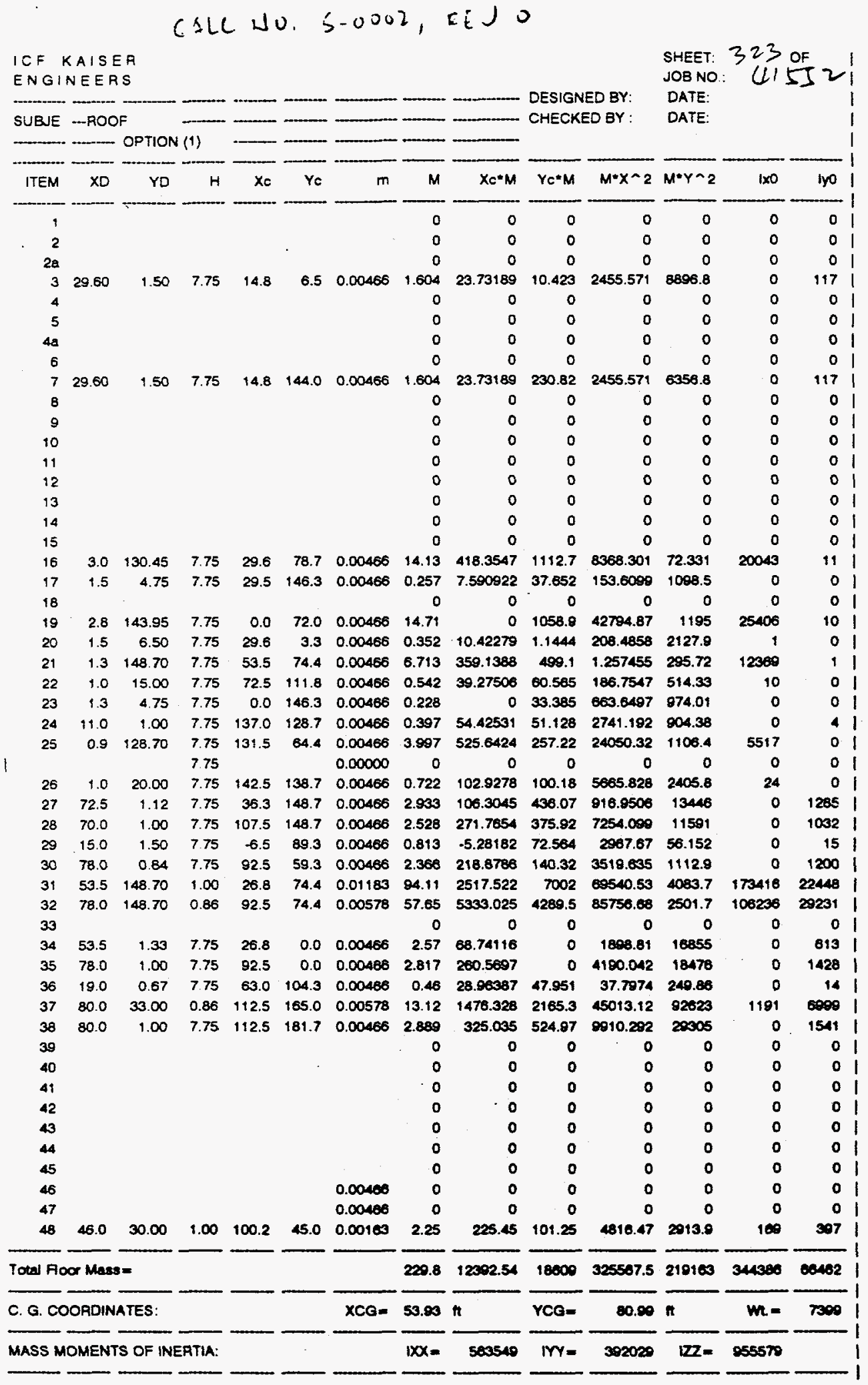

Units: Kips, Feet, Second 


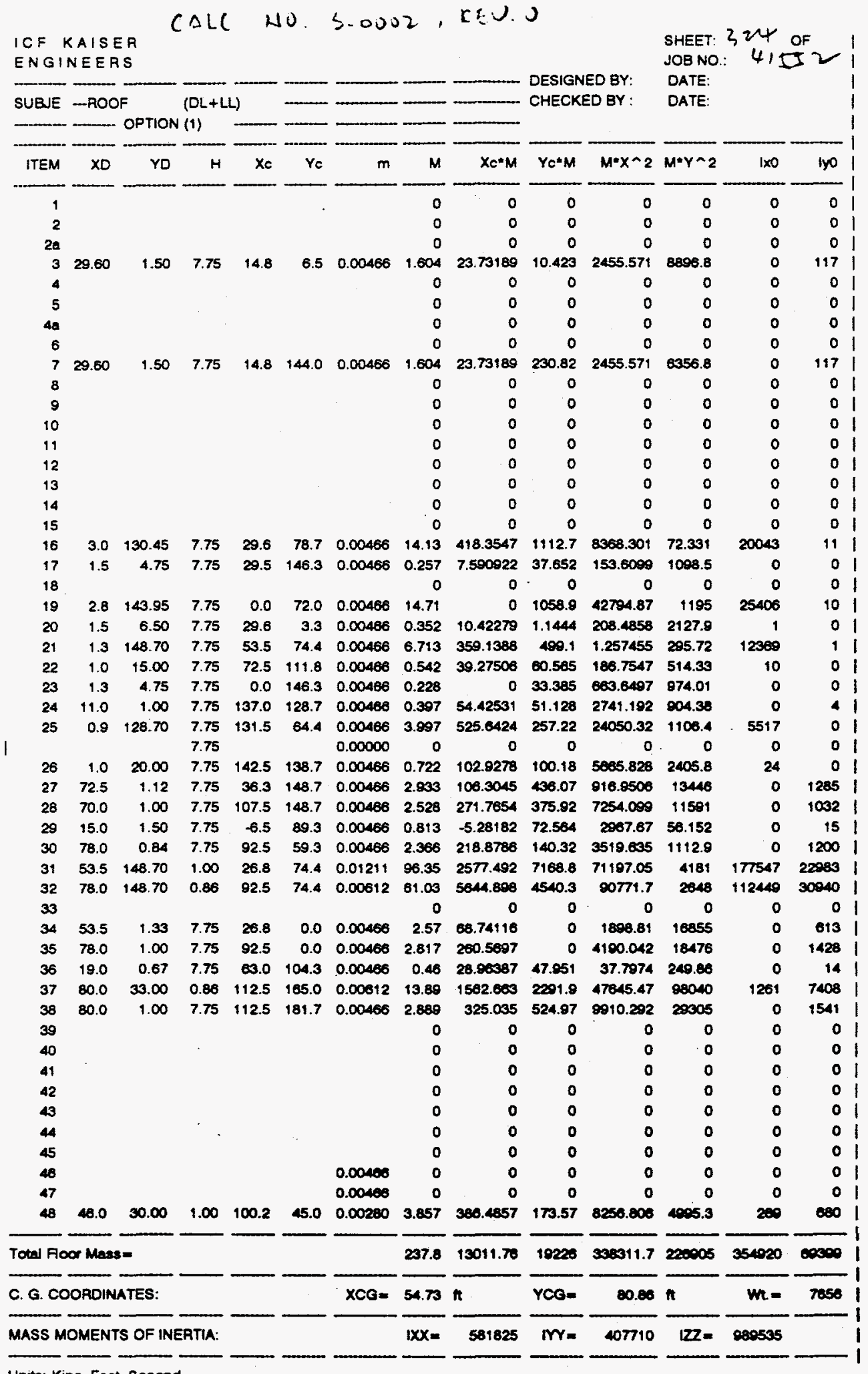

Units: Kips, Feet, Second 


\begin{tabular}{|c|c|c|c|c|c|c|c|c|c|c|c|c|c|c|}
\hline 4 & & & & & & & 0 & 0 & 0 & 0 & 0 & 0 & 0 & 01 \\
\hline 5 & & & & & & & 0 & 0 & 0 & 0 & 0 & 0 & 0 & $0 i$ \\
\hline $4 a$ & & & & & & & 0 & $0^{\prime}$ & 0 & 0 & 0 & 0 & 0 & 0 \\
\hline 6 & & & & & & & 0 & 0 & 0 & 0 & 0 & 0 & 0 & 0 \\
\hline 7 & 29.60 & 1.50 & 7.75 & 14.8 & 144.0 & 0.00652 & 2.245 & 33.22464 & 323.15 & 3437.8 & 8899.5 & 0 & 164 & 4 \\
\hline 8 & & & & & & & 0 & 0 & 0 & 0 & 0 & 0 & 0 & 01 \\
\hline 9 & & & & & & & 0 & 0 & 0 & 0 & 0 & 0 & 0 & 01 \\
\hline 10 & & & & & & & 0 & 0 & 0 & 0 & 0 & 0 & 0 & 01 \\
\hline 11 & & & & & & & 0 & 0 & 0 & 0 & 0 & 0 & 0 & 0 \\
\hline 12 & & & & & & & 0 & 0 & 0 & 0 & 0 & 0 & 0 & 0 \\
\hline 13 & & & & & & & 0 & 0 & 0 & 0 & 0 & 0 & 0 & 01 \\
\hline 14 & & & & & & & 0 & 0 & 0 & 0 & 0 & 0 & 0 & 0 \\
\hline 15 & & & & & & & 0 & 0 & 0 & 0 & 0 & 0 & 0 & 01 \\
\hline 16 & 3.0 & 130.45 & 7.75 & 29.6 & 78.7 & 0.00652 & 19.79 & 585.8968 & 1557.7 & 11715.62 & 101.26 & 28080 & 15 & 51 \\
\hline 17 & 1.5 & 4.75 & 7.75 & 29.5 & 146.3 & 0.00652 & 0.36 & 10.62729 & 52.713 & 215.0538 & 1537.9 & 1 & 0 & 01 \\
\hline 18 & & & & & & & 0 & 0 & 0 & 0 & 0 & 0 & 0 & 01 \\
\hline 19 & 2.8 & 143.95 & 7.75 & 0.0 & 72.0 & 0.00652 & 20.6 & 0 & 1482.5 & 59912.82 & 1672.8 & 35568 & 14 & 41 \\
\hline 20 & 1.5 & 6.50 & 7.75 & 29.6 & 3.3 & 0.00652 & 0.493 & 14.5919 & 1.6022 & 291.8802 & 2979.1 & 2 & 0 & 01 \\
\hline 21 & 1.3 & 148.70 & 7.75 & 53.5 & 74.4 & 0.00652 & 9.398 & 502.7944 & 698.74 & 1.780437 & 414.01 & $173+7$ & 1 & 11 \\
\hline 22 & 1.0 & 15.00 & 7.75 & 72.5 & 111.8 & 0.00852 & 0.758 & 54.98500 & 84.791 & 261.4586 & 720.06 & 14 & 0 & 01 \\
\hline 23 & 1.3 & 4.75 & 7.75 & 0.0 & 146.3 & 0.00652 & 0.319 & 0 & 46.739 & 829.1005 & 1363.6 & 1 & 0 & 01 \\
\hline 24 & 11.0 & 1.00 & 7.75 & 137.0 & 128.7 & 0.00652 & 0.556 & 76.19543 & 74.579 & 3837.688 & 1288.1 & 0 & 6 & 61 \\
\hline 25 & 0.9 & 128.70 & 7.75 & 131.5 & 64.4 & 0.00852 & 5.596 & 735.8993 & 300.11 & 33670.44 & 1549 & 7724 & 0 & 01 \\
\hline ! & & & 7.75 & & & 0.00000 & 0 & 0 & 0 & 0 & 0 & 0 & 0 & 01 \\
\hline 26 & 1.0 & 20.00 & 7.75 & 142.5 & 138.7 & 0.00652 & 1.011 & 144.0989 & 140.26 & 7832.159 & 3368.1 & 34 & 0 & 01 \\
\hline 27 & 72.5 & 1.12 & 7.75 & 36.3 & 148.7 & 0.00652 & 4.106 & 148.8223 & 610.5 & 1283.731 & 18824 & 0 & 1788 & 81 \\
\hline 28 & 70.0 & 1.00 & 7.75 & 107.5 & 148.7 & 0.00652 & 3.539 & 380.4715 & 526.29 & 10155.74 & 16228 & 0 & 1445 & 51 \\
\hline 29 & 15.0 & 1.50 & 7.75 & -6.5 & 89.3 & 0.00652 & 1.138 & -7.39455 & 101.50 & 4154.738 & 78.612 & 0 & 21 & 1 \\
\hline 30 & 78.0 & 0.84 & 7.75 & 92.5 & 59.3 & 0.00652 & 3.313 & 308.43 & 198.45 & 4927.480 & 1558.1 & 0 & 1680 & 01 \\
\hline 34 & 53.5 & 148.70 & 1.00 & 26.8 & 74.4 & 0.01724 & 137.1 & 3667.900 & 10202 & 101318.9 & 5949.9 & 252864 & 32706 & 61 \\
\hline 32 & 78.0 & 148.70 & 0.86 & 92.5 & 74.4 & 0.00888 & 88.6 & 8195.131 & 6501.5 & 131780.2 & 3844.3 & 163251 & 44918 & 31 \\
\hline 33 & & & & & & & 0 & 0 & 0 & 0 & 0 & 0 & 0 & 01 \\
\hline 34 & 53.5 & 1.33 & 7.75 & 26.8 & 0.0 & 0.00852 & 3.598 & 98.23782 & 0 & 2658.334 & 23597 & 1 & 858 & 81 \\
\hline 35 & 78.0 & 1.00 & 7.75 & 92.5 & 0.0 & 0.00652 & 3.944 & 364,7970 & 0 & 5888.058 & 25887 & 0 & 1999 & 91 \\
\hline 36 & 19.0 & 0.67 & 7.75 & 63.0 & 104.3 & 0.00352 & 0.644 & 40.54042 & 67.132 & 52.91038 & 349.81 & 0 & 19 & 01 \\
\hline 37 & 80.0 & 33.00 & 0.86 & 112.5 & 165.0 & 0.00888 & 20.17 & 2288.037 & 3327.3 & 60170.58 & 142332 & 1830 & 10755 & 51 \\
\hline 38 & 80.0 & 1.00 & 7.75 & 112.5 & 181.7 & 0.00852 & 4.045 & 455.040 & 734.85 & 13874.41 & 41027 & 0 & 2157 & 71 \\
\hline 39 & & & . & & & . & 0 & 0 & 0 & 0 & 0 & 0 & 0 & 01 \\
\hline 40 & & & & & & & 0 & 0 & 0 & 0 & 0 & 0 & 0 & 01 \\
\hline 41 & & & & & & & 0 & 0 & 0 & 0 & 0 & 0 & 0 & 01 \\
\hline 42 & & & & & & & 0 & 0 & 0 & 0 & 0 & 0 & 0 & 01 \\
\hline 43 & & & & & & & 0 & 0 & 0 & 0 & 0 & 0 & 0 & $0 i$ \\
\hline 44 & & & & & & & 0 & 0 & 0 & 0 & 0 & 0 & 0 & 01 \\
\hline 45 & & & & & & & 0 & 0 & 0 & 0 & 0 & 0 & 0 & 01 \\
\hline 46 & & & & & & & 0 & 0 & 0 & 0 & 0 & 0 & 0 & 01 \\
\hline 47 & & & & & & & 0 & 0 & 0 & 0 & 0 & 0 & 0 & 01 \\
\hline 48 & 46.0 & 30.00 & 1.00 & 100.2 & 45.0 & 0.00183 & 2.057 & 200.2457 & 119.57 & 5888.022 & 3441.2 & 100 & 400 & 01 \\
\hline Total Fo & or Mass & & & & & & 356.2 & 18374.20 & 27312 & 470574.7 & 310424 & 500057 & 99100 & \\
\hline c. G. $\mathrm{CO}$ & DOROINA & ATES: & & & & $X \subset G=$ & 54.05 & $n$ & YCG $=$ & 81.23 & $n$ & $m=$ & 10827 & \\
\hline MASS $M$ & IOMENTS & S OF iN & ERTIA: & & & & $1 D X=$ & 820001 & $m=$ & 575705 & $12 Z=$ & 1401856 & & \\
\hline
\end{tabular}




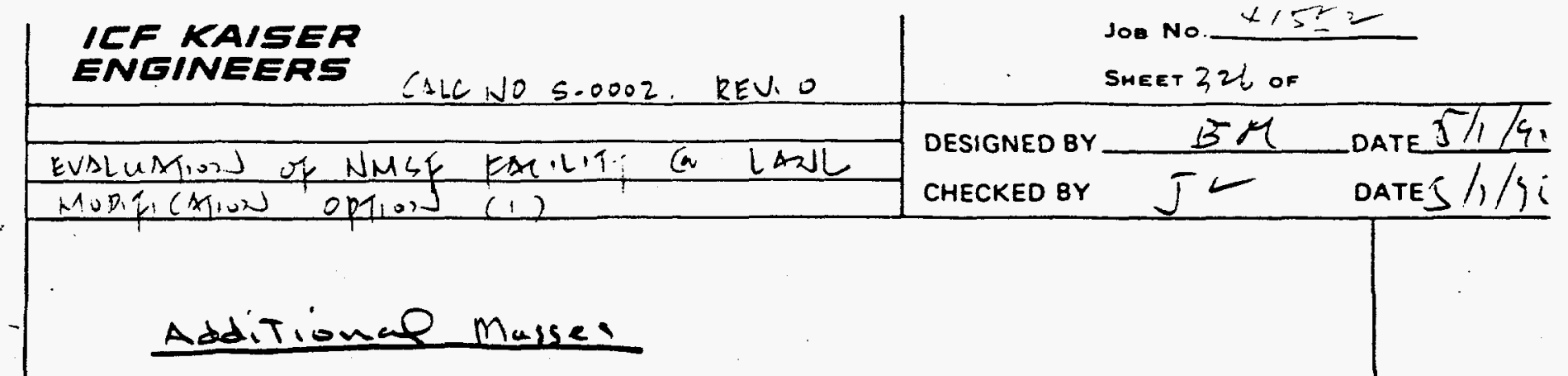

I) Security Grille/Louver on South wall $=26,000^{16}$

II) Detection/Secinty Grate on outlet stuck $=26,000^{16}$

Distribute $(I)$ over the woden D Roof level:

$\begin{array}{cccc}\text { NoDE } & & D L(K) & \text { Mass }\left(K-F^{-1}-S^{2}\right) \\ 7001 & 25 \% & 6.5 & 0.2019 \\ 7002 & 50 \% & 13.0 & 0.4037 \\ 7003 & 25 \% & 6.5 & 0.2019\end{array}$

Distribute (II) oven The nodes D Top of stacks

NODE

$$
\begin{aligned}
& (9101 \rightarrow 9103),(9091 \rightarrow 9093) \\
& \Delta L=26 / 6=4.3 \mathrm{~K}\left(\mathrm{Kr}^{-1} \mathrm{~s}^{2}\right) \text { (Each Node) } \\
& \text { Mass }=26 / 6 / 32.2=0.1346^{\text {each Nods) }}
\end{aligned}
$$




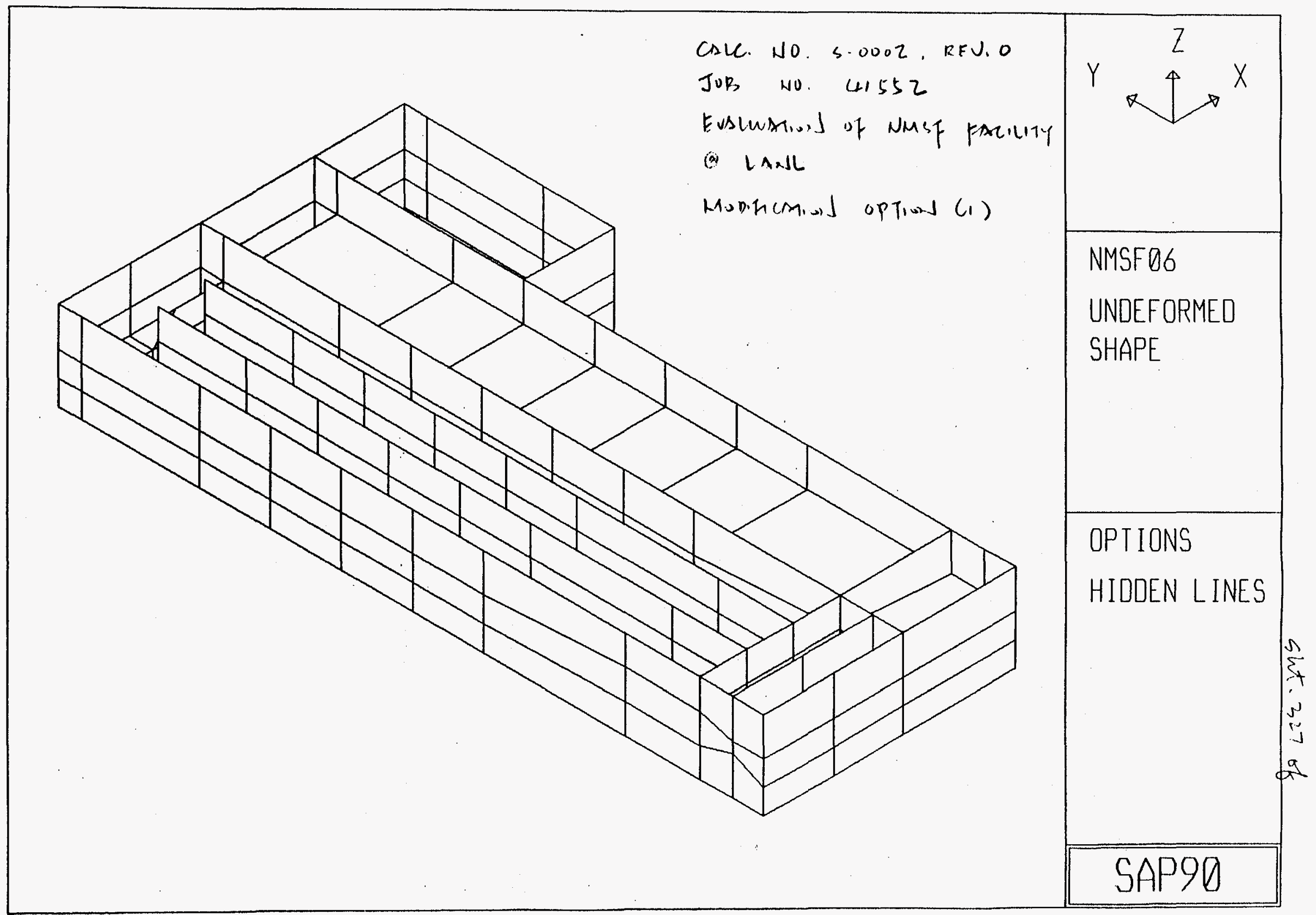




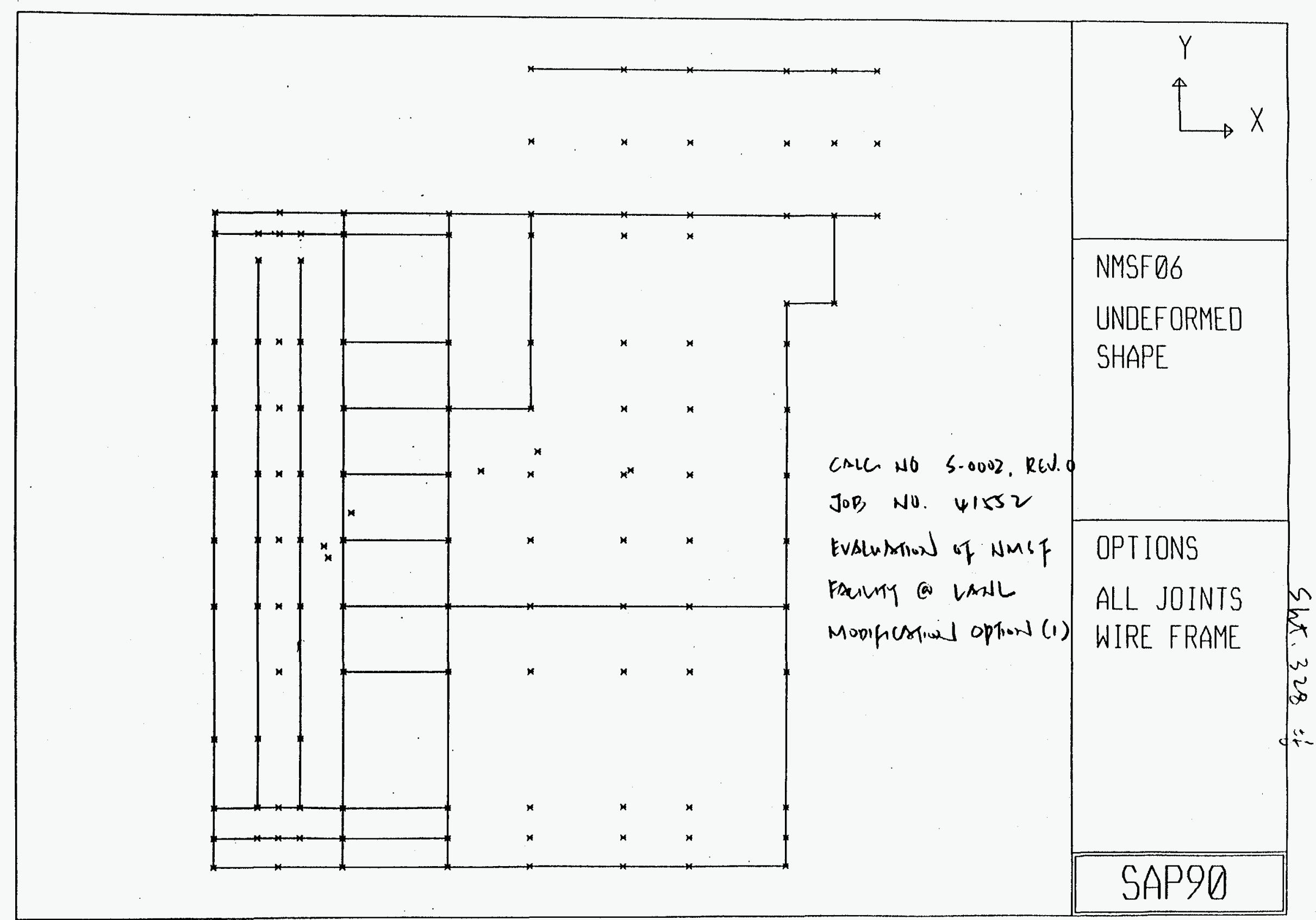




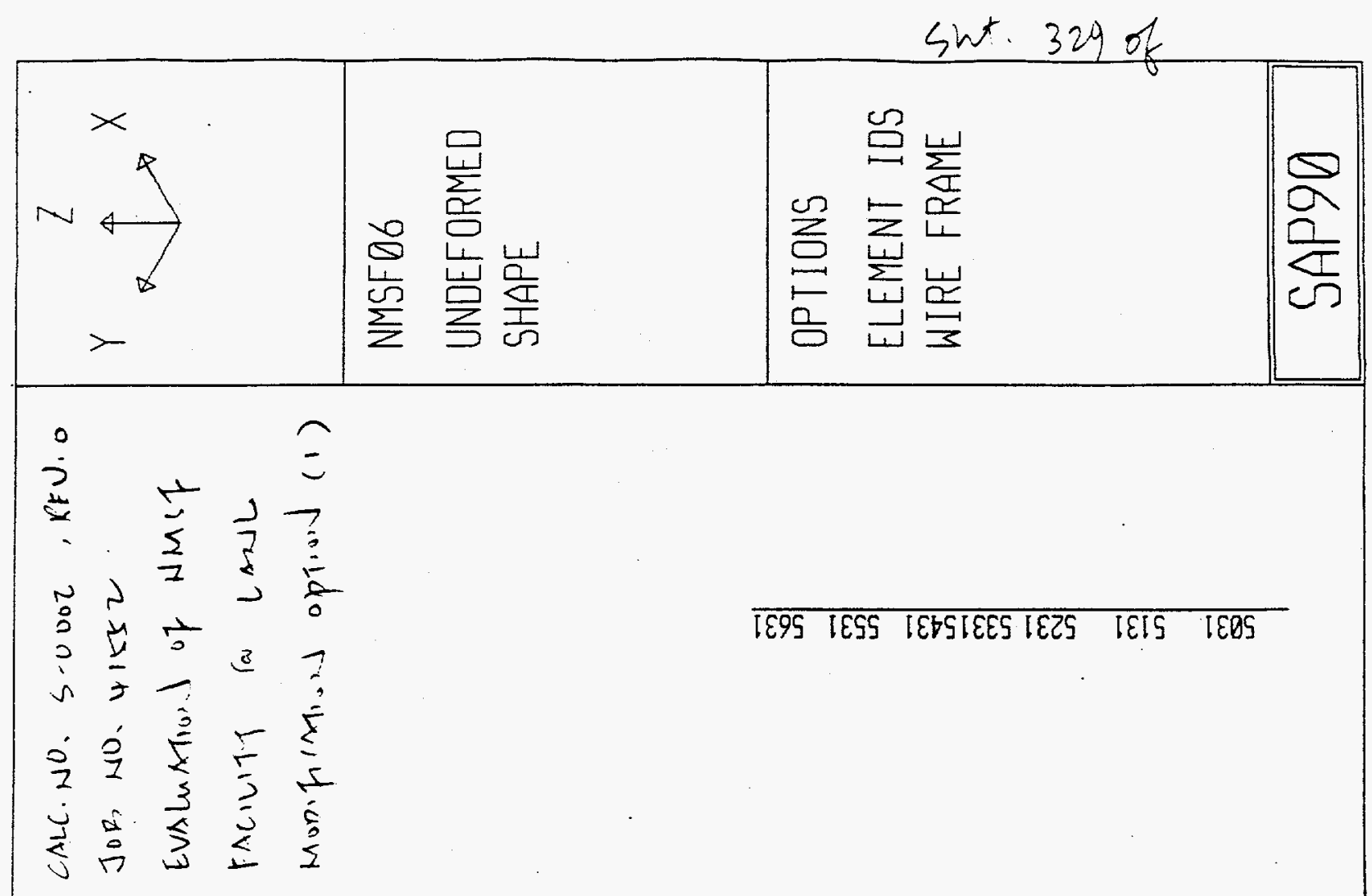

I69S ItSS IbESIEES IVZS IbIS IbOS

1995 1955 1965I9ES 1925 1915 1905

I895 I895 I86518E5 I825 I8I5 I885 
$\operatorname{sint} .330$ of
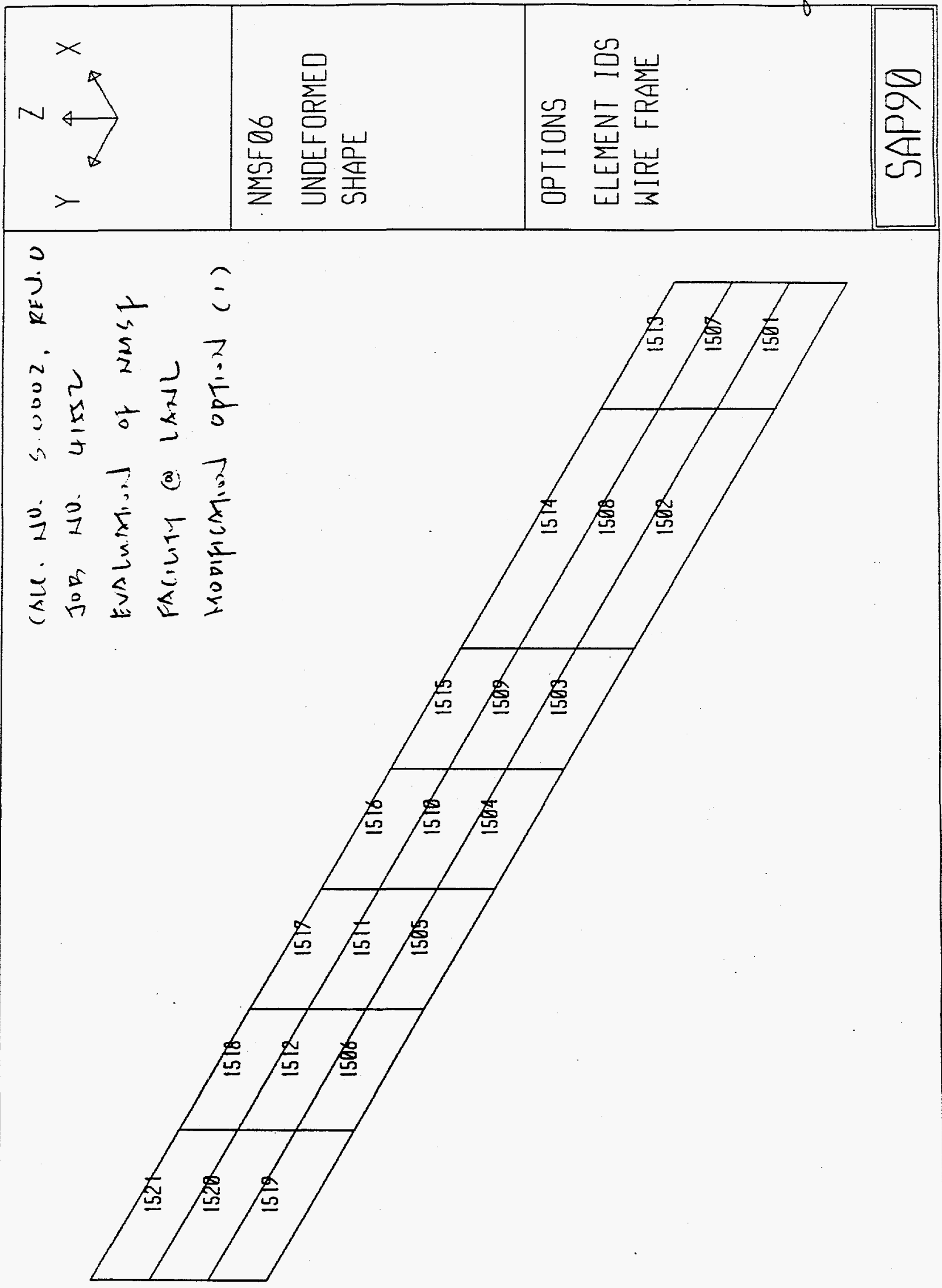
$\sin t .33: 5 f$

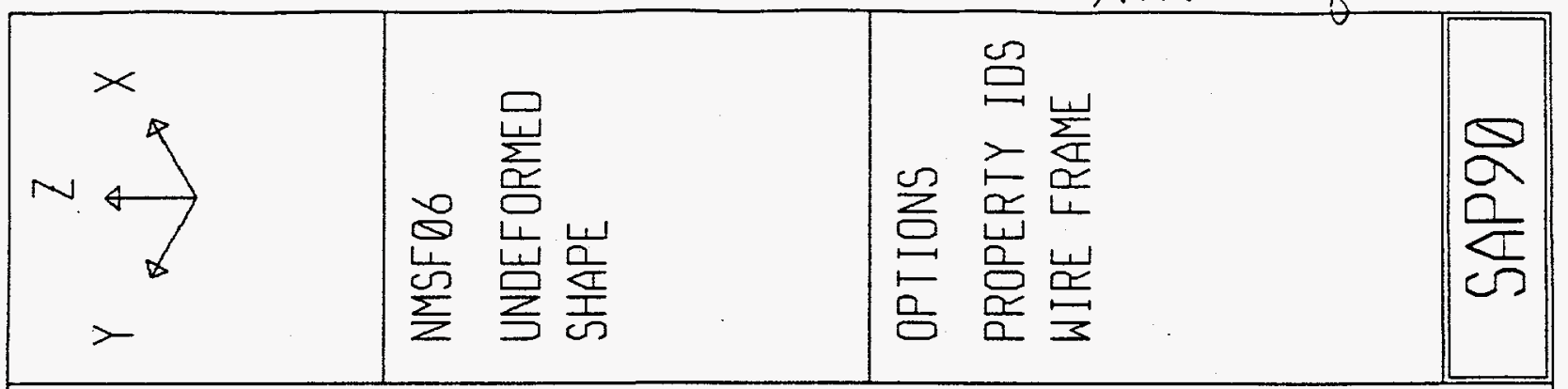

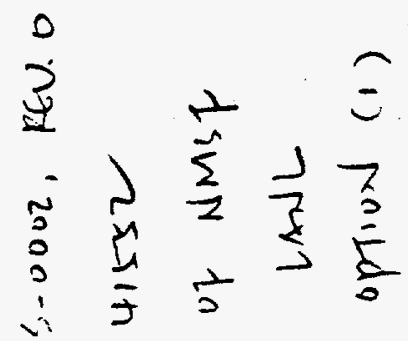

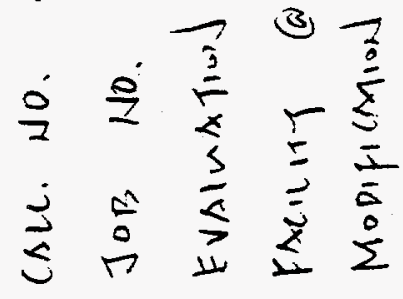
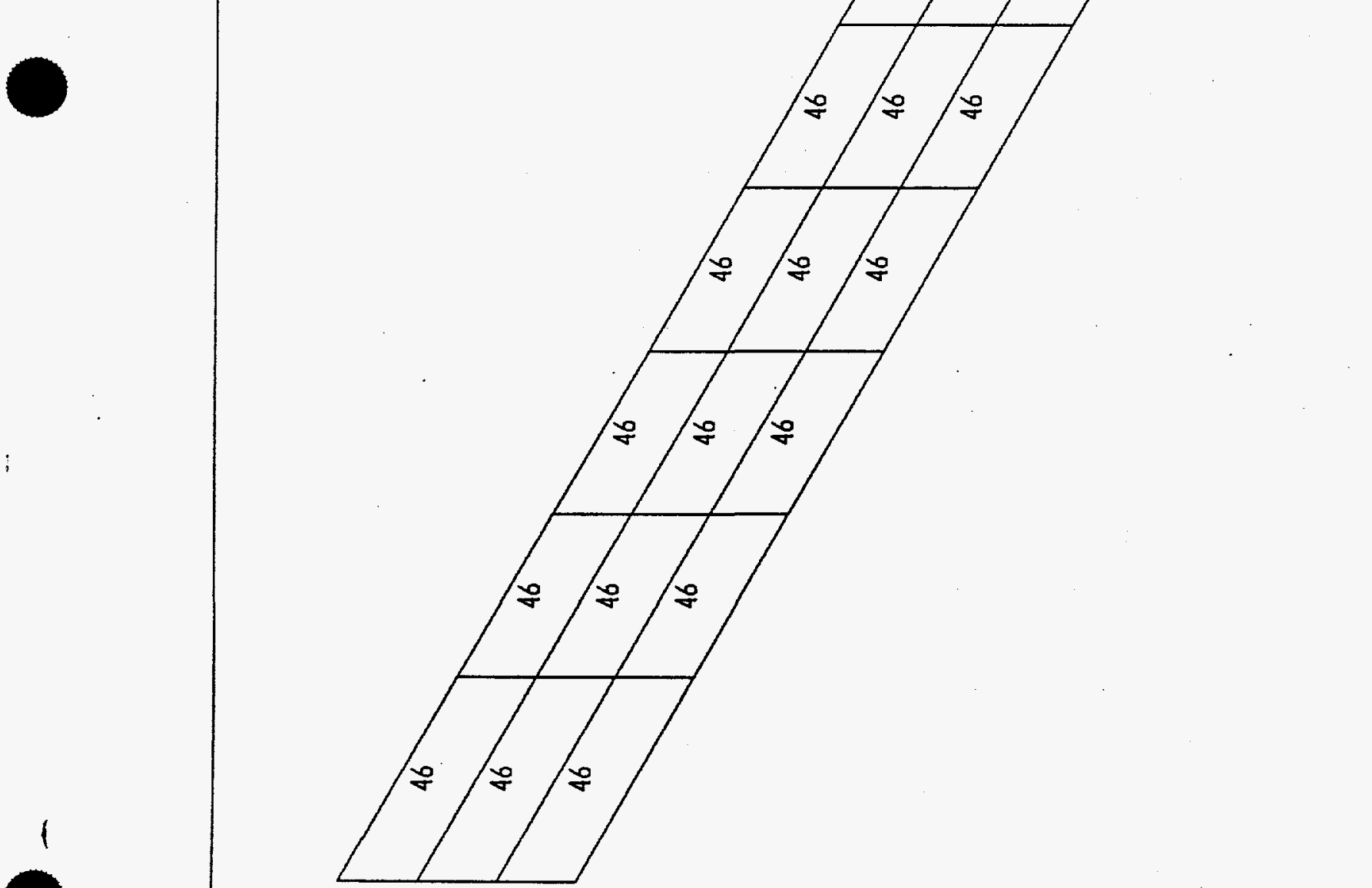


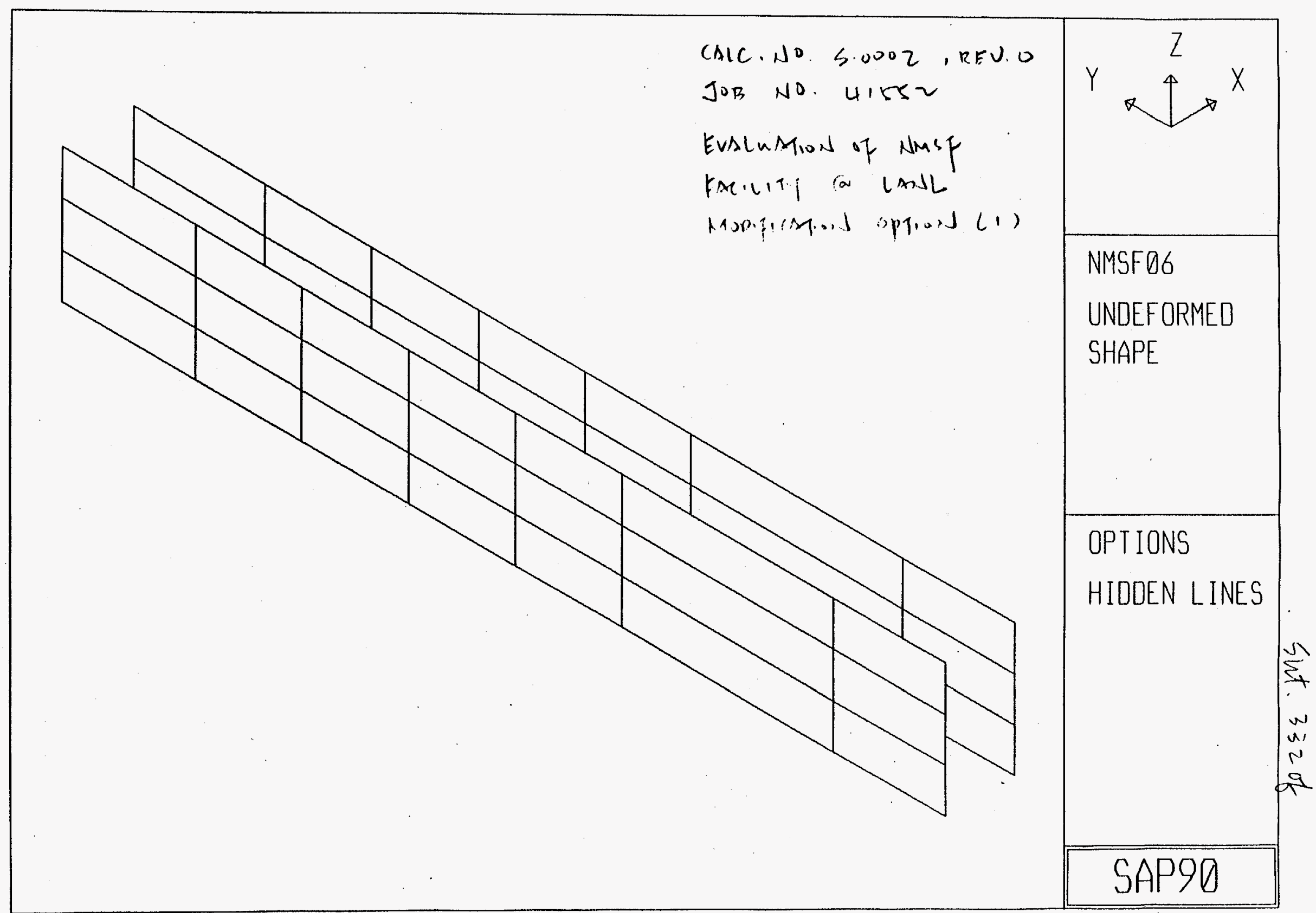


- -

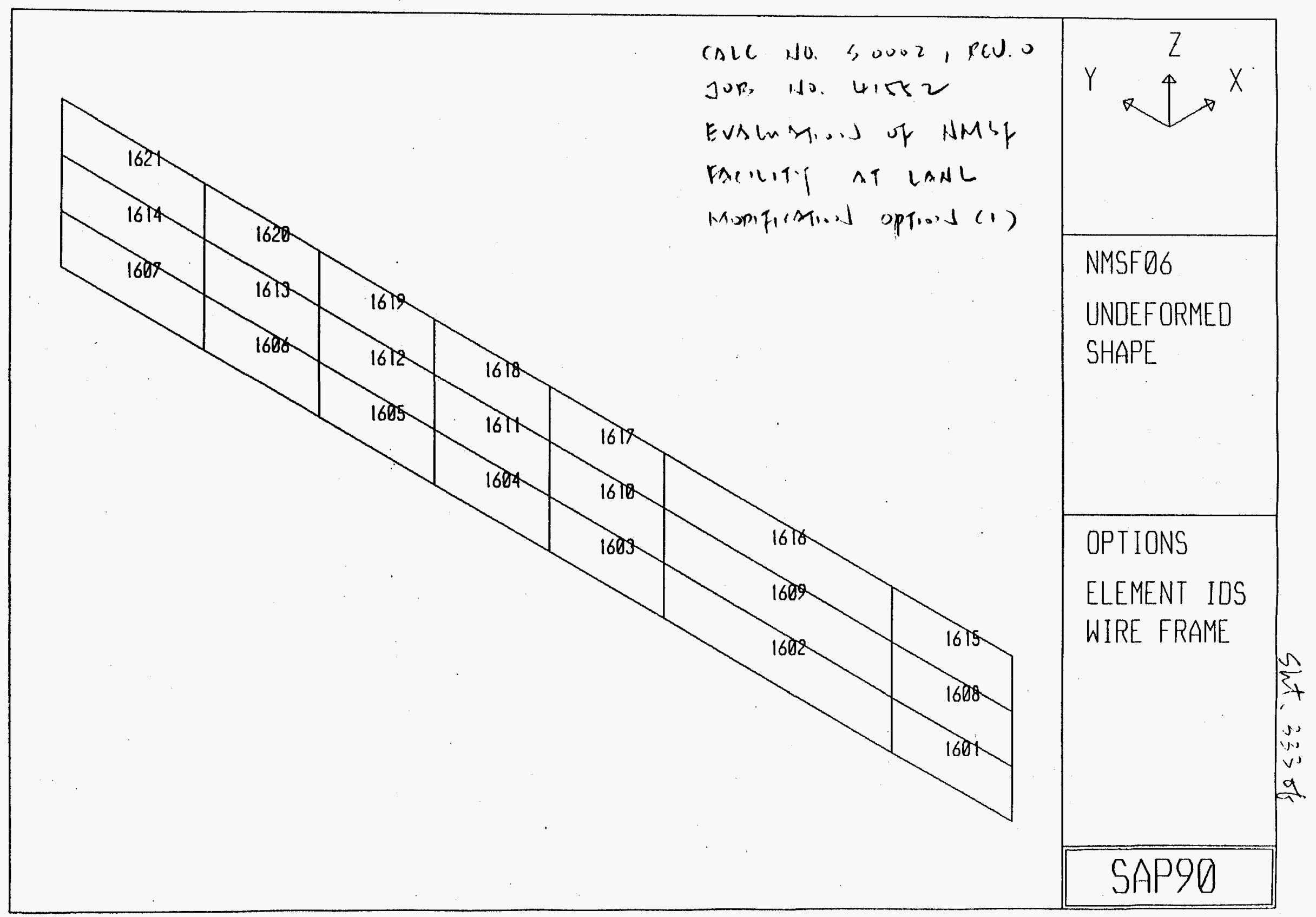


$\operatorname{sint} .334 \sigma_{6}$

FREQ ANALYS:

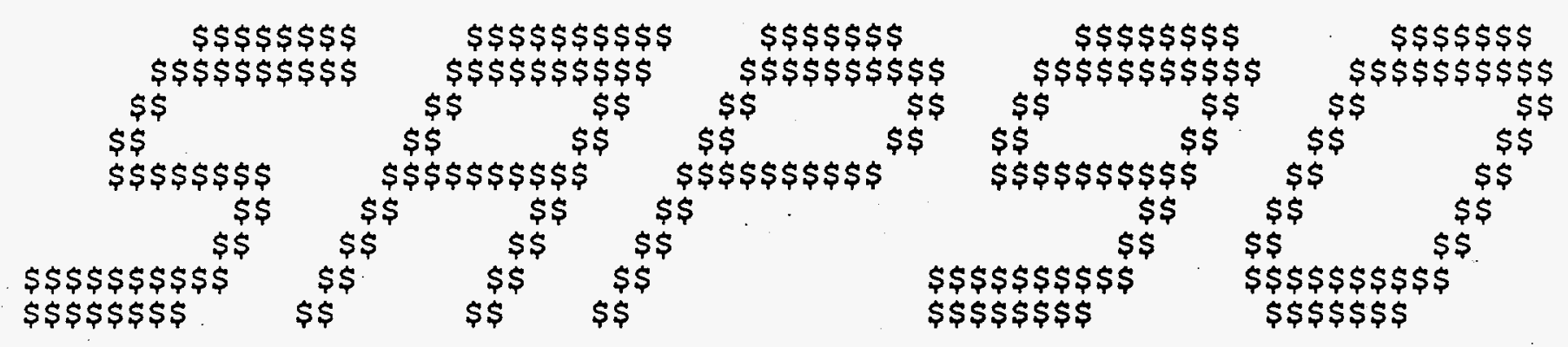

STRUCTURAL ANALYSIS PROGRAMS

VERSION P5.40

Copyright (C) 1978-1992

EDWARD L. WILSON

All rights reserved

CALC. NO: S-0002, REVID

JOB NO. 41552

EvaluMion of NMSF

Faxilify (a) LANL

Mopiparaus oppiod (1) 


$$
\sin t=35=5
$$

URS CONSULTANTS, INC.

SEISMIC ANALYSIS OF NMSF BUILDING (MODIFIED) PAGE 1

PROGRAM : SAP90/FILE : NMSF06.RIT

-SAP9O FILENAME: NM

I T Z S Y S T E M P A R A M E T E R S

NUMBER OF EQUATIONS

NUMBER OF MASSES

NUMBER OF RITZ VECTORS

NUMBER OF DIRECTION VECTORS

$=3138$

$=\quad 237$

$=\quad 10$

$=3$

CAU. NO. S-0002, REU.O JOB NO. 41552 
IRS CONSULTANTS, INC.

SEISMIC ANALYSIS OF MSG BUILDING (MODIFIED)

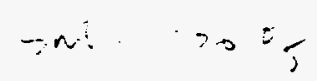

PAGE 2
PROGRAM : SAP90/FILE: NMSF06.RIT -SAP9O FILENAME: NM

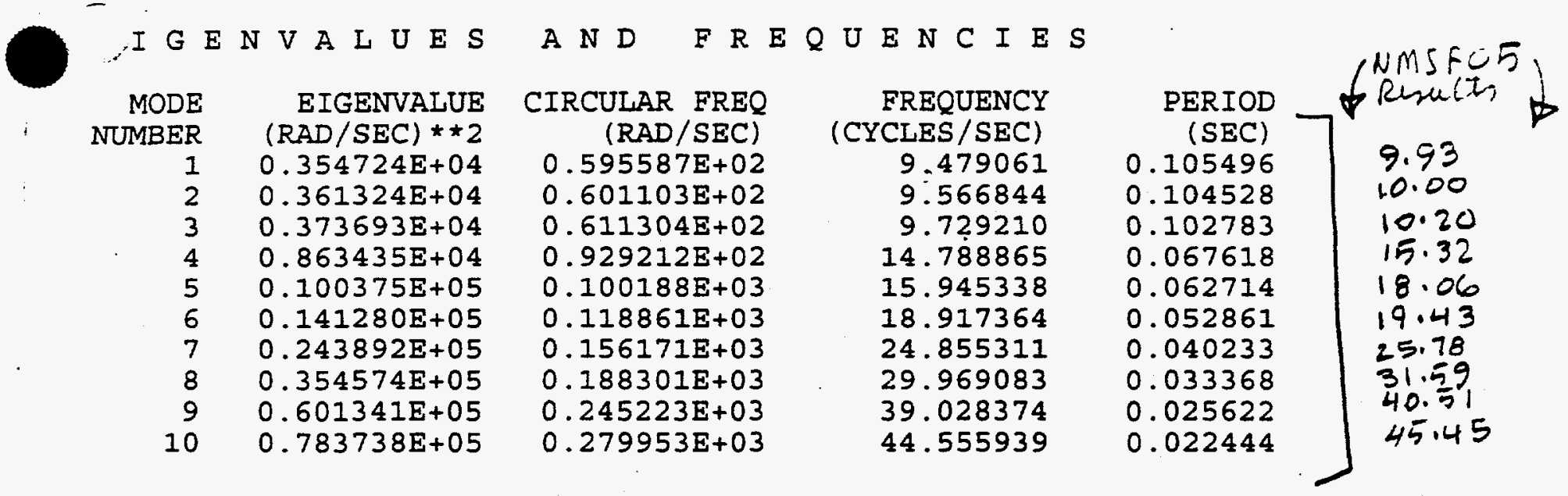

CAME. NO. S.0002, REL. 0 JOB NO WIST 
URS CONSULTANTS, INC.

SEISMIC ANALYSIS OF NMSF BUILDING (MODIFIED)

\section{Sut. 337 of}

PAGE

PROGRAM : SAP9 O/FILE : NMSF06. RIT -SAP90 FILENAME : NM
A S E
$F \circ R \subset E$
$R E A C T I O N$
$F A \subset T O R S$

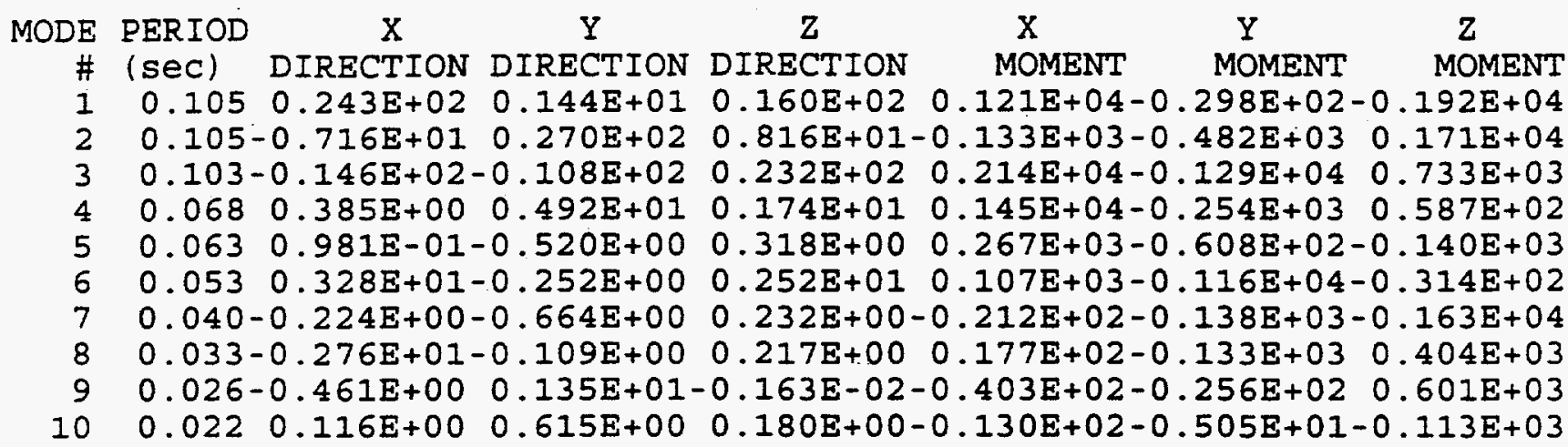

CALC. NO S-OOON, REU. O

JOB NU. 41552 


$$
\text { sut. } 330 \text { of }
$$

URS CONSULTANTS, INC.

SEISMIC ANALYSIS OF NMSF BUILDING (MODIFIED)
PAGE 4

PROGRAM : SAP90/FILE : NMSF06.RIT -SAP9O FILENAME: NM A R T I I P A T I N G MAS S - (percent)

$\begin{array}{rrrrrrr}\text { MODE } & \text { X-DIR } & \text { Y-DIR } & \text { Z-DIR } & \text { X-SUM } & \text { Y-SUM } & \text { Z-SUM } \\ 1 & 67.648 & 0.239 & 29.330 & 67.648 & 0.239 & 29.330 \\ 2 & 5.877 & 83.288 & 7.622 & 73.525 & 83.527 & 36.952 \\ 3 & 24.317 & 13.319 & 61.909 & 97.842 & 96.847 & 98.861 \\ 4 & 0.017 & 2.778 & 0.345 & 97.859 & 99.624 & 99.206 \\ 5 & 0.001 & 0.031 & 0.012 & 97.860 & 99.655 & 99.218 \\ 6 & 1.232 & 0.007 & 0.725 & 99.092 & 99.662 & 99.943 \\ 7 & 0.006 & 0.051 & 0.006 & 99.097 & 99.713 & 99.949 \\ 8 & 0.876 & 0.001 & 0.005 & 99.973 & 99.714 & 99.955 \\ 9 & 0.024 & 0.208 & 0.000 & 99.997 & 99.922 & 99.955 \\ 10 & 0.002 & 0.043 & 0.004 & 99.999 & 99.965 & 99.958\end{array}$

CALC. NO. S-0002, REU.O JOB No. UISS2 


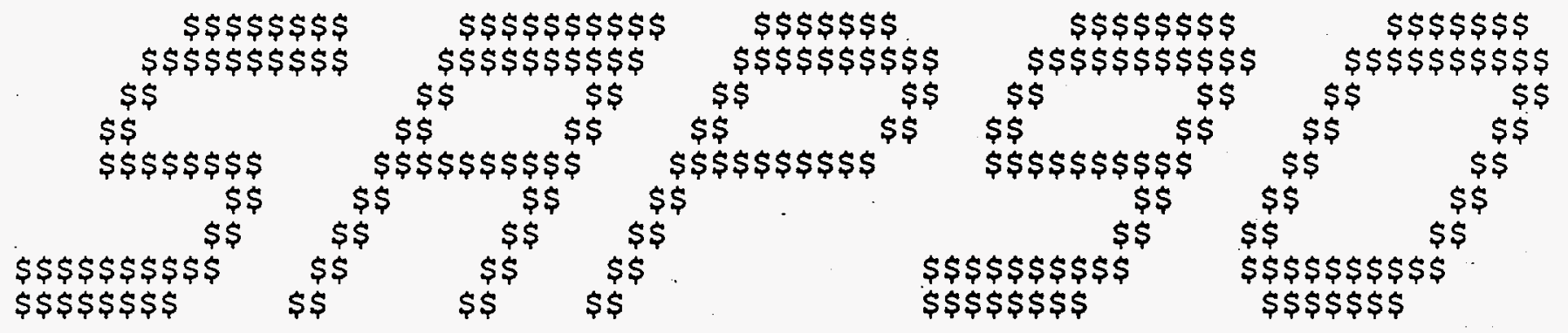

STRUCTURAL ANALYSIS PROGRAMS

VERSION P5.40

Copyright (C) 1978-1992

EDWARD I. WILSON

All rights reserved

CALC. NO. S-0002, REV.O I.B NO. U1552 


$$
\sin \lambda .340=5
$$

URS CONSULTANTS, INC.

SEISMIC ANALYSIS OF NMSF BUILDING (MODIFIED)
PAGE

PROGRAM : SAP90/FILE : NMSF06. SPC -SAP9O FILENAME : NM $\vdots \mathrm{P} E \mathrm{E} C \mathrm{~T} R \mathrm{R}$ U $\mathrm{M}$ I N P U T D A TA

AMPLITUDE MULTIPLIER --- "S"DAMPING RATIO _......- "D" ANGLE OF S1 WITH X-AXIS - "A"-
9.660

0.070

0.000

CALC. NO. S-0002, REN.O JOB NO. UIKS工 
URS CONSULTANTS, INC.

SEISMIC ANALYSIS OF NMSE BUILDING (MODIFIED)

$$
\text { sti. 就 to }
$$

PAGE

PROGRAM : SAPQO/FTIE : NMSF06 SPC -SAP9O FILENAME: NM

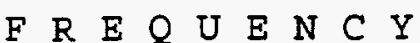
JUMBER RAD./SEC CYCLES/SEC PERIOD-SEC

\begin{tabular}{|c|c|c|c|c|c|c|}
\hline 1 & 59.56 & 9.48 & $\begin{array}{r}0.105496(1) \\
(2) \\
(\mathrm{Z})\end{array}$ & $\begin{array}{l}21.222 \\
21.222 \\
14.148\end{array}$ & $\begin{array}{l}0.356 \\
0.356 \\
0.238\end{array}$ & $\begin{array}{l}0.006 \\
0.006 \\
0.004\end{array}$ \\
\hline 2 & 60.11 & 9.57 & $\begin{array}{r}0.104528(1) \\
(2) \\
(z)\end{array}$ & $\begin{array}{l}21.099 \\
21.099 \\
14.066\end{array}$ & $\begin{array}{l}0.351 \\
0.351 \\
0.234\end{array}$ & $\begin{array}{l}0.006 \\
0.006 \\
0.004\end{array}$ \\
\hline 3 & 61.13 & 9.73 & $\begin{array}{r}0.102783(1) \\
(2) \\
(\mathrm{Z})\end{array}$ & $\begin{array}{l}20.875 \\
20.875 \\
13.917\end{array}$ & $\begin{array}{l}0.341 \\
0.341 \\
0.228\end{array}$ & $\begin{array}{l}0.006 \\
0.006 \\
0.004\end{array}$ \\
\hline 4 & 92.92 & 14.79 & $\begin{array}{r}0.067618(1) \\
(2) \\
(Z)\end{array}$ & $\begin{array}{l}16.029 \\
16.029 \\
10.686\end{array}$ & $\begin{array}{l}0.172 \\
0.172 \\
0.115\end{array}$ & $\begin{array}{l}0.002 \\
0.002 \\
0.001\end{array}$ \\
\hline 5 & 100.19 & 15.95 & $\begin{array}{r}0.062714(1) \\
(2) \\
(Z)\end{array}$ & $\begin{array}{l}15.285 \\
15.285 \\
10.190\end{array}$ & $\begin{array}{l}0.153 \\
0.153 \\
0.102\end{array}$ & $\begin{array}{l}0.002 \\
0.002 \\
0.001\end{array}$ \\
\hline 6 & 118.86 & 18.92 & $\begin{array}{r}0.052861(1) \\
(2) \\
(Z)\end{array}$ & $\begin{array}{r}13.719 \\
13.719 \\
9.146\end{array}$ & $\begin{array}{l}0.115 \\
0.115 \\
0.077\end{array}$ & $\begin{array}{l}0.001 \\
0.001 \\
0.001\end{array}$ \\
\hline 7 & 156.17 & 24.86 & $\begin{array}{r}0.040233(1) \\
(2) \\
(\mathrm{z})\end{array}$ & $\begin{array}{r}11.551 \\
11.551 \\
7.700\end{array}$ & $\begin{array}{l}0.074 \\
0.074 \\
0.049\end{array}$ & $\begin{array}{l}0.000 \\
0.000 \\
0.000\end{array}$ \\
\hline 8 & 188.30 & 29.97 & $\begin{array}{r}0.033368(1) \\
(2) \\
(Z)\end{array}$ & $\begin{array}{r}10.263 \\
10.263 \\
6.842\end{array}$ & $\begin{array}{l}0.055 \\
0.055 \\
0.036\end{array}$ & $\begin{array}{l}0.000 \\
0.000 \\
0.000\end{array}$ \\
\hline 9 & 245.22 & 39.03 & $\begin{array}{r}0.025622(1) \\
(2) \\
(Z)\end{array}$ & $\begin{array}{l}9.660 \\
9.660 \\
6.440\end{array}$ & $\begin{array}{l}0.039 \\
0.039 \\
0.026\end{array}$ & $\begin{array}{l}0.000 \\
0.000 \\
0.000\end{array}$ \\
\hline 10 & 279.95 & 44.56 & $\begin{array}{r}0.022444(1) \\
(2) \\
(Z)\end{array}$ & $\begin{array}{l}9.660 \\
9.660 \\
6.440\end{array}$ & $\begin{array}{l}0.035 \\
0.035 \\
0.023\end{array}$ & $\begin{array}{l}0.000 \\
0.000 \\
0.000\end{array}$ \\
\hline
\end{tabular}

CALC. NO, S-0002, REU.O JOB NO. 41002 
IRS CONSULTANTS, INC.

SEISMIC ANALYSIS OF NMSF BUILDING (MODIFIED)

OD AL AM PI IT U DE FACTOR S

$\begin{array}{rrrrr}\text { AT } & 0.00 \text { AND } & \text { - } 90.00 & \text { DEGREES } & \\ \text { MODE } & \text { PERIOD } & \text { I-DIRECTION } & \text { 2-DIRECTION } & \text { Z -DIRECTION } \\ 1 & 0.105 & 0.145368 & 0.008642 & 0.063813 \\ 2 & 0.105 & -0.041820 & 0.157431 & 0.031750 \\ 3 & 0.103 & -0.081378 & -0.060227 & 0.086564 \\ 4 & 0.068 & 0.000715 & 0.009140 & 0.002149 \\ 5 & 0.063 & 0.000149 & -0.000792 & 0.000323 \\ 6 & 0.053 & 0.003184 & -0.000245 & 0.001629 \\ 7 & 0.040 & -0.000106 & -0.000315 & 0.000073 \\ 8 & 0.033 & -0.000800 & -0.000032 & 0.000042 \\ 9 & 0.026 & -0.000074 & 0.000216 & 0.000000 \\ 10 & 0.022 & 0.000014 & 0.000076 & 0.000015\end{array}$

PAGE 3

PROGRAM : SAP90/FILE: NMSF06.SPC

- SAP OO FILENAME : NM

CALL. NO. S-0002, RENO

JOB NO. 41552 
sit. 343 of

PROGRAM : SAP90/FILE : NMSF06. SPA

IRS CONSULTANTS, INC.

SEISMIC ANALYSIS OF NMSF BUILDING (MODIFIED)

- SAP90 FILENAME : NM

OD AL CORRELATION FACTORS

$\begin{array}{rrrrrrrrrrrr} & 1 & 2 & 3 & 4 & 5 & 6 & 7 & 8 & 9 & 10 \\ 1 & 1.00 & 1.00 & 0.97 & 0.09 & 0.06 & 0.04 & 0.02 & 0.01 & 0.01 & 0.01 \\ 2 & 1.00 & 1.00 & 0.99 & 0.09 & 0.07 & 0.04 & 0.02 & 0.01 & 0.01 & 0.01 \\ 3 & 0.97 & 0.99 & 1.00 & 0.10 & 0.07 & 0.04 & 0.02 & 0.01 & 0.01 & 0.01 \\ 4 & 0.09 & 0.09 & 0.10 & 1.00 & 0.78 & 0.24 & 0.06 & 0.03 & 0.02 & 0.01 \\ 5 & 0.06 & 0.07 & 0.07 & 0.78 & 1.00 & 0.40 & 0.09 & 0.04 & 0.02 & 0.01 \\ 6 & 0.04 & 0.04 & 0.04 & 0.24 & 0.40 & 1.00 & 0.21 & 0.08 & 0.03 & 0.02 \\ 7 & 0.02 & 0.02 & 0.02 & 0.06 & 0.09 & 0.21 & 1.00 & 0.36 & 0.08 & 0.05 \\ 8 & 0.01 & 0.01 & 0.01 & 0.03 & 0.04 & 0.08 & 0.36 & 1.00 & 0.22 & 0.11 \\ 9 & 0.01 & 0.01 & 0.01 & 0.02 & 0.02 & 0.03 & 0.08 & 0.22 & 1.00 & 0.53 \\ 10 & 0.01 & 0.01 & 0.01 & 0.01 & 0.01 & 0.02 & 0.05 & 0.11 & 0.53 & 1.00\end{array}$

CAUL. NO. S.0002, REV .O

JOB NO. $4155^{2}$ 
IRS CONSULTANTS, INC.

SEISMIC ANALYSIS OF NMSF BUILDING (MODIFIED) $\therefore s^{t} j+40$

PAGE

PROGRAM : SAP90/FILE : NMSF06.SPC

-SAP90 FILENAME : NM

$\begin{array}{rlrllllll}\text { B A S E } & R \text { EA C T I O N F F R CE S } \\ \text { AT } & & 0.00 & \text { AND } & -90.00 & \text { DEGREES } & & \end{array}$

i

MODE

NUMBER

$1-D I R$

2 -DIR

FORCE

Z -DIR

FORCE

$1-D I R$

$10.13704 \mathrm{E}+05$

MOMENT

2-DIR

$Z-D I R$

$40.31333 \mathrm{E}+02 \quad 0.40031 \mathrm{E}+03 \quad 0.14115 \mathrm{E}+03 \quad 0.11791 \mathrm{E}+06-0.20640 \mathrm{E}+05 \quad 0.47754 \mathrm{E}+04$

$50.85487 E+00-0.45284 E+01 \quad 0.27723 E+01 \quad 0.23272 E+04-0.52949 E+03-0.12233 E+04$

$60.16605 \mathrm{E}+03-0.12779 \mathrm{E}+02 \quad 0.12740 \mathrm{E}+03 \quad 0.54247 \mathrm{E}+04-0.58980 \mathrm{E}+05-0.15900 \mathrm{E}+04$

$7-0.18593 E+01-0.55071 E+01 \quad 0.19269 E+01-0.17579 E+03-0.11419 E+04-0.13549 E+05$

$8-0.78587 E+02-0.31059 E+01 \quad 0.61811 E+01 \quad 0.50241 E+03-0.37802 E+04 \quad 0.11487 E+05$

$90.63401 \mathrm{E}+01-0.18515 \mathrm{E}+02 \quad 0.22383 \mathrm{E}-01 \quad 0.55444 \mathrm{E}+03 \quad 0.35184 \mathrm{E}+03-0.82657 \mathrm{E}+04$

$10 \quad 0.71480 E+00 \quad 0.37872 E+01 \quad 0.11101 E+01-0.79929 E+02-0.31110 E+02-0.69525 E+03$

$\begin{array}{llllllll}\text { QC } & 0.17992 E+05 & 0.17835 E+05 & 0.12188 E+05 & 0.10926 E+07 & 0.65963 E+06 & 0.16519 E+07\end{array}$

;

CAL. NO. S-0002, REV.

JOR NO. HISS 
Sht. 345 t!

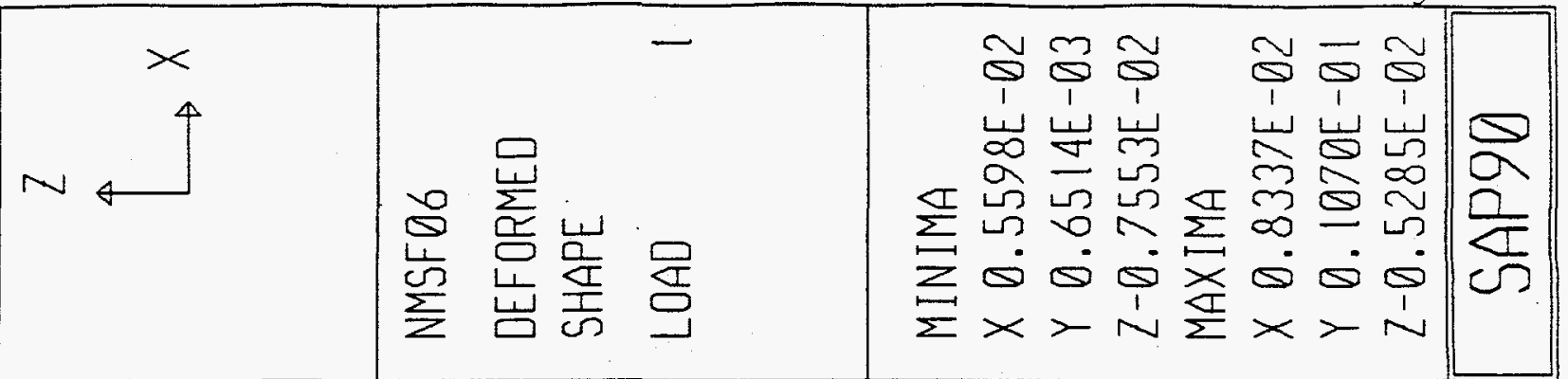

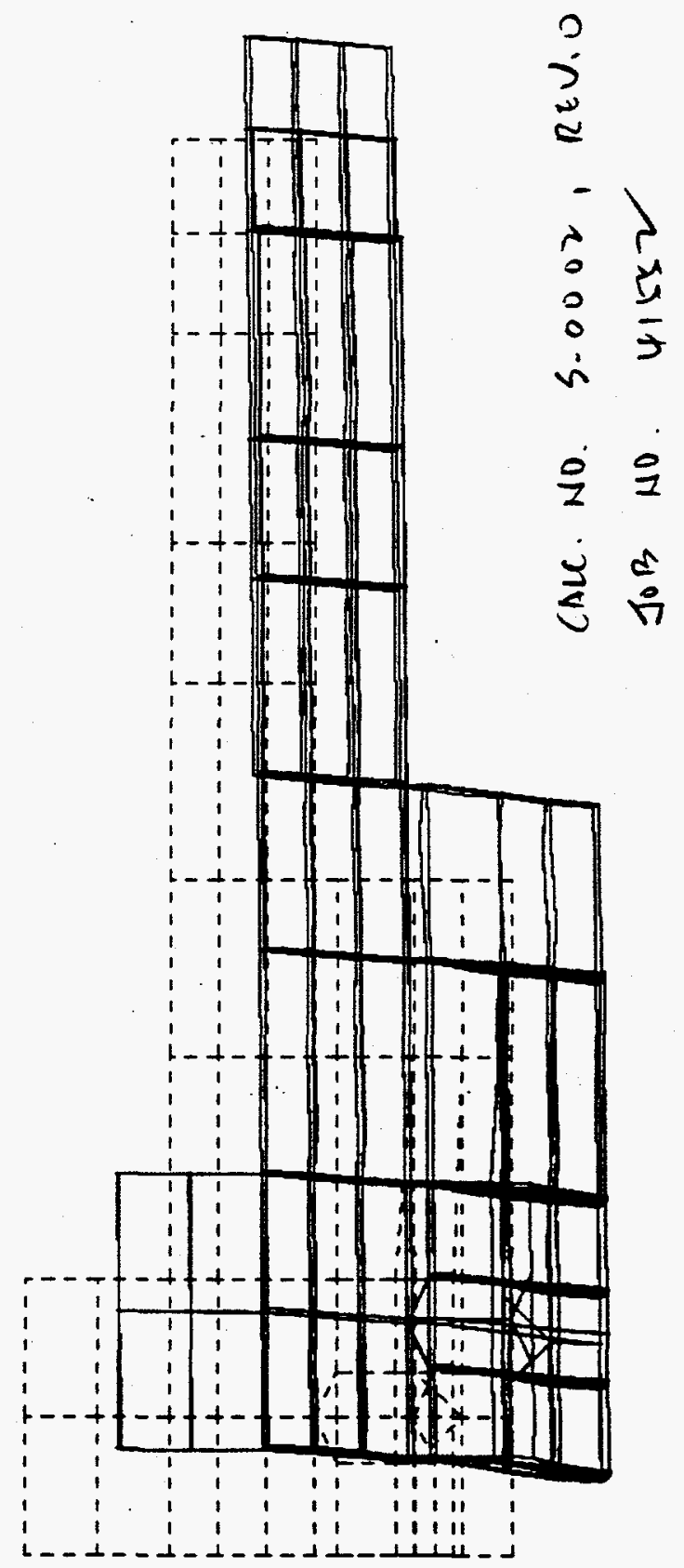


sint. 346 of

\begin{tabular}{|c|c|c|c|}
\hline & 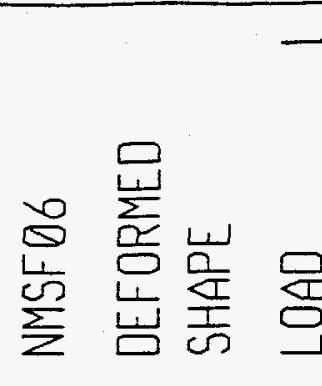 & 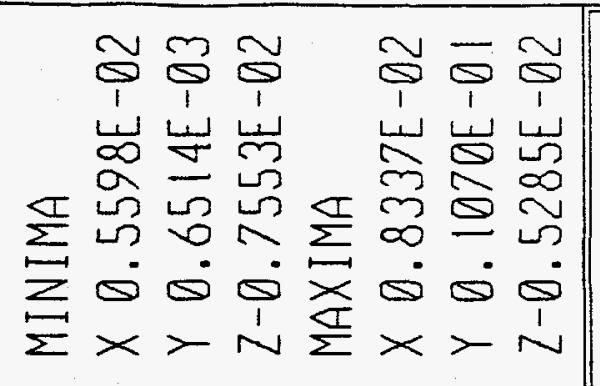 & 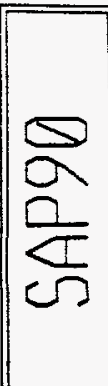 \\
\hline
\end{tabular}

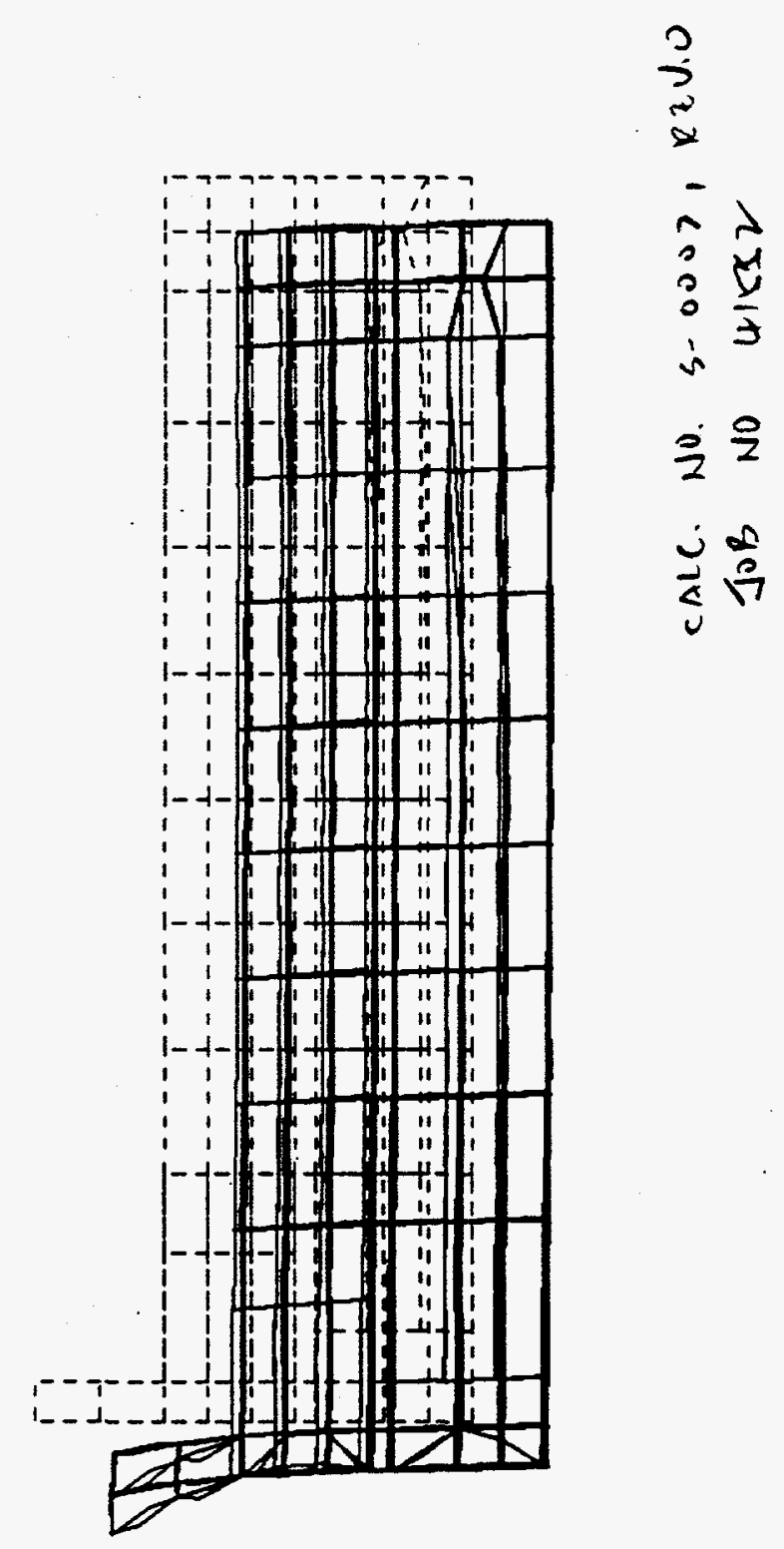




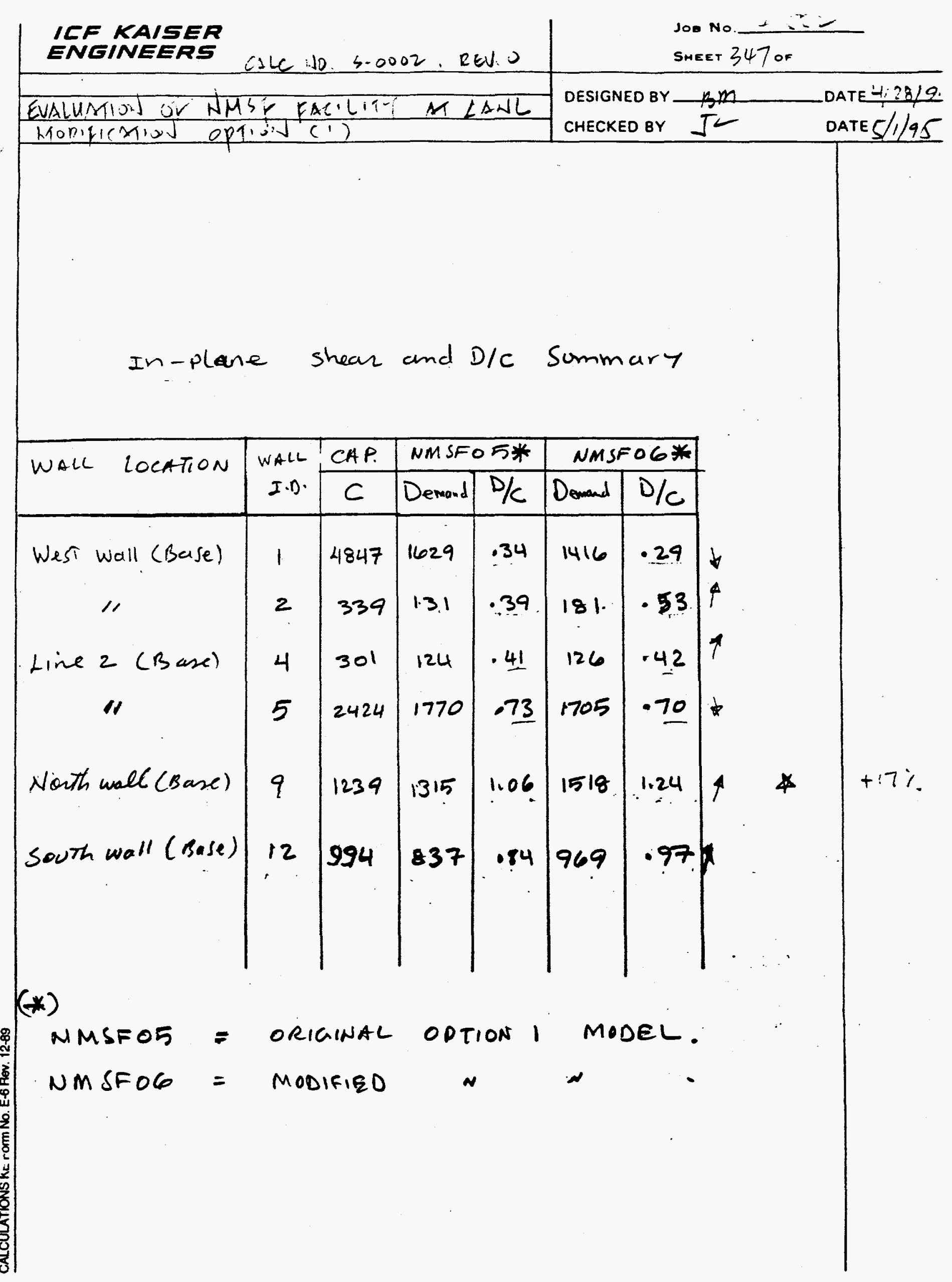




\section{ICF KAISER \\ ENEINEERS}

Jos No. 4.52

EUALUATION OF NMSE D LANL Mopipitris oplion (!)

REV.O

SHEET 348 OF

DESIGNED BY DATE

CHECKED BY

WALL IN-PLANE SHEAR REF. SAP9O FILE:

WALL: 1,2 line (1) Basment

REF SAPGO FIIE: NMSFOS.FEF

\begin{tabular}{|c|c|c|c|c|}
\hline $\begin{array}{l}\text { SHELL } \\
\text { ELEMENT }\end{array}$ & JOINT & $\begin{array}{l}\text { LCI } \\
\text { FY }\end{array}$ & $\begin{array}{l}L C_{2} \\
F Y \\
\end{array}$ & $\begin{array}{l}\angle C 3 \\
F\end{array}$ \\
\hline \multirow{2}{*}{1101} & 1 & 40.5 & 2 & \\
\hline & 11 & 35.0 & 5 & \\
\hline \multirow{2}{*}{1102} & 11 & 41.6 & -6 & \\
\hline & 21 & 29.0 & -17 & \\
\hline \multirow{2}{*}{1121} & $2 !$ & 111. & -38 & \\
\hline & 31 & 91 & -56 & \\
\hline \multirow{2}{*}{1122} & 31 & 184 & -105 & \\
\hline & $4^{\prime}$ & 157 & -134 & \\
\hline \multirow{2}{*}{23} & 41 & 96 & -50 & \\
\hline & 51 & 72 & -73 & \\
\hline \multirow{2}{*}{24} & 51 & 98 & -49 & \\
\hline & 61 & 71 & -74 & \\
\hline \multirow{2}{*}{25} & 61 & 98 & -48 & \\
\hline & 71 & 71 & -75 & \\
\hline \multirow{2}{*}{26} & 71 & 100 & -49 & \\
\hline & BI & 69 & -76 & \\
\hline \multirow{2}{*}{27} & BI & 150 & -80 & \\
\hline & 91 & 119 & -107 & \\
\hline \multirow{2}{*}{1111} & 91 & 27 & -4 & \\
\hline & 101 & 7 & $\therefore-20$ & \\
\hline & & $i^{-\cdots}$ & 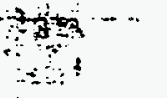 & \\
\hline WALL & & & & \\
\hline BASE SHGAR & & 1591 & 1068 & \\
\hline
\end{tabular}


IEF KAISER

ENEINEERS

CsLC No. 3.0002, EEN. 0

Joe No

SHEET 349 OF

EUALUATION OF NMSE Q LANL Merikicmion oplin (1)

DESIGNED BY

DATE

CHECKED BY

WALL IN-PLANE SHEAR REF: SAP9O FILE:
WALL: LINe (2) Buremet

REF: NMSFO6.FEF

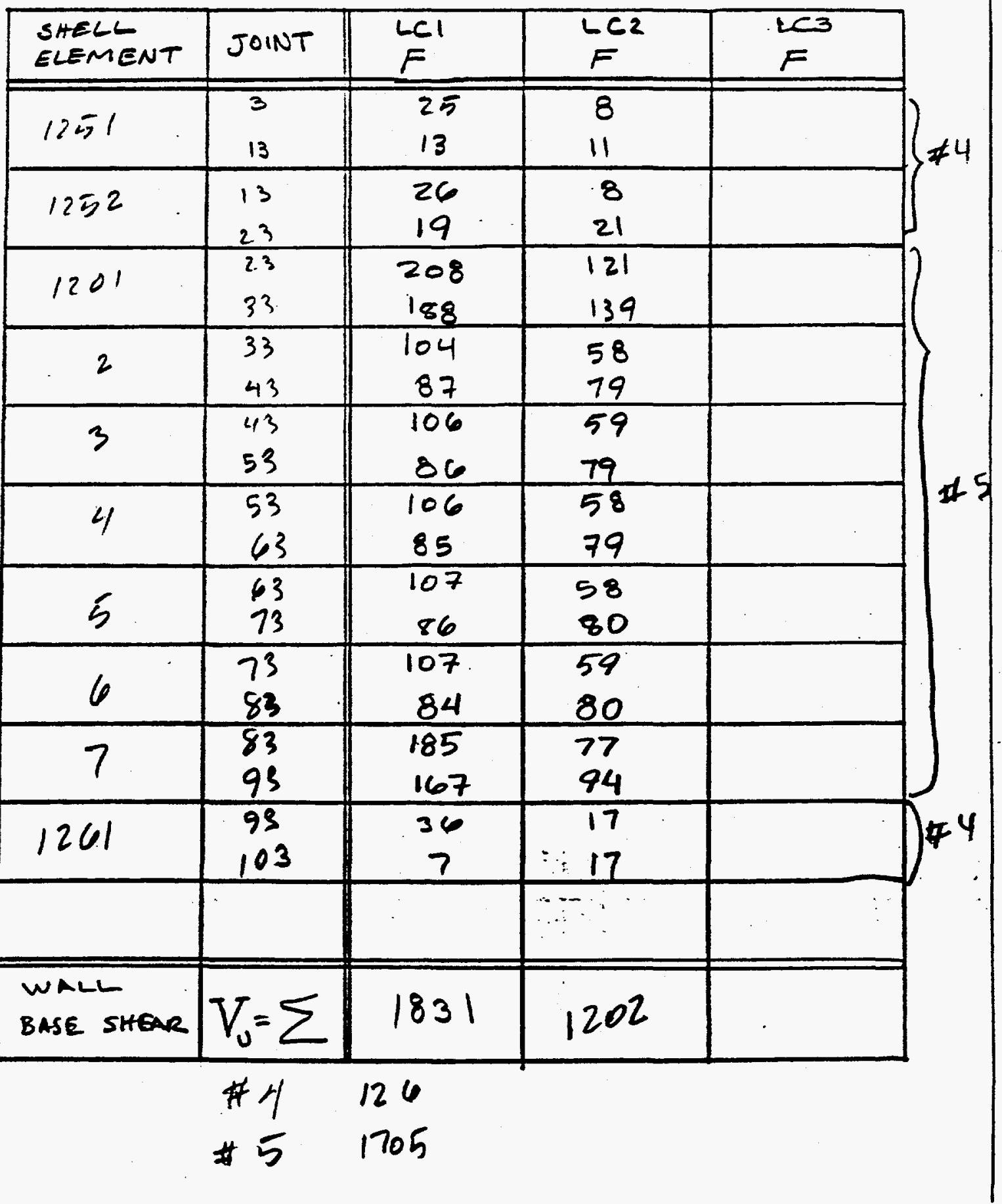




\section{ICF KAISER ENGINEERS}

evaluation of nMSE a lane hophimen op pion (i)

WALL IN-PLANE SHEAR

REF. SAPGO FILE: WALL: $(12)$ line $G$ Buernert

REF: NMSFOGIFEF

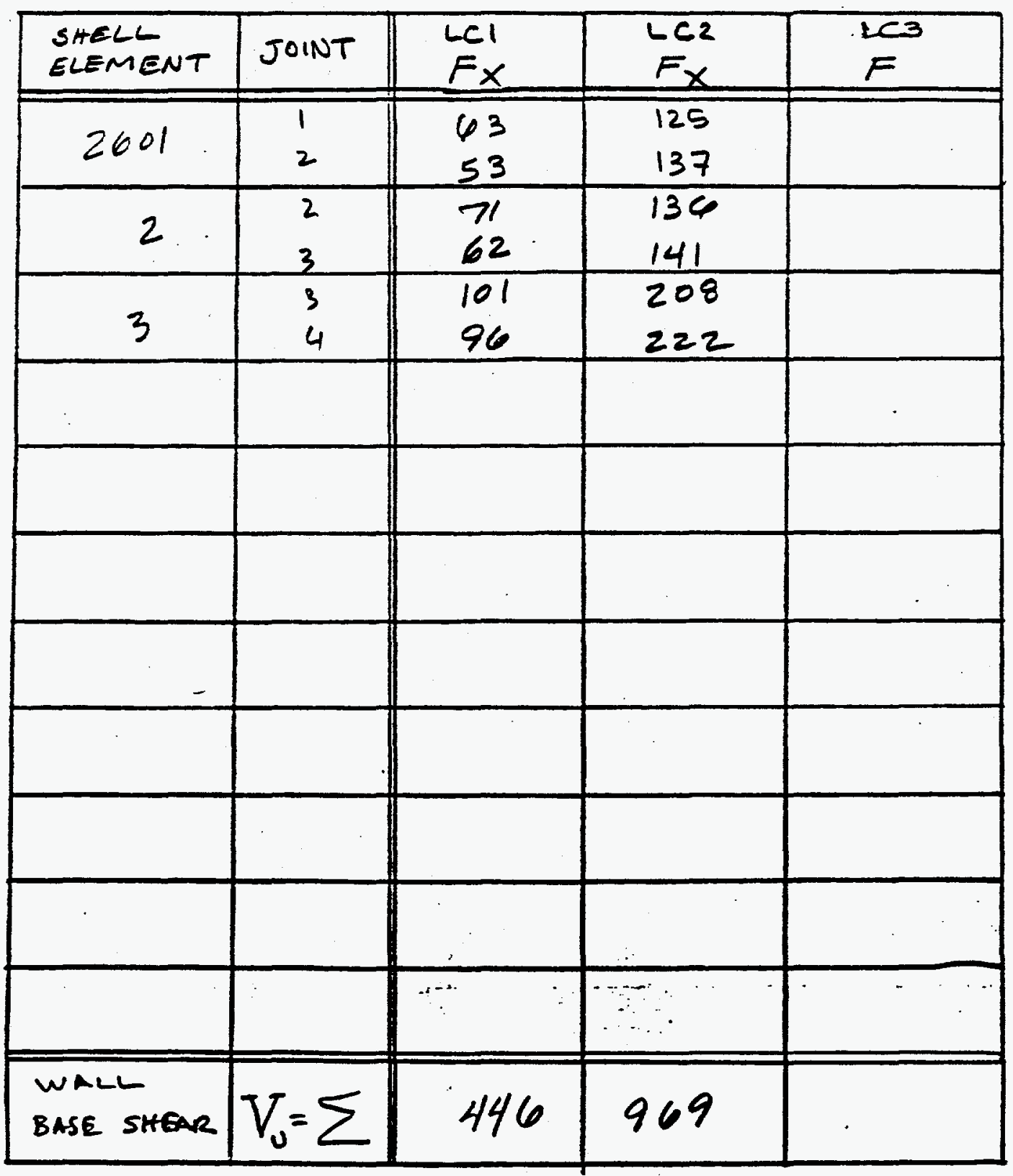




\section{ICF KAISER \\ ENEINEERS}

CALC NO S.0002 KEJ O

Jog No. $-1:-$

EUALUATION OF NMSE O WANL MODIfCM.ON OPII.N (1)

DESIGNED BY

CHECKED BY

REF. SAPQO FILE:

WALL IN-PLANE SHEAR

Wall: (9) $\operatorname{limp} B$ (Busenat lenel)

REF NMSFOG.FEF

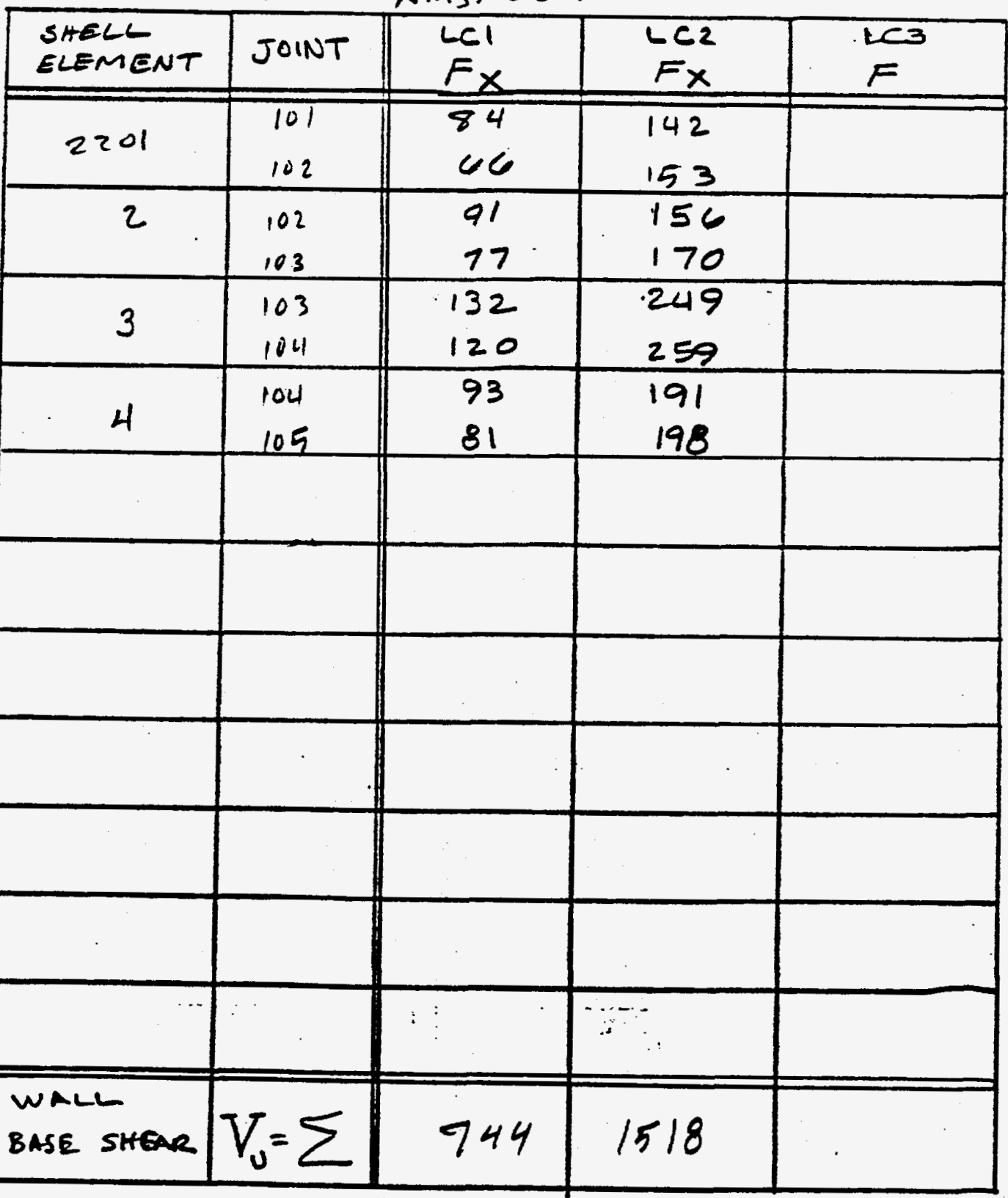


WALL IN-PLANE SHEAR

REF. SAP9O FILE:

WALL: Line (1) Basement

$$
\text { REF: NMSFGI.FFE (STATIG) }
$$

\begin{tabular}{|c|c|c|c|c|}
\hline $\begin{array}{l}\text { SHELL } \\
\text { ELEMENT }\end{array}$ & JOINT & $\begin{array}{l}\text { LCI } \\
F_{Y}\end{array}$ & $\begin{array}{l}\text { LC2 } \\
F Y\end{array}$ & $\begin{array}{l}\angle C 3 \\
F\end{array}$ \\
\hline 1101 & 11 & $\begin{array}{r}14 \\
1\end{array}$ & $\begin{array}{l}34 \\
34\end{array}$ & \\
\hline 2 & $\begin{array}{l}11 \\
21\end{array}$ & $\begin{array}{r}17 \\
9 \\
\end{array}$ & $\begin{array}{l}27 \\
12 \\
\end{array}$ & \\
\hline 1121 & $\begin{array}{l}2 ! \\
3 !\end{array}$ & $\begin{array}{l}21 \\
13\end{array}$ & $\begin{array}{l}58 \\
32\end{array}$ & \\
\hline 2 & $\begin{array}{l}31 \\
41 \\
\end{array}$ & $\begin{array}{l}20 \\
19 \\
\end{array}$ & $\begin{array}{l}64 \\
23 \\
\end{array}$ & \\
\hline 3 & $\begin{array}{l}4 . \\
5.1\end{array}$ & $\begin{array}{l}17 \\
19 \\
\end{array}$ & $\begin{array}{r}36 \\
2 \\
\end{array}$ & \\
\hline 4 & $\begin{array}{l}51 \\
61\end{array}$ & $\begin{array}{l}18 \\
20 \\
\end{array}$ & $\begin{array}{c}36 \\
1 \\
\end{array}$ & \\
\hline 5 & $\begin{array}{l}6 ! \\
7 ! \\
\end{array}$ & $\begin{array}{l}19 \\
20 \\
\end{array}$ & $\begin{array}{r}39 \\
0 \\
\end{array}$ & \\
\hline 6 & $\begin{array}{l}71 \\
81\end{array}$ & $\begin{array}{l}23 \\
21 \\
\end{array}$ & $\begin{array}{l}39 \\
2\end{array}$ & \\
\hline 7 & $\begin{array}{l}81 \\
91 \\
\end{array}$ & $\begin{array}{l}5 \\
48\end{array}$ & $\begin{array}{l}60 \\
19 \\
\end{array}$ & \\
\hline 1111 & $\begin{array}{l}91 \\
101 \\
\end{array}$ & $\begin{array}{r}3 \\
19 \\
\end{array}$ & $\begin{array}{l}19 \\
8 \\
\end{array}$ & \\
\hline & 40 & & $\varepsilon$ & \\
\hline $\begin{array}{l}\text { WALL } \\
\text { BASE SHEAR }\end{array}$ & & & & \\
\hline
\end{tabular}


IEF KAISER

ALC. NO. S-0002, REU 0

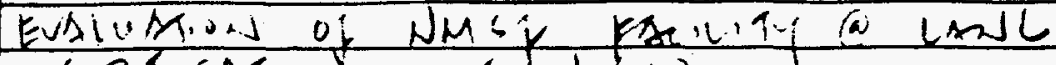
Monificmion op l.w.J (1)

JoE No

SHEET $\sum_{3 \text { OF }}^{3 / 3}$

$\begin{array}{lll}\text { DESIGNED BY } & B M & \text { DATE } 3 / 8 / 95 \\ \text { CHECKED BY } & T \_ & \text {DATE } 3 / 1 / 4 \pi\end{array}$

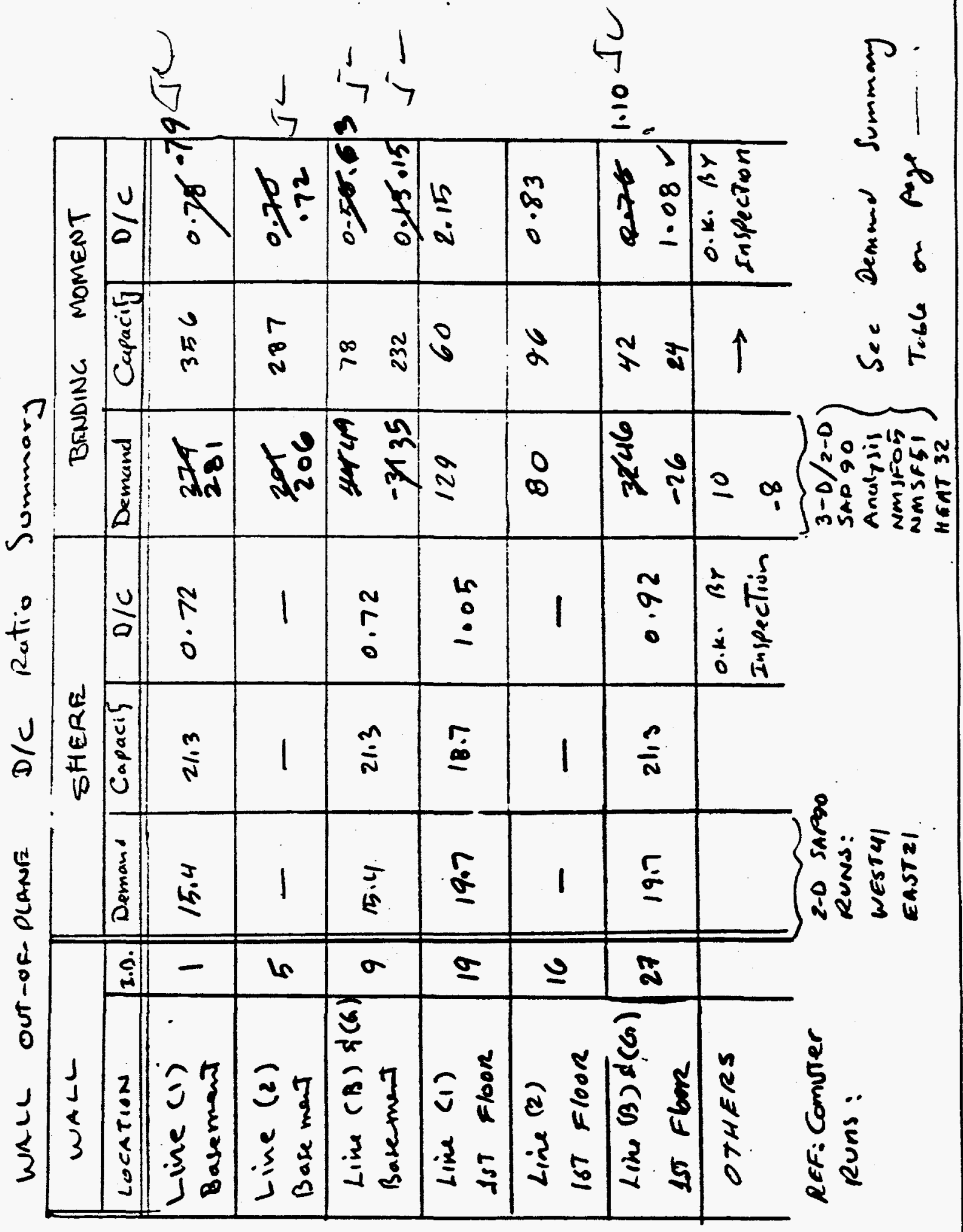




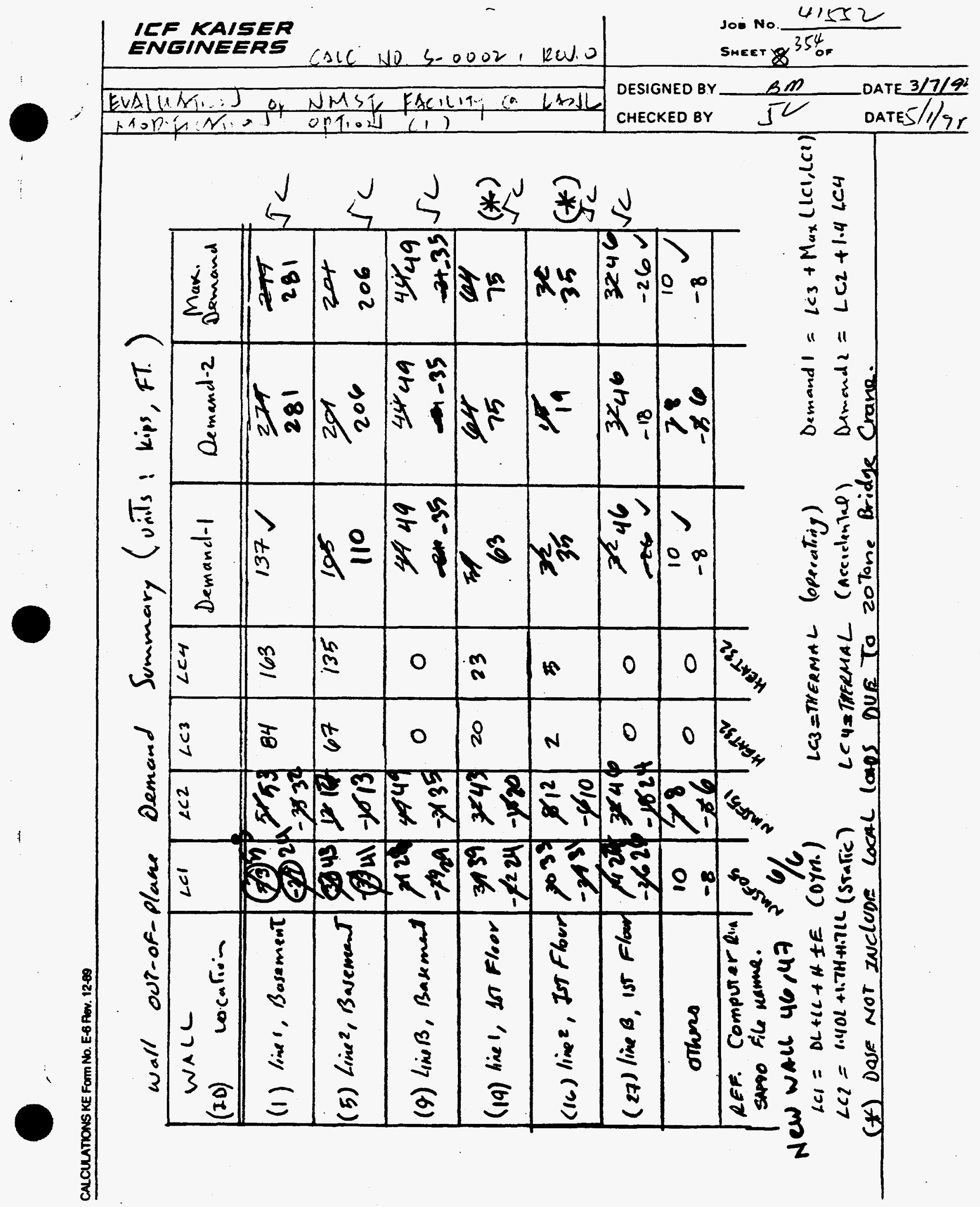


ICF KAISER

$$
\text { Joo No. } 41552
$$

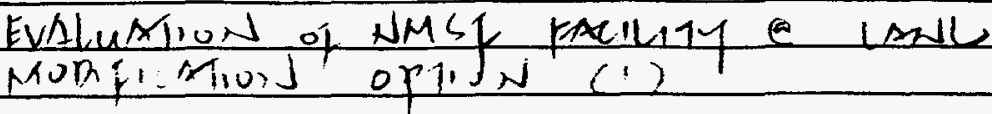

SHEET 355 OF

DESIGNED BY $\triangle M$

DATE $5 / / / 91 / 94$

wad-ot - ff-Plane Demanel

New watls in Busemet (woll I.D.'s 46 2 47 )

LCI: $D L+L+H \pm E$ [REF: NMSFOG]

LE2: $1.4 D L+1.7 H+1.7 L L$ CREF: NMSFG1]

LC3: Themal orenciting [REF: HEAT 32]

LCH: $N$ ACCidental [REF: N]

$$
\begin{aligned}
& \text { Demandl }=\text { LCS }+\operatorname{Max}(L C 1, L C 2) \\
& \text { Demand2 }=\text { LC } 2+1.4 L C C_{4}
\end{aligned}
$$

$\begin{aligned} \text { LC1 } & \text { Max Mom. } & =5.6 & \text { M-F } \\ \text { LC2: } & N & & =3.2 \\ \text { LC3: } & N & & =3.4 \mathrm{~N} \\ \text { LC3: } & N & & =6.7 \mathrm{~N} \\ D_{1}: & N & & =3.4+5.6=9.1 \mathrm{~K}-\mathrm{FT} \\ D_{2}: & N & & =3.2+1.4 \times 6.7=12.6\end{aligned}$

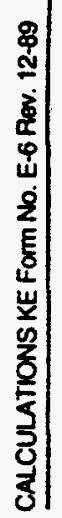$$
D=12.6
$$

Wall Thicknen $=1^{\prime}-6^{\prime \prime}$

use Min sjeel, $A_{s}=-\frac{200}{60,600} \times 12 \times 18$

$$
\begin{aligned}
=0.7 \dot{m}^{2} & (\neq 6,006) \\
& A=2 x \cdot 44=.88
\end{aligned}=^{2}
$$




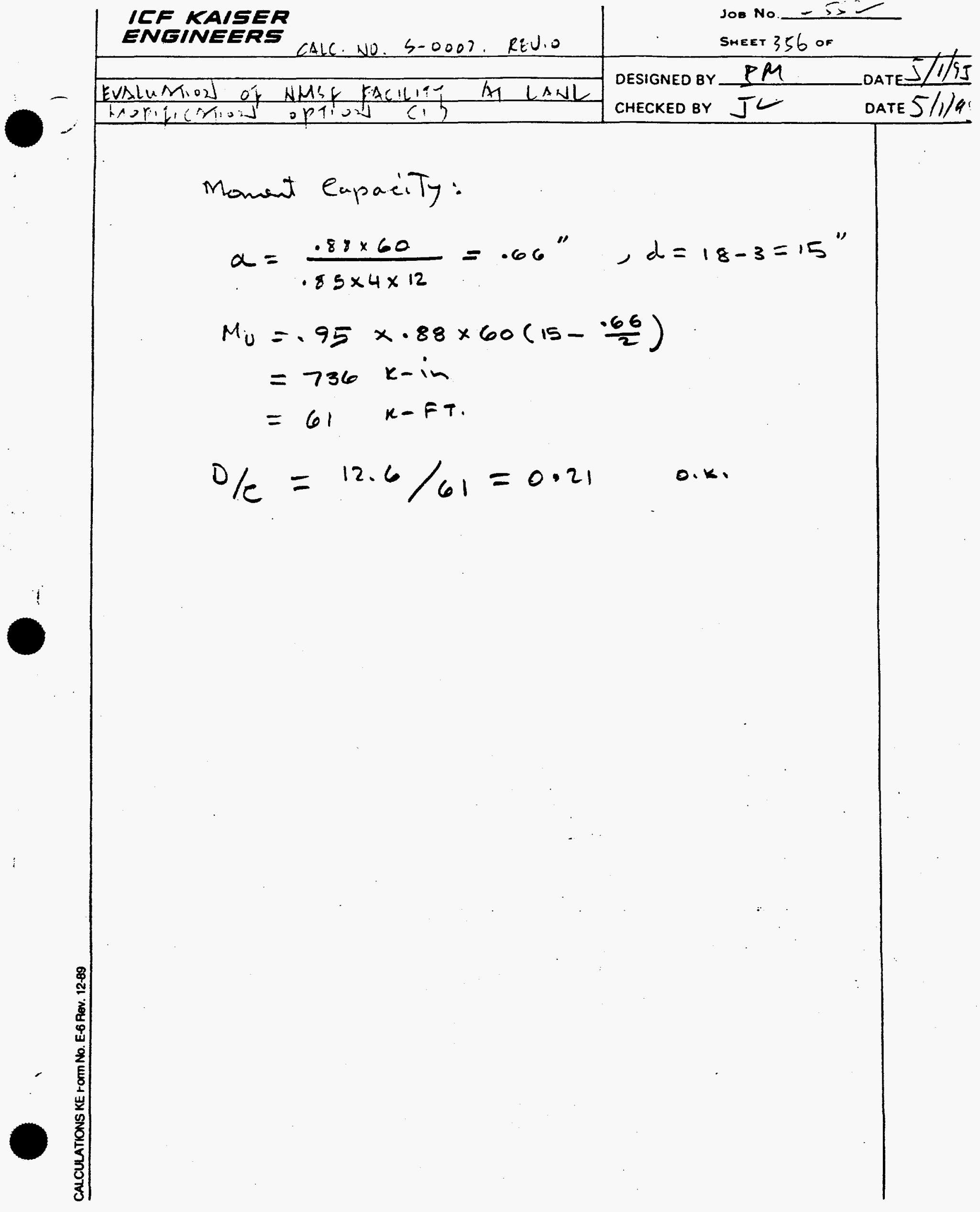



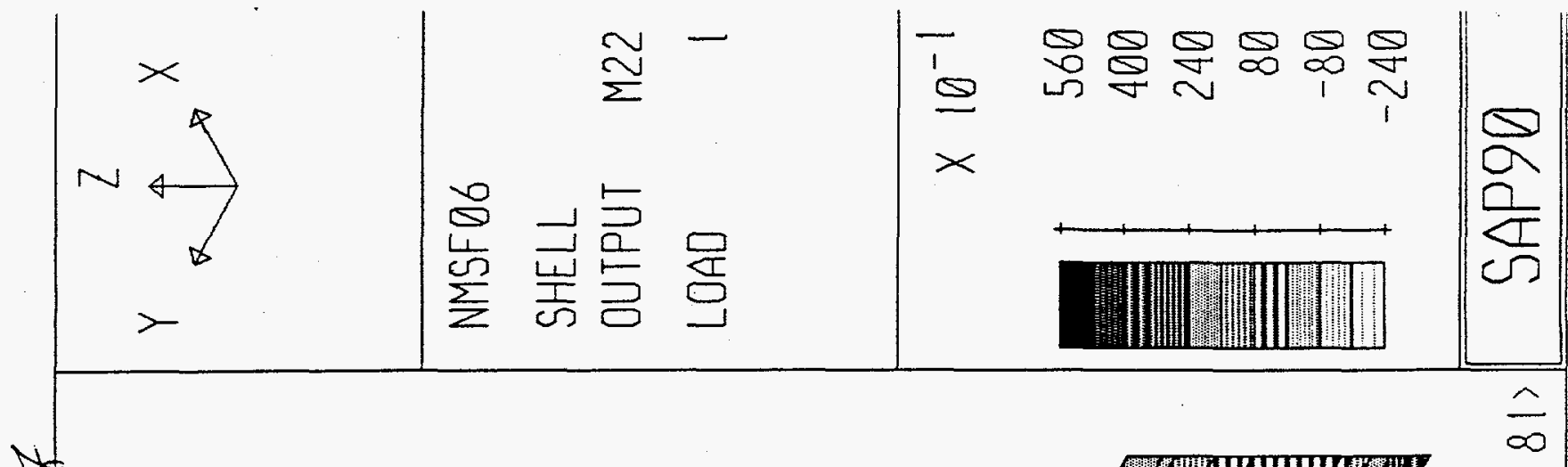

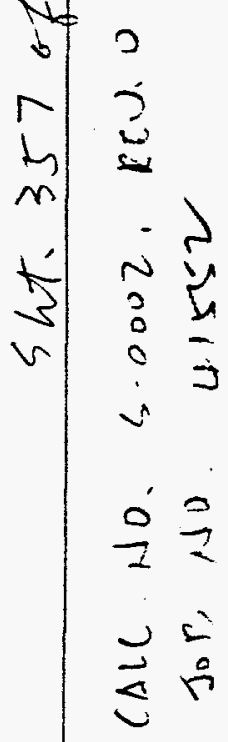


sut. 358 of

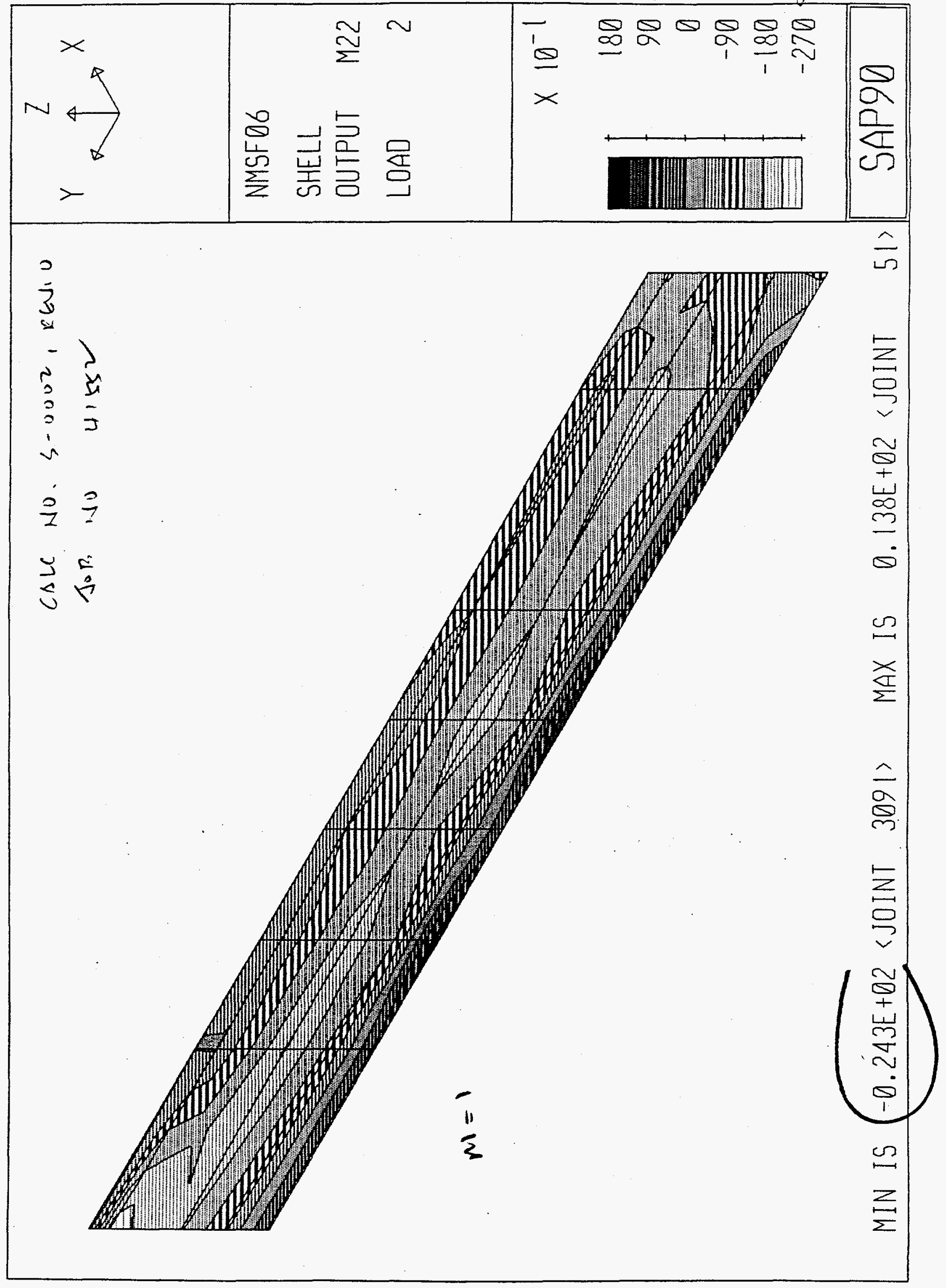


Sht. 359 of
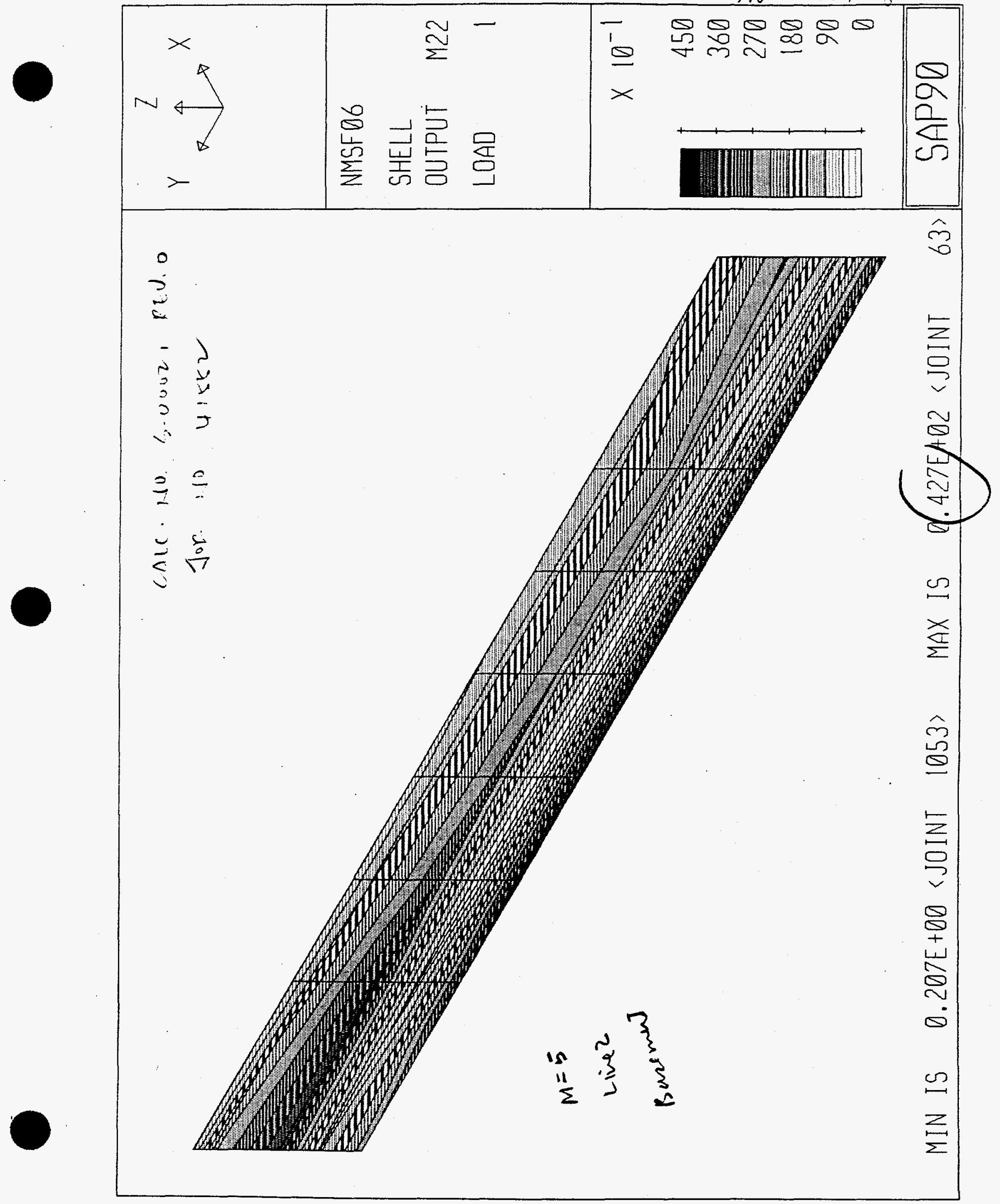


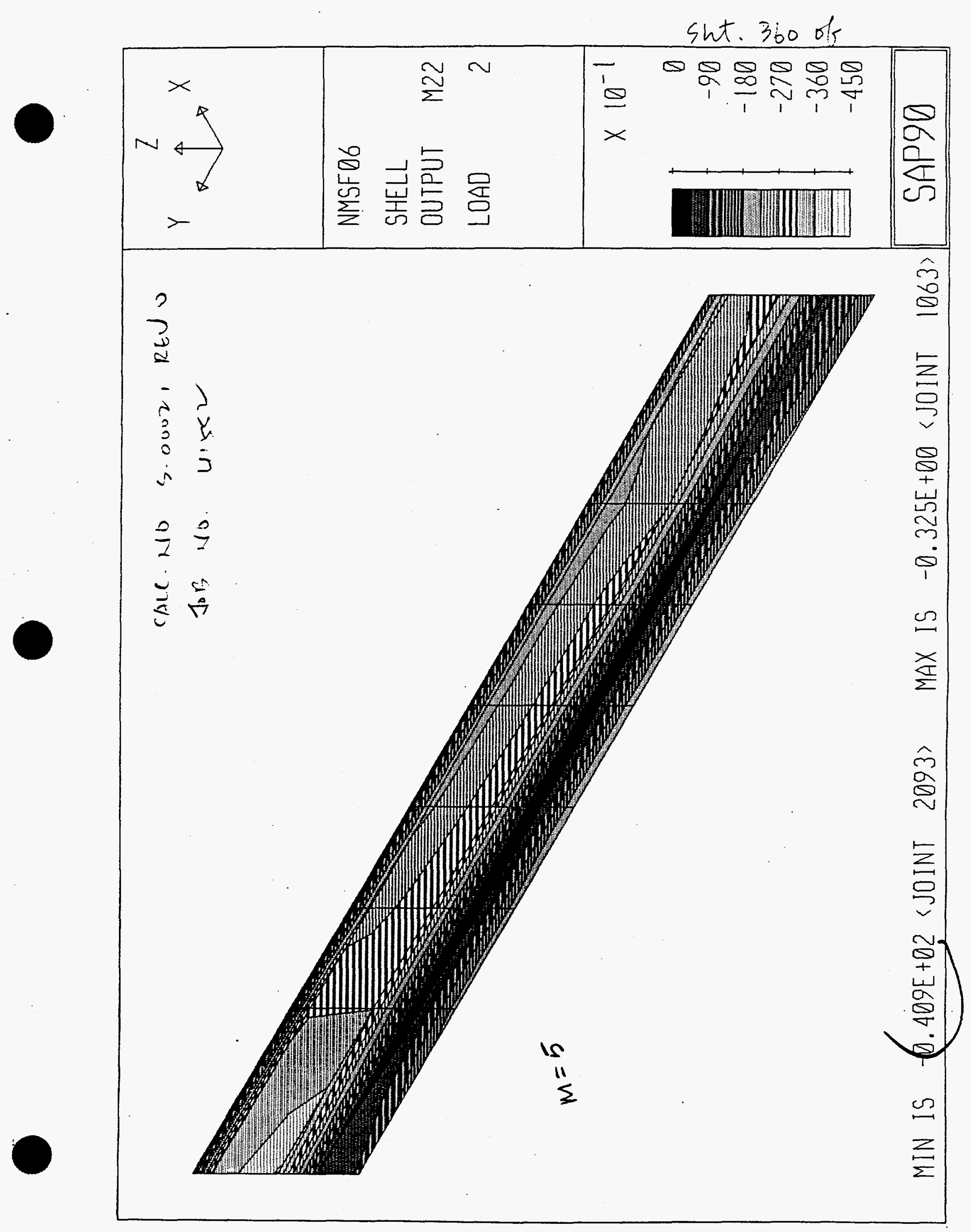




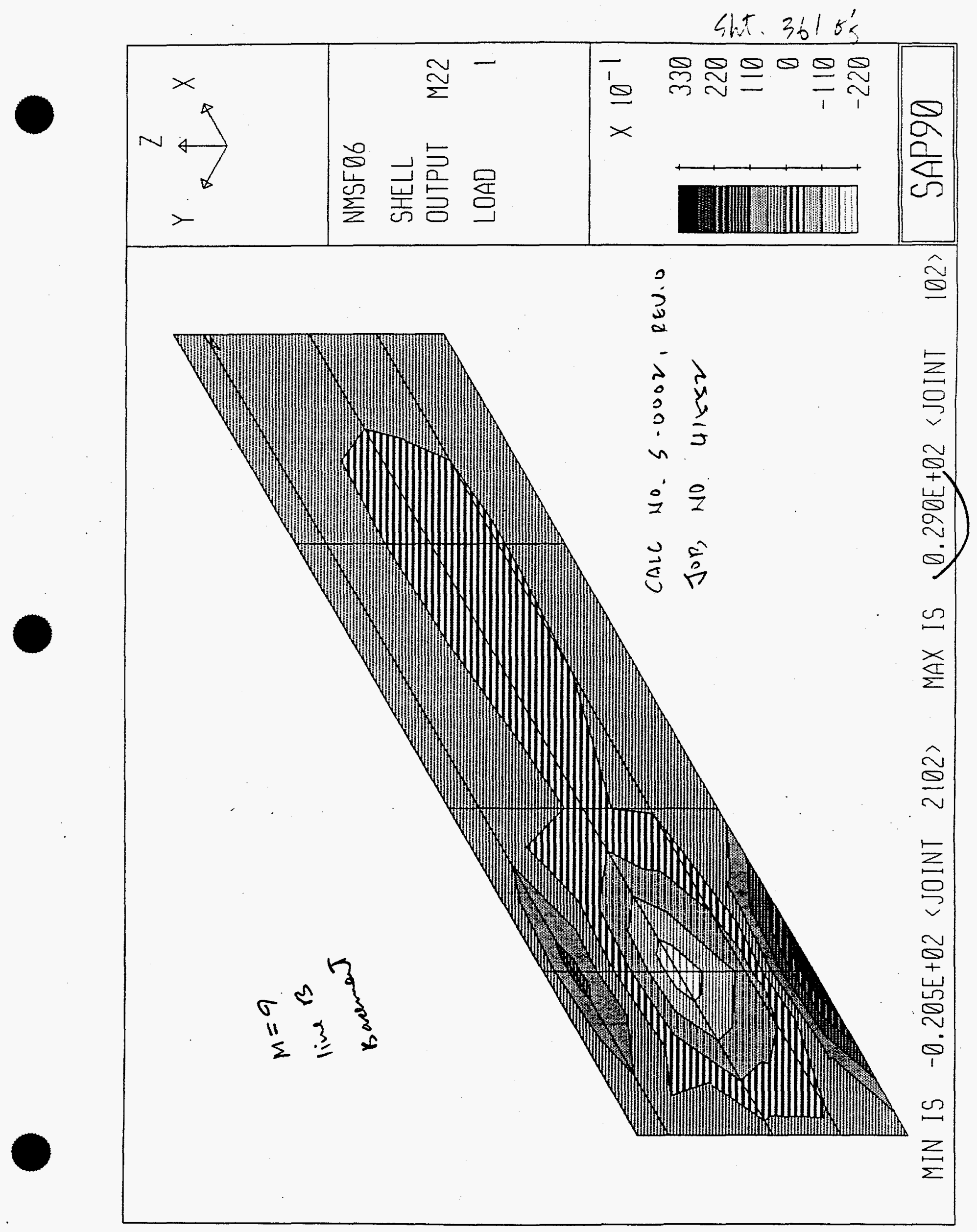


Sht. 362 of

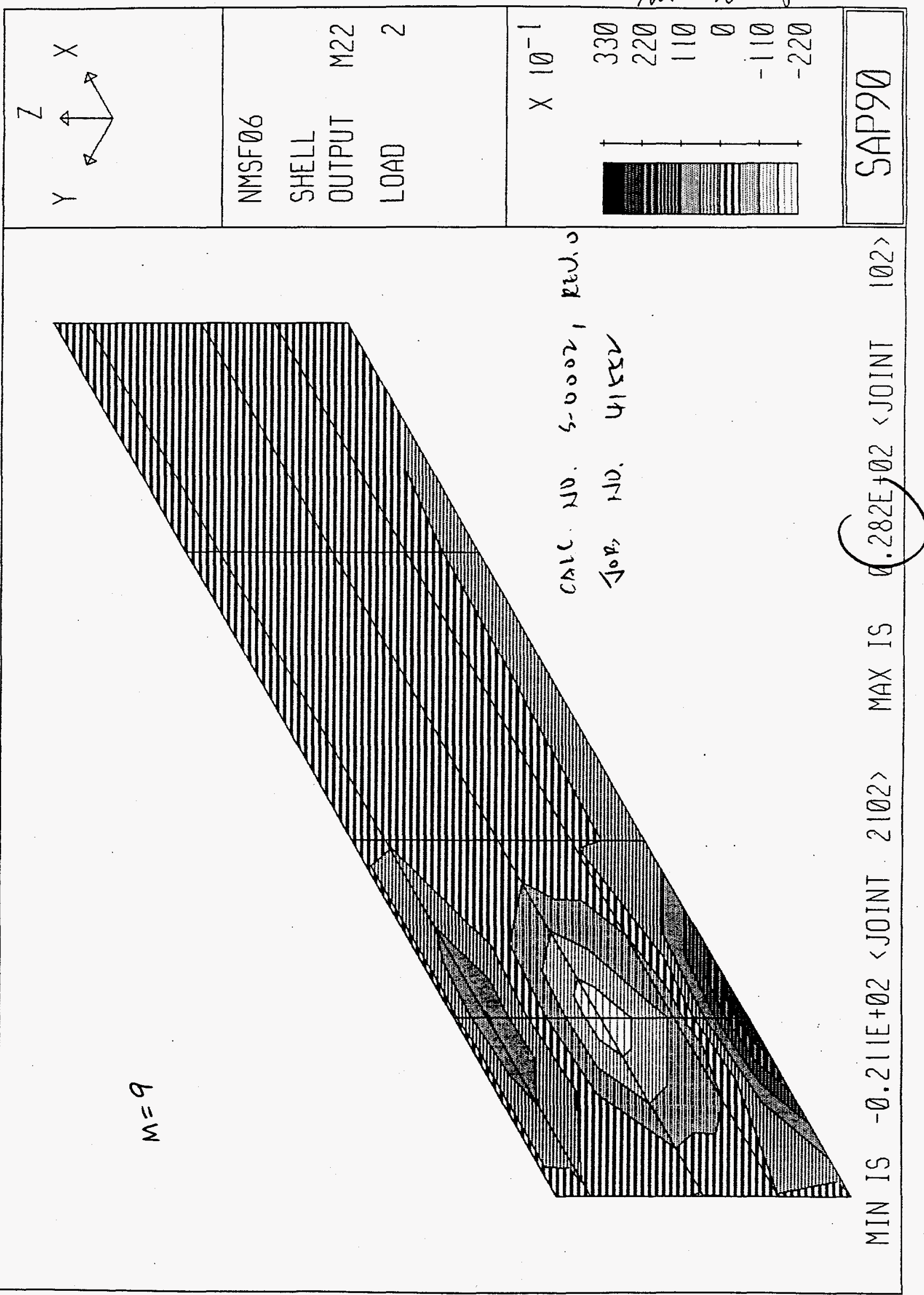




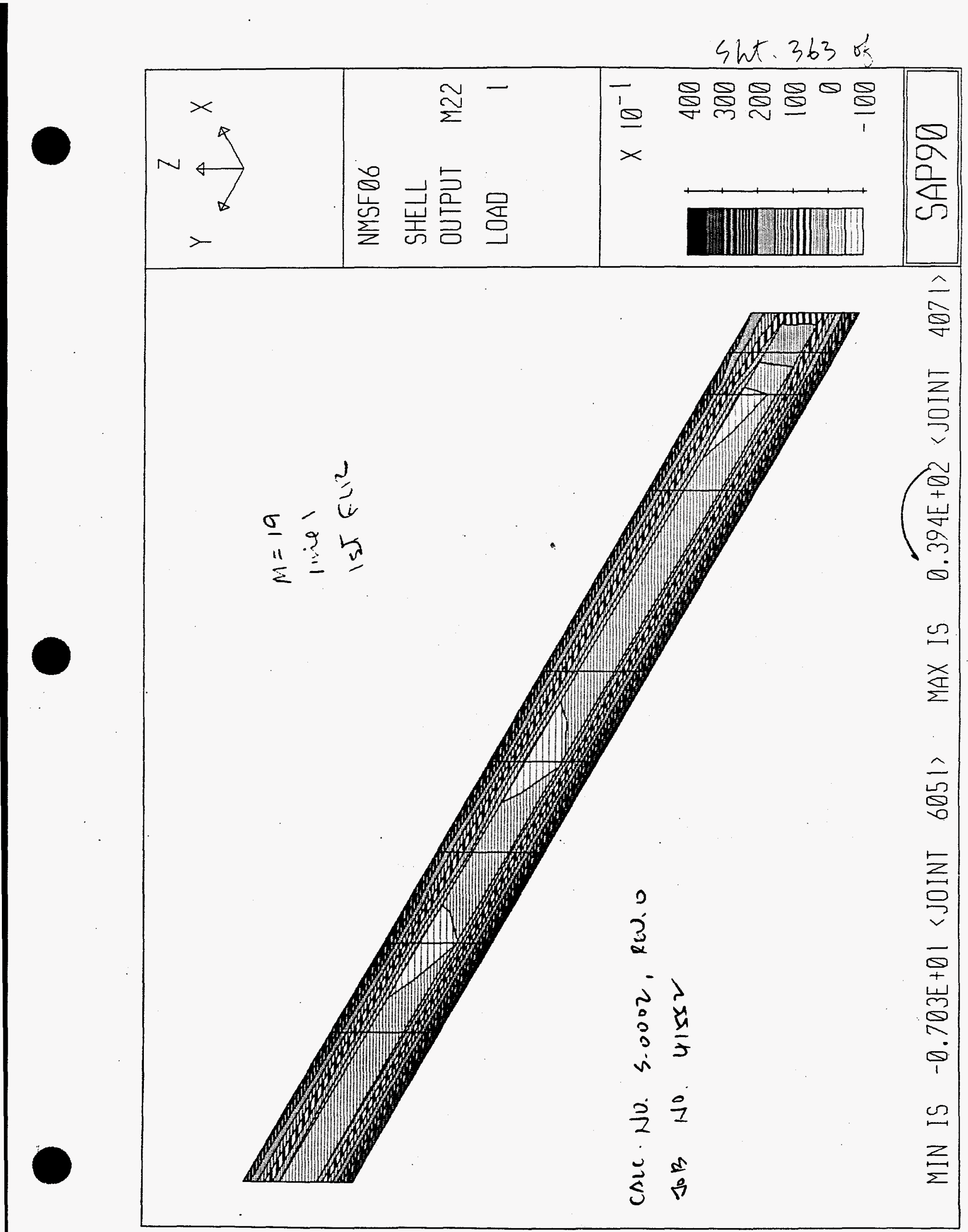




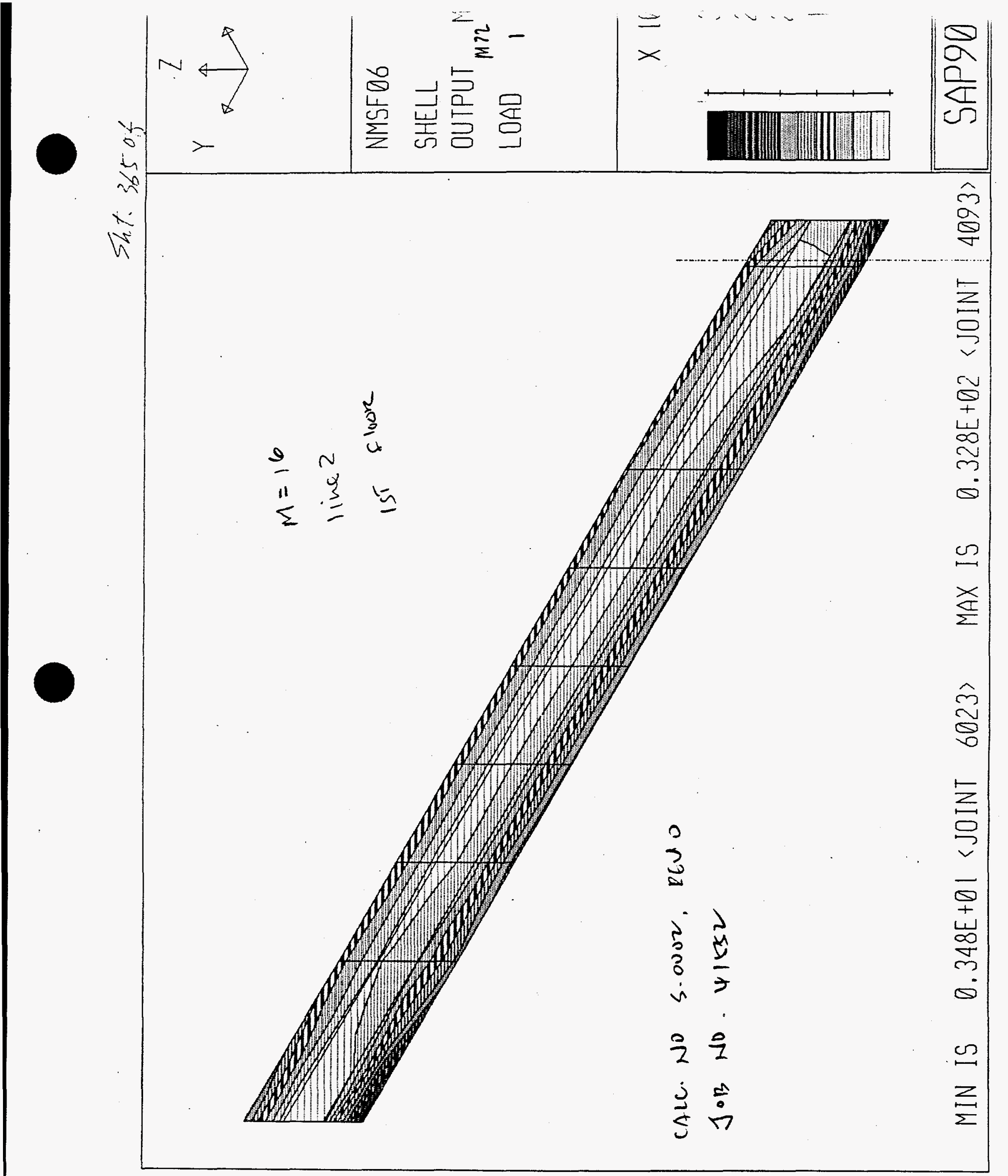




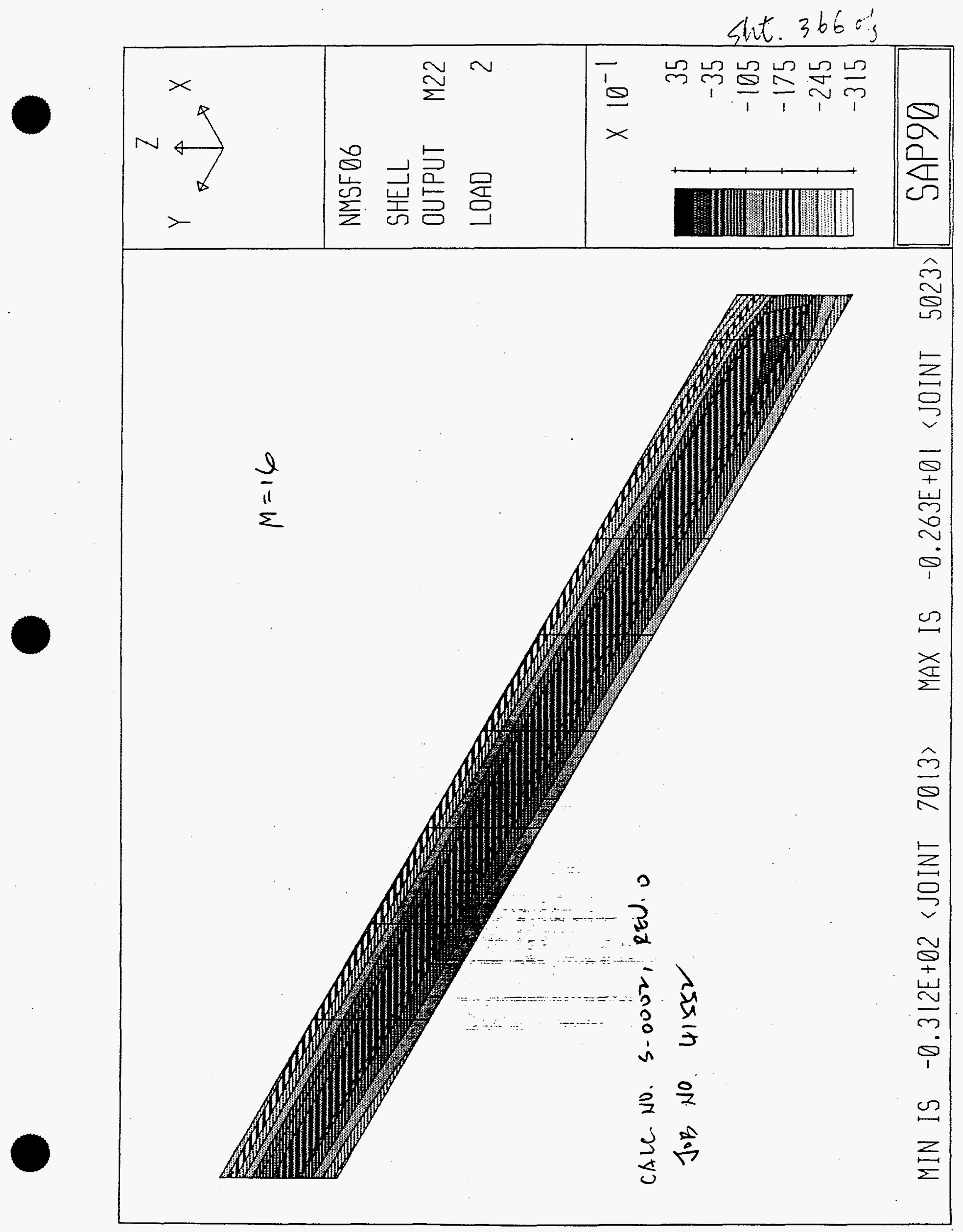


Sht. 367 of

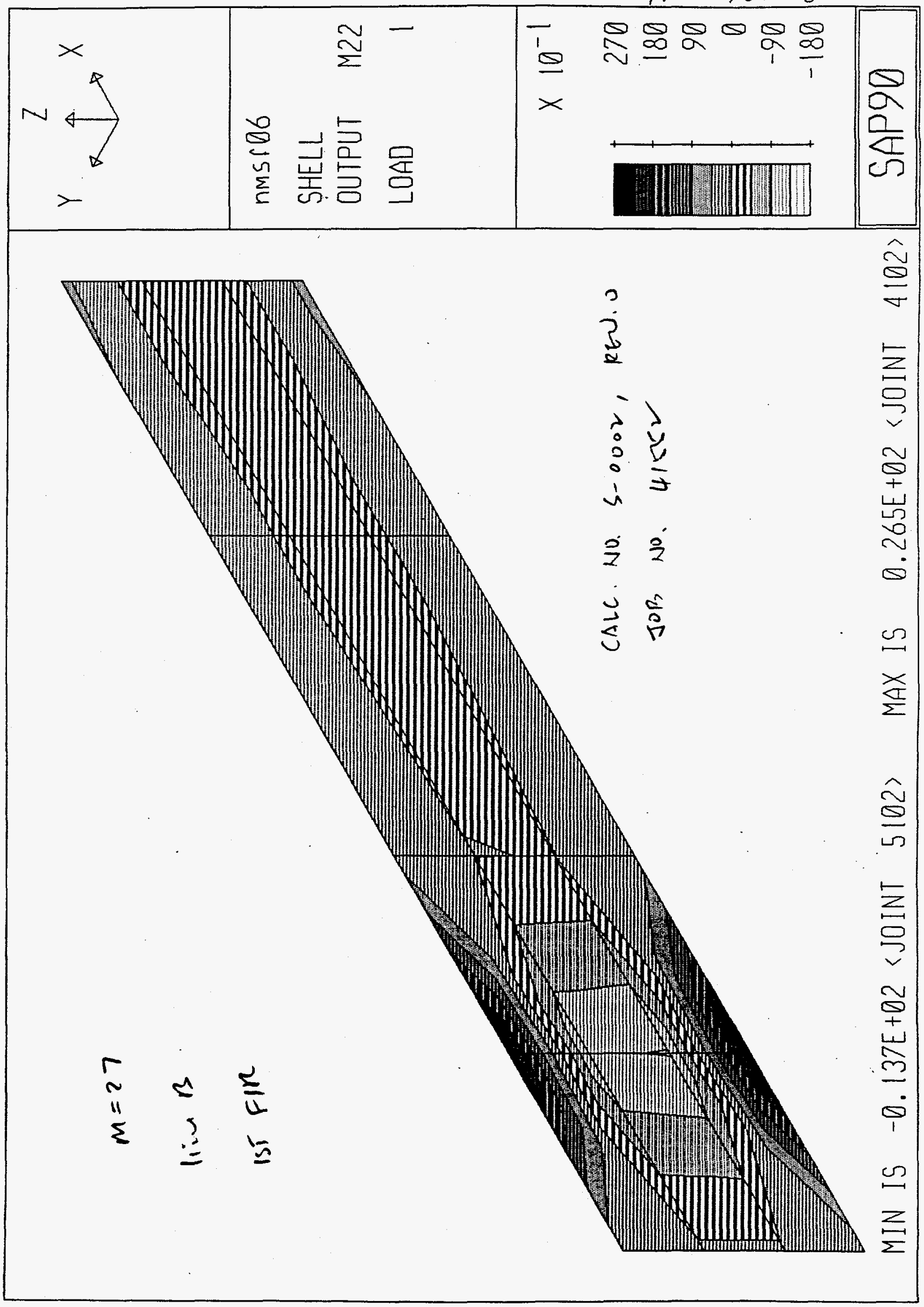




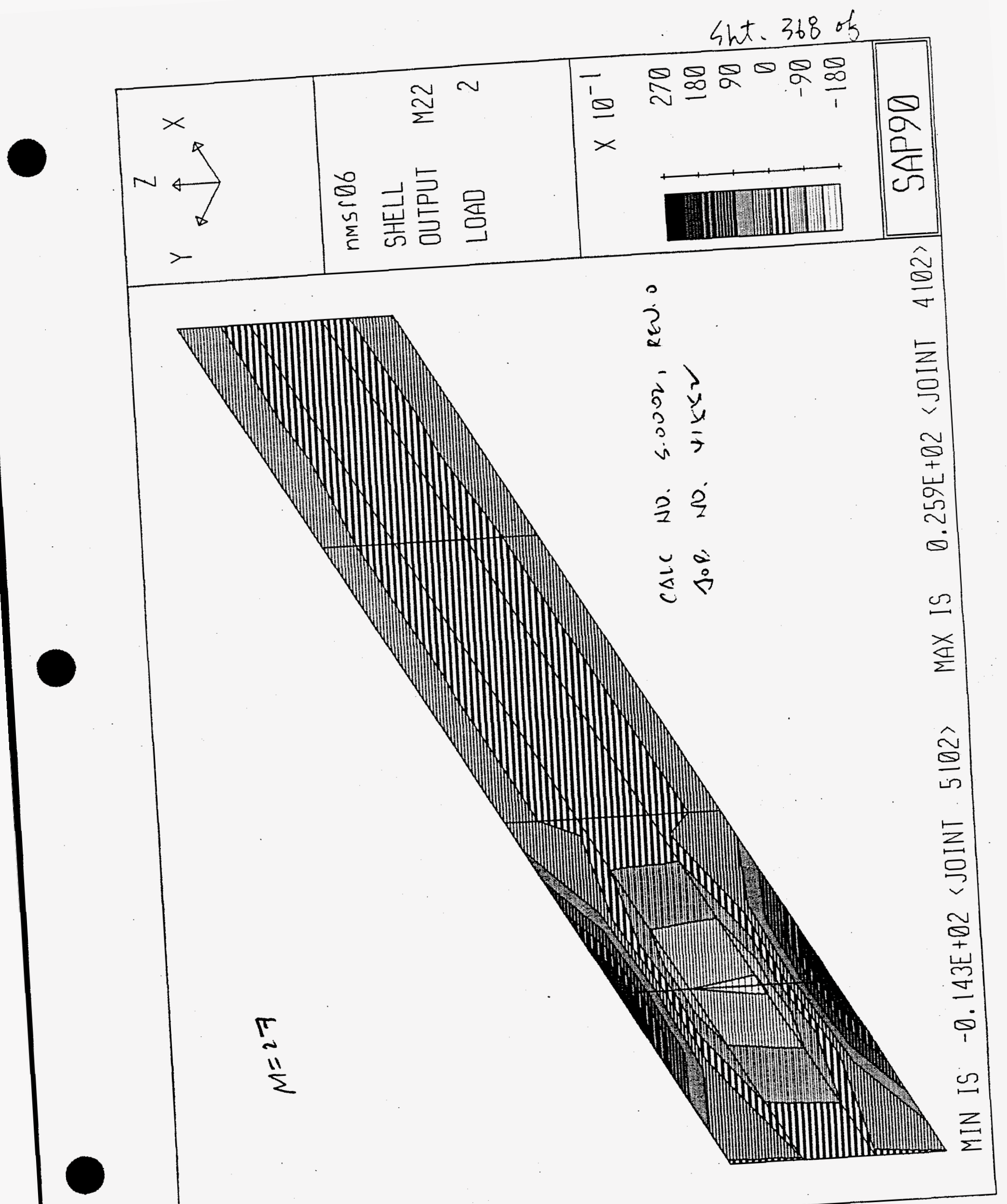


Sht. 369 of

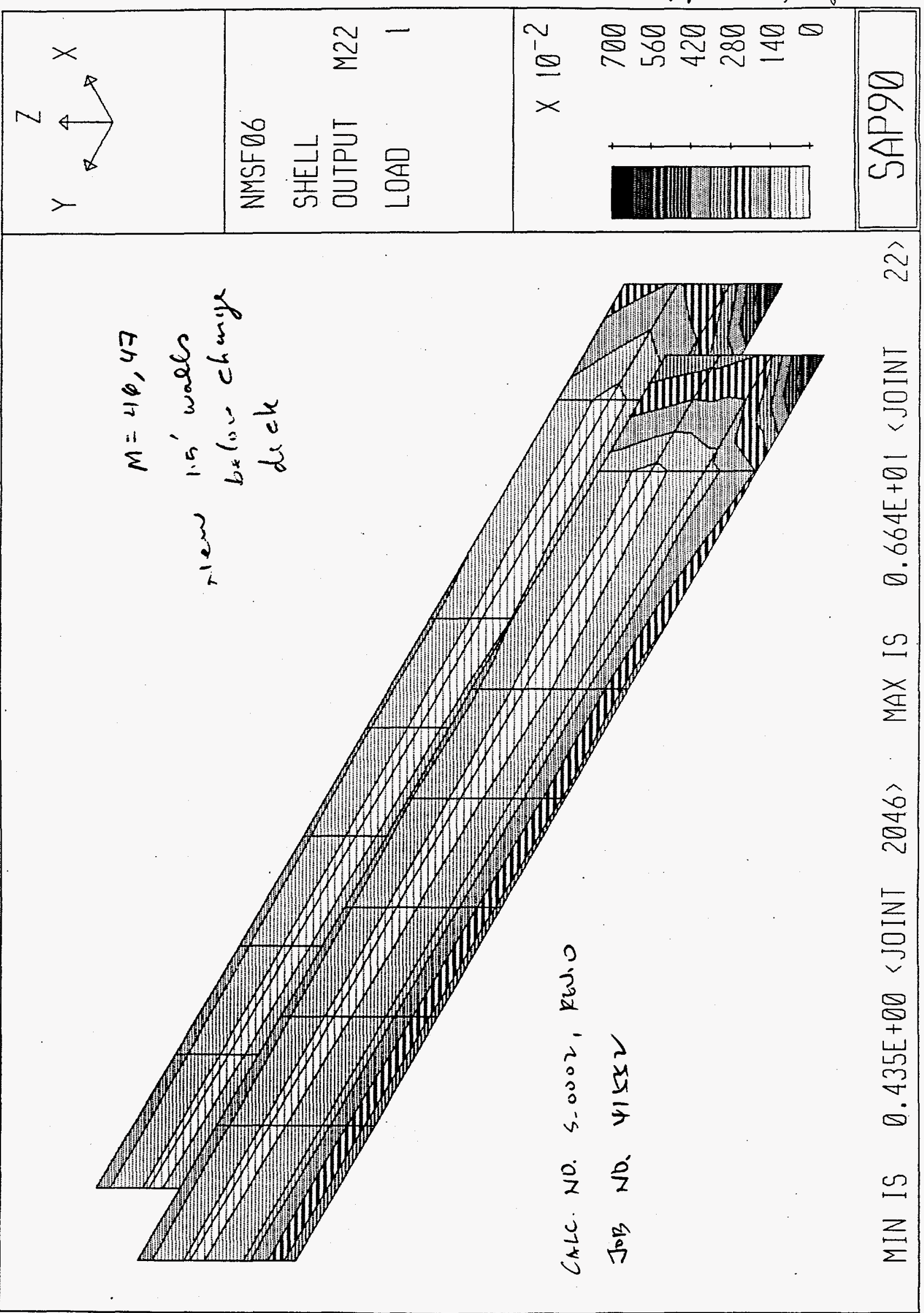


Sint. $370 \mathrm{tt}$

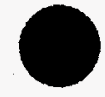

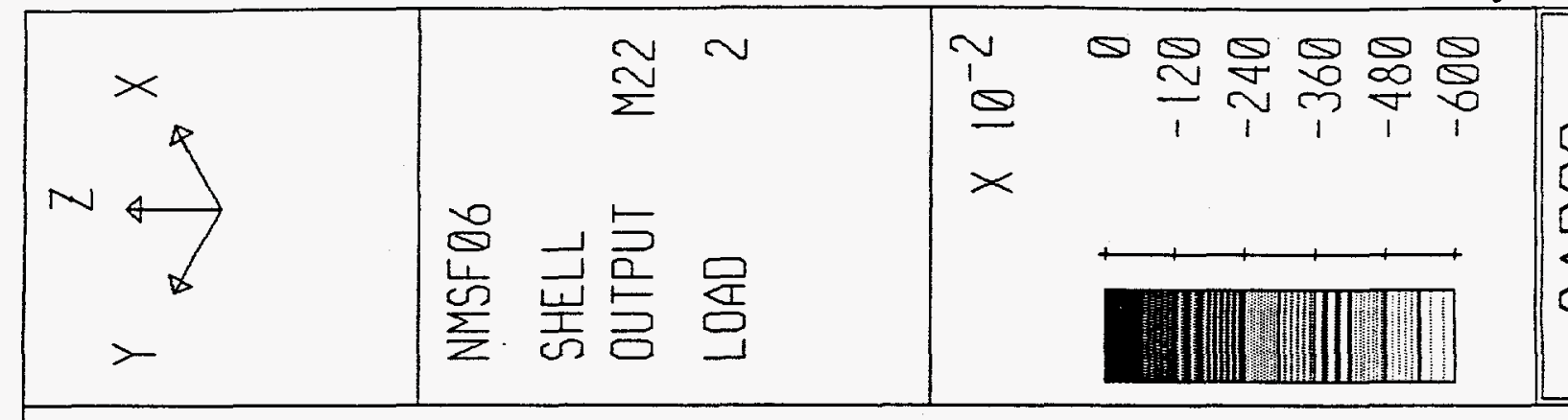

$\frac{D_{0}^{2}}{20}$

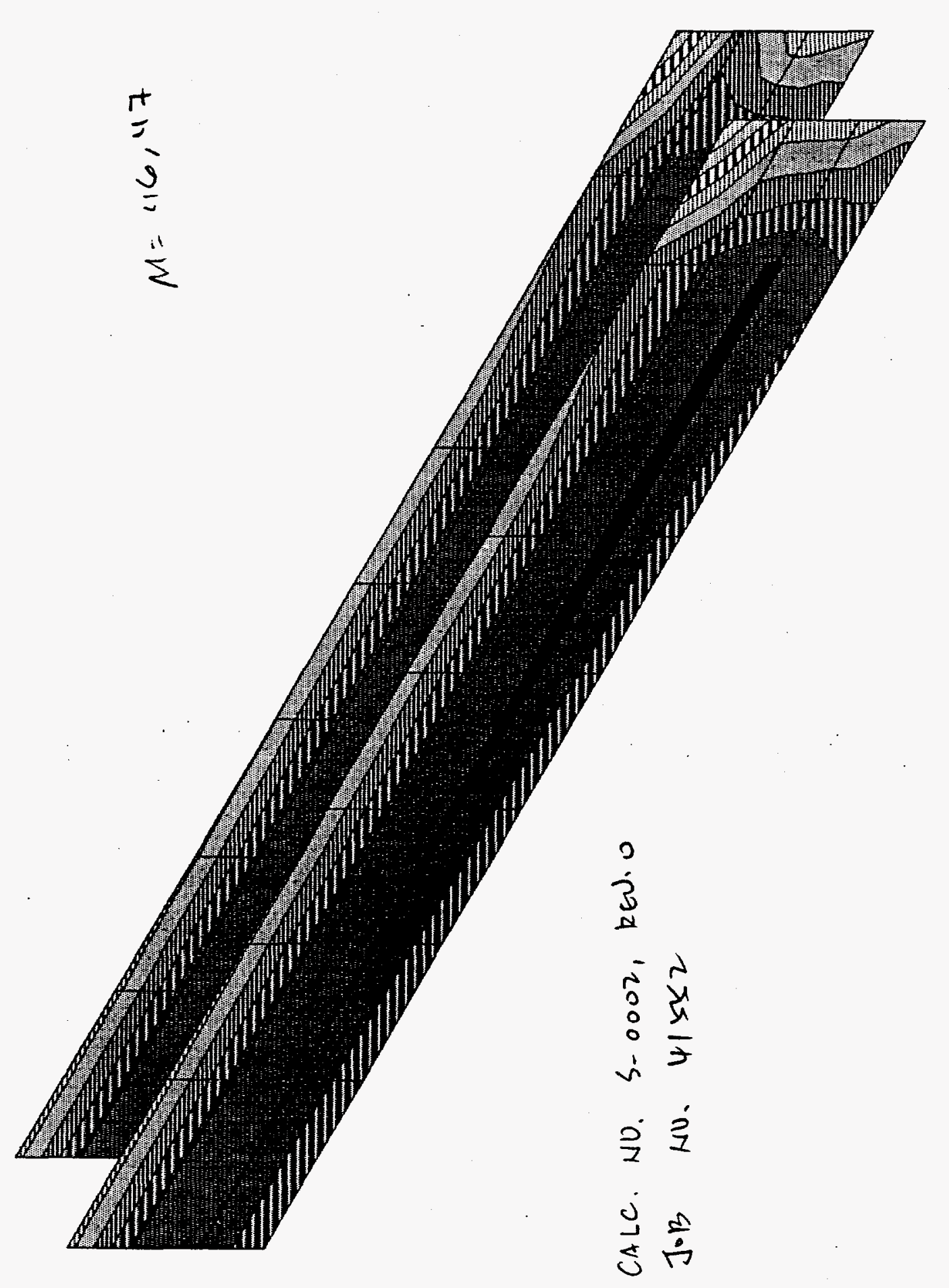

$\widehat{\vartheta}$

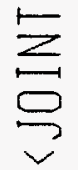

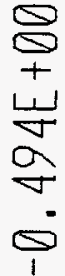




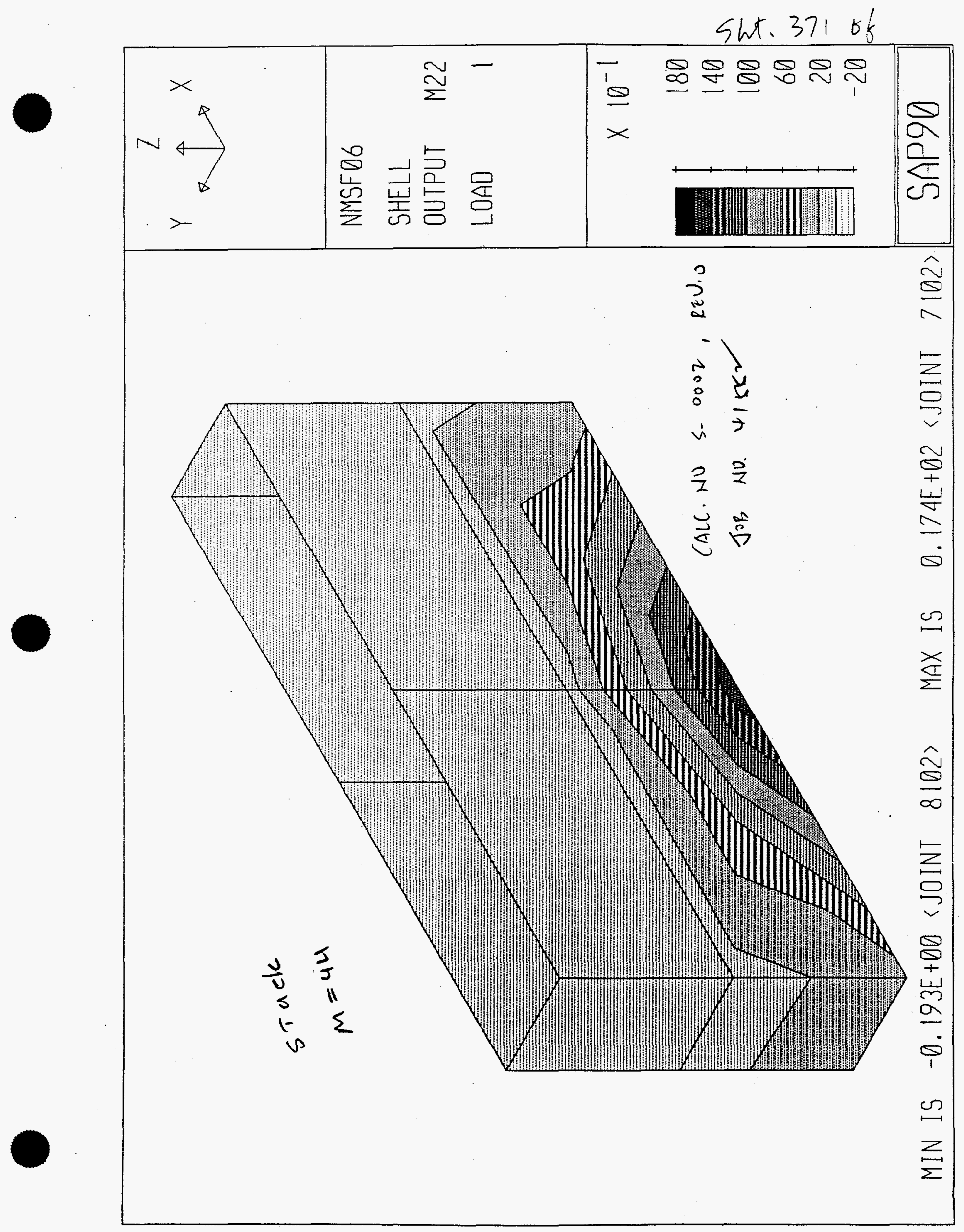


Sht. 312 of

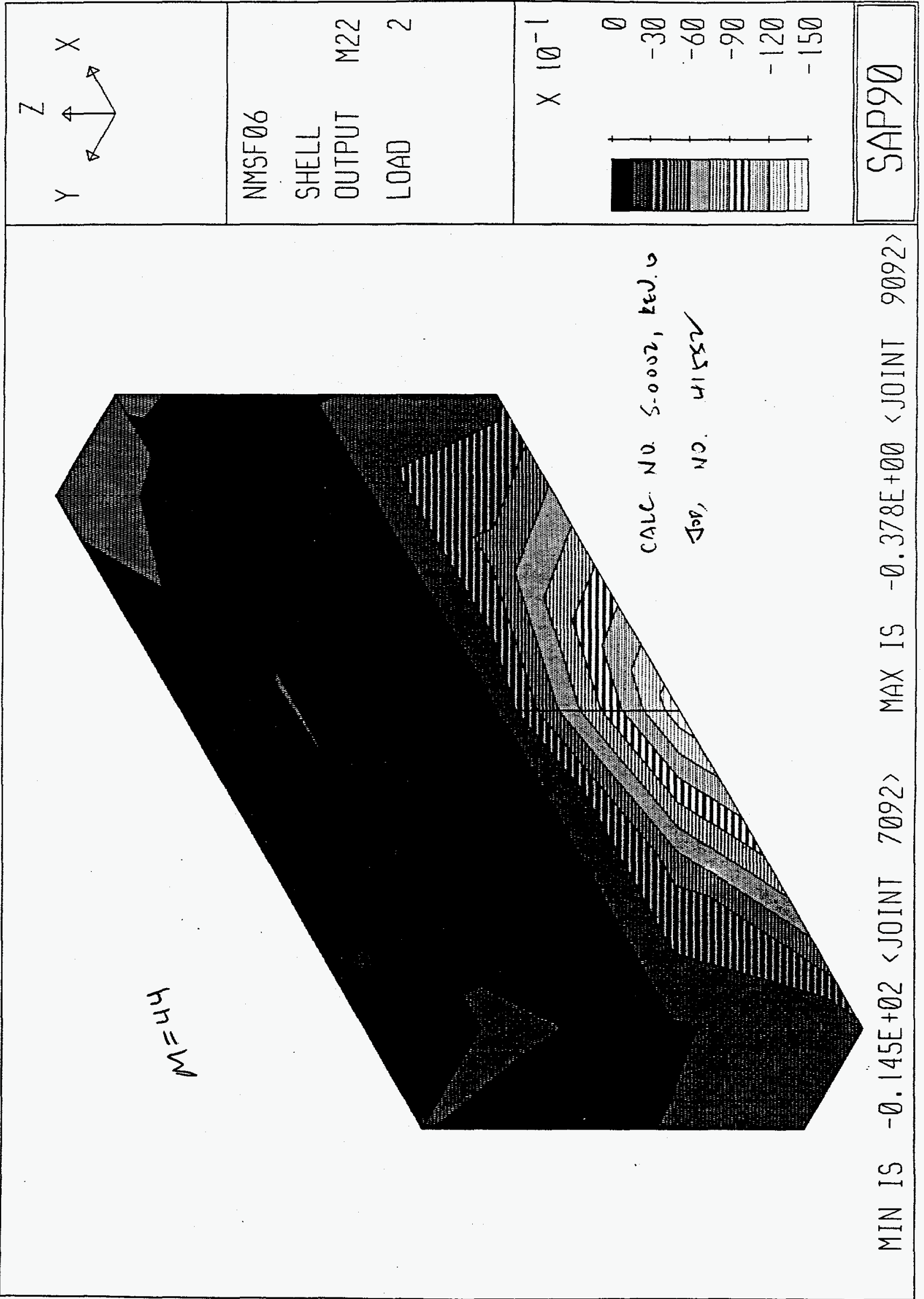




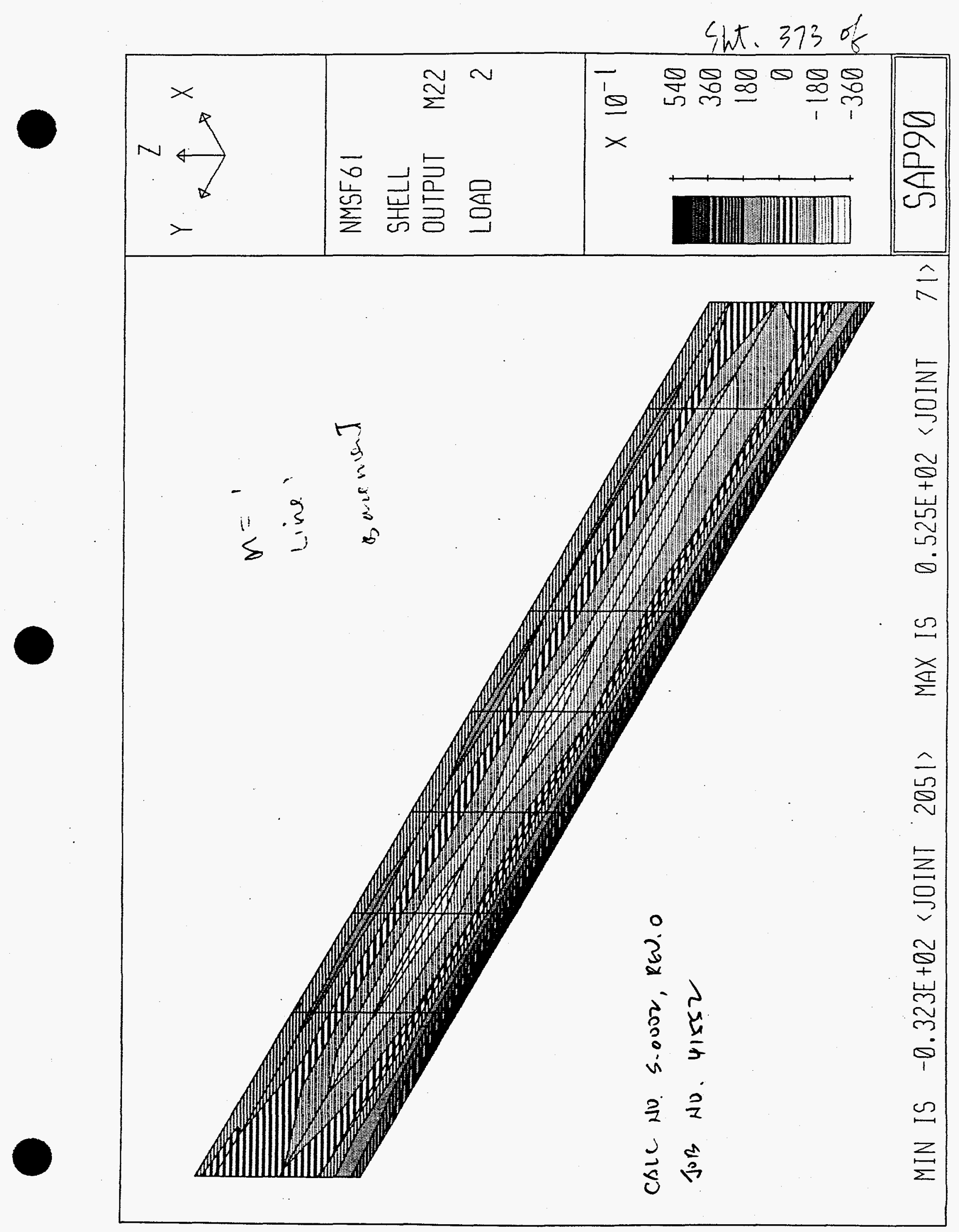




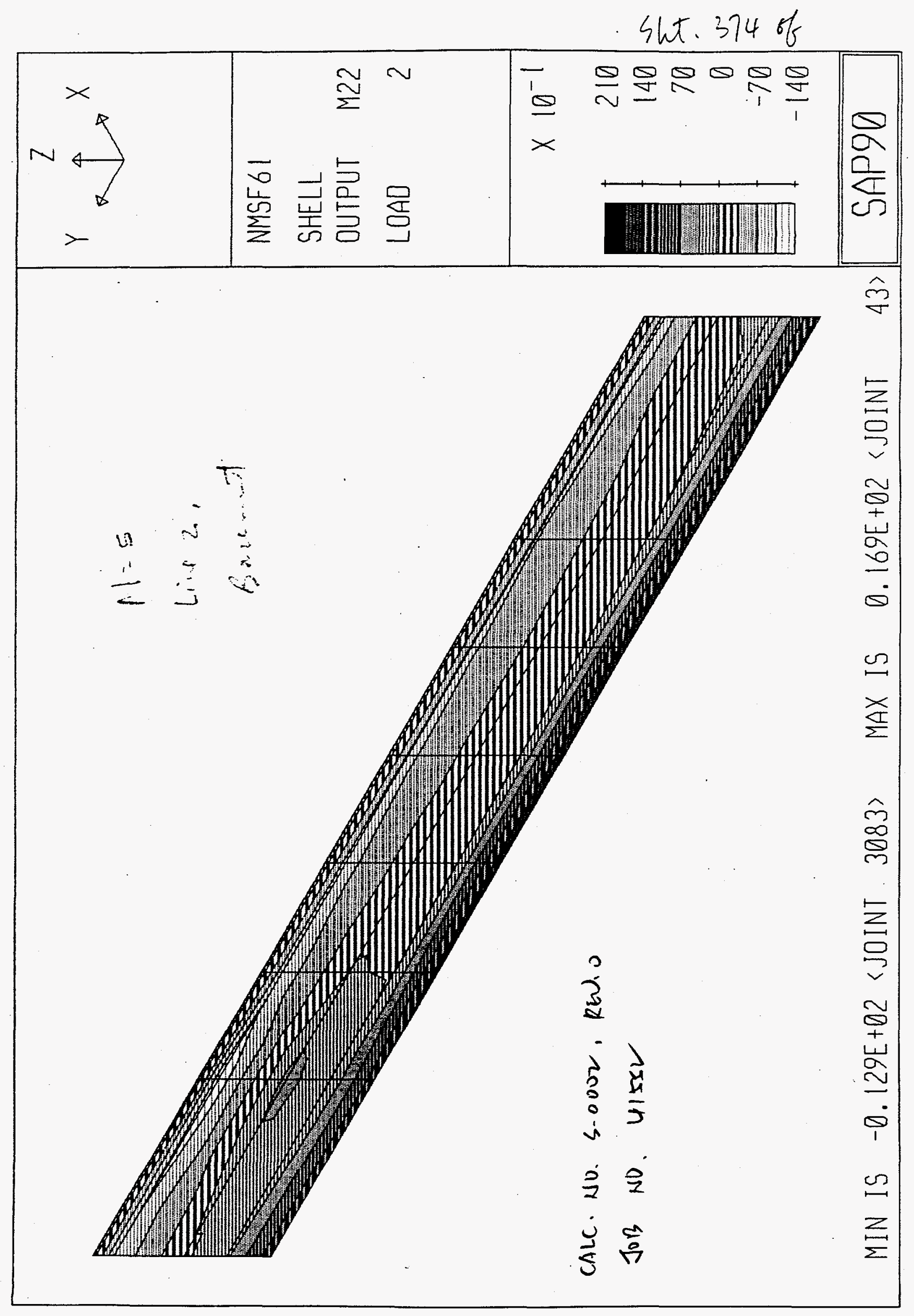




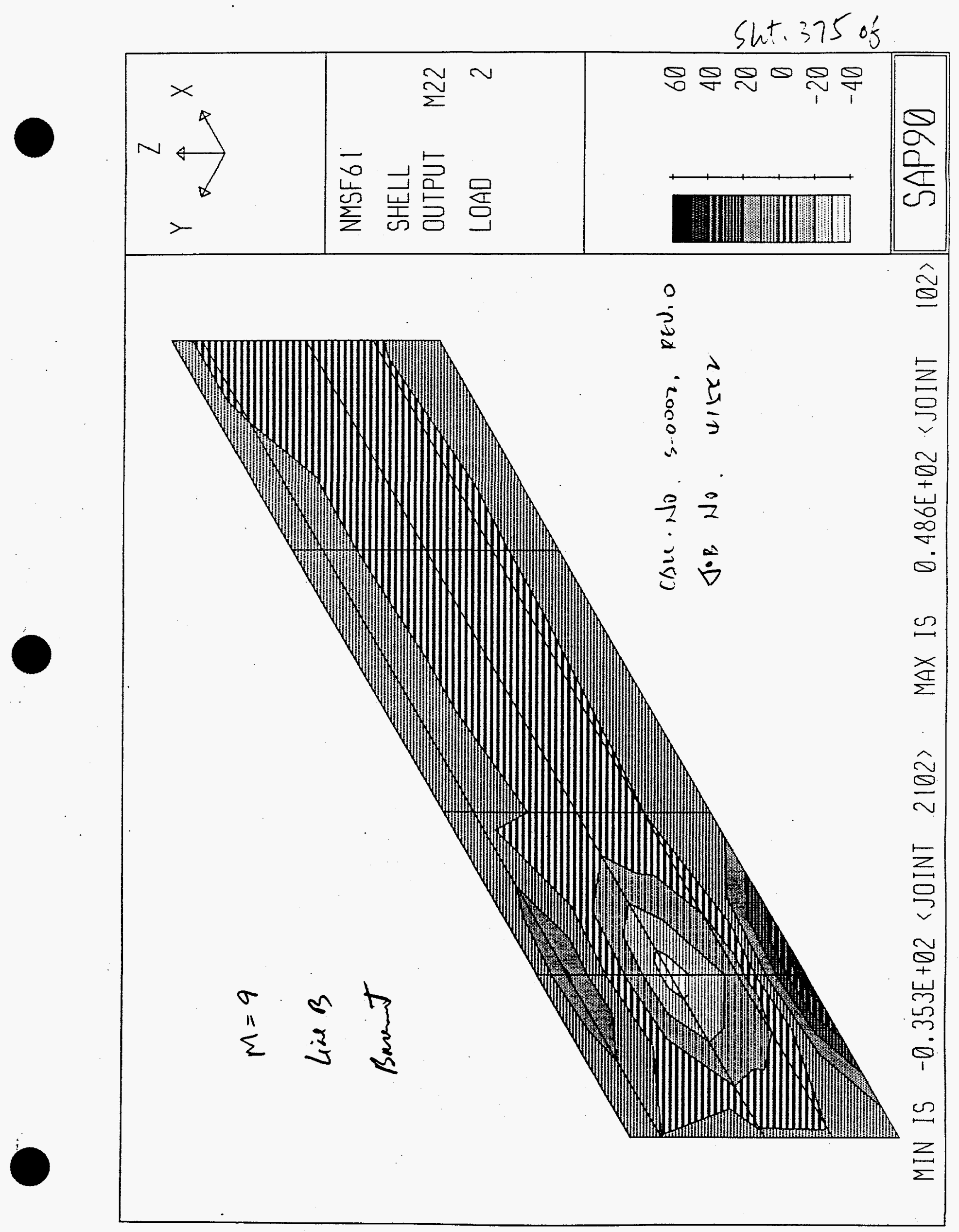


Sht: 376 of

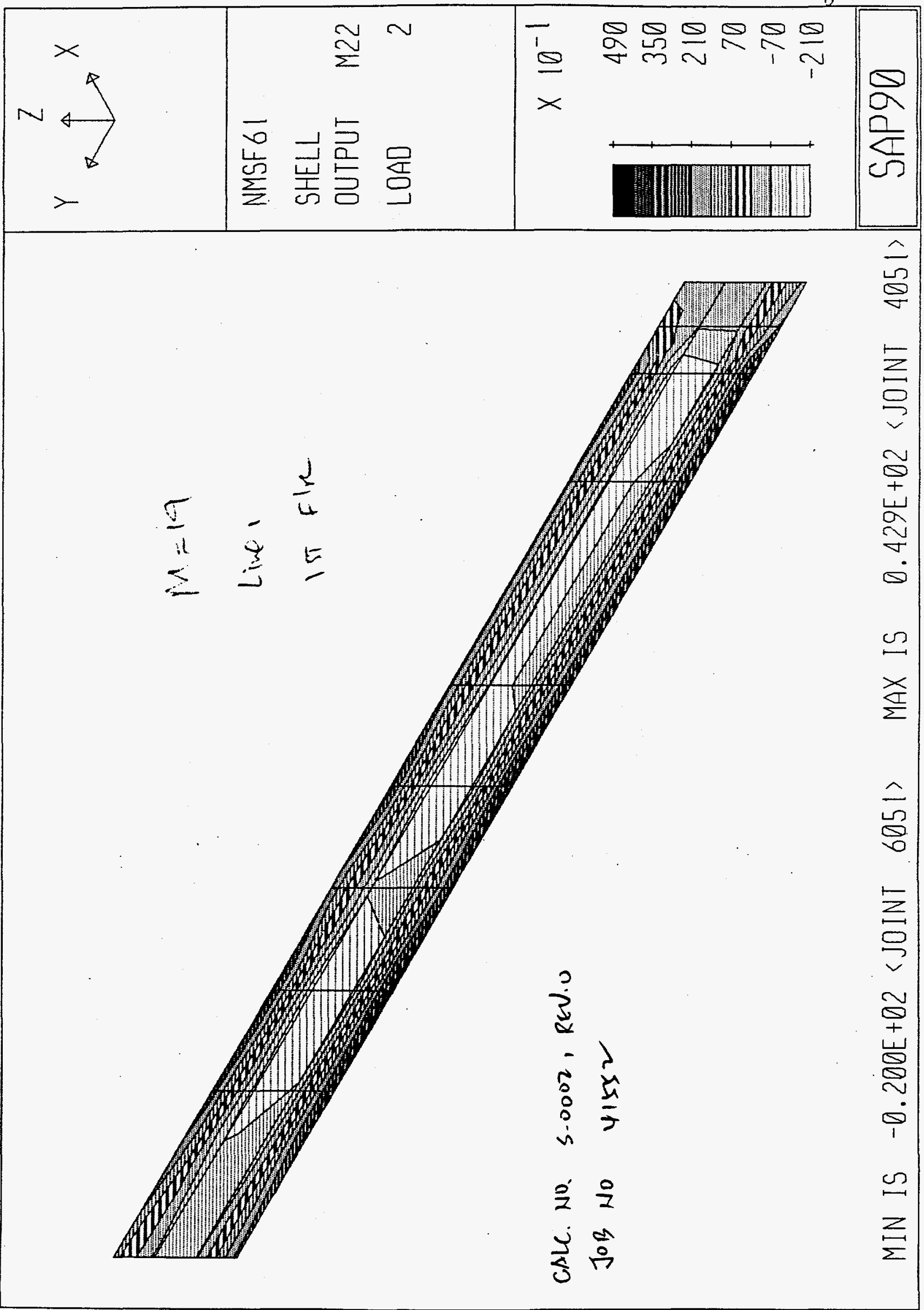


Sint. 377 of

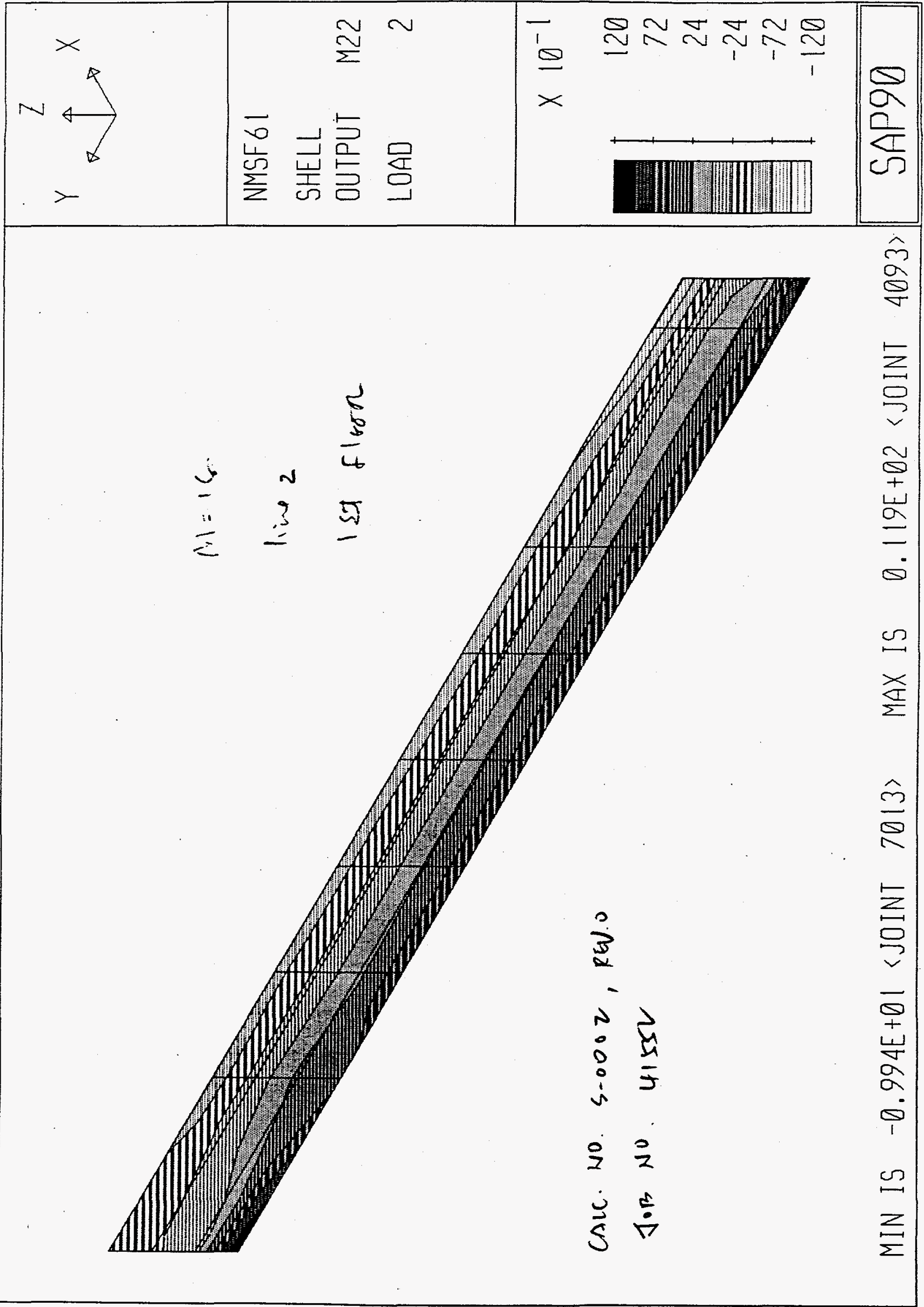




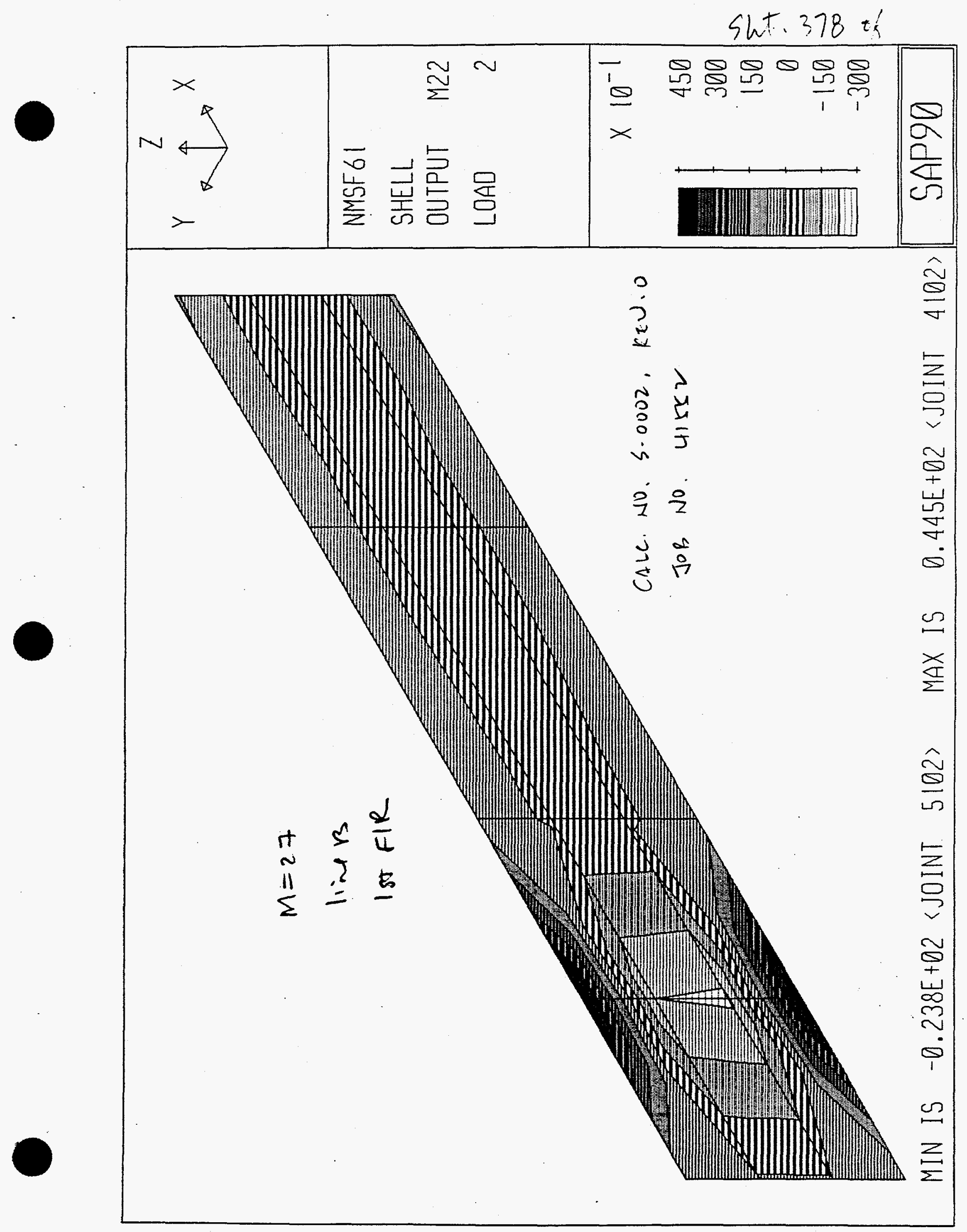




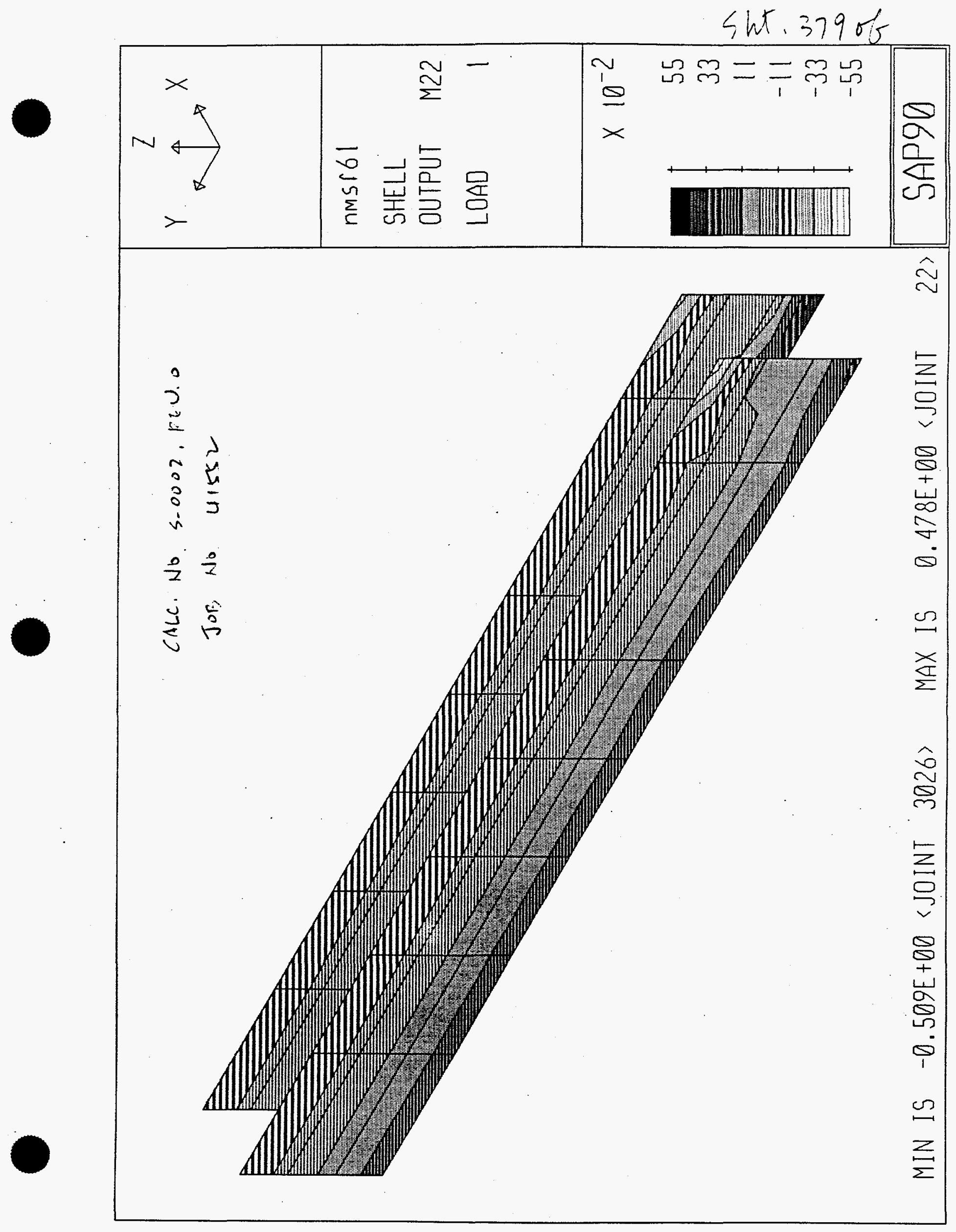




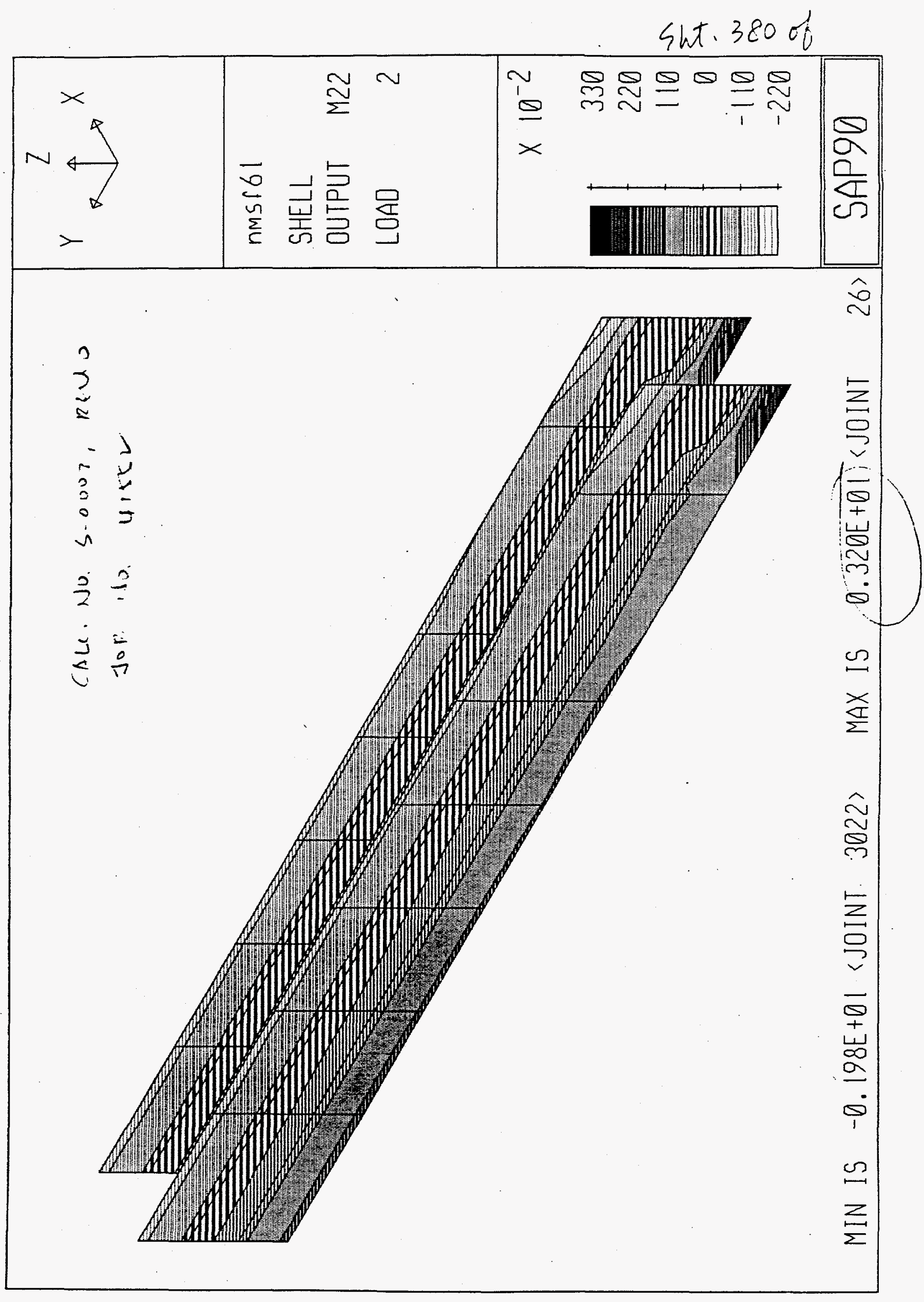




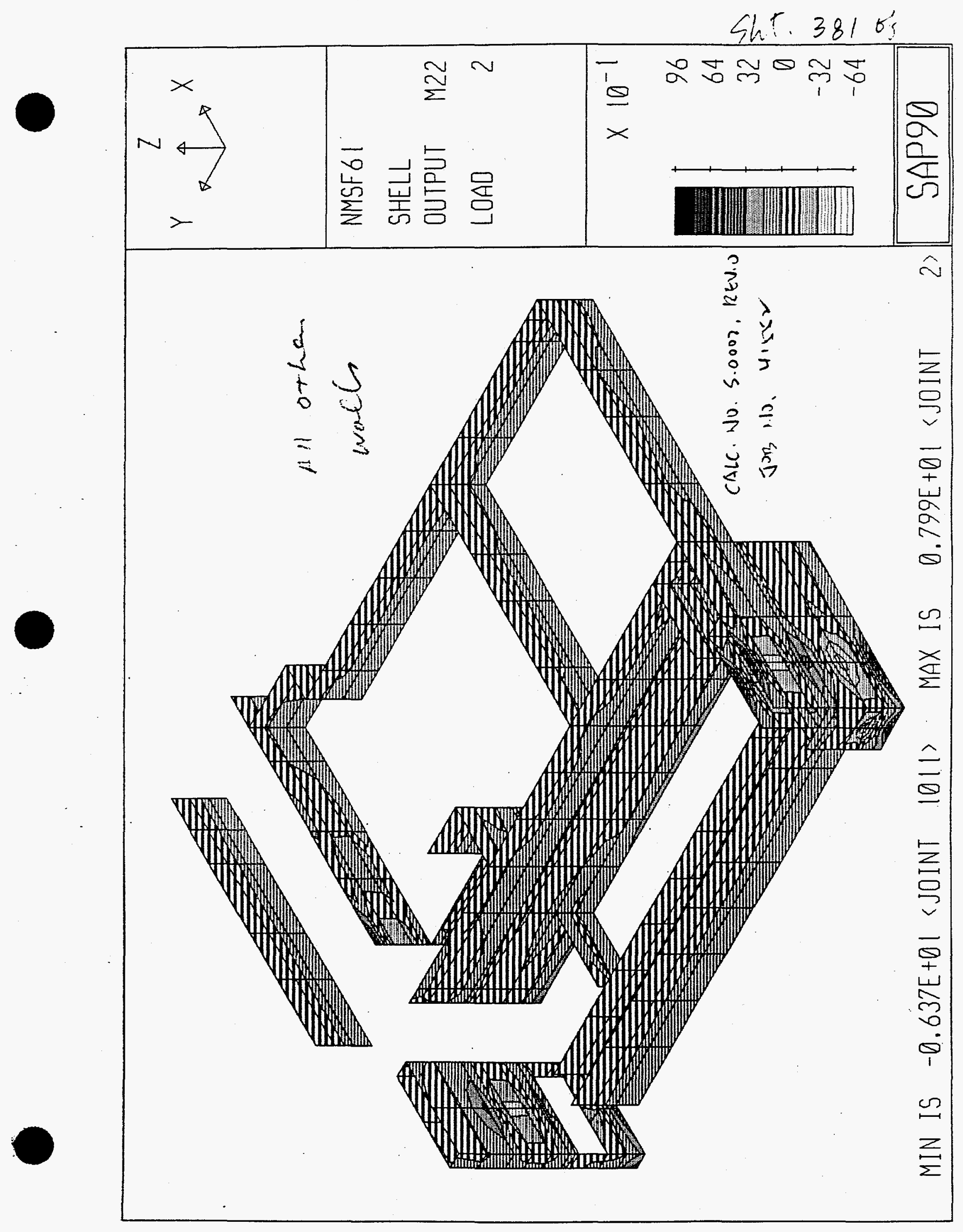




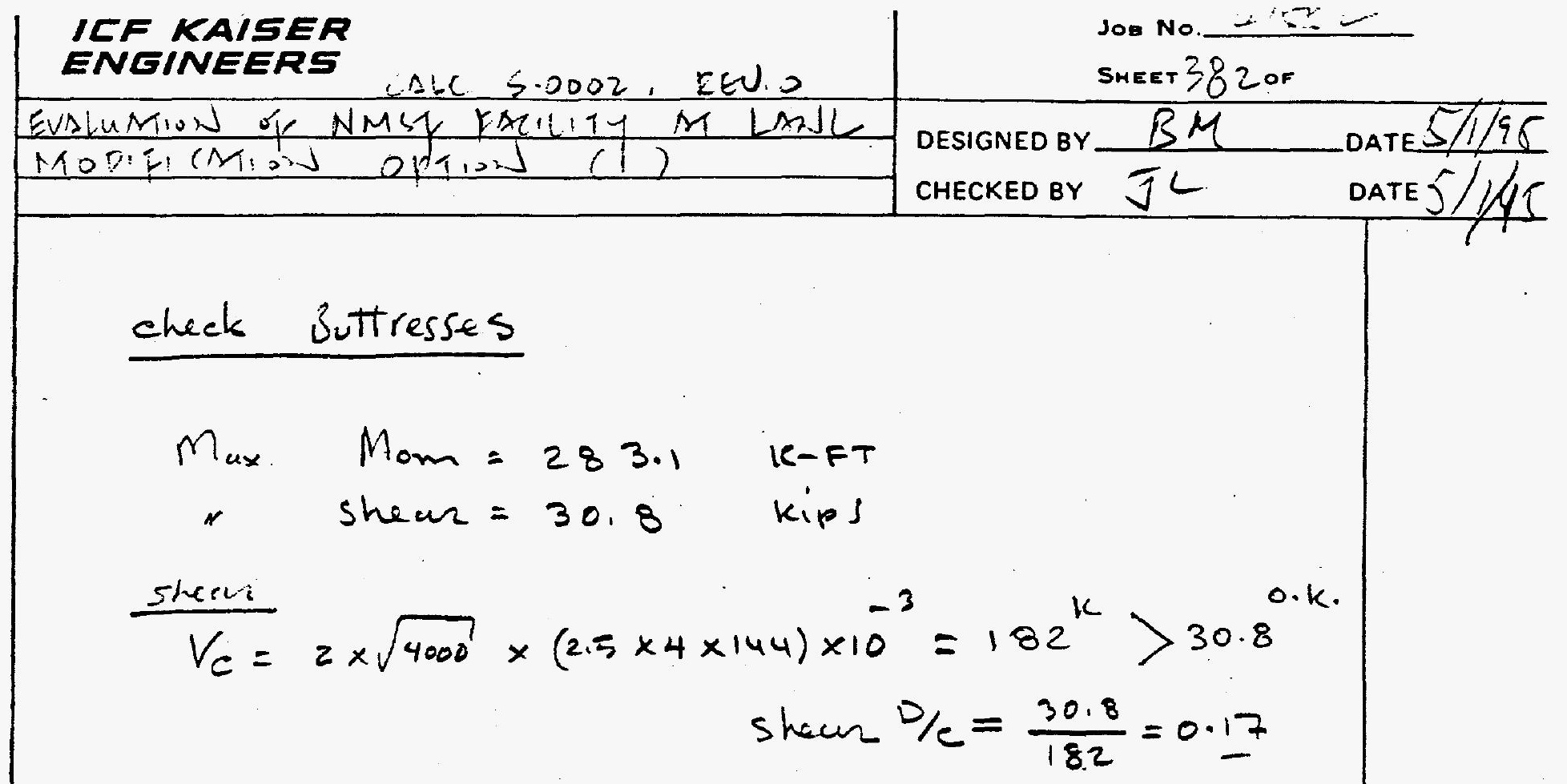

Eencling

Use minimun steal

$$
\begin{aligned}
& \rho=\frac{f_{y}}{200}=\frac{60}{200}=-3 \% \\
& A_{3}=\frac{.3}{100} \times 30 \times 48=4.32 \mathrm{in}^{2} \\
& a=\frac{4.32 \times 60}{.85 \times 4 \times 12}=6.35 \mathrm{in}, d=48-4=44^{\prime \prime} \\
& M_{u}=.9 \times 4.32 \times 60\left(44-\frac{6.35}{2}\right) \\
& =9524 \mathrm{k}-\therefore \\
& =794 \text { K.FT } \geq 283.1 \text { K-FT. O.K. }
\end{aligned}
$$




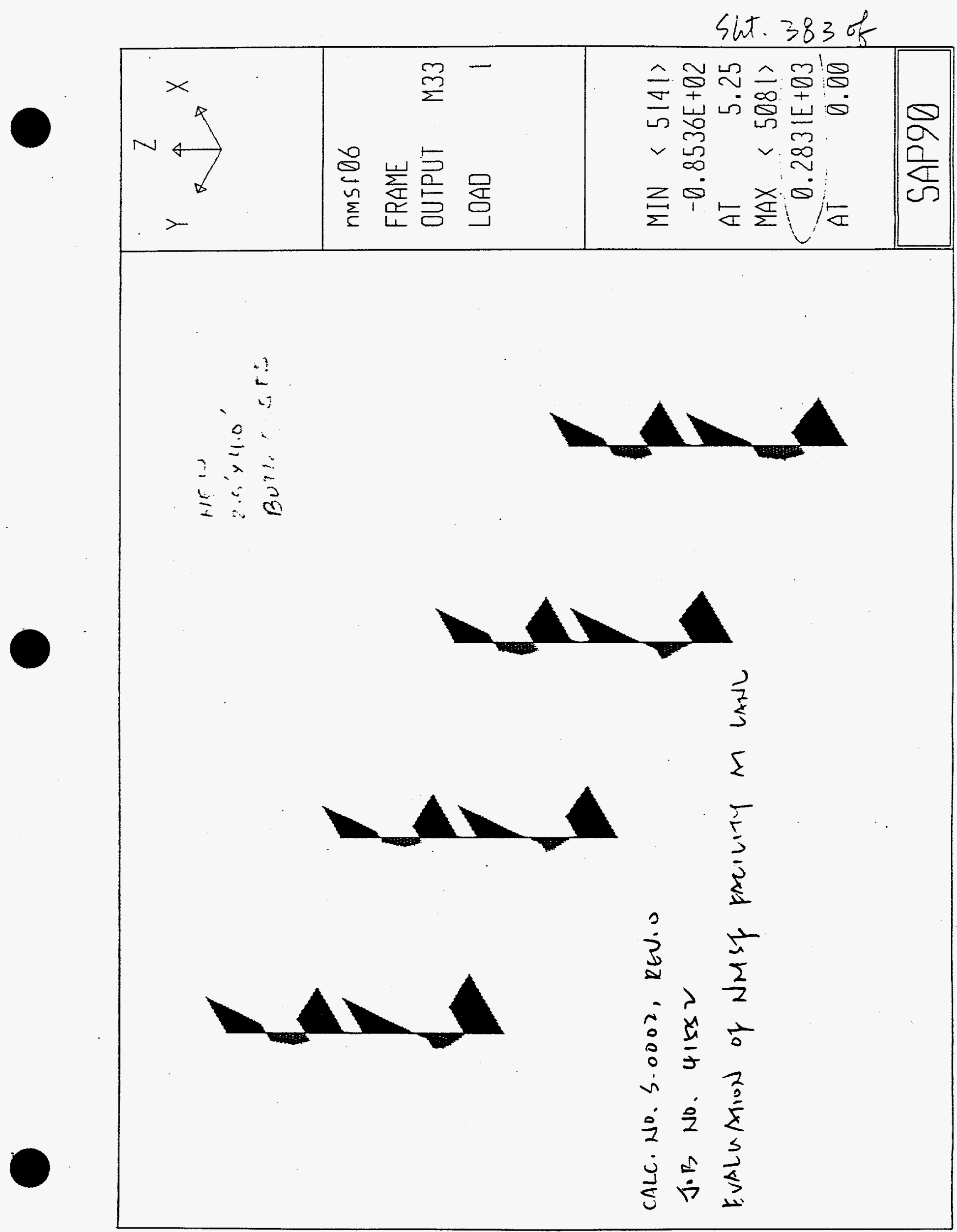


Sint. $384 \mathrm{~s}_{5}^{\prime}$

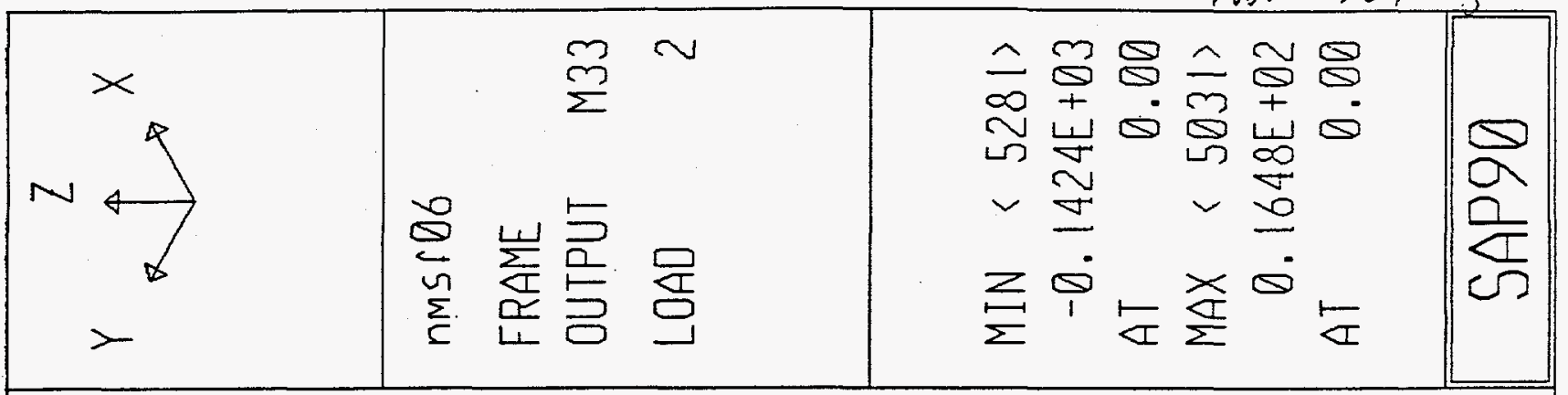
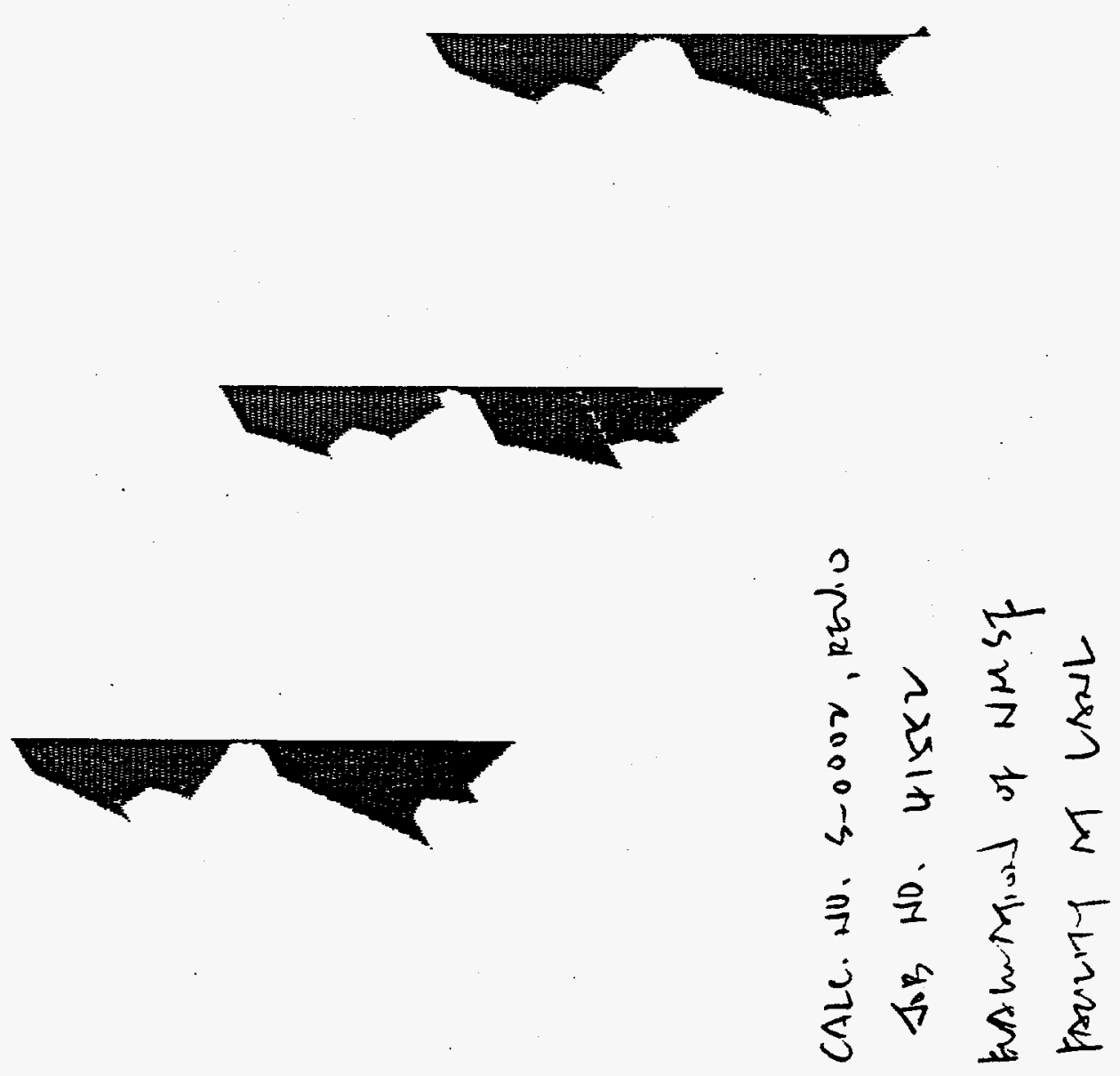


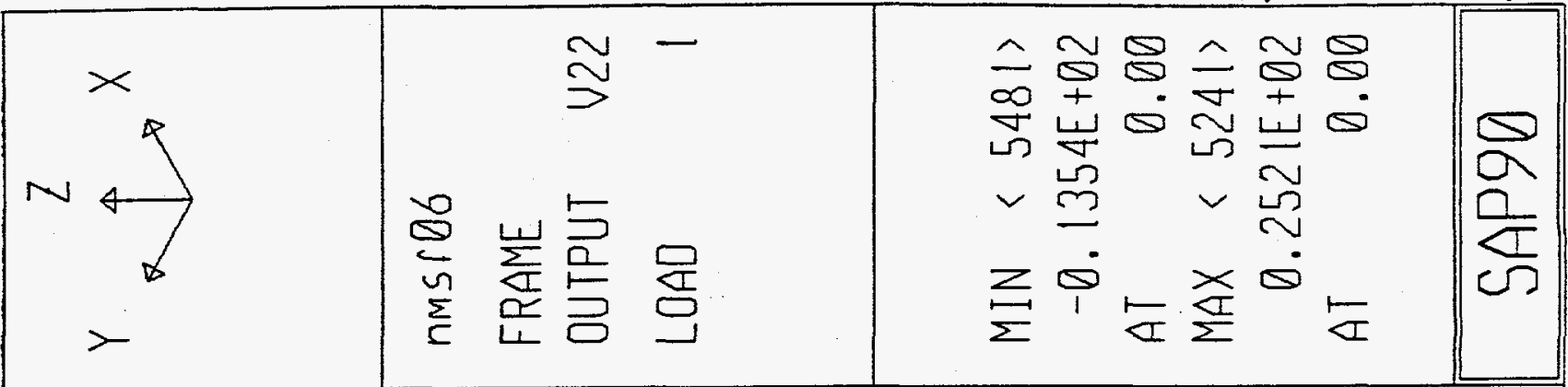
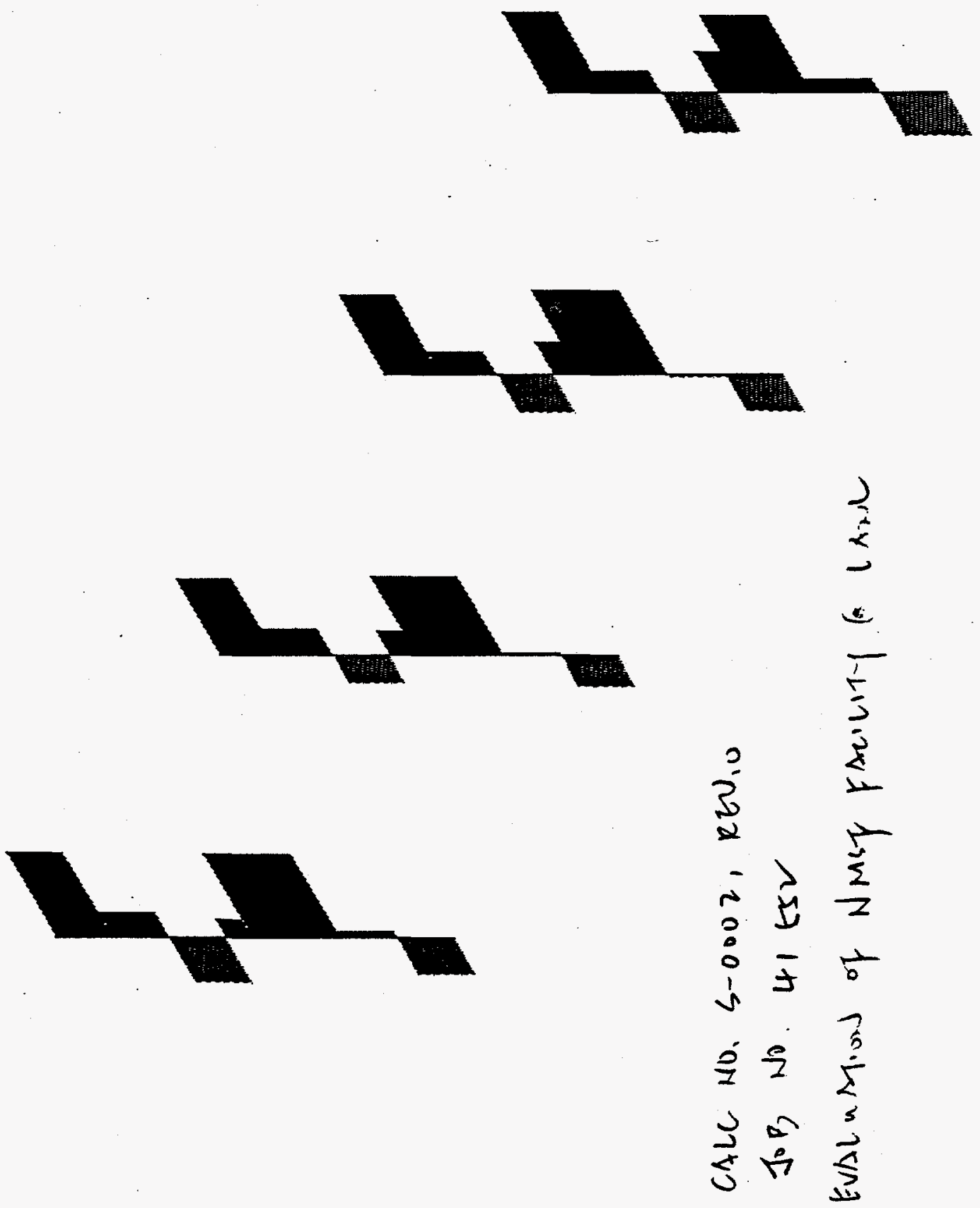


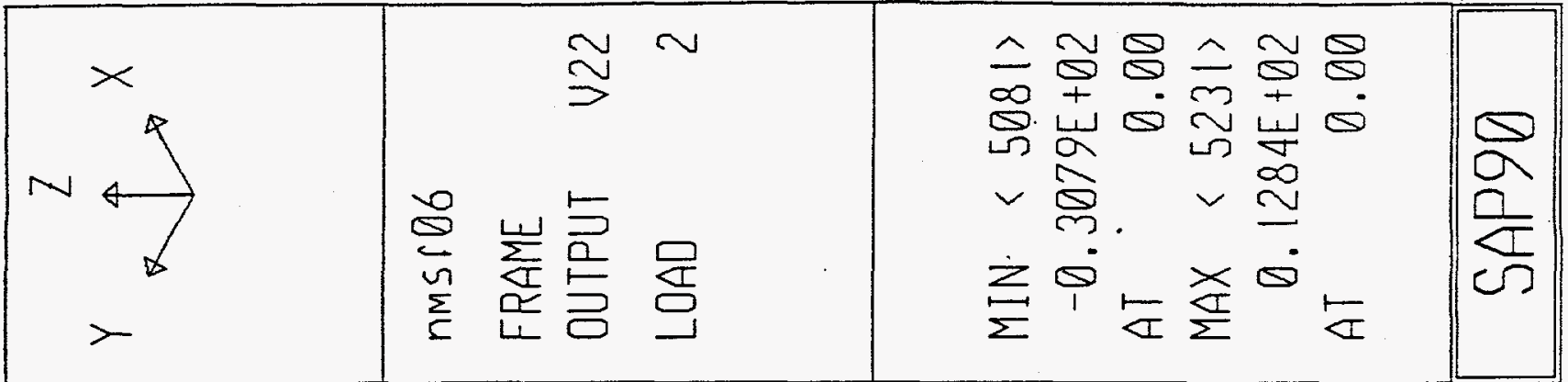

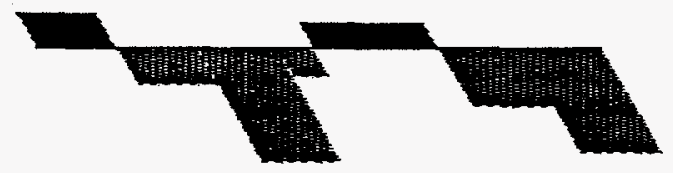

$\frac{3}{3}$
5
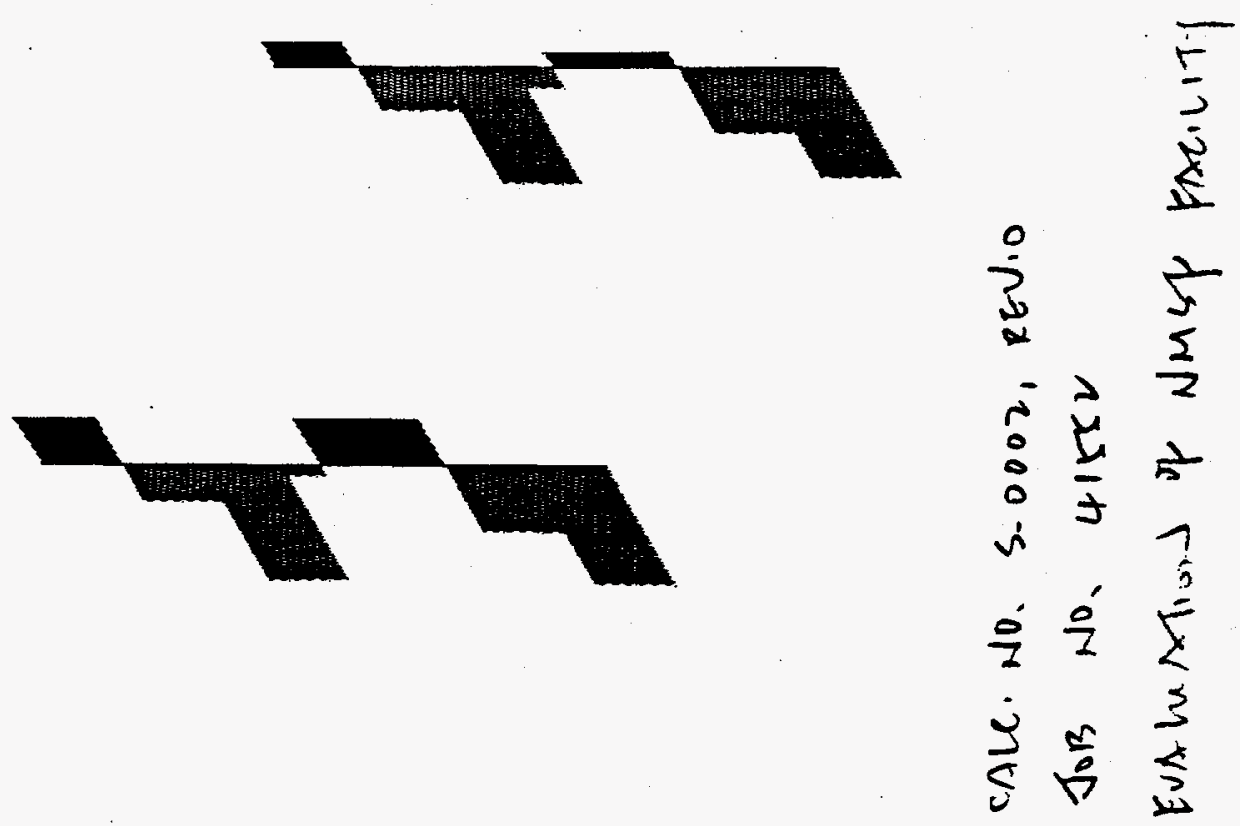


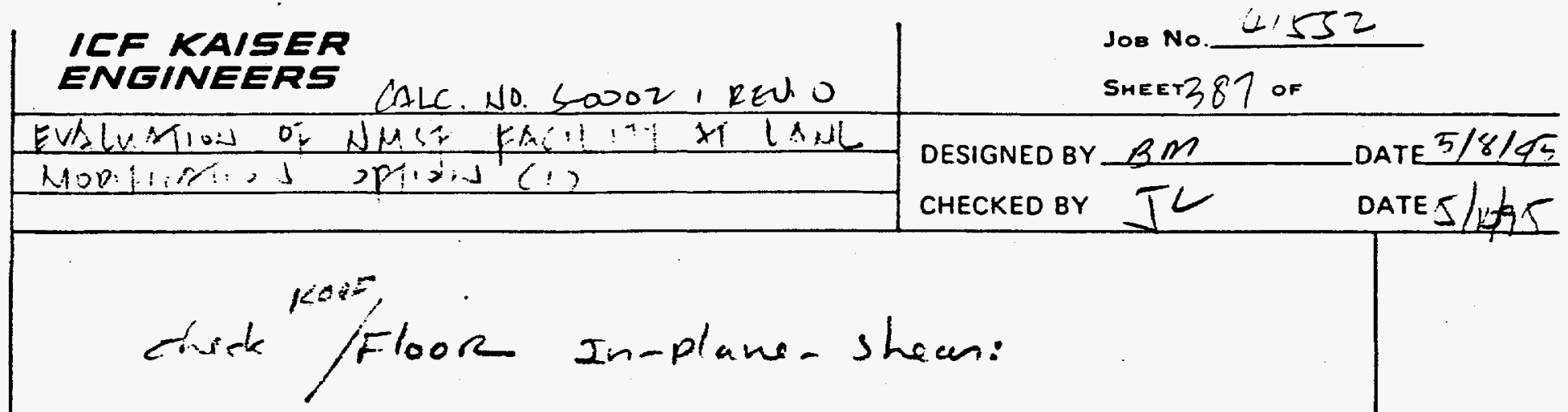

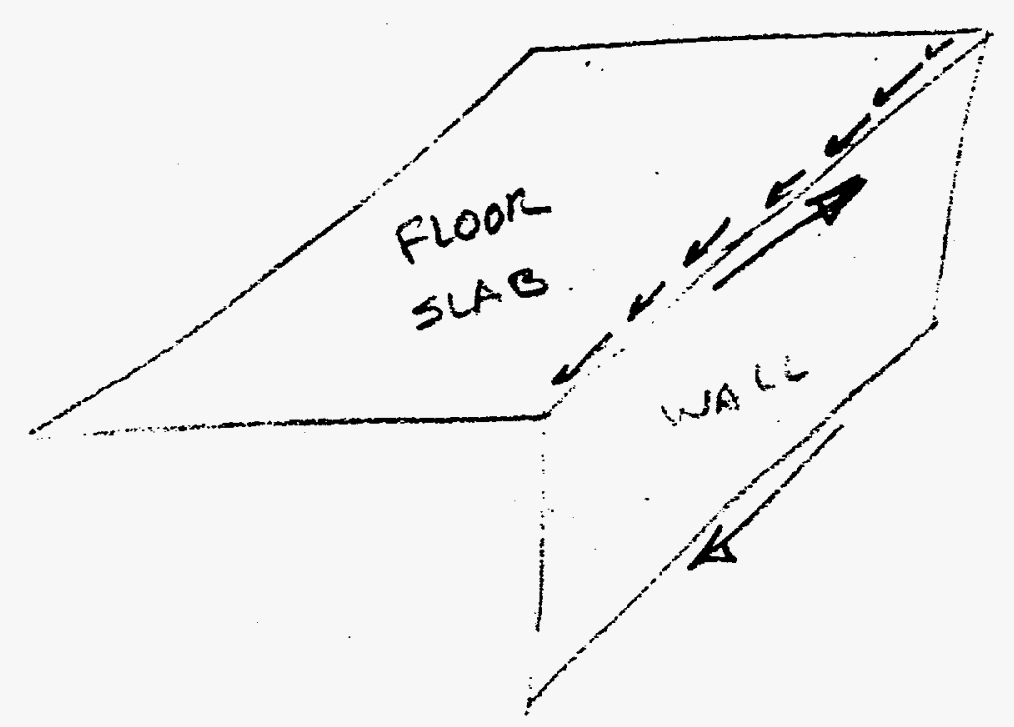

- check slab strength vs amount of steen That is Tranfered from shear wall.

The Concrete slab of th Rob. Dst flour, and Mezsanion Floor are al 1 Font dap.

shear capacity of $1^{\prime}$ slab (excluding steal) can Conservatively estimated as

$$
\begin{aligned}
V_{c} & =2 \sqrt{f_{c}^{\prime}} \times b d \\
& =2 \sqrt{4000} \times 12 \times 12 \times 10^{-3}=18.2 \mathrm{kips} / \mathrm{L}_{\mathrm{F}} .
\end{aligned}
$$

The maximum shem/unit length of the wall access on call on line $B$ at Baseman level: which is $18.1^{\mathrm{K} / \mathrm{F}}$ and in $\operatorname{len}$ than $18.2^{\mathrm{k}} / \mathrm{L}$. F. 


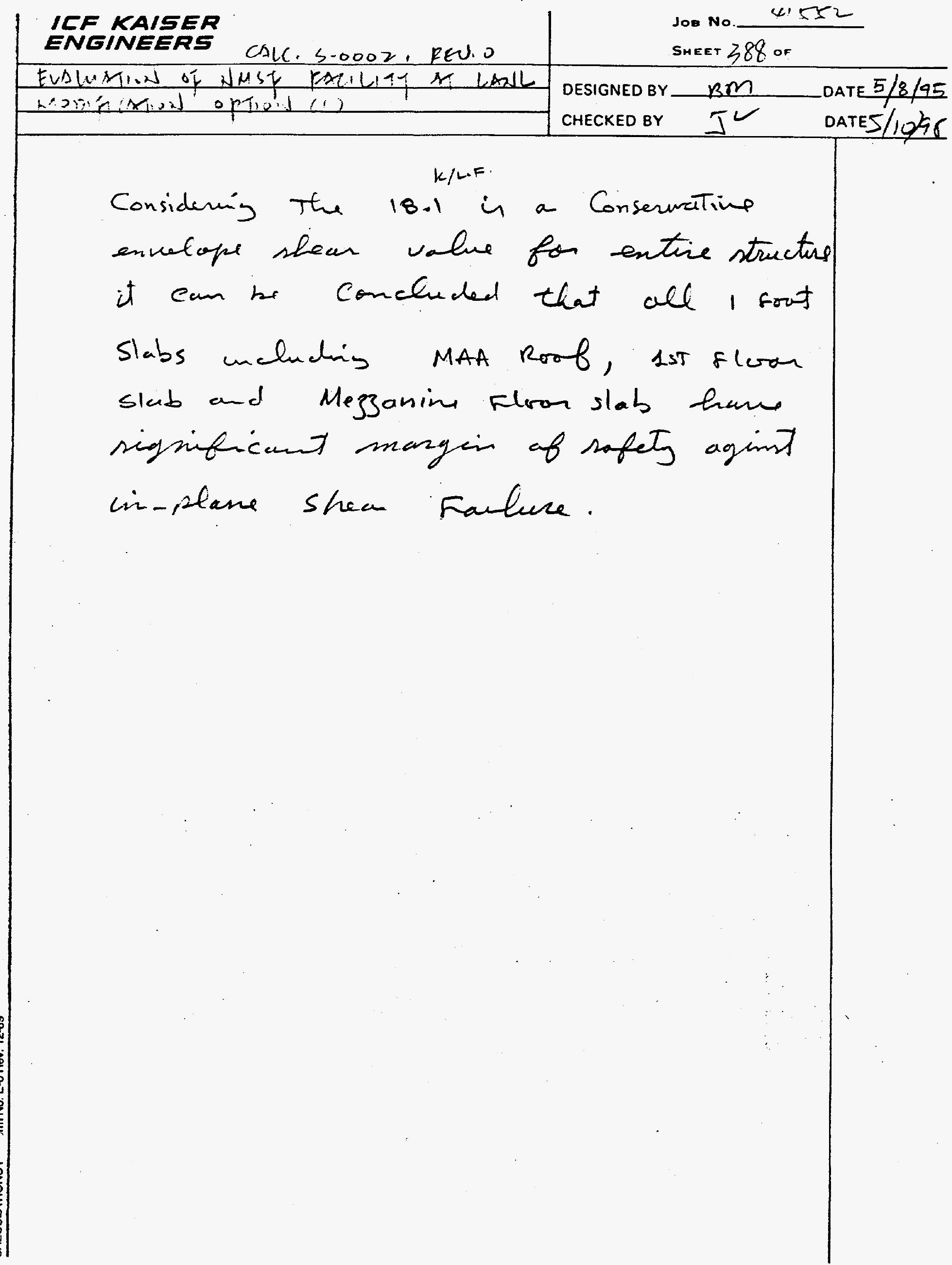


CAL. NOS S.0002, ELO O

KAISER

Jor, 20. $4 / 552$

ENGINEER

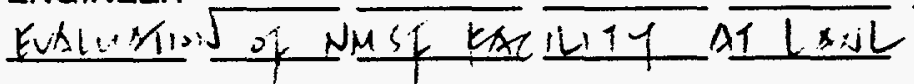

SHEET

3890F ㄴ.

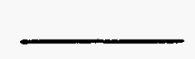

CHKD:

BY: $B m$

DATE: $3 / 10 / 95$

DATE:

\section{SUMMARY OF IN-PLANE SHEAR D/C RATIOS (OPTION 1)}

\begin{tabular}{|c|c|c|c|c|c|}
\hline $\begin{array}{l}\text { WALL } \\
\text { SEGME }\end{array}$ & $\begin{array}{r}\text { LENGTH } \\
L \\
\text { (FEET) } \\
\end{array}$ & $\begin{array}{r}\text { WIDTH } \\
\text { Be( }\left(^{\star}\right) \\
\text { (FEET) }\end{array}$ & $\begin{array}{r}\text { CAPACITY } \\
\text { (Vc) } \\
\text { (KIPS) } \\
\end{array}$ & $\begin{array}{c}\text { DEMAND } \\
\text { (Vu) } \\
\text { (KIPS) }\end{array}$ & $\begin{array}{l}(D / C) \\
V u / V c\end{array}$ \\
\hline 1 & 130.45 & 3 & 4847 & 1629 & 0.34 \\
\hline 2 & 18.25 & 1.5 & 339 & 131 & 0.39 \\
\hline 4 & 18.25 & 1.33 & $\begin{array}{r}301 \\
3424\end{array}$ & 124 & 0.41 \\
\hline 5 & 130.45 & 2.83 & & 1770 & 0.39 \\
\hline 6 & 148.7 & 1.45 & 2671 & 1080 & 0.40 \\
\hline 9 & 72.5 & 1.38 & 1239 & 1315 & 1.06 \\
\hline 12 & 53.5 & 1.5 & 994 & 837 & 0.84 \\
\hline 8 & 44.4 & 1 & 550 & 215 & 0.39 \\
\hline 10 & 19 & 1 & 235 & 230 & 0.98 \\
\hline 18 & 29.6 & 1.5 & 550 & 509 & 0.93 \\
\hline 33 & 23.9 & 1 & 296 & 279 & 0.94 \\
\hline 11 & 23.9 & 1 & 296 & 227 & 0.77 \\
\hline 7 & 29.6 & 1.5 & 550 & 274 & 0.50 \\
\hline 29 & 15 & 1.5 & 279 & 250 & 0.90 \\
\hline 3 & 29.6 & 1.5 & 550 & 70 & 0.13 \\
\hline 21 . & 148.7 & 1.25 & 2302 & 1072 & 0.47 \\
\hline 28 & 80 & 1 & 991 & 791 & 0.80 \\
\hline 30 & 78 & 0.84 & 812 & 578 & 0.71 \\
\hline 35 & 78 & 1 & 966 & 751 & 0.78 \\
\hline 22 & 25 & 1 & 310 & 71 & 0.23 \\
\hline 36 & 19 & 0.67 & 158 & 114 & 0.72 \\
\hline 25 & 128.7 & 0.86 & 1371 & 826 & 0.60 \\
\hline 38 & 80 & 1 & 991 & 721 & 0.73 \\
\hline $\begin{array}{l}\mathrm{Vc}=.85^{*} \\
\mathrm{~V} c=.8 \\
\mathrm{Be}=\mathrm{Effe}\end{array}$ & 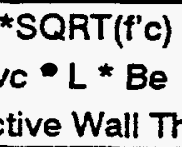 & $=$ & 108 & PSI & \\
\hline
\end{tabular}

(*) wall 5 is $1.5^{\prime}$ wide

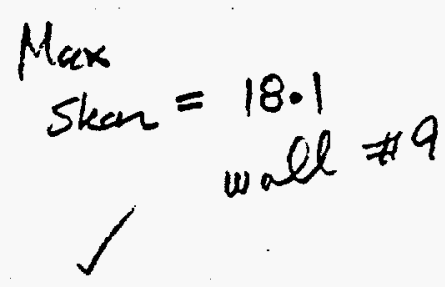




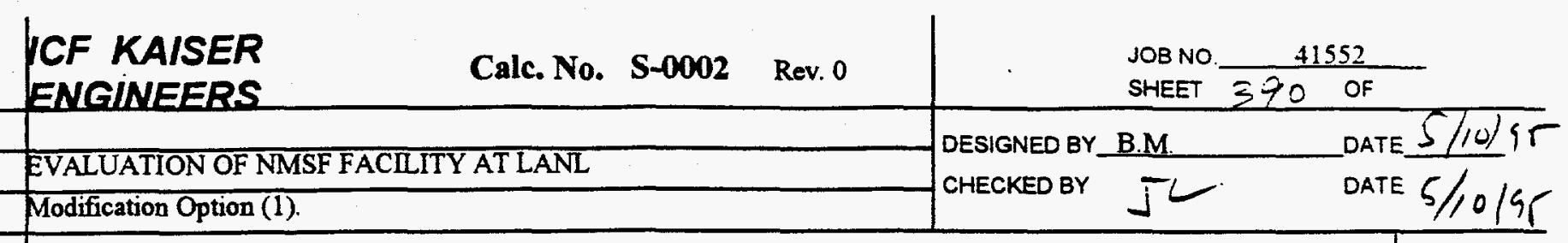

\subsubsection{Vertical Analysis.}

The objective of this analysis was to evaluate the out-of-plane response of the floor and roof slabs of the as built as well as the modified NMSF Building.

A two dimensional transverse strip of the building was modeled using SAP90 program and was subjected to $100 \%$ of the ground motion for DBE criteria in vertical direction phus DL and $L L$ per the evaluation criteria covered earlier in this calculation. The 2-D model were analyzed for dynamic and static (UBC) load combinations. To capture a realistic level of ground acceleration the vertical soil spring were selected such that the resulting fundamental modal frequency matches that of the 3-d model The detail of calculations and evaluations of the floor slabs, charge deck and roof slab are presented in the following pages.

The following conchusions can be drawn from the results of this analysis:

1) The roof slab and supporting beam on line 2 are overstressed under the vertical loadings. The roof slab and beam will be adequate, however, if the 2 foot deep top soil is removed from the roof of the building.

2) In the modified structure the open space between the columns on line two, between the 1st floor slab and the roof, is proposed to be infilled with new 18" concrete walls. With these walls added the roof can safely withstand the vertical loads and there is no need to remove the topside soils.

3) The 1st floor slab is adequate to resist out-of-pane loads.

4) The new 1 foot slab at mezzanine level and the new 30" composite charge deck are adequate. 
IF KAISER

Jos No $4155=$

SHEET 391 or

$-$

EVALUATION OF NMSF FAULTY AT LAN

DESIGNED BY BM DATE $5 / 26 / 9$.

CHECKED BY DATE $5 / 26 / 5$

Summary of ard Vertical Analysis results

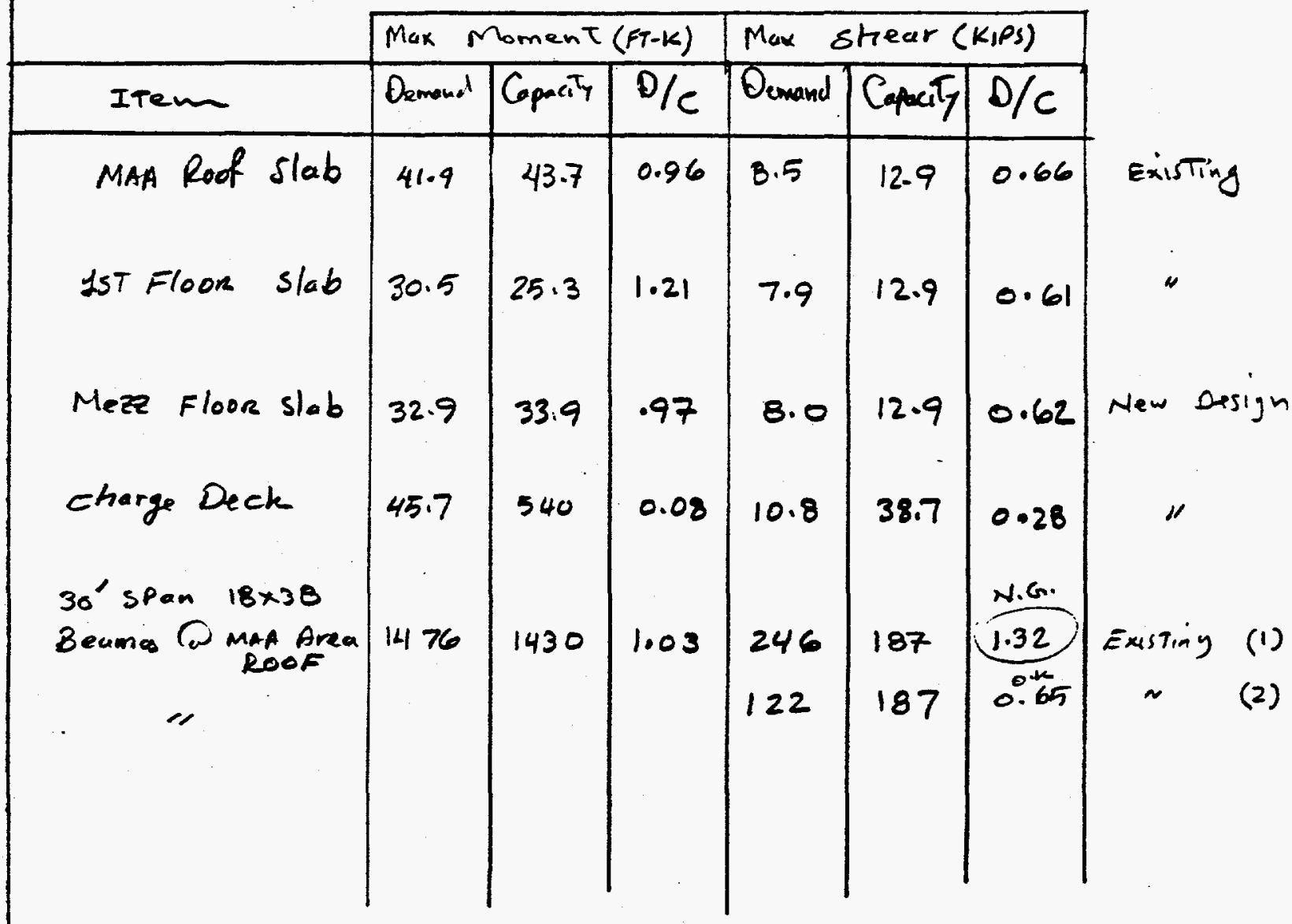

(1) Beam inadequate with topside soil in-place.

(2) Beam oik. with Topside sails removed.

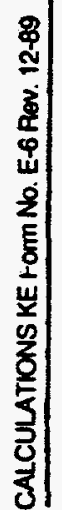


IF KAISER

sos No. $\quad S_{2}$

WISE CD LANE

SHEET 392 or

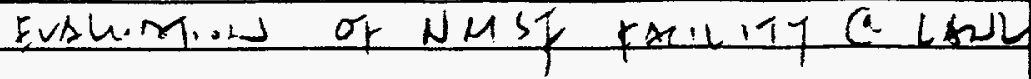

DESIGNED BY_ $\quad \beta M$

DATE $4 / 27 / 9$.

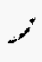

CHECKED BY TL DATE $J / 3 / 4$

VERTICAL ANALYSIS of FLOOR and ROOF SLABS

lIst Floon/Mezz slab (1'. Concrete slabs)

mass: $\quad m=\left(0.15+\frac{0.01+.25 \times .25}{1.0}\right) / 32.2=.00691 \ldots$

$\Delta L+L L: \quad \omega_{1}=(0.15+0.01+0.25)=0.41$

$1.40 L+1.746: \quad \omega_{2}=1.4(0.15+0.01)+1.7(0.25)=0.649$

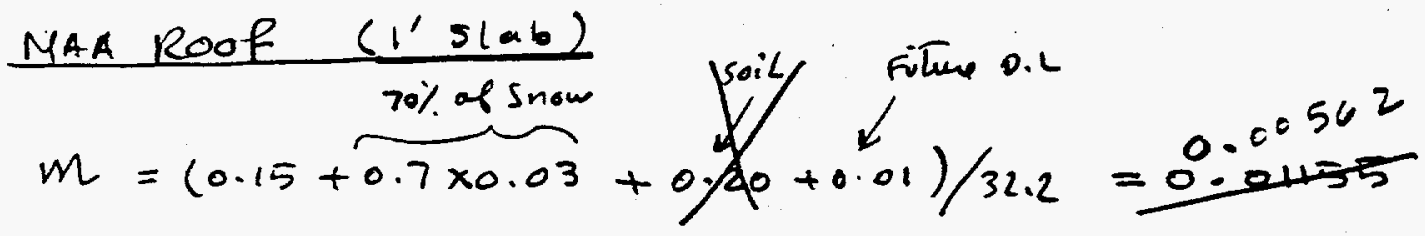

$\omega_{1}=(0.15+0.03+0.20+0.01)$

$=0.39$

$w_{2}=1.4(0.15+0.01+0.72)+1.7 \times 0.03=\frac{0.275}{0.555}$

Basement $\left(1.5^{\prime}\right.$ Slab $)$

$$
\begin{aligned}
& m=0.00691+\left(\frac{0.15 \times .5}{32.2}\right)=0.0092 \\
& \omega_{1}=0.410+(0.15 \times .5)=0.485 \\
& \omega_{2}=0.649+(0.15 \times .5) \times 1.4=0.754
\end{aligned}
$$

Change Deck ( $30^{\circ}$ Composite)

$$
\begin{aligned}
& m_{1}=0.02865 \\
& \omega_{1}=1.110 \mathrm{k} / \mathrm{FT} \\
& \omega_{2}=1.629 \mathrm{k} / \mathrm{FT}
\end{aligned}
$$


I CF KAISER

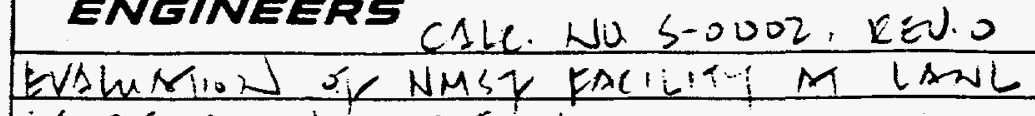

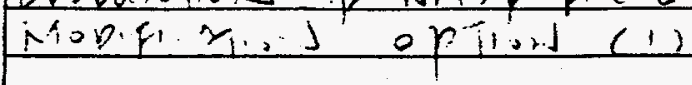

DESIGNED BY

CHECKED BY JuL.

41552

SHEET 293 OF

Change Deck STiffness ProPs.

$$
\begin{aligned}
& E_{c}=518400 \mathrm{KSF} \\
& E_{S}=29000 \times 12^{2}=4176000 \mathrm{KSF} \\
& E_{s} / E_{c}=8.1 \\
& A=2.5 \times 1+2 \times 1 . \times \frac{.5}{12} \times 8.1=3.175 \mathrm{FT}^{2} \\
& I=2.5 / 12+2 \times(.5 \times 8.1 / 12) \times\left(\frac{2.5}{2}\right)^{2}=1.30+1.05=2.35
\end{aligned}
$$




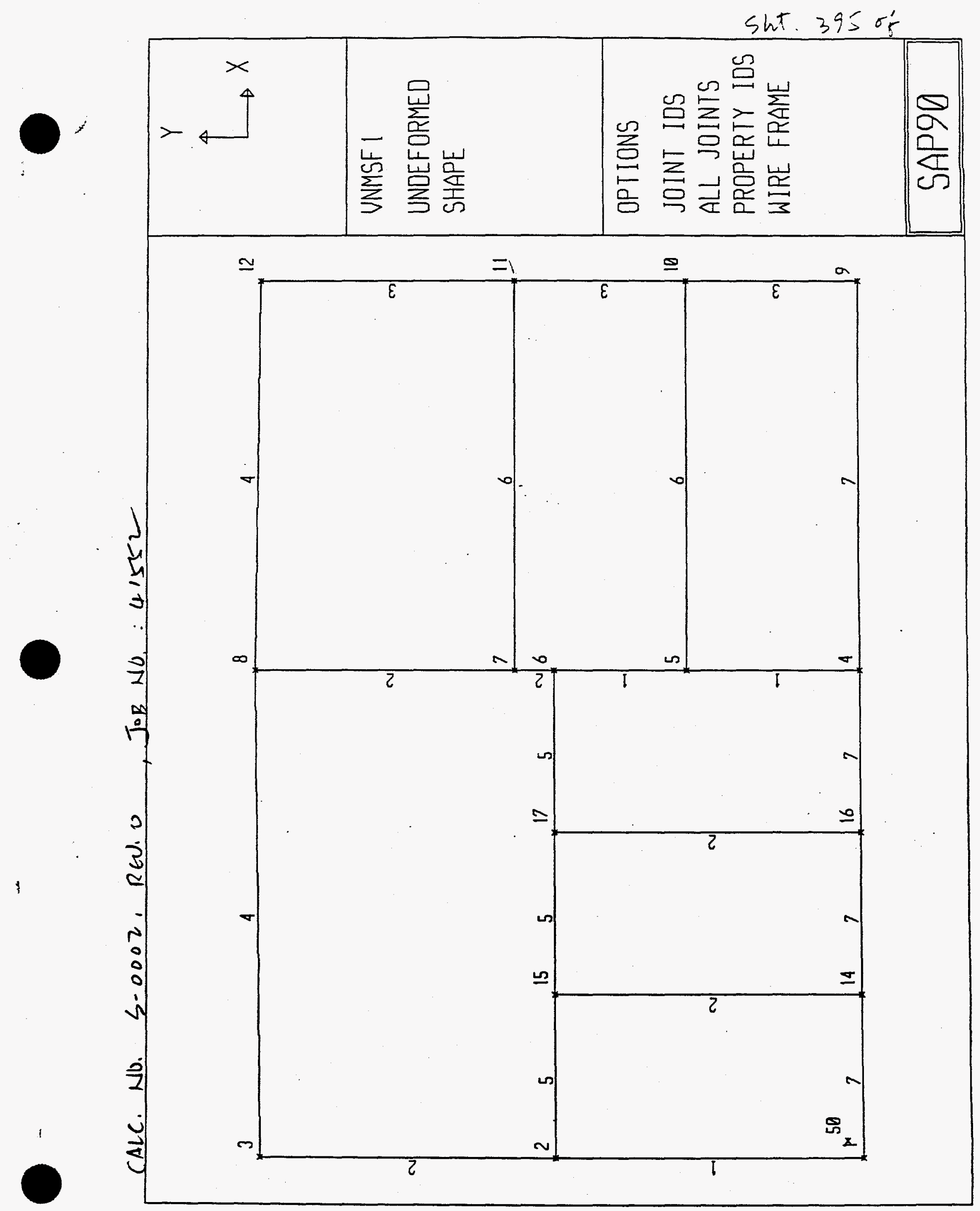


(LANL NMSF BUILDING) 2-D VERTICAL ANALYSIS OF ROOF - (VNMSF2) Lift. 396 of

$\mathrm{C}$

C-----REV. DATE----FILE------DESCRIPTION

4-27-95 BM VNMSF1 -ASSUME SOIL MOD. OF SUBGRADE OF 1 KSF

-adjust soil springs to match vertical

$\mathrm{C}$ from 3-D Model (NMSF06)

C $4-27-95$ BM TO DO:

C

SYSTEM

$L=2 \quad Z=10$

JOINTS

$1 \quad x=0$

$2 \mathrm{X}=0$

$3 \quad x=0$

$Y=0$

$Y=18.6$

$4 \quad X=29.6 \quad Y=0$

$5 \quad X=29.6 \quad Y=10.5$

$6 \quad \mathrm{X}=29.6 \quad \mathrm{Y}=18.6$

$7 \mathrm{X}=29.6 \quad \mathrm{Y}=21$.

$8 \quad X=29.6 \quad Y=36.8$

C

$9 \mathrm{X}=53.5 \quad \mathrm{Y}=0$

$10 \quad X=53.5 \quad Y=10.5$

$11 \mathrm{X}=53.5 \mathrm{Y}=21$.

$12 X=53.5 \quad Y=36.5$

$14 \quad \mathrm{X}=29.6 / 3 \quad \mathrm{Y}=0$

$\mathrm{X}=29.6 / 3 \quad \mathrm{Y}=18.6$

$X=29.6 / 3 * 2 \quad Y=0$

$7 \mathrm{X}=29.6 / 3 * 2 \quad \mathrm{Y}=18.6$

$X=1 \quad Y=1$

RESTRAINTS

1 17. $1 \mathrm{R}=0,0,1,1,1,0$

$1 \quad R=0,0,1,1,1,0$

$4 \quad R=0,0,1,1,1,0$

$9 \quad \mathrm{R}=0,0,1,1,1,0$

$14162 \mathrm{R}=0,0,1,1,1,0$

$09121 \quad R=1,0,1,1,1,0$

$50 \quad \mathrm{R}=1,1,1,1,1,1$

$131 \quad R=1,0,1,1,1,0$

C SET SOIL SPRINGS TO MATCH VERTICAI FREQUENCY OF 3D ANALYSIS SPRINGS

$\begin{array}{ll}1 & \mathrm{~K}=0,05000 / 3 . \\ 4 & \mathrm{~K}=0,17000 / 3 . \\ 9 & \mathrm{~K}=0,12000 / 3 .\end{array}$

$14162 \mathrm{~K}=0,10000 / 3$.

\section{FRAME}

$\mathrm{NM}=7 \quad \mathrm{NL}=6 \quad \mathrm{NSEC}=5$

$1 \mathrm{SH}=\mathrm{R} \quad \mathrm{T}=3.00,1$

$\therefore \mathrm{SH}=\mathrm{R} \quad \mathrm{T}=1.50,1$

$H=R \quad T=1.00,1$.

$E=518400 . * 0.75 \quad M=.15 * 3 / 32.2 \quad W=.15 * 3$

$E=518400 * 0.75 \mathrm{M}=.15 * 1.5 / 32.2 \mathrm{~W}=1.5 * .15$

$W=.15$

$E=518400 * 0.75 M=.15 / 32.2$

$W=0$.

$A=2.5 * 1 \quad I=2.35,1000 \quad E=518400$

$\mathrm{SH}=\mathrm{R} \quad \mathrm{T}=1.0,1 . \quad \mathrm{E}=518400 . * 75$

$M=0.01155$

$W=0$.

$M=0.00691 \quad W=0$.

7 SH=R $T=1 \cdot 5,1$.

$E=518400 *$ *.75

$M=0.0092$

$\mathbf{W}=0$.

3' WALLS

$1.5^{\prime}$ WALL

1. WALL

1. MAA ROOF

: $2.5^{\prime}$ CHARGE DECK

: 1' 1ST FLR/MEZZ FLR

: $1.5^{\prime}$ BASEMENT FLOOR 


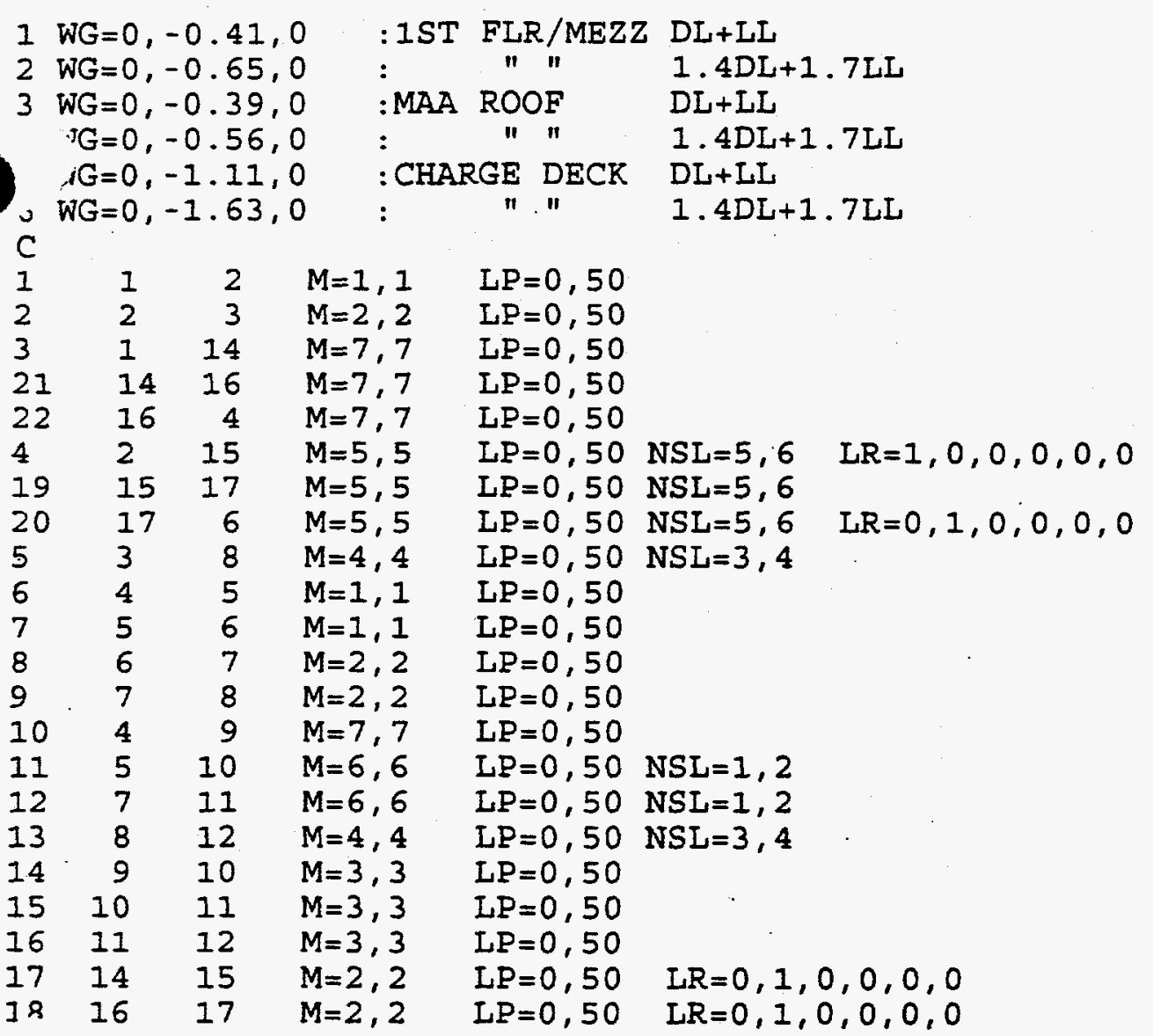

REG. GUIDE 1.60 (REV.1，73) RESPONSE SPECTRUM (Sa VS. FREQ); 1.0g; 7\% DAMPING TOTAL 87 POINTS

SPEC

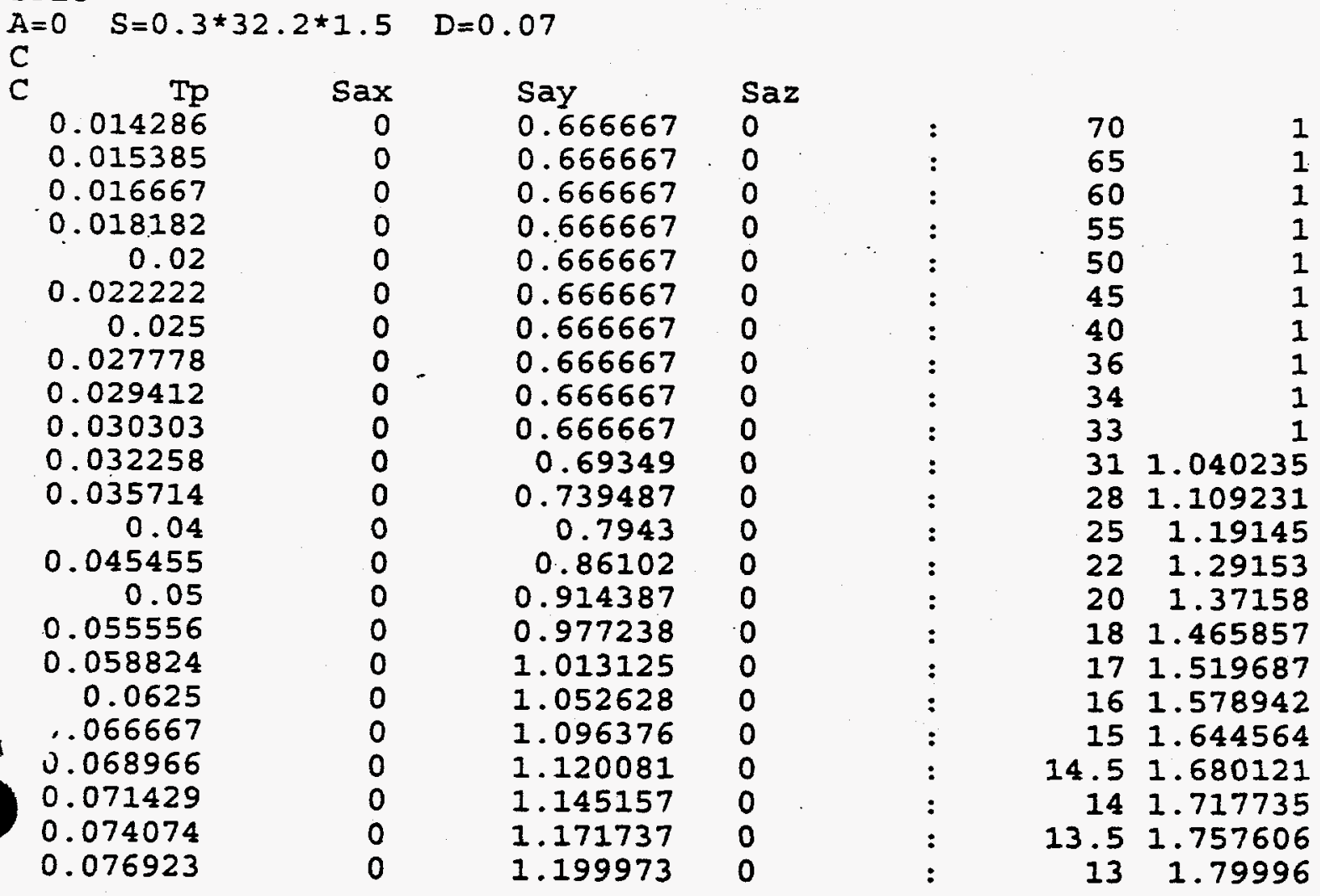




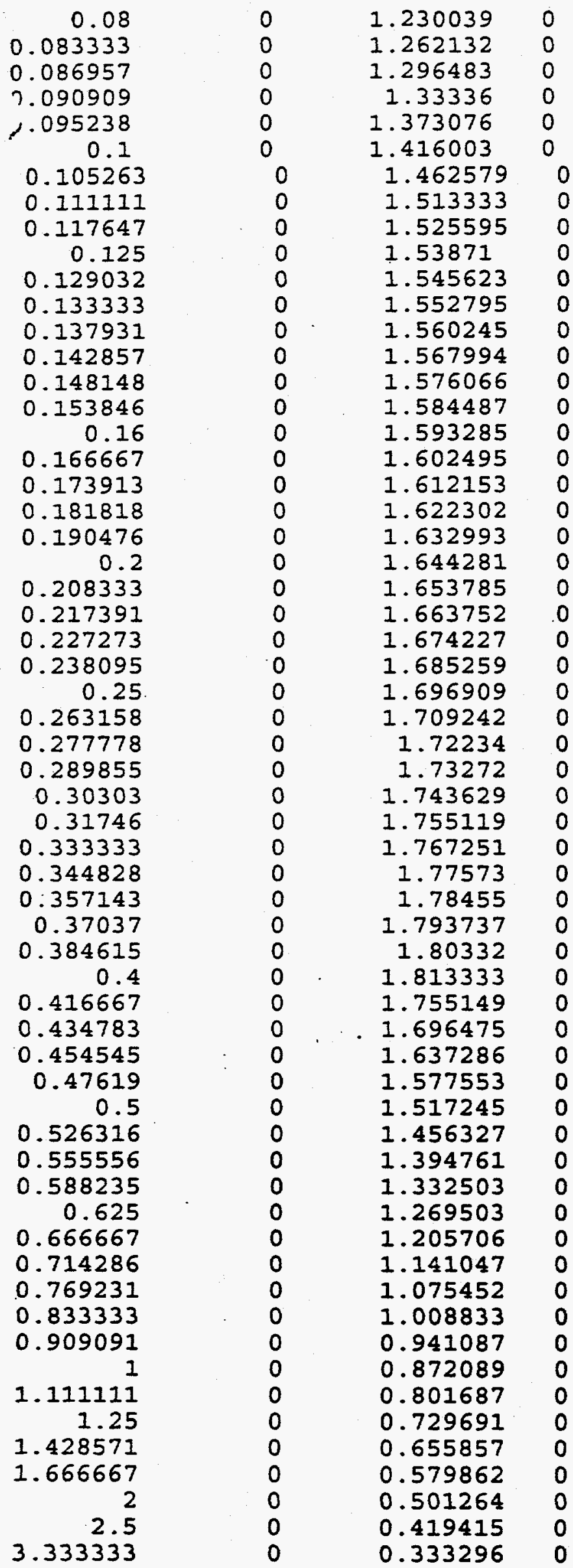

$12.5 \quad 1.845058$ 121.893198

11.51 .944725 $11 \quad 2.00004$ $10.5 \quad 2.059614$ $10 \quad 2.124004$ 9.52 .193868 $\begin{array}{ll}9 & 2.27\end{array}$

8.52 .288393

82.308065

$7.75 \quad 2.318434$

$7.5 \quad 2.329192$

$7.25 \quad 2.340367$ 72.351991
.75

6.752 .364099

$\begin{array}{ll}6.5 & 2.37673\end{array}$

$\begin{array}{ll}6.25 & 2.389927\end{array}$

62.403742
6

5.752 .418229

5.52 .433453

$5.25 \quad 2.449489$

52.466421

$4.8 \quad 2.480677$

4.62 .495628

$4.4 \quad 2.51134$

4.22 .527889

42.545363

3.82 .563863

$3.6 \quad 2.58351$

$3.45 \quad 2.59908$

3.32 .615444

$3.15 \quad 2.632678$

32.650876

2.92 .663595

2.82 .676825

2.72 .690605

$\begin{array}{ll}2.6 & 2.70498\end{array}$

$2.5 \quad 2.72$

$2.4 \quad 2.632724$

2.32 .544713

2. $2 \quad 2.455929$

2.12 .366329

22.275867

1.92 .184491

1.82 .092141

$1.7 \quad 1.998754$

$1.6 \quad 1.904254$

1.51 .808559

$1.4 \quad 1.711571$

1.31 .613178

$1.2 \quad 1.51325$

1.11 .411631

11.308134

0.91 .202531

$0.8 \quad 1.094537$

$0.7 \quad 0.983785$

$0.6 \quad 0.869793$

0.50 .751896

$0.4 \quad 0.629123$

$\begin{array}{ll}0.3 & 0.499944\end{array}$
CAL NO. S.OOO? REJ.O

Jor wo 4!: 


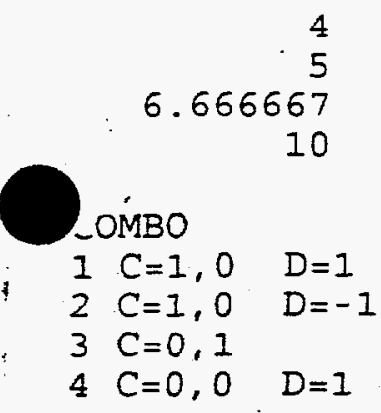

$\begin{array}{ll}0.288119 & 0 \\ 0.184396 & 0 \\ 0.103723 & 0 \\ 0.046099 & 0\end{array}$

0.250 .432178

$\operatorname{sint} 3 \div 3=$

0.20 .276594

$0.15 \quad 0.155584$

0.10 .069149

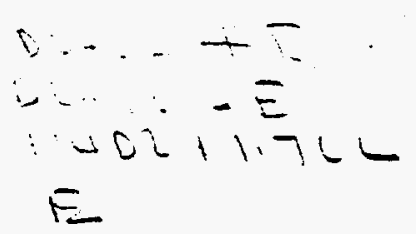

$$
\begin{aligned}
& \text { CALC. NO. S-0002, REU.O } \\
& \text { JOF NO. } 4.552
\end{aligned}
$$


List 400 of

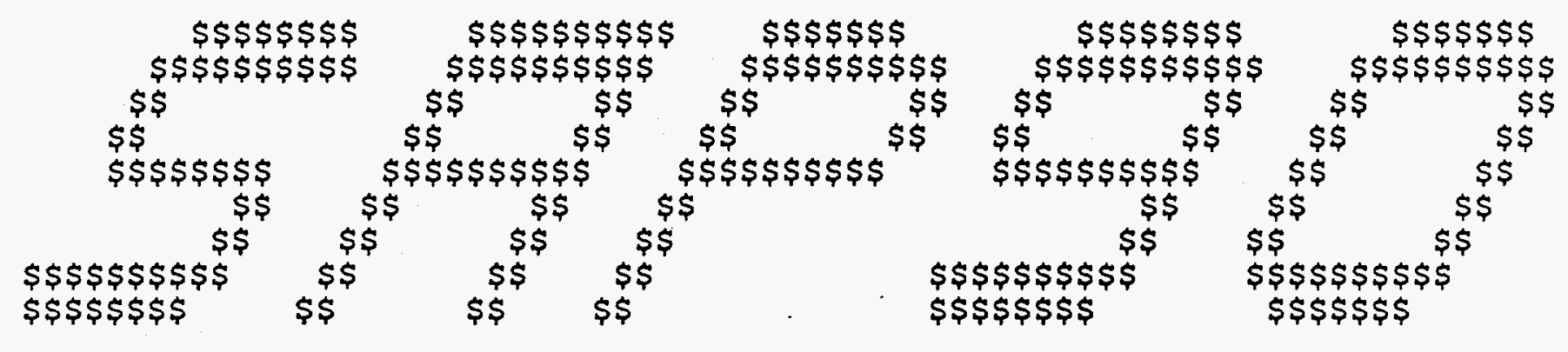

STRUCTURAL ANALYSIS PROGRAMS

VERSION P5.40

Copyright (C) 1978-1992

EDWARD L. WILSON

All rights reserved

CALL NO: S-0002, RENI O

JOB ND: 41552 
PROGRAM: SAP9O/FILE: VNMSF2.RIT

(LANL NMSF BUILDING) 2-D VERTICAI ANALYSIS OF ROOF - (VNMSF1)

R I T Z S Y S TEM P A R A M E TER S

sht. in of

- TUMBER of eOUATIONS

.UMBER OF MASSES

NUMBER OF RITZ VECTORS

NUMBER OF DIRECTION VECTORS

$$
\begin{array}{lr}
= & 41 \\
= & 25 \\
= & 10 \\
= & 2
\end{array}
$$

CALC. NO: S-0002, REN.O JOB NO: 4155 
PROGRAM : SAP90/FILE : VNMSE2.RII

(LANL NMSF BUILDING) 2-D VERTICAL ANALYSIS OF ROOF - (VNMSF1)

E I G E N V A L U E S

$$
\begin{array}{rrr}
\text { MODE } & \begin{array}{r}
\text { EIGENVALUE } \\
\text { MBER }
\end{array} & \text { CIRCULAR FREQ } \\
\text { (RAD/SEC) } \star * 2 & (R A D / S E C) \\
1 & 0.306951 E+04 & 0.554032 E+02 \\
2 & 0.501209 E+04 & 0.707961 E+02 \\
3 & 0.636291 E+04 & 0.797678 E+02 \\
4 & 0.122092 E+05 & 0.110495 E+03 \\
5 & 0.738465 E+05 & 0.271747 E+03 \\
6 & 0.871656 E+05 & 0.295238 E+03 \\
7 & 0.101422 E+06 & 0.318468 E+03 \\
8 & 0.129779 E+06 & 0.360248 E+03 \\
9 & 0.149116 E+06 & 0.386156 E+03 \\
10 & 0.360255 E+06 & 0.600213 E+03
\end{array}
$$

Sht. $402=\frac{1}{\delta}$

PERIOD

(SEC)

(CYCLES (SEC)

8.817687

11. 267554

12.695443

17.585855

43.249913

46.988609

50.685768

57.335317

61.458578

0.113408

0.088750

0.078768

0.056864

0.023121

0.021282

0.019729

0.017441

$95.526813 \quad 0.010468$

CALC. HO: S-0002. REU.O

J.B ND. 4/55V 
(LANL NMSF BUILDING) 2-D VERTICAL ANALYSIS OF ROOF - (VNMSFI)

B A S E FORCE REACTION FACTORS

$\sin .4035$

$\begin{array}{rlcccccc}\cdots \text { PDE } & \text { PERIOD } & X & Y & Z & X & Y & Z \\ \# & (S E C) & \text { DIRECTION } & \text { DIRECTION } & \text { DIRECTION } & \text { MOMENT } & \text { MOMENT } & \text { MOMENT } \\ 1 & 0.113 & 0.223 E-02 & 0.959 E+00 & 0.000 E+00 & 0.000 E+00 & 0.000 E+00-0.737 E+00 \\ 2 & 0.089-0.107 E-04 & 0.145 E+01 & 0.000 E+00 & 0.000 E+00 & 0.000 E+00 & 0.393 E+02 \\ 3 & 0.079-0.423 E-02 & 0.619 E+00 & 0.000 E+00 & 0.000 E+00 & 0.000 E+00 & 0.370 E+02 \\ 4 & 0.057 & 0.139 E-02 & 0.296 E+00 & 0.000 E+00 & 0.000 E+00 & 0.000 E+00 & 0.346 E+01 \\ 5 & 0.023 & 0.194 E+00-0.194 E-02 & 0.000 E+00 & 0.000 E+00 & 0.000 E+00-0.101 E+02 \\ 6 & 0.021 & 0.101 E+01 & 0.312 E-02 & 0.000 E+00 & 0.000 E+00 & 0.000 E+00-0.252 E+02 \\ 7 & 0.020 & 0.826 E+00-0.107 E-01 & 0.000 E+00 & 0.000 E+00 & 0.000 E+00-0.671 E+01 \\ 8 & 0.017-0.383 E+00-0.554 E-01 & 0.000 E+00 & 0.000 E+00 & 0.000 E+00-0.125 E+01 \\ 9 & 0.016-0.420 E+00 & 0.421 E-01 & 0.000 E+00 & 0.000 E+00 & 0.000 E+00 & 0.380 E+01 \\ 10 & 0.010 & 0.222 E+00 & 0.202 E-01 & 0.000 E+00 & 0.000 E+00 & 0.000 E+00-0.254 E+01\end{array}$

CALC. NO. S.0002, REJ.O J.R NO. WSK2 
(LANL NMSF BUILDING) 2-D VERTICAL ANALYSIS OF ROOF - (VNMSFI) PARTICI P T T N G MAS S - (percent)

$\mathrm{X}-\mathrm{SUM}$

0.000

Y-SUM

Z-SUM

0.000

0.000

26.360

0.000

0.000

0.001

0.000

0.001

0.000

1.737

0.000

49.233

0.000

80.764

87.548

0.000

95.699

0.000

97.972

86.329

0.000

97.311

99.828

99.828

0.000

0.000

0.000

99.828

99.832

0.000

99.920

0.000

99.970

0.000

99.982

0.000

0.000

CALC. NO. S-OOO2, REU.O JoB NO: 41552 


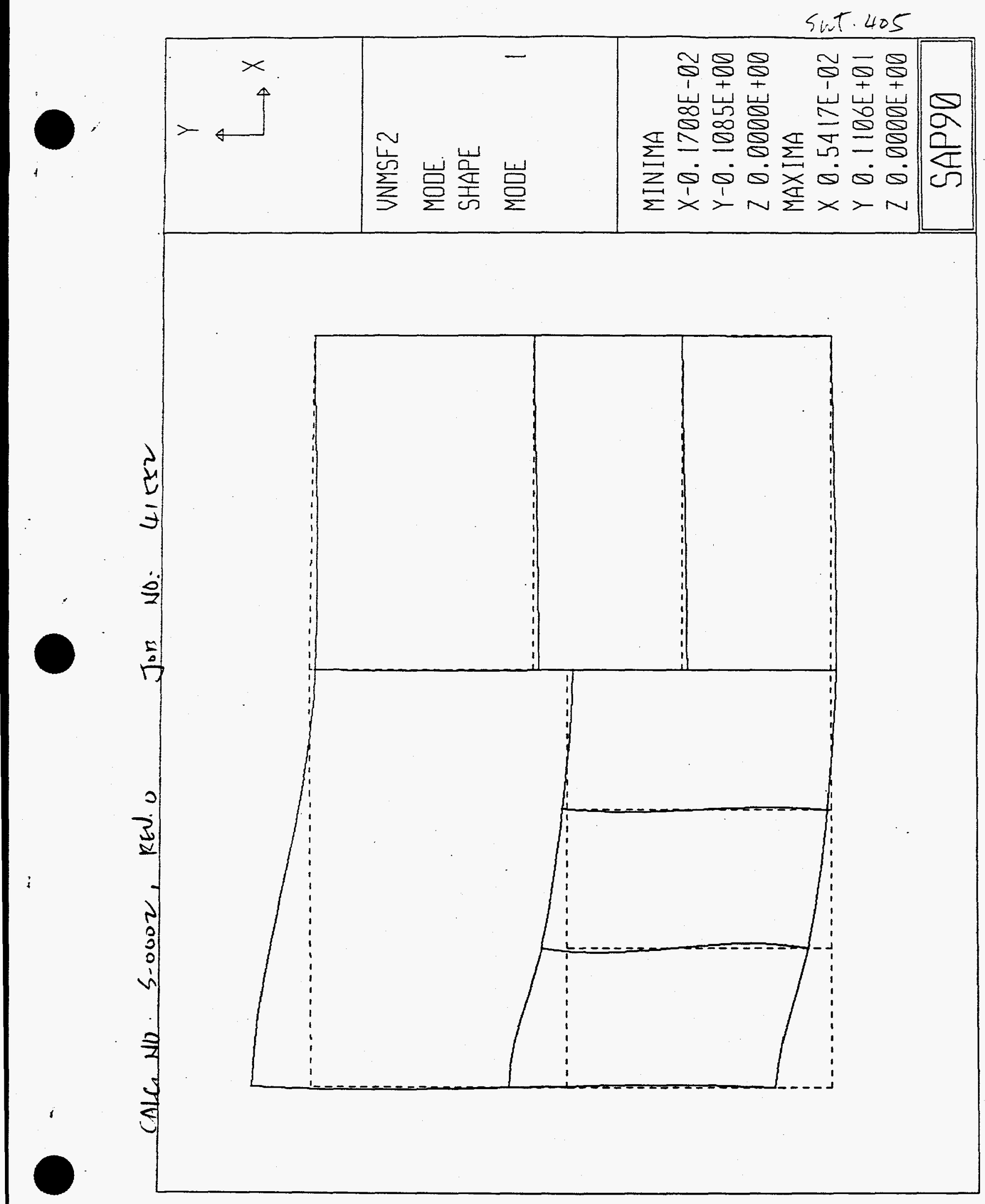


CALCNO.S-0002, RGN.O JOB NO. $4155^{2}$

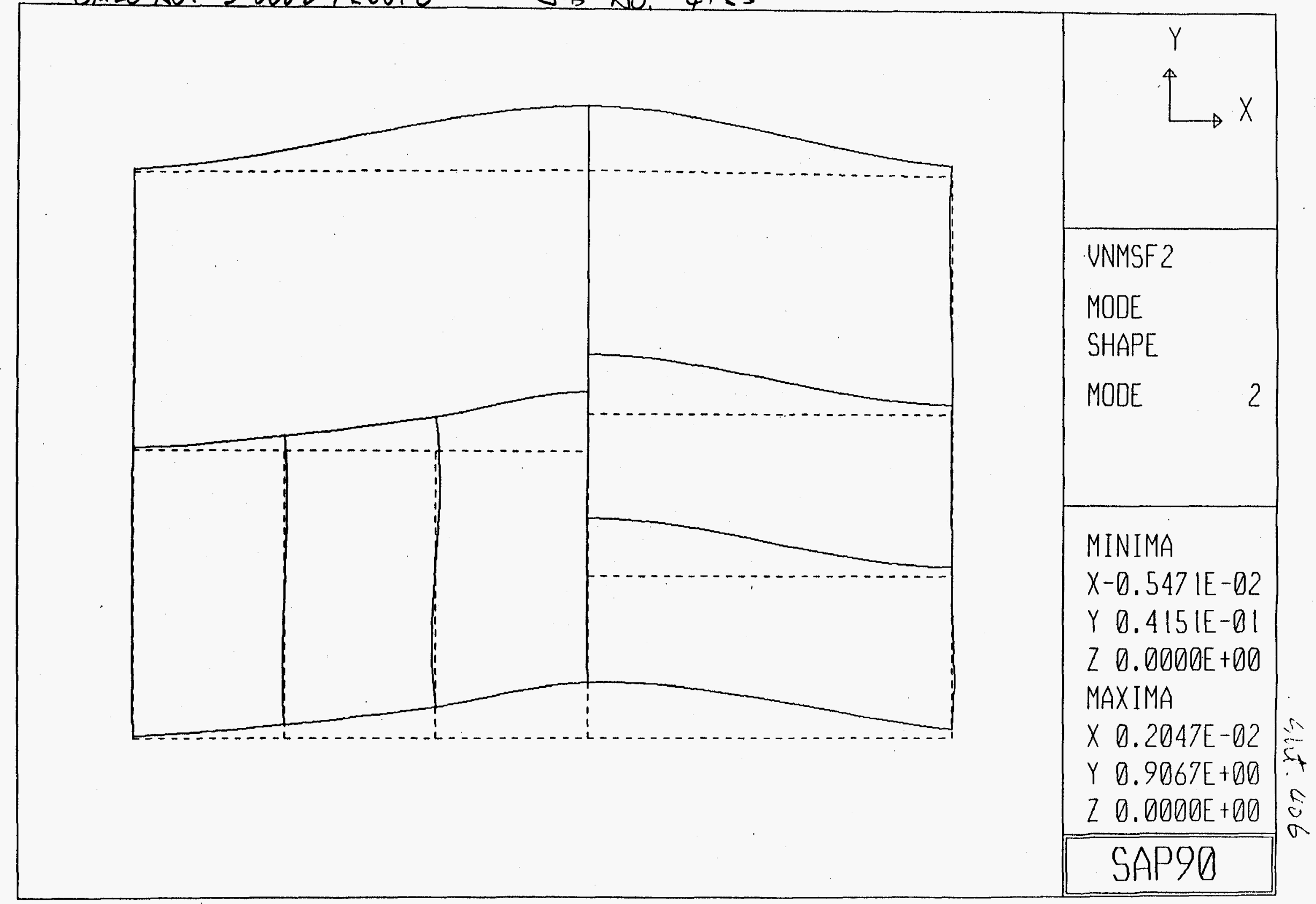


ShT. 4.97 of

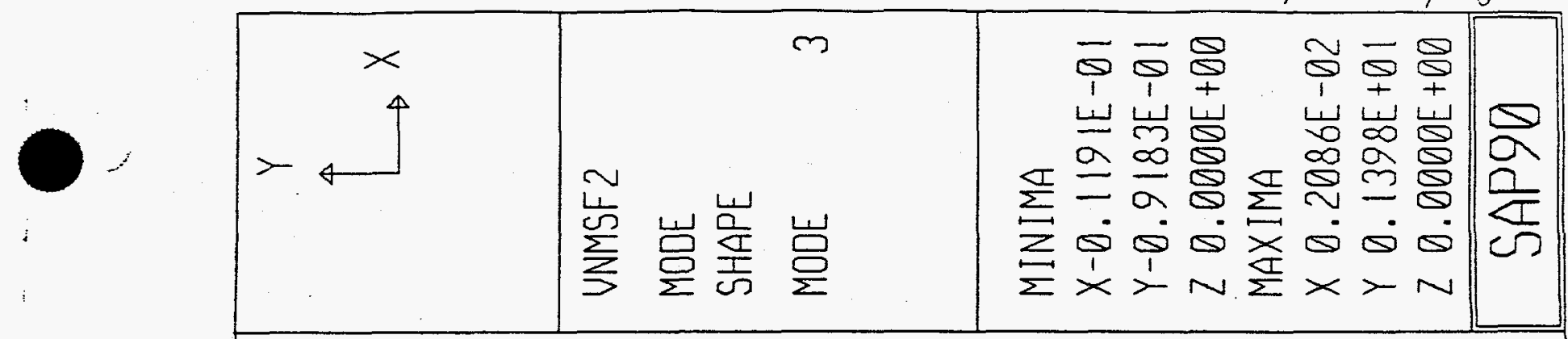
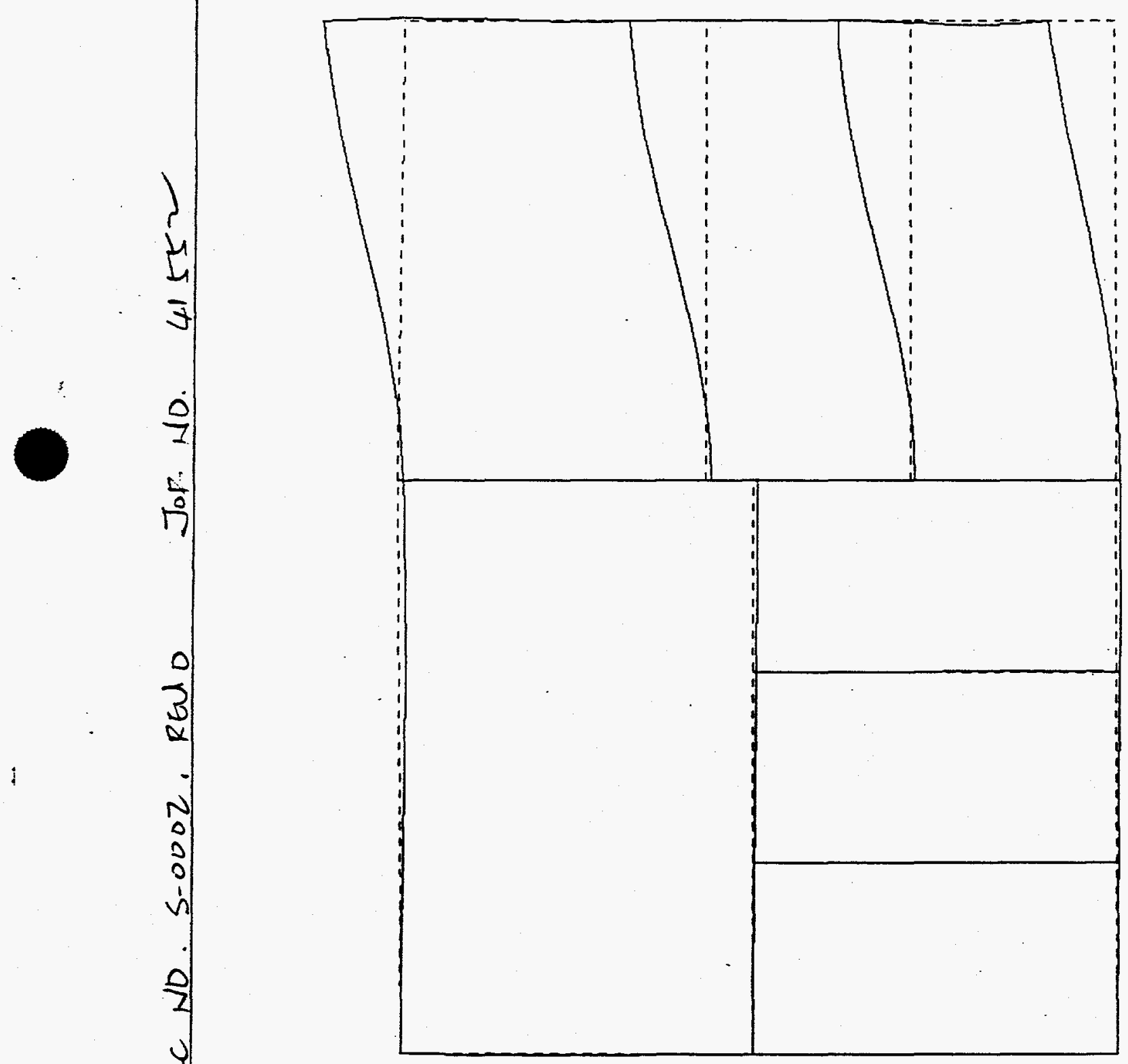

i

3
4
3
7
1
5
7

0
3
2

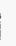

-
0
0
0
1
$n$
0
7

$\sqrt{4}$ 
CALC NO S-OOOZ, REV.O JUB NO 41552

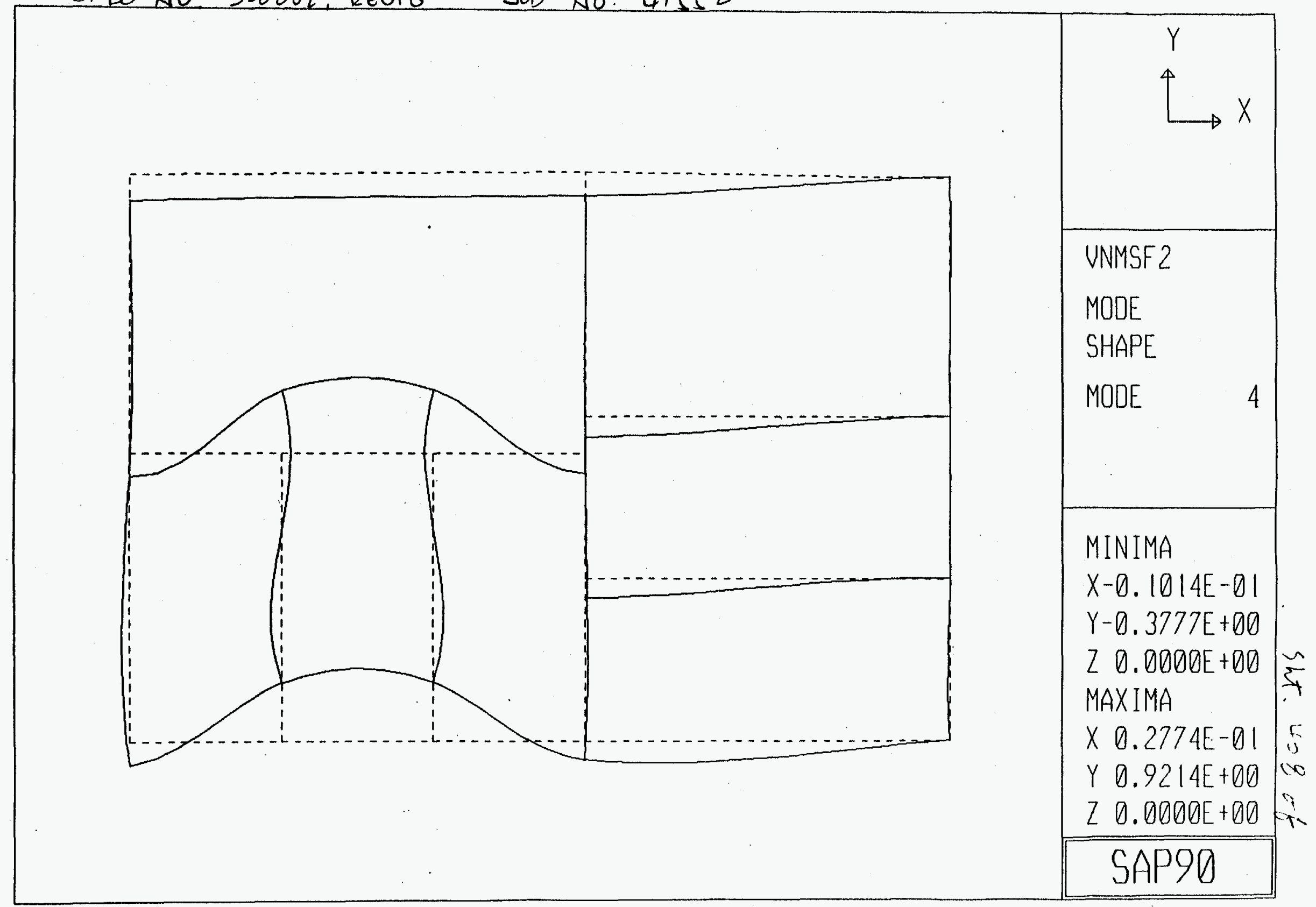


Sht. $409=15$

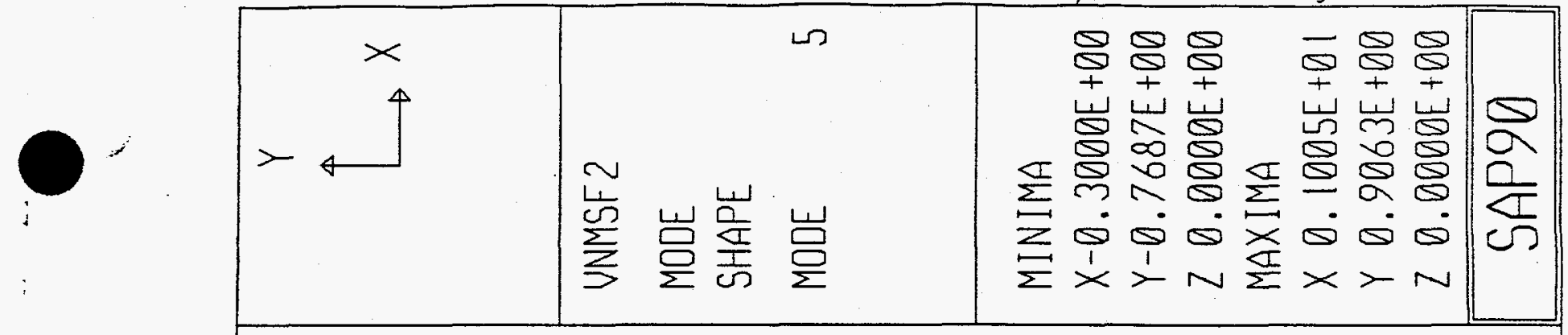

2
5
2
2
2
2
17

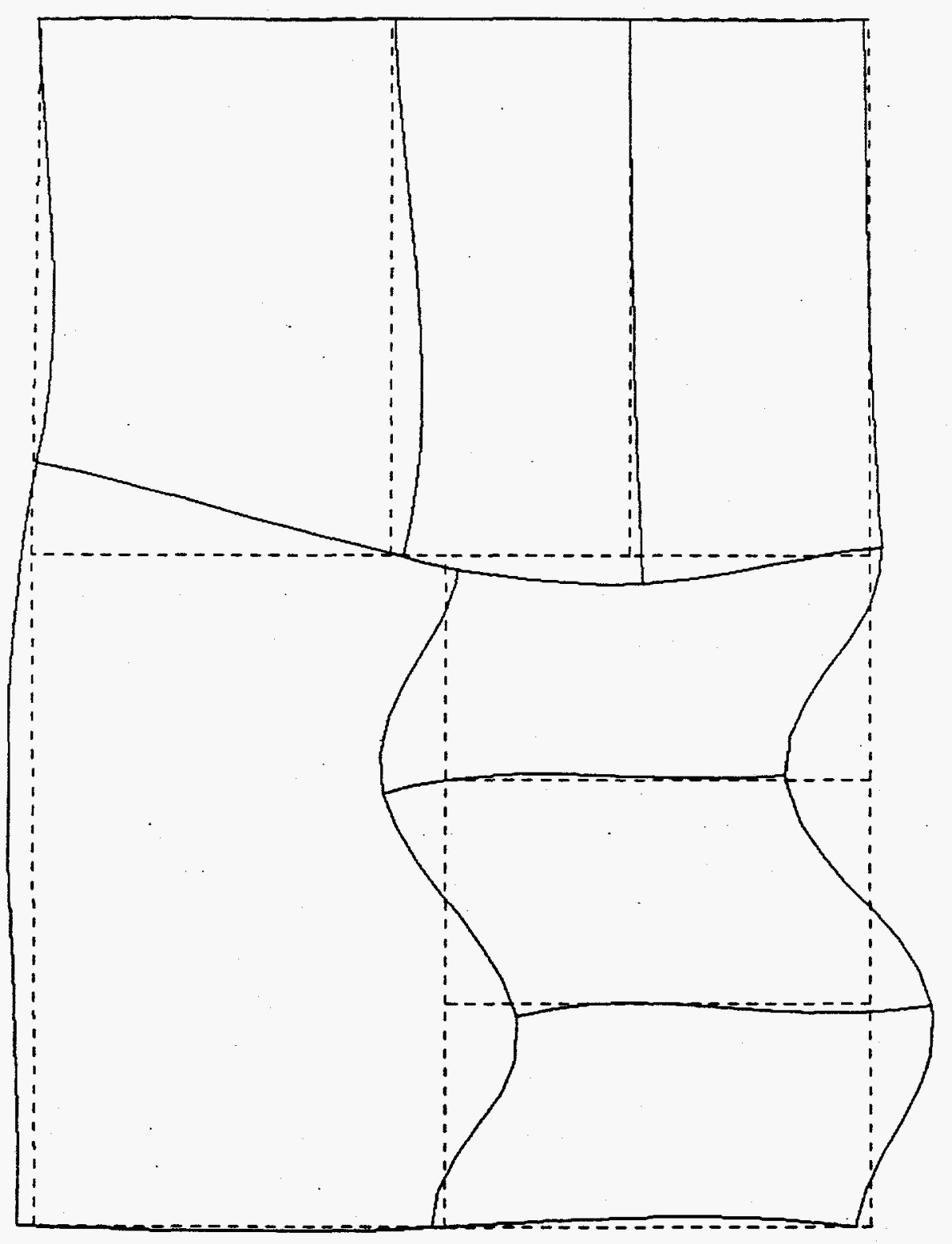




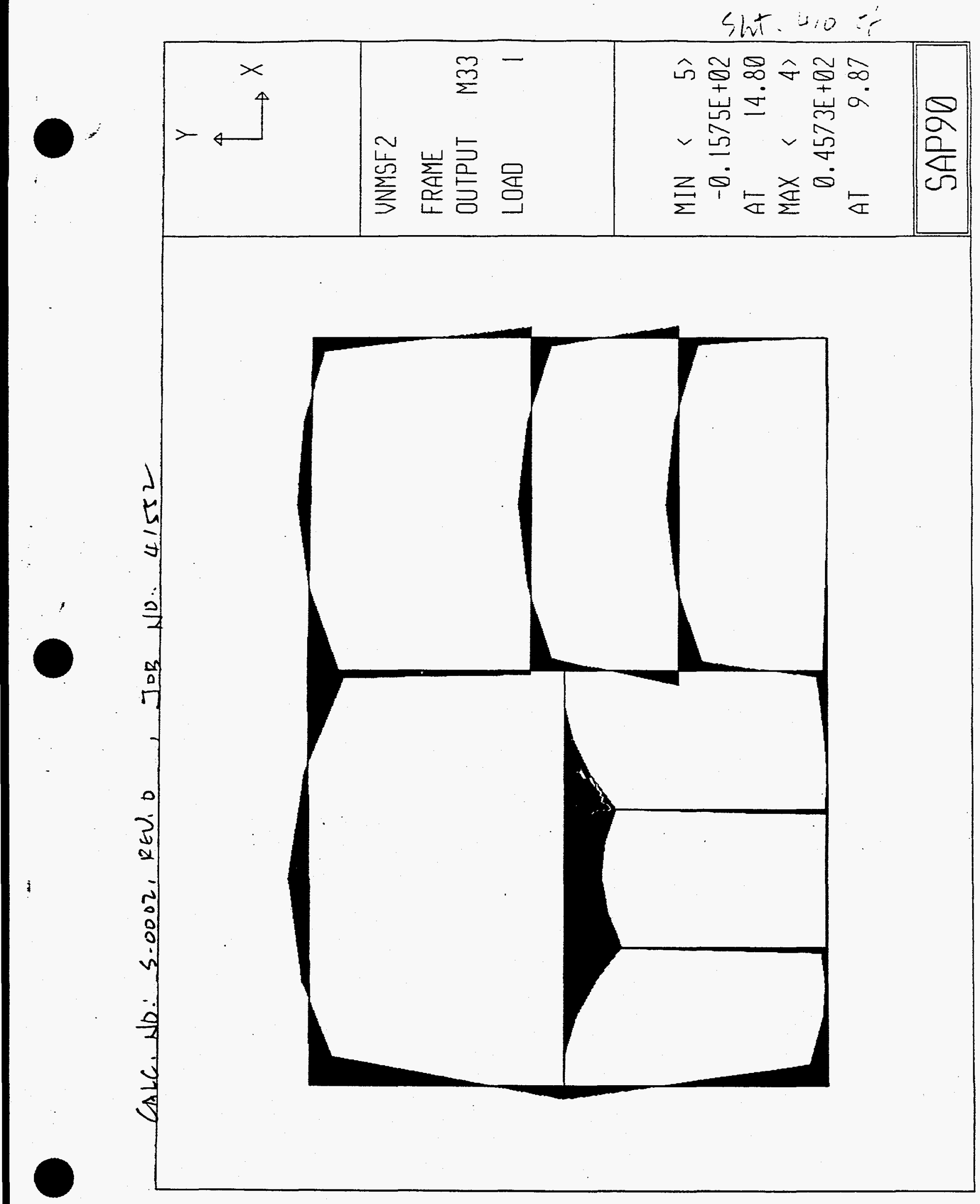




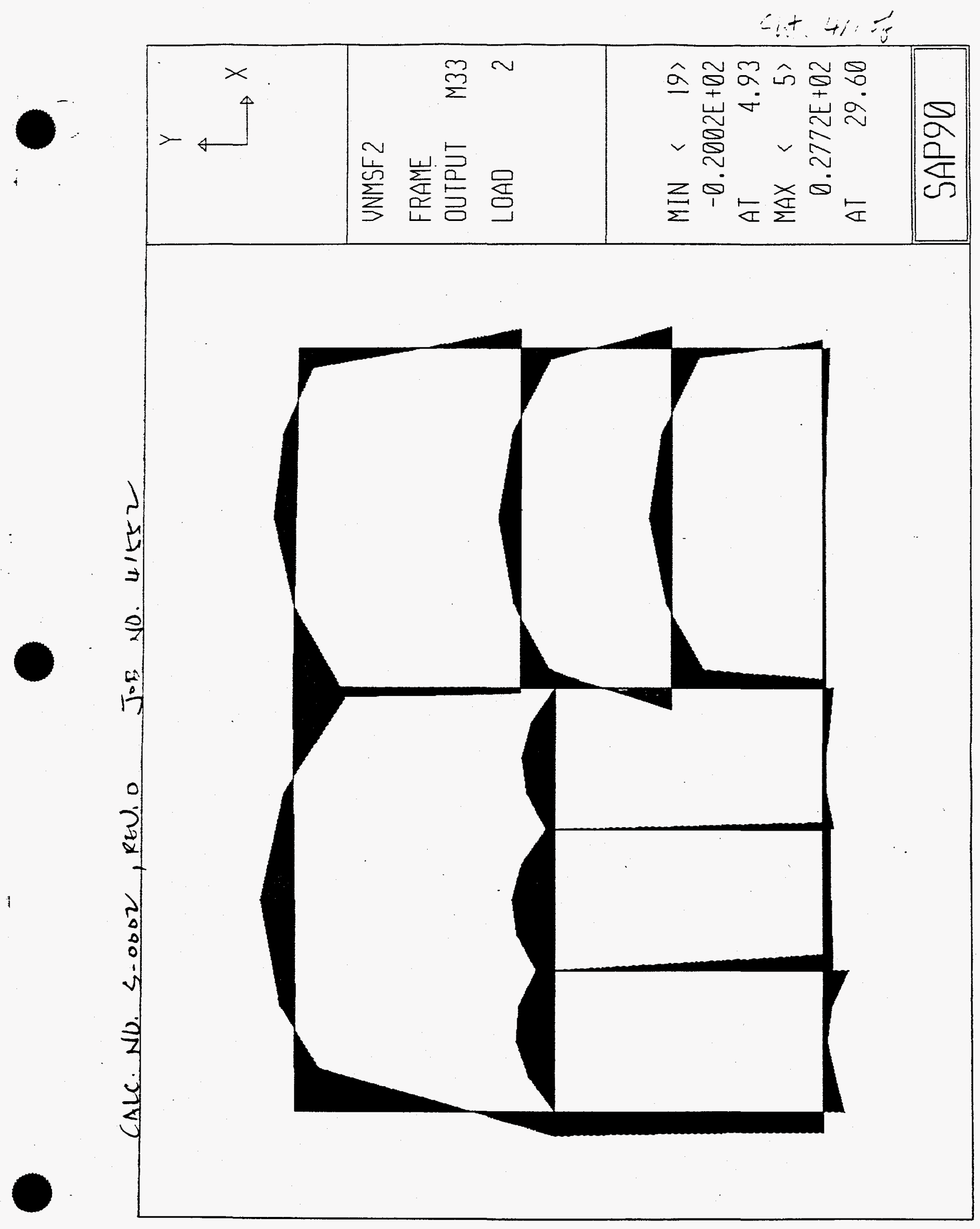




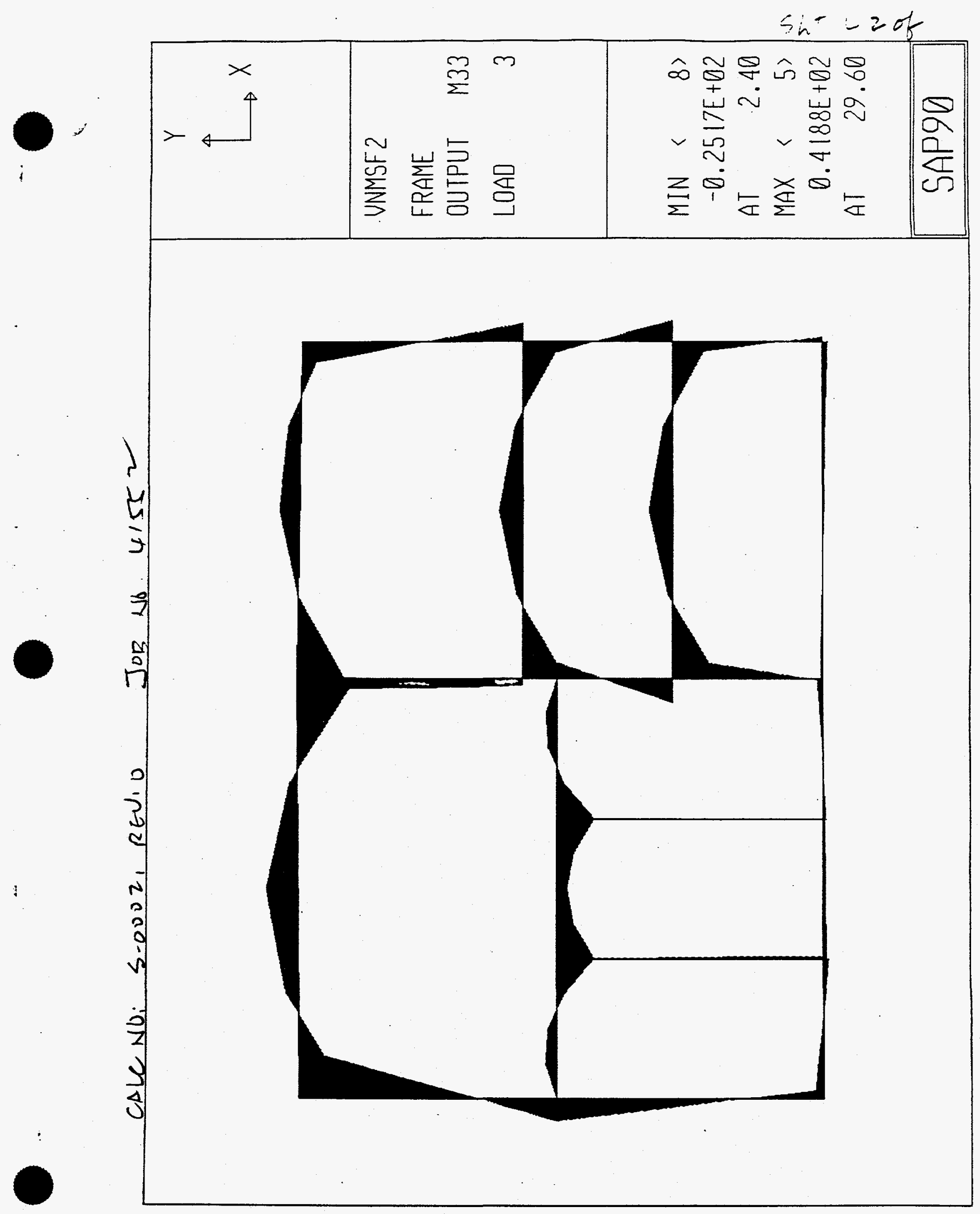




$$
\text { Sift. = os }
$$

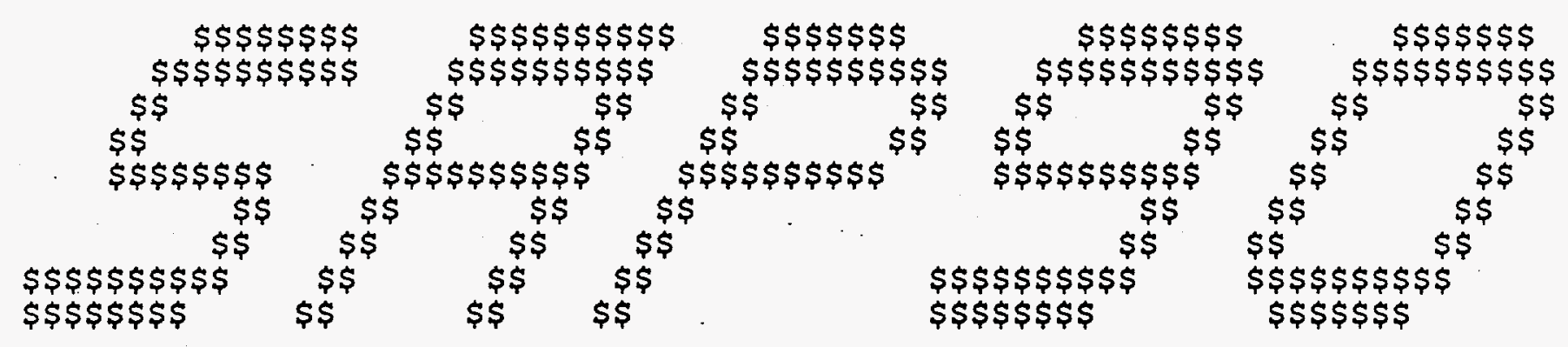

STRUCTURAL ANALYSIS PROGRAMS

VERSION P5.40

Copyright (C) 1978-1992 EDWARD L. WILSON

All rights reserved

CALL NO. S-0002, REU.O

JOB NO. 41552

EVALUMTION of NMSF

FATUITY M LAN 
URS CONSULTANTS, INC.

$$
C, T: 4 \sigma^{\prime}
$$

(LANL NMSF BUILDING) 2-D VERTICAL ANALYSIS OF ROOF - (VNMSF2) R A M E E L E M E N T F O R C E S

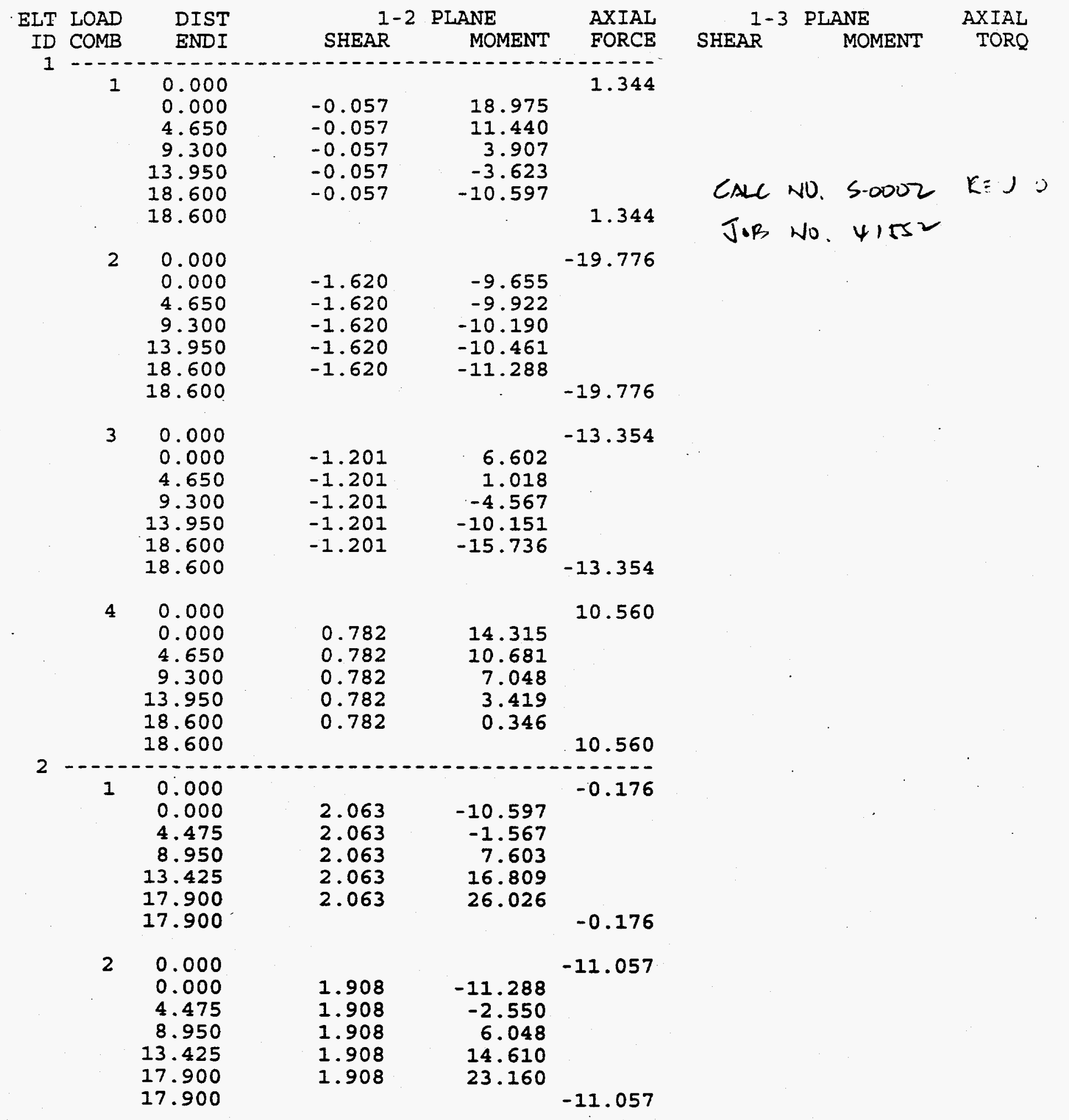


URS CONSULTANTS, INC.

$$
\text { Sint 4is of }
$$

(LANL NMSF BUILDING) 2-D VERTICAL ANALYSIS OF ROOF - (VNMSF2)

PROGRAM : SAP $90 / F I L E$ : VNMSF2.F3F

\begin{tabular}{|c|c|c|c|c|c|c|c|c|c|}
\hline $\begin{array}{l}\text { ELT } \\
\text { ID }\end{array}$ & $\begin{array}{l}\text { LOAD } \\
\text { COMB }\end{array}$ & $\begin{array}{l}\text { DIST } \\
\text { ENDI }\end{array}$ & SHEAR ${ }^{1-2}$ & $\begin{array}{l}\text { PLANE } \\
\text { MOMENT }\end{array}$ & $\begin{array}{l}\text { AXIAL } \\
\text { FORCE }\end{array}$ & SHEAR & PLANE & MENT & $\begin{array}{l}\text { AXIAL } \\
\text { TORQ }\end{array}$ \\
\hline & 3 & $\begin{array}{r}0.000 \\
0.000 \\
4.475 \\
8.950 \\
13.425 \\
17.900 \\
17.900\end{array}$ & $\begin{array}{l}2.856 \\
2.856 \\
2.856 \\
2.856 \\
2.856\end{array}$ & $\begin{array}{r}-15.736 \\
-2.957 \\
9.822 \\
22.600 \\
35.379\end{array}$ & -8.070 & CAL & No. & 50002. & $r=3.0$ \\
\hline & 4 & $\begin{array}{r}0.000 \\
0.000 \\
4.475 \\
8.950 \\
13.425 \\
17.900 \\
17.900\end{array}$ & $\begin{array}{l}0.078 \\
0.078 \\
0.078 \\
0.078 \\
0.078\end{array}$ & $\begin{array}{l}0.346 \\
0.492 \\
0.778 \\
1.099 \\
1.433\end{array}$ & 5.440 & J.B & No & 41552 & \\
\hline 3 & 1 & $\begin{array}{l}0.000 \\
0.000 \\
2.467 \\
4.933 \\
7.400 \\
9.867 \\
9.867\end{array}$ & $\begin{array}{l}1.582 \\
1.582 \\
1.582 \\
1.582 \\
1.582\end{array}$ & $\begin{array}{r}18.975 \\
11.316 \\
3.685 \\
2.107 \\
5.981\end{array}$ & 0.159 & & & 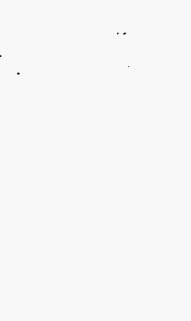 & \\
\hline & 2 & $\begin{array}{l}0.000 \\
0.000 \\
2.467 \\
4.933 \\
7.400 \\
9.867 \\
9.867\end{array}$ & $\begin{array}{l}-3.107 \\
-3.107 \\
-3.107 \\
-3.107 \\
-3.107\end{array}$ & $\begin{array}{r}-9.655 \\
-5.759 \\
-1.890 \\
-4.074 \\
-11.710\end{array}$ & -0.983 & & & & \\
\hline & 3 & $\begin{array}{l}0.000 \\
0.000 \\
2.467 \\
4.933 \\
7.400 \\
9.867 \\
9.867\end{array}$ & $\begin{array}{l}-1.084 \\
-1.084 \\
-1.084 \\
-1.084 \\
-1.084\end{array}$ & $\begin{array}{r}6.602 \\
3.929 \\
1.256 \\
-1.417 \\
-4.089\end{array}$ & -0.680 & & & & \\
\hline & 4 & $\begin{array}{l}0.000 \\
0.000 \\
2.467 \\
4.933 \\
7.400 \\
9.867 \\
9.867\end{array}$ & $\begin{array}{l}2.345 \\
2.345 \\
2.345 \\
2.345 \\
2.345\end{array}$ & $\begin{array}{r}14.315 \\
8.538 \\
2.788 \\
3.091 \\
8.845\end{array}$ & 0.571 & & & & \\
\hline
\end{tabular}


URS CONSULTANTS, INC.

(LANL NMSF BUILDING) 2-D VERTICAL ANALYSIS OF ROOF - (VNMSF2)

PROGRAM : SAP90/FILE : VNMSF2. F3F

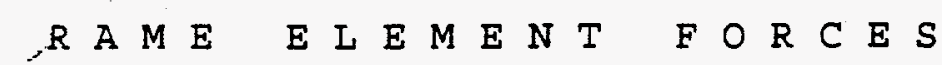

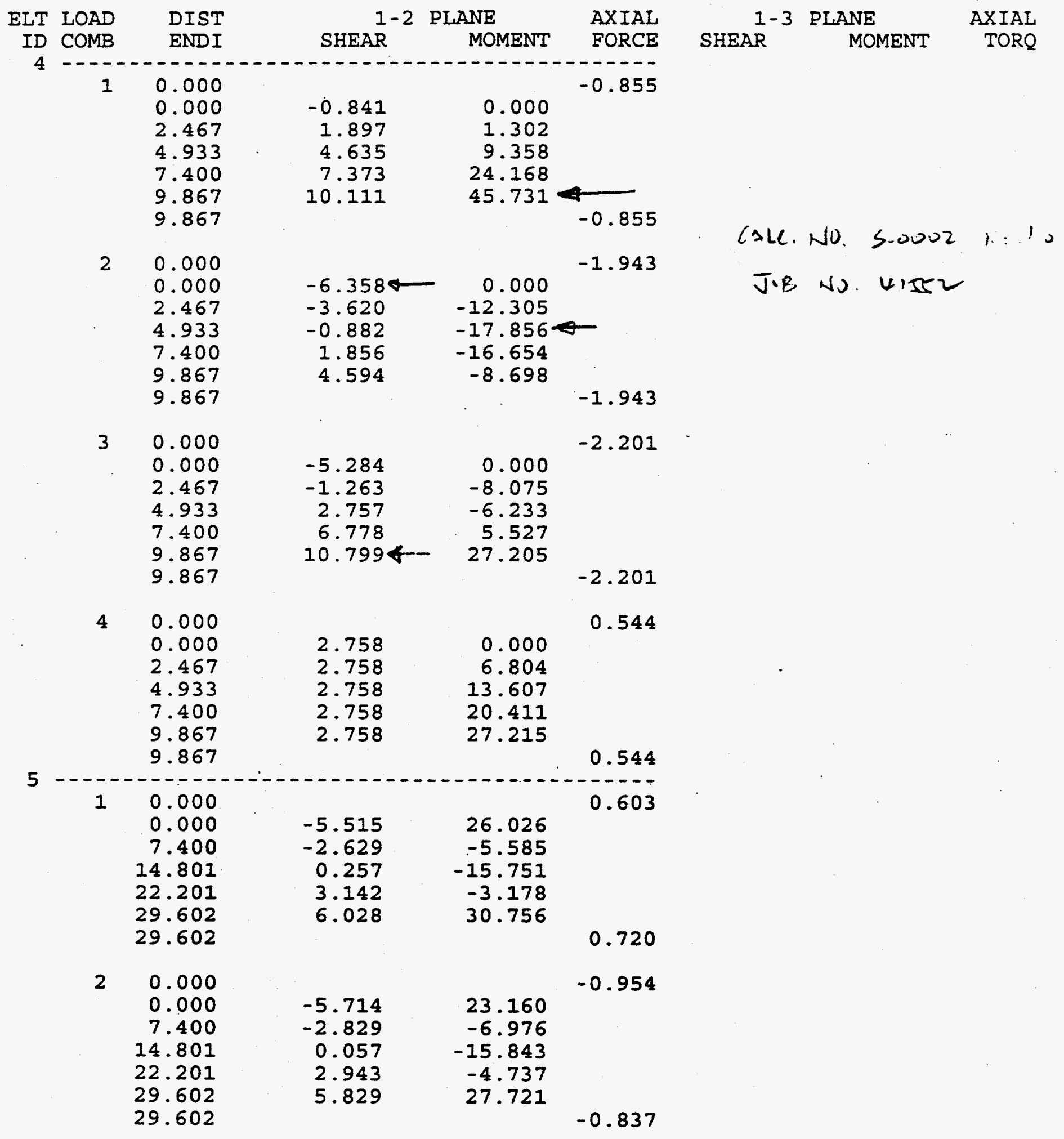


URS CONSULTANTS, INC.

$$
\text { Sh. . 417 of }
$$

PAGE 4 (LANL NMSF BUILDING) 2-D VERTICAL ANALYSIS OF ROOF - (VNMSF2)

PROGRAM : SAP90/FILE : VNMSF2 . F3F R A M E E L E M E N T FOR C E S

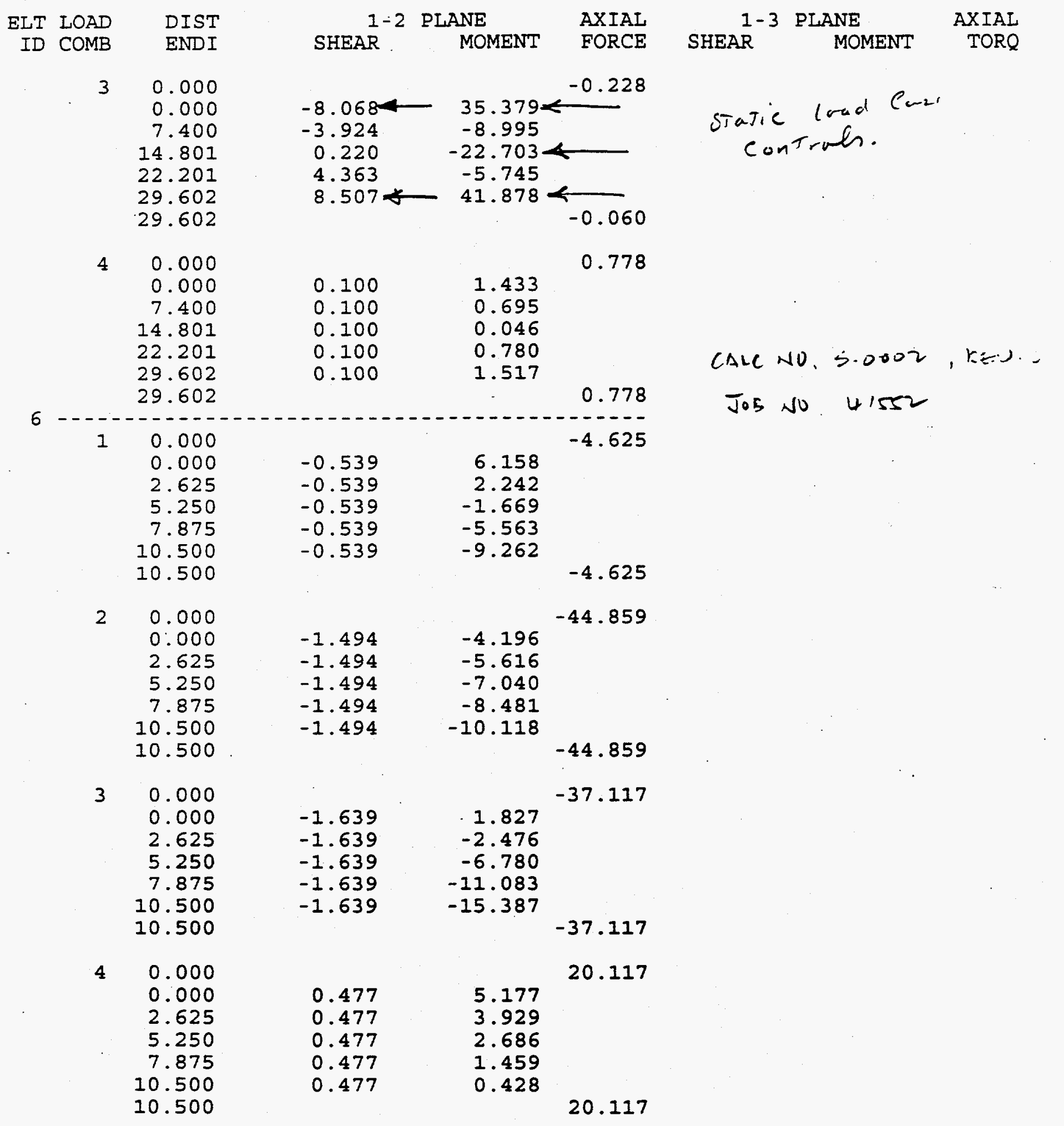


URS CONSULTANTS, INC.

(LANL NMSF BUILDING) 2-D VERTICAL ANALYSIS OF ROOF - (VNMSF2)

PROGRAM : SAP90/EILE : VNMSF2 . F3F $\mathrm{R}$ R A M E L E M E N T F O R C E S

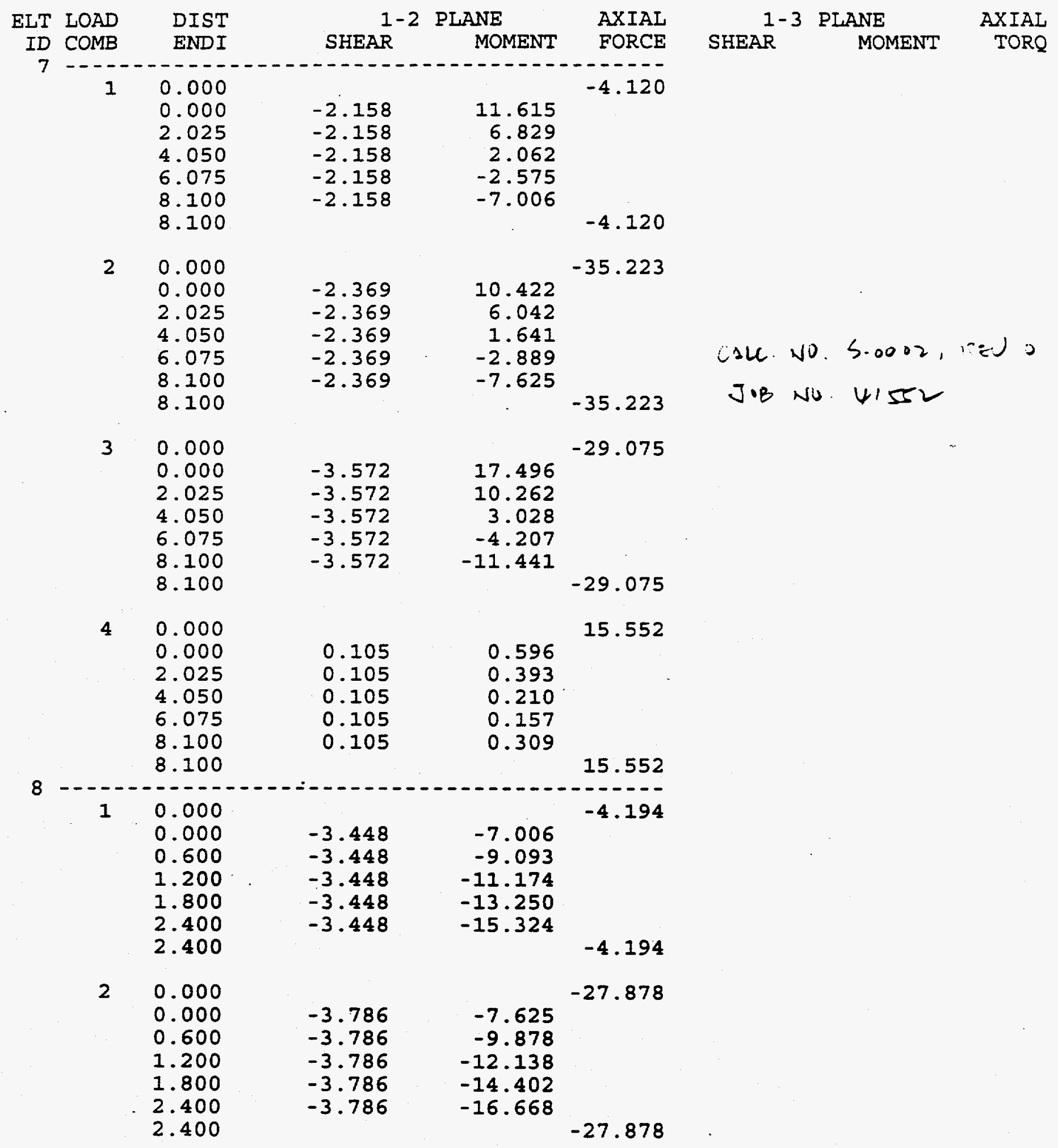


URS CONSULTANTS, INC.

$$
\text { Sht } 419=
$$

(LANL NMSF BUILDING) 2-D VERTICAL ANALYSIS OF ROOF - (VNMSF2)

PROGRAM : SAP9O/FILE: VNMSF2 . F3F RAME ELEMENT FORCES

\begin{tabular}{|c|c|c|c|c|c|c|c|c|}
\hline $\begin{array}{l}\text { ELT } \\
\text { ID }\end{array}$ & $\begin{array}{l}\text { LOAD } \\
\text { COMB }\end{array}$ & $\begin{array}{l}\text { DIST } \\
\text { ENDI }\end{array}$ & ${ }_{\text {SHEAR }}^{1-2}$ & $\begin{array}{l}\text { PLANE } \\
\text { MOMENT }\end{array}$ & $\begin{array}{l}\text { AXIAL } \\
\text { FORCE }\end{array}$ & $\begin{array}{l}1-3 \quad P 1 \\
\text { SHEAR }\end{array}$ & $\begin{array}{l}\text { MLANE } \\
\text { MOMENT }\end{array}$ & $\begin{array}{r}\text { AXIAL } \\
\text { TORQ }\end{array}$ \\
\hline & 3 & $\begin{array}{l}0.000 \\
0.000 \\
0.600 \\
1.200 \\
1.800 \\
2.400 \\
2.400\end{array}$ & $\begin{array}{l}-5.720 \\
-5.720 \\
-5.720 \\
-5.720 \\
-5.720\end{array}$ & $\begin{array}{l}-11.441 \\
-14.873 \\
-18.305 \\
-21.737 \\
-25.168\end{array}$ & -23.775 & & & \\
\hline & 4 & $\begin{array}{l}0.000 \\
0.000 \\
0.600 \\
1.200 \\
1.800 \\
2.400 \\
2.400\end{array}$ & $\begin{array}{l}0.169 \\
0.169 \\
0.169 \\
0.169 \\
0.169\end{array}$ & $\begin{array}{l}0.309 \\
0.392 \\
0.482 \\
0.576 \\
0.672\end{array}$ & 11.842 & $\begin{array}{l}\text { CALE. NO. } \\
\text { JOB No }\end{array}$ & $\begin{array}{l}\text { s-0002, } \\
41552\end{array}$ & $K=J U$ \\
\hline 9 & $-\overline{1}$ & $\begin{array}{r}0.000 \\
0.000 \\
3.950 \\
7.900 \\
11.850 \\
15.800 \\
15.800\end{array}$ & $\begin{array}{l}0.265 \\
0.265 \\
0.265 \\
0.265 \\
0.265\end{array}$ & $\begin{array}{l}3.565 \\
3.987 \\
4.855 \\
5.876 \\
6.915\end{array}$ & -2.502 & & & \\
\hline & 2 & $\begin{array}{r}0.000 \\
0.000 \\
3.950 \\
7.900 \\
11.850 \\
15.800 \\
15.800\end{array}$ & $\begin{array}{l}0.075 \\
0.075 \\
0.075 \\
0.075 \\
0.075\end{array}$ & $\begin{array}{l}2.657 \\
3.580 \\
4.058 \\
4.382 \\
4.688\end{array}$ & -19.671 & & & \\
\hline & 3 & $\begin{array}{r}0.000 \\
0.000 \\
3.950 \\
7.900 \\
11.850 \\
15.800 \\
15.800\end{array}$ & $\begin{array}{l}0.183 \\
0.183 \\
0.183 \\
0.183 \\
0.183\end{array}$ & $\begin{array}{l}5.308 \\
6.031 \\
6.754 \\
7.476 \\
8.199\end{array}$ & -15.909 & & & \\
\hline & 4 & $\begin{array}{r}0.000 \\
0.000 \\
3.950 \\
7.900 \\
11.850 \\
15.800 \\
15.800\end{array}$ & $\begin{array}{l}0.095 \\
0.095 \\
0.095 \\
0.095 \\
0.095\end{array}$ & $\begin{array}{l}0.454 \\
0.204 \\
0.399 \\
0.747 \\
1.113\end{array}$ & 8.585 & & & \\
\hline
\end{tabular}


URS CONSULTANTS, INC.

(LANL NMSF BUILDING) 2-D VERTICAL ANALYSIS OF ROOF - (VNMSF2)

PROGRAM : SAP90/FILE: VNMSF2. F3F R A M E E L E M E N T FOR C E S

\begin{tabular}{|c|c|c|c|c|c|c|c|c|c|}
\hline \multirow{5}{*}{$\begin{array}{r}\text { ELT } \\
\text { ID } \\
10\end{array}$} & $\begin{array}{l}\text { LOAD } \\
\text { COMB }\end{array}$ & $\begin{array}{l}\text { DIST } \\
\text { ENDI }\end{array}$ & SHEAR & $\begin{array}{l}\text { PLANE } \\
\text { MOMENT }\end{array}$ & $\begin{array}{l}\text { AXIAL } \\
\text { FORCE }\end{array}$ & SHEAR & 3 PL & $\begin{array}{l}\text { MNE } \\
\text { MOMENT }\end{array}$ & $\begin{array}{r}\text { AXIAL } \\
\text { TORQ }\end{array}$ \\
\hline & 1 & $\begin{array}{r}0.000 \\
0.000 \\
5.975 \\
11.950 \\
17.925 \\
23.900 \\
23.900\end{array}$ & $\begin{array}{l}0.012 \\
0.012 \\
0.012 \\
0.012 \\
0.012\end{array}$ & $\begin{array}{r}4.065 \\
2.090 \\
0.117 \\
-1.041 \\
-0.975\end{array}$ & $\begin{array}{l}0.950 \\
0.950\end{array}$ & & & & \\
\hline & 2 & $\begin{array}{r}0.000 \\
0.000 \\
5.975 \\
11.950 \\
17.925 \\
23.900 \\
23.900\end{array}$ & $\begin{array}{l}-0.331 \\
-0.331 \\
-0.331 \\
-0.331 \\
-0.331\end{array}$ & $\begin{array}{l}-1.257 \\
-1.188 \\
-1.120 \\
-1.868 \\
-3.839\end{array}$ & 0.168 & $\begin{array}{l}C \Delta L C . \\
\bar{J} \circ B\end{array}$ & $\begin{array}{l}\text { No. } \\
\text { No }\end{array}$ & $\begin{array}{l}5.0002 \\
41552\end{array}$ & $x \equiv J$ \\
\hline & 3 & $\begin{array}{r}0.000 \\
0.000 \\
5.975 \\
11.950 \\
17.925 \\
23.900 \\
23.900\end{array}$ & $\begin{array}{l}-0.241 \\
-0.241 \\
-0.241 \\
-0.241 \\
-0.241\end{array}$ & $\begin{array}{r}2.067 \\
0.626 \\
-0.816 \\
-2.257 \\
-3.698\end{array}$ & 0.906 & & & & \\
\hline & 4 & $\begin{array}{r}0.000 \\
0.000 \\
5.975 \\
11.950 \\
17.925 \\
23.900 \\
23.900\end{array}$ & $\begin{array}{l}0.171 \\
0.171 \\
0.171 \\
0.171 \\
0.171\end{array}$ & $\begin{array}{l}2.661 \\
1.639 \\
0.619 \\
0.413 \\
1.432\end{array}$ & 0.391 & & . & & \\
\hline 11 & 1 & $\begin{array}{r}0.000 \\
0.000 \\
5.975 \\
11.950 \\
17.925 \\
23.900 \\
23.900\end{array}$ & $\begin{array}{r}-4.987 \\
-2.537 \\
-0.087 \\
2.362 \\
4.812\end{array}$ & $\begin{array}{r}21.712 \\
-1.766 \\
-10.603 \\
-3.817 \\
17.616\end{array}$ & 1.838 & & & & $=$ \\
\hline & 2 & $\begin{array}{r}0.000 \\
0.000 \\
5.975 \\
11.950 \\
17.925 \\
23.900 \\
23.900\end{array}$ & $\begin{array}{r}-5.154 \\
-2.705 \\
-0.255 \\
2.195 \\
4.645\end{array}$ & $\begin{array}{r}19.706 \\
-2.773 \\
-10.618 \\
-4.811 \\
15.622\end{array}$ & 0.656 & & & & \\
\hline
\end{tabular}


URS CONSULTANTS, INC.

$$
\sin ^{-} .42105
$$

PROGRAM : SAP9O/FILE : VNMSF2 . F3F

(LANL NMSF BUILDING) 2-D VERTICAL ANALYSIS OF ROOF - (VNMSF2) RA ME ELEMENT FORCES

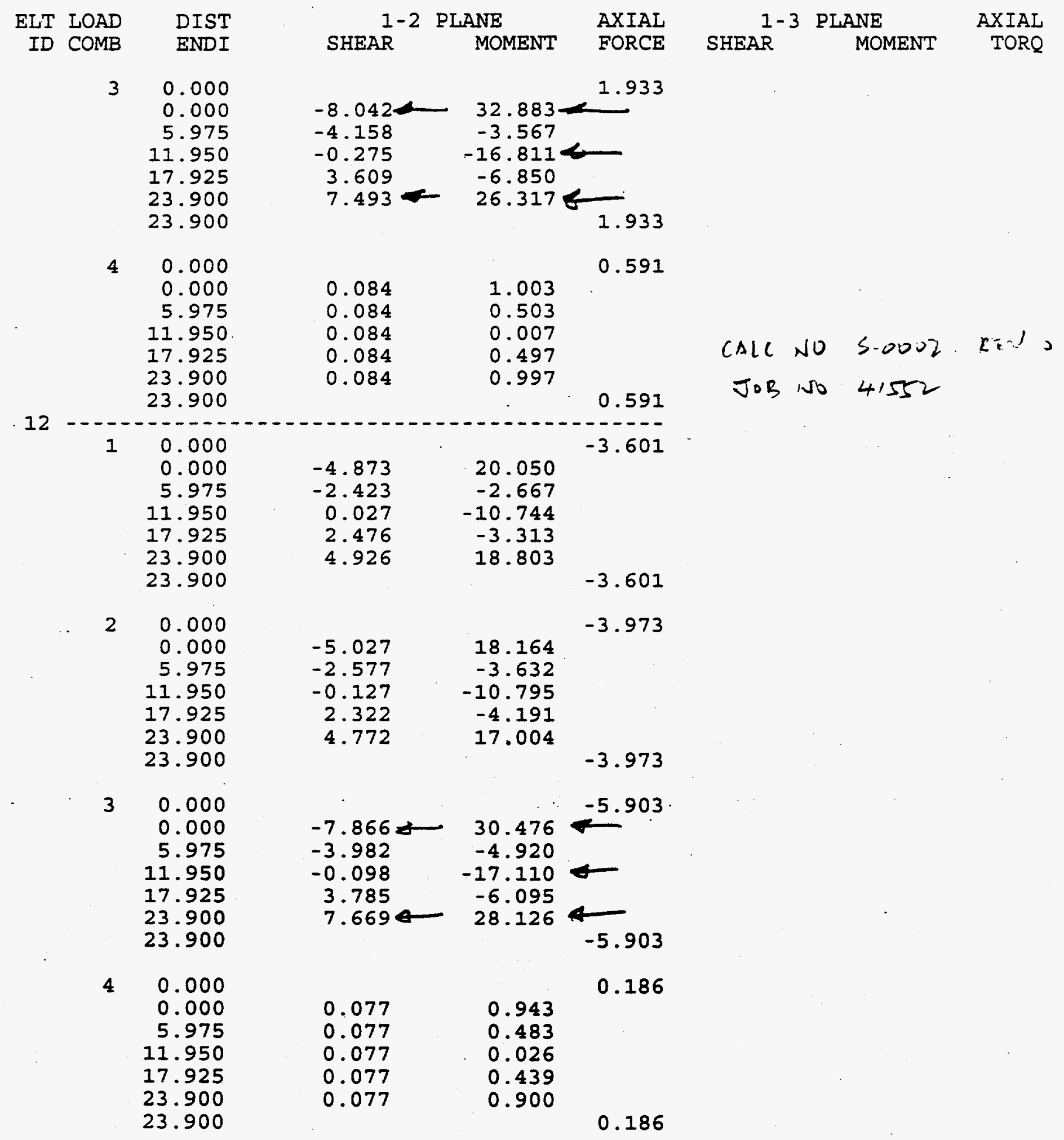


URS CONSULTANTS, INC.

(LANL NMSF BUILDING) 2-D VERTICAL ANALYSIS OF ROOF - (VNMSF2)

PROGRAM : SAP90/FILE : VNMSF2. F3F R A M E E L E M E N T FOR C E S

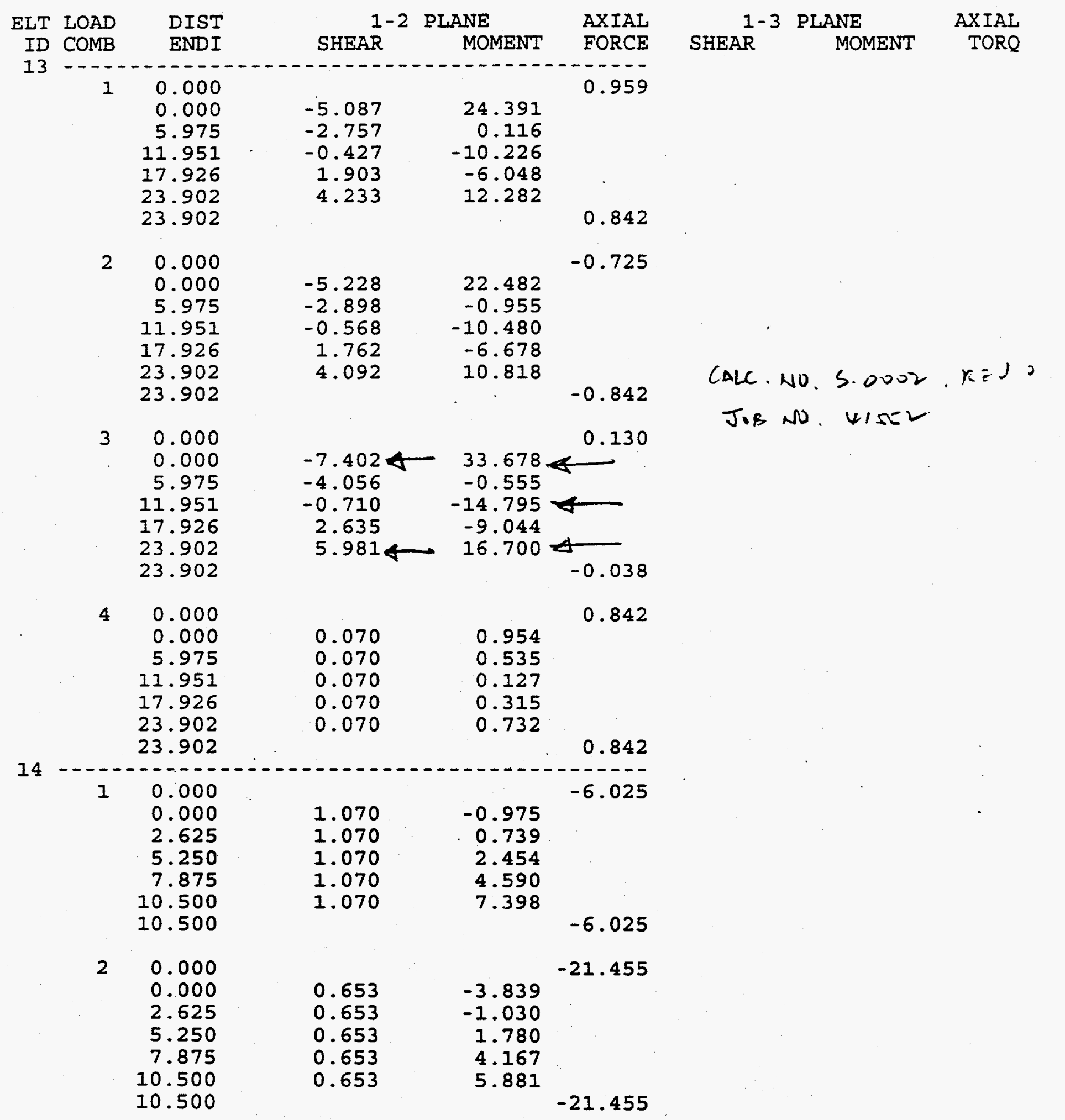


URS CONSULTANTS, INC.

Snt $423 \mathrm{gj}^{\circ}$

PAGE 10

(LANL NMSF BUILDING) 2-D VERTICAL ANALYSIS OF ROOF - (VNMSF2)

PROGRAM : SAP90/FILE : VNMSF2. F3F R A M E E L E M E N T FO R C E S

\begin{tabular}{|c|c|c|c|c|c|c|c|c|}
\hline $\begin{array}{r}\text { ELT } \\
\text { ID }\end{array}$ & $\begin{array}{l}\text { LOAD } \\
\text { COMB }\end{array}$ & $\begin{array}{l}\text { DIST } \\
\text { ENDI }\end{array}$ & SHEAR $^{1-2}$ & $\begin{array}{l}\text { PLANE } \\
\text { MOMENT }\end{array}$ & $\begin{array}{l}\text { AXIAL } \\
\text { FORCE }\end{array}$ & SHEAR & $\begin{array}{l}\text { NE } \\
\text { MOMENT }\end{array}$ & $\begin{array}{r}\text { AXIAL } \\
\text { TORQ }\end{array}$ \\
\hline & 3 & $\begin{array}{r}0.000 \\
0.000 \\
2.625 \\
5.250 \\
7.875 \\
10.500 \\
10.500\end{array}$ & $\begin{array}{l}1.343 \\
1.343 \\
1.343 \\
1.343 \\
1.343\end{array}$ & $\begin{array}{r}-3.698 \\
-0.174 \\
3.350 \\
6.875 \\
10.399\end{array}$ & -21.143 & . & & \\
\hline & 4 & $\begin{array}{r}0.000 \\
0.000 \\
2.625 \\
5.250 \\
7.875 \\
10.500 \\
10.500\end{array}$ & $\begin{array}{l}0.209 \\
0.209 \\
0.209 \\
0.209 \\
0.209\end{array}$ & $\begin{array}{l}1.432 \\
0.884 \\
0.337 \\
0.211 \\
0.759\end{array}$ & 7.715 & cale. No. & 5.0002. & REบ. 0 \\
\hline 15 & $\begin{array}{c}\cdots \\
1\end{array}$ & $\begin{array}{r}0.000 \\
0.000 \\
2.625 \\
5.250 \\
7.875 \\
10.500 \\
10.500\end{array}$ & $\begin{array}{l}1.888 \\
1.888 \\
1.888 \\
1.888 \\
1.888\end{array}$ & $\begin{array}{r}-9.738 \\
-5.088 \\
-0.310 \\
4.644 \\
9.599\end{array}$ & -3.433 & JoR NO & 4152 & \\
\hline & 2 & $\begin{array}{r}0.000 \\
0.000 \\
2.625 \\
5.250 \\
7.875 \\
10.500 \\
10.500\end{array}$ & $\begin{array}{l}1.771 \\
1.771 \\
1.771 \\
1.771 \\
1.771\end{array}$ & $\begin{array}{r}-10.221 \\
-5.266 \\
-0.440 \\
4.210 \\
8.860\end{array}$ & -14.590 & & & \\
\hline & 3 & $\begin{array}{r}0.000 \\
0.000 \\
2.625 \\
5.250 \\
7.875 \\
10.500 \\
10.500\end{array}$ & $\begin{array}{l}2.942 \\
2.942 \\
2.942 \\
2.942 \\
2.942\end{array}$ & $\begin{array}{r}-15.918 \\
-8.195 \\
-0.472 \\
7.250 \\
14.973\end{array}$ & -13.650 & & & \\
\hline & 4 & $\begin{array}{r}0.000 \\
0.000 \\
2.625 \\
5.250 \\
7.875 \\
10.500 \\
10.500\end{array}$ & $\begin{array}{l}0.058 \\
0.058 \\
0.058 \\
0.058 \\
0.058\end{array}$ & $\begin{array}{l}0.241 \\
0.089 \\
0.065 \\
0.217 \\
0.370\end{array}$ & 5.579 & & & \\
\hline
\end{tabular}


URS CONSULTANTS, INC.

PAGE 11

(LANL NMSF BUILDING) 2-D VERTICAL ANALYSIS OF ROOF - (VNMSF2)

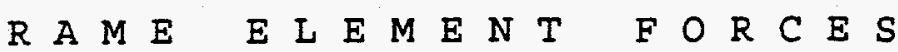

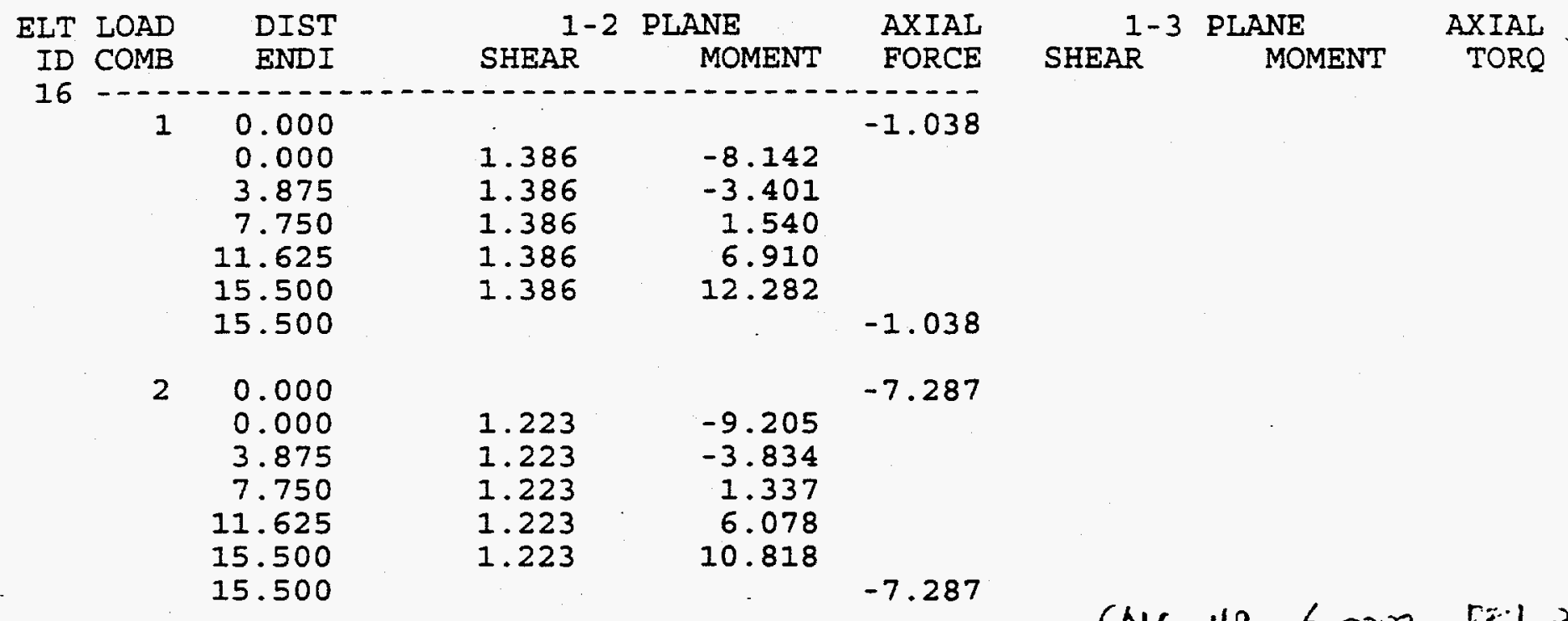

$3 \quad 0.000$

0.000

3.875

1.926

$-13.153$

$-5.981$

7.750

1.926

$-5.689$

1.926

1. 774

11.625

1.926

9.237

15.500

1.926

16.700

15.500

$4 \quad 0.000$

0.000

3.875

7.750

11.625

15.500

15.500

17

0.000

0.000

4.650

9.300

13.950

18.600

18.600

0.081

0.081

0.081

0.081

0.081

CALC. NO, S-0002. E:-J. O

J०8 D. 41552

$-5.981$

3.125

0.532

0.216

0.102

0.416

0.732

3.125

$-5.177$

0.406

4.836

$0.406 \quad 3.627$

$0.406 \quad 2.418$

$0.406 \quad 1.209$

0.406

0.000

$-5.177$

20.000

0.000

$-0.260$

$-20.553$

4.650

$-0.260$

$-7.549$

$-5.661$

9.300

$-0.260$

$-3.774$

13.950

$-0.260$

$-1.887$

18.600

$-0.260$

0.000

18.600

$-20.553$ 
URS CONSULTANTS, INC.

$$
\text { Sint } 425 \text { of }
$$

PAGE 12

(LANL NMSE BUILDING) 2-D VERTICAL ANALYSIS OF ROOF - (VNMSF2)

PROGRAM : SAP9O/FILE : VNMSF2 . F3F R A M E E L E M E N T FOR C E S

\begin{tabular}{|c|c|c|c|c|c|c|c|c|}
\hline $\begin{array}{r}\text { ELT } \\
\text { ID }\end{array}$ & $\begin{array}{l}\text { LOAD } \\
\text { COMB }\end{array}$ & $\begin{array}{l}\text { DIST } \\
\text { ENDI }\end{array}$ & SHEAR & $\begin{array}{l}\text { PLANE } \\
\text { MOMENT }\end{array}$ & $\begin{array}{l}\text { AXIAL } \\
\text { FORCE }\end{array}$ & SHEAR & $\begin{array}{l}\text { PLANE } \\
\text { MOMENT }\end{array}$ & $\begin{array}{r}\text { AXIAL } \\
\text { TORQ }\end{array}$ \\
\hline & 3 & $\begin{array}{r}0.000 \\
0.000 \\
4.650 \\
9.300 \\
13.950 \\
18.600 \\
18.600\end{array}$ & $\begin{array}{l}0.101 \\
0.101 \\
0.101 \\
0.101 \\
0.101\end{array}$ & $\begin{array}{r}-1.883 \\
-1.412 \\
-0.941 \\
-0.471 \\
0.000\end{array}$ & -18.856 & ' & & \\
\hline 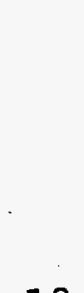 & 4 & $\begin{array}{r}0.000 \\
0.000 \\
4.650 \\
9.300 \\
13.950 \\
18.600 \\
18.600\end{array}$ & $\begin{array}{l}0.333 \\
0.333 \\
0.333 \\
0.333 \\
0.333\end{array}$ & $\begin{array}{r}6.193 \\
4.644 \\
3.096 \\
1.548 \\
0.000\end{array}$ & 7.688 & CALC & $5-0002$ & KEJ.o \\
\hline 18 & 1 & $\begin{array}{r}0.000 \\
0.000 \\
4.650 \\
9.300 \\
13.950 \\
18.600 \\
18.600\end{array}$ & $\begin{array}{l}0.167 \\
0.167 \\
0.167 \\
0.167 \\
0.167\end{array}$ & $\begin{array}{l}4.118 \\
3.089 \\
2.059 \\
1.030 \\
0.000\end{array}$ & -6.583 & JOR & No. 4152 & 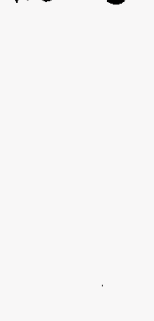 \\
\hline & 2 & $\begin{array}{r}0.000 \\
0.000 \\
4.650 \\
9.300 \\
13.950 \\
18.600 \\
18.600\end{array}$ & $\begin{array}{l}-0.221 \\
-0.221 \\
-0.221 \\
-0.221 \\
-0.221\end{array}$ & $\begin{array}{r}-3.101 \\
-2.326 \\
-1.551 \\
-0.775 \\
0.000\end{array}$ & -18.930 & & & \\
\hline & 3 & $\begin{array}{r}0.000 \\
0.000 \\
4.650 \\
9.300 \\
13.950 \\
18.600 \\
18.600\end{array}$ & $\begin{array}{l}-0.048 \\
-0.048 \\
-0.048 \\
-0.048 \\
-0.048\end{array}$ & $\begin{array}{l}0.890 \\
0.668 \\
0.445 \\
0.223 \\
0.000\end{array}$ & -18.809 & & & \\
\hline & 4 & $\begin{array}{r}0.000 \\
0.000 \\
4.650 \\
9.300 \\
13.950 \\
18.600 \\
18.600\end{array}$ & $\begin{array}{l}0.194 \\
0.194 \\
0.194 \\
0.194 \\
0.194\end{array}$ & $\begin{array}{l}3.610 \\
2.707 \\
1.805 \\
0.902 \\
0.000\end{array}$ & 6.174 & & & \\
\hline
\end{tabular}


URS CONSULTANTS, INC.

PAGE 13 (LANL NMSF BUILDING) 2-D VERTICAL ANALYSIS OF ROOF - (VNMSF2)

PROGRAM : SAP90/FILE : VNMSF2.F3F -

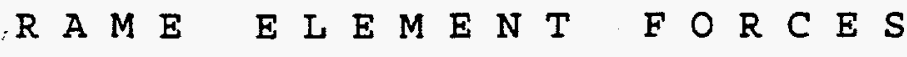

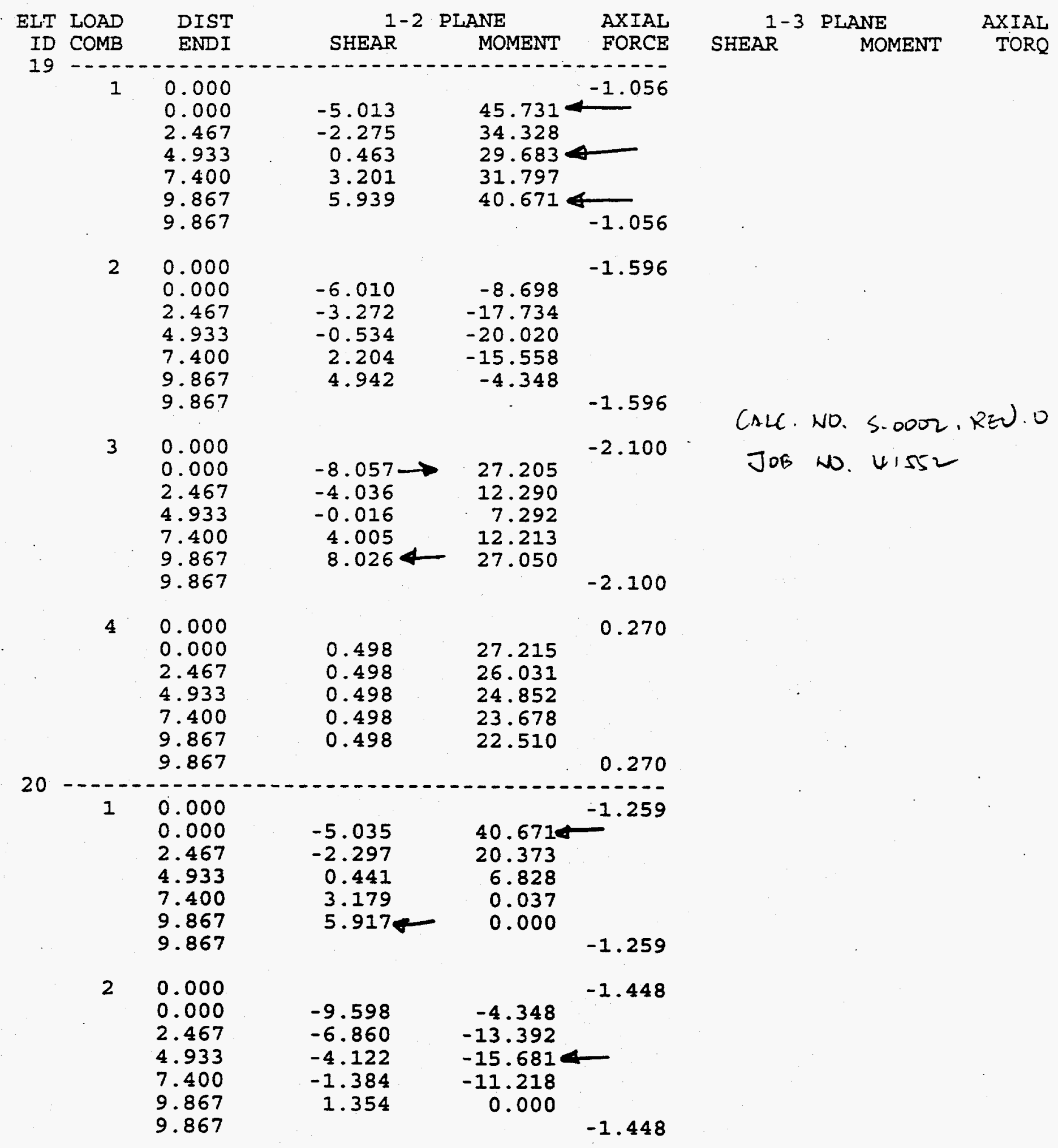


URS CONSULTANTS, INC.

(LANL NMSF BUILDING) 2-D VERTICAL ANALYSIS OF ROOF - (VNMSF2)

$\triangle R$ A M E E L E M E N T FOR C E S

\begin{tabular}{|c|c|c|c|c|c|c|c|c|c|}
\hline $\begin{array}{r}\text { ELT } \\
\text { ID }\end{array}$ & $\begin{array}{l}\text { LOAD } \\
\text { COMB }\end{array}$ & $\begin{array}{l}\text { DIST } \\
\text { ENDI }\end{array}$ & SHEAR & $\begin{array}{l}\text { ANE } \\
\text { MOMENT }\end{array}$ & $\begin{array}{l}\text { AXIAL } \\
\text { FORCE }\end{array}$ & SHEAR & 3 PL & $\begin{array}{l}\text { ANE } \\
\text { MOMENT }\end{array}$ & $\begin{array}{r}\text { AXIAL } \\
\text { TORQ }\end{array}$ \\
\hline & 3 & $\begin{array}{l}0.000 \\
0.000 \\
2.467 \\
4.933 \\
7.400 \\
9.867 \\
9.867\end{array}$ & $\begin{array}{l}-10.783 \\
-6.762 \\
-2.742 \\
1.279 \\
5.300\end{array}$ & $\begin{array}{r}27.050 \\
5.411 \\
-6.310 \\
-8.114 \\
0.000\end{array}$ & -2.147 & & & & \\
\hline & 4 & $\begin{array}{l}0.000 \\
0.000 \\
2.467 \\
4.933 \\
7.400 \\
9.867 \\
9.867\end{array}$ & $\begin{array}{l}2.281 \\
2.281 \\
2.281 \\
2.281 \\
2.281\end{array}$ & $\begin{array}{r}22.510 \\
16.882 \\
11.255 \\
5.627 \\
0.000\end{array}$ & 0.095 & CALC. & No. & $S .0002$ & $r^{2}=J$ \\
\hline 21 & 1 & $\begin{array}{l}0.000 \\
0.000 \\
2.467 \\
4.933 \\
7.400 \\
9.867 \\
9.867\end{array}$ & $\begin{array}{l}0.150 \\
0.150 \\
0.150 \\
0.150 \\
0.150\end{array}$ & $\begin{array}{l}1.551 \\
1.526 \\
1.502 \\
1.481 \\
1.463\end{array}$ & -0.174 & $J \circ \beta$ & No. & 4152 & \\
\hline & 2 & $\begin{array}{l}0.000 \\
0.000 \\
2.467 \\
4.933 \\
7.400 \\
9.867 \\
9.867\end{array}$ & $\begin{array}{l}-0.015 \\
-0.015 \\
-0.015 \\
-0.015 \\
-0.015\end{array}$ & $\begin{array}{l}-4.568 \\
-4.211 \\
-3.855 \\
-3.502 \\
-3.152\end{array}$ & -0.795 & & & & \\
\hline & 3 & $\begin{array}{l}0.000 \\
0.000 \\
2.467 \\
4.933 \\
7.400 \\
9.867 \\
9.867\end{array}$ & $\begin{array}{l}0.093 \\
0.093 \\
0.093 \\
0.093 \\
0.093\end{array}$ & $\begin{array}{l}-2.207 \\
-1.978 \\
-1.750 \\
-1.522 \\
-1.293\end{array}$ & -0.781 & & & & \\
\hline & 4 & $\begin{array}{l}0.000 \\
0.000 \\
2.467 \\
4.933 \\
7.400 \\
9.867 \\
9.867\end{array}$ & $\begin{array}{l}0.083 \\
0.083 \\
0.083 \\
0.083 \\
0.083\end{array}$ & $\begin{array}{l}3.059 \\
2.868 \\
2.679 \\
2.492 \\
2.307\end{array}$ & 0.310 & & & & \\
\hline
\end{tabular}


URS CONSULTANTS, INC.

$$
\sin ^{+} \operatorname{cic} 6
$$

PAGE 15 (LANL NMSF BUILDING) 2-D VERTICAL ANALYSIS OF ROOF - (VNMSF2)

PROGRAM : SAP9O/FILE : VNMSF2 . F3F R A M E E L E M E N T FOR C E S

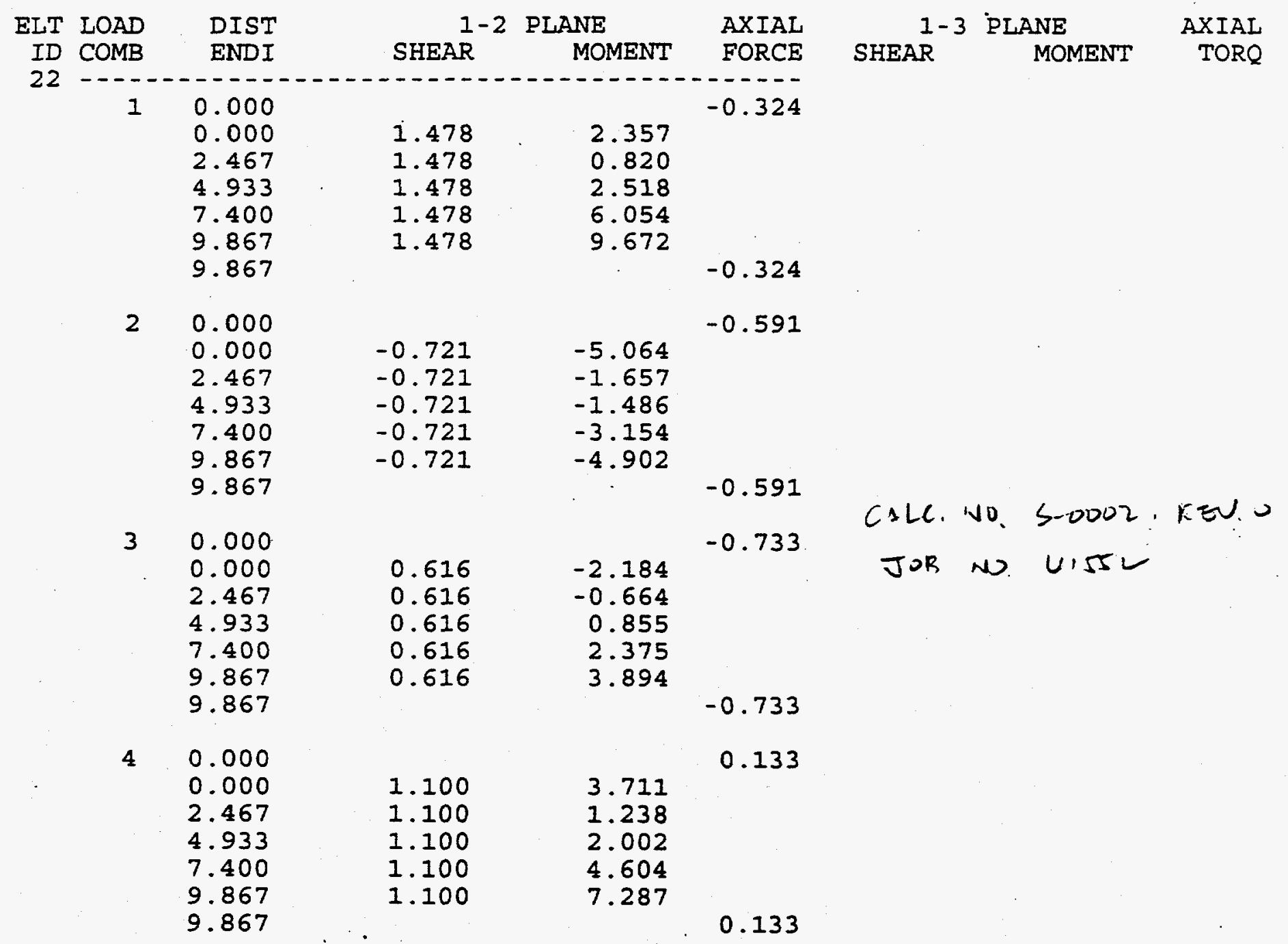




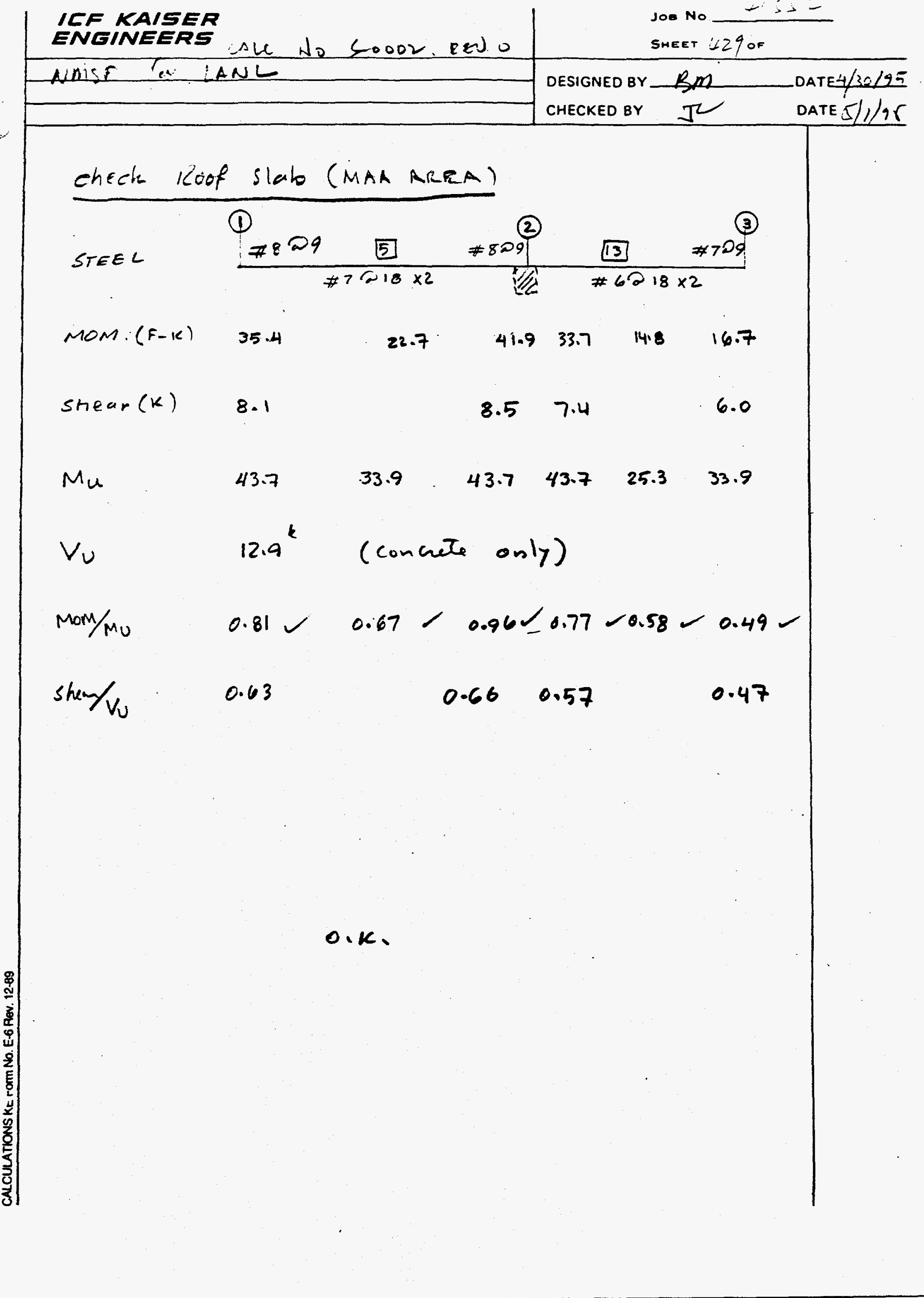




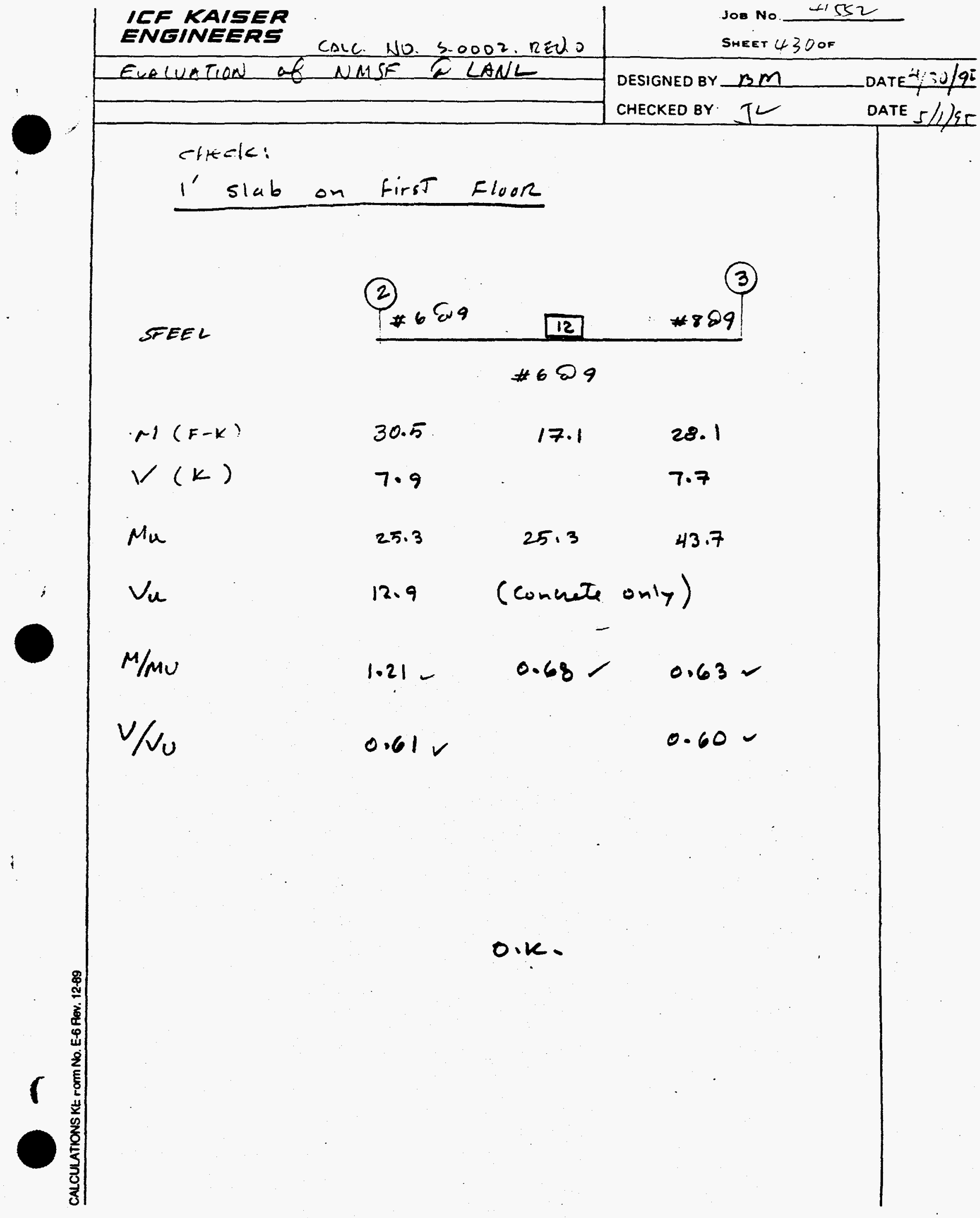




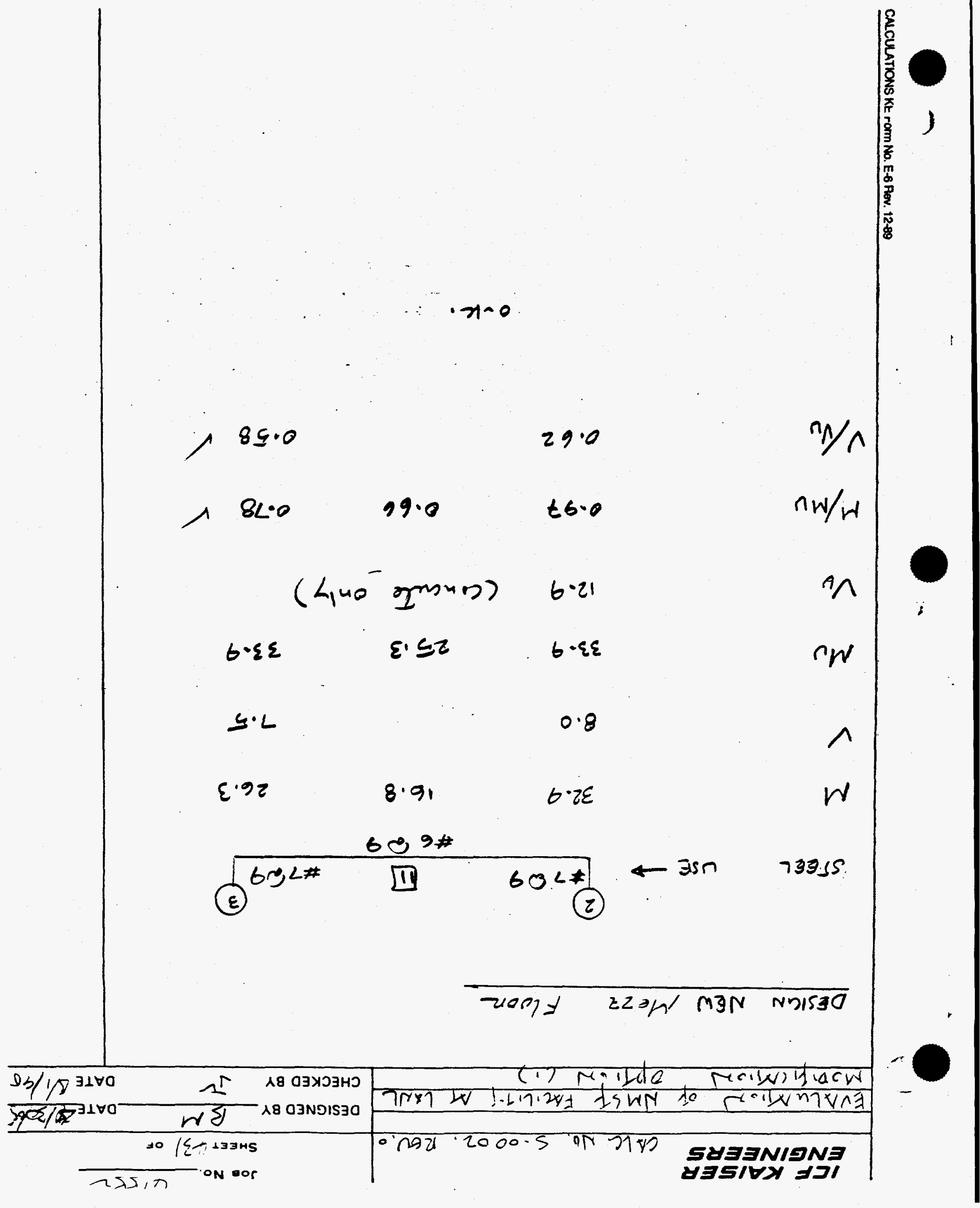




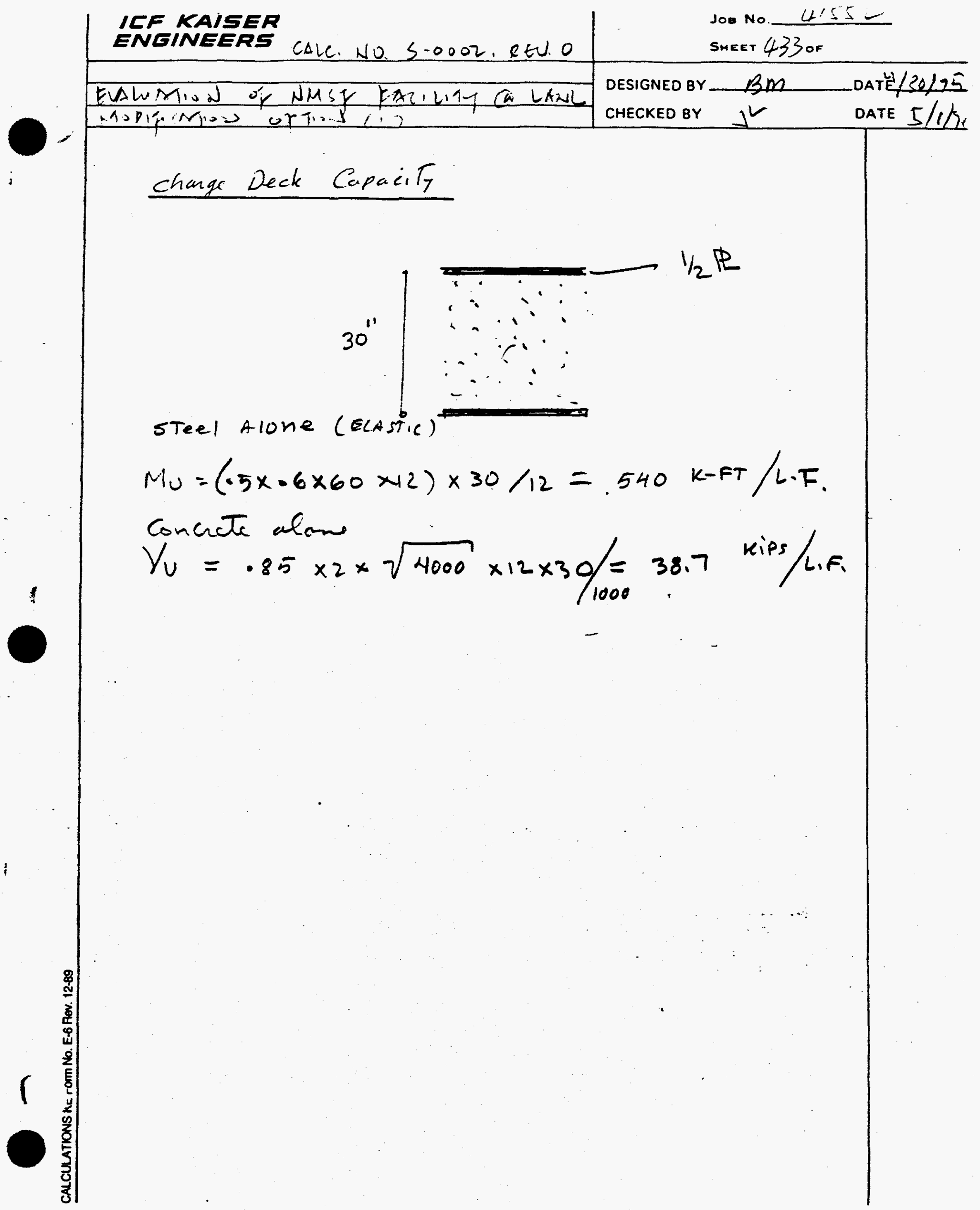


CALC. NO. S.0002, EU.J LAISER

JOB NO. 41552-056-03

SHEET 434 o

-ENGINEER

DESIGNED BY:

CHECKED BY:

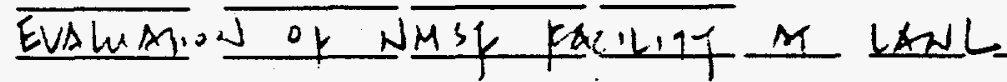

DATE:

DATE:

ROOF AND FLOOR SLAB CAPACITIES (MODIFIED NMSF BLDG)

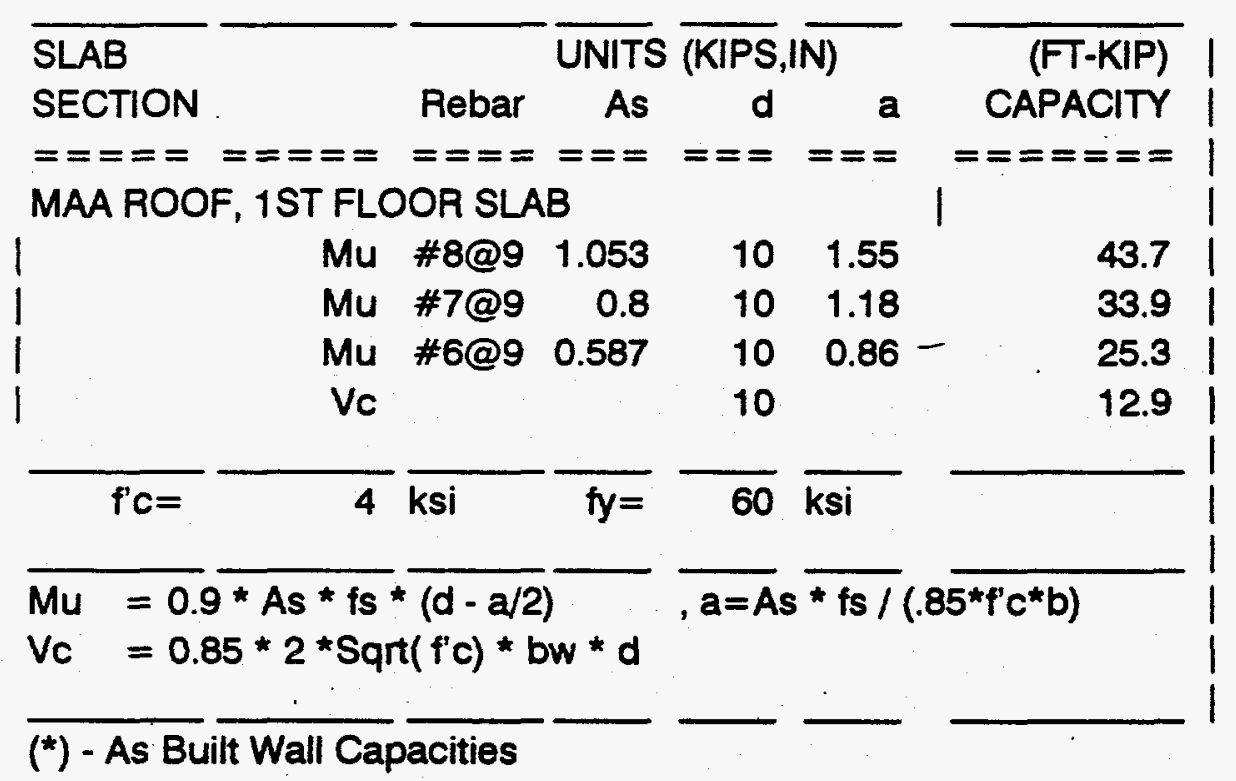


IVF KAISER

ENGINEERS

Jos No. T' Bi

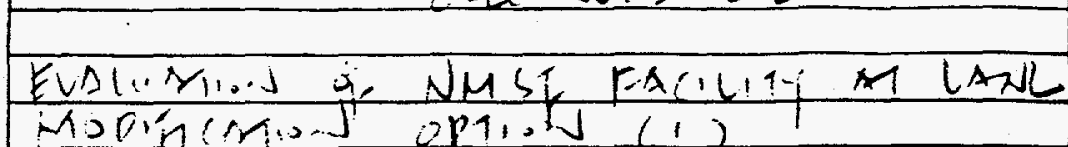

DESIGNED BY $\mathrm{rm}$

DATE $5 / 2 / 95$

CHECKED BY IL

DATE 5 (II) ar

check $30^{\prime}$ SPAN ROOF BEAMS (RB-8)

REF: $\angle A-2 J-B-5-3.2$

using Tributary of si le.

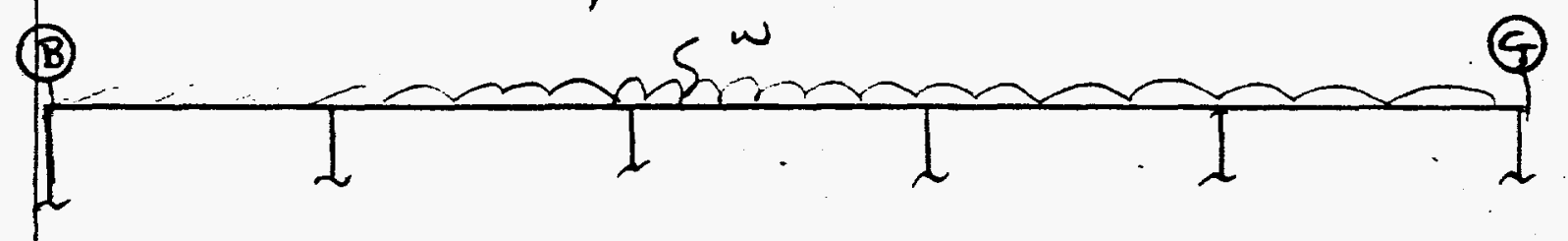

Tribe wed Th $=\left[(20-4)+\left(28-8^{\prime \prime}\right)\right] / 2=29.5^{\prime}$

$1.4 D L+1.74$ This L.C. Controls

$$
\omega=1.4 D L+1.7 L L=0.555 \times 29.5=16.4 \mathrm{~K} / \mathrm{C} . \mathrm{F} .
$$

Max Pos. Mam $=\omega \ell^{2} / 16$

$$
\begin{aligned}
M_{n} & =16.4 \times 30^{2} / 16 \\
& =923 \quad \mathrm{FT}-\mathrm{KIP}
\end{aligned}
$$

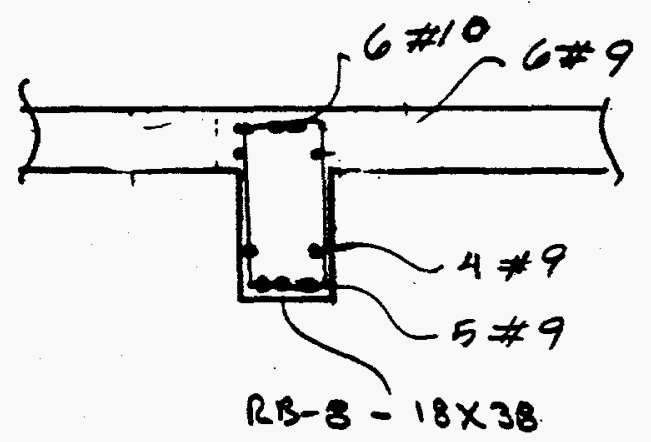

Max Neg Mom $=$

$$
M_{n}=w e^{2} / 10=1476 \text { Fr-kins }
$$

positive mom: $\quad A_{s}=9 \times 1=9 \mathrm{in}^{2}$.

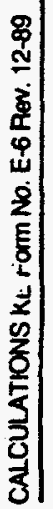

$b=30 / 4=7.5$

$a=\frac{9 \times 60}{.85 \times 4 \times 7.5 \times 12}=1.76^{\prime \prime}, d \approx 38-8=30^{\circ}$ Cons.

$M_{u}=.9 \times 9 \times 60 \times\left(30-\frac{1.76}{2}\right)=14,15.3 \mathrm{k}$-in

$=1179 \mathrm{~K}-\mathrm{FT}$

$D / c=1023 / 1179=0.780 . k$. 


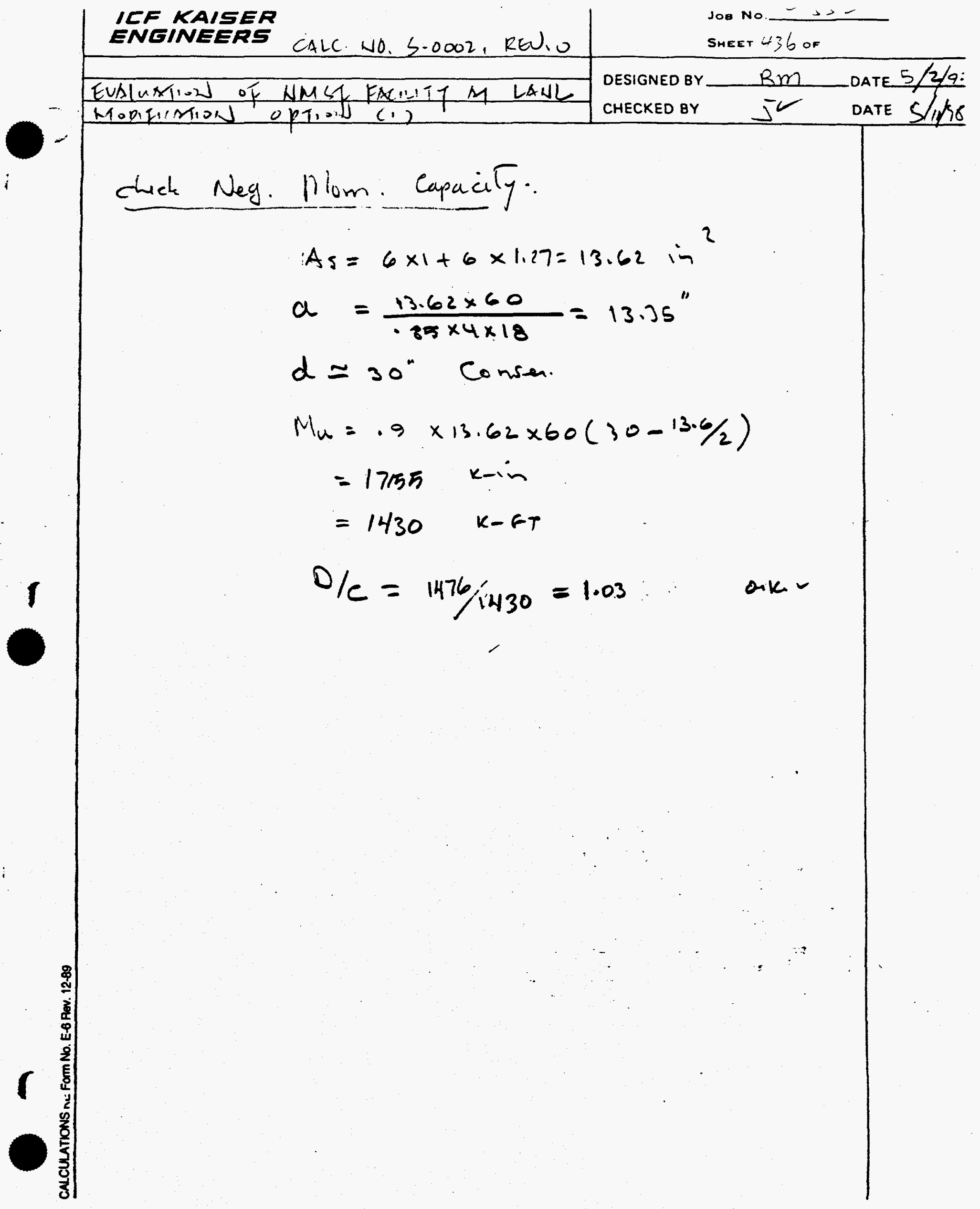


IVF KAISER
ENGINEERS

$-$

EVALUMiva of NMSF FrailTY AT LONL Mopigi:Mioz OPTion (1)

DESIGNED BY

Joe No. $415=$

$-$

click shear of Rear locums

vertical stieecus \#3/

$$
\begin{aligned}
& V_{n}=V_{c}+V_{s} \\
&=2 \sqrt{f_{c}^{\prime}} b_{w} d+\frac{A_{w} f_{y d}}{3} \\
&=2 \sqrt{4000} \times 18 \times(38-5) \times 16+\frac{.11 \times 2 \times 60 \times 33}{3} \\
&=75.1+145.2=220.3 \mathrm{kips} \\
& V_{n}=\frac{30}{2} \times 16.4=246>0.85 \times 220.3=187 \\
& \mathrm{NGG}
\end{aligned}
$$

IF Remove Top soil:

$$
\begin{aligned}
& w=8.275 \\
& V_{n}=15 \times(0.275 \times 29.5)=122^{\text {kips }}<187 \quad 0 . k
\end{aligned}
$$

$\because$ Roof beam is As- BuiLT streocture sonly ok. if Top soil is Removed.

- In the modified structure shear malls are added between supporting Columns and Top soil need nut be removed. 


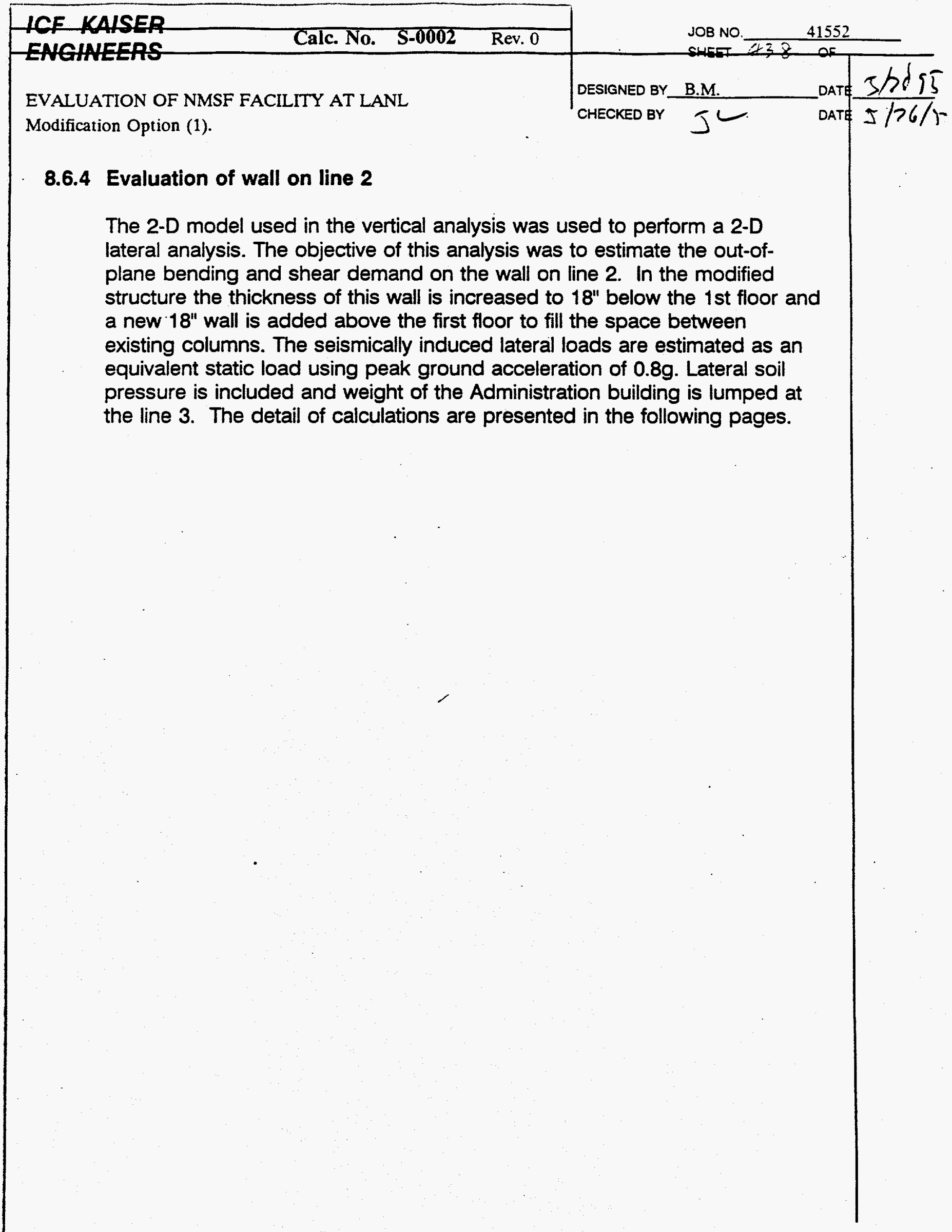


| IEF KAISER

ENEINEERS

CALC. NO. S-0002 REV.O

JOB No

$+5$

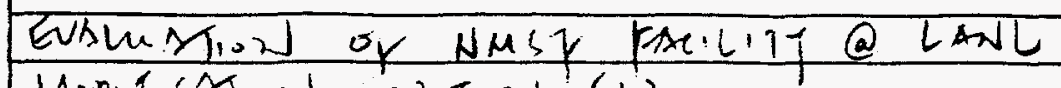

SMEET 439 OF

Mopipeniond option (1)

DESIGNED BY BM

DATE $5 / x / 4 c$

CHECKED BY JL DATES $/ 2 C h$ Ar

2D Equivatent Static Analyis of umsF Facitity

- use a Transverse strip of NMSF Blds.

- Neglect STiffress of Admin Area

- Lump mass of Aelmin area to maA BLdy.

- Perform Equivalent lateral static andysis using Peack sreciral acculeratim.

- Include hateral soil Pressurs Computes earher

- Investigite adequacer be Barement eural on line 2

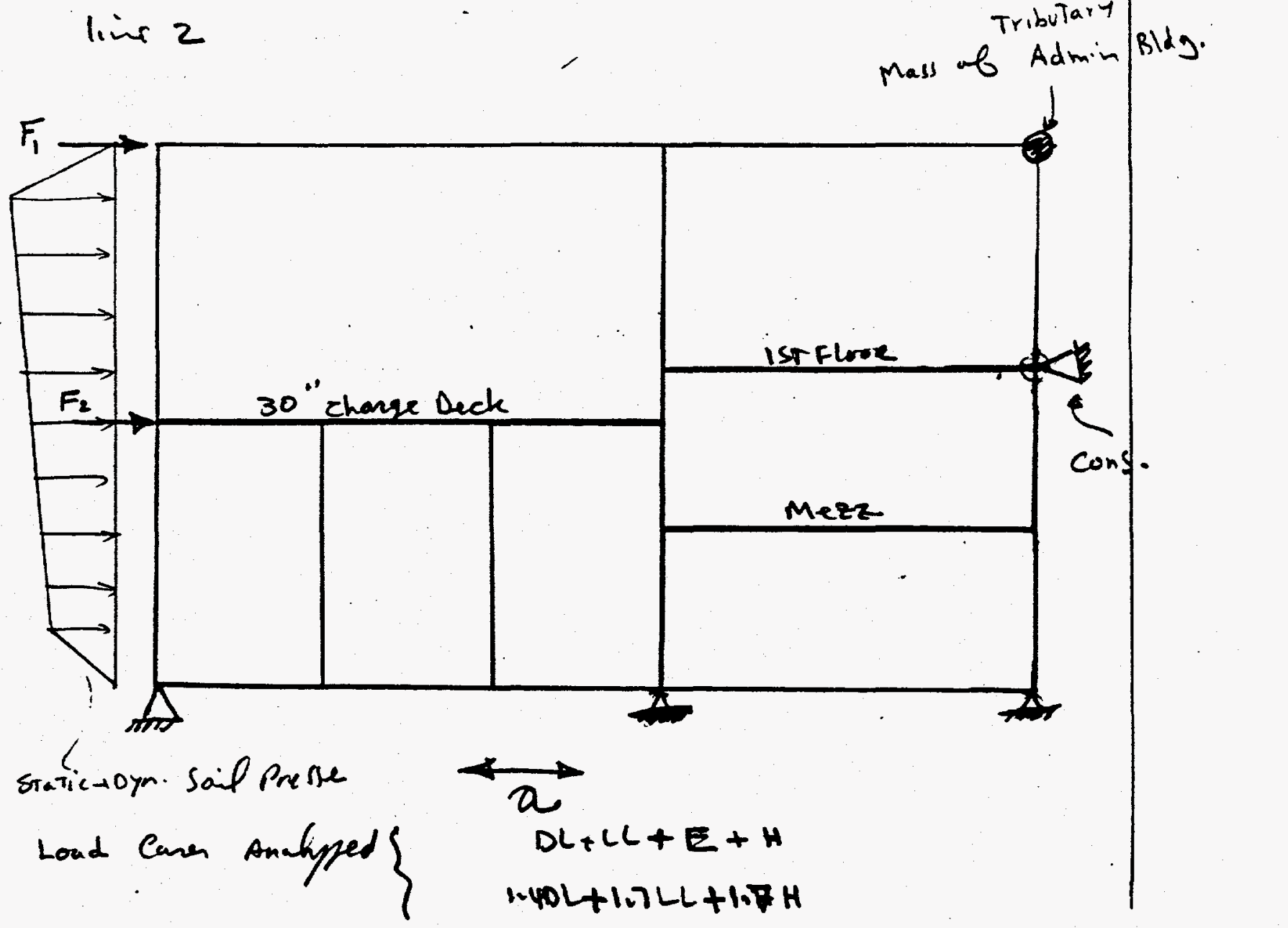




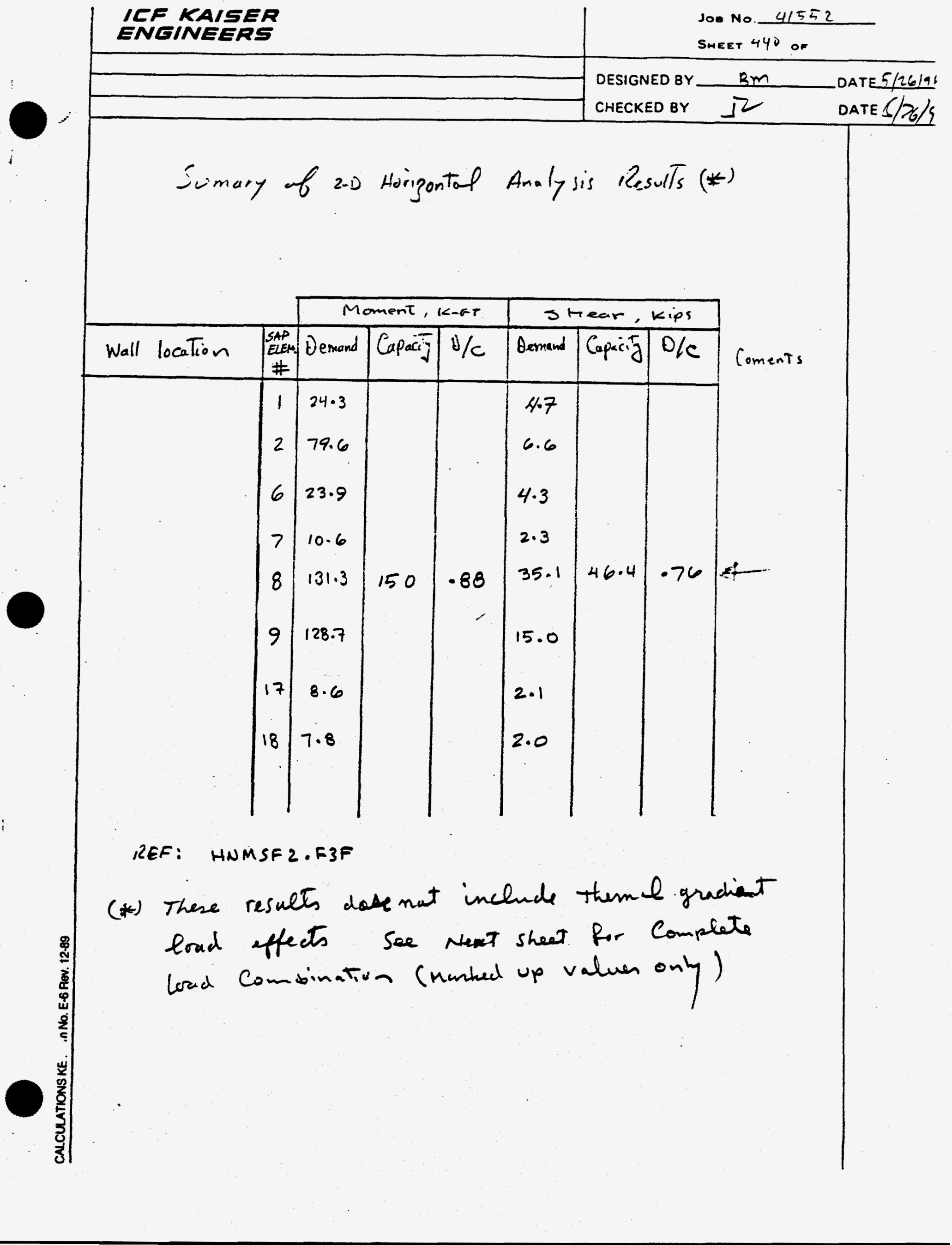


IEF KAYSER
ENGINEERS

Joe No. 41552

SHEET \&U/ OF

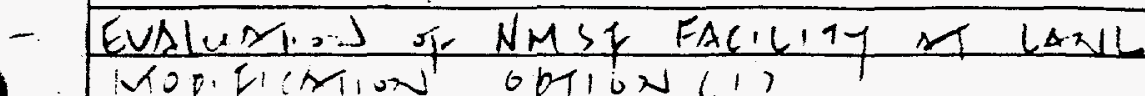

DESIGNED BY $\Omega M$

DATE $526 / 5$

MOD. Finis option (1)

CHECKED BY $\widetilde{J L}$

DATE $/ / 2 / 5$

Laterul Soit Pressure.

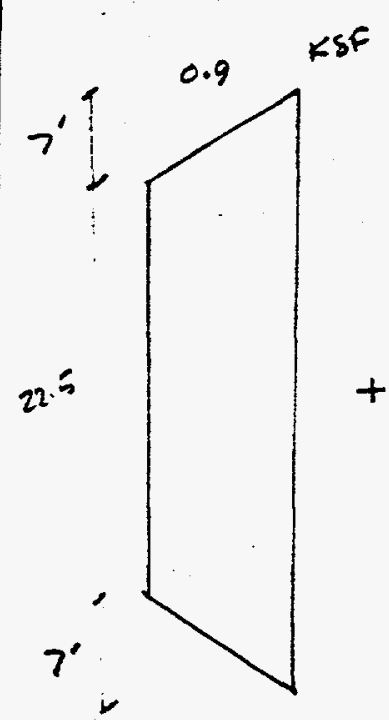

STATIC

$D\{M$

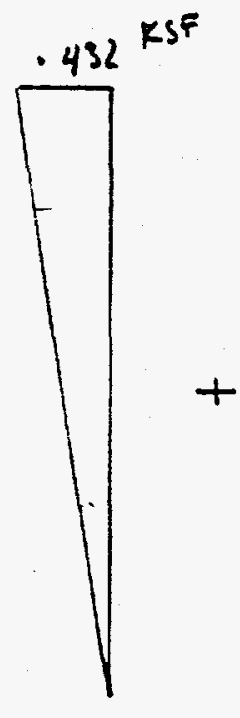

Dyn.

Mononube -

Okabe

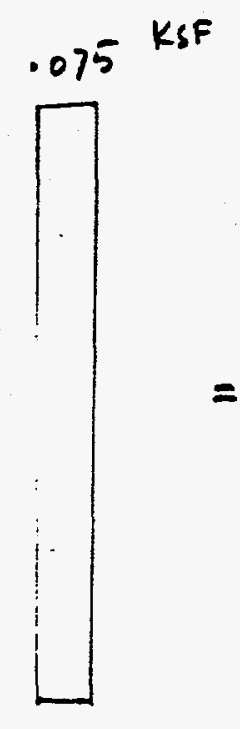

ssatic

surchang

$.250 \times \cdot 3=.075$

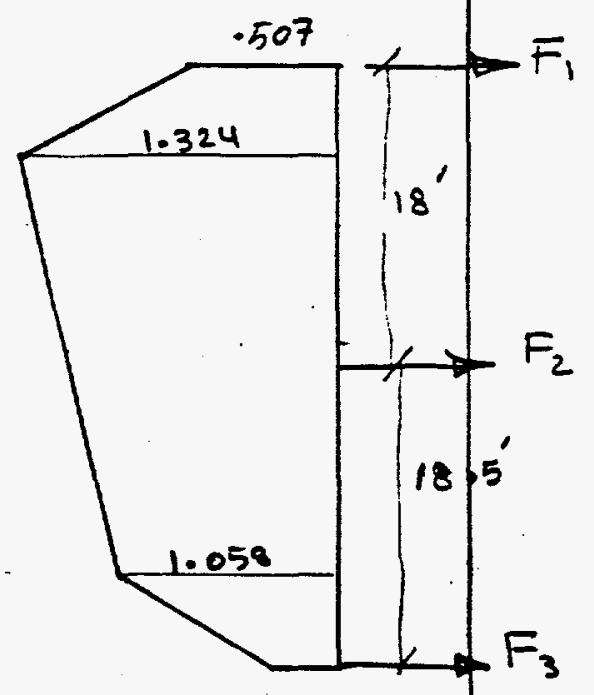

.075

$F_{1}=\left(\left(\frac{10}{2}-7\right) \times .9+\frac{7}{2} \times .9\right)+\left(.432+\frac{18.5+9}{36.5} \times .432\right) \frac{9}{2}+9 \times .075$ $=4.95+3.409+.675=9.034 \mathrm{kips} / \mathrm{LF}$

$F_{2}=\left(\frac{18+18.5}{2}\right) \cdot 9+\left((18.5+9) \times \frac{-432}{36.5}+\frac{18.5}{2} \times \frac{.432}{36.5}\right) \times \frac{18+18.5}{2 \times 2}+\frac{18+1855}{2} \times \cdot 075$

$=16.425+3.969+1.369=21.763^{K}$

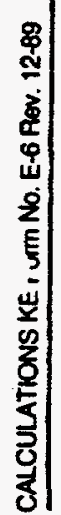




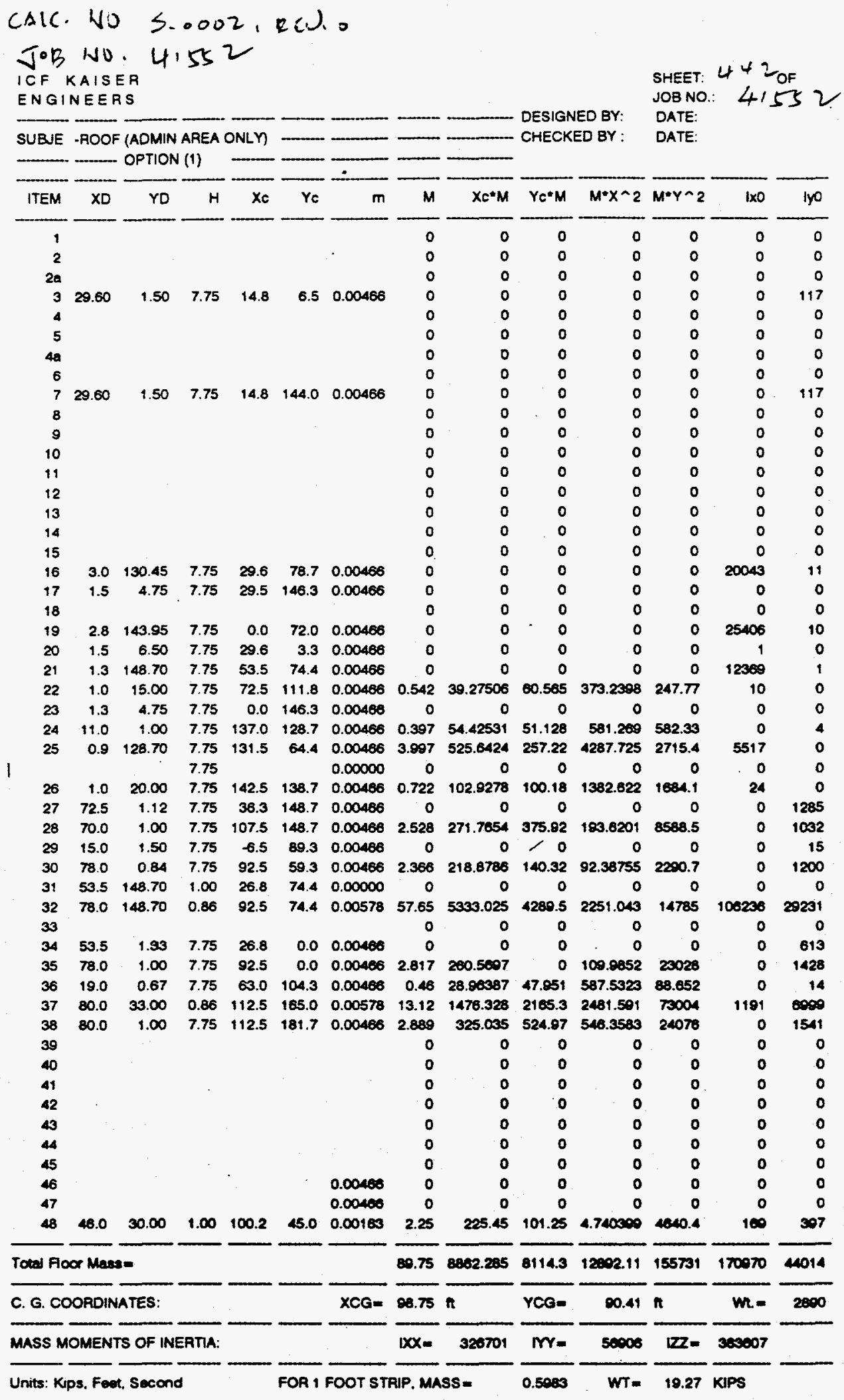




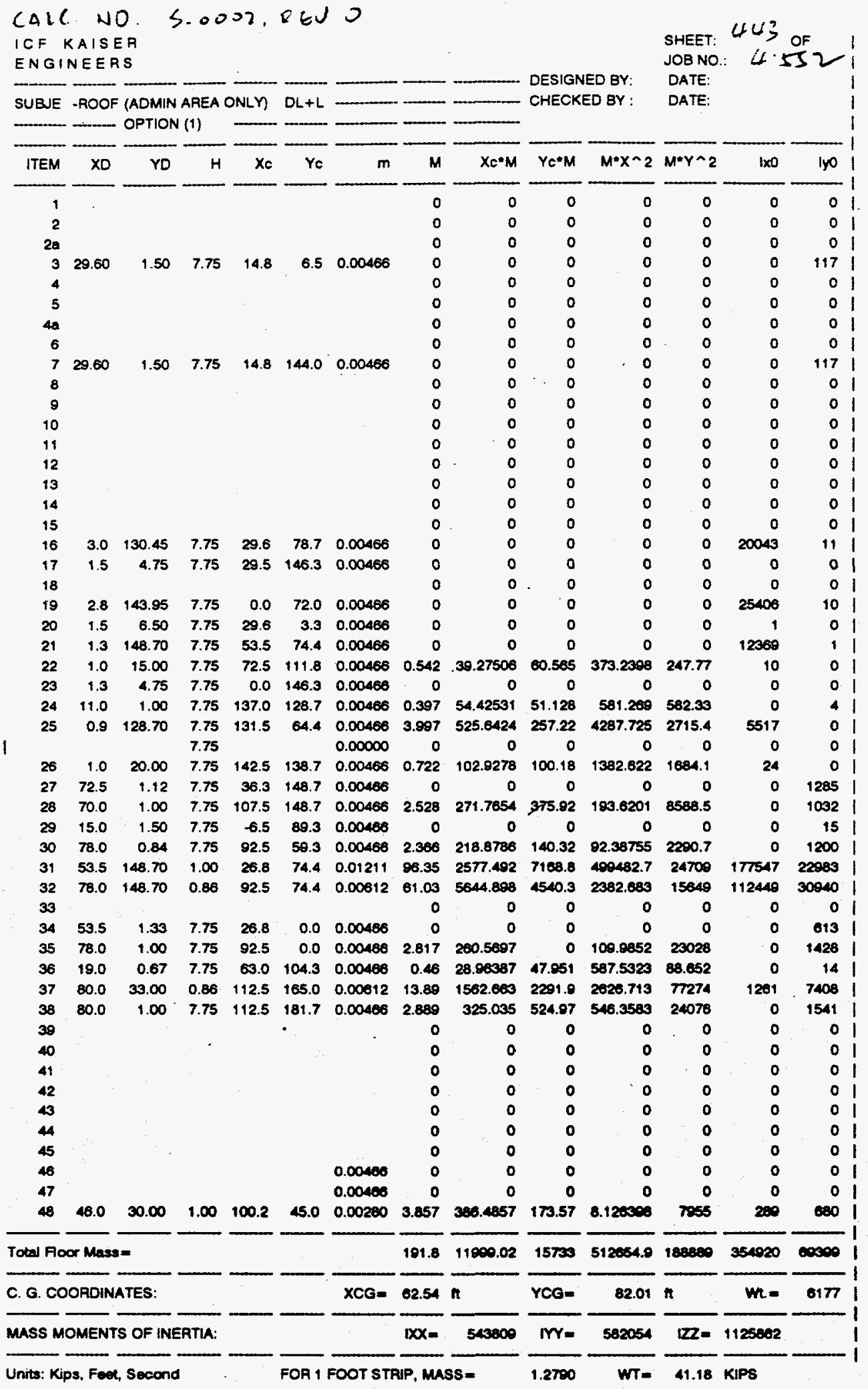




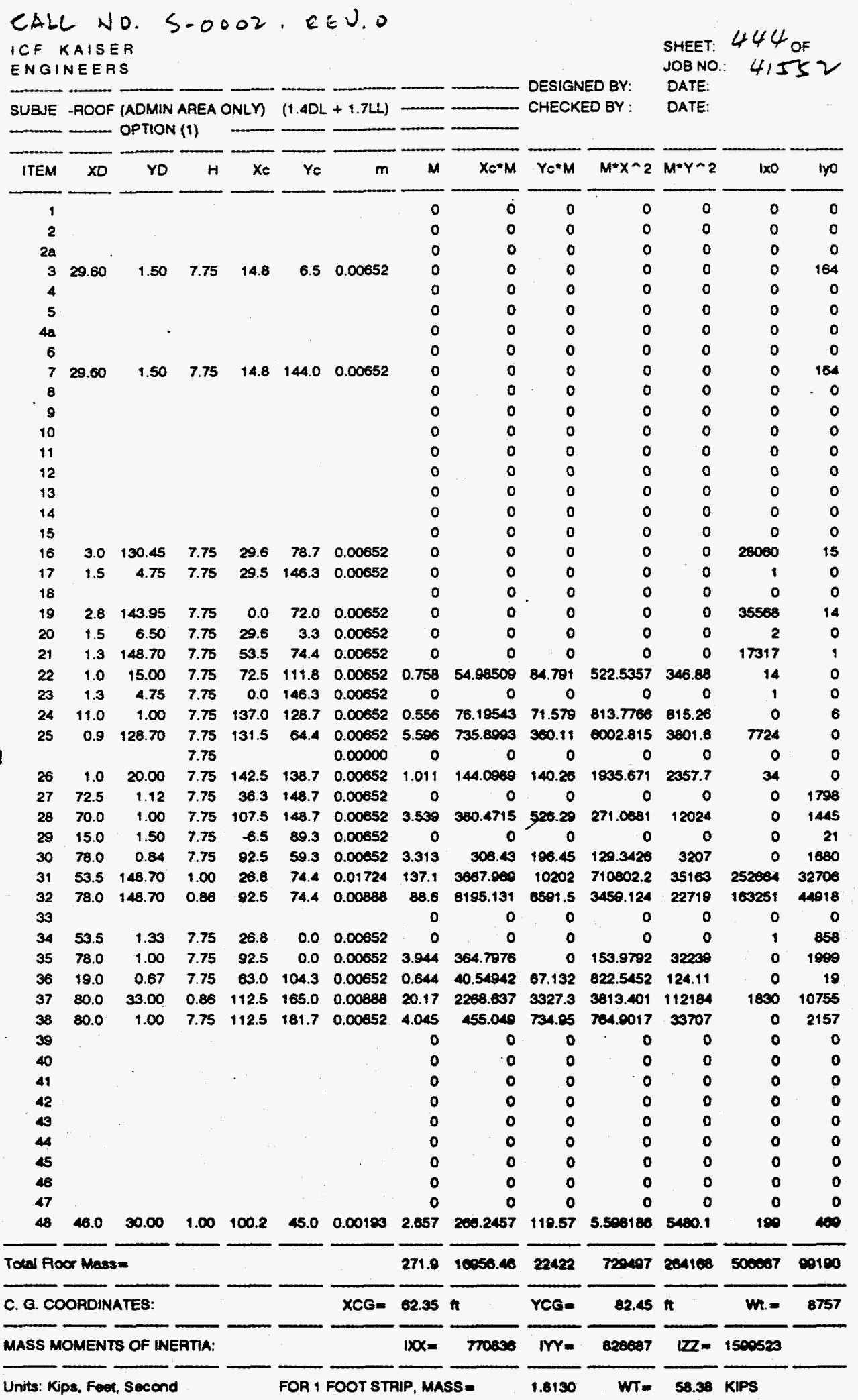


(ILANL NMSF BUILDING) 2-D VERTICAL ANALYSIS - (HNMSF3) Sht. $445 \sigma_{k}$

C C.

4-27-95 BM VNMSF1

4-28-95 BM VNMSF2

5-3-95 BM HNMSF1

5-3-95 BM HNMSF2
-ASSUME SOIL MOD. OF SUBGRADE OF $1 \mathrm{KSF}$

- ADUUST SOIL SPRING PROPERTIES TO MATCH VERTICAL FREQ. OF 3-D MODEL (NMSFO6) -2D EQUIVALENT STATIC ANALYSIS

- ADD LATERAL SOIL PRESSURE

- ASSUME MAX HORIZ. PEAK ACCELERATION.

-ANALYZE DL+LL+E+H AND 1.4DL+1.7LI+1.7H

- CHARGE DECK WITH MOM CONNECTIONS TO WALLS

$$
\begin{array}{lll}
\mathrm{L}=4 & Z=00 & \\
\text { JOINTS } & \\
1 & X=0 & Y=0 \\
2 & X=0 & Y=18.6 \\
3 & X=0 & Y=36.5 \\
C & & \\
4 & X=29.6 & Y=0 \\
5 & X=29.6 & Y=10.5 \\
6 & X=29.6 & Y=18.6 \\
7 & X=29.6 & Y=21.5 \\
8 & X=29.6 & Y=36.8 \\
3 & & \\
& X=53.5 & Y=0 \\
Y & X=53.5 & Y=10.5 \\
1 & X=53.5 & Y=21.5 \\
12 & X=53.5 & Y=36.5 \\
14 & X=29.6 / 3 \\
15 & X=29.6 / 3 & Y=0 \\
16 & X=29.6 / 3 \star 2 & Y=18.6 \\
17 & X=29.6 / 3 * 2 & Y=18.6 \\
50 & X=1 & Y=1
\end{array}
$$

$$
\text { CALC NO: S-ODOZ. REU.O }
$$$$
\text { J०B wo: } 41552
$$

\section{RESTRAINTS}

$\begin{array}{llll}1 & 17 & 1 & R=0,0,1,1,1,0 \\ 1 & & R & R=1,1,1,1,1,1 \\ 4 & & & R=1,1,1,1,1,1 \\ 9 & & R & R=1,1,1,1,1,1 \\ 14 & 16 & 2 & R \\ 11 & & R=1,1,1,1,1,1 \\ 50 & & R=1,0,1,1,1,0 \\ R & =1,1,1,1,1,1\end{array}$

\section{FRAME}

$\mathrm{NM}=7 \quad \mathrm{NL}=12 \quad \mathrm{NSEC}=5 \quad \mathrm{Y}=-1.0$

$1 \mathrm{SH}=\mathrm{R} \quad \mathrm{T}=3.00,1 \quad E=518400 * 0.75 \quad M=.15 \star 3 / 32.2 \quad W=.15 * 3 \quad: 3^{\prime}$ WALLS

$2 \mathrm{SH}=\mathrm{R} \quad \mathrm{T}=1.50,1 \quad \mathrm{E}=518400.0 .75 \mathrm{M}=.15 * 1.5 / 32.2 \mathrm{~W}=1.5 * .15: 1.5^{\prime}$ WALL

$3 \mathrm{SH}=\mathrm{R} \quad \mathrm{T}=1.33,1 . \mathrm{E}=518400 . * 0.75 \mathrm{M}=.15 * 1.33 / 32.2 \mathrm{~W}=.15 * 1.33: 1.33^{\prime}$ WALL

$\triangle \mathrm{SH}=\mathrm{R} \mathrm{T}=1.0,1 . \quad \mathrm{E}=518400 . \star 0.75 \mathrm{M}=0.01155 \quad \mathrm{~W}=0 . \quad$ : 1 MAA ROOF

, $=2.5 * 1 \quad I=2.35,1000 \quad E=518400 \quad M=0.02865 \quad: \quad W=0.5^{\prime}$ CHARGE DECK

6 $S H=R \quad T=1.0,1 . \quad E=518400 . * 75 \quad M=0.00691 \quad W=0 . \quad$ : 1 ; $1 S T$ FLR/MEZZ FLR $\mathrm{SH}=\mathrm{R} \quad \mathrm{T}=1.5,1 . \quad \mathrm{E}=518400{ }^{\star} .75 \quad \mathrm{M}=0.0092 \quad \mathrm{~W}=0 . \quad: 5^{\prime}$ BASEMENT FLOOR WG $=0.00691 * 32.2 * .8,-0.41,0: 1 S T$ FLR $/ \mathrm{MBZZ}$ DL+LL WG $=0,-0.65,0: " 1,1.4 \mathrm{DL}+1.7 \mathrm{LL}$ 
$3 W G=0.01155 * 32.2 * .8,-0.39,0$
$4 W G=0,-0.56,0: 1 "$

$5 \mathrm{WG}=0.02865 * 32.2 * .8,-1.11,0$

=WG $=0,-1.63,0$ :

$G=0.00691 * 32.2 * .8,0,0$

WG $=0.01155 * 32.2 * .8,0,0$

WG $=0.02865 * 32.2 * .8,0,0$

$10 W G=.15 * 3.0 * .8,0,0$

$11 \mathrm{WG}=.15 * 1.5 * .8,0,0$

12 WG $=.15 * 1.33 * .8,0,0$

C

1

2

3

21

22

4

$\begin{array}{lll}19 & 15 & 17\end{array}$

$\begin{array}{lll}20 & 17 & 6\end{array}$

$\begin{array}{lll}5 & 3 & 8\end{array}$

$\begin{array}{lll}6 & 4 & 5\end{array}$

$7 \quad 5 \quad 6$

$8 \quad 6 \quad 7$

$\begin{array}{lll}9 & 7 & 8\end{array}$

$\begin{array}{lll}10 & 4 & 9\end{array}$

1155

$\begin{array}{lll}12 & 7 & 11\end{array}$

$\begin{array}{lll}13 & 8 & 12\end{array}$

149910

$10 \quad 11$

$\begin{array}{lll} & 10 & 11 \\ 7 & 11 & 12 \\ 7 & 14 & 15 \\ 3 & 16 & 17\end{array}$

$\begin{array}{lll} & 10 & 11 \\ 7 & 11 & 12 \\ 7 & 14 & 15 \\ 3 & 16 & 17\end{array}$

$\begin{array}{lll} & 10 & 11 \\ 7 & 11 & 12 \\ 7 & 14 & 15 \\ 3 & 16 & 17\end{array}$

$M=1,1$
$M=3,3$
$M=7,7$
$M=7,7$
$M=7,7$
$M=5,5$
$M=5,5$
$M=5,5$
$M=4,4$
$M=2,2$
$M=2,2$
$M=2,2$
$M=2,2$
$M=7,7$
$M=6,6$
$M=6,6$
$M=4,4$
$M=2,2$
$M=2,2$
$M=3,3$
$M=2,2$
$M=2,2$

$L P=0,50$

$L P=0,50$

$L P=0,50$

$L P=0,50$
:MAA ROOF

1. 4DL+1.7LL

:CHARGE DECK DL+LL

1. $4 \mathrm{DL}+1.7 \mathrm{LL}$

$D L+L L$

\section{ShT.L. O of}

\section{: IST FLR/MEZZ}

:MAA ROOF

: CHARGE DECK

\section{E \\ E \\ $\mathrm{E}$}

$\begin{array}{ll}: 3^{\prime} \text { WALLS } & \mathbf{E} \\ : 1.5^{\prime} \text { WALLS } & \mathrm{E} \\ : 1.33^{\prime} \text { WALLS } & \mathrm{E}\end{array}$

$\mathrm{LP}=0,50 \quad \mathrm{NSL}=0,10$

$\mathrm{LP}=0,50$ NSL $=0,12$

$\mathrm{LP}=0,50 \mathrm{NSI}=5,9,0,6$

$: \quad L R=1,0,0,0,0,0$

$L P=0,50 \quad N S L=5,9,0,6$

$L P=0,50$ NSI $=5,9,0,6$

: $L R=0,1,0,0,0,0$

$L P=0,50$ NSI $=3,8,0,4$

$L \mathrm{P}=0,50 \quad \mathrm{NSI}=0,11$

$L P=0,50$ NSI $=0,11$

$L P=0,50 \quad N S L=0,11$

$L P=0,50 \quad N S L=0,11$

CAUE. NO. S-0002, RES. O

$\mathrm{LP}=0,50$ NSL $=1,7,0,2$

$L P=0,50 \quad N S L=1,7,0,2$

$\mathrm{LP}=0,50$ NSL $=3,8,0,4$

$L P=0,50$ NSL $=0,11$

$L P=0,50$ NSL $=0,11$

$L P=0,50$ NSL $=0,12$

$L P=0,50$ NSL $=0,11: L R=0,1,0,0,0,0$

J०B NO. 41552

$L P=0,50$ NSL $=0,11 \div L R=0,1,0,0,0,0$

CASES

$\mathrm{I}=1$

$\mathrm{L}=2$

$L=3$

$\mathrm{L}=4$
$\mathrm{DL}+\mathrm{LL}$

E

H

1.4DL+1.7LL

C

LOADS

12

12

12

2

3

$F=0,-41.2,0$
$F=.5963 * 32.2 *$
$F=0,-58.4,0$
$F=21.76,0,0$
$F=9.03,0,0$
$0,0 \quad: \quad D=1$
$0,0 \quad:$
$1,0 \quad:$
$1,0 \quad:$
$1.7,1 \quad:$

$L=1$

$I=2$

$I=4$

$\mathrm{L}=3$

$I=3$

:ADMIN DL+LL

: ADMIN $\quad \mathrm{E}$

:ADMIN $1.4 \mathrm{DL}+1.7 \mathrm{LL}$

: SOIL PRESSURE

:SOIL PRESSURE

\section{COMBO}

$\begin{array}{ll}1 & C=1,0,0,0 \\ 2 & C=0,1,0,0 \\ 3 & C=0,0,1,0 \\ 4 & C=1,1,1,0 \\ 5 & C=0,0,1.7,1\end{array}$

DL+LL

E

HI

$\mathrm{DL}+\mathrm{LL}+\mathrm{E}+\mathrm{H}$

I. DL+1.7LL+1.7H 
sut. $4470_{5}^{\circ}$

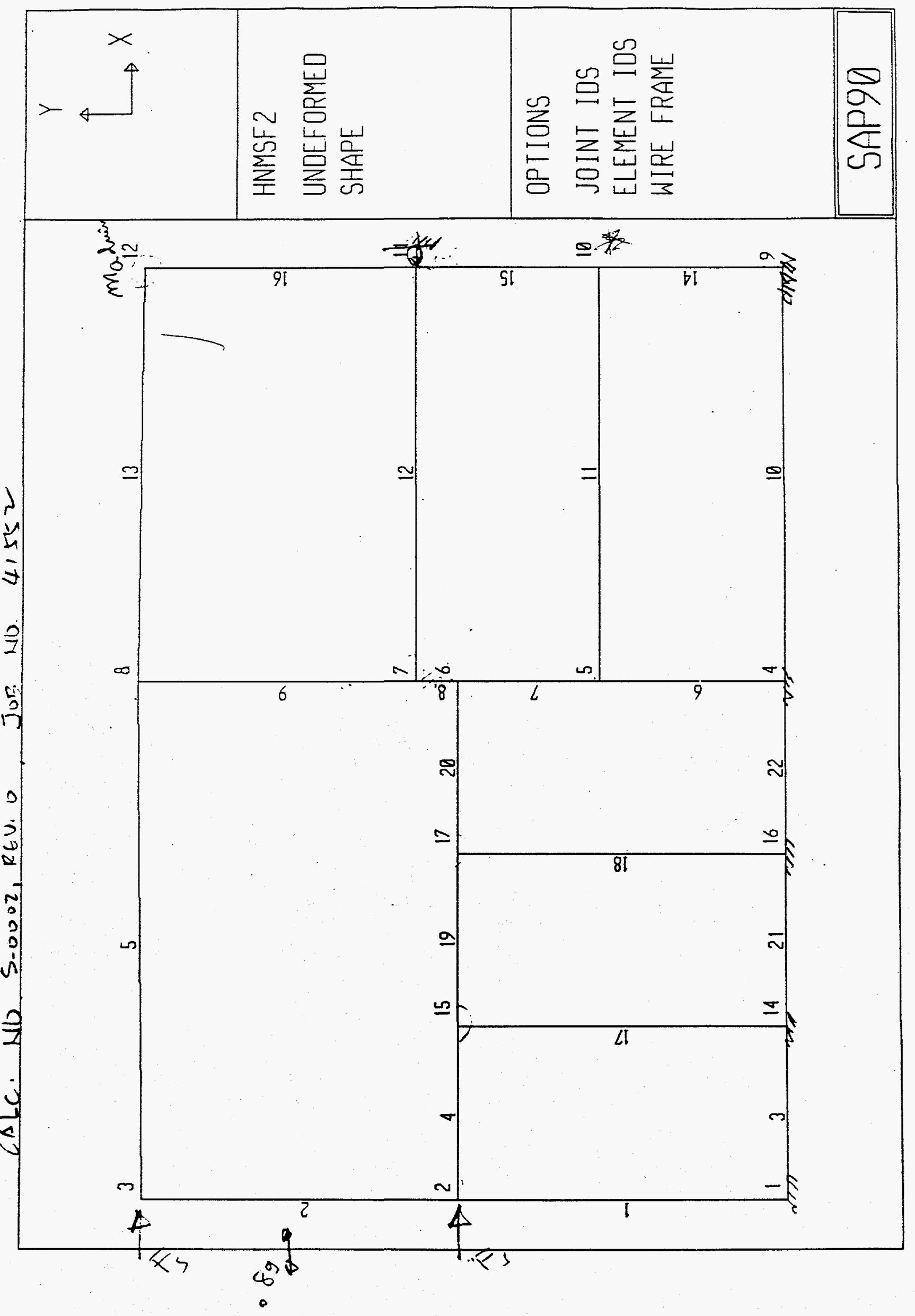


sint 44305

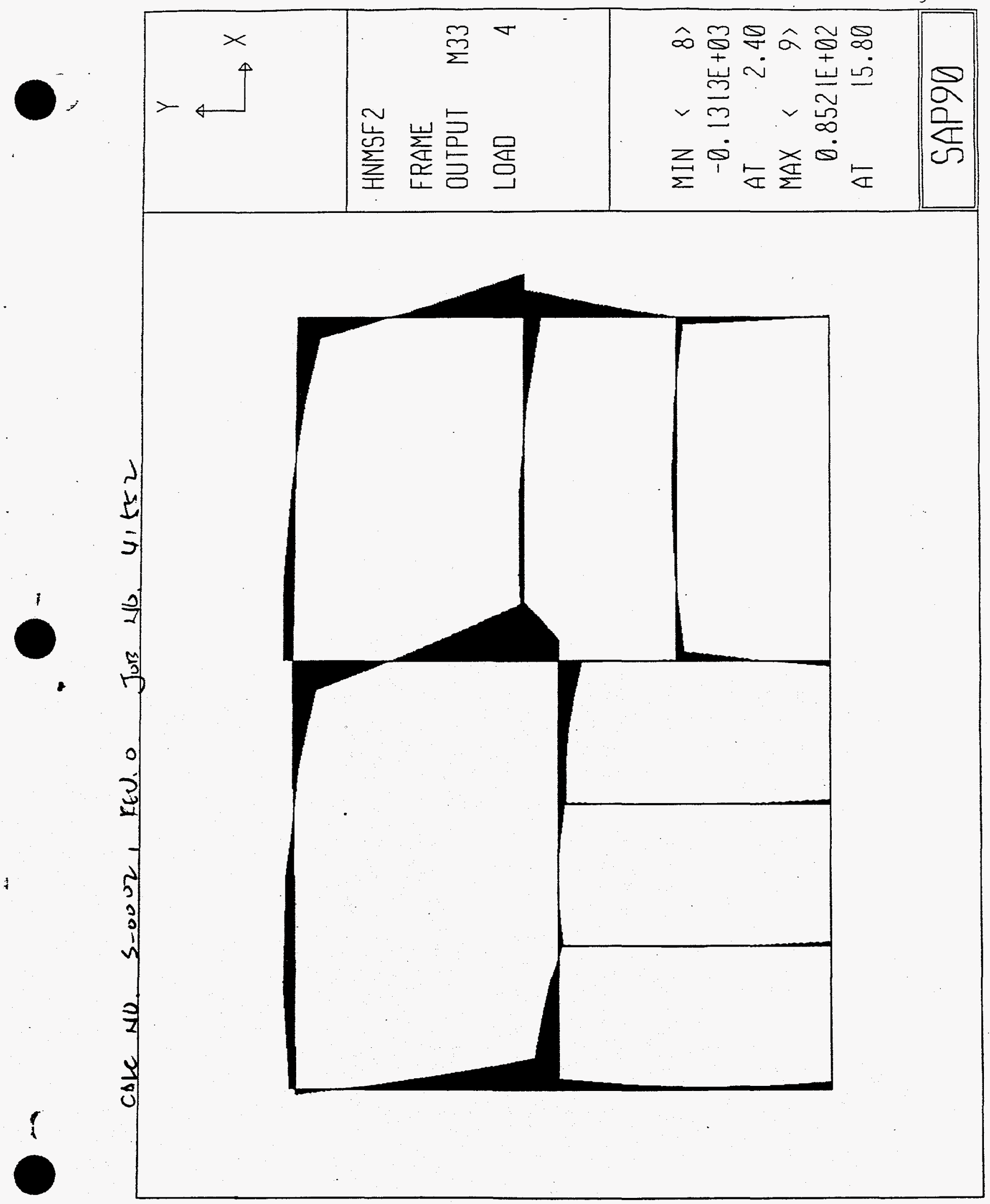




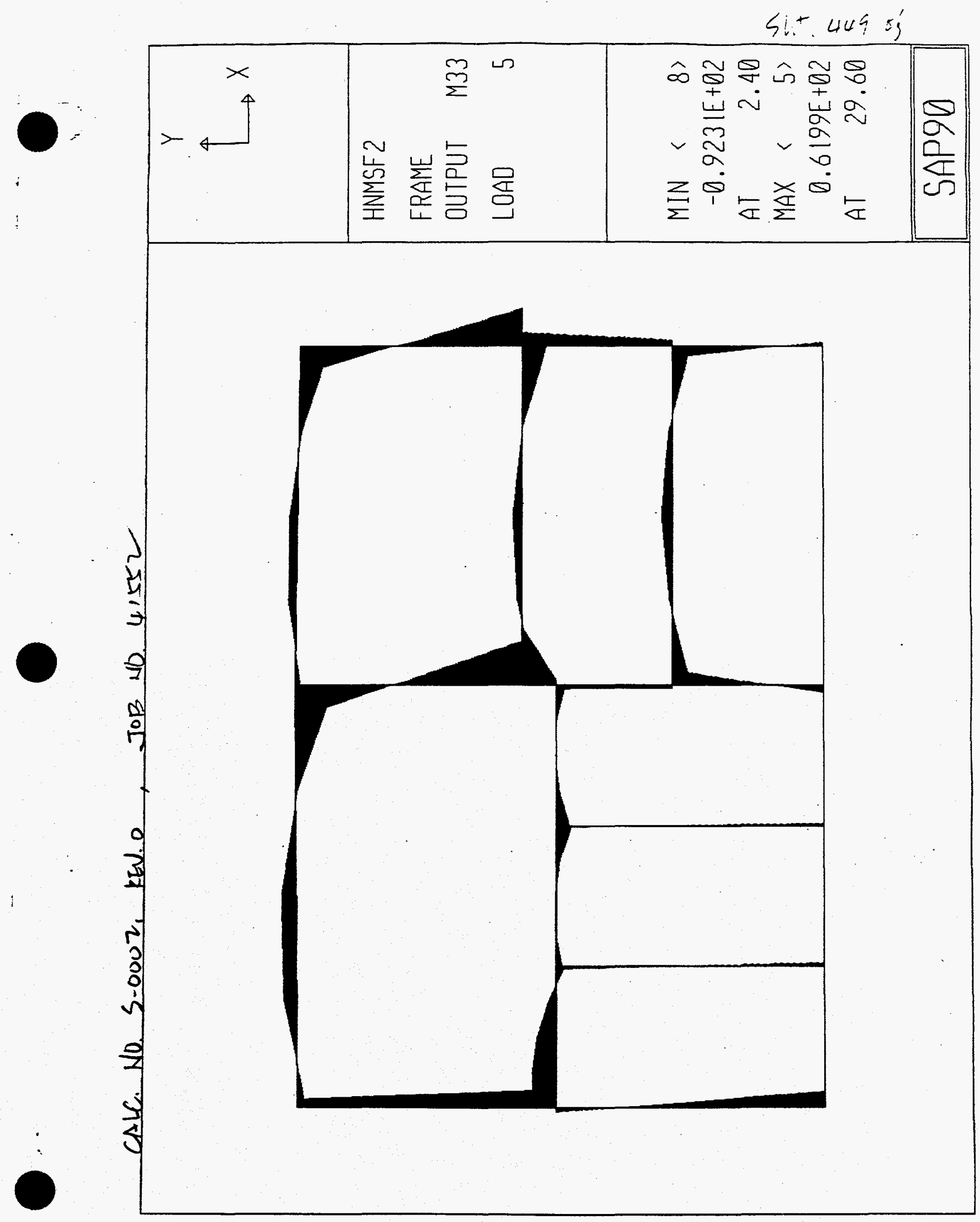




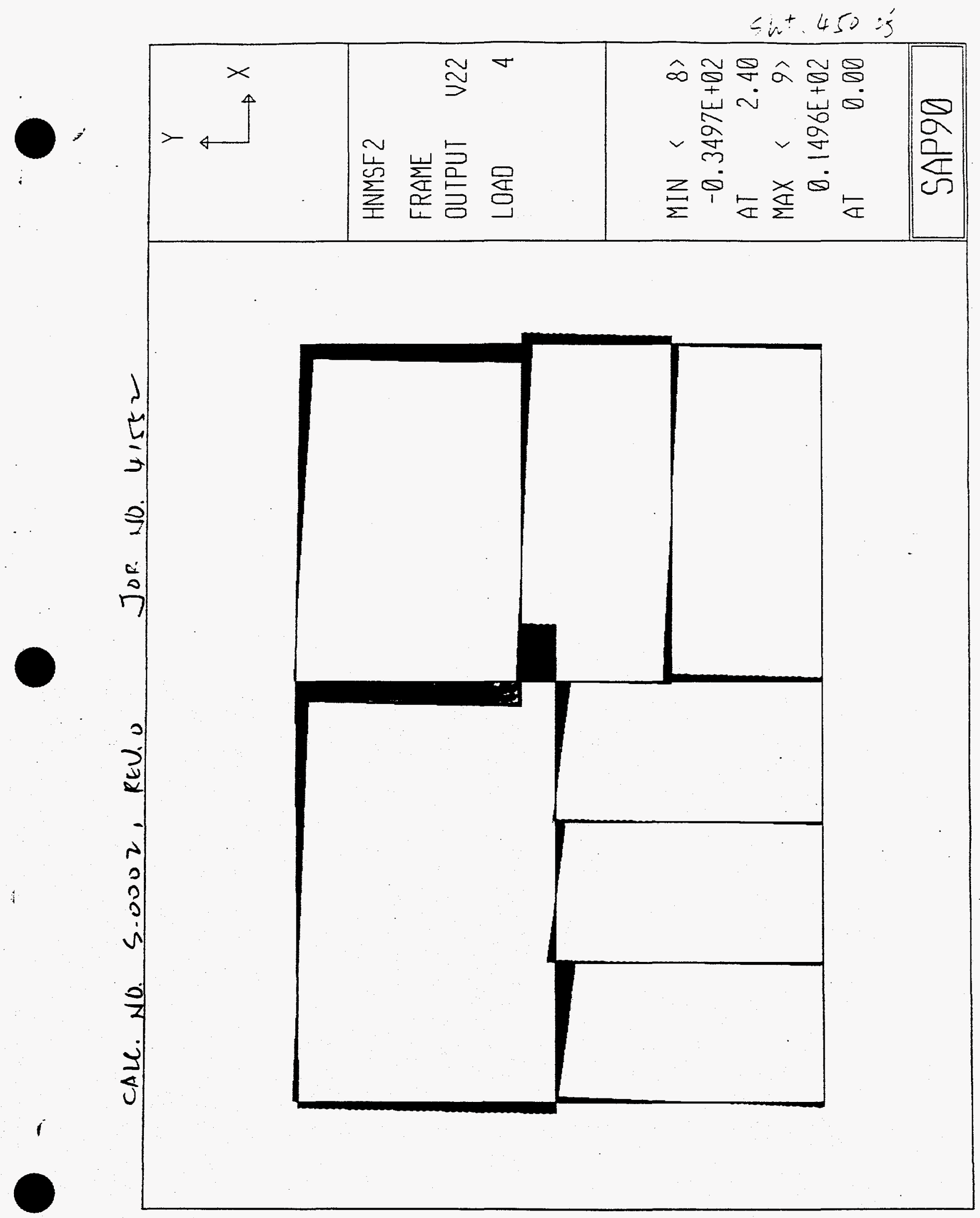


Sit. 451 sij

0

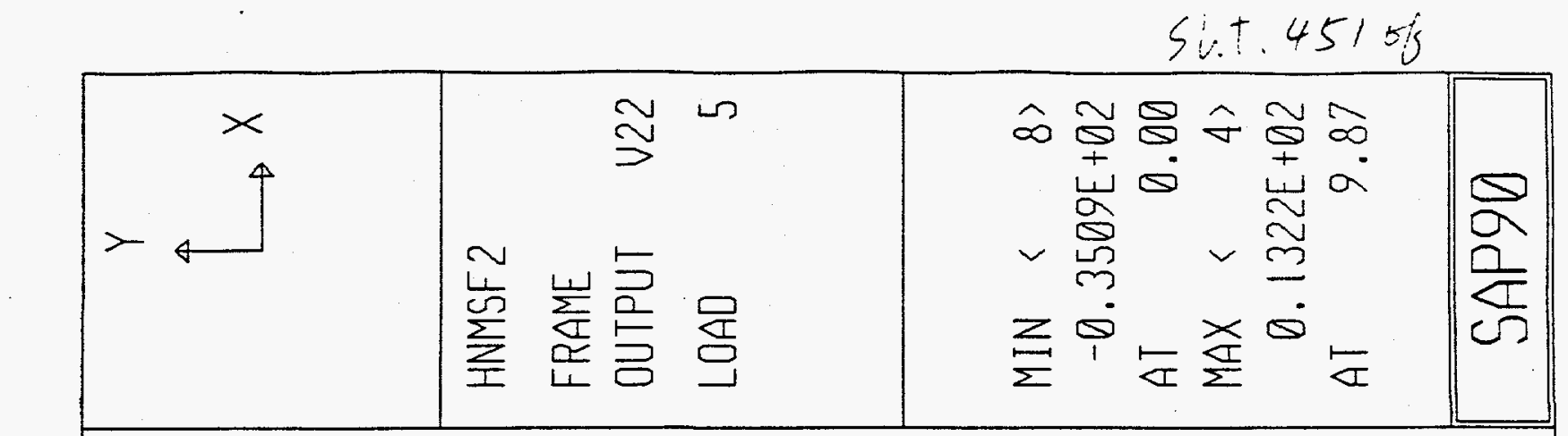

;

$\lambda$
\pm
7
7
7

- $\frac{n}{3}$

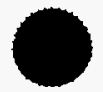

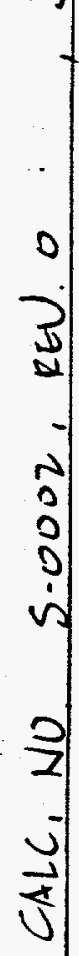

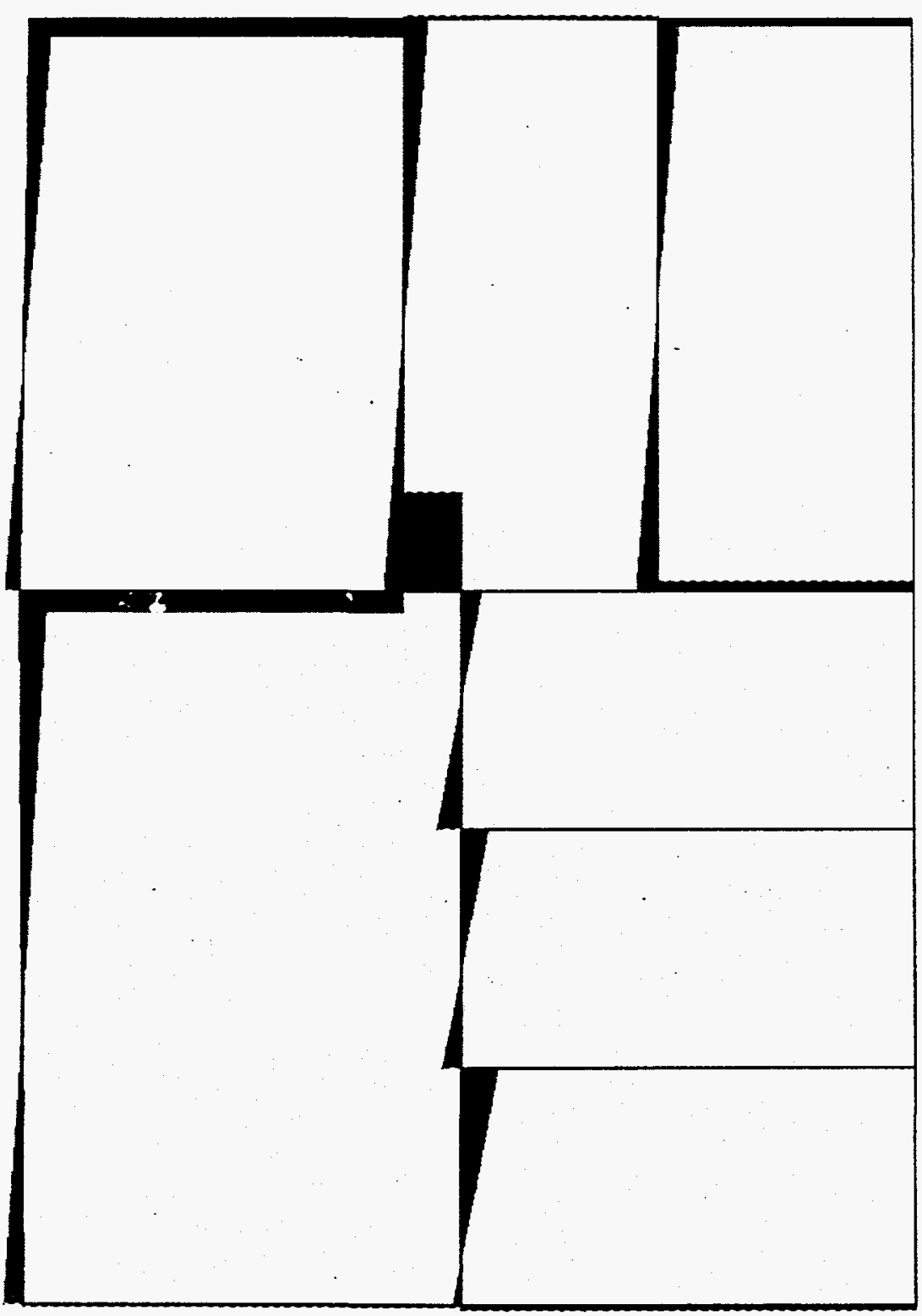




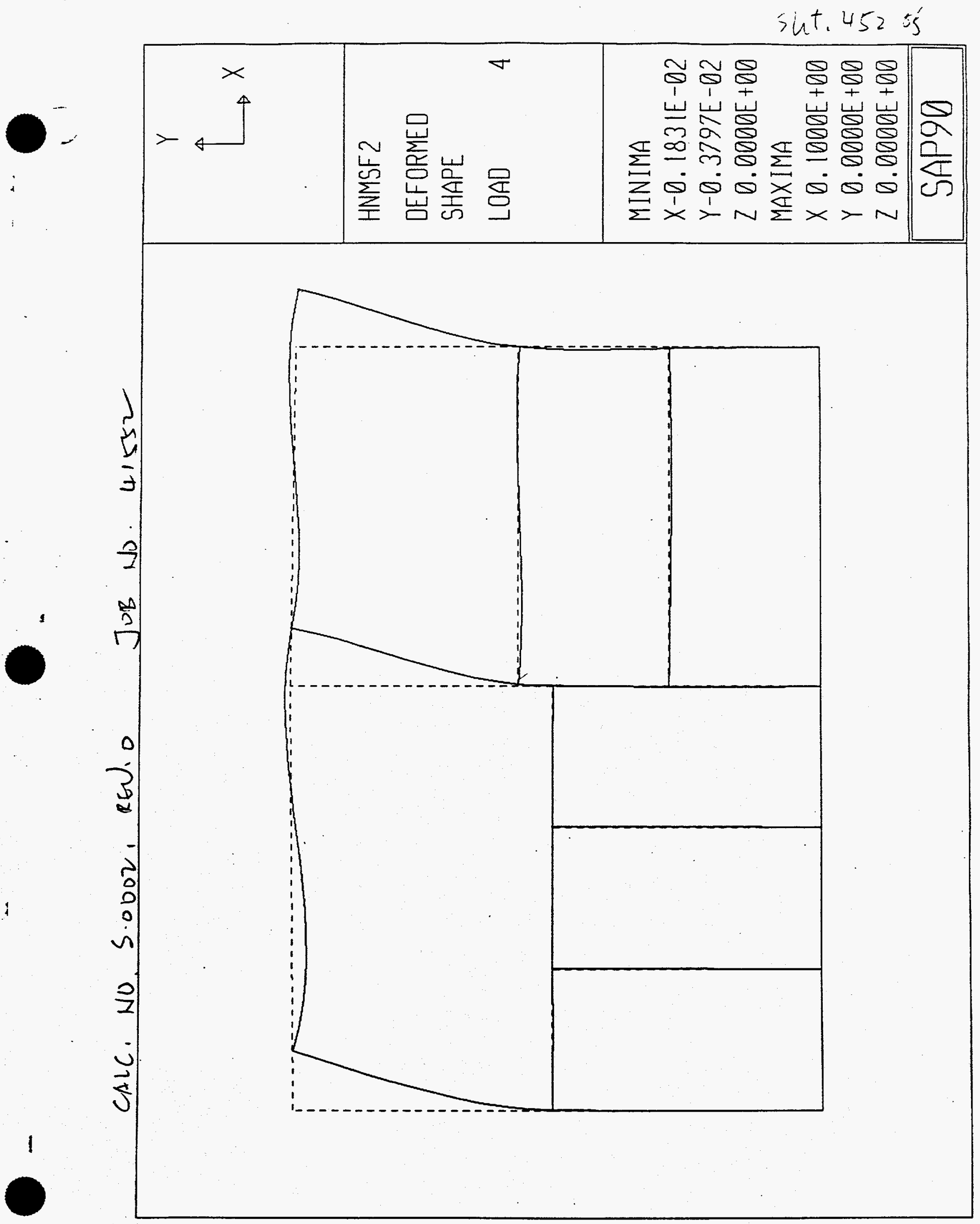


CALC. NO S-000Z, REU. U JOB NO 41552

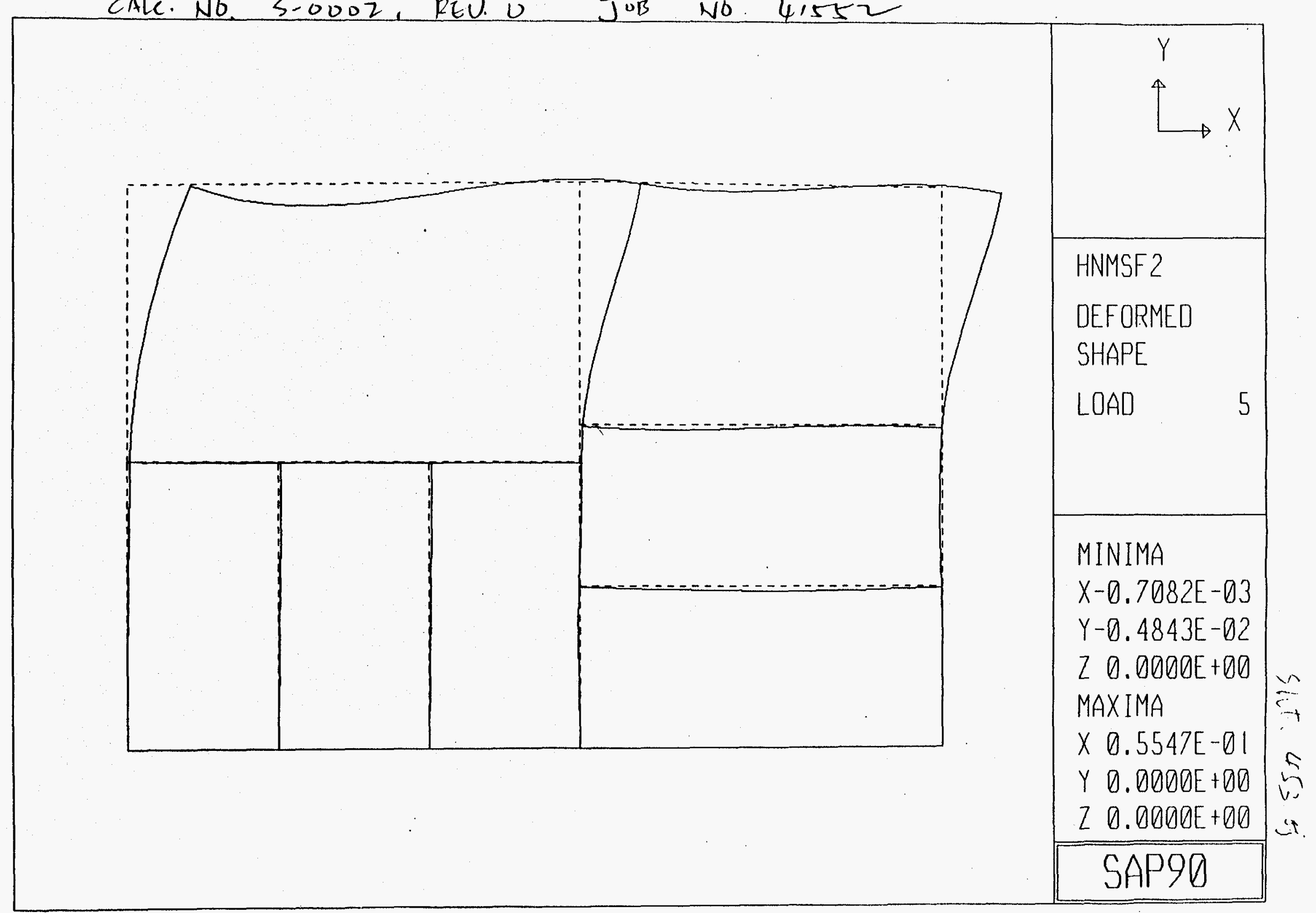


| IF KAISER

CALL NO. $S-0002$, RE O. 0

Joe No. $\times 155=$

EvaluMias of NM Bf EATLII1 $M$ LAN

DESIGNED BY $B M$

Date $5 / 26 / 5$

Mopinglyion option (i) CHECKED BY. IV DATES /26/4S

Design New wall on line 2

$$
\begin{aligned}
& M=\overbrace{132}^{F i x-F i x} \text { keck } \\
& V=35 \text { kips }
\end{aligned}
$$

Bending:

$$
\begin{aligned}
& \text { fry \#10.06 } A_{s}=2 \times 1.27=2.54 \mathrm{in}^{2} \\
& d=18-3=15^{\prime \prime} \\
& \alpha=\frac{2.54 \times 60}{.85 \times 4 \times 12}=3.74^{11} \\
& M_{\nu}=.9 \times 2.54 \times 60\left(15-\frac{3.74}{2}\right) \times \frac{1}{12} \\
& =150 \mathrm{FT} \text {-Kips } / \mathrm{L} \text {.F. } \\
& D / C=0.88
\end{aligned}
$$

Shear:

$$
\begin{aligned}
V_{c} & =2 \phi \sqrt{\xi_{e}^{\prime}} b_{\nu} d \times 10^{-3} \\
& =2 \times .85 \sqrt{4000} \times 12 \times 18 \times 10^{-3}=23.2^{\mathrm{kip} /} / \mathrm{L}_{1} .
\end{aligned}
$$

wall under Compression due to DL USE

$$
\begin{gathered}
V_{c}=4 \phi \sqrt{f_{c}^{\prime}} b_{w d} \times 10^{-3} \\
=46.4 \mathrm{Kips} / \mathrm{L}_{\mathrm{F}}>43 . \\
\mathrm{k} / \mathrm{c}=0.93
\end{gathered}
$$

NoTe: This encoluation excludes out-of-plane demands due to Thermal loads. An accurate mere ob thermal loads is due to be Finalized. 
Sht. 455 of

HNMSF2

20 - Houre Analysis

Ficed Enel change Dock

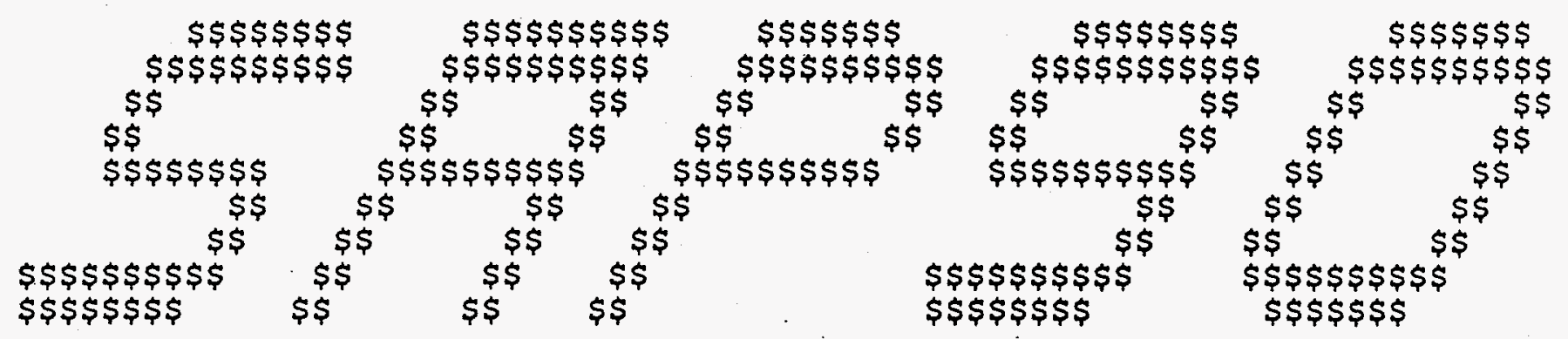

STRUCTURAI ANALYSIS PROGRAMS

VERSION P5.40

Copyright (C) 1978-1992

EDWARD I?' WILSON

All rights reserved

CALC. NO S.0002, REU.O

JOB NO: 41552

EUAMMA..N of NMSF FACILTY C LANL

Monificmions option(1) 
(IANL NMSF BUILDING) 2-D VERTICAL ANALYSIS - (HNMSF3)

FR A M E E L E M E N T FOR C E S

\section{sint. $456 \mathrm{~g}$}

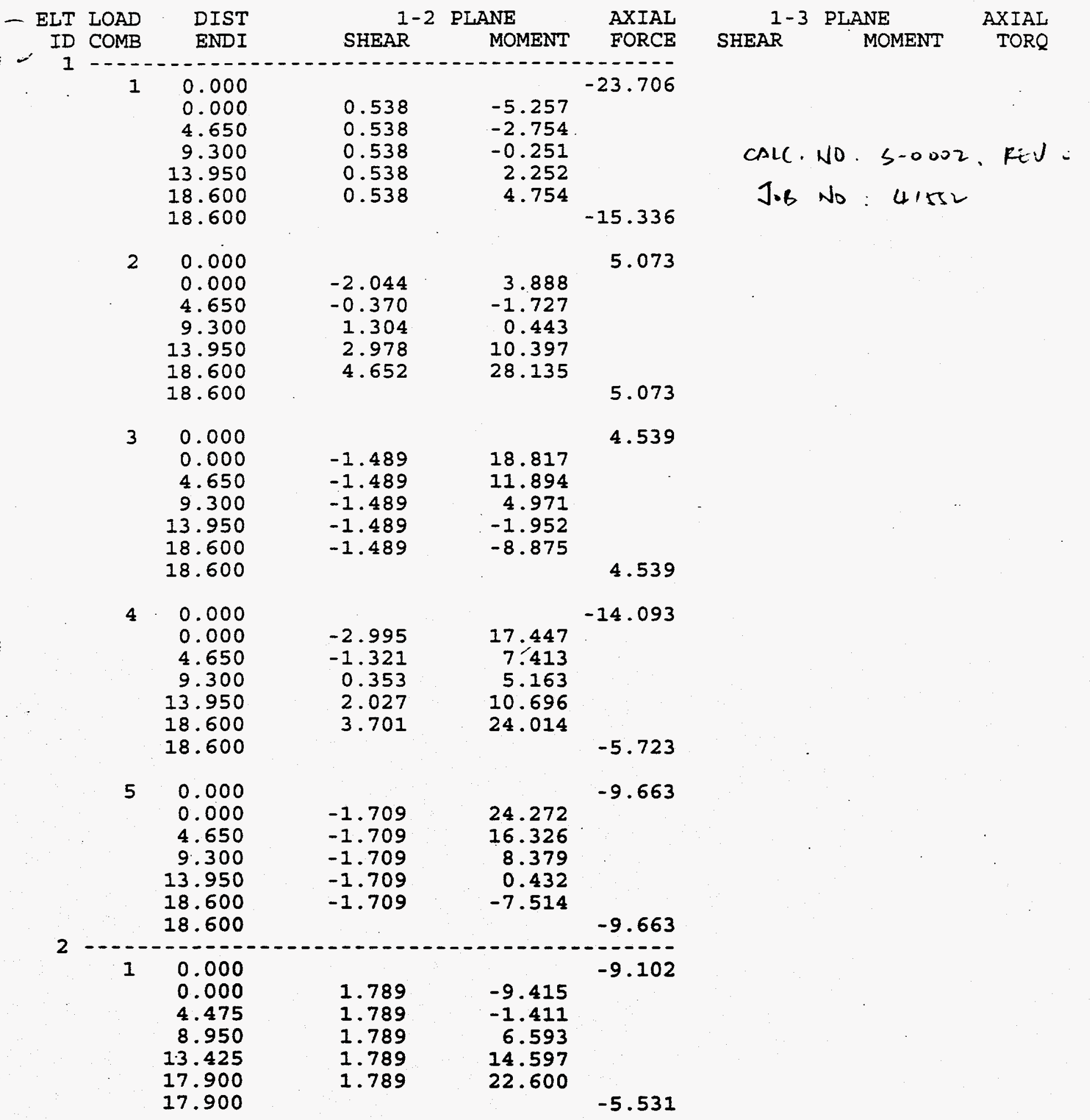




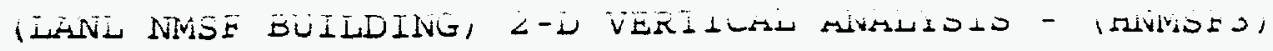

F R A M E E I E M E N T F O R C E S

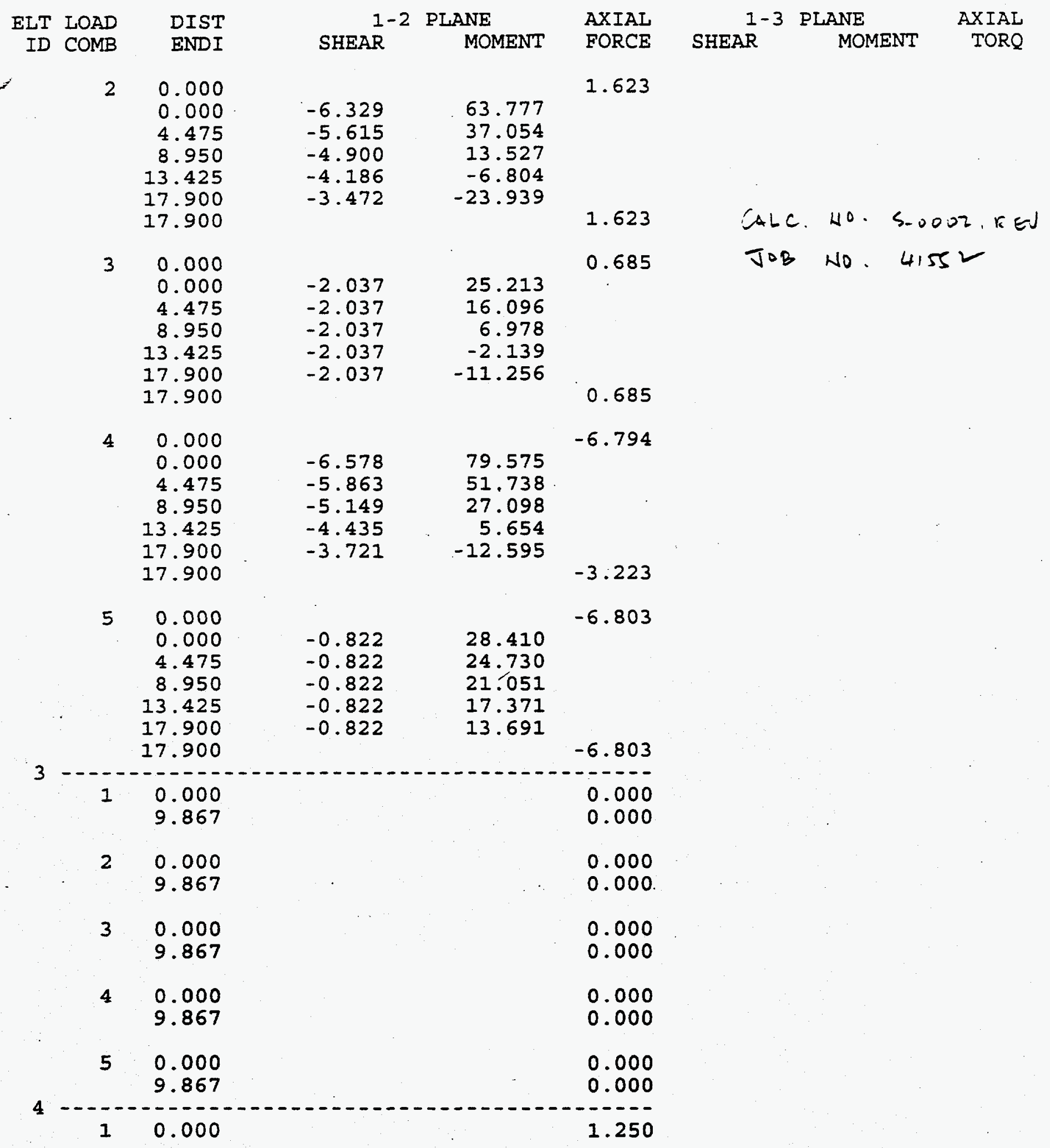


(IANL NMSF BUILDING) 2-D VERTICAL ANALYSIS - (HNMSF3)

PROGRAM: SAF9O/EILE: HNVISE . ESE FRAME ELEMEN T FOR C E S

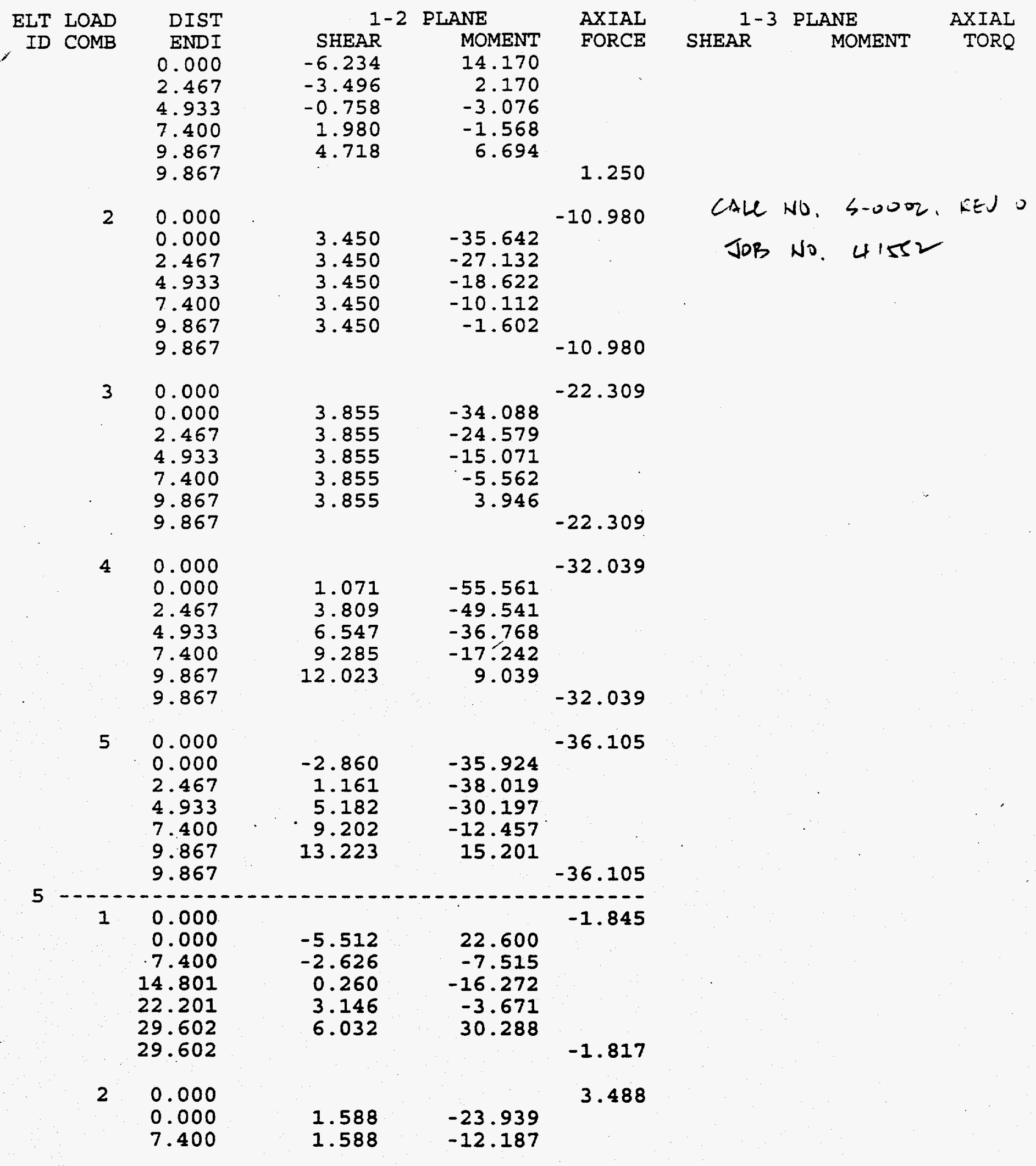




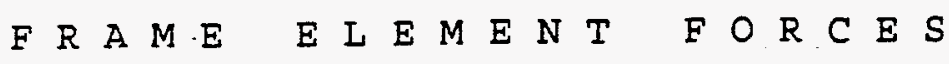

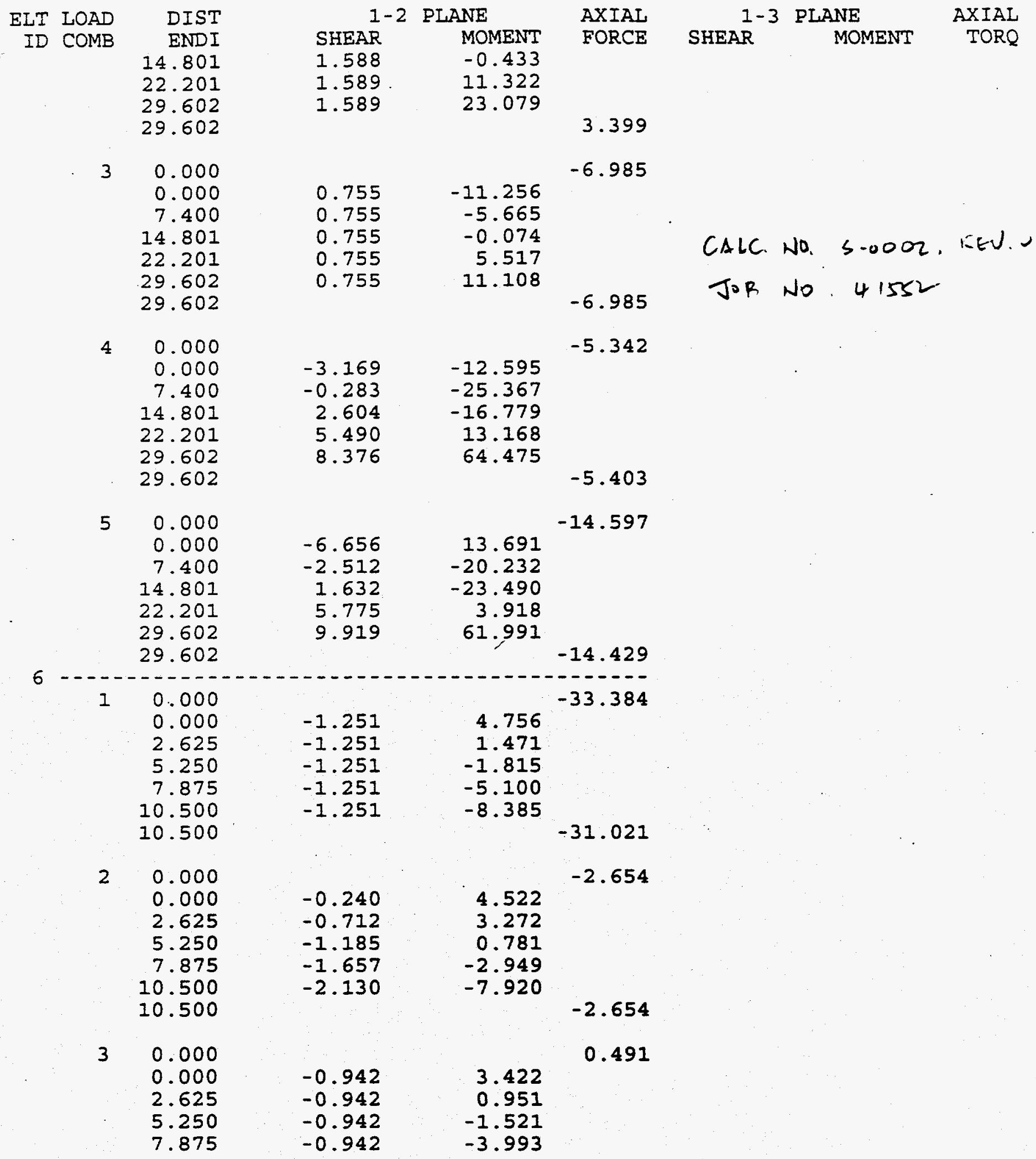

(LANL NMSF BUILDING) 2-D VERTICAL ANALYSIS

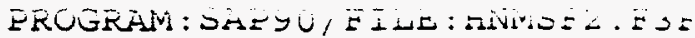
(HNMSF3)

$$
\text { sht. } 459.6 \%
$$

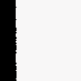
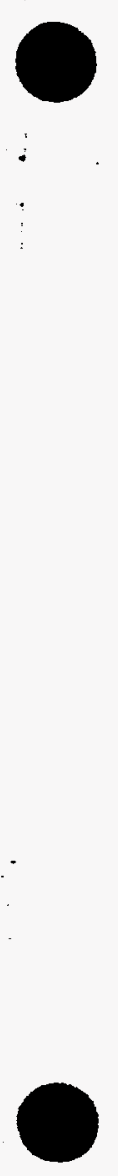


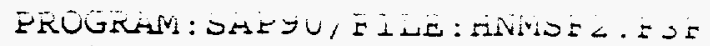

(LANL NMSF BUILDING) 2-D VERTICAL ANALYSIS - (HNMSF3)

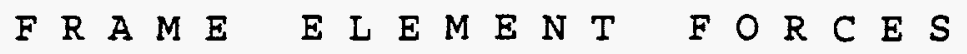

\section{$\operatorname{sint} .460$ of}

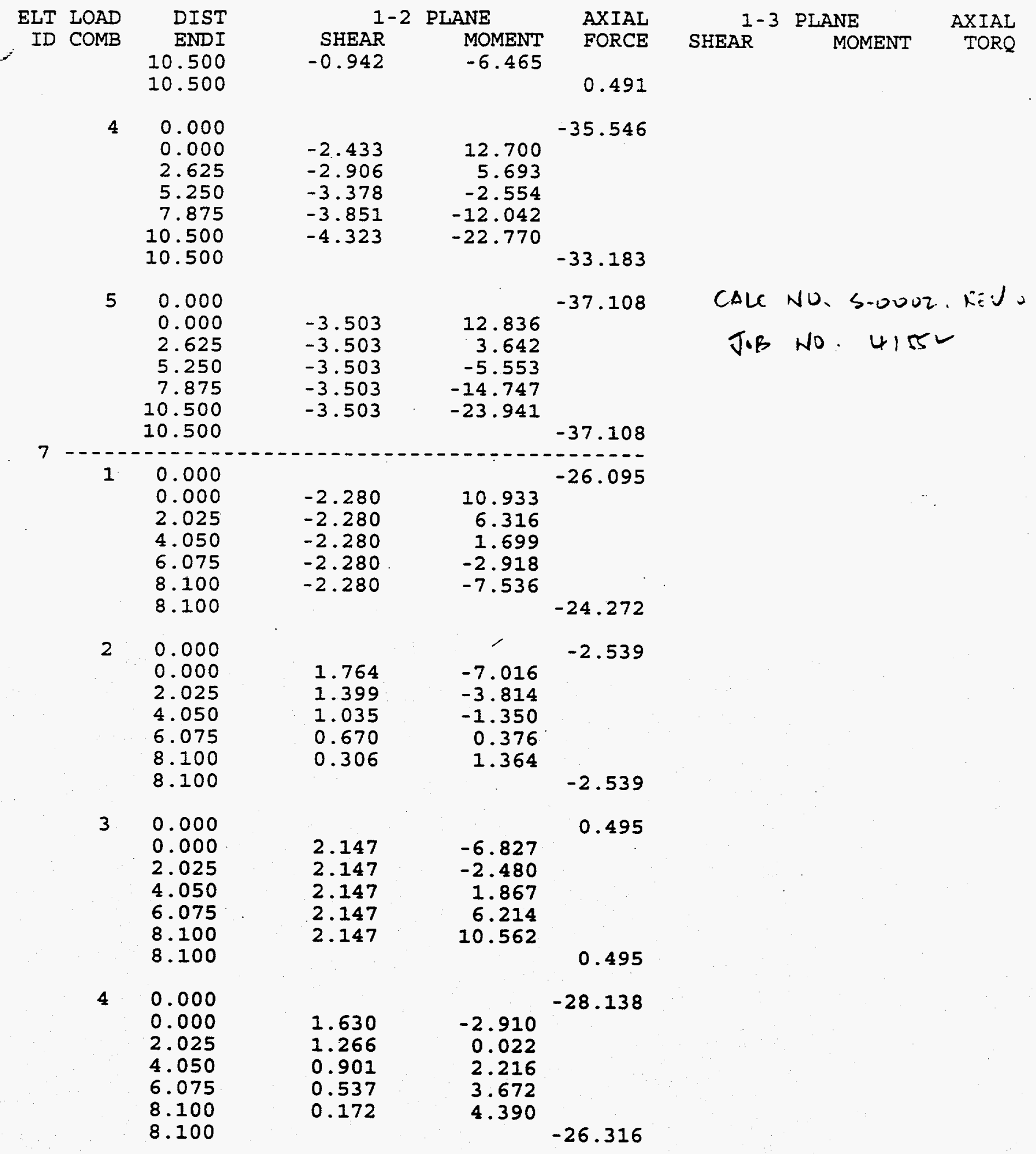


(LANL NMSF BUILDING) 2-D VERTICAL ANALYSIS - (HNMSF3)

F R A M E E L E M E N T FOR C E S

\begin{tabular}{|c|c|c|c|c|c|c|c|c|}
\hline $\begin{array}{r}\text { ELT } \\
\text { ID }\end{array}$ & $\begin{array}{l}\text { LOAD } \\
\text { COMB }\end{array}$ & $\begin{array}{l}\text { DIST } \\
\text { ENDI }\end{array}$ & SHEAR & $\begin{array}{l}\text { PLANE } \\
\text { MOMENT }\end{array}$ & $\begin{array}{l}\text { AXIAL } \\
\text { FORCE }\end{array}$ & SHEAR ${ }^{1-3}$ PI & $\begin{array}{l}\text { ANE } \\
\text { MOMENT }\end{array}$ & $\begin{array}{r}\text { AXIAL } \\
\text { TORQ }\end{array}$ \\
\hline & 5 & $\begin{array}{l}0.000 \\
0.000 \\
2.025 \\
4.050 \\
6.075 \\
8.100 \\
8.100\end{array}$ & $\begin{array}{l}0.087 \\
0.087 \\
0.087 \\
0.087 \\
0.087\end{array}$ & $\begin{array}{l}5.961 \\
6.138 \\
6.314 \\
6.490 \\
6.667\end{array}$ & -29.301 & & & \\
\hline 8 & $---\frac{-}{1}$ & $\begin{array}{l}0.000 \\
0.000 \\
0.600 \\
1.200 \\
1.800 \\
2.400 \\
2.400\end{array}$ & $\begin{array}{l}-1.250 \\
-1.250 \\
-1.250 \\
-1.250 \\
-1.250\end{array}$ & $\begin{array}{l}-13.475 \\
-14.225 \\
-14.975 \\
-15.725 \\
-16.475\end{array}$ & -19.932 & $\begin{array}{l}\text { CAle no } \\
\text { Jir no }\end{array}$ & $\begin{array}{l}5.0002 \\
4.562\end{array}$ & RETS. O \\
\hline & 2 & $\begin{array}{l}0.000 \\
0.000 \\
0.600 \\
1.200 \\
1.800 \\
2.400 \\
2.400\end{array}$ & $\begin{array}{l}-13.852 \\
-13.960 \\
-14.068 \\
-14.176 \\
-14.284\end{array}$ & $\begin{array}{l}-41.489 \\
-49.832 \\
-58.241 \\
-66.714 \\
-75.252\end{array}$ & 1.740 & & . & \\
\hline & 3 & $\begin{array}{l}0.000 \\
0.000 \\
0.600 \\
1.200 \\
1.800 \\
2.400 \\
2.400\end{array}$ & $\begin{array}{l}-19.437 \\
-19.437 \\
-19.437 \\
-19.437 \\
-19.437\end{array}$ & $\begin{array}{r}7.054 \\
-4.608 \\
-16.270 \\
-27.932 \\
-39.594\end{array}$ & 0.897 & . & & \\
\hline & 4 & $\begin{array}{l}0.000 \\
0.000 \\
0.600 \\
1.200 \\
1.800 \\
2.400 \\
2.400\end{array}$ & $\begin{array}{l}-34.539 \\
-34.647 \\
-34.755 \\
-34.863 \\
-34.971\end{array}$ & $\begin{array}{r}-47.910 \\
-68.665 \\
-89.486 \\
-110.371 \\
-131.321\end{array}$ & -17.295 & & & \\
\hline & 5 & $\begin{array}{l}0.000 \\
0.000 \\
0.600 \\
1.200 \\
1.800 \\
2.400 \\
2.400\end{array}$ & $\begin{array}{l}-35.089 \\
-35.089 \\
-35.089 \\
-35.089 \\
-35.089\end{array}$ & $\begin{array}{r}-8.102 \\
-29.155 \\
-50.209 \\
-71.262 \\
-92.315\end{array}$ & -21.974 & & & \\
\hline
\end{tabular}




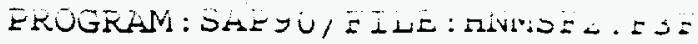
(LANL NMSF BUILDING) 2-D VERTICAL ANALYSIS - (HNMSF3) F R A E E L E M E N T FOR C E S

\section{Sht. $4 t=05$}

\begin{tabular}{|c|c|c|c|c|c|c|c|c|}
\hline $\begin{array}{r}\text { ELT } \\
\text { ID }\end{array}$ & $\begin{array}{l}\text { LOAD } \\
\text { COMB }\end{array}$ & $\begin{array}{l}\text { DIST } \\
\text { ENDI }\end{array}$ & SHEAR & $\begin{array}{l}\text { PLANE } \\
\text { MOMENT }\end{array}$ & $\begin{array}{l}\text { AXIAL } \\
\text { FORCE }\end{array}$ & ${ }_{\text {SHEAR }}^{1-3}$ PL & $\begin{array}{l}\text { ANE } \\
\text { MOMENT }\end{array}$ & $\begin{array}{r}\text { AXIAL } \\
\text { TORQ }\end{array}$ \\
\hline 9 & --- & $\begin{array}{r}0.000 \\
0.000 \\
3.950 \\
7.900 \\
11.850 \\
15.800 \\
15.800\end{array}$ & $\begin{array}{l}0.395 \\
0.395 \\
0.395 \\
0.395 \\
0.395\end{array}$ & $\begin{array}{l}1.668 \\
3.227 \\
4.787 \\
6.346 \\
7.906\end{array}$ & -14.552 & & & \\
\hline & 2 & $\begin{array}{r}0.000 \\
0.000 \\
3.950 \\
7.900 \\
11.850 \\
15.800 \\
15.800\end{array}$ & $\begin{array}{r}10.231 \\
9.520 \\
8.809 \\
8.098 \\
7.387\end{array}$ & $\begin{array}{r}-87.047 \\
-48.041 \\
-11.842 \\
21.547 \\
52.129\end{array}$ & 0.739 & $\begin{array}{l}\text { Cale No } \\
\text { Jo B NO }\end{array}$ & $\begin{array}{l}s-0002 \\
41552\end{array}$ & בEJ.J. \\
\hline & 3 & $\begin{array}{r}0.000 \\
0.000 \\
3.950 \\
7.900 \\
11.850 \\
15.800 \\
15.800\end{array}$ & $\begin{array}{l}4.336 \\
4.336 \\
4.336 \\
4.336 \\
4.336\end{array}$ & $\begin{array}{r}-43.337 \\
-26.209 \\
-9.080 \\
8.049 \\
25.177\end{array}$ & 0.549 & & & \\
\hline & 4 & $\begin{array}{r}0.000 \\
0.000 \\
3.950 \\
7.900 \\
11.850 \\
15.800 \\
15.800\end{array}$ & $\begin{array}{l}14.962 \\
14.251 \\
13.540 \\
12.829 \\
12.118\end{array}$ & $\begin{array}{r}-128.717 \\
-71.022 \\
-16.135 \\
35.943 \\
85.212\end{array}$ & -9.709 & & & \\
\hline & 5 & $\begin{array}{r}0.000 \\
0.000 \\
3.950 \\
7.900 \\
11.850 \\
15.800 \\
15.800\end{array}$ & $\begin{array}{l}7.780 \\
7.780 \\
7.780 \\
7.780 \\
7.780\end{array}$ & $\begin{array}{r}-69.562 \\
-38.833 \\
-8.103 \\
22.626 \\
53.355\end{array}$ & -14.863 & & & . \\
\hline 10 & 1 & $\begin{array}{r}0.000 \\
23.900\end{array}$ & ------ & & $\begin{array}{l}0.000 \\
0.000\end{array}$ & & & \\
\hline & 2 & $\begin{array}{r}0.000 \\
23.900\end{array}$ & & & $\begin{array}{l}0.000 \\
0.000\end{array}$ & & & \\
\hline & 3 & $\begin{array}{r}0.000 \\
23.900\end{array}$ & & & $\begin{array}{l}0.000 \\
0.000\end{array}$ & & & \\
\hline
\end{tabular}


(LANL NMSF BUILDING) 2-D VERTICAL ANALYSIS - (HNMSF3)

FRAME ELEMENT FORCES

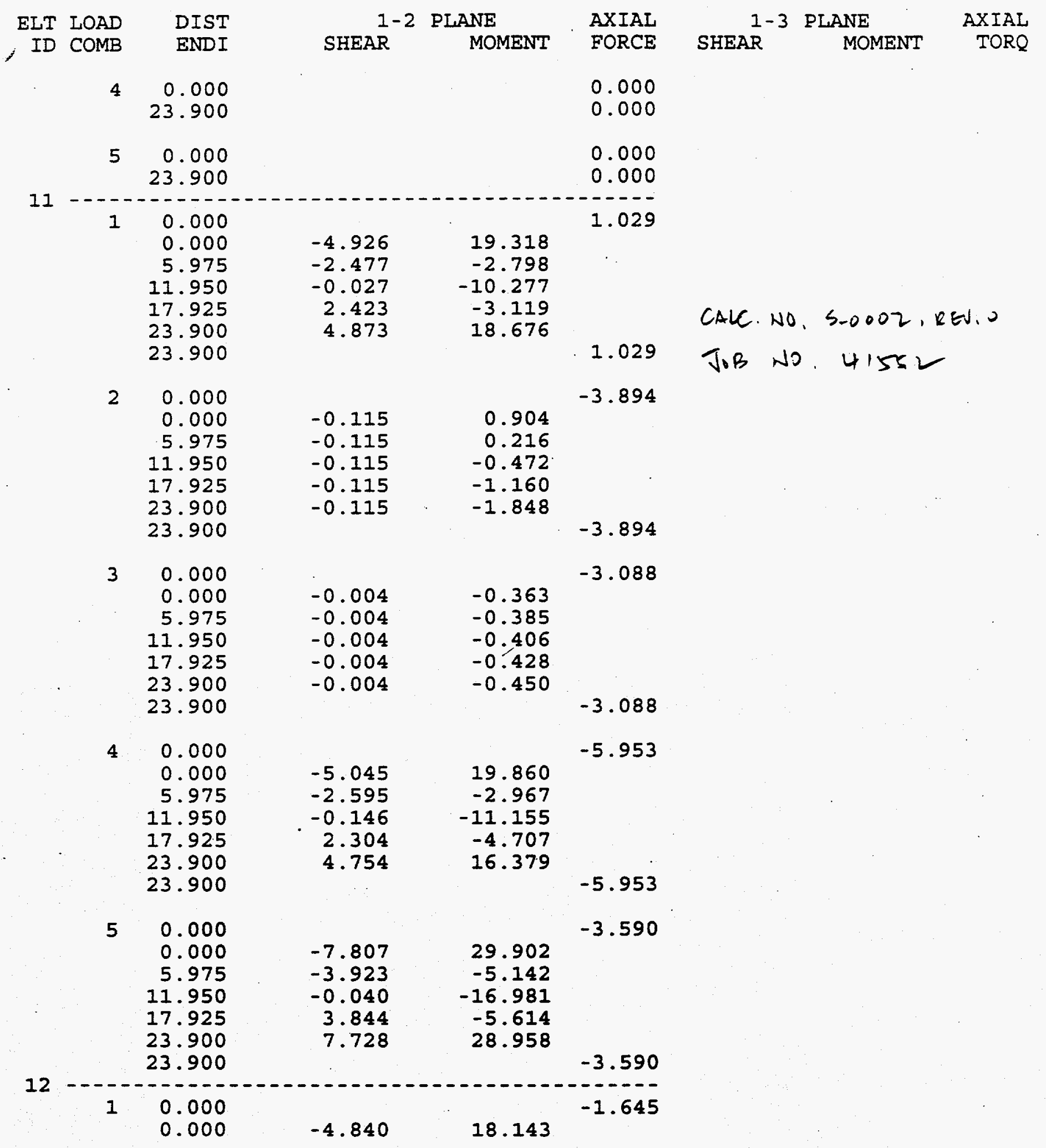




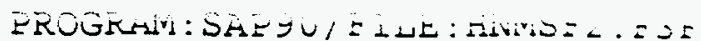
(LANL NMSF BUILDING) 2-D VERTICAL ANALYSIS - (HNMSF3)

$F R A E$ E L E M E N T FORCES cist. 461 of

\begin{tabular}{|c|c|c|c|c|c|c|c|c|c|}
\hline $\begin{array}{l}\text { ELT } \\
\text { ID }\end{array}$ & $\begin{array}{l}\text { LOAD } \\
\text { COMB }\end{array}$ & $\begin{array}{r}\text { DIST } \\
\text { ENDI } \\
5.975 \\
11.950 \\
17.925 \\
23.900 \\
23.900\end{array}$ & $\begin{array}{r}1- \\
\text { SHEAR } \\
-2.390 \\
0.060 \\
2.510 \\
4.959\end{array}$ & $\begin{array}{l}\text { LANE } \\
\text { MOMENT } \\
-3.456 \\
-10.417 \\
-2.741 \\
19.572\end{array}$ & -1.645 & SHEAR & $-3 \mathrm{P}$ & $\begin{array}{l}\text { MANE } \\
\text { MOMENT }\end{array}$ & $\begin{array}{r}\text { AXIAL } \\
\text { TORQ }\end{array}$ \\
\hline & 2 & $\begin{array}{r}0.000 \\
0.000 \\
5.975 \\
11.950 \\
17.925 \\
23.900 \\
23.900\end{array}$ & $\begin{array}{l}1.001 \\
1.001 \\
1.001 \\
1.001 \\
1.001\end{array}$ & $\begin{array}{r}-11.795 \\
-5.816 \\
0.162 \\
6.141 \\
12.120\end{array}$ & -24.515 & CALC. & NO. & 5.0002 & ce-s. 0 \\
\hline & 3 & $\begin{array}{r}0.000 \\
0.000 \\
5.975 \\
11.950 \\
17.925 \\
23.900 \\
23.900\end{array}$ & $\begin{array}{l}0.348 \\
0.348 \\
0.348 \\
0.348 \\
0.348\end{array}$ & $\begin{array}{r}-3.743 \\
-1.666 \\
0.412 \\
2.490 \\
4.567\end{array}$ & -23.773 & $J \circ B$ & No. & 41552 & \\
\hline & 4 & $\begin{array}{r}0.000 \\
0.000 \\
5.975 \\
11.950 \\
17.925 \\
23.900 \\
23.900\end{array}$ & $\begin{array}{r}-3.491 \\
-1.042 \\
1.408 \\
3.858 \\
6.308\end{array}$ & $\begin{array}{r}2.605 \\
-10.938 \\
-9.843 \\
5.890 \\
36.259\end{array}$ & -49.933 & & & & \\
\hline & 5 & $\begin{array}{r}0.000 \\
0.000 \\
5.975 \\
11.950 \\
17.925 \\
23.900 \\
23.900\end{array}$ & $\begin{array}{r}-7.111 \\
-3.228 \\
0.656 \\
4.540 \\
8.424\end{array}$ & $\begin{array}{r}22.753 \\
-8.135 \\
-15.817 \\
-0.294 \\
38.434\end{array}$ & -42.868 & & & & \\
\hline 13 & $\begin{array}{r}---- \\
1\end{array}$ & $\begin{array}{r}0.000 \\
0.000 \\
5.975 \\
1.1 .951 \\
17.926 \\
23.902 \\
23.902\end{array}$ & $\begin{array}{r}-5.002 \\
-2.672 \\
-0.343 \\
1.987 \\
4.317\end{array}$ & $\begin{array}{r}22.382 \\
-0.548 \\
-9.556 \\
-4.642 \\
14.192\end{array}$ & -1.627 & & & & \\
\hline & 2 & $\begin{array}{r}0.000 \\
0.000 \\
5.975 \\
11.951\end{array}$ & $\begin{array}{l}2.497 \\
2.497 \\
2.497\end{array}$ & $\begin{array}{r}-29.050 \\
-14.127 \\
0.794\end{array}$ & 10.739 & & & & \\
\hline
\end{tabular}


(LANL NMSF BUILDING) 2-D VERTICAL ANALYSIS - (HNMSF3)

PROGRAM : SAP90/FILE : HNMSF2 . F3F

$F R A M E$ E L E M E N T FOR C E S

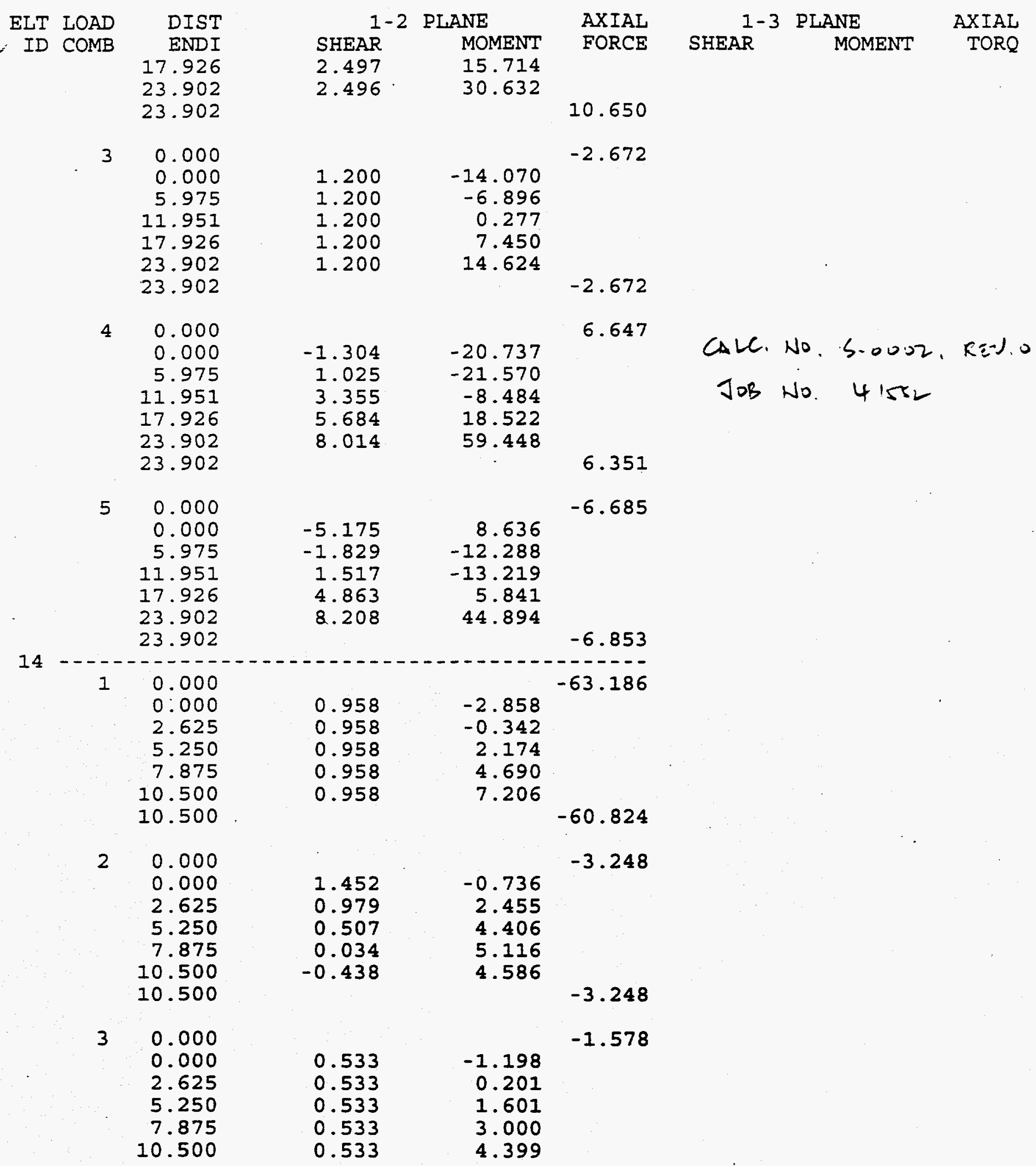

sht. 465 of

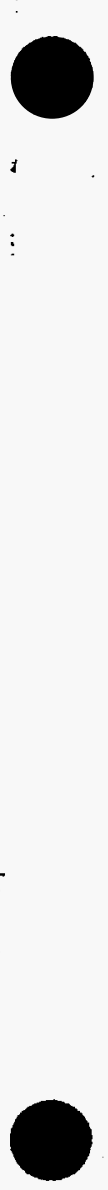


(LANL NMSF BUILDING) 2-D VERTICAL ANALYSIS - (HNMSF3)

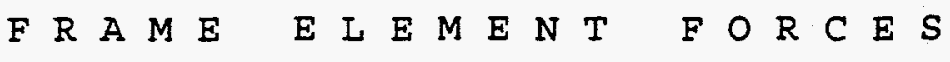

\begin{tabular}{|c|c|c|c|c|c|c|c|c|c|}
\hline $\begin{array}{r}\text { ELT } \\
\text { ID }\end{array}$ & $\begin{array}{l}\text { LOAD } \\
\text { COMB }\end{array}$ & $\begin{array}{r}\text { DIST } \\
\text { ENDI } \\
10.500\end{array}$ & SHEAR & $\begin{array}{l}\text { PLANE } \\
\text { MOMENT }\end{array}$ & $\begin{array}{r}\text { AXIAL } \\
\text { FORCE } \\
-1.578\end{array}$ & SHEAR & 3 & $\begin{array}{l}\text { ANE } \\
\text { MOMENT }\end{array}$ & $\begin{array}{l}\text { AXIAL } \\
\text { TORQ }\end{array}$ \\
\hline & 4 & $\begin{array}{r}0.000 \\
0.000 \\
2.625 \\
5.250 \\
7.875 \\
10.500 \\
10.500\end{array}$ & $\begin{array}{l}2.943 \\
2.471 \\
1.998 \\
1.526 \\
1.053\end{array}$ & $\begin{array}{r}-4.793 \\
2.314 \\
8.180 \\
12.806 \\
16.191\end{array}$ & -65.650 & & & & \\
\hline & 5 & $\begin{array}{r}0.000 \\
0.000 \\
2.625 \\
5.250 \\
7.875 \\
10.500 \\
10.500\end{array}$ & $\begin{array}{l}2.496 \\
2.496 \\
2.496 \\
2.496 \\
2.496\end{array}$ & $\begin{array}{r}-7.053 \\
-0.501 \\
6.051 \\
12.603 \\
19.156\end{array}$ & -82.845 & 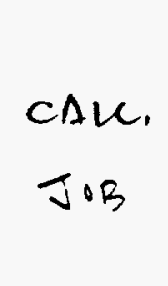 & $\begin{array}{l}\text { No } \\
\text { Wo }\end{array}$ & $\begin{array}{l}5-0002 \\
41552\end{array}$ & REN. D \\
\hline 15 & $---\frac{-}{1}$ & $\begin{array}{r}0.000 \\
0.000 \\
2.625 \\
5.250 \\
7.875 \\
10.500 \\
10.500\end{array}$ & $\begin{array}{l}1.987 \\
1.987 \\
1.987 \\
1.987 \\
1.987\end{array}$ & $\begin{array}{r}-11.470 \\
-6.254 \\
-1.038 \\
4.178 \\
9.394\end{array}$ & -55.951 & & & $\therefore$ & \\
\hline & 2 & $\begin{array}{r}0.000 \\
0.000 \\
2.625 \\
5.250 \\
7.875 \\
10.500 \\
10.500\end{array}$ & $\begin{array}{l}-4.332 \\
-4.804 \\
-5.277 \\
-5.749 \\
-6.222\end{array}$ & $\begin{array}{r}6.434 \\
-5.557 \\
-18.788 \\
-33.259 \\
-48.971\end{array}$ & -3.363 & & & & \\
\hline & 3 & $\begin{array}{r}0.000 \\
0.000 \\
2.625 \\
5.250 \\
7.875 \\
10.500 \\
10.500\end{array}$ & $\begin{array}{l}-2.555 \\
-2.555 \\
-2.555 \\
-2.555 \\
-2.555\end{array}$ & $\begin{array}{r}4.849 \\
-1.858 \\
-8.566 \\
-15.274 \\
-21.981\end{array}$ & -1.582 & & & & \\
\hline & 4 & $\begin{array}{r}0.000 \\
0.000 \\
2.625 \\
5.250 \\
7.875 \\
10.500 \\
10.500\end{array}$ & $\begin{array}{l}-4.900 \\
-5.372 \\
-5.845 \\
-6.317 \\
-6.790\end{array}$ & $\begin{array}{r}-0.187 \\
-13.669 \\
-28.392 \\
-44.355 \\
-61.558\end{array}$ & -58.533 & & & & \\
\hline
\end{tabular}


(LANL NMSE BUILDING) 2-D VERTICAL ANALYSIS - (HNMSF3)

F R A E E L E M E N T FOR C E S

$\sin .46 ? \sigma_{j}$

\begin{tabular}{|c|c|c|c|c|c|c|c|c|c|}
\hline ELT & $\begin{array}{l}\text { LOAD } \\
\text { COMB }\end{array}$ & $\begin{array}{l}\text { DIST } \\
\text { ENDI }\end{array}$ & SHEAR & $\begin{array}{l}\text { PLANE } \\
\text { MOMENT }\end{array}$ & $\begin{array}{l}\text { AXIAL } \\
\text { FORCE }\end{array}$ & SHEAR $^{1-3}$ & PLAI & $\begin{array}{l}\text { IE } \\
\text { IOMENT }\end{array}$ & $\begin{array}{r}\text { AXIAL } \\
\text { TORQ }\end{array}$ \\
\hline & 5 & $\begin{array}{r}0.000 \\
0.000 \\
2.625 \\
5.250 \\
7.875 \\
10.500 \\
10.500\end{array}$ & $\begin{array}{l}-1.094 \\
-1.094 \\
-1.094 \\
-1.094 \\
-1.094\end{array}$ & $\begin{array}{r}-9.802 \\
-12.672 \\
-15.543 \\
-18.413 \\
-21.284\end{array}$ & -75.117 & & & & \\
\hline 16 & $--\frac{-}{1}$ & $\begin{array}{r}0.000 \\
0.000 \\
3.875 \\
7.750 \\
11.625 \\
15.500 \\
15.500\end{array}$ & $\begin{array}{l}1.572 \\
1.572 \\
1.572 \\
1.572 \\
1.572\end{array}$ & $\begin{array}{r}-10.178 \\
-4.085 \\
2.007 \\
8.100 \\
14.192\end{array}$ & -48.629 & $\begin{array}{l}\text { CALC. } \\
\text { JOR }\end{array}$ & No & $\begin{array}{c}5-000 \\
415\end{array}$ & $\begin{array}{l}\text {. REU.O } \\
: 2\end{array}$ \\
\hline & 2 & $\begin{array}{r}0.000 \\
0.000 \\
3.875 \\
7.750 \\
11.625 \\
15.500 \\
15.500\end{array}$ & $\begin{array}{l}7.154 \\
6.536 \\
5.918 \\
5.299 \\
4.681\end{array}$ & $\begin{array}{r}-61.091 \\
-34.565 \\
-10.436 \\
11.296 \\
30.632\end{array}$ & -2.363 & & & & \\
\hline & 3 & $\begin{array}{r}0.000 \\
0.000 \\
3.875 \\
7.750 \\
11.625 \\
15.500 \\
15.500\end{array}$ & $\begin{array}{l}2.656 \\
2.656 \\
2.656 \\
2.656 \\
2.656\end{array}$ & $\begin{array}{r}-26.549 \\
-16.256 \\
-5.962 \\
4.331 \\
14.624\end{array}$ & $\begin{array}{l}-1.234 \\
-1.234\end{array}$ & & & & \\
\hline & 4 & $\begin{array}{r}0.000 \\
0.000 \\
3.875 \\
7.750 \\
11.625 \\
15.500 \\
15.500\end{array}$ & $\begin{array}{r}11.383 \\
10.765 \\
10.146 \\
9.528 \\
8.909\end{array}$ & $\begin{array}{r}-97.817 \\
-54.906 \\
-14.391 \\
23.727 \\
59.448\end{array}$ & -49.133 & & & & . \\
\hline & 5 & $\begin{array}{r}0.000 \\
0.000 \\
3.875 \\
7.750 \\
11.625 \\
15.500 \\
15.500\end{array}$ & $\begin{array}{l}6.749 \\
6.749 \\
6.749 \\
6.749 \\
6.749\end{array}$ & $\begin{array}{r}-59.718 \\
-33.565 \\
-7.412 \\
18.741 \\
44.894\end{array}$ & -66.694 & & & & \\
\hline
\end{tabular}


(LANL NMSF BUILDING) 2-D VERTICAL ANALYSIS - (HNMSF3) F R A M E E L E M E N T FOR C E S

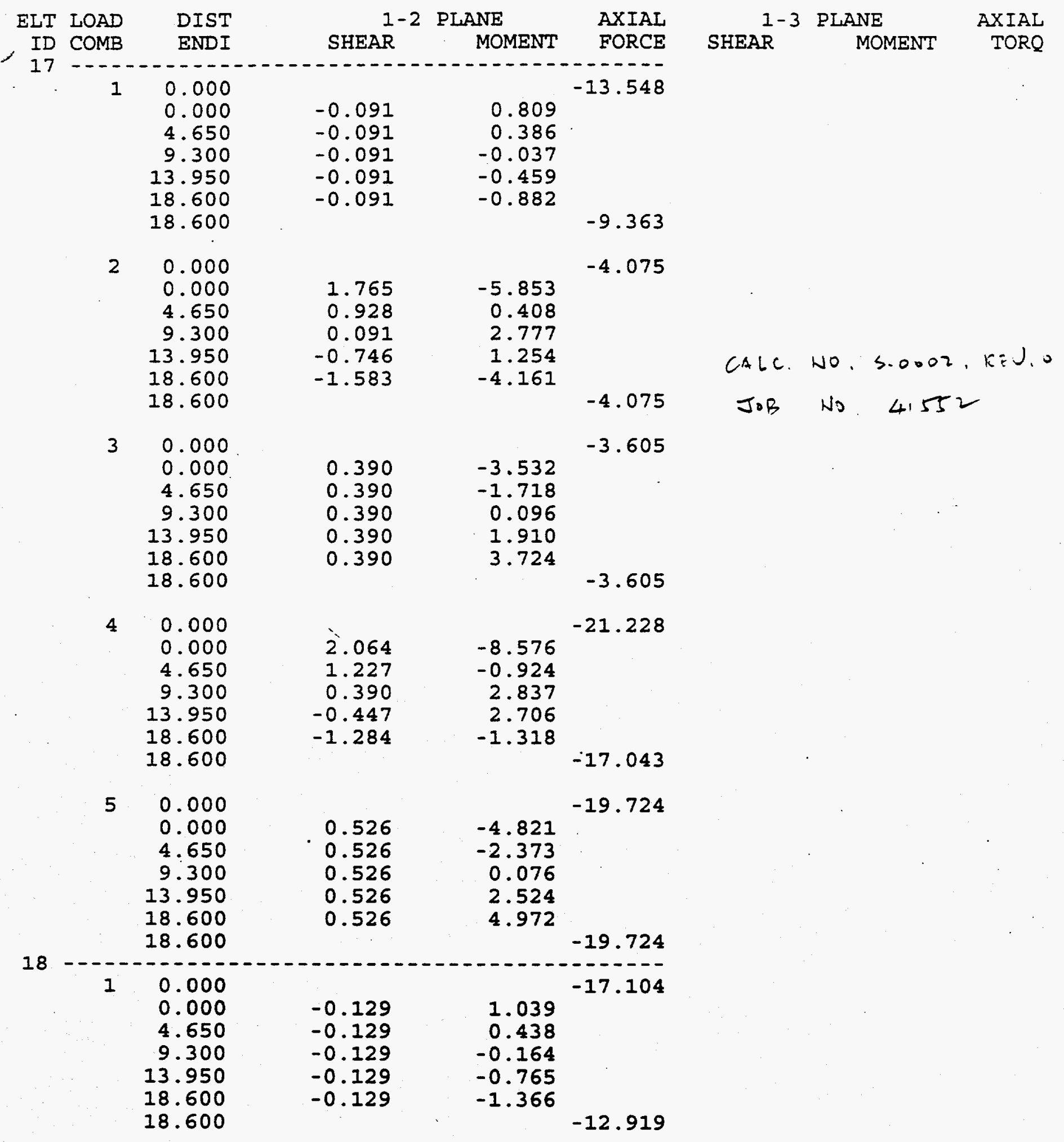




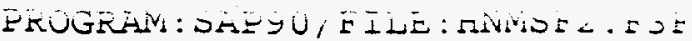

(LANL NMSF BUILDING) 2-D VERTICAL ANALYSTS - (HNMSF3)

F R A M E L E M E N T FOR C E S

$\sin 5,4690$

\begin{tabular}{|c|c|c|c|c|c|c|c|c|c|}
\hline $\begin{array}{r}\text { ELT } \\
\text { ID }\end{array}$ & $\begin{array}{l}\text { LOAD } \\
\text { COMB }\end{array}$ & $\begin{array}{l}\text { DIST } \\
\text { ENDI }\end{array}$ & SHEAR & $\begin{array}{l}\text { ANE } \\
\text { MOMENT }\end{array}$ & $\begin{array}{l}\text { AXIAL } \\
\text { FORCE }\end{array}$ & SHEAR & $-3 P I$ & $\begin{array}{l}\text { ANE } \\
\text { MOMENT }\end{array}$ & $\begin{array}{r}\text { AXIAL } \\
\text { TORQ }\end{array}$ \\
\hline & 2 & $\begin{array}{r}0.000 \\
0.000 \\
4.650 \\
9.300 \\
13.950 \\
18.600 \\
18.600\end{array}$ & $\begin{array}{r}1.754 \\
0.917 \\
0.080 \\
-0.757 \\
-1.594\end{array}$ & $\begin{array}{r}-5.723 \\
0.486 \\
2.802 \\
1.227 \\
-4.241\end{array}$ & 4.903 & & & & \\
\hline & 3 & $\begin{array}{r}0.000 \\
0.000 \\
4.650 \\
9.300 \\
13.950 \\
18.600 \\
18.600\end{array}$ & $\begin{array}{l}0.335 \\
0.335 \\
0.335 \\
0.335 \\
0.335\end{array}$ & $\begin{array}{r}-3.088 \\
-1.530 \\
0.027 \\
1.584 \\
3.142\end{array}$ & 0.152 & CALC. & No. & 5.0002 & (ai) \\
\hline & 4 & $\begin{array}{r}0.000 \\
0.000 \\
4.650 \\
9.300 \\
13.950 \\
18.600 \\
18.600\end{array}$ & $\begin{array}{r}1.959 \\
1.122 \\
0.285 \\
-0.552 \\
-1.389\end{array}$ & $\begin{array}{r}-7.772 \\
-0.607 \\
2.666 \\
2.046 \\
-2.465\end{array}$ & -7.863 & JOR & ND & 4152 & \\
\hline & 5 & $\begin{array}{r}0.000 \\
0.000 \\
4.650 \\
9.300 \\
13.950 \\
18.600 \\
18.600\end{array}$ & $\begin{array}{l}0.403 \\
0.403 \\
0.403 \\
0.403 \\
0.403\end{array}$ & $\begin{array}{r}-3.892 \\
-2.018 \\
-0.143 \\
1.731 \\
3.605\end{array}$ & -18.337 & & & & \\
\hline 19 & $\begin{array}{c}1 \\
1\end{array}$ & $\begin{array}{l}0.000 \\
0.000 \\
2.467 \\
4.933 \\
7.400 \\
9.867 \\
9.867\end{array}$ & $\begin{array}{r}-4.645 \\
-1.907 \\
0.831 \\
3.569 \\
6.307\end{array}$ & $\begin{array}{r}7.575 \\
-0.504 \\
-1.831 \\
3.597 \\
15.778\end{array}$ & 1.159 & & & & \\
\hline & 2 & $\begin{array}{l}0.000 \\
0.000 \\
2.467 \\
4.933 \\
7.400 \\
9.867 \\
9.867\end{array}$ & $\begin{array}{l}-0.625 \\
-0.625 \\
-0.625 \\
-0.625 \\
-0.625\end{array}$ & $\begin{array}{r}2.559 \\
1.018 \\
-0.522 \\
-2.063 \\
-3.604\end{array}$ & -12.563 & & & & \\
\hline & 3 & 0.000 & & & -21.918 & & & & \\
\hline
\end{tabular}


(IANL NMSF BUILDING) 2-D VERTICAL ANALYSIS - (HNMSF3)

F R A E E L E M E N T FOR C E S

\section{$\operatorname{sh} T \cdot 4705^{5} !$}

\begin{tabular}{|c|c|c|c|c|c|c|c|c|c|}
\hline $\begin{array}{r}\text { ELT } \\
\text { ID }\end{array}$ & $\begin{array}{l}\text { LOAD } \\
\text { COMB }\end{array}$ & $\begin{array}{r}\text { DIST } \\
\text { ENDI } \\
0.000 \\
2.467 \\
4.933 \\
7.400 \\
9.867 \\
9.867\end{array}$ & $\begin{array}{r}1 \\
\text { SHEAR } \\
0.250 \\
0.250 \\
0.250 \\
0.250 \\
0.250\end{array}$ & $\begin{array}{l}\text { ANE } \\
\text { MOMENT } \\
0.222 \\
0.838 \\
1.453 \\
2.069 \\
2.684\end{array}$ & -21.918 & $\begin{array}{r}1 \\
\text { SHEAR }\end{array}$ & -3 & $\begin{array}{l}\text { MOME } \\
\text { MOMENT }\end{array}$ & $\begin{array}{r}\text { AXIAL } \\
\text { TORQ }\end{array}$ \\
\hline & 4 & $\begin{array}{l}0.000 \\
0.000 \\
2.467 \\
4.933 \\
7.400 \\
9.867 \\
9.867\end{array}$ & $\begin{array}{r}-5.020 \\
-2.282 \\
0.456 \\
3.194 \\
5.932\end{array}$ & $\begin{array}{r}10.357 \\
1.351 \\
-0.900 \\
3.602 \\
14.858\end{array}$ & -33.322 & CALC. & No. & S.0002, & REU. O \\
\hline & 5 & $\begin{array}{l}0.000 \\
0.000 \\
2.467 \\
4.933 \\
7.400 \\
9.867 \\
9.867\end{array}$ & $\begin{array}{r}-6.502 \\
-2.481 \\
1.540 \\
5.560 \\
9.581\end{array}$ & $\begin{array}{r}10.229 \\
-0.850 \\
-2.010 \\
6.747 \\
25.422\end{array}$ & $\begin{array}{r}-35.579 \\
-35.579\end{array}$ & $J \cdot B$ & No & 41512 & \\
\hline 20 & $\begin{array}{r}1 \\
1\end{array}$ & $\begin{array}{l}0.000 \\
0.000 \\
2.467 \\
4.933 \\
7.400 \\
9.867 \\
9.867\end{array}$ & $\begin{array}{r}-6.612 \\
-3.874 \\
-1.136 \\
1.602 \\
4.340\end{array}$ & $\begin{array}{r}17.145 \\
4.213 \\
-1.966 \\
-1.390 \\
5.939\end{array}$ & 1.030 & & & & \\
\hline & 2 & $\begin{array}{l}0.000 \\
0.000 \\
2.467 \\
4.933 \\
7.400 \\
9.867 \\
9.867\end{array}$ & $\begin{array}{l}4.279 \\
4.279 \\
4.279 \\
4.279 \\
4.279\end{array}$ & $\begin{array}{r}0.636 \\
11.190 \\
21.744 \\
32.299 \\
42.853\end{array}$ & -14.158 & & & & . \\
\hline & 3 & $\begin{array}{r}0.000 \\
0.000 \\
2.467 \\
4.933 \\
7.400 \\
9.867 \\
9.867\end{array}$ & $\begin{array}{l}0.402 \\
0.402 \\
0.402 \\
0.402 \\
0.402\end{array}$ & $\begin{array}{r}-0.458 \\
0.534 \\
1.525 \\
2.516 \\
3.508\end{array}$ & -21.584 & & & & \\
\hline & 4 & $\begin{array}{l}0.000 \\
0.000 \\
2.467\end{array}$ & $\begin{array}{r}-1.931 \\
0.807\end{array}$ & $\begin{array}{l}17.323 \\
15.937\end{array}$ & -34.711 & & & & \\
\hline
\end{tabular}


(LANL NMSF BUILDING) 2-D VERTICAL ANALYSIS - (HNMSF3)

PROGRAM : SAP90/FILE : HNMSE2 . E3F F R A E E L E M E N T FOR C E S

\begin{tabular}{|c|c|c|c|c|c|c|c|c|c|}
\hline $\begin{array}{l}\text { ELT } \\
\text { ID }\end{array}$ & $\begin{array}{l}\text { LOAD } \\
\text { COMB }\end{array}$ & $\begin{array}{r}\text { DIST } \\
\text { ENDI } \\
4.933 \\
7.400 \\
9.867 \\
9.867\end{array}$ & $\begin{array}{l}1 \\
\text { SHEAR } \\
3.545 \\
6.283 \\
9.021\end{array}$ & $\begin{array}{l}\text { ANE } \\
\text { MOMENT } \\
21.304 \\
33.425 \\
52.299\end{array}$ & $\begin{array}{l}\text { AXIAL } \\
\text { FORCE }\end{array}$ & SHEAR & PLA & $\begin{array}{l}\text { NE } \\
\text { MOMENT }\end{array}$ & $\begin{array}{l}\text { AXIAL } \\
\text { TORQ }\end{array}$ \\
\hline & 5 & $\begin{array}{l}0.000 \\
0.000 \\
2.467 \\
4.933 \\
7.400 \\
9.867 \\
9.867\end{array}$ & $\begin{array}{r}-8.756 \\
-4.735 \\
-0.714 \\
3.306 \\
7.327\end{array}$ & $\begin{array}{r}21.816 \\
5.178 \\
-1.543 \\
1.654 \\
14.769\end{array}$ & -35.176 & & . & & \\
\hline 21 & 1 & $\begin{array}{l}0.000 \\
9.867\end{array}$ & $\cdots \cdots$ & & $\begin{array}{l}0.000 \\
0.000\end{array}$ & CALC & No & 5.0002 & REN.O \\
\hline & 2 & $\begin{array}{l}0.000 \\
9.867\end{array}$ & & & $\begin{array}{l}0.000 \\
0.000\end{array}$ & $J D B$ & Wo & 41552 & \\
\hline & 3 & $\begin{array}{l}0.000 \\
9.867\end{array}$ & & & $\begin{array}{l}0.000 \\
0.000\end{array}$ & - & & .. & \\
\hline & 4 & $\begin{array}{l}0.000 \\
9.867\end{array}$ & & & $\begin{array}{l}0.000 \\
0.000\end{array}$ & & & & \\
\hline & 5 & $\begin{array}{l}0.000 \\
9.867\end{array}$ & $\therefore$ & & $\begin{array}{l}0.000 \\
0.000\end{array}$ & & & & \\
\hline 22 & 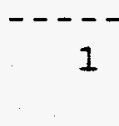 & $\begin{array}{l}0.000 \\
9.867\end{array}$ & & & $\begin{array}{l}0.000 \\
0.000\end{array}$ & & & & \\
\hline & 2 & $\begin{array}{l}0.000 \\
9.867\end{array}$ & & & $\begin{array}{l}0.000 \\
0.000\end{array}$ & & & & \\
\hline & 3 & $\begin{array}{l}0.000 \\
9.867\end{array}$ & & & $\begin{array}{l}0.000 \\
0.000\end{array}$ & & & & \\
\hline & 4 & $\begin{array}{l}0.000 \\
9.867\end{array}$ & & & $\begin{array}{l}0.000 \\
0.000\end{array}$ & & & & \\
\hline & 5 & $\begin{array}{l}0.000 \\
9.867\end{array}$ & & & $\begin{array}{l}0.000 \\
0.000\end{array}$ & & & & \\
\hline
\end{tabular}


ICF KAISER

ENEINEERS

$$
\text { CALC. S-0002.REV.O } \quad \text { JOE No. } 41552
$$

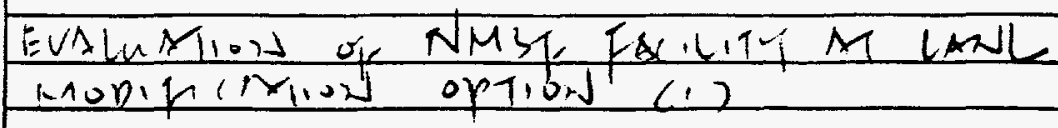

DESIGNED BY SLlus

DATEنंL-198

CHECKED BY 5

DATE5//19T

Deflectios] CALC.

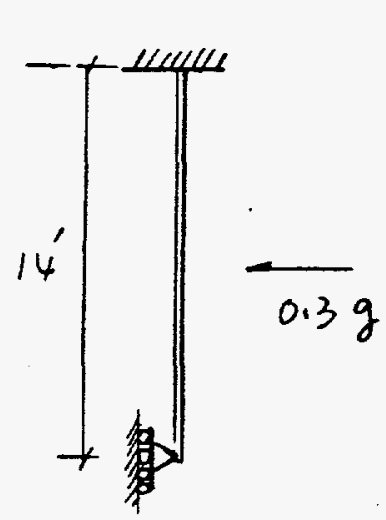

LENGTH $=14^{\prime}=168^{\prime \prime}$

Wi. of CONTENTS $=500$ (101/XL)

CRoss section

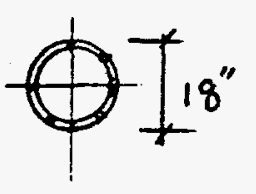

$$
\begin{aligned}
0 . D & =18^{\prime \prime}, I . D=17^{\prime \prime} \\
t & =0.5^{\prime \prime}
\end{aligned}
$$

MATERIML = STEEL $E: 29 \times 10^{6} \mathrm{ps}$.

LELT10)J PROPERTIES:

$$
\begin{aligned}
& A=\frac{\pi\left(0.0^{2}-1.0^{2}\right)}{4}=\frac{\pi\left(18^{*}-17^{*}\right)}{4}=27.49 \mathrm{n}^{2} \\
& I=\frac{\pi\left(0 . D^{4}-1 . D^{4}\right)}{64}=\frac{\pi\left(18^{*}-17^{*}\right)}{64}=1053.17 \mathrm{n}^{4} \\
& S=\frac{\pi\left(0 . D^{4}-1.0^{*}\right)}{320.0}=\frac{\pi\left(18^{4}-17^{4}\right)}{32 \times 18}=117.02 \mathrm{n}^{3}
\end{aligned}
$$

WT. of $18^{\prime \prime}$ STEL P.PE W/ $1 / 2$ " wsul TUICKNESS

$$
=93.45 \% / \mathrm{KI}=7.7875 \%
$$

SPPLIED FORCE (UNIFORMII DISTRIBUTED LOAO)

$$
w_{F}=(0.3 \times 1.5) \times\left(7.7975+\frac{500}{168^{\prime \prime}}\right)=4.8437 \%
$$


ICF KAISER

Jos No. U'JE L

EnmMind of NMA, Fariv197 C LANL

DESIGNED BY

SHEET 473 OF

-10pincmios optiod (1)

CHECKED BY

$$
\begin{aligned}
& \text { DATE }: 1 /-195 \\
& \text { DATE } / 1 / 96
\end{aligned}
$$

EOR BEAM FIXED AT ONE END, SUPPORTED AT OIUER, SURJETTED TO UNIFORALLY RSSTRIBUTES LOAD,

InE MAX. DEFlECTION OCCUREO (a)

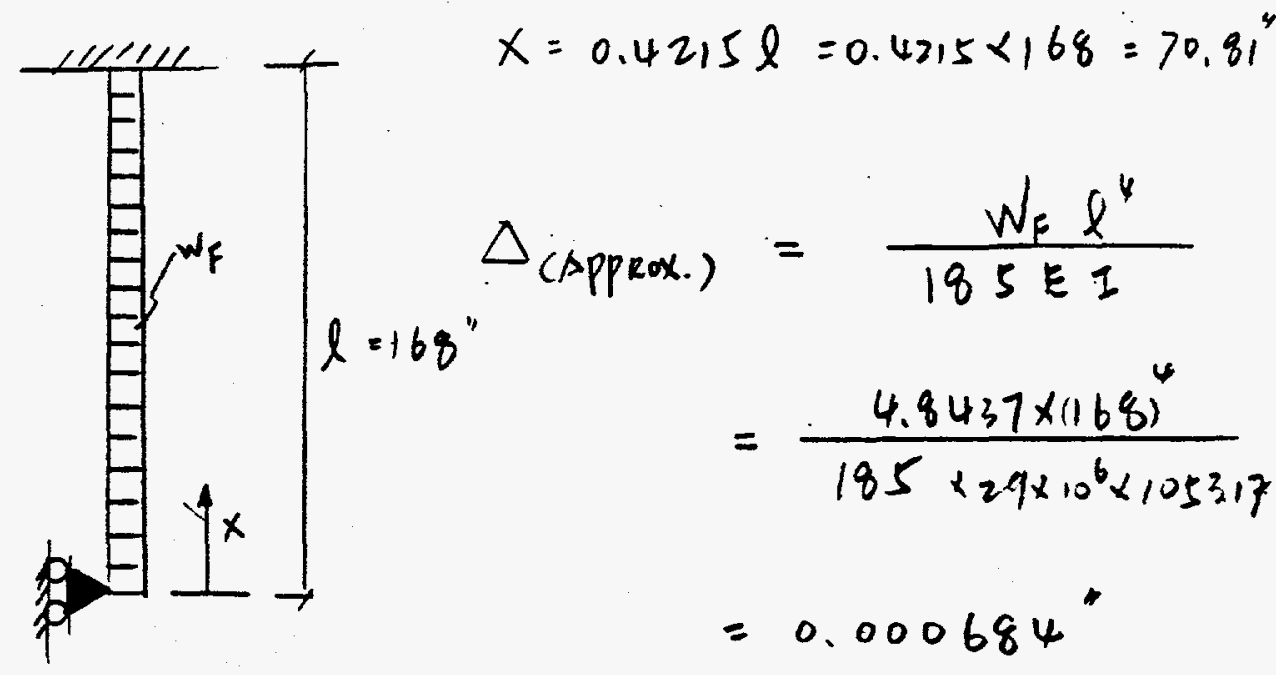

